Göttingen Series in Social and Cultural Anthropology

Wiebke Grimmig

\title{
Die Kunst zu leben
}

Die biographische Verarbeitung von Katastrophenerfahrungen und Emotionen von Künstlern im gegenwärtigen Japan

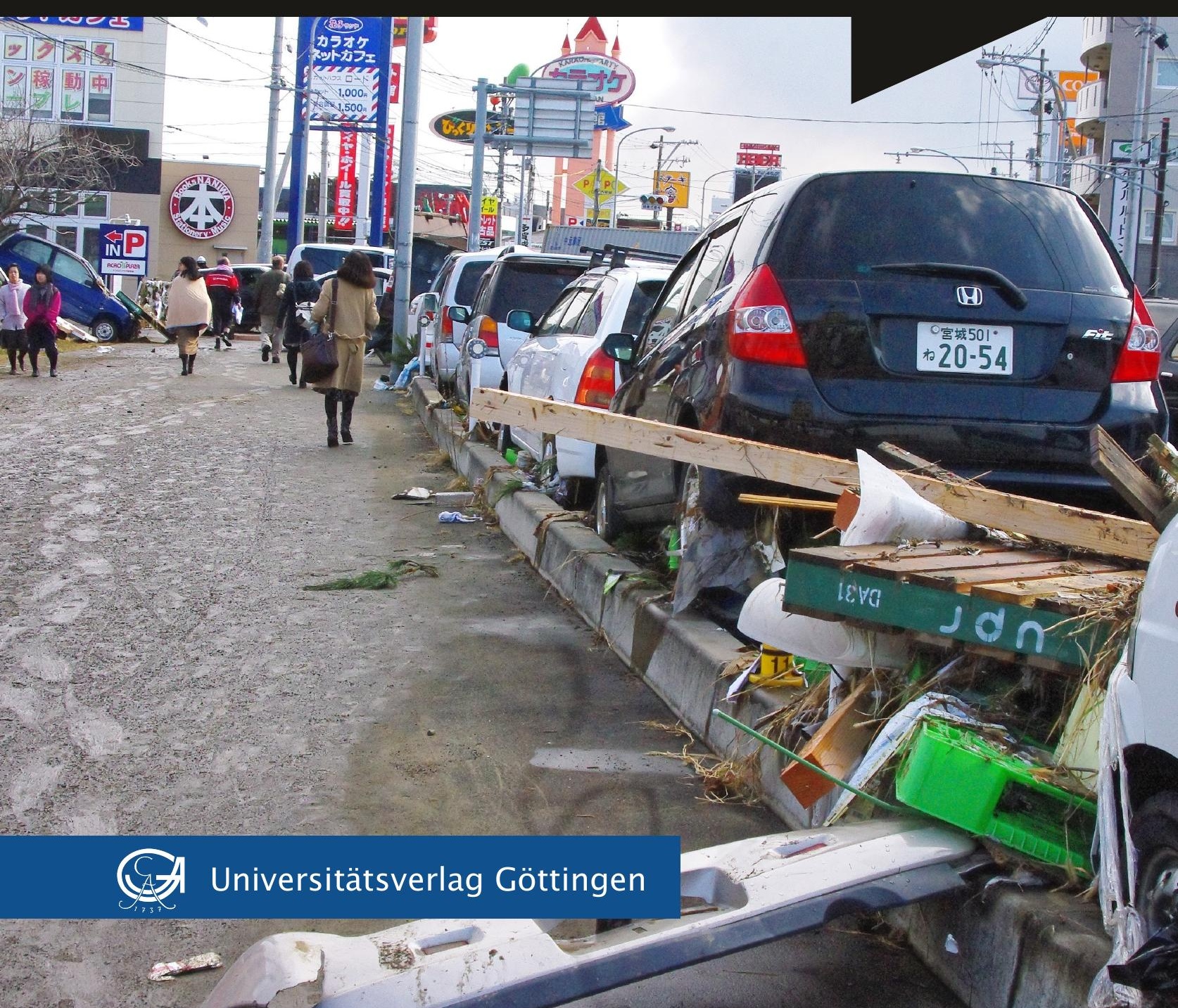



Wiebke Grimmig

Die Kunst zu leben

Dieses Werk ist lizenziert unter einer

Creative Commons

Namensnennung - Weitergabe unter gleichen Bedingungen

4.0 International Lizenz.

(c) (i) (2) 
erschienen als Band 22 in der Reihe „Göttinger Reihe zur Ethnologie Göttingen Series in Social and Cultural Anthropology“"

im Universitätsverlag Göttingen 2022

Diese Reihe ist eine Fortsetzung der „Göttinger Beiträge zur Ethnologie“ 
Wiebke Grimmig

\section{Die Kunst zu leben}

Die biographische Verarbeitung von Katastrophenerfahrungen und Emotionen von Künstlern im gegenwärtigen Japan

Band 22

Göttinger Reihe zur Ethnologie Göttingen Series in Social and Cultural Anthropology

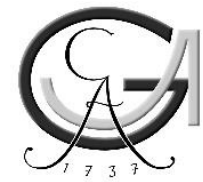

Universitätsverlag Göttingen 2022 


\title{
Bibliografische Information
}

Die Deutsche Nationalbibliothek verzeichnet diese Publikation in der Deutschen Nationalbibliografie; detaillierte bibliografische Daten sind im Internet über $<$ http://dnb.dnb.de $>$ abrufbar.

\author{
„Göttinger Reihe zur Ethnologie - Göttingen Series in Social and Cultural Anthropology" \\ herausgegeben von \\ Prof. Dr. Elfriede Hermann \\ Prof. Dr. Andrea Lauser \\ Prof. Dr. Roman Loimeier \\ Prof. Dr. Nikolaus Schareika \\ Institut für Ethnologie \\ Georg-August-Universität Göttingen \\ Theaterplatz 15 \\ D-37073 Göttingen
}

Dissertation, Georg-August-Universität Göttingen

Dieses Buch ist auch als freie Onlineversion über die Homepage des Verlags sowie über den Göttinger Universitätskatalog (GUK) bei der Niedersächsischen Staats- und Universitätsbibliothek Göttingen (https://www.sub.uni-goettingen.de) erreichbar. Es gelten die Lizenzbestimmungen der Onlineversion.

Satz und Layout: Steffen Herrmann

Coverabbildung: Earthquake Memorial Museum, Tohoku Regional Bureau, Ministry of Land, Infrastructure, Transport and Tourism.

Elektronische Ressource. <https://infraarchive311.jp/en $/$ ?view $=305418 \&$ fbclid $=$ IwAR3BVfDNDK1oSctjL2j31BBjd5umA3y-R8hspszPz0iEkEdDQOQbVC-z8s>

No. 305418: Miyagi Tagajo. Cars and rubble washed away by tsunami. Tagajo City (National Highway No. 45)

(C) 2022 Universitätsverlag Göttingen https://univerlag.uni-goettingen.de ISBN: 978-3-86395-518-2

DOI: https://doi.org/10.17875/gup2022-1861

ISSN: $2199-5346$

eISSN: 2512-6881 


\section{Inhalt}

Danksagung …................................................................................................ 9

$1 \quad$ Einleitung ............................................................................................... 11

$2 \quad$ Forschungsstand ................................................................................. 19

2.1 „Fukushima“ im internationalen Wissenschaftsdiskurs ......................... 21

2.2 Arbeiten über die Narrative zu „Fukushima“ ........................................ 25

2.2.1 Die Aushandlung der „Fukushima“-Diskurse durch Eliten ......... 27

2.2.2 Die Diskursalternative - Kulturelle Akteure in der Forschung ..... 30

2.3 Zusammenfassung ..................................................................... 35

3 Theoretische und methodische Rahmungen ........................................ 37

3.1 Katastrophen und ihre Folgen - Eine theoretische Verortung ................ 37

3.2 Diskursanalyse ........................................................................... 39

3.2.1 Der Diskurs des Selbst - Die Herstellung des Selbst im Diskurs . 43

3.2.2 Die Politik im Diskurs ............................................................. 46

3.2.3 Das Ringen um diskursive Macht .......................................... 48

3.2.4 Der Zusammenhang von Wahrheit und Biographie .................... 49

3.2.5 Zusammenfassung ………...................................................... 50

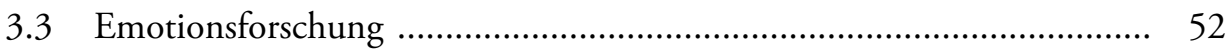

3.3.1 Der Emotionsdiskurs - Individuum und Gesellschaft ................ 53

3.3.2 Die Perspektiven der Emotionsforschung ................................... 54

3.3.3 Emotionen, das Selbst und Erfahrung - Bezüge zum
biographischen Narrativ ......................................................... 58

3.3.4 Empathie - wissenschaftliche Perspektiven auf den „social glue“ . 60

3.4 Die Erforschung von Emotionen im Feld - Methodisches Vorgehen ..... 63

3.4.1 Das narrative Interview .......................................................... 66

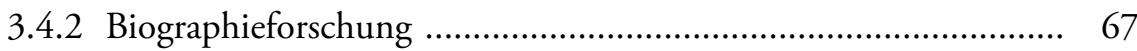

3.4.3 Biographieforschung in Japan .................................................... 69

3.4.4 Teilnehmende Beobachtung ..................................................... 71

3.5 Meine Gesprächspartner .......................................................... 72

3.5.1 Zugänge zur Kunstszene ......................................................... 73

3.5.2 Empathie als Leitlinie meiner Interaktion im Feld -

3.5.3 Die Auswahlkriterien meiner Gesprächspartner: Alter, Gender, Lokalität ................................................................................ 76 
$4 \quad$ Der sozio-kulturelle Kontext der Dreifachkatastrophe ....................... 79

4.1 Der kulturspezifische Ausdruck des Selbst im sozialen Gefüge .............. 81

4.1.1 Der Kompass der Werte - Ethik und Selbst im japanischen

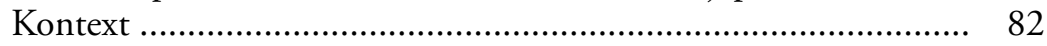

4.1.2 Das Verhältnis vom Individuum zur Gruppe ........................... 87

4.1.3 Konfliktlösungen in der sozialen Praxis ....................................... 90

4.2 Ein diskursiver Blickwinkel auf die japanische Gesellschaft ................... 95

4.2.1 Die „polarisierende Differenzgesellschaft“ - Eine Diskussion ...... 96

4.2.2 Die verstärkte Individualisierung und ihre Folgen für die soziale Mobilität .................................................................... 102

4.2.3 Eine Schwächung der sozialen Bindungen ................................ 104

5 Vertrauen und Risiko - Die vorläufige Bilanz einer gesellschaftlichen Katastrophe

5.1 Der „soziale Tsunami“ - Auswirkungen der Dreifachkatastrophe auf die japanische Bevölkerung

5.1.1 Gesundheitliche Folgen der Verstrahlung und das Ringen um die Wahrheit

5.1.2 Der mentale Stress: Die Lage der Betroffenen fünf Jahre danach . 112

5.2 Kulturelle Spezifika als Katastrophenrisiko? 116

5.2.1 Gestaltung der vertikalen Beziehungen ..................................... 117

5.2.2 Politik, Ökonomie und Verantwortung - eine Frage der Aushandlung

5.3 Die Akteure der Diskurse um den 11.03.2011 ................................... 120

5.3.1 „Das Atomdorf“ (genpatsu mura) .............................................. 121

5.3.2 Medien, die staatliche Öffentlichkeit und ihre Alternativen ........ 125

6 Kunst und Künstler in Japan - Eine Milieuanalyse ............................. 131

6.1 Was ist Kunst? ................................................................................. 132

6.2 Die Gegenwartskunst in Japan: Eine historische Einordnung ................ 134

6.3 Gegenwartskunst heute ................................................................... 136

7 Kogoro aus Tokyo - „Fukushima ist ein Röntgenbild der japanischen Gesellschaft“

7.1 Kogoro, der Künstler ........................................................................ 140

7.1.1 Die lokale Verortung der Katastrophendiskurse .......................... 141

7.1.2 Die Aufgabe der Kunst in Krisenzeiten ..................................... 142

7.1.3 Authentizität als emotionaler Anker ........................................... 147 
7.2 Eine Biographie der Emotionen ........................................................... 149

7.2.1 Isolation und Einsamkeit .......................................................... 149

7.2.2 Die emotionalen Parallelen zwischen den Generationen ............. 151

7.3 Traurigkeit und Wut: Die Verknüpfung biographischer Emotionen mit aktuellen Ereignissen ................................................................. 153

7.3.1 Empathische Identifikation: Kogoros Sicht auf Atomkraft

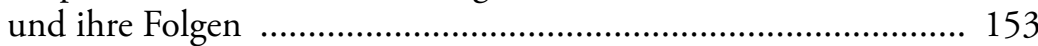

7.3.2 Identifikationspunkte von Diskriminierung und Stigmatisierung. 156

7.3.3 „Es gibt keinen Platz für mich“: Der Selbstmordversuch ............. 157

7.4 Die persönliche Bedeutung des 11. März 2011 - „I am one of the seeds of change"

8 Ayako von Shiogama - Der Tsunami im lokalen Kontext der Ostküste .... 163

8.1 Ayako, die Künstlerin ................................................................... 164

8.2 Eine lokal geprägte Lebensgeschichte .................................................. 165

8.2.1 Erinnerungen und lokale Verbundenheit ................................... 166

8.2.2 Rollenkonflikte .................................................................... 169

8.3 Biographische Hintergründe ........................................................... 171

8.4 Das Erleben des 11. März 2011 - Eine andere Realität ......................... 178

8.4.1 Direkt nach dem Tsunami - Ein neuer Alltag ............................ 178

8.4.2 Die Verarbeitung von Ohnmacht, Verlust und Trauer ................ 180

8.4.3 Die Zeit danach: Gestärktes Zugehörigkeitsgefühl und Reflexion . 182

8.4.4 Ayakos persönliche Lektionen des 11. März 2011 ....................... 184

8.4.5 Die Historisierung des Erlebten: Aus Mitleid wird Stolz ............. 187

8.5 Die Kunst als Form persönlichen Handlungsvermögens .......................... 189

9 Die biographischen Antworten der Gegenwartskünstler im Kontext des nationalen Post-Fukushima-Diskurses ....................... 193

9.1 Tsunami und Atomkraft:

Eine Gegenüberstellung der emotionalen Diskurse ................................ 195

9.2 Vor und nach 2011 - Biographische Antworten

auf gesellschaftliche Fragen ................................................................... 199

9.2.1 Identität und Zugehörigkeit ...................................................... 200

9.2.2 Sichtbarkeit von Emotionen ...................................................... 204

9.2.3 Widerstand gegen gesellschaftliche Konventionen ...................... 210

9.3 Die Ausnahme der Regel - Tasha aus Tokyo ......................................... 217 
9.4 Weitere essentielle Facetten der gesellschaftlichen Verortung ................. 221

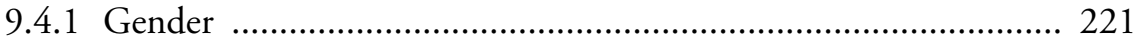

9.4.2 Prekäre Lebensumstände ......................................................... 224

10 Gegenwartskünstler als Experten der Bedeutungsherstellung ........... 229

10.1 Die Rolle des Gegenwartskünstlers in der gesellschaftlichen

Aushandlung von Emotionen ............................................................. 230

10.1.1 Künstler als Erschaffer eines sozialen Aushandlungsraumes ....... 230

10.1.2 Kreativität als eine Ressource für Empathie und Handlungsvermögen .............................................................. 233

10.2 Biographische Lösungswege für die Gesellschaft? ................................. 238

10.2.1 Japans Künstler als ethisches Korrektiv der Gesellschaft? ........... 238

10.2.2 Ist ein Künstler ein politischer Aktivist? .................................... 241

10.3 Emotionen als moralischer Kompass für eine Gesellschaft ..................... 243

10.4 Das Prinzip der narrativen Transparenz ………...................................... 246

11 Schlussfolgerungen - Die Kunst zu leben ........................................... 251

12 Literaturverzeichnis .......................................................................... 257 


\section{Danksagung}

All children are born artists. The problem is to stay one as we grow up.

(Picasso)

Ich habe die Möglichkeit, diese Danksagung zu schreiben, weil Menschen aus ganz unterschiedlichen Bereichen meines Lebens mich unterstützt und mich ermutigt haben. Ohne diese Menschen wäre dieses Buch nie entstanden. Ich möchte den Beteiligten aber nicht nur für ihre konstante und geduldige Unterstützung danken, sondern vor allem für den Raum, den sie mir gaben. Den Raum für Fehler, neue Gedanken und eine gewisse Entschleunigung, die manche dieser Gedanken brauchten, um richtig reifen zu können.

Meiner Erstbetreuerin Prof. Dr. Elfriede Hermann, die mich in zahlreichen Sprechstunden, Kolloquien, Stipendiumsanträgen und Thesis-Committee-Sitzungen von Beginn an in diesem Projekt unterstützt und angeleitet hat. Herzlichen Dank für diese enge Betreuung. Prof. Dr. Katja Triplett, die mir nicht nur mit ihrer Expertise, wichtigen Literaturhinweisen und Ratschlägen, sondern auch mit ihrer Unterstützung bei der Bewerbung für ein Stipendium des Deutschen Instituts für Japanstudien in Tokyo unter die Arme gegriffen hat. Mit ihrer Hilfe bekam ich bereits im Vorfeld der Forschungsphase wichtige Hinweise, die mir einen guten Start in das Dissertationsprojekt ermöglichten. Prof. Dr. Gerrit Jasper Schenk, der mich vor, während und nach der Forschungs- und Schreibphase ermutigt und mit wichtigen Hinweisen und Perspektiven aus der Katastrophenforschung versorgt hat.

Für die finanzielle und fachliche Unterstützung danke ich dem Deutschen Institut für Japanstudien in Tokyo (DIJ), das mir für meine einjährige Forschung von 
2014 bis 2015 ein Stipendium gewährt hat. Dank dieser finanziellen Unterstützung war es mir überhaupt erst möglich, mein Dissertationsvorhaben in dieser Weise durchzuführen.

Ich möchte meine, MitstreiterInnen' nicht vergessen, die so viele Versionen meiner Kapitel gelesen und ihre Perspektive beigesteuert haben: Carolin Landgraf, Isabel van Manen, Julia Racz, Anna Wiemann, Antonie Fuhse und Serena Müller. Danke auch an Paul Christensen und Carolin Landgraf für eure moralische und technische Unterstützung am Tag meiner Disputation. Für die Korrekturen und den Blick fürs Detail danke ich Anna Wiemann und Steffen Herrmann.

So viele aufmunternde Worte bekam ich auf den Fluren, beim Kaffee oder einer gemeinsamen Mittagspause von Jelka Günther, Julia Vorhölter, Christina Schneider, Christiane Goos, Maike Lange, Arite Heuck-Richter und Philipp Kleinert. Danke, dass ihr für mich da ward. Ebenso gilt mein Dank Michael Bonn-Gerdes für seine Flexibilität und sein Verständnis, insbesondere in den letzten Schreibphasen.

Meiner Familie, meinen Eltern und meiner Schwester, danke ich für ihr Verständnis und ihre Geduld, gerade in Zeiten des Zweifels.

Besondere Rückendeckung und Stärkung bekam ich von Katja Winkler. Danke für die innige Verbundenheit, die meinen Weg seit unserem Zusammentreffen prägt. Du bist eine der Personen, die mich dabei unterstützen, mein Leben neben der Promotion aktiv zu gestalten und mutig meinen Weg zu gehen.

Zum Schluss geht mein besonderer Dank an die vielen KünstlerInnen in Japan, die mir so bereitwillig die Tür zu ihrem Leben, ihrer Expertise und ihren Erfahrungen geöffnet haben. Ihr seid das Herz dieser Arbeit.

Danke. 


\section{Einleitung}

Der 11. März 2011, 14:46 Uhr in Tokyo. 370 km nordöstlich vor Japans Ostküste erschüttert ein Beben mit der Stärke von 9,0 auf der Richterskala den Meeresboden. Diese ungeheure Kraft entsteht, als sich der Druck zwischen der pazifischen und nordamerikanischen Landplatte entlädt. Der in Folge des Bebens ausgelöste Tsunami trifft auf die Ostküste Japans und damit auch auf das Atomkraftwerk „Fukushima Daiichi“. Das eindringende Wasser unterbricht die Stromversorgung zur Steuerung des Reaktors und die atomare Kettenreaktion kann nicht mehr kontrolliert werden. Die so entstandene Kernschmelze mit anschließender Wasserstoffexplosion entlässt radioaktive Bestandteile wie Cäsium und Uran in die unmittelbare Umgebung und verstrablt weite Teile der Präfektur Fukushima und der an sie angrenzenden Gebiete. Die Entladung der Naturgewalten in Form des Erdbebens und des dadurch ausgelösten Tsunamis trifft am 11. März 2011 auf die Technologie der Atomkraft, die dieser Kraft nicht standhalten kann.

Wichtige Entscheidungen, die Jahrzehnte zuvor beim Bau des Kraftwerks Fukushima Daiichi gefällt wurden, stehen seit Bekanntwerden massiver konzeptioneller Mängel in der öffentlichen Kritik. Diese Mängel betreffen sowohl den konkreten Standort als auch die notwendigen Sicherheitsmaßnahmen bezüglich eines möglichen Tsunamis. Die Folgen des Erdbebens, des Tsunamis und der atomaren Verstrahlung (auch als „Dreifachkatastrophe“ bezeichnet) werden in den japanischen Diskursen folglich in zwei Kategorien unterteilt: Die Naturkatastrophen (jap.: tensai), in der Erdbeben 
und Tsunami zusammengefasst werden, und die menschengemachte Katastrophe (jap.: jinsai). ${ }^{1}$ Es gibt Vorschläge, die Atomkatastrophe umzubenennen. Statt wie bisher den Namen „Fukushima“, der sowohl der Name der Präfektur, der Stadt und des AKWs ist, beizubehalten und damit zur Stigmatisierung der Anwohner beizutragen, sollen die Verursacher in den Fokus gestellt werden. Daher schlägt Dr. Martin Repp vom Zentrum Oekumene in Frankfurt den Namen „TEPCO-Katastrophe“ vor (siehe Repp 2015). Dieser Vorschlag hat sich meines Wissens nicht durchgesetzt. Aus diesem Grund bleibe ich bei der Bezeichnung „Fukushima“, ohne damit eine Wertung vornehmen zu wollen.

Als Ethnologin interessiere ich mich insbesondere für die Bedeutungsherstellung und die Interpretationen des Alltags in der Folgezeit der Dreifachkatastrophe und die Konsequenzen für die gesamtgesellschaftlichen japanischen Diskurse, die sich daraus ergaben. Katastrophenereignisse wie die am 11. März 2011 sind im Allgemeinen von großem erkenntnistheoretischem Wert, da sie die sozialen Auswirkungen von und den Umgang mit schrecklichen Ereignissen in ihrer Kulturspezifik beobachtbar und analysierbar machen (siehe Oliver-Smith und Hoffman 2002; Kimura 2012). Darüber hinaus gewähren die durch Katastrophen ausgelösten Erschütterungen der Gesellschaft Japans einmalige Einblicke sowohl in die Struktur des sozialen Gefüges als auch in die Konstruktionsprozesse von Vergangenheit, Gegenwart und Zukunft.

Aufgrund meiner früheren Arbeit im Bereich der traditionellen japanischen Künste (siehe Grimmig 2012) erschien mir ein Zugang zur Kunst als Form der Auseinandersetzung mit den Ereignissen und ihren Folgen als besonders gewinnbringend. Erste Vorgespräche mit Gegenwartskünstlern ${ }^{2}$ und das Studium entsprechender, vorwiegend deutscher und englischer Literatur im Jahr 2013 sowie der Rat meiner Zweitbetreuerin Frau Prof. Dr. Katja Triplett bestätigten diesen Eindruck. Die bereits in den Gesprächen dieser frühen Forschungsphase zutage getretenen Emotionen wie Wut und Trauer veranlassten mich dazu, den Fokus der Arbeit auf den emotionalen Ausdruck der Künstler zu legen.

Die 12-monatige Feldforschungsphase in den Jahren 2014 und 2015 habe ich entsprechend dieser ersten Rechercheergebnisse gestaltet. So war es mir wichtig, die Erhebungsmethodik so auszuwählen, dass den Künstlern viele Möglichkeiten blie-

\footnotetext{
1 Zur genaueren Ausdifferenzierung des Begriffs „Naturkatastrophe“ siehe Felgentreff und Glade (2008). Die Autoren vertreten die Auffassung, dass der Begriff der Katastrophe eine zutiefst menschliche Kategorie ist, also auch im Fall einer Naturkatastrophe die menschliche Verantwortung eine Rolle spielt. Daher empfehlen sie den Begriff „Sozialkatastrophe“ zu verwenden (2008: 3). Obwohl ich diese Argumentation nachvollziehen kann, verwende ich aufgrund der Nähe zum japanischen Diskurs weiterhin die oben genannten Kategorien. Ein Nachhall des Vorschlags von Felgentreff und Glade findet sich in der Titelgebung des Kapitels 5.1 „Der ,soziale Tsunami - Auswirkungen der Dreifachkatastrophe auf die japanische Bevölkerung".

2 Aus Gründen der Vereinfachung verzichte ich auf die gegenderte Schreibweise der Bezeichnung, die sich jedoch stets auf männliche und weibliche Gesprächspartner bezieht. Des Weiteren verwende ich im Folgenden auch den Begriff „Künstler“, mit dem ausschließlich, falls nicht anders gekennzeichnet, Gegenwartskünstler gemeint sind (siehe Kapitel 6 „Kunst und Künstler in Japan - Eine Milieuanalyse“).
} 
ben, Verlauf und Inhalt der Gespräche selbst zu bestimmen. Es stellte sich zudem im Laufe der ersten Recherche heraus, dass Werke des einzelnen Künstlers in signifikanter Weise mit biographischen Erfahrungen verknüpft sind, was eine entsprechende Erhebung lebensgeschichtlicher Daten notwendig machte. Ich wählte eine narrative Interviewform, die es meinen Gesprächspartnern erlaubte, ihre eigenen Themenschwerpunkte zu setzen. Es ist dieser offenen Gestaltung der Interviews geschuldet, dass ich nicht nur relevante biographische Daten und emotionale Erfahrungsbeschreibungen in Bezug auf die Katastrophe sammeln konnte, sondern auch unvorhersehbare Facetten zum Verständnis der Selbstpositionierung der jeweiligen Personen im japanischen Sozialgefüge finden konnte. Des Weiteren bieten die erhobenen Narrative die Möglichkeit, die Schnittstellen von Individuum und Gesellschaft zu beleuchten (siehe Fischer-Rosenthal 2000) und den Konstruktionsprozess eines Selbst in einer konkreten Forschungssituation zu analysieren.

Die Gespräche fanden teilweise auf Englisch, teilweise in japanischer Sprache statt. Im letzteren Fall erhielt ich Unterstützung durch einen Dolmetscher, der ins Englische übersetzte. Satoshi, wie meine Gesprächspartner als Künstler tätig, begleitete mich streckenweise während meines Forschungsaufenthalts und war mir über seine Übersetzungsdienste hinaus ein wichtiger Diskussionspartner bei der Erörterung der geschilderten Eindrücke und Erfahrungen. Er war mir in Tokyo von einer Bekannten empfohlen worden und nach einer kurzen Erläuterung meines Vorhabens konnte ich ihn für das Projekt gewinnen. Als vielfältig interessierte Persönlichkeit bewegte sich Satoshi an der Schnittstelle zwischen persönlichem Interesse, beruflicher Neugier und einer distanzierteren Rolle als Übersetzer. Meine gemeinsam mit ihm vollzogene Reflexion über diese Rollenwechsel war besonders fruchtbar und schärfte meinen Blick für die unterschiedlichen Handlungsebenen der verschiedenen Gesprächspartner innerhalb und außerhalb der Interviewsituation.

Die Vorteile eines Dolmetschers aus der Kunstszene zeigten sich bereits beim ersten gemeinsamen Interviewtermin, bei dem meine vorrangigen Identifikationsmerkmale als ausländische Forscherin in den Hintergrund treten konnten und damit einen ungezwungenen Gesprächsfluss ermöglichten. Wie von mir erhofft, verschwanden die Hemmungen des Gesprächspartners, sich gegenüber einer gesellschaftsfremden Person artikulieren zu müssen, durch Satoshis Anwesenheit fast völlig und es entstand eine unverkrampfte Atmosphäre. Satoshis positiver Einfluss auf den Verlauf der Erhebungsphase, seine Expertise als Kenner der Szene und unsere gemeinsamen Reflexionen zu den Interviewinhalten sind für die vorliegende Analyse von großem Wert.

Für Tokyo als Ausgangsbasis der Forschung entschied ich mich aufgrund der genannten ersten Rechercheergebnisse. Die Hauptstadt Japans erlaubte es mir, Künstler vieler verschiedener Genres anzutreffen und in ihrem Alltag zu begleiten. Zudem liegt Tokyo circa 270 Kilometer vom Katastrophengebiet an Japans Ostküste entfernt und ist der Hauptverbraucher des in Fukushima erzeugten Stroms. Die geographische wie emotional-moralische Verbundenheit Tokyos mit den Ereignissen vom 11. März 2011 machte die Stadt für mich als Forschungsbasis attraktiv. 
Um jedoch das emotionale Spektrum der Dreifachkatastrophe erfassen zu können, entschied ich mich dafür, meinen Zugang durch multilokale Einblicke zu erweitern. Zum einen wollte ich die Stimmungen, Erfahrungen und Meinungen der Anwohner und Künstler in den unmittelbar betroffenen Gebieten einbeziehen und bei der Auswahl meiner Gesprächspartner berücksichtigen. Zum anderen wollte ich die Erfahrungen meiner Tokyoter Gesprächspartner, sofern sie die betroffenen Gebiete selbst im Rahmen ihrer Recherche besucht hatten, nachvollziehen können. Mehrfache Reisen in verschiedene Orte entlang der Ostküste waren daher ebenso Bestandteil der Forschungsmonate wie Besuche relevanter Gebiete zum Verständnis der atomaren Vergangenheit Japans wie beispielsweise Hiroshima. Dabei erweiterten Gespräche mit Künstlern in Ausstellungen und Galerien mein Netzwerk aus Gesprächspartnern beständig um weitere Zugänge und Forschungsfacetten. So konnte ich das alltägliche Handeln meiner Gesprächspartner teilnehmend beobachten und wichtige Rückfragen zum Verständnis ihrer Motivationen und konkreten Vorgehensweise stellen.

Dabei wurde schnell deutlich, dass sich ein wesentlicher Bestandteil der Arbeit meiner Gesprächspartner auf emotionaler Ebene vollzog. Indem die Künstler zum Großteil selbst in den Krisengebieten der Dreifachkatastrophe gewesen waren beziehungsweise zu Recherchezwecken immer wieder dorthin reisten, erlebten und verfolgten sie die Schicksale der unmittelbar Betroffenen direkt. Mir wurde klar, dass sich die Verarbeitung ihrer emotionalen Erfahrungen in diesem Zusammenhang sowohl auf ihre künstlerischen Tätigkeiten als auch auf ihre Repräsentation ihrer selbst in den Interviewsituationen mit mir auswirkte. Die Fähigkeit meiner Gesprächspartner, sowohl die Emotionen der unmittelbar Betroffenen als auch ihre eigenen in ihre künstlerische Tätigkeit zu integrieren, schien eine entscheidende Rolle zu spielen. Mit anderen Worten: Die Fähigkeit zur Empathie war von essentieller Bedeutung bei der Analyse ihrer persönlichen Motivationen, Selbstkonstruktionen und gesellschaftlichen Anliegen. Damit war auch eine Betonung von Authentizität verbunden, die für meine Gesprächspartner sowohl für ihr Selbstbild von Bedeutung war als auch für ihre Rollenzuschreibung innerhalb der japanischen Gesellschaft. Darauf komme ich später erneut zu sprechen.

Vor dem Hintergrund der Dreifachkatastrophe und unter Berücksichtigung des biographisch-narrativen Ansatzes ergibt sich folgende zentrale Forschungsfrage: Welche emotionalen Kopplungseffekte mit den durch die Katastrophe aufgeworfenen gesellschaftlichen Themen werden in den Lebensgeschichten der einzelnen Gesprächspartner deutlich?

Um diese Frage beantworten zu können, ist es zielführend, folgende sich daran anschließende Aspekte genauer zu betrachten, die sich einerseits an der biographischen Konstruktion des Selbst des jeweiligen Akteurs orientieren und die andererseits die Einflechtung der Ereignisse vom 11. März 2011 in die biographische Repräsentation hervorheben: Welche Thematiken werden von den Interviewten als prägend für ihr jeweiliges biographisches Narrativ beurteilt? Welche Ereignisse werden in diesem Reflexionsprozess als Zäsur empfunden und wie wird dies begründet? Und inwiefern ergeben sich daraus entscheidende Rahmungen für ihr Selbstverständnis als Gegen- 
wartskünstler, insbesondere nach dem 11. März 2011? Inwiefern leisten sie einen Beitrag zur emotionalen Verarbeitung der Katastrophe auf gesellschaftlicher Ebene?

Auf dieser empirischen Grundlage basiert die These für die vorliegende Arbeit: Die Gegenwartskünstler Japans stellen der japanischen Gesellschaft ihre biographischen Lösungswege zur Verfügung, indem sie durch ihre Werke einen öffentlichen Raum zur Auseinandersetzung mit ambivalenten Themen generieren. Ich betrachte Gegenwartskünstler als eine Akteursgruppe, die die dominanten Narrative hinterfragt und weitere Perspektiven auf die katastrophalen Ereignisse und ihre Folgen bereitstellt.

Das Ziel meiner Arbeit ist es, zur Schließung einer sozialwissenschaftlichen Lücke in der Untersuchung der psychosozialen Auswirkungen atomarer Verstrahlung beizutragen. Indem ich weitere narrative Facetten japanischer Diskurse rund um die Dreifachkatastrophe auf der Basis von 20 Interviews analysiere, erörtere ich die hier aufgeworfenen Fragen mit Blick auf meine Gesprächspartner. Dabei beziehe ich mich auf die von Samuels (2013) und anderen erbrachten gesellschaftspolitischen und soziologischen Beiträge zur Dreifachkatastrophe vom 11. März 2011, die sich jedoch fast ausschließlich mit der strukturellen Ebene beschäftigen. Indem ich mich der Verarbeitung der Dreifachkatastrophe mithilfe von Ansätzen aus der Emotionsforschung nähere, kann ich weitere gesellschaftspolitische Prozesse abbilden, mit denen laut Stephens (2002) alle beteiligten Akteure aus Politik, Wirtschaft und Gesellschaft zu kämpfen haben. Dabei erläutere ich sowohl die Diskursführungen und deren Verlauf als auch die für die Diskussionen wichtigen Begrifflichkeiten vor ihrem kulturspezifischen Hintergrund und beziehe wichtige Facetten gesellschaftlicher Aushandlung wie ethische Vorstellungen ein (vgl. Lambek 2010).

In dieser Arbeit verwende ich überwiegend das ethnographische Präsens. Die Vergangenheitsform verwende ich davon abweichend an Stellen, an denen ich aus der Perspektive des Erscheinungsjahres 2021 auf die einzelnen Schritte des Forschungsprozesses blicke oder zur zeitlichen Kontextualisierung der Aussagen meiner Gesprächspartner.

Die Motive für die subversiven Tätigkeiten einzelner kultureller Akteure gewähren einen erweiterten perspektivischen Blick auf die gerade stattfindenden Abläufe der Diskursgenerierung in Bezug auf 2011 und eröffnen die Möglichkeit einer aktiven Auseinandersetzung mit innerjapanischen Wahrnehmungen. Dies ist zum Zeitpunkt der Endredaktion des vorliegenden Textes in der Literatur weitestgehend unbeachtet geblieben.

Vor dem Hintergrund meiner Erkenntnisse wird deutlich, was ich als „Prinzip der narrativen Transparenz" bezeichne. Ich meine damit eine Form der Emotionsarbeit, durch die meine Gesprächspartner ihre persönlichen biographischen Erfahrungen einer Öffentlichkeit zur Verfügung stellen und für die Auseinandersetzung mit ambivalenten gesellschaftlichen Thematiken nutzen. Dies stellt besonders im japanischen Kontext eine Besonderheit dar. Meine Gesprächspartner finden sowohl im direkten Dialog mit ihren Rezipienten als auch im übertragenen Sinne mithilfe ihrer Werke einen Ausdruck für Emotionen, der bei den Rezipienten eine unmittel- 
bare Resonanz auslöst. Auf diese Weise machen meine Gesprächspartner Emotionen als wichtigen Bestandteil der Katastrophendiskurse sichtbar und versinnbildlichen darüber hinaus deren Vielfalt und Komplexität.

Entsprechend dieser Überlegungen habe ich die Struktur der vorliegenden Arbeit aufgebaut: In Kapitel 2 beschäftige ich mich zunächst mit dem derzeitigen Stand der Forschung in Bezug auf die Dreifachkatastrophe. Ich betrachte darin insbesondere die Atomkatastrophe „Fukushima“ als wissenschaftlich diskutierte Thematik und bette sie in einen internationalen Vergleichskontext ein. Diese Rahmung auf Basis der vorliegenden Literatur führt mich anschließend in Kapitel 3 zu einer Diskussion relevanter Theorien, die sich sowohl mit dem Begriff des Selbst als auch mit der Beeinflussung desselben durch den vorherrschenden Diskurs beschäftigen. Darauf aufbauend stelle ich wichtige Ansätze der Emotionsforschung (Lutz und Abu-Lughod 1990; Rosaldo 1984; Hermann 2011; Röttger-Rössler 2004) vor. Emotionen lege ich im Hinblick auf ihre verbindende Wirkung von Individuum und Gesellschaft, ihrer Rolle bei der Aushandlung des Selbst und ihrer impulsgebenden Wirkung bei gesellschaftlichen Gestaltungsprozessen dar. Die Verknüpfung von Emotionen und Selbst mit dem Diskursbegriff bildet die verständnistheoretische Basis der vorliegenden Arbeit, in der deutlich wird, inwiefern diese konzeptionelle Basis auch ethisch-moralische Vorstellungen tangiert (Geertz 1968; Collier und Lakoff 2005). Vor diesem Hintergrund gehe ich genauer auf die methodischen Zugänge zum Feld ein, indem ich die verwendeten ethnologischen Forschungsansätze umreiße und für den japanischen Kontext aufbereite. Die Vorstellung meiner Gesprächspartner schließt sich daran an und rundet einen ersten Überblick über die für die Thematik relevanten Überlegungen und Erkenntnisse ab.

In Kapitel 4 führe ich in den konkreten gesellschaftlichen Kontext der Dreifachkatastrophe mit seinen kulturellen Spezifika ein und nehme essentielle Konzepte wie Wertvorstellungen, Ethik und Selbstverortung im japanischen Sozialgefüge auf. Mit der Erörterung der Frage, inwieweit sich die kulturspezifische Konstruktion des Selbst wiederum entscheidend auf die emotionale Bewertung von Erfahrungen auswirkt (Kitayama und Markus 1994; Hardcastle 2008), schließe ich diesen Teil der Arbeit ab.

Im sich anschließenden 5. Kapitel setze ich mich mit den konkreten Folgen der Dreifachkatastrophe vom 11. März 2011 im gesellschaftlichen Kontext auseinander. Dabei spreche ich die gesundheitlichen wie mentalen Belastungen für die Bevölkerung ebenso an wie die aktuellen Diskurse zur Beteiligung unterschiedlicher politisch-wirtschaftlicher Akteure an den weichenstellenden Entscheidungen der Atompolitik. Im Zuge einer Aufarbeitung des historischen Entscheidungsfindungsprozesses seit den 1970er-Jahren, der zur Etablierung der atomaren Stromerzeugung in Japan geführt hat, mache ich die aktuellen japanischen Diskurse und Meinungsäußerungen in ihrer Komplexität verständlich.

Nachdem ich die Hintergründe und Diskurse zur Dreifachkatastrophe in dieser Weise aufgeschlüsselt habe, nähere ich mich in Kapitel 6 dem Milieu der Gegenwartskünstler, indem ich ihre Rolle in ihren Spezifika aufzeige. Vor dem Hintergrund 
gesellschaftlicher und diskursiver Rahmungen der Rolle eines Gegenwartskünstlers steige ich in die Analysekapitel 7 und 8 ein, in denen ich die biographischen Narrative zweier Gesprächspartner im Hinblick auf die genannten Forschungsschwerpunkte untersuche. Die beiden Akteure Kogoro und Ayako habe ich aufgrund einiger Besonderheiten für die Tiefenanalyse ausgewählt. Sie ergänzen sich in Hinblick auf die Facetten der Verarbeitung der Dreifachkatastrophe und ermöglichen die Betrachtung der sozialen Folgen aus unterschiedlichen Perspektiven. Kogoros Fokus auf die Folgen des atomaren GAUs für die Anwohner berührt diejenigen Bereiche des Katastrophendiskurses in Japan, der zusätzlich zur Katastrophe die Frage der Opfer- und Täterschaft verhandelt. Ayako weiß als Anwohnerin eines Ortes an der Ostküste von der unmittelbaren Zerstörungskraft des Erdbebens und des Tsunamis zu berichten. Im Erleiden von persönlichen Verlusten bewegt sie sich in einem stark lokal verankerten Aushandlungsprozess mit ihrer unmittelbaren Nachbarschaft.

Neben den genannten lokalen Zugehörigkeiten bilden Gender, Alter, Klasse und die Verschiedenartigkeit der Arbeitsfoki wichtige Differenzierungsmerkmale in der von mir untersuchten Akteursgruppe.

Eine Zusammenfassung der ergänzenden Facetten zu den zwei Hauptanalysen von Ayako und Kogoro bilde ich in Kapitel 9 ab. In der Beschäftigung mit den biographischen Narrativen weiterer 18 Gesprächspartner zeige ich, auf welche Weise Gegenwartskünstler mithilfe ihrer Werke die gesellschaftliche Verarbeitung der Ereignisse beeinflussen und wie ihre persönlichen Erfahrungen in ihre Arbeit einfließen.

Die bei ihrer Arbeit zutage tretende Emotionsarbeit auf persönlicher wie kollektiver Ebene bereite ich in Kapitel 10 auf. In diesem Teil der Arbeit fließen alle rollenspezifischen Facetten des Gegenwartskünstlers zu einem Gesamtbild zusammen, das aufzeigt, wie Emotionen, Katastrophe und Rollenzuschreibung in der japanischen Gesellschaft das Selbstverständnis eines Gegenwartskünstlers prägen und inwiefern deren Beiträge zum aktuellen Katastrophendiskurs Bezüge zu ihren jeweiligen Biographien aufweisen.

Das Abschlusskapitel gibt schließlich eine zusammenfassende Antwort auf die eingangs gestellte Frage nach der emotionalen Kopplung der Katastrophe mit den persönlichen Lebensgeschichten meiner Gesprächspartner. Die vorliegende Studie ist also eine sowohl historische wie auch kulturspezifische und in diverse kulturtheoretische Perspektiven eingebettete Analyse, in der ich alle Themen der aktuellen Diskurse, die von meinen Gesprächspartnern als entscheidend betrachtet wurden, aufgegriffen habe.

Der Titel Die Kunst zu leben bezieht sich folglich nicht nur auf die Ereignisse vom 11. März 2011 und den Umgang mit den Auswirkungen der Dreifachkatastrophe, sondern nimmt auch Bezug zur Lebensweise der Gegenwartskünstler innerhalb ihrer sozialen Rahmung. Ihr Wirklichkeitsverständnis wirft ein Schlaglicht auf Japan im neuen Alltag der Post-Fukushima-Diskurse. 



\section{Forschungsstand}

Die große wissenschaftliche Herausforderung bei der Untersuchung der Auswirkungen von Katastrophen besteht in ihrer ungeheuren Komplexität. Am 11. März 2011 wurden durch das große Erdbeben und den Tsunami weite Teile der Ostküste Japans zerstört, doch dieses lokale und zeitlich überschaubare Desaster wird durch den andauernden und ungewissen Verlauf des atomaren Super-GAUs des Kraftwerks Fukushima Daiichi verschärft. Lokale wie internationale Diskurse drehen sich seither um die Fragen nach den gesundheitlichen Langzeitfolgen, Wiederaufbau- und Besiedelungsmaßnahmen und um die Suche nach Antworten bezüglich der Verantwortung einzelner Akteure und Institutionen. In diesem Kapitel stelle ich jene wissenschaftlichen Zugänge vor, die wesentlich zum Verständnis der diskursiven Auseinandersetzung mit Katastrophen im Allgemeinen und mit technologischinduzierten Desastern beitragen.

In der Katastrophenforschung werden desaströse Ereignisse spätestens seit den 1970er-Jahren nicht nur unter den Vorzeichen der Adaption der lokalen Bevölkerung an die Umwelt oder unter dem Aspekt der Bedeutungsherstellung betrachtet. Auch die sozio-kulturellen Voraussetzungen von Risiko- und Verwundbarkeitsverteilung einzelner Bevölkerungsgruppen stehen zunehmend im Fokus der Untersuchungen (siehe Kimura 2012; Oliver-Smith 2002). Dabei wird in Betracht gezogen, inwiefern einzelne Bevölkerungsanteile, wie beispielsweise ältere Menschen, ein stärkeres Risiko tragen, an den Folgen von Katastrophen zu leiden. 
In diesem Sinne verorte ich die vorliegende Arbeit im Kontext der ethnologischen Katastrophenforschung und untersuche die Dreifachkatastrophe Japans von 2011 im Hinblick auf sozio-kulturelle Prozesse. Das heißt konkret, dass ich die Aussagen meiner Gesprächspartner in einen Bezug zu gesellschaftlichen Diskursen, Wahrnehmungen und Standpunkten setze und die persönlichen Auslegungen kultureller Wert- und Normenvorstellungen meiner Gesprächspartner reflektiere.

Der japanische Diskurs um die Dreifachkatastrophe tritt, wie eingangs bereits angedeutet, im Wesentlichen mit zwei unterschiedlichen Konnotationen auf: Zum einen besteht eine ständige Bedrohung durch Erdbeben und Tsunami aufgrund der besonderen geographischen Lage Japans. Die Inselkette liegt genau auf der Bruchzone von vier tektonischen Erdplatten, deren Bewegungen täglich kleinere Erdbeben auslösen. Von dieser permanenten Gefahr durch Naturgewalten wird die atomare Verstrahlung, ausgelöst durch die Havarie des Kernkraftwerkes „Fukushima Daiichi“ am 11. März 2011, diskursiv abgespalten. Deren Auftreten wird nicht den Naturgewalten, sondern menschlichem Versagen zugeschrieben (siehe Kapitel 2.2). Bei diesem „menschlichen Faktor“ des dritten Teils der Katastrophe vom 11. März 2011 handelt es sich um einen Aspekt, der mithilfe der Diskursanalyse besonders spannende Einblicke in die japanische Gesellschaft erlaubt.

In der Katastrophenforschung geht man grundsätzlich von einem Zusammentreffen von gesellschaftlich geprägten materiellen Praktiken („material practices“), wie beispielsweise Städteplanung, und Umweltbedingungen (beispielsweise Erdbeben) aus, die die Verwundbarkeit einzelner Individuen, Bevölkerungsgruppen und Lokalitäten beim Eintreten einer Katastrophe maßgeblich bestimmen (Oliver-Smith 2002: 36). Laut den Vertretern dieses Forschungszweiges gestalten die wechselseitigen Prägungen von Kultur und Umwelt das gesellschaftliche Verständnis einer Katastrophe auf mentaler wie materieller Ebene. Dies ist die erkenntnistheoretische Basis, aufgrund der ich zu der Auffassung gelange, dass die materielle Zerstörung in Folge eines Desasters von einer Erschütterung der Gesellschaftslandschaft begleitet wird, die sich in der Hinterfragung ideologischer, moralisch-ethischer und machtpolitischer Rahmungen manifestiert. Für die folgenden Ausführungen ist des Weiteren wichtig, dass die „[...] Unterscheidung von Naturkatastrophen versus menschengemachte versus technische versus Verbundkatastrophen $\mathrm{u}$. Ä. in Bereichen der Rechtsprechung und dem Versicherungswesen von Belang [...]" (Felgentreff und Glade 2008: 3; Hervorhebung im Original) ist. Der atomare GAU nimmt unter anderem deshalb eine Sonderrolle sowohl im japanischen Diskurs als auch in dieser Arbeit ein, weil die Aushandlung der Verantwortlichkeit in besonderem Maße präsent ist.

Im Hinblick auf die wissenschaftliche Auseinandersetzung mit Gesellschaftsdiskursen wie denjenigen rund um „Fukushima“ mache ich zwei grundsätzliche Forschungsrichtungen aus: Erstens gibt es Forschungszweige, die sich mit der gesellschaftsstrukturellen Machtausübung der japanischen Eliten und deren interessengesteuertem Einfluss auf die öffentliche Meinung befassen. Und zweitens steht dem ein deskriptiverer Argumentationszweig gegenüber, der im Vergleich zu gesamt- 
gesellschaftlichen Studien das Handlungsvermögen der einzelnen oppositionellen Akteure verstärkt in den Fokus rückt.

Dementsprechend habe ich das Kapitel 2 so strukturiert, dass ich mich zunächst den Besonderheiten des nuklearen Desasters und dessen Einordnung in den internationalen Vergleichsrahmen widme. In Kapitel 2.1 kontrastiere ich die verschiedenen naturwissenschaftlichen Erkenntnisse und Standpunkte mit dem ethnologischen Fokus der vorliegenden Arbeit auf die akteurszentrierte Perspektive und erläutere die sich daraus ergebenden Konsequenzen für meine Arbeit. Anschließend setze ich mich mit der japanologischen Forschung bezüglich der kulturellen Akteure auseinander und setze sie in Bezug zu meinen eigenen Forschungsergebnissen.

\section{1 „Fukushima“ im internationalen Wissenschaftsdiskurs}

Eine der Besonderheiten eines Austritts von radioaktivem Material in die Umgebung hat bereits Ulrich Beck in seinem Klassiker der Soziologie „Risikogesellschaft: Auf dem Weg in eine andere Moderne“ im Jahr 1986 beschrieben. Kurz vor dem Reaktorzwischenfall in Tschernobyl veröffentlicht, zeichnet Becks Werk die gesellschaftlichen Zusammenhänge zwischen marktwirtschaftlichen Modernisierungsprozessen und Risiken nach. Dabei nennt er explizit die Radioaktivität als eines jener Risiken, die sich dem menschlichen Wahrnehmungsvermögen entziehen und daher besondere Merkmale aufweisen:

„Sie [die Risiken] setzen systematisch bedingte, oft irreversible Schädigungen frei, bleiben im Kern meist unsichtbar, basieren auf kausalen Interpretationen, stellen sich also erst und nur im (wissenschaftlichen bzw. antiwissenschaftlichen) Wissen um sie her, können im Wissen verändert, verkleinert oder vergrößert, dramatisiert oder verharmlost werden und sind insofern im besonderen Maße offen für soziale Definitionsprozesse." (Beck 1986: 29-30; Hervorhebung im Original)

Beck zufolge ist bereits die Inbetriebnahme von Kernreaktoren zur Stromgewinnung ein kollektiv eingegangenes Risiko einer Gesellschaft, die zum wirtschaftlichen Vorteil einen langfristigen Schaden in Kauf nimmt. Für umso wichtiger erachtet er daher die Kommunikation eines solchen Wirtschaftszweiges mit der Öffentlichkeit und fährt fort: „Damit werden Medien und Positionen der Risikodefinition zu gesellschaftlich-politischen Schlüsselstellungen" (Beck 1986: 30).

Damit verweist er auf einen wichtigen Aspekt im Vorfeld einer nuklearen Katastrophe: Nicht nur die radioaktiven Stoffe sind unsichtbar, sondern auch das sie umgebende gesundheitliche Risiko für Mensch und Umwelt. Die Vermittlung dieses Risikos ist stark vom Informationsgehalt und den Informationsquellen abhängig, die der Öffentlichkeit zur Verfügung gestellt werden, weshalb Beck die Rolle der Medien so stark betont. 
Der sozialwissenschaftlichen Katastrophenforschung zufolge (siehe Oliver-Smith und Hoffman 2002: 7) treten nach Verwirklichung des Risikos in Form einer Katastrophe diejenigen Zusammenhänge und Widersprüche ans Licht, die in jeder Gesellschaft Teil des sozialen Miteinanders sind (siehe Kapitel 3.1 „Katastrophen und ihre Folgen - Eine theoretische Verortung"). Im Fall der Diskussion um die Nutzung, Gefahren und Risiken der Atomenergie werden diese Widersprüche allerdings auch im naturwissenschaftlichen Umfeld sichtbar.

Diese Widersprüche in den verschiedenen Wissenschaftsdisziplinen lassen sich vor allem mit den nur schwer abschätzbaren Folgen atomarer Verstrahlung für Land, Meer und Menschen begründen. Internationale Experten ziehen zur Beurteilung der gemessenen Strahlenwerte die einzigen ihnen zur Verfügung stehenden Vergleichsfälle heran. ${ }^{3}$ Dazu gehören neben den Messdaten, die nach 1945 in Hiroshima und Nagasaki erhoben wurden, auch die gesundheitlichen Langzeitstudien in Tschernobyl in der ehemaligen Sowjetunion. Die Menschen dort waren dabei ähnlich wie in den Gebieten um das Atomkraftwerk (AKW) „Fukushima Daiichi“ nicht nur der äußerlich einwirkenden Strahlung ausgesetzt. Die Kontaminierung von Boden, Wasser und damit auch der angebauten Lebensmittel durch strahlende Partikel bildet die Basis für erste Prognosen bezüglich der gesundheitlichen Langzeitfolgen einer inneren Verstrahlung durch kontaminierte Nahrung und Trinkwasser.

Erste sozialwissenschaftliche Auseinandersetzungen mit „Fukushima“, den international stark unterschiedlich ausfallenden Einschätzungen der beschriebenen Folgen atomarer Strahlung sowie den soziologischen Beobachtungen in Japan nach 2011 finden sich unter anderem bei Japanologen wie Coulmas und Stalpers (2011), Richter (2015) und Samuels (2013).

Im Folgenden möchte ich zunächst die Vergleichbarkeit von Tschernobyl und „Fukushima“ in den Vordergrund stellen, bevor ich mich im darauffolgenden Kapitel mit der konkreten wirtschaftspolitischen Agenda von Japans Eliten und damit einem dominanten Diskurs in Bezug auf „Fukushima“ auseinandersetze.

Die Japanologen Florian Coulmas und Judith Stalpers beschäftigten sich schon sehr früh mit der Vergleichbarkeit von Tschernobyl und „Fukushima“, indem sie die 2011 bereits zur Verfügung stehenden technischen Messdaten näher untersuchten. Die in ihrem Buch zitierten ersten Experteneinschätzungen unmittelbar nach dem atomaren GAU bezeichnen „Fukushima“ als weniger schwerwiegend als Tschernobyl, was von den Experten unter anderem mit den stark unterschiedlich ausfallenden Zeiträumen bis zur Evakuierung begründet wird. Die Bevölkerung der betroffenen Gebiete Japans sei sofort evakuiert worden, wohingegen es in Tschernobyl erst nach zwei Tagen zu entsprechenden Maßnahmen gekommen sei (Coulmas und Stalpers 2011: 75ff.).

Anders als diese frühen Prognosen geben sich Coulmas und Stalpers wesentlich skeptischer: In Anbetracht der Ungewissheit, wie viel Radioaktivität nach wie vor

\footnotetext{
${ }_{3} \mathrm{Zu}$ den bekanntesten dieser internationalen Expertengruppe gehört Dr. Sebastian Pflugbeil, Physiker und Bürgerrechtler sowie seit 1999 Präsident der Gesellschaft für Strahlenschutz e.V.
} 
austrete, zum Beispiel in den Pazifischen Ozean, werde es Jahre dauern „[...] bis exakte Angaben gemacht werden können. Das wird erst möglich sein, wenn man weiß, wie viel Brennstoff in den Reaktorbehältern verblieben ist. Bis das durch die Inspektion des Reaktorinneren überprüft werden kann, werden voraussichtlich mindestens zehn Jahre vergehen" (Coulmas und Stalpers 2011: 77).

In diesem Zitat wird deutlich, was Beck (1986: 29-30) eingangs mit der „Offenheit für soziale Definitionsprozesse“ meint. Zum Zeitpunkt der Veröffentlichung des Werks von Coulmas und Stalpers im Jahr 2011 war noch nicht abzusehen, wie die Daten zu interpretieren sind, geschweige denn, dass eine verlässliche Zukunftsprognose hätte erstellt werden können. Die dennoch frühzeitig erfolgte Einordnung des gegenwärtigen wie zukünftigen Gefahrenpotentials von „Fukushima“ wirft daher Fragen nach deren Stoßrichtung auf.

Auf der Internationalen Bewertungsskala für Nukleare Ereignisse (INES) wird „Fukushima“ zwar auf der höchsten Stufe 7 eingeordnet, ${ }^{4}$ also als Zwischenfall verstanden, bei dem große Mengen Radioaktivität austraten, die langfristige Gegenmaßnahmen nötig machen. Der Zwischenfall wird jedoch aktuellen Einschätzungen des japanischen Ministry of Economy, Trade and Industry (METI) zufolge dennoch weit unterhalb von Tschernobyl eingeordnet.

Dabei bleiben jedoch spezifische Charakteristika der verschiedenen Bevölkerungsgruppen unberücksichtigt, die sich entscheidend auf eine konkrete Risikoeinschätzung auswirken. Alter, Gender oder der Wohnort zum Zeitpunkt der Katastrophe werden dabei ebenso ausgeschlossen wie die den Evakuierten zur Verfügung stehenden Ressourcen. Diese Faktoren der Verwundbarkeit (vulnerability) finden keinerlei Niederschlag in den genannten Einschätzungen und legen aus meiner Sicht den Verdacht einer wirtschaftspolitischen Agenda nahe.

Um mir ein genaueres Bild von der Diskussion um die Einschätzung möglicher gesundheitlicher Folgen zu machen, besuchte ich 2014 eine Veranstaltung des United Nations Scientific Committee on the Effects of Atomic Radiation (UNSCEAR) in Tokyo. In ihrem Bericht kamen die Mitglieder des UNSCEAR-Komitees zu der Einschätzung, dass es zu keinem signifikanten Anstieg von Krebserkrankungen aufgrund des Reaktorunfalls kommen werde. ${ }^{5}$

Auf der Veranstaltung folgten der Vorstellung des Berichts eine Reihe von internationalen Beiträgen, die zur Diversifizierung der Thematik beitrugen. So kamen

\footnotetext{
${ }^{4}$ „INES Rating on the Events in Fukushima Dai-ichi NPS“, Seite 8 von Ministry of Economy, Trade and Industry (METI): „Japan Faces an Unprecedented Challenge“, 08. März 2012, https://www.meti. go.jp/english/earthquake/nuclear/japan-challenges/pdf/japan-challenges_a.pdf.

5 Eines der Mitglieder des Komitees, Dr. Wolfgang Weiss, sprach sich ausdrücklich gegen die Veröffentlichung des Dokuments aus. Er begründete seinen Widerspruch damit, dass der Bericht die Feststellung einer kausalen Verbindung zwischen erhöhten Krebsraten und der durch „Fukushima Daiichi“ freigesetzten Strahlung (siehe Asanuma-Brice 2014) verweigere. Dies ist ein weiterer Hinweis darauf, wie umstritten sowohl die wissenschaftlichen Erhebungsmethoden wie auch die Auswertung und Beurteilung der Strahlungsdaten sind. Mittlerweile liegt eine aktualisierte Version des Berichtes aus dem Jahr 2015 vor.
} 
auch japanische Stimmen aus betroffenen Präfekturen wie Tochigi zu Wort, die die Einschätzung des UNSCEAR nicht teilten und einen erheblichen Anstieg der Schilddrüsenkrebsrate bei Kindern feststellten. Als unmittelbare Nachbarn der Präfektur Fukushima hatten die Anwohner einen ganz anderen Eindruck von ihrer persönlichen Situation als sie der wissenschaftliche Bericht der UNSCEAR vermittelte.

Dies verdeutlicht erneut die hohe Brisanz der Thematik angesichts einer großen Diskrepanz zwischen den Interpretationen der Realitäten. Auf der einen Seite steht die Experteninterpretation erhobener Strahlendaten und auf der anderen die Wahrnehmung persönlich betroffener Menschen, die sich angesichts der sich zum Teil stark widersprechenden Experteneinschätzungen große Sorgen um ihre Gesundheit machen. Die zu berücksichtigenden gesundheitlichen Aspekte betreffen nicht nur die physischen, sondern auch die psychischen Folgen der Verstrahlung angesichts von heimatlicher Entwurzelung und der Trennung von Familie und gewohnter Umgebung.

Die für „Fukushima“ sichtbar gewordene Abhängigkeit diskursiver Positionen von der wissenschaftlichen Interpretation der Strahlendaten und des spezifischen Risikos für Bevölkerungsanteile verdeutlicht die interessengesteuerte Vielschichtigkeit der Thematik. Die Kulturwissenschaftler Oliver-Smith und Hoffman (2002: 9) beschreiben diese Vielschichtigkeit als einen Teil des Katastrophenprozesses, der die Sozialstruktur einer Gesellschaft inklusive ihrer Bindungen, ihrer Widerstandsfähigkeit und anderer Allianzen offenlegt. Für mich ergibt sich nach der Einarbeitung in die Thematik folgendes Bild eines Kontinuums an Positionen, auf denen sich die einzelnen gesellschaftlichen Akteure befinden: Auf der einen Seite stehen machtpolitische Akteure. Damit meine ich auf meinen konkreten Forschungskontext bezogen vor allem Vertreter aus Japans Politik und Wirtschaft, die eine Interpretationsweise der Vorkommnisse favorisieren, die das Ausmaß der Katastrophe auf einem zu verwaltenden Level einstuft. Dies wiederum erlaubt es ihnen, bisherige Verfahren der Entscheidungsfindung beizubehalten. Dazu ziehen machtpolitische Akteure wissenschaftliche Erhebungen wie die der INES heran, um ihre Interpretation zu unterstreichen, die Bevölkerung zu beruhigen und ihre Machtposition zu halten. Auf der anderen Seite des Kontinuums stehen die Erfahrungen der unmittelbar Betroffenen, die ihre eigene körperliche wie psychische Gesundheit und die nachkommender Generationen gefährdet sehen und entsprechende personelle wie strukturelle Konsequenzen erwarten und fordern. Zwischen diesen sich gegenüberstehenden Positionen definiert sich das Realitätsverständnis meiner Gesprächspartner ebenso wie das der restlichen japanischen Bevölkerung.

In meiner ethnologischen Untersuchung der Katastrophe nehme ich insbesondere diejenigen Kommunikationsprozesse in den Fokus, die das Risiko vor, während und nach dem Desaster in den öffentlichen Diskursen rahmen. Hinzu kommen die entsprechenden Hintergründe der Handelnden und moralisch-ethische Fragestellungen im kulturspezifischen Kontext. 


\subsection{Arbeiten über die Narrative zu „Fukushima“}

Vor dem Hintergrund der internationalen Einordnung von „Fukushima“ sowohl als Ereignis als auch als andauernde Katastrophe geht es mir in den folgenden Kapiteln um bisherige wissenschaftliche Untersuchungen des japanischen Diskursverlaufs und dessen Gestaltung durch verschiedene nationale wie internationale Akteure aus Politik, Wirtschaft und den Wissenschaften. Die wissenschaftliche Auseinandersetzung mit „Fukushima“ ist aufgrund der Aktualität der Ereignisse noch nicht sehr weit fortgeschritten und bezieht sich in erster Linie auf die dominante Argumentation wirtschaftlicher und politischer Eliten. Die naturwissenschaftliche wie auch gesellschaftliche Interpretation biologischer wie psychosozialer Effekte von Radioaktivität wird indes in den Sozialwissenschaften vielseitig thematisiert. In ihren Beiträgen setzen sich Soziologen wie Beck (1986) und Stephens (2002), Kulturwissenschaftler wie Oliver-Smith and Hoffman (2002) sowie Japanologen wie Samuels (2013) mit der auch für „Fukushima“ wichtigen Verbindung von wissenschaftlichem Wissen über die Effekte radioaktiver Strahlung auf den menschlichen Körper und den besonderen Interessen aus Politik und Wirtschaft an atomarer Energiegewinnung auseinander. Dabei ist für die verschiedenen Autoren von besonderer Bedeutung, wie die Forderungen der Bevölkerung nach Schutzmaßnahmen umgesetzt werden, um dem jeweiligen Sicherheitsbedürfnis gerecht zu werden. Die herangezogenen Experten weisen dabei andere Zugänge zum Thema Sicherheit in der Atomenergie auf als die im öffentlichen Raum existierenden Auffassungen. Daher entstehen im Fall „Fukushima“ verstärkt gesellschaftliche Konflikte bezüglich getroffener Maßnahmen zum Schutz der Bevölkerung vor atomarer Verstrahlung.

Meiner Analyse zufolge bewegen sich die Auffassungen der involvierten Akteure auf einem Kontinuum zwischen zwei Polen: Auf der einen Seite stehen diejenigen naturwissenschaftlichen Experten, die in Gremien wie der Internationalen Strahlenschutzkommission (ICRP) die Balance zwischen notwendigen Schutzmaßnahmen und Vorteilen der atomaren Stromerzeugung finden sollen. Auf der anderen Seite befinden sich NGOs und kulturelle Akteure.

Naturwissenschaftliche Experten stehen laut Stephens (2002) vor der Herausforderung, das verloren gegangene Vertrauen der Bevölkerung in die zuständigen Behörden zurückzugewinnen und gleichzeitig die Sicherheitsmaßnahmen wirtschaftlich rentabel zu gestalten. Angesichts des erhöhten Bewusstseins der Menschen für die Gefahren, die auch die „friedliche Nutzung" der Atomenergie mit sich bringt (siehe Bauer 2013), und den weiterhin zu zahlenden Entschädigungen an die unmittelbar Betroffenen, stellt dies eine große Herausforderung dar. Das Gleichgewicht zwischen Sicherheit und Wirtschaftlichkeit zu erlangen, wird jedoch durch verschiedene spezifische Eigenschaften der Atomenergie erschwert.

Zum einen wird das Maß einer gesundheitlich unbedenklichen Strahlendosis in den einzelnen Staaten festgelegt und Grenzwerte können daher stark voneinander abweichen. Dies führt im Falle eines atomaren Zwischenfalls zu großer Verunsiche- 
rung und Misstrauen in den verschiedenen Teilen der Bevölkerung. Zum anderen liegt den öffentlichen Diskursen, im Gegensatz zu wissenschaftlichen Diskursen, ein heterogenes Gesellschaftsbild zugrunde. Darin werden entscheidende Faktoren zur Risikowahrnehmung wie Alter, Gender, Klasse etc. (Stephens 2002: 111) berücksichtigt - wohingegen dies in den wissenschaftlichen Diskursen vernachlässigt wird (Stephens 2002: 105). Für das Individuum ist eine solche Einschätzung des radioaktiven Strahlenaustritts ohne die Berücksichtigung der persönlich als wichtig empfundenen Faktoren im Zweifelsfall nur von geringer Aussagekraft.

Die Kulturwissenschaftlerin Stephens (Stephens 2002: 101) nimmt darüber hinaus Bezug zu den psychosozialen Faktoren einer Verstrahlung. Die Umsiedlung und ungewissen Konsequenzen einer erhöhten Strahlendosis für die Gesundheit der eigenen und zukünftiger Generationen führt zu erhöhtem Stressempfinden bei den unmittelbar Betroffenen, das sich in negativen Lebensstilveränderungen wie erhöhtem Alkoholkonsum manifestieren kann.

Diese und andere Effekte radioaktiver Verstrahlung wurden bereits im Rahmen der Nachuntersuchungen von Tschernobyl 1986 festgestellt (Stephens 2002: 102). Die Befunde weiter auszuführen würde an dieser Stelle zu weit gehen, doch ich halte fest, dass sich die Diskurse um „Fukushima“ zwischen Polen aus naturwissenschaftlichen Experten, Politik und Wirtschaft auf der einen, und anderen aktiven Gestaltern der Gesellschaft, wie NGOs und kulturellen Akteuren, auf der anderen Seite auftun. Stephens zeigt die Interessen und Zielrichtungen der jeweiligen Akteursgruppen auf und vermittelt das Bild eines „credibility gap“ (Stephens 2002: 106) zwischen beiden Parteien. Diese „Lücke“ entsteht aufgrund der oben ausgeführten unterschiedlichen Risikoeinschätzung seitens der Öffentlichkeit und den herangezogenen Experten, was zu einem sehr geringen Vertrauen gegenüber Letzteren führt.

Erste Hinweise auf die Involvierung von Emotionen in den Debatten rund um die Atomenergie liefert Stephens Aussage (Stephens 2002: 110), die Einschätzung der Experten bezüglich der öffentlichen Meinung sei geprägt von Charakterisierungen, die sich entlang von „irrational“, „,ungebildet“, ,emotional“ und „hysterisch“ bewegen. Diese Bezeichnungen beziehen sich überwiegend auf Personen und Personengruppen, die ihre Wohngebiete verlassen haben, ohne einen offiziellen Evakuierungsaufruf erhalten zu haben. Ein solcher Evakuierungsbefehl wird ausgestellt, wenn man von offizieller Seite zu der Überzeugung gelangt ist, es bestehe Gefahr für die Bewohner einer bestimmten Gegend. Menschen, die ihre Wohngegend ohne Evakuierungsaufruf verlassen, gelten als sogenannte „freiwillig Geflüchtete“. 6 Diese Personen müssen damit rechnen, dass Entschädigungszahlungen früher oder später eingestellt werden. ${ }^{7}$ Aus Sicht der unmittelbar Betroffenen jedoch stehen ihnen kei-

\footnotetext{
${ }^{6}$ Siehe Justin McCurry: „Fukushima evacuees face ,forced“ return as subsidies withdrawn“, The Guardian, 11.3.2017, https:/www.theguardian.com/world/2017/mar/10/japan-fukushima-nuclear-di saster-evacuees-forced-return-home-radiation.

7 So geschehen im März 2017 (Aniatom-fuku: Fukushima heute Berichte 2018, https://antiatom-fu ku.de/fukushima-heute-bericht-2018.html).
} 
ne anderen Möglichkeiten offen, ihre Gesundheit zu schützen. Ganze Dorfgemeinschaften entscheiden sich für das Verlassen ihrer Wohnhäuser, weil sie von Angst, Unsicherheit und Verwirrung angesichts der oben genannten sich widersprechenden Stimmen aus Wissenschaft und anderen Quellen getrieben sind. ${ }^{8}$

Angesichts des dargelegten diskursiven Hintergrunds verwundert es nicht weiter, dass sich die Risikoeinschätzung in Politik und Wirtschaft von Ansichten in der Öffentlichkeit unterscheidet und auch die Reaktionen der Akteure auf atomare Zwischenfälle entsprechend ihrer Interessen und Motivationen unterschiedlich ausfallen. Die Sozialwissenschaften können die diskursspezifischen Rahmenbedingungen für die Bewertung von Atomenergie sowie die dahinter liegenden gesellschaftlichen Aushandlungsprozesse offenlegen und auf diese Weise zum besseren Verständnis des Akteursverhaltens beitragen.

Die sozialwissenschaftliche Erforschung der gesellschaftlichen Auswirkungen macht einen Gesamtüberblick über die Positionen der entsprechenden Akteure erforderlich, die in der Wissenschaft behandelt und diskutiert werden. Im Folgenden berücksichtige ich daher zunächst den soziologischen Zugang zu den Narrativen von „Fukushima“, die von Personen in Machtpositionen (wie Regierungsvertretern, Gremien, Experten, Wirtschaftsvertretern etc.) verstärkt vertreten werden. Anschließend widme ich mich denjenigen wissenschaftlichen Beiträgen, die sich mit den „alternativen" Akteursperspektiven (Stephens 2002: 94) auf Radioaktivität und ihren Folgen befassen.

\subsubsection{Die Aushandlung der „Fukushima“-Diskurse durch Eliten}

Die Wissensbasis des Diskurses um den 11. März 2011 besteht im Allgemeinen aus dem vorläufigen Wissen um die Lebensumstände der 160.000 Evakuierten („displaced persons"), die aus Fukushima und den Nachbarregionen vor den strahlenden Partikeln fliehen mussten (siehe Asanuma-Brice 2014). So kann man in vielen internationalen Medien verfolgen, wie die mehrheitlichen Stimmen japanischer Politik eine Rückkehr der Geflohenen in ihre Heimatgebiete favorisieren, auch wenn diese zum Zeitpunkt der Fertigstellung dieser Dissertationsschrift noch oder wieder kontaminiert sind. Japanische Experten und Behörden argumentieren unter anderem, dass die Strahlung nicht gefährlich sei und das Kernkraftwerk stabilisiert sei (siehe Gebhardt 2013: 316). Je nach Auslegung der erhobenen Strahlenmessdaten bewegt sich die Beurteilung dieser Herangehensweise durch Dritte zwischen „vertretbar“ und „unverantwortlich“. Die dominanten Argumentationslinien, insbesondere der japanischen Behörden, erfordern daher eine gesonderte Betrachtung.

Die Narrative der Katastrophe in den japanischen Diskursen werden vom Japanologen und Politikwissenschaftler Richard J. Samuels in seinem Werk 3.11 Disaster and Change in Japan von 2013 zusammengefasst. Er liefert einen ersten

\footnotetext{
8 Weiteren Bezug auf die emotionalen Verhandlungsweisen nehme ich in Kapitel 5 „Vertrauen und Risiko - Die vorläufige Bilanz einer gesellschaftlichen Katastrophe“ vor.
} 
Überblick über die gesamtgesellschaftlichen Verarbeitungsprozesse zwei Jahre nach den Ereignissen. Samuels (2013: 26) beschreibt drei Hauptnarrativstränge in Bezug auf die japanische Atompolitik:

1. Das „forward-leaning-narrative“: Eine Zukunftsausrichtung auf einen neuen gesellschaftlichen Kurs nach Eintreten des Traumas. Dabei soll die Qualität des Staates, also insbesondere seine Effektivität und die Transparenz seiner Entscheidungen, verbessert werden.

2. Die „stay-on-course-narrative“: Vertreter dieser Narrative vertreten die Ansicht, dass es keinen Anlass zur Veränderung gibt.

3. Den „reverse-course“, das radikalste der drei Narrative, bei dem davon ausgegangen wird, dass sich die japanische Gesellschaft auf einem Irrweg befindet, der einer radikalen Korrektur bedarf.

Die Untersuchung der Effektivität dieser Narrative im Hinblick auf die politische Instrumentalisierung der Katastrophe macht den Reiz von Samuels' Analyse aus, in der er aus soziologischer Sicht die diskursive Konstruktion eines desaströsen Ereignisses nachvollziehbar macht. Die Instrumentalisierung macht Samuels an der Stärkung eines japanischen Nationalgefühls, der Verbesserung des bereits etablierten Systems sowie der Rückkehr zu einer idealisierten Vergangenheit aus: „Crisis are constructed and manipulated by stake holders and are constituted in and through such narratives" (Samuels 2013: 26).

Samuels nähert sich der japanischen Gesellschaft aus einer Vogelperspektive und beleuchtet das Einwirken gesellschaftlicher Eliten auf den Diskursverlauf. Laut Samuels versuchen Personen in einflussreichen Positionen, die öffentliche Meinung zugunsten ihrer wirtschaftlichen Interessen zu beeinflussen, indem sie auf gesellschaftliche Vorstellungen und Werte Bezug nehmen. Zu diesen gehören zum Beispiel gesellschaftlich hoch geschätzte Werte wie Durchhaltevermögen (gaman zuyoi) und soziale Solidarität. So wird in den japanischen Medien die Rolle der Opfer beispielsweise mit heldenhaften Attributen ausgestattet und mit einem Gefühl des nationalen Stolzes verknüpft: „On this account, the people of Tohoku embodied what it meant to be Japanese - they formed a community (komyunitei) connected (tsunagu) by bonds (kizuna) and human contact (fureai) that sustains solidarity (renkei) through a common battle (Ganbaro Nippon!)" (Samuels 2013: 39). In diesem Zitat wird deutlich, dass die kulturspezifischen Artikulationsmöglichkeiten und -wege die Prozesse der Bedeutungszuschreibung bis zum heutigen Tage beeinflussen, auch wenn diese nationalen Narrative im Lokalen durchaus starke Kritik erfahren. So repräsentieren einzelne Akteure den „[...] nur scheinbar duldsamen japanischen Bürger, der sich nicht selten als mutiger Sprecher erweist" (Gebhardt 2013: 316). Auf diese Aspekte werde ich in Kapitel 5.2 „Kulturelle Spezifika als Katastrophenrisiko?“ genauer eingehen.

Ich halte Samuels Werk für einen entscheidenden Beitrag, weil er die Bedeutung der machtvollen diskursiven Basis betont, vor deren Hintergrund sich Gegenpositio- 
nen entwickeln. Samuels bezieht sich auf diskursive Rahmungstheorien der Politikwissenschaftler Chong und Druckman (2007). Deren Perspektive zufolge können Ereignisse entsprechende Gegeneffekte in der Gesellschaft hervorrufen („counterframing"). Samuels (2013: xiii) zielt auf die Beantwortung der Frage ab, wie die Aussichten eines strukturellen wie institutionellen Wandels im Fall der Dreifachkatastrophe vom 11. März 2011 einzuschätzen sind. Transformationen der japanischen Gesellschaft gehen seiner Meinung nach unter anderem vom Handlungsvermögen junger Menschen aus, die sich gemäß den Erwartungen wissenschaftlicher Beobachter veranlasst sehen könnten, verstärkt in der Öffentlichkeit zu erscheinen (Samuels 2013: 31).

An dieser Stelle setzt meine eigene Forschung an. Indem ich nach den biographischen Hintergründen einzelner gesellschaftspolitisch engagierter Akteure und ihren individuellen Handlungsmöglichkeiten frage, gelange ich zu Erkenntnissen über das Selbstverständnis japanischer sozio-politischer Akteure nach dem 11. März 2011, die die Ergebnisse von Samuels (2013) und den anderen genannten sozialwissenschaftlichen Beiträgen auf qualitativer Ebene ergänzen. ${ }^{9}$ Dabei berücksichtige ich auch das von Samuels erörterte Feld kulturspezifisch ausgeformter Wertekategorien. Im nationalen Diskurs Japans werden lokale Vorbilder instrumentalisiert, um eine Gemeinschaftsstimmung zu erzeugen, die als Basis für einen Wiederaufbau der Gemeinden, Beziehungsnetzwerke und der Normalität dienen soll (siehe Kapitel 5.3.1 „Das Atomdorf"). ${ }^{10}$ Die Betroffenen zu gesamtgesellschaftlichen Vorbildern zu stilisieren, verdeutlicht einen Umgang von Japans Behörden mit Schicksalsschlägen, der der Analyse meiner Forschungsdaten zufolge den Samen für das „counter-framing“ im Sinne von Chong und Druckman (2007) legen kann.

Samuels Analyse zufolge wurden in den Post-Fukushima-Diskursen bereits existierende Narrationsstränge und Konflikte weitergeführt:

„To be sure, 3.11 was a crisis, but it stimulated battles within existing pathways and generated solutions consistent with existing goals. There was very little that was novel in the 3.11 discourse, apart from the horrific experience itself." (Samuels 2013: 184)

Diese Aussage Samuels verstehe ich vor dem in Kapitel 2.2 dargelegten Hintergrund der wissenschaftlichen Debatte um die gesellschaftliche Bewertung von Strahlendosen so, dass sich die Stimmen des „Fukushima“-Diskurses entlang der dort sichtbar gewordenen Argumentationsstränge orientieren. Die wirkmächtigen Akteure würden dementsprechend kaum „neue“ Aspekte einfließen lassen. Samuels mag mit seiner Ansicht Recht haben, dass der 11. März 2011 zum Zeitpunkt der Veröffentlichung seiner Publikation keinen Wendepunkt in den politischen Hauptfragen Japans dar-

\footnotetext{
9 Siehe Kapitel 5.2 „Kulturelle Spezifika als Katastrophenrisiko?“

${ }^{10}$ Die Rolle der japanischen Experten (sog. „Gefälligkeits- oder Regierungsgelehrten“, jap.: goyo gakusha) in diesem Prozess wird unter anderem von John (2013) dargestellt.
} 
stellte (Samuels 2013: 193). Dennoch halte ich diese Annahme vor dem kulturspezifischen Hintergrund Japans für überhöht.

Wie meine Studie zeigt, sind die gesellschaftlichen Strukturen der Entscheidungsund Bewertungsfindung selbstverständlich auch nach einer solchen Katastrophe präsent. Sie wirken sich sowohl restriktiv als auch ermutigend aus, beispielsweise auf kulturelle Akteure, die ihre Wahrnehmung und Beurteilung öffentlich äußern. Samuels Fokus auf die Eliten verzichtet auf die Einbeziehung weniger sichtbarer Akteure. Dementsprechend entgehen ihm die sich im Stillen vollziehenden kleinen Veränderungen in den Haltungen, Anschauungen und Wahrnehmungen der japanischen Bevölkerung. In meiner eigenen Forschung hingegen setze ich den Fokus auf die Perspektive des „Grassroot-Levels“, um neue erkenntnistheoretische Zugänge auf die japanische Gesellschaft nach dem 11. März 2011 zu eröffnen. Samuels (2013: 200) abschließende Aussage, er beobachte durchaus Kreativität in der politischen Klasse, gibt einen wichtigen Hinweis auf vorhandene Handlungspotentiale, die meine Gesprächspartner nutzen können.

Ich werde nicht nur die zerstörerischen Aspekte der Dreifachkatastrophe darstellen, sondern auch die entstandenen Möglichkeiten zur gesellschaftlichen Gestaltung am Beispiel der Akteursgruppe der Gegenwartskünstler erörtern. Insofern schließe ich mich Samuels Aussage an, wenn er sagt: „3.11 is no ,game changer but it also empowered and opened up opportunities" (Samuels 2013: 200).

\subsubsection{Die Diskursalternative - Kulturelle Akteure in der Forschung}

Nach dem aufgezeigten Spannungsverhältnis aus naturwissenschaftlich, wirtschaftspolitisch und gesellschaftlich geprägten Realitäten nähere ich mich nun den Untersuchungen derjenigen Stimmen, die eine Gegenposition zu den wirkmächtigen Akteuren der japanischen Gesellschaft vertreten. Diese Akteursgruppe umfasst unter anderem Literaten, Schauspieler und Künstler, die sich angesichts des Streits um Grenzwerte und differenzierte Risikocharakteristika der Gesellschaft mit den daraus folgenden sozio-politischen Konsequenzen auseinandersetzen.

Aus soziologischer Perspektive gibt Samuels bereits erste Hinweise auf die für den gesellschaftlichen Aushandlungsprozess entscheidenden Faktoren der Emotionalität als Motivator, indem er die negativen Gefühle des lokalen Kontexts als eine Ausgangsbasis für die Auseinandersetzung mit entsprechend artikulierten Narrativen heranzieht. Auf diese Weise manifestiere sich anhand der lokalen Unzufriedenheit ein weiterer Diskursstrang, in dem die Zivilgesellschaft die Inkompetenz der politischen Führung Japans ins Zentrum stelle (Samuels 2013: 33).

Hommerich (2014) stellt mit ihrer Untersuchung eines beobachtbaren Vertrauensverlustes in die gesellschaftlichen Eliten eine Ergänzung aus soziologischer Perspektive zu Samuals Beitrag dar. Meiner Ansicht nach bildet dieser Vertrauensverlust die Basis für den eingangs erwähnten Prozess des „counter-framings" nach Chong und Druckman (2007), welcher ein starkes gesellschaftliches Spannungsverhältnis erzeugt. 
Sozialwissenschaftliche Auseinandersetzungen mit demokratischen Bestrebungen, Protestbewegungen (Stekelenburg und Klandermans 2010; Twine 1994), dem Begriff der „Zivilgesellschaft" und seiner Anwendbarkeit auf Japan beschäftigen sich spätestens seit den 1980er-Jahren mit den Zusammenschlüssen zivilgesellschaftlicher Akteure in Organisationen wie Nichtregierungsorganisationen (Steinhoff 1984; Pharr 1984; Vosse 1999; Liscutin 2012; Wiemann 2017). All diese Aspekte auszuführen, würde an dieser Stelle zu weit führen. Dennoch verdeutlichen die verschiedenen Ansätze der genannten sozialwissenschaftlichen Autoren die Vielschichtigkeit der akteurszentrierten sozialwissenschaftlichen Forschung.

$\mathrm{Zu}$ diesen Forschungsansätzen zählen auch Forschungsbereiche der Japanologie. Die bereits erwähnten soziologisch-politischen Erörterungen Samuels (2013) und Hommerichs (2014) zur Desillusionierung der japanischen Bevölkerung sowohl in sozio-politischer Hinsicht wie auch in Bezug auf die Sicherheit der atomaren Kernenergie werden durch Beiträge von Richter (2012), Mundt (2013) und Gebhardt (2015) ergänzt.

Die auch von Richter behandelte diskursive Unterteilung der Dreifachkatastrophe in ein Desaster natürlichen Ursprungs (jap.: tensai), bestehend aus Erdbeben und Tsunami, und des vom Menschen verursachten Atomunglücks (jap.: jinsai) stellt wichtige Aspekte in der Auseinandersetzung mit dem massiven Vertrauensverlust der japanischen Bevölkerung in die japanischen Behörden dar. Die Differenzierung der Ereignisfacetten in natürliche und menschliche Ursprünge der Dreifachkatastrophe ist maßgebend für die von mir untersuchten Herangehensweisen der Gegenwartskünstler, der Gesellschaft in dieser Zeit der Verunsicherung neue Orientierungspunkte anzubieten. Darauf komme ich an entsprechender Stelle wieder zu sprechen.

Mit ihren Beiträgen im Sammelband „Japan nach ,Fukushima - Ein System in der Krise" (2012) bieten Richter, Gebhardt und andere einen ersten Überblick über den Diskurs aus der Sicht kultureller Akteure. Die in Richters Beitrag zur Sprache kommenden Stimmen japanischer Soziologen klingen in der Beurteilung potentieller Transformationen sowohl optimistisch als auch äußerst skeptisch. Für Richter selbst sind beide Aspekte notwendige Voraussetzungen für Wandel, da:

„[...] Skepsis, gar ,Pessimismus des Verstandes‘, wie auch ,Optimismus des Willens' (Gramsci) vonnöten ist, um wirklich einen Systemwechsel zu bewirken (wobei dann immer noch danach zu fragen ist, was mit ,System' eigentlich gemeint ist - Reformen des politischen Systems oder der Verhältnisse, die in der Produktionssphäre, z. B. von Energie und Strom, vorherrschen).“

(Richter 2012: 104)

Während sich Richter (2012: 101) also mit den kritischen Stimmen japanischer Aktivisten zur Reaktorkatastrophe beschäftigt und dabei die Aspekte der elitären Kontrolle und Abhängigkeit vom System einbezieht, beschäftigt sich Gebhardt (2012) mit den Stimmen der zeitgenössischen japanischen Literatur. Sie beschreibt das Feld 
einer kulturschaffenden Szene, die ihren eigenen Beitrag zum Themenkomplex „Fukushima" verfasst.

Insgesamt gibt es wenige aktuelle Forschungen zum Wirken von Gegenwartskünstlern in Japan. Gegenwartskunst wird nach wie vor in ihren Wechselwirkungen mit einer globalisierten Welt diskutiert. In ihren unterschiedlichen Rollen der Diskursherstellung beziehungsweise -beeinflussung werden Gegenwartskünstler jedoch nur selten entsprechend kontextualisiert.

Vor dem Hintergrund der nationalen wie auch kulturellen Identitätsdiskurse wird am Beispiel der Künstler der rasche Wandel und die Verschiedenartigkeit ihrer Rollen in diesen Veränderungsprozessen erläutert (siehe Mathews 2000). Mathews beschreibt aus kulturwissenschaftlicher Sicht die Handlungskonditionen und -umstände japanischer Künstler in einer von der Moderne geprägten Zeit, weshalb er an dieser Stelle nicht unerwähnt bleiben soll. Mathews widmet sich wie keine andere der mir zur Verfügung stehenden literarischen Quellen den Identitätsaushandlungen der Künstler vor dem Hintergrund der nationalen Diskurse, der globalisierten Welt und des gesellschaftlichen Konformitätsdrucks. Die in seinem Werk zutage tretenden Aushandlungsprozesse um eine öffentliche Auseinandersetzung mit öffentlichen und zivilgesellschaftlichen Belangen haben den Fokus meiner Arbeit beeinflusst. Ich gehe davon aus, dass Künstler gesellschaftliche Stimmungen, Emotionen und Realitäten aufgreifen und die Frage nach möglichen Veränderungen angesichts der inzwischen eingetretenen Katastrophe von 2011 im öffentlichen Diskurs forcieren. Autoren der Japanologie wie Gebhardt behandeln in ihren Werken zwar die ersten Reaktionen berühmter Schriftsteller Japans auf die im Rahmen der Katastrophe zutage getretenen Schwachstellen des "Japanese system“ (Gebhardt 2015: 245 ff.), gehen jedoch nicht auf weitere kulturelle Akteure ein, die ebenfalls versuchen, den japanischen Diskurs zu beeinflussen. Ihre Ausführungen stellen nichtsdestotrotz einen wichtigen Ausgangspunkt zur kontextuellen Einordnung der von mir beschriebenen Gegenwartskünstler dar.

Japanische Autoren und Literaten werden von Gebhardt (2015) in zwei Kategorien unterteilt. Die Autoren der „bibliotherapeutischen“ Ausrichtung befassen sich mit Trost- und Wiederaufbautexten, die sich bemühen, ein Gefühl der Hoffnung zu vermitteln. Die Literaten der Bestandsaufnahmen des Status quo nach 2011 bilden die zweite Kategorie.

Die Beiträge beider Literaturvertreter zur „Erdbebenliteratur“ (shinsai bungaku) spiegeln die unterschiedlichen Emotionen bei den nach und nach zur Verfügung stehenden Informationen aus den unmittelbar betroffenen Gebieten wieder: Das Erleben des „Zerfalls der sozialen Strukturen“, „Drohende Umsiedlung und Angst vor der Radioaktivität" sowie „Selbstmorde aus Verzweiflung “ finden in diesen literarischen Werken ebenso ihren Ausdruck (Gebhardt 2012: 179) wie die Hoffnung im Dialog (Gebhardt 2012: 195) und die Botschaft, „,...] dass Glück auch ,nach Fukushima" noch möglich ist" (Gebhardt 2012: 172). Richter kommt zu dem Schluss, dass die Literatur Japans ihre „Aufgabe“, die Menschen zum Nachdenken anzuregen, ein Jahr nach Fukushima erfüllt habe: „Man attestiert ihr, dass sie, wenn sie auch noch 
keinen Richtungswechsel im Sinne eines political turn vollzogen habe, zumindest ihren Standpunkt verantwortungsvoll überdenke" (Gebhardt 2012: 197).

Die wissenschaftlichen Beiträge zur literarischen Szene Japans (Gebhardt 2012, 2015 u.a.) machen bereits deutlich, wie stark die Folgen der atomaren Verstrahlung und ihrer soziopolitischen Folgen alle Ebenen des gesellschaftlichen Lebens in Japan berühren und dementsprechend emotional aufgeladen sind.

Gebhardts Ausführungen zur künstlerischen Auseinandersetzung mit dem „System Japan“ werden durch Mundts Beitrag „Ungehorsam, die erste Künstlerpflicht - 'Fukushima' als Zäsur in der zeitgenössischen japanischen Theater- und Performanceszene“ (2013) ergänzt. Wie der Titel ihres Artikels vermuten lässt, dreht sich Mundts Betrachtung um den wichtigen Beitrag der Kunst zur gesellschaftlichen Verarbeitung und Auseinandersetzung mit dem bereits von Gebhardt und Richter aufgeworfenen Themenkomplex des „Systems Japan“11.

„Kunst ist etwas, das der gesellschaftlichen Wirklichkeit etwas Starkes entgegensetzen kann. Und dieses Etwas ist für eine Gesellschaft notwendig - für eine Gesellschaft, in der solche Katastrophen wie in Japan geschehen sind, erst recht. Denn wenn es dieses Etwas, eine Antithese, nicht gibt, dann müssen die Menschen glauben, es gebe nur diese einzige Wirklichkeit. Dann ist die Richtung des Denkens festgelegt." (Okada 2011: 19-20, zitiert in Mundt 2013: 102)

Besonders wichtig ist Lisa Mundt daher die Erörterung der Frage nach einer Repolitisierung der japanischen Theaterszene, einem Bereich der Kunstwelt, der seit jeher dafür prädestiniert ist, sich kritisch mit den gesamtgesellschaftlichen Entwicklungen auseinanderzusetzen. Obwohl sich Mundt dabei auf die Performancekunst fokussiert, decken sich einige ihrer Ergebnisse grundsätzlich mit den Standpunkten vieler meiner Gesprächspartner. So gehe es Theaterproduzenten darum, „[...] eine Vielfalt an Meinungen und Stimmen zu sammeln und diesen via Internet zu einer eigenen Öffentlichkeit zu verhelfen“ (Mundt 2013: 107). Die damit zusammenhängende Reflexionsleistung schließt eine Kritik an der medialen Abdeckung der Katastrophe ebenso ein wie die Ironisierung der staatlichen Wiederaufbaumaßnahmen: „Hier schafft die Kunst [...] ein Gegengewicht zu den etablierten Medien, die nach Meinung der Künstler ihrer Aufgabe einer kritischen Berichterstattung nicht gerecht werden" (Mundt 2013: 112).

\footnotetext{
${ }^{11}$ Unter diesem von Gebhardt und Richter (2013: 9) verwendeten Begriff verstehe ich das enge Zusammenspiel und das in der Zeit nach dem 11.03.2011 besonders sichtbar gewordene enge Beziehungsnetzwerk zwischen Wirtschafts- und Politikmächten (genpatsu mura), das die Geschicke Japans lenkt (siehe Kapitel 5.3.1 „Das Atomdorf"). Dabei spielen Hierarchiedenken, interdependente Beziehungsnetzwerke und mangelndes politisches Bewusstsein in der japanischen Bevölkerung eine entscheidende Rolle bei der wissenschaftlichen Beurteilung der Signifikanz dieses Beziehungsnetzwerkes für den Verlauf der Katastrophe.
} 
Diese Aussagen der Künstler werden von Mundt in einem geschichtlichen Rahmen kontextualisiert, indem sie auf die Protestbewegung der 1960er-Jahre gegen die atomare Aufrüstung eingeht. In dieser Zeit hätten sich vor allem die bildenden und darstellenden Künste mit diesen Protesten auseinandergesetzt (Mundt 2013: 118). Besonders spannend für meine vorliegende Untersuchung der Haltung der Gegenwartskünstler in den Jahren 2014 und 2015 ist Mundts Darstellung (2013: 119) eines sich etablierenden Ungehorsams „gegenüber Strukturen und Autoritäten“, der sich auch in meinen eigenen Erhebungen finden lässt.

Im Bereich der japanischen Gegenwartskunst nach dem Desaster von 2011 stellen die japanologischen Untersuchungen von Richter, Gebhardt und Mundt wichtige Beiträge zur kulturellen Verarbeitung der Ereignisse dar. ${ }^{12}$ Mit Blick auf Individuum und Gesellschaft, der Reflexion von Lebenswirklichkeiten und dem Verhältnis von Kunst und Öffentlichkeit liefern sie wichtige Hinweise auf das Wirken japanischer Künstler in sozio-politischen Bereichen (siehe Kapitel 6 „Kunst und Künstler in Japan - Eine Milieuanalyse“). Die Kreativität im Umgang mit der gesellschaftlichen Situation und die Hinterfragung des dominanten Diskurses spielen dabei eine entscheidende Rolle.

Analytisch betrachtet stimmen alle hier zitierten Autoren aus den Bereichen der Soziologie, den Politikwissenschaften und der Japanologie im Wesentlichen in einem entscheidenden Punkt überein: Ein öffentlicher, nicht-staatlich dominierter Raum mit unabhängigen Medien ist entscheidend für die Artikulation und Diskussion gegenwärtiger wie auch zukünftiger Entwicklungen sowie für die sich daraus ergebenen Veränderungsprozesse.

Aus meiner Sicht sind alle sich in diesem Raum entfaltenden Aktivitäten im weitesten Sinn politisch motiviert. Meine akteurszentrierte Arbeit beleuchtet eine spezifische Perspektive auf die genannten sozio-politischen Herausforderungen. Ich zeige auf, wie Gegenwartskünstler sowohl ihre Empathiefähigkeit als auch ihre Kreativität nutzen, um die Belange der unmittelbar Betroffenen der Dreifachkatastrophe in den gesellschaftlichen Diskursen Japans dauerhaft zu platzieren.

Meine Betrachtung der Motivation des Wirkens der Künstler stellt dabei eine wichtige Ergänzung zum Verständnis des von Richter, Gebhardt und Mundt dargelegten Handelns der kulturschaffenden Akteure Japans dar. Meine Arbeit gibt Aufschluss darüber, warum und auf welche Weise Gegenwartskünstler die soziale Ordnung Japans im Post-Fukushima-Diskurs auf den Prüfstand stellen. Die von Richter und anderen Autoren genannten Aspekte ergänze ich um Facetten aus Teilbereichen des japanischen Kontextes wie die der Individualität, der Emotionalität, der Werte, der Normen, der Moral, des Alters, des Genders und der Erwartungen . Erst vor dem Hintergrund biographischer Erzählweisen werfen die persönlichen Perspektiven meiner Gesprächspartner ein Schlaglicht auf die sozialen Praktiken in Japan

\footnotetext{
12 Mit den Effekten der Katastrophe auf die japanische Medienlandschaft und die Kulturindustrie im Allgemeinen beschäftigen sich unter anderem Furukawa und Denison (2015).
} 
nach 2011 und tragen dazu bei, das entstandene „credibility gap“ (Stephens 2002: 106) zwischen Bevölkerung und Entscheidungsträgern begreifbar zu machen. ${ }^{13}$

Dazu führe ich eine „Rückkopplungsanalyse“ durch, indem ich die von Richter eingangs erwähnte Differenzierung zwischen der Naturkatastrophe (tensai) und dem menschengemachten Desaster (jinsai) berücksichtige und in einen Bezug zum emotionalen Umgang des einzelnen Gesprächspartners mit der Dreifachkatastrophe setze. Ich betrachte also nicht nur die Auswirkungen der Ereignisse vom 11. März 2011 auf die Arbeit des jeweiligen Künstlers, sondern beziehe auch dessen Aussagen bezüglich einer möglichen Veränderung in die Betrachtung seiner Selbstwahrnehmung ein. Dies ermöglicht ein tieferes Verständnis für die jeweilige selbstdefinierte Rolle meiner Gesprächspartner als Gegenwartskünstler im sozialen Gefüge Japans.

Meine Arbeit rezipiert folglich soziologische Perspektiven zu Modernität (Beck 1986) und akteursabhängigen Risikowahrnehmungen (Beck 1986; Samuels 2013; Steineck 2015) sowie aktuelle japanologische Beiträge (Richter 2012; Gebhardt 2015 u.a.) und ergänzt sie durch eine ethnopsychologische Perspektive auf individuelle Akteure vor dem Hintergrund ihrer Biographien.

\subsection{Zusammenfassung}

Die aufgeführten wissenschaftlichen Beiträge aus Soziologie, Japanologie und Philosophie, die den derzeitigen Diskursverlauf um das große ostjapanische Seebeben und seine akuten und längerfristigen sozialen Folgen diskutieren, machen eines deutlich: Die aktuellen Vorkommnisse werden von den verschiedenen Akteuren nicht nur rekonstruiert, erörtert und in einen kulturspezifischen Kontext gesetzt, sondern es werden darüber hinaus grundsätzliche ethisch-moralische Antworten auf die Fragen gesucht, die eine Atomkatastrophe in besonderem Maße aufwirft. Diese Fragen beziehen sich auf die wechselseitigen Verantwortlichkeiten zwischen einem Staat und dessen Bürgern. Ebenso eröffnet eine Atomkatastrophe einen Blick auf die ethische Neutralität von Technik, deren kulturelle Konstitution (Steineck 2015: 28) und des gesellschaftlich verteilten Risikos atomarer Verstrahlung (Steineck 2015: 40; Beck 1986). Dementsprechend vielfältig äußern sich die Autoren zur Beurteilung „Fukushimas“ im Vergleich zu Tschernobyl oder in der gesamthistorischen Beurteilung des Vorfalls. Die einen - wie Coulmas und Stalpers (2011) - fällen kein endgültiges Urteil über die Reaktion der japanischen Behörden, halten deren Maßnahmen jedoch für angemessen. Andere wiederum halten die Atomenergie im Allgemeinen vor dem Hintergrund der Geschehnisse vom 11. März 2011 für „unverantwortlich“: „In the absence of responsible agents, nuclear energy utilisation takes on the form of organised irresponsibility“ (Steineck 2015: 26).

\footnotetext{
${ }^{13}$ Die stark von einander abweichenden Wahrnehmungen der verschiedenen gesellschaftlichen Akteure bezüglich der Dreifachkatastrophe und deren Folgen werden auch als „kognitive Dissonanz“ bezeichnet (Castells 2009: 169).
} 
Die sowohl in Japan als auch international emotional aufgeladenen Debatten bringen ans Licht, wie die Verletzungen der ethischen Wurzeln durch den Umgang mit „Fukushima“ in unterschiedlichen kulturellen Kontexten wahrgenommen und diskutiert werden. An diesem Vorfall entzünden sich Grundsatzdiskussionen des gesellschaftlichen Zusammenlebens, die Fragen nach Verantwortung, Solidarität und angemessener Kompensation aufwerfen. Gleichzeitig wird die Suche nach einer angemessenen öffentlichen Repräsentation des individuellen Erlebens und emotionalen Verarbeitens der japanischen Gesellschaft in den Mittelpunkt gestellt.

Trotz aller Bemühungen seitens der Wissenschaft, die Folgen der atomaren Verstrahlung für die Gesundheit von Umwelt und Menschen in physischer wie psychologischer Weise einzuschätzen und mit soziologischen Theorien und kulturellen $\mathrm{Zu}$ gängen zu erklären, bleiben die Konsequenzen atomarer Verstrahlung schlussendlich bis auf Weiteres unabsehbar. Die Lebenspraxis des Einzelnen wird dabei in einer Weise beeinflusst, die sowohl auf individueller als auch auf gesellschaftlicher Ebene historischen Vorbildern entbehrt.

In den Beiträgen der genannten Autoren wurde deutlich, dass der Artikulationsund Aushandlungsprozess der Dreifachkatastrophe erst am Anfang steht, zu dem meine Arbeit einen entscheidenden Beitrag liefert. 


\section{Theoretische und methodische Rahmungen}

\subsection{Katastrophen und ihre Folgen - Eine theoretische Verortung}

Katastrophen, gleichgültig ob sie langsam voranschreiten wie Dürren oder einen schnellen Verlauf haben wie Erdbeben, ermöglichen einen einzigartigen Einblick in die betroffene Gesellschaft. Deren soziale Struktur und die ihr zugrunde liegenden Werte werden in einem solchen Ausnahmezustand offengelegt. Ein Desaster fordert die Gesellschaft materiell, sozial und ideologisch heraus und eröffnet den Blick für die gesellschaftlichen Prozesse im sozialen, ökologischen, kulturellen, politischen und technologischen Bereich (siehe Oliver-Smith 2002: 25-26). Dem einzigartigen Charakter eines Verarbeitungsprozesses und der Erholung nach einem katastrophalen Ereignis widmen sich Sozialwissenschaftler auf ebenso vielen Ebenen. OliverSmith und Hoffmans Definition einer Katastrophe folgend verstehe ich unter einem desaströsen Ereignis für eine Gesellschaft

„[...] a process/event combining a potentially destructive agent/force from the natural, modified, or built environment and a population in a socially and economically produced condition of vulnerability, resulting in a perceived disruption of the customary relative satisfactions of individual and social needs for physical survival, social order, and meaning." (Oliver-Smith und Hoffman 2002: 4) 
Die verschiedenen Dimensionen eines solchen Ereignisses und seiner Folgeprozesse zu erfassen und die Realitäten der Interviewpartner gleichermaßen zu berücksichtigen, stellt eine der großen Herausforderungen der Katastrophenforschung und dieser Arbeit dar. Die im Feld von meinen Gesprächspartnern geschilderten Erfahrungen müssen in einen individuellen und sozialen Kontext gesetzt und vor diesem Hintergrund von mir reflektiert werden. Dies ist im Besonderen deshalb von großer Bedeutung, weil nur auf diese Weise die sozial konstruierte Deutung der gemachten Erfahrungen in den Vordergrund der ethnologischen Arbeit treten und die Stimme der Gesprächspartner intersubjektiv nachvollziehbar hörbar werden kann. Katastrophen begreife ich demzufolge auch als Diskurse, die kulturspezifisch ablaufen und einer lokalen Deutung unterliegen (siehe Hornbacher 2013: 97): „Die Konstruktivität von Krisen und Katastrophen gründet darin, dass sie nicht einfach vorgegeben oder ,da' sind, sondern von den Menschen und Medien, die darüber berichten, gemacht werden" (Nünning 2013: 125). ${ }^{14}$

In Theorizing Disasters. Nature, Power, and Culture beschreibt Oliver-Smith (2002) die Grundhaltung der sozialwissenschaftlichen Katastrophenforschung, indem er die Naturphänomene in ihrer kulturellen Übersetzung im Zusammenhang mit Politik, Ideologie und Wirtschaft aufführt. Dazu gehören auch moralisch-ethische Vorstellungen und Glaubenssysteme bezüglich Gerechtigkeit (siehe Oliver-Smith 1996: 308). Darüber hinaus werden in der wissenschaftlichen Betrachtung von Katastrophen bei Oliver-Smith und Susanna Hoffman (2002) die lokalen Risiken in ihrer Wechselwirkung mit globalen Prozessen in Zusammenhang gebracht.

Das heißt, dass sowohl die materielle wie auch die kulturelle Entwicklung der Landschaft über die Dauer des konkreten katastrophalen Ereignisses hinaus in ihrer Prozesshaftigkeit betrachtet wird. In diesem fortlaufenden Prozess werden nicht nur die bestehenden Sozialstrukturen mit ihren Widersprüchen für den sozialwissenschaftlichen Forscher genauer analysierbar, sondern auch die gesellschaftlichen Neuverhandlungen von Ideologie, Identität, kollektiven Erinnerungen und Geschichtsschreibung beobachtbar. Dennoch sind Oliver-Smith und Hoffman (2002: 9) der Auffassung: ,[...] the role of hazards and disasters in mobilizing forces of cultural change is vastly understudied."

An den Schnittpunkten zwischen Individuum und Gesellschaft wird darüber hinaus die spezifische Verwundbarkeit von Individuen, Gruppen und Lokalitäten thematisiert. So können beispielsweise im Fortlauf einer Katastrophe aufgrund unterschiedlicher emotionaler Bewertungen ideologische, moralisch-ethische und letztendlich auch machtpolitische Instanzen in Konflikt geraten und damit Widersprüchlichkeiten in der Lebenspraxis offenbaren. Kultureller Wandel wird von Auto-

\footnotetext{
${ }^{14}$ Krisen und Katastrophen unterliegen damit einer Erzählstruktur, die Nünning (2013) auch als „Narratologie der Krise“ bezeichnet. Eine Katastrophe definitiert Nünning unter anderem durch „[...] ein bereits eingetretenes extrem ereignishaftes, irreversibles und folgenreiches Desaster“, wohingegen eine Krise einen „Zustand der Latenz“ aufweise (Nünning 2013: 126). Meiner Ansicht nach ist damit das Eintreten der Dreifachkatastrophe vom 11.03.2011 demzufolge als Katastrophe zu bezeichnen wobei die Folgen des atomaren GAUs Merkmale eines krisenhaften Verlaufs aufweisen.
} 
ren wie Oliver-Smith (1996, 2002) und Oliver-Smith und Hoffman (2002) auf der Grundlage solcher Prozesse verortet.

In jedem Fall stellen die verschiedenen Interpretationen der Ereignisse einen wichtigen Teil der Erforschung von Katastrophen dar, die über den akuten Notzustand hinaus für die Betroffenen und ihr gesellschaftliches Selbstverständnis von essentieller Bedeutung sind:

„Disasters create contexts in which power relations and arrangements can be more clearly perceived and confronted, which transforms political consciousness, shapes individual actions, and strengthens or dissolves institutional power arrangements." (Oliver-Smith 1996: 309)

Die Katastrophenforschung kann durch ein induktives Vorgehen nicht nur die kulturellen Merkmale einer Gesellschaft freilegen, sondern auch die Komplexität gesellschaftlichen Zusammenwirkens darstellen, die Widersprüche im sozialen Gefüge aufdecken und die gelebte Praxis analytisch aufbereiten. Kulturelle Transformationsprozesse einer Post-Desaster-Gesellschaft (Oliver-Smith 1996: 312) können so in Anfängen auf individueller Ebene beobachtet und bis auf gesellschaftliche Level nachvollziehbar gemacht werden.

In diesem sozialkonstruktivistischen Kontext ist die vorliegende Arbeit zu lesen, in der ich all die genannten Aspekte in ihrer Komplexität für die Dreifachkatastrophe von Japan im März 2011 untersucht und ausgewertet habe. Den Ansätzen der Katastrophenforschung (Oliver-Smith und Hoffman 2002) folgend, nähere ich mich im folgenden Abschnitt der Dreifachkatastrophe als Prozess an, in welchem das menschliche Handlungsvermögen ausgehandelt wird. Mithilfe der Diskursanalyse kann ich sowohl die aufgezeigten Themen in ihrer Prozesshaftigkeit bei der Verhandlung zwischen einzelnen Akteuren und den gesellschaftlichen Strukturebenen aufzeigen als auch den Rückfluss der Diskurse in den Darstellungen der jeweiligen biographischen Narrative verdeutlichen.

\subsection{Diskursanalyse}

Wie bereits angesprochen, gehe ich in der vorliegenden Arbeit von sozialkonstruktivistischen Prämissen aus, das heißt ich leite die Sinnhaftigkeit der Ereignisse rund um den 11. März 2011 im gesellschaftlichen Gefüge vor Japans kulturspezifischen Hintergrund her. Auf der sozialkonstruktivistischen Annahme basieren viele theoretische Konzepte, die ich zum Verständnis der Narrative meiner einzelnen Gesprächspartner und der Ereignisse selbst als zielführend erachte. Als besonders aufschlussreiche theoretische Perspektive betrachte ich dabei die Diskursanalyse, die ich im Folgenden in ihren Grundannahmen darstelle. Dabei beziehe ich mich vor allem auf die sozialwissenschaftliche Perspektive von Jørgensen und Phillips (2002), da diese beiden Autoren in ihrer Betrachtung der Diskursgestaltung und dessen Ablauf 
bereits die für mich zentralen Konzepte des Selbst, der Emotion und deren Auswirkungen auf die soziale Interaktion aufgreifen. Ergänzende Beiträge aus den Bereichen der Ethnopsychologie - Lutz und Abu-Lughod (1990) und Geoffrey White (1990, 1994) - sowie der interpretativen Ethnologie - Geertz (1983) - werden in den entsprechenden Abschnitten ebenfalls hinzugezogen.

Unter Diskurs verstehe ich Artikulationswege, die „[...] not neutrally reflect our world, identities and social relations but, rather, play an active role in creating and changing them" (Jørgensen and Phillips 2002: 1). Es ist wichtig, das konstruktivistische und wechselseitige Element der Artikulation einzubeziehen. In der Äußerung entsteht erst die soziale Welt meiner Gesprächspartner, deren individuelles gesellschaftliche Setting ich beleuchte. Die „Kunst“ verstehe ich im Folgenden als eine der Sprach- bzw. Kommunikationsformen, die der Diskurs annehmen kann. Die in diesem Diskursraum agierenden Individuen nutzen den Nachklang der Ereignisse vom März 2011 verstärkt, um den japanischen Diskursraum aus einem anderen Blickwinkel zu beleuchten. Die Kunst als Teil des öffentlichen Diskurses ist damit einer der kulturellen Ordnungsräume (Birkhan 2012: 70), der sich einer gewissen Ausdruckweise, also der Sprache, bedient. Gerade die Sprache bildet bei Foucault als Vertreter der kontextualen-konstruktivistischen Position ein ganz entscheidendes Element bei der Strukturierung des Diskurses: „Die Formation der Aussagen einer diskursiven Praxis wird durch eine Gesamtheit von Beziehungen, Regeln oder Normen charakterisiert. [....] Dabei betont Foucault besonders, dass das Subjekt im Bereich des Wissens angesiedelt und eine davon abhängige Figur ist [...]" (Birkhan 2012: 131-132).

In der sozialen Praxis wird folglich das Wissen des Individuums widergespiegelt und in Abhängigkeit von seinen Beziehungen im sozialen Gefüge diskursiv abgebildet. Anhand des geführten Diskurses lassen sich dementsprechende Rückschlüsse sowohl über das Subjekt selbst wie auch über das gesamtgesellschaftliche ,Beziehungsnetzwerk' (Foucault nennt es ,Formationssystem') von Aussagen treffen. Die Bedeutung der Sprache für das Artikulieren eines Sachverhaltes stellt Foucault so dar: „Es [Das Formationssystem] schreibt vor, was in einer diskursiven Praxis in Beziehung gesetzt werden musste [...]" (Foucault 1973: 108-109), um Begriffe, Gegenstände und Sachverhalte in den Diskurs einzuführen und/oder ihnen entsprechende Bedeutung zukommen zu lassen. Es ist diese Verbindung von Wissen und dessen Artikulation, die die Wichtigkeit der Position des Individuums in einem Beziehungsnetzwerk verdeutlicht. Zur Erinnerung: Ich verwies in der Einleitung dieser Arbeit auf den Soziologen Beck (1986: 30), der auf einen „sozialen Definitionsprozess“ bei der Bewertung von Wissen aufmerksam machte. Durch die diskursanalytischen $\mathrm{Zu}$ gänge wird deutlicher, dass es erst die Position des Individuums ist, die dem Gesagten die entsprechende Macht verleiht und folgerichtig den „sozialen Definitionsprozess“ entscheidend prägen kann:

„[...] Aussagen haben ihr Gewicht als Äußerung von Personen mit spezifischem Status, sind an bestimmte Institutionen gebunden, haben dadurch Geltung, 
werden dort gebildet oder gesammelt. Es sind epistemologische Positionen für das Subjekt eines Diskurses festzustellen, die die Wissensproduktion bestimmen. Ein konkreter Diskurs artikuliert sich über das Zusammenwirken der Äußerungsmodalitäten, deren spezifisches Beziehungssystem er als Praxis herstellt [...].“ (Birkhan 2012: 191-192)

Die Beziehungen zwischen den einzelnen Akteuren bestimmen dementsprechend die Zusammenhänge zwischen Wissensquellen und üben eine entsprechende Macht auf die Diskursführung aus. Wie genau dabei die Begriffsbildung und die Organisationsform der Beziehungen entsteht, ist Gegenstand der Diskursanalyse.

Wie bereits angedeutet, spielt die Macht des Einzelnen in diesem Beziehungsgeflecht eine Rolle, die dazu führt, dass bestimmte Gegenstände in den Diskurs einfließen und andere nicht. Die Ethnologin Birkhan (2012: 193) beschreibt hier einen gelenkten Filtermechanismus durch Entscheidungsinstanzen, der die Vielfalt der diskursiven Realisierung eingrenzt. Dieses Prinzip der Knappheit in der Artikulation macht laut Foucault (1973) wiederum die Spezifität einer Kultur aus.

Für die vorliegende Arbeit ergeben sich daraus folgende Fragen: Welche Sachverhalte werden in den Post-Fukushima-Diskursen artikuliert und welche nicht? Was erfährt besondere Betonung, während anderes beiläufig mitschwingt? Welche Machtinstanzen haben die japanischen Diskurse bisher gestaltet und wie wird dies in der Retroperspektive von meinen Gesprächspartnern bewertet? Wie verändert sich daraufhin die Wahrnehmung ihrer Subjektivität im sozialen Gefüge Japans und welche Handlungsoptionen ergeben sich für sie daraus?

Vor dem Hintergrund kulturspezifischer Subjektivität und Artikulationsweise betrachte ich die Form der Wissensproduktion meiner Gesprächspartner unter Berücksichtigung ihrer Position im gesellschaftlichen Kräfteverhältnis. Ich gehe davon aus, dass die Produktion von Wissen eng mit der Herstellung von Macht verbunden ist. Die Genese einer Diskontinuität im Diskurs stellt damit aus meiner Sicht eine besonders reizvolle Perspektive dar. Birkhan formuliert dies wie folgt:

„Für die Formation von Aussagen zu Diskursen, also die Etablierung von Regelmäßigkeiten im Existenzmodus der Diskontinuität, ist entscheidend, dass Aussagen beschränkt und selektiert werden, also die Seltenheit von Aussagen und dass sich umgekehrt diese wenigen Aussagen wiederholen, ähnliche Aussagen sich häufen, akkumulieren. Ihr Wert ergibt sich dabei über die Stellung im differentiellen Kontext." (Birkhan 2012: 225)

In der sozialen Praxis wird die zur Verfügung stehende Sprache entsprechend der jeweiligen kulturspezifischen Regeln subjektiv eingesetzt. Es ist folglich die Praxis, die den Umgang des Sprechers mit diesem Regelwerk widerspiegelt.

Es stellen sich also zusätzlich zu den oben genannten Fragen noch folgende weitere: Wo werden von meinen Gesprächspartnern innergesellschaftliche Grenzen gezogen und/oder aufgelöst? Vor welchem Wissenshintergrund geschieht dies und 
welche Folgen könnten diese Grenzen für die Post-Fukushima-Diskurse in Japan haben?

Die Sprechweise des Individuums vermag es, Widersprüche in den Diskursen aufzudecken, deren Auswirkungen laut Birkhan (2012: 236) ganz unterschiedlich sein können: „Sie können eine Dynamik additioneller Entwicklung implizieren, eine Neuorganisation bewirken oder eine kritische Funktion haben, die Existenz und Akzeptabilität des Diskurses aufs Spiel setzt." Diese diversen Optionen der Einflussnahme auf den diskursiven Verlauf beleuchte ich in der vorliegenden Analyse anhand des konkreten Falles der Gegenwartskünstler Japans. Den diskursanalytischen Zugängen folgend, berücksichtige ich dabei die spezifische gesellschaftliche Rolle der von mir untersuchten Akteursgruppe für die gegenwärtigen Diskursverläufe. $\mathrm{Zu}$ diesem Zweck untersuche ich ihre Verortung im japanischen Sozialgefüge sehr genau (siehe Kapitel 6 „Kunst und Künstler in Japan - Eine Milieuanalyse“).

Auf der Basis der Akteursverortung im Beziehungsnetzwerk zeigen sich auch die Transformationspotentiale, die den Diskursen innewohnen: Jørgensen und Philipps zufolge können Diskurse dazu beitragen, die Wege zu entdecken „[... in which people's selves, thoughts and emotions are formed and transformed through social interaction and to cast light on the role of these processes in social and cultural reproduction and change" (Jørgensen und Phillips 2002: 7).

Die Möglichkeiten zur Veränderung der Diskurse verorte ich demnach auf der Ebene des Individuums, das mittels des ihm zur Verfügung stehenden Artikulationsweges in eine wechselseitige Beziehung zum öffentlichen Diskurs und damit zur Gesellschaft tritt. So betrachtet repräsentiert der Begriff des Diskurses den Raum, das Medium und das Machtinstrument gesellschaftlicher Aushandlungsprozesse. Aufgrund dieser Eigenschaften von Diskursen betrachte ich die Kunst als wichtiges Kommunikationsmittel dieses gesellschaftlichen Prozesses und untersuche ihre Funktion für die Bereicherung des Diskurses mit verschiedenen Perspektiven.

In Kapitel 5 werde ich mich mit der Katastrophe von 2011 und ihren Folgen auseinandersetzen. Dabei wird die Wahrheit, wie sie von den einzelnen Akteuren in ihrer Pluralität definiert wird, zum Maßstab für die moralisch bewertete "richtige“ Nutzung von Macht- und damit von Wissensressourcen. In der Betrachtung des derzeitigen Wissensstandes zum Thema Dreifachkatastrophe und „Fukushima“ in Kapitel 2 habe ich bereits darauf verwiesen, dass sich soziologische Betrachtungen mit Emotionalität und ethischen Grundsatzfragen in den japanischen Diskursen um „Fukushima“ verbinden. In den folgenden Abschnitten führe ich nun elementare Begrifflichkeiten ein, die für die Wirkkraft der verschiedenen Akteure auf die öffentlichen Diskurse und deren Gestaltung von entscheidender Bedeutung sind. $\mathrm{Zu}$ diesen Begriffen gehört insbesondere die Wahrheit als tragende Säule der Machtstruktur in den japanischen Post-Fukushima-Diskursen: Wer Anspruch auf die wahre Deutung der Ereignisse erhebt, bestimmt den „sozialen Definitionsprozess“ im Sinne Becks (1986). Wie oben ausgeführt, ist jedoch die Position des Einzelnen entscheidend für die Machtentfaltung der entsprechenden Artikulation. Daher nehme ich mich den Termini des „Selbst“ als individuelle Verortung im sozialen Gefüge ebenso an wie 
der Politik als Austragungsraum für gesellschaftliche Kämpfe um diskursive Macht. Der „Wahrheit“ als wichtiges Instrument dieses Machtkampfes widme ich mich anschließend.

\subsubsection{Der Diskurs des Selbst - Die Herstellung des Selbst im Diskurs}

Die Katastrophen-Narrative der Gegenwartskünstler betrachte ich vor dem Hintergrund der biographischen Konstruktion des einzelnen Gesprächspartners. Es ist zu beachten, dass mir meine Gesprächspartner nicht nur Informationen über die Ereignisse und deren Verarbeitungen vermittelten, sondern auch ihren jeweiligen individuellen Bewertungsrahmen für die Ereignisse in ihrem Leben. Dabei schwangen implizite Annahmen über die Beschaffenheit von Selbst und Person als kulturspezifische Konzepte mit, die ich in Kapitel 4.1 „Der kulturspezifische Ausdruck des Selbst im sozialen Gefüge" ausführe.

Auf theoretischer Ebene bilden die Zusammenhänge von Macht und Selbst die Perspektive vieler bedeutender Werke von Autoren wie Foucault $(1973,1974)$ und Bourdieu (1987). Identität, Subjektivität, Hegemonie und ihre Wechselwirkungen stehen dabei jeweils in unterschiedlichen, wechselseitigen Beziehungen zueinander. Sie versetzen je nach erkenntnistheoretischem Hintergrund das Subjekt der Macht entweder in eine mögliche Opposition durch Handlungsvermögen (agency) oder rücken das Selbst in einen Rahmen geprägt von Wissen und Erfahrung, der entscheidend dazu beiträgt, dass Hegemonien internalisiert und naturalisiert werden.

In meiner Verwendung der Begrifflichkeiten des „Selbst“ und von „Person“ beziehe ich mich auf den ethnopsychologischen Ansatz, der keine scharfe Trennlinie zwischen diesen beiden Begrifflichkeiten zieht (siehe Hermann und Röttger-Rössler 2003). Mit meinem Fokus auf biographische Narrative betrachte ich das Selbst grundsätzlich als sozialen Akteur, der „[...] gemäß der jeweiligen sozio-kulturellen Vorgaben Handlungsvermögen ausüben" kann (Hermann und Röttger-Rössler 2003: 8). Aus der ethnopsychologischen Perspektive werden das Selbst und die Person in der konkreten Interviewsituation konstruiert. Dies ist eine soziale Praktik, die sowohl die Vergangenheit, Gegenwart und, im vorliegenden Fall, auch die Zukunft umfasst. Die Macht, die diese soziale Praxis des Erzählens entfaltet, wirkt sowohl auf das Individuum selbst als auch im gesellschaftlichen Kontext. Diesem Aspekt gehen Jeannette Marie Mageo und Bruce M. Knauft in ihrer „Introduction: Theorizing Power and the Self“ (2002) nach. Ihre dem Selbst zugrunde liegende Definition fällt wie folgt aus:

„Self we take to be as an encompassing domain term that includes within it virtually all aspects of personhood and subjectivity. The self is constituted by acts of identification with internal elements of experience and with persons, groups, and representations in the cultural world.“

(Mageo und Knauft 2002: 3) 
Die Autoren betonen die wechselseitige Konstitution von Macht und Selbst in der aktiven Aushandlung. So wird der Einzelne zwar durch Macht (bspw. einem Diskurs) beeinflusst, kann jedoch durch Widerstand seine eigene Macht zur Geltung bringen. Die Machtstrukturen im Sinne Foucaults werden also verinnerlicht, werden aber gleichzeitig durch die Subjekte einer Gesellschaft geformt, transformiert und umgewandelt.

Mageo und Knauft (2002: 3) zufolge werden dabei Gefühle und Erfahrungen mit bereits existierenden Werten und Mächten abgeglichen - und entweder angenommen oder abgelehnt. Dies ist ein weiteres Argument für die Fokussierung meiner Analyse auf den Vorgang der Selbstkonstruktion: Das Selbst wird zum Ausgangspunkt für das ethnologische Verständnis von Wahrnehmung, Erfahrungen und Handlungsmöglichkeiten. Die dabei entstehenden Realitäten „[...] are created and recreated by human choices at the same time that human beings are shaped by political ideologies incorporated as modes of thinking, feeling, relating, performing, and embodiment" (Mageo und Knauft 2002: 8).

Die Verinnerlichung von gesellschaftlichen Bewertungskriterien für Gefühle und Erfahrungen stabilisiert also eine bestimmte Realitätsvorstellung und legitimiert gleichzeitig eine Form der Machtausübung wie beispielsweise die der Politik. Das Individuum baut auf diese grundlegenden Bewertungskriterien sein Selbst auf und verbindet sie auf diese Weise mit gesellschaftlich geltenden Moralvorstellungen. Taylor (1989), politischer und sozialwissenschaftlicher Philosoph, behandelt diesen Aspekt des kulturspezifischen Selbst in seinem Werk zu Sources of Self-The Making of Modern Identity. Darin geht er auf den Herstellungsprozess von Bedeutung des Lebens für den Einzelnen als ein spezifisch modernes Phänomen der westlichen Gesellschaften ein. So ist Taylor der Ansicht, der persönliche Ausdruck sei von entsprechender Bedeutung für das individuelle Selbst und sei aufs Engste mit der individuellen Interpretation des Status quo verbunden: „It depends on the modern sense, invoked in the previous section, that what meaning there is for us depends in part on our powers of expression, that discovering a framework is interwoven with inventing" (Taylor 1989: 22).

Die Wahrnehmung bestimmt dabei in hohem Maße die Realität ihrer Subjekte. Mageo und Knauft betrachten die Inkorporierung von hegemonialen Kräften im foucaultschen Sinne, berücksichtigen aber ebenso die dem Menschen innewohnende Fähigkeit der Vorstellungskraft („Imagination“) und ihrer Wirkung auf die Innovation und Neuinterpretation des Selbst und der Möglichkeit zum Widerstand. Mageo und Knauft beschreiben „,...] that forms of agency are intimately bound-up with the human capacity to innovate upon if not to reimagine existing schemata; these innovations and reimaginings are integral to the activity of self-making" (Mageo und Knauft 2002: 8). Damit stehen sie im Einklang mit den kulturwissenschaftlichen Betrachtungen von Collier und Lakoff (2005), die ebenfalls die Wahrnehmung als handlungsinitiierenden Faktor bestimmen.

Um mich dem Verständnis von Handlungsvermögen (agency) als Ausgangspunkt für gesellschaftliche Veränderungen zu nähern, einschließlich des sich möglicherwei- 
se daraus generierenden Widerstands gegen bestehende Regeln und Konventionen, beziehe ich mich auf die Kulturanthropologin Ortner (2006). Ihre Betrachtungen zu Handlungsvermögen auch außerhalb institutionalisierter Formen spielen beim Engagement der Künstler und ihres Anspruches an sich selbst eine besondere Rolle. Ortner argumentiert, dass Widerstand als eine Form von Handlungsvermögen (agency) die Präsenz und Wirkungsweise von Macht in Beziehungen erhellt (Ortner 2006: 44). Dies schließt alle Ebenen der Gesellschaft ein und eröffnet ein transformierendes Potential: In einer Machtbeziehung habe der Dominante stets etwas anzubieten, wenn auch zu einem Preis (Ortner 2006: 45). Jeder Einzelne jedoch kann sich dazu entscheiden, diesen Preis nicht mehr zahlen zu wollen.

Das auf diese Weise angestoßene Ausdehnen des allgemein gesellschaftlich akzeptierten und legitimierten Diskursraumes auf unangenehme, da zur Diskussion anhaltende Art und Weise stellt einen wichtigen Meilenstein bei der Etablierung und Legitimierung eines öffentlichen Raumes dar, der die politischen Akteure im demokratischen Sinne in die Verantwortung zu nehmen vermag. ,[...] art sometimes produces social lectures uncomfortably resistant to interpretation, containing complex significations and seeming to embody uglification and threat" (Rosler 1994: 56). Die bei der Künstlerin und Kulturkritikerin Rosler anklingende Bedrohung des Status quo durch Kritik an gesellschaftlichen Zuständen steht im engen Bezug zum Leben des Künstlers im Allgemeinen, wie ich in den Analysekapiteln weiter ausführen werde. Ortner hingegen bezieht die Bedrohung oder den Konflikt nicht nur auf eine nach außen wirkende Kraft von agency, sondern verortet sie auch im Inneren: „Finally, individual acts of resistance, as well as large-scale resistance movements, are often themselves conflicted, internally contradictory, and affectively ambivalent, in large part due to these internal political complexities" (Ortner 2006: 48-49).

In Verbindung mit den Ausführungen zum Diskurs anhand von Jørgensen und Phillips (2002) sowie Birkhan (2012) habe ich bereits hervorgehoben, dass die Interpretation der Inhalte solcher Diskurse zu ganz entscheidendem Anteil von persönlichen Erfahrungen, Selbst-Konzepten und den sich daraus ergebenden moralischen Vorstellungen abhängen. Es ist erst das Selbst, das den verschiedenen Konstruktionen von Historizität und Wahrheit die notwendige Kontinuität, den Sinnzusammenhang und die diskursive Macht verleiht. Die enge Beziehung zwischen Selbst und Diskurs ist folglich von entscheidender Bedeutung bei der Analyse der Aussagen meiner Gesprächspartner und den diesen zugrunde liegenden Selbst-Narrativen.

Die Beziehung zwischen Selbst und Diskurs bildet die Ausgangsbasis für meine Überlegung, die Aussagen meiner Gesprächspartner in diesen Kontext einzuordnen und Foucaults Anspruch an die Diskursanalyse gerecht zu werden, „[...] diese materiellen und diskursiven Bedingungen ihres Auftretens freizulegen" (Sarasin 2006: 107). In Verbindung mit dem biographischen Kontext kann die Frage beantwortet werden, warum bestimmte Aussagen in einer bestimmten Weise getätigt wurden und nicht anders (siehe Swann 1983). Die Platzierung der Artikulation in einem bestimmten Kontext spiegelt dabei die Macht des Diskurses wider: „Er [der Diskurs] 
ist dasjenige, worum und womit man kämpft; er ist die Macht, deren man sich zu bemächtigen such"“ (Foucault 1974: 11).

Diese von Foucault und Anderen beschriebene Verknüpfung von Diskurs, Macht, Wissen und Moral bezieht sich auch auf die Subjekte selbst: „Discourses contribute centrally to producing the subjects we are, and the objects we can know something about (including ourselves as subjects)" (Jørgensen and Phillips 2002: 14). Es handelt sich hierbei also um eine Denkrichtung, die im Vergleich zu Taylor (1989) die umgekehrte Wirkungsweise in den Fokus nimmt: Hatte Taylor sich vor allem auf die Genese eines Selbst in einem diskursiven Kontext fokussiert, betrachten Jørgensen und Phillips (2002) die Auswirkungen des Diskurs auf das Selbst.

„Ein Formationssystem in seiner besonderen Individualität zu definieren heißt also, einen Diskurs oder eine Gruppe von Aussagen durch die Regelmäßigkeit einer Praxis zu charakterisieren. [...] sie setzt das Artikulationsprinzip zwischen einer Serie von Ereignissen, von Transformationen, von Veränderungen und Prozessen fest." (Foucault 1973: 108-109)

In meinem Verständnis betont die Generierung eines Subjekts im und durch den Diskurs demzufolge zwei Dinge, die für meine Analyse eine besondere Rolle spielen:

1. Die Perspektive der Historizität des Selbst, das heißt zum einen, das jeweilige Individuum als „Kind seiner Zeit“ im historischen Kontext einzuordnen, und zum anderen, die Generierung der persönlichen Geschichte des jeweiligen Gesprächspartners in der Betrachtung seines sozialen Umfelds, seiner Handlungsmöglichkeiten und gesellschaftlichen Rahmungen in der Analyse zu berücksichtigen, und

2. sich als Forscher für die Transformationspotentiale des jeweiligen Akteurs durch die wechselseitigen Wirkungen der gesellschaftlichen Diskurse zu sensibilisieren. Das heißt, sich wandelnde Begrifflichkeiten und Bedeutungszuschreibungen in ihrer gesellschaftshistorischen Genese zu betrachten.

\subsubsection{Die Politik im Diskurs}

Wie ich noch darlegen werde, wird die wechselseitige Wirkung zwischen der individuellen und gesellschaftlichen Ebene von meinen Gesprächspartnern in besonderer Weise genutzt, um ihre Anliegen in den japanischen Diskurs einzubringen. Die Aufsprengung des diskursiven Rahmens durch ihre kreative Arbeit nach 2011 stellt, mal mehr mal weniger explizit, ein erklärtes Ziel meiner Gesprächspartner dar. Durch ihren Beitrag zu den Katastrophendiskursen, insbesondere aber zu den Diskursen um „Fukushima“, stellen sie einen Zugang zu den Vorkommnissen auch für nicht unmittelbar Betroffene her. Die Gegenwartskünstler ermöglichen damit eine Beteiligung Betroffener an der parallel ablaufenden Herstellung einer Realität nach „Fu- 
kushima“. Ich sehe darin Jørgensens und Phillips (2002: 34) Aussage untermauert, die Artikulation der Aushandlungsprozesse auf politischer Ebene „[...] determine how we act and think and thereby how we create society."

Meiner Auffassung nach sind damit auch politische Prozesse als eine Form der Gesellschaftsgestaltung gemeint. Jørgensen und Phillips verstehen Politik als artikulativen Ausdruck sozialer Organisation und damit als eine Form des diskursiven Prozesses. „Reproduction and chance of meaning ascriptions are, in general terms, political acts" (Jørgensen and Phillips 2002: 36). Diese sehr breit gefasste Politikdefinition lässt eine enge Verbindung zwischen einem individuellen Akteur und der Strukturebene zu. Sie erlaubt darüber hinaus konzeptionelle Freiräume für die sich auf allen gesellschaftlichen Ebenen auswirkenden Artikulationen und damit auch für potentielle Veränderungen. Ich vertrete die Ansicht, dass meist erst das Sichtbarwerden von alternativen Diskursen einen Blick auf ablaufende Steuerungsversuche freigibt, die Objekte, Subjekte, wissenschaftlichen „Fakten“ und Symbole betreffen.

Aus theoretischer Perspektive vergrößert meist ein Großereignis wie eine Katastrophe den Raum für alternative Diskursfacetten, die bislang zwar vorhanden waren, aber keine Hauptrollen spielten. Dass auf hoher Ebene getroffene Maßnahmen kritisierbar werden, legt den diskursiven Charakter der Gesellschaftsstrukturen frei und macht sie anfälliger für Transformationsbestrebungen. Akteure, die in dieser Weise aktiv sind, arbeiten damit gegen eine Naturalisierung einer einzigen Perspektive in den Diskursen an. Das bedeutet, dass solche Akteure gegen den Ausschluss anderer Narrative, die vor dem Event stark von einer staatlichen Öffentlichkeit dominiert wurden, arbeiten. ${ }^{15}$ Jørgensen und Phillips verdeutlichen, warum die Diskurs- und Machtfrage eine solch wichtige Rolle in der Betrachtung von Gesellschaften spielt: „It is power that creates knowledge, our identities and how we relate to one another as groups or individuals" (Jørgensen und Phillips 2002: 37). Die von Taylor aufgezeigte Verbindung von Identität, Macht und Diskurs lässt mich annehmen, dass Aussagen einen bestimmten Diskurs betreffend immer auch Artikulationen des eigenen Selbst beinhalten, die bei genauer Betrachtung Hinweise auf die Selbst-Narrative und die Frage „Wer bin ich?“ einschließen.

In einen konkreten Katastrophenkontext übertragen, kann die diskursive Interpretation des wissenschaftlichen Faktenwissens und die Beschäftigung mit den unmittelbar Betroffenen in den Fokus der öffentlichen Aufmerksamkeit rücken und Druck auf die mächtigen Instanzen ausüben. Die verschiedenen Akteure können sich aufgrund dessen zunehmend in die Pflicht genommen sehen, sich dieser Themen anzunehmen und sie auch in den Diskursen zu behandeln.

${ }^{15}$ Siehe Kapitel 5.3.2 „Medien, die staatliche Öffentlichkeit und ihre Alternativen“. 


\subsubsection{Das Ringen um diskursive Macht}

Die enge Verbindung zwischen Macht und Beziehungen habe ich bereits erörtert. Der nun folgende Abschnitt beschäftigt sich mit der Beeinflussung des weitläufigen Diskurses durch Beziehungsnetzwerke. So kann unter anderem die öffentliche Meinung, welcher ich eine wichtige Rolle im Diskurs einräume, stark von Einschätzungen von machtvollen Akteuren wie Experten, Politikern und Wirtschaftsvertretern abhängen. Umso wichtiger ist die Diversität der Artikulationen, die einer zu starken Vereinfachung der Bedeutungszuschreibung von Erfahrungen, „Wahrheiten “16 und Emotionen entgegenwirken. Nicht zuletzt deshalb geht der Soziologe Castells (2009: 3) in seinem Werk davon aus, dass die Macht der Kommunikation das Herzstück einer jeden Gesellschaft und ihrer Dynamiken ist. Die Kommunikation ist demnach der Kanal, durch den Macht fließt und ihre Wirkung bei den Individuen einer Gesellschaft entfaltet. Wie oben bereits erörtert, wird dies in besonderem Maße bei der Aushandlung gesellschaftlicher Entwicklungen deutlich, zum Beispiel in der Politik. Demnach bilden Institutionen und Diskussionsplattformen als Schnittstelle zwischen Metaebene und "grass-root-level“ den Raum für die Äußerung von Ansichten, Perspektiven und Werten. Die Möglichkeiten des Aufeinandertreffens von Individuum und sozialer Struktur sind die Aushandlungsbasis des gesellschaftlichen Zusammenlebens. Die einzelnen Mitglieder einer Gesellschaft bilden dabei „Netzwerke der Kommunikation" (Castells 2009: 4), die zur Vermittlung von verschiedenen diskursiven Positionen beitragen. Die Ausformung der Kommunikationsnetzwerke sowohl innerhalb als auch außerhalb eines institutionalisierten Rahmens geben wichtige Hinweise auf die Diversität des betreffenden Diskurses.

Meine Analyse zeigt anhand der von mir ausgewählten Akteursgruppe exemplarisch auf, wie Artikulationskanäle generiert und genutzt werden, um sich beispielsweise mit den Werten einer Gesellschaft auseinanderzusetzen und Hoffnungen für politische Veränderungen zu inspirieren (siehe Castells 2009: 8). In Verbindung mit den aufgeführten Quellen von diskursiver Macht, beispielsweise Beziehungsnetzwerken, wird das „empowerment" des Einzelnen spürbar: „Power is the relational capacity that enables a social actor to influence asymmetrically the decisions of other social actor(s) in ways that favor the empowered actor(s) will, interests, and values" (Castells 2009: 10).

Bei der Betrachtung des individuellen Akteurs wird die Verbindung zwischen der Artikulations- und Kommunikationsfähigkeit des Einzelnen und der Verteilung von Macht innerhalb einer Gesellschaft deutlich. Castells (2009) veranschaulicht in seinen Ausführungen, dass sich das jeweilige Individuum auch außerhalb eines in-

\footnotetext{
${ }^{16}$ Ich setze „Wahrheit“ bewusst in Anführungszeichen, um drauf zu hinzuweisen, dass es keine einzige, allgemeingültige Definition von Wahrheit gibt. Ich gehe davon aus, dass es so viele Realitäten und "Wahrheiten“ wie Personen gibt und dass jede Biographie Aufschlüsse über die Herleitung der persönlichen Wahrheit gibt.
} 
stitutionellen Rahmens Handlungsvermögen aneignen kann und damit seine eigene Macht entfaltet.

Vor dem Hintergrund dieser Überlegungen betrachte ich alle von den japanischen Post-Fukushima-Diskursen aufgegriffenen Themen unter dem Vorzeichen der Kommunikation: Welcher Akteur artikuliert was zu welchem Zeitpunkt und warum? Inwiefern werden darin Realitätsvorstellungen deutlich, die die Diskursrahmen anderer Akteursgruppen beeinflussen? Was sagen diese Vorstellungen von der Welt über das Individuum und seine Selbstwahrnehmung aus?

Da in einer Demokratie die Herstellung einer möglichst mehrheitlich getragenen Realität das politische System legitimiert, bestimmt nicht zuletzt das Ringen um die öffentliche Meinung das Handeln der Akteure. „The public mind“, wie Castells sie nennt, „[...] is ultimately what influences individual and collective behavior [...]" (Castells 2009: 53). In diesem Zitat klingt bereits an, dass Akteure die Bedeutung, die Ereignissen, Erfahrungen, „Wahrheiten“ und den damit zusammenhängenden Emotionen zugeschrieben werden, im öffentlichen Diskurs stark diversifizieren können. Damit gefährden Akteure gleichzeitig das Monopol auf die Beeinflussung des Diskurses durch mächtige Beziehungs- und Kommunikationsnetzwerke, die beispielsweise wirtschaftspolitischer Art ${ }^{17}$ sein können.

Am Beispiel des Ringens um die Definitionsmacht von „Wahrheit“ werde ich diesen Punkt noch genauer ausführen. Er bildet die Basis zum Verständnis um die diskursive Praxis in Japan nach der Dreifachkatastrophe und den sich auf individueller Ebene zeigenden Emotionen.

\subsubsection{Der Zusammenhang von Wahrheit und Biographie}

In diesem Abschnitt geht es mir um ein wichtiges Kernelement der japanischen Diskurse, das in der Folgezeit nach dem 11. März 2011 an Bedeutung gewonnen hat. Die „Wahrheit“ als Konstruktion erfährt insbesondere während der Aufarbeitung von Katastrophen eine erhöhte Aufmerksamkeit. Die sich daraus ergebenden Chancen für die Wandlung der dominanten Diskurse stehen vor der Herausforderung, eine gewisse Trägheit auszuhalten und zu überwinden: „Both people and society are understood as historical phenomena that are compelled to work on the basis of the existing structures, presupposing and ensuring continuity in the social“ (Jørgensen and Phillips 2002: 38).

Zuvor habe ich bereits veranschaulicht, wie eng Diskurs, Politik, gesellschaftliche Macht und das Selbst miteinander verbunden sind. Was in diesem konstant stattfindenden Aushandlungsprozess zwischen den verschiedenen gesellschaftlichen Ebenen als „Wahrheit“ definiert wird, ist entscheidend für die Selbstpositionierung des Individuums im sozialen Gefüge und dessen Beurteilung seines Handlungsvermögens. Für die Betroffenen ist es eine Herausforderung, ihren neuen Alltag in den Katastrophendiskursen zu platzieren. Für sie zeigt sich hier, ob die Werte der Gesell-

\footnotetext{
17 Siehe Kapitel 5.3.1 „Das Atomdorf (genpatsu mura)“.
} 
schaft wie Solidarität auch tatsächlich gelebt werden. So bemessen beispielsweise meine Gesprächspartner ihren beruflichen Erfolg daran, ob sie in der Auseinandersetzung mit sozialen, gesundheitlichen und wirtschaftlichen Folgen des ostjapanischen Erdbebens die für sie relevanten Themen im Diskurs platzieren und halten können. Auf diese Weise werden Bereiche, Themen und Realitäten in Diskursen angesprochen, die bis vor einigen Jahren noch in der Peripherie des öffentlichen Raumes existierten, so unter anderem psychologische Phänomene sowie die Diskussion wichtiger Identitätsaspekte wie „Heimat“ und Stigmatisierung. Die entsprechend aktiven Akteure nehmen also Einfluss auf die bis dato geltende diskursive Praxis und decken ein wesentliches Element der Diskursführung auf, das Foucault (1974: 16) mit „dem Willen zur Wahrheit“ umschrieb: Die Wahrheit für sich zu beanspruchen ist ein Ausschließungsmechanismus, der ,[...] dazu tendiert, auf die anderen Diskurse Druck und Zwang auszuüben“" (Foucault 1974: 11).

Wie im vorangegangenen Kapitel bereits beschrieben, ist die Konstruktion „der Wahrheit" mit einem Machteffekt verbunden. Folglich ringen viele parallel existierende Wahrheitsdefinitionen um die Dominanz in Diskursen und der damit einhergehenden Macht, einen Bewertungsmaßstab zu setzen. „Denn ohne derartige Machtwirkungen der ,Wahrheit' gibt es keinen der Kritik entzogenen Grund für die Aktualisierung einer bestimmten und den Ausschluss anderer diskursiver Tatsachen und auch keinen Maßstab für die richtige Repräsentation" (Birkhan 2012: 314 315). Die Wahrheiten und Tatsachen haben einen sozialen Wert und befinden sich im ständigen wechselseitigen Austausch im Ringen um die Definitionsmacht (siehe Birkhan 2012: 316-317). Dementsprechend geht es in Diskursen „der Wahrheit“ immer auch um Diversität, deren Artikulation und den dabei stattfindenden Aushandlungsprozess zwischen den entsprechenden Akteursgruppen.

Meine Arbeit beleuchtet folglich die aktuellen japanischen Diskurse mit Blick auf eine spezifische Akteursseite und deren Wahrheitskonstruktion. Ich erörtere wichtige, diskursiv geprägte Begriffe wie „Selbst“ und „Emotion“ ebenso wie ich den gesellschaftshistorischen Hintergrund eines „Gegenwartskünstlers“ in seiner sozialen Position betrachte. Die Verortung des Sinns und der Bedeutung der Konstruktion der eigenen Lebensgeschichte im Zusammenhang mit den aktuellen Ereignissen liegt dabei beim Individuum (siehe Birkhan 2012: 330).

\subsubsection{Zusammenfassung}

Der Diskurs als Konzept bietet eine hervorragende Möglichkeit, den Austragungsraum des aktuellen kulturellen Ringens um Bedeutungszuschreibungen und Artikulationen zu beschreiben, die sich auch auf Aspekte wie die Identitätskonstruktion auswirken. Bisherige, durch die Hierarchie der diskursiven Beziehungsebenen am Rande behandelte Themenkomplexe werden in der Folgezeit des 11. März 2011 zunehmend in den Mittelpunkt der Diskussion über das gesellschaftliche Zusammenleben gestellt. So sind zum Beispiel emotional geformte Realitäten von Einzelnen - wie ein starkes Isolationsgefühl inmitten der Gesellschaft - bisher als Randphä- 
nomene vergleichsweise wenig behandelt worden. Die Auseinandersetzung mit dem „Anderen“ wird jedoch durch die Betroffenheit ganzer Bevölkerungsgruppen nach „Fukushima“ mehr und mehr zu einer Pflicht aller. Um Foucaults Worte zu benutzten, beschreibe ich hier also ein bisher peripheres „Formationssystem“ (Foucault 1973): Ein System, das ein Beziehungssystem vorschreibt, um Bezüge, Aussagen und Strategien zum Vorschein kommen zu lassen. Die von Foucault beschriebene „Regelmäßigkeit der Praxis" verorte ich sowohl in den wiederkehrenden Themen in den biographischen Aussagen meiner Gesprächspartner wie auch in der diskursiven Behandlung relevanter Themen in der weiterführenden wissenschaftlichen Literatur.

Dem sich mit dem Begriff „Fukushima“ verbundenen Prozess der Konstruktion von Sinn- und Bedeutungszusammenhängen, unter anderem durch sich verschiebende Machtkonstellationen, trage ich in dieser Arbeit Rechnung. Die von individuellen Akteuren empfundene „Wahrheit“ betrachte ich dabei als im Diskurs entstehend, die einige allgemeingültige Ansprüche emotionaler Art zwar erhebt, jedoch nie vollkommen durchsetzen kann. Beispielsweise wurde die Atomenergie in der Vergangenheit als „sicher“ und „gewinnbringend“ angesehen. Diese „Wahrheit" wird nach dem 11. März 2011 in den japanischen wie internationalen Diskursen zunehmend in Frage gestellt. Die Diskursanalyse ist dabei viel mehr als eine Methode. Sie ist eine perspektivische Ansicht der Dinge, die genau diesen, ständigen Veränderungen unterworfenen Aspekt gesellschaftlichen Zusammenlebens besonders betont.

Dies gilt folgerichtig auch für die vorliegende Arbeit und spiegelt mein wissenschaftliches Selbstverständnis als Ethnologin wider: In der wissenschaftlichen Praxis werden sowohl ich als Forscherin als auch die mit mir interagierenden Personen im wechselseitigen Austausch konstruiert. Die dabei ausgehandelte Wahrheit ist eine spezifische und verkörpert keinen allumfassenden Anspruch (siehe Birkhan 2012: 315). Von diesen Prämissen ausgehend, betrachte ich sowohl meine Perspektive als auch die Aussagen meiner Gesprächspartner als aus unterschiedlichen Diskursfeldern erwachsene Selbst- und Fremdverständnisse. Unsere jeweiligen Betrachtungsweisen sind kulturspezifische Momentaufnahmen.

Ich zeige, dass die mir präsentierten Artikulationen meiner Gesprächspartner einen diskursiven Bruch im Vergleich zum bis dato herrschenden Diskurs darstellen. Logik, biographische Aspekte und Wahrheiten der Diskursbeiträge meiner Gesprächspartner mache ich dabei transparent. Mein Anspruch, die unterschiedlichen Standpunkte im Post-Fukushima-Diskurs zu präsentieren, bedeutet laut Birkhan, „[...] ausgeschlossenes, ,unterworfenes` Wissen zum Vorschein und zu Gehör bringen, die Kräfteverhältnisse zu verschieben und somit den Raum für Veränderungen offen zu halten" (Birkhan 2012: 378).

Es handelt sich bei meiner Arbeit also um die Beobachtung eines Prozesses, der die Handlungsmöglichkeiten von Subjekten in Bezug auf sich verschiebende Machtkonstellationen und die Nutzung derselben in unterschiedlichen Bereichen des gesellschaftlichen Zusammenlebens berücksichtigt. Auf theoretischer Ebene habe ich bisher die Komplexität des Diskurses und der ihn generierenden Kräfte, bestehend aus der Position des Selbst im sozialen Gefüge sowie den Kommunikations- und 
Handlungsoptionen, aufgezeigt. Das „moderne Identitätsdenken“ (Birkhan 2012: 309) umschließt dabei die von Foucault favorisierte Anschauung, auch den Objekten des Diskurses Artikulations- und Handlungsmöglichkeiten einzuräumen, sie also an ihrer eigenen Identitätskonstruktion mitwirken zu lassen. Im folgenden Abschnitt nehme ich verstärkt Emotionen und Erfahrungen in den Blick, die die jeweilige Biographie und damit die persönliche Wahrnehmung eines Diskursverlaufs maßgeblich beeinflussen.

\subsection{Emotionsforschung}

Die Aspekte des Selbst in Verbindung mit Wissen, Macht, sozialer Position und Wahrheit standen im vorangegangenen Kapitel im Vordergrund. Die Klärung des Einflusses von Emotionen auf die gegenseitige Konstitution der genannten Teilaspekte der Diskurse steht im Mittelpunkt der nun folgenden Ausführungen.

Dementsprechend möchte ich an dieser Stelle wichtige Aspekte einführen, die sowohl für die Auswertung der erhobenen biographischen Daten als auch für den erkenntnistheoretischen Hintergrund der aktuellen japanischen Diskurse von großer Bedeutung sind. Ich möchte darauf verweisen, dass sich Emotionen auf die unterschiedlichen Diskursstränge bezüglich eines Ereignisses auswirken. In Verbindung mit einer spezifischen Position im sozialen Gefüge, der damit verbundenen Macht zur Bedeutungszuschreibung und des zur Verfügung stehenden Wissens ergibt sich ein individuelles Narrativ. Auf diese Weise betrachtet, wird das Zusammenspiel der Diskurse „[...] an important focal point in the analysis“ (Jørgensen and Phillips 2002: 145).

Ich begreife persönliche Erfahrungen und Emotionen als den Ansatzpunkt für die Analyse der individuellen Motivation meiner Gesprächspartner, die verschiedenen Erzählstränge zu sammeln, zu kontrastieren und zu hinterfragen. In der Gesprächspraxis wird ein öffentlicher Raum erschaffen, in dem bisher Undenkbares angesprochen, Selbstverständliches in Zweifel gezogen und neue Grenzziehungen ausgehandelt werden. Die Professorin für Psychologie Valerie Hardcastle beschreibt die so in Gang gesetzte Erschaffung einer sozialen Realität durch das Subjekt als „self-verification process“, welches seine Selbst-Konzeptionen verifiziere: „Some of these processes are designed to bring people's friends, colleagues, and intimates to see them as they see themselves. Others are designed to make the social environment seem more compatible with their self-conceptions than it really is " (Hardcastle 2008: 33). Hardcastle folgend gehe ich davon aus, dass der Umgang mit emotionalen Erfahrungen entscheidend ist für die Positionierung des Individuums im Diskurs. 


\subsubsection{Der Emotionsdiskurs - Individuum und Gesellschaft}

Im Zuge dieser theoretischen Vorüberlegungen betrachte ich die Auseinandersetzung mit japanischen Diskursen nach dem 11.03.2011 und mit der Akteursgruppe der Gegenwartskünstler nicht nur als „emotionale Diskurse“, sondern auch als „Diskurse über Emotionen“. Diese Unterscheidung eröffnet Perspektiven sowohl auf die Prägung von Emotionen durch kulturelle Werte und Normen als auch die Wirkungsweise von Emotionen auf die Diskurse (siehe Abu-Lughod und Lutz 1990: 11). Im folgenden Kapitel möchte ich hervorheben, dass es aus meiner Sicht nicht nur darum geht, die im Feld vorgefundenen Emotionen zu dokumentieren und auszuwerten. Vielmehr ziehe ich zusätzlich die sozialen Rahmenbedingungen, Regeln und Normen zur Bewertung von Erfahrungen in ihrer kulturellen Spezifik in meine Analyse mit ein. Auf diese Weise möchte ich dem Ausdruck und den Artikulationen von Emotionen einen besonderen Stellenwert einräumen. Wenn zum Beispiel jemand Traurigkeit empfindet, kann es je nach kultureller Rahmung als angebracht oder unangebracht betrachtet werden, wenn geweint wird.

Die „kulturellen Kodierungen“, wie Röttger-Rössler (2004: 68) sie nennt, sind dabei entscheidend für „das Management emotionalen Erlebens sowie die Kommunikation von Emotionen, sie prägen aber nicht das emotionale Erleben selbst“ (2004: 68). Es geht also im Folgenden maßgeblich um den Ausdruck und damit auch um den wahrnehmbaren Raum der legitimen Äußerung von Emotionen in einem bestimmten Kontext.

Die von White $(1990,1994)$ bereits betonte Subjektivität eines Gesellschaftsmitglieds beim Erleben und Ausdrücken von Empfindungen findet sich auch bei Röttger-Rössler und erklärt unterschiedliches Verhalten im selben kulturellen Kontext. Die enge Verknüpfung von Emotionen mit einem spezifischen kulturellen Modell vollzieht sich in der Sozialisation, in der das akzeptierte Bewertungssystem im Individuum verankert wird (Röttger-Rössler 2004: 82). Zur Selbstverständlichkeit wird die Verbindung aus Emotion und Ausdruck - Röttger-Rössler bezeichnet sie als Emotionskodex - durch die Wiederholung und Imitation. Dadurch erweist sie sich für den Einzelnen als „wahr“ und wird nicht mehr ständig hinterfragt. Das Wissen um den Ausdruck von Emotionen wird zum Wissen um Regeln und Normen.

In diesem Kapitel werde ich Überlegungen bezüglich der Verflechtung von Emotionen, Selbst und Moral als Teil des sozialen Lebens anstellen, die für die weiteren Ausführungen von zentraler Bedeutung sind. So stellen die Psychologen Kitayama und Markus (1994: 13) beispielsweise die Frage zur Diskussion, ob Emotionen in irgendeiner Weise mit dem moralischen Charakter von kulturellen Glaubenssystemen, Werten und Konstrukten verbunden sind. Weitere Verbindungen von Emotionalität zu Handlungsvermögen (agency) und Kreativität als Ausdruck von Empathie werde ich im Kapitel 3.3.4 „Empathie - wissenschaftliche Perspektiven auf den ,social glue“" darlegen.

Die zeitliche Nähe meiner Forschungsergebnisse aus den Jahren 2014 und 2015 zur Dreifachkatastrophe vom 11. März 2011 ermöglicht es, verlässliche Angaben 
darüber zu machen, inwieweit die artikulierten Emotionen meiner Gesprächspartner als ein individuell relevantes Event eingeschätzt und welche gesellschaftlichen Themen dabei gleichzeitig angeschnitten werden (vgl. Frijda und Mesquita 1994: 52 ff.). Die persönlichen Bewertungen der Akteure geben wiederum Aufschluss über deren ethisch-moralischen Rahmen:

„[...] norms exist with regard to having or not having particular emotions [...], to having or not having particular emotions in particular situations [...], and to showing or not showing the expression of particular emotions in either general or particular circumstances [...]. Such norms can be assumed to be of both social and individual origin." (Frijda and Mesquita 1994: 60)

Die nähere Untersuchung der Emotionalität und ihrer Genese im kulturspezifischen Kontext sowie ihre Bedeutungszuschreibung innerhalb einer lebensgeschichtlichen Erzählung können also Aufschluss darüber geben, wie eine Person ihr Selbst definiert beziehungsweise wie sie ihre Position innerhalb des sozialen Gefüges wahrnimmt. Über die Verschränkung der individuellen mit der strukturellen Analyseebene hinaus stellen emotionale Artikulationen auch soziale Statements über die Einschätzung konkreter Situationen dar (siehe Frijda und Mesquita 1994: 83) und eröffnen so das Feld zur Beschreibung persönlicher Praxis in einem sozio-kulturell geprägten Rahmen. In dieser Arbeit möchte ich zeigen, wie meine Gesprächspartner in ihrer Rolle als Gegenwartskünstler „[...] the hard and fast boundaries between the inner and the outer, the ideational and the material, the self and society" (Markus und Kitayama 1994b: 341) auflösen.

\subsubsection{Die Perspektiven der Emotionsforschung}

Nach der Beschäftigung mit der Verbindung von Individuum und Gesellschaft durch Emotionen geht es mir in diesem Unterkapitel um eine Einführung in die Emotionsforschung als Forschungsfeld. Ich mache dabei verschiedene Analyserichtungen aus, die sich jeweils verstärkt mit einem Aspekt des Emotionsdiskurses auseinandersetzen:

Emotionen werden als Ausdruck verstanden, der durch Sprache konstruiert wird. Dies erlaubt einen ersten Zugang zu einem Individuum und lässt einen Einblick in dessen Realität zu (Abu-Lughod und Lutz 1990):

„Rather, we should view emotional discourse as a form of social action that creates effects in the world, effects that are read in a culturally informed way by the audience for emotional talk. Emotion can be said to be created in, rather than shaped by, speech in the sense that it is postulated as an entity in language where its meaning to social actors is also elaborated. To say this is not to reduce the concept of emotion to the concept of speech, even though 
a discourse-centered approach might be construed as a rejection or obscuring of the body." (Abu-Lughod und Lutz 1990: 12)

In diesem Ansatz werden die engen Verbindungen zum Begriff des Diskurses als sprachlicher Ausdruck sozialer Praxis besonders augenscheinlich. Mit der Abwendung vom Paradigma des Essentialismus in den 1980er Jahren, in dem Emotionen als natürlich auftretendes Phänomen des menschlichen Körpers verstanden wurden, erfuhr die Kulturspezifik im Ausdruck von Emotionalität und ihren gesellschaftlichen Umgangsformen nun mehr Aufmerksamkeit.

Diesem Ansatz zufolge sind es Emotionen und ihre kulturspezifischen Ausdrucksweisen, die auf zwischenmenschlicher Ebene als Kommunikationsmittel dienen. Dabei eröffnet sich ein Raum für die Aushandlung des Umgangs mit positiv wie negativ konnotierten Emotionen, der einen Rahmen für die Interaktion entstehen lässt.

Mit der Fokussierung auf Emotionalität als soziokulturelles Konstrukt flossen weitere Aspekte des sozialen Zusammenlebens in die wissenschaftliche Debatte ein. Dazu zählen der gesellschaftlich-moralische Rahmen (Taylor 1989), das Verständnis von Emotionen als Teil von Machtbeziehungen und Ökonomie (Abu-Lughod und Lutz 1990) sowie nicht zuletzt des Zusammenhangs von Emotionen und Historizität (Hermann 1995; Hermann und Röttger-Rössler 2003).

Anhand dieser Verbindungen verschiedener theoretischer Konzepte wird deutlich, dass Emotionen seither als Antrieb für soziale Transformationsprozesse begriffen werden. Persönliche wie kollektive Zugänge sowie wechselseitige Beeinflussung und Positionierungen sind in einem komplexen Geflecht aus sozialen Netzwerkverbindungen miteinander verwoben. Die Ebenen des Privaten und des Öffentlichen verschwimmen und verlieren folglich an Trennschärfe. Die gesellschaftshistorisch gewachsenen Spezifika des kulturellen Selbst spielen demzufolge eine entscheidende Rolle bei der Analyse und berücksichtigen die vom Ethnologen Clifford Geertz (1983) geforderte sorgfältige Analyse des Reichtums einer spezifischen sozialen Situation.

Der wissenschaftliche Paradigmenwechsel in den 1980er Jahren erschloss die Emotionalität als breit gefächertes Forschungsfeld und löste sie aus dem Bereich der Psychoanalyse heraus (Lutz 1988; Abu-Lughod und Lutz 1990; Rosaldo 1984). Emotionen werden seither in der Ethnologie als ein Zusammenfluss von kultureller Weltanschauung und sozialen Verhaltensskripten angesehen (siehe Lutz 1988: 10). Darüber hinaus verbinden sie die private mit der öffentlichen Sphäre „[...] by its construal of emotion as about social life rather than internal states, and its exploration of the close involvement of emotion talk with issues of sociability and power - in short, with the politics of everyday life" (Abu-Lughod und Lutz 1990: 1-2). Die kulturspezifische Modellierung des Gefühls wurde von der Ethnologin Birgitt Röttger-Rössler (2004: 2) auch als „emotionale Wende“ in der ethnographischen Forschung bezeichnet, die sich bis dato weitestgehend auf die amerikanische Ethnologie beschränkte. Im Folgenden führe ich drei Möglichkeiten auf, sich auf analytisch fundierte Weise mit Emotionen auseinanderzusetzen. Bei diesen Zugängen 
beziehe ich mich auf die Ethnologinnen und Genderforscherinnen Lila Abu-Lughod und Catherine Lutz (1990: 3ff.), die sich mit Emotionen in forschungsgeschichtlicher Weise auseinandergesetzt haben.

Ein Zugang geht von der Grundhaltung aus, die Existenz und Konnotation von Emotionen nicht als universell zu betrachten. Dadurch wird Raum für soziale Praktiken eröffnet, die dem Forscher andernfalls verborgen bleiben könnten.

Die zweite Perspektive des ethnopsychologischen Forschungszweiges betrifft die Historisierung der Auseinandersetzung mit Emotionen als soziokulturelles Phänomen (Abu-Lughod und Lutz 1990: 5). Dabei wird der Emotionsdiskurs, die Subjektivität und das Selbst eines Individuums in den Mittelpunkt der Analyse gestellt und in seinen Transformationen thematisiert. Die von mir in Kapitel 3.2 „Diskursanalyse" bereits aufgeworfenen Fragen nach der Rolle des Einzelnen in der wechselseitigen Wirkung von Diskurs und Subjekt sind dabei von essentieller Bedeutung und werden um die Analyse der Bedeutungsgenese von Emotionen ergänzt. Emotionen und ihre Bewertungen werden auf diese Weise nicht als Zustand, sondern als gesellschaftlicher Prozess verstanden.

Noch spezifischer ist der dritte Zugang zu emotionalen Diskursen, in dem konkrete Situationen in einem kulturellen Kontext untersucht werden (Abu-Lughod und Lutz 1990: 6). Dabei wird versucht, anhand bestimmter zeitlicher und lokaler Verortung den sozialen Diskurs über Emotionen in seiner Tiefe zu begreifen und verständlich zu machen. Erst aufgrund dieses Bemühens um ein holistisches Verständnis der emotionalen Bedeutungsherstellung können von Seiten des Forschers zugrunde liegende Strukturen einer Gesellschaft wie beispielsweise Wert- und Normvorstellungen sichtbar gemacht werden.

Gleichzeitig bilden diese Grundlagen die Basis für die Analyse des spezifischen sozio-kulturellen Kontextes, in dem sich befragte Individuen verorten. Anhand einer konkreten Situation werden also bestimmende Faktoren der Realitätserfahrung sowohl auf struktureller wie auch individueller Ebene sichtbar.

Gemeinsam ist allen drei Aspekten die Berücksichtigung von Positivität, Angemessenheit oder Zulässigkeit je nach Status, Rolle und Gender etc. und die kontextuelle Generierung von Emotionen auch innerhalb einer Kultur (siehe Hardcastle 2008). Aktuellen Forschungsansätzen zufolge werden Emotionen folglich als ein Phänomen betrachtet, das in seiner Spezifik sowohl im kulturspezifischen Feld als auch in seiner konkreten gesellschaftlichen Diskurssituation eingebettet werden muss. Abu-Lughod und Lutz gehen bereits von dieser grundlegenden Prämisse aus und betrachten Emotionen als diskursive Praxis (1990: 10).

Bei den von Abu-Lughod und Lutz aufgezeigten drei Strategien der Herangehensweise an Emotionsforschung (das Negieren von Essentialismus, die Berücksichtigung von Historizität und das Verständnis von Emotionen als sozialer Diskurs) interessieren mich vor allem die im japanspezifischen Kontext deutlich werdenden Schnittstellen zwischen der individuellen Bedeutungszuschreibung an Emotionen und deren diskursive Beeinflussung angesichts aktueller gesellschaftskritischer Themen. Anhand der Tätigkeiten meiner Gesprächspartner lassen sich Emotionen in ihrem 
aktivierenden Aspekt in der diskursiven Performanz im konkreten Fall analysieren. Auf mein Forschungsfeld übertragen, betonen die hier aufgeführten ethnologischen Zugänge zu Emotionen die Wichtigkeit der sich im Wandel der Zeit befindenden Emotionsdiskurse in Japan in der Folgezeit des 11. März 2011. Ich sehe mich in der Tradition jenes Forschungsansatzes, vertreten von Michelle Rosaldo, Catherine Lutz, Lila Abu-Lughod und Elfriede Hermann, der Emotionen als kulturell konstruiert und stark von kulturellen Konzeptionen abhängig betrachtet. Emotionale Diskurse können demzufolge ,[...] serve, in other instances, for the relatively powerless as loci of resistance and idioms of rebellion, [...] as means of establishing relationships and coercing gifts [...], or even as means of establishing complementarity with status superiors [...]" (Abu-Lughod und Lutz 1990: 15).

$\mathrm{Da}$ ich mich des Weiteren für einen biographischen Zugang zur subjektiven Position meiner Gesprächspartner entschieden habe, spielt die sprachliche Konstruktion des Selbst bei der Analyse der Emotionen eine entscheidende Rolle. Dabei machen die Bezüge des jeweiligen Individuums zum näheren gesellschaftlichen Umfeld und nicht zuletzt zu mir als Forscherin die Einzigartigkeit des jeweiligen biographischen Konstrukts aus.

An dieser Stelle will ich betonen, dass es sich bei allen von mir ausgewerteten Artikulationen um spezifische Manifestationen handelt. Das heißt, dass ich in den geführten Interviews auch ein Verhaltensskript dokumentiert habe, das die emotionalen Leitlinien der japanischen Gesellschaft, das heißt Emotionskonzepte, berücksichtigt. Wichtige Aspekte sind dabei unter anderem die Hierarchisierung des Sozialgefüges, die an Interdependenz ausgerichtete Konstruktion des Individuums, ${ }^{18}$ sowie die Prägung des öffentlichen Raumes durch den japanischen Staat. ${ }^{19}$ Der Schlüssel zum Verständnis dieses Verhaltensskripts, insbesondere in Bezug auf Emotionen, liegt laut Hardcastle in dem Wissen „[...] how it is we organize our own experiences. Some of what we do is absolutely central to our very being; some is not" (2008: 18).

Es ist davon auszugehen, dass aufgrund der engen Verknüpfung von Emotionen, Erfahrungen und der biographischen Konstruktion des Selbst eine Bewertung von Erfahrungen ohne emotionale Konnotation nicht möglich ist. Diese wiederum prägen die jeweilige Persönlichkeit und ihre Selbstwahrnehmung in essentieller Weise, was sich erneut in emotionalen Äußerungen während des biographischen Interviews manifestiert.

Diese Wechselwirkungen in einer biographischen Erzählung nachzuvollziehen und im aktuellen Generierungsprozess analytisch aufzubereiten, stellt die Herausforderung meiner Arbeit dar. Dabei stellt sich die Frage, wie genau Emotionen wirken und wie sie gesellschaftliche Bedeutung erlangen können. Was also macht die Macht von Emotionen auf kollektiver wie individueller Ebene aus?

\footnotetext{
${ }^{18}$ Siehe Kapitel 4.1.1 „Der Kompass der Werte - Ethik und Selbst im japanischen Kontext“ und 4.1.2 „Das Verhältnis vom Individuum zur Gruppe“.

19 Siehe Kapitel 5.3.2 „Medien, die staatliche Öffentlichkeit und ihre Alternativen“.
} 


\title{
3.3.3 Emotionen, das Selbst und Erfahrung - Bezüge zum biographischen Narrativ
}

Wie bereits erläutert, betrachte ich Emotionen im Folgenden als treibende Kraft im menschlichen Miteinander, die über ihre identifikatorische Wirkung hinaus sowohl das Individuum in seiner Lebensperspektive verortet als auch die individuelle gesellschaftliche Rahmung verdeutlicht. Um die gelebte Praxis meiner Gesprächspartner nachvollziehen zu können, ist ein Verständnis dafür vonnöten „, [...] how our emotions are coupled with our thoughts" (Hardcastle 2008: 130). In Hardcastles "Constructing the Self" verbinden sich Emotionen mit Wahrnehmung, Erinnerung und Erfahrung und bilden ein komplexes Konglomerat, aus dem sich das Selbst generiert: , $[. .$.$] cognition, emotion, memory, and experience are all tightly bound up$ with one another. As a result there are not going to be simple answers to difficult psychological questions" (Hardcastle 2008: 158). Das Selbst eines Gegenwartskünstlers in Japan in den Jahren 2014 und 2015 zu erfassen, bedeutet folglich auch, sich mit den Erfahrungen auseinanderzusetzen, auf deren Basis die jeweilige Persönlichkeit ihre aktuellen Einschätzungen gesellschaftlicher Themen vornimmt. Welche diskursiven Narrative wurden im Laufe der Sozialisation verinnerlicht und damit zu einem Teil des Selbst? Welchen Erfahrungen schreiben die Akteure selbst entscheidende Bedeutung für ihren späteren Lebensweg zu? Wie wird dabei im biographischen $\mathrm{Zu}$ sammenhang argumentiert? "To be a self, then, is to ascribe experiences to oneself as one`s own experiences" (Hardcastle 2008: 22). Welche Erfahrungen wurden derart emotional aufgeladen, dass es in das Bild des Selbst überging und nach außen repräsentiert wird?

Wie ich in den Kapiteln zur biographischen Analyse noch verdeutlichen werde, ist die Präsentationsform eines Narrativs keineswegs dem Zufall überlassen, sondern verfolgt immer bestimmte Zielrichtungen und Intentionen. Die Suche nach einem Sinn in Erfahrungen sei der Grund der Existenz des Selbst, so Hardcastle (2008: 24). Nach Meinung von Markus und Kitayama (1994b: 349) können scheinbar subjektive Gefühle durch den individuellen Prozess der Bedeutungsgenerierung auch immer etwas über den Kern an kulturellen Ideen aussagen.

\begin{abstract}
„The basis of this variability in emotional experience is the individual interpretive structures or schemas that have developed as a result of previous emotional conditioning, both conscious and nonconscious and of past efforts of meaning making." (Markus und Kitayama 1994b: 349)
\end{abstract}

Die persönliche Bedeutungssuche setzt die artikulierende Person also nicht nur in Bezug zu sich selbst und den eigenen Erfahrungen, sondern auch zu anderen im näheren und gesellschaftlichen Kontext. Als Gemeinsamkeit oder Widerspruch formuliert setzt sich das Selbst in seiner Sinnsuche unausweichlich mit „dem Anderen“ auseinander und bestätigt damit seine eigene Existenz. Die Art und Weise dieser Auseinandersetzung ist im kulturellen Skript festgelegt und beschreibt das Verhalten 
einer „guten“ Person. ${ }^{20}$ In persönlichen Orientierungsrahmen, Entscheidungen und Erfahrungen spiegeln sich demzufolge auch gesellschaftliche Konventionen, Normvorstellungen und moralische Anforderungen wider. Diesen spezifischen Formen kultureller Realitäten spüre ich mit meiner Analyse des biographischen Materials meiner Gesprächspartner nach. Es zeichnen sich darin auch die kulturellen Emotionsskripte und aktuelle diskursive Aushandlungen ab.

Bei der Biographie handelt es sich darüber hinaus um eine an Raum und Zeit gebundene Erinnerung an die eigene Person und die für sie wichtigen Ereignisse und Erfahrungen (Hardcastle 2008: 64). Im Prozess des Erinnerns spielen Emotionen eine weitere entscheidende Rolle: „Emotional response is obviously at the core of our narrative selves. In order to understand how we construct our narratives, we need to understand our emotional reactions" (Hardcastle 2008: 67). Die Perspektive auf Emotionen, die mit bestimmten Erfahrungen verknüpft maßgeblich an der Geschichte unseres Selbst mitwirken, erweist sich angesichts des von mir generierten Datenmaterials als sehr zielführend. In den Analysekapiteln 7 bis 9 werde ich darauf zurückkommen.

Es sind die von Emotionen unterlegten Erinnerungen, die die Basis für ein Selbst bilden, das in der Lage ist, sich in den Erlebnissen anderer wiederzufinden, emotional zu identifizieren und entsprechende Handlungsweisen abzuleiten. Die Sprache der dominanten Diskurse wird in diesem Prozess ebenso hinterfragt wie die darin verwendeten Begrifflichkeiten. Dabei entsteht eine neue Vielfalt an diskursiven Strängen, die in unterschiedlicher Konstellation zusammenfinden und dabei ständig Neues erschaffen:

„In talk, language users select elements from different discourses which they draw on from mass mediated and interpersonal communication. This may result in new hybrid discourses. Through producing new discourses in this way, people function as agents of discursive and cultural change."

(Jørgensen and Phillips 2002: 17)

Ich betrachte die Zusammenführung diskursiver Stränge als kreativen Akt, der angesichts von einschneidenden kollektiven Erfahrungen an gesellschaftlicher Bedeutung gewinnen kann. ${ }^{21}$ An dieser Stelle möchte ich jedoch mit der Verknüpfung von Biographie, Selbst und Emotion fortfahren und eine weitere wichtige Dimension im Zusammenhang mit der Analyse biographischer Erfahrungen hervorheben.

Eine Lebensgeschichte wird von der Gegenwart aus immer wieder aufs Neue konstruiert und erzeugt so Kontinuität und Beständigkeit. „In this way, through our narratives we reflect social norms in our self-conception" (Hardcastle 2008: 45).

Damit ist nicht nur der Zeitpunkt der Forschung von entscheidender Bedeutung bei der Einbettung der Forschungsergebnisse in den kulturellen Kontext, sondern

${ }^{20}$ Siehe Kapitel 4.1.1 „Der Kompass der Werte - Ethik und Selbst im japanischen Kontext“.

${ }^{21}$ Siehe Kapitel 10 „Gegenwartskünstler als Experten der Bedeutungsherstellung“. 
auch die Zeit als Teil der biographischen Selbstkonstruktion. Mit einer stringenten und in sich schlüssigen Selbstdarstellung kann der Erzählende seiner eigenen Vergangenheit und Gegenwart Logik und Authentizität verleihen.

Zudem kann die Geschichte des Selbst nur dann erzählt werden, wenn das entsprechende Umfeld dafür die Gelegenheit und die Legitimation bietet. Dies gilt sowohl für den Forschungskontext als auch für die alltägliche Lebensrahmung des Erzählenden. Hardcastle (2008: 45) verwendet für die alltägliche Lebensrahmung den Begriff des „sozialen Milieus“, den ich unter vergleichbarer Konnotation in Kapitel 6 bei der Beschreibung der japanischen Kunstszene verwende. Hardcastle beschreibt die Wirkung von Normvorstellungen und Verhaltensregeln als wichtige Elemente des sozialen Milieus, die sich in den jeweiligen Beschreibungen des Selbst widerspiegeln.

Der sozialen Praxis der Emotionsäußerung werde ich im folgenden Abschnitt besondere Aufmerksamkeit widmen. Aus meiner Sicht spielt dieser Raum für meine Gesprächspartner auch deshalb eine so entscheidende Rolle, weil ihre emotionale Arbeit beziehungsweise ihre Emotionsarbeit in Form ihrer Werke einen Platz in der Öffentlichkeit finden muss, um Wirkung zu entfalten. Ihr Selbstnarrativ, das sie als Gegenwartskünstler verifiziert, ist also unter anderem von den zur Verfügung stehenden Möglichkeiten der Ausdrucksäußerung abhängig. Die unauflösliche wechselseitige Verknüpfung von ihrer Person mit ihrer Arbeit, Leben und Beruf, wie ich sie ausführlicher in Kapitel 6 beschreibe, stellt meine Gesprächspartner vor einige Herausforderungen. Nicht nur äußern sie ihre Meinungen weitestgehend auf der Basis gesellschaftsspezifischer Konventionen, sondern bringen gleichzeitig die subversive Kraft ihrer individuellen Ansichten, Emotionen und Erfahrungen zur Entfaltung.

Die enge Verbindung zwischen dem Konzept der Person und ihres Selbstnarrativs wirft im spezifischen Kontext einer Untersuchung die Frage nach dem entsprechenden sozialen Raum auf, in dem Emotionen empfunden, artikuliert, ausgedrückt und ausgehandelt werden können. Diese Wirkungsmöglichkeiten von Emotionen auf gesellschaftlicher Ebene können für die Lebenszufriedenheit der Akteure bestimmend sein. Im Laufe der Analyse meines Datenmaterials kristallisierte sich daher die Empathie als eine wesentliche Fähigkeit im Umgang mit Emotionen heraus.

\subsubsection{Empathie - wissenschaftliche Perspektiven auf den „social glue“}

Bisher habe ich die jeweiligen Konzepte der individuellen Emotionalität in Bezug auf ihre diskursive Beeinflussung dargestellt. Es ist deutlich geworden, dass aus dieser theoretischen Sicht eine enge wechselseitige Verbindung zwischen dem individuellen Selbst und der gesellschaftlichen Ebene erkannt werden kann.

In diesem Kapitel möchte ich auf einen Aspekt von Emotionen eingehen, der entscheidend ist für das menschliche Zusammenleben und darüber hinaus auf gesellschaftlicher Ebene stabilisierend wirkt: Empathie. Die Ethnologen Hollan und Throop verweisen auf die von Halpern (2001: 85) formulierte Definition von Empathie: 
„[...] as a first person-like, experiential understanding of another person's perspective. For Halpern, empathy is a type of reasoning in which a person emotionally resonates with the experience of another while simultaneously attempting to imaginatively view a situation from that other person's perspective." (Hollan and Throop 2011: 2)

Insbesondere im japanischen Kontext mit einer besonders ausgeprägten Fokussierung auf Harmonie ${ }^{22}$ hat die Empathie einen besonderen Stellenwert inne. Sie kann meiner Meinung nach, ähnlich wie Glück als „social glue“ (Matsumoto 1996: 36) fungieren. Gemeint ist damit, dass durch Empathie zwischenmenschliche Bindungen erschaffen und erhalten werden. Der Japanologe Matsumoto (1996) betont die Wichtigkeit der sozialen Bindungskraft von Emotionen in Japans Kultur, was er in der Ausrichtung der Gesellschaftsstruktur auf das Kollektiv begründet sieht: „A Japanese person is never fully independent; being Japanese means that one must always be conscious of others" (Matsumoto 1996: 9). Diese Facette führe ich in Kapitel 4.1 „Der kulturspezifische Ausdruck des Selbst im sozialen Gefüge“ weiter aus. Im vorliegenden Abschnitt weise ich nur auf die Verbindung der verschiedenen Begrifflichkeiten, Diskurse, dem Verständnis des Selbst im japanischen Sozialgefüge und die wichtige Rolle der Emotionen hin.

Empathie begreife ich als essentielle Fähigkeit im Umgang mit den Emotionen anderer. Dies gilt meiner Meinung nach in besonderem Maße für die Arbeit meiner Gesprächspartner, in der Emotionen in den verschiedenen Kunstgenres ihren Ausdruck finden.

Insbesondere in Ausnahmesituationen wie Katastrophen spielt Empathie in der Herstellung von Solidarität und Hilfsbereitschaft eine entscheidende Rolle, kann aber auch über die akute Notsituation hinaus eine wichtige Rolle spielen. ${ }^{23}$

Hollan und Throop unterscheiden Empathie von Projektion, also der Übertragung eigener Emotionen auf Andere. Sie betrachten, unter Rückbezug auf Halpern (2001) Empathie als Prozess, der einen lang anhaltenden Dialog voraussetzt: „This concern with accuracy, the willingness, indeed the necessity, to alter one's impression of another's emotional state as one engages with the other and learns more about his or her perspective, is what distinguishes empathy from simple projection [...]" (Hollan und Throop 2011:3). Die beiden Autoren problematisieren die Analyse von Empathie sowohl in der jeweiligen kulturspezifischen Kontextgebundenheit, die viel Wissen voraussetzt, als auch in der theoretischen Verortung von Forschungsansätzen. Das Konzept der Empathie überlappe sich mit Konzepten wie agency, Intentionalität, Intersubjektivität und moralischen Gefühlen wie Sympathie, Mitgefühl und Mitleid (Hollan and Throop 2011: 6). Die enge Verbindung mit dem kulturspezifischen und situativen Kontext schließt demzufolge auch ethische Vorstellungen und historisches Verständnis ein: „,...] the fact that the subjects of our empathy are people who think

22 Siehe Kapitel 4.1 „Der kulturspezifische Ausdruck des Selbst im sozialen Gefüge“.

${ }^{23}$ Näheres dazu in den empirischen Analysekapiteln 7, 8 und 9. 
and feel and act in very specific culturally and historically constituted moral worlds while we ourselves, as empathizers, are similarly bound and constrained" (Hollan and Throop 2011: 5). Dabei birgt das Vorhaben, Empathie im Feld zu erforschen, auch Potential. Schließlich, so Hollan and Throop (2011: 17) weiter, könnten Wissenschaftler aufgrund dieser Bemühungen, so schwierig sie auch sein mögen, die interessantesten Daten erheben (siehe auch Lepowsky 2011). Oder um es mit den Worten des Ethnologen Lohmann auszudrücken: „Since anthropology is in the business of knowing both others and selves so that we might understand humankind as a whole, empathy is a major tool of the trade" (Lohmann 2011: 109).

Nicht das Thematisieren und Nutzen von Empathie in der Forschung ist laut Lohmann zu problematisieren. Das entscheidende Qualitätsmerkmal sei von einer fähigen Differenzierung der vielen Perspektiven abhängig, die in den empirischen Daten enthalten seien (Lohmann 2011: 112). Dies vorausgesetzt liefert die Thematisierung der Empathie die „[...] most valuable products of the discipline“ (Lohmann 2011: 112).

Auf Basis der angegebenen Literatur verstehe ich Empathie als eine Fähigkeit, durch die subjektive Realitätsvorstellungen von Emotionen erweitert werden können, so dass zum Beispiel Solidarisierung mit anderen Mitgliedern der Gesellschaft auch über große Differenzen in der Lebenssituation möglich wird.

„After emotionally engaging with another, an emphathizer must begin to imagine how and why the other acts or feels the way he or she does. This imaginative process is guided by the empathizer's emotional engagement, activating an associational network of memories, images, and meanings in his or her mind, which in turn are mapped onto the experiences and perspectives of the other in an attempt to understand them." (Hollan and Throop 2011: 14)

Gerade im Kontext der Katastrophenforschung wird Empathie auf eine gesellschaftliche Ebene übertragen, die zu Engagement motivieren kann (siehe Beck 1986). Die kulturspezifischen Emotionen sind dabei in den oben beschriebenen Komplex von Identitäten und Szenarien eingebettet: „As a personal response mediating social action and reaction, specific emotions such as ,anger ' designate interactive scenarios with known evaluative and behavioral implications" (White 1990: 48).

Weitere Untersuchungen für die verbindende Kraft von Emotionen im gesamten sozialen Spektrum einer Gesellschaft finden sich unter anderem beim Ethnologen White, der folgende Worte findet: „My intention is not to promote a constructivist or interpretivist theory of emotion to the exclusion of physiological or psychological processes, but to suggest that emotions are, if nothing else, semiotic mediators linking the body, the subject, and the social" (White 1994: 220).

Man könnte also argumentieren, dass das Individuum mithilfe der durch Empathie entstandenen Solidarität die Rolle des aktiv gestaltenden Mitglieds einer Gesellschaft einnehmen kann. Dies ist das zweite Element der Emotionen, dessen Wirkung sich in den biographischen Narrativen meiner Gesprächspartner mani- 
festiert. Die von White (1990: 47) beschriebene Verbindung zwischen Emotionen und sozialem Handeln spielen eine ganz besondere Rolle in der vorliegenden Analyse, erklärt diese Verbindung doch viele von mir beobachteten Reaktionen der Gegenwartskünstler Japans auf die zu Tage geförderten Kommunikations- und Diskursabläufe der mächtigen gesellschaftlichen Akteure. Die in den Interviewdaten deutlich werdenden Emotionen meiner Gesprächspartner beeinflussen demzufolge nicht nur deren eigene Realitätskonstruktion und Perspektive, sondern auch ihre Selbstpositionierung in Relation zu bestehenden Machtkonstellationen. ${ }^{24}$ In einem kulturspezifischen komplexen Gebilde aus Identiät, Handlungszusammenhängen, Erfahrungen und Selbstbezug spiegelt sich Abu-Lughods Begriff der „politics of sentiment", der beschreibt „,...] how emotional discourses are implicated in the play of power and the operation of a historically changing system of social hierarchy" (AbuLughod and Lutz 1990: 15).

\subsection{Die Erforschung von Emotionen im Feld - Methodisches Vorgehen}

Sich als Forscher in einem anderen kulturellen Kontext zu bewegen als dem eigenen stellt gewisse Herausforderungen dar. Vom Einfinden in das Setting und dem Knüpfen von Kontakten bis hin zur Darstellung der mitwirkenden Akteure und ihrer Aussagen im zeit- und ortsgebundenen Rahmen nach der Datenerhebung: Die verschiedenen Phasen der Forschung erfordern ein tiefes Eintauchen in andere Gesellschaftsstrukturen, deren Werte und Normen, Vorstellungen und historisch erklärbare gesellschaftliche Spannungsfelder und Widersprüche. Um die Lebensperspektive meiner Gesprächspartner verstehen und in möglichst holistischer Weise widerspiegeln zu können, nutze ich unterschiedliche methodische Ansätze.

Eine Besonderheit meines Vorgehens liegt im offenen Zugang zum Forschungsfeld. Das heißt, dass während des gesamten Forschungszeitraumes meine Gesprächspartner die thematischen Schwerpunkte meiner Arbeit gesetzt haben. Anhand dieser Foki erarbeite ich die Perspektive meiner Gesprächspartner in Bezug auf ihre emotionalen Erfahrungen, Selbstwahrnehmungen und das daraus resultierende Verhalten im sozialen Gefüge Japans. Um dem Ziel einer umfassenden Darstellung subjektiver Narrative gerecht zu werden, habe ich, wie jeder ethnologische Forscher, einen Lernprozess durchlaufen, der dem einer erneuten Sozialisierung gleichkommt: Durch Beobachtung, Aufstellen und Widerlegung von Annahmen und der Adaptierung von Sprache habe ich ein spezifisches kulturelles Bedeutungssystem erfasst und eine adäquate Beschreibung angestrebt. Das kulturelle Wissen des jeweiligen Akteurs ist unterteilt in „explizites“, also leicht zugängliches Wissen, und „implizites“, das heißt latent verwendetes, wenig artikuliertes Wissen. Letzteres ist nur erlernbar durch genaues Beobachten und unter Anwendung ethnologischer Methoden wie dem nar-

${ }^{24}$ Siehe Analysekapitel 7, 8 und 9. 
rativen Interview, um die zu Tage tretenden Verhaltensweisen in einen kulturellen Bedeutungszusammenhang zu stellen und die dahinter verborgenen Grundannahmen über das menschliche Zusammenleben herauszufiltern. Die mir als Forscherin in einem fremden Umfeld unweigerlich begegnenden Interferenzen mit meinem eigenen kulturellen Hintergrund machen deutlich, dass eine Ethnographie stets kulturgebunden bleibt. Jedoch trägt die Einhaltung der notwendigen Transparenz entscheidend zum Verständnis der kulturellen Spezifika bei:

„However, it [ethnography] provides descriptions that reveal the range of explanatory models created by human beings. [...] It says to all investigators of human behavior, ,Before you impose your theories on the people you study, find out how those people define the world"." (Spradley 1979: 11)

Der hier vom Ethnologen Spradley zusammengefasste Ansatz, von den Bedeutungssystemen der Untersuchten auszugehen, bildet einen wesentlichen Ausgangspunkt für alle meine folgenden Ausführungen: Durch die Kenntlichmachung meiner eigenen Annahmen, begrifflichen Abweichungen und Thesen bin ich bemüht, dem Leser einen offenen Zugang zu den Lebensrealitäten meiner Gesprächspartner zu ermöglichen.

Die aus den Aussagen meiner Gesprächspartner resultierenden theoretischen Überlegungen basieren auf meinen Eindrücken und werden durch entsprechende wissenschaftliche Literatur ergänzt. Damit verfolge ich den Ansatz der „Grounded Theory", wie er von den Soziologen Glaser, Strauss und Paul (1972) ausgeführt wurde, und der sich mit der Exploration menschlicher Erfahrungen als Basis für wissenschaftliches Arbeiten beschäftigt. Mit einem deskriptiven Fokus auf eine Akteursgruppe handelt es sich in der folgenden Analyse um eine sogenannte "Case Study“, also um eine Einzelfallanalyse von Individuen, die nach bestimmten Kriterien ausgewählt wurden. ${ }^{25}$

Im Zuge der methodischen Vorüberlegungen habe ich mir auch die grundsätzliche Frage nach der Übertragbarkeit von qualitativen Daten auf die Gesamtheit der untersuchten Gesellschaft gestellt. In der Auseinandersetzung mit den verschiedenen Möglichkeiten eines Feldzuganges wird in der Literatur neben der bereits erwähnten Subjektivität des Forschers insbesondere das Verhältnis von Phänomenologie zur Struktur und ihrer Interdependenz hinterfragt. Deshalb befasse ich mich zunächst mit der Problematisierung der Forscherrolle im Feld und der Datenauswertung, bevor ich auf die von mir gewählten Methoden eingehe.

Wie bereits in den Abhandlungen zu meiner theoretischen Perspektive deutlich geworden ist, verfolge ich einen sozialkonstruktivistischen Ansatz, dem die Annahme zugrunde liegt, dass sich das Selbst, der Diskurs und die Gesellschaft über die soziale Praxis generieren. Das Individuum und sein kulturspezifisches Umfeld sind untrennbar miteinander verbunden und bedingen sich gegenseitig. Individuelle Aus-

\footnotetext{
25 Siehe Kapitel 3.5 „Meine Gesprächspartner“.
} 
sagen werden zu validen Artikulationen eines gesellschaftlichen Akteurs. Die Artikulationen des Einzelnen sagen damit immer auch etwas über dessen Wahrnehmung der gesellschaftlichen Diskurse aus.

Der Wert der qualitativen Methoden ist daher mit den Ergebnissen der quantitativen Forschung gleichzusetzen. Qualitative Aussagen beleuchten eine Form der Wissensgenerierung, die ergänzend und inspirierend für quantitative Erhebungen wirken kann: „Qualitative description is a kind of measurement, an integral part of the complex whole that comprises scientific research" (Bernard 2011: 20).

Ich möchte die subjektiven Stimmen einzelner Akteure hervorheben und anhand ihrer Aussagen die japanischen Diskurse um „Fukushima“ beleuchten. Dazu bedarf es nicht nur eines entsprechenden Netzwerkes an Gesprächspartnern, sondern auch einer klaren Rollenzuschreibung von Informanten und Forscher. Angesichts meiner Schwerpunktsetzung auf Emotionen war es mir besonders wichtig, die Beziehungen zum einzelnen Künstler positiv und vertrauensvoll zu gestalten. Ich hatte die Hoffnung, dass sich auf diese Weise die Rollen meiner Gesprächspartner vom Informant und/oder „gatekeeper“ eines Netzwerkes zu der eines Lehrers und/oder Vertrauten wandeln würden - eine Hoffnung, die sich im Laufe der Forschung erfüllte. Indem ich mich beispielsweise in der Rolle der Lernenden präsentierte, konnte ich auch manche kontroversen Themen im Laufe des sich entwickelnden Vertrauensverhältnisses besprechen und diskutieren. So gewann ich während des einen oder anderen Interviews den Eindruck, der mir gegenübersitzende Akteur habe nur auf eine Gelegenheit gewartet, die im japanischen Diskurs kontrovers behandelten oder verschwiegenen Themen zur Diskussion zu stellen und meine außenperspektivische Sicht der Dinge zu hören.

Auch meine persönlichen Eindrücke über meine Gesprächspartner finden ihren Weg in die Darstellung des jeweiligen Akteurs. Dennoch behandle ich die mir anvertraute persönliche Geschichte als „off the record“ (siehe Spradley 1979: 36). Das heißt, einzelne sehr brisante Aussagen habe ich auf Wunsch meiner Gesprächspartner nicht aufgezeichnet, sondern lediglich in einem Gedächtnisprotokoll festgehalten. Diese Möglichkeit hat zusätzlich zur Entspannung der Gesprächssituation und zum Aufbau von gegenseitigem Vertrauen beigetragen. Zusammen mit kulturspezifischen Hintergründen kann der Leser die entsprechenden Aussagen besser einordnen und nachvollziehen.

Hinzu kommt auch der sich mit zunehmender Offenheit wandelnde sprachliche Ausdruck meiner Gesprächspartner, der wichtige Anhaltspunkte für die Verknüpfungspunkte von professioneller Tätigkeit, gesellschaftlicher Rolle und individuellem Selbstverständnis des einzelnen Gesprächspartners gibt. Näheres dazu folgt in den entsprechenden Analyseabschnitten.

In den folgenden Kapiteln gehe ich nun auf die verschiedenen Methoden ein, durch die ich das analysierte Datenmaterial generiert habe. Dabei spielen die oben geschilderten Vorüberlegungen eine wichtige Rolle. Sie bestimmten im Wesentlichen meinen Zugang zum Personenkreis der Gegenwartskünstler und den von ihnen als wichtig empfundenen Thematiken. Es folgen allgemeine Erläuterungen der signifi- 
kanten Foki der Biographieforschung im Allgemeinen. Erst nach dieser Einführung gehe ich auf die methodischen Spezifika ein, die ich im japanischen Kontext berücksichtigt habe.

\subsubsection{Das narrative Interview}

Die Darstellung des biographischen Werdegangs des jeweiligen Künstlers vor dem Hintergrund der emotionalen Verarbeitung der Dreifachkatastrophe sowohl auf individueller als auch auf gesellschaftlicher Ebene bildet den Hauptfokus meiner ethnologischen Forschungsarbeit. Ich möchte durch die entsprechende Methodik auf wichtige kulturelle Themen aufmerksam machen: „Life histories are another kind of description that offers an understanding of alien cultures. They reveal the details of a single person's life and in the process show important parts of the culture" (Spradley 1979: 24; Hervorhebung im Original). Für die Erhebung von Lebensgeschichten entschied ich mich unter anderem deshalb, weil sie ein hervorragendes Material zur kulturellen wie individuellen Rahmung darstellen. Die jeweiligen persönlichen Aussagen verankern das Individuum nicht nur im sozialen Gefüge, sondern verorten es gleichzeitig in einem persönlichen Bewertungskontext. Der Forscher kann dadurch eine differenzierte Analyse vornehmen.

Für die Erhebung der biographischen Daten habe ich mich für die narrative, unstrukturierte Interviewführung entschieden. Mit dem unstrukturierten Interview ist eine Gesprächseinleitung ohne Zuhilfenahme bereits konzipierter Fragebögen gemeint, die dennoch nicht ohne Vorbereitung und Plan auskommt. Bernard beschreibt die Vorteile dieses Vorgehens folgendermaßen:

„Unstructured interviews are based on a clear plan that you keep constantly in mind, but are also characterized by a minimum of control over the people's responses. The idea is to get people to open up and let them express themselves in their own terms, and at their own pace." (Bernard 2011: 157)

Dem einzelnen Gesprächspartner weitestgehend die Kontrolle über die inhaltliche Rahmung des Interviews zu überlassen, hat Aussagen zutage gefördert, die ich in einem formalen Fragebogen nicht erhoben hätte. So trat beispielsweise die große Bedeutung einzelner Emotionen wie Einsamkeit für die biographische Verortung des jeweiligen Gesprächspartners im sozialen Gefüge erst dadurch zutage, dass derjenige sie immer wieder in unterschiedlicher Art zum Ausdruck brachte.

Hinzu kommt, dass das narrative Interview es dem Forscher ermöglicht „[...] das zu untersuchende Thema aus der Perspektive der Interviewten zu erfassen und darüber hinaus verstehen und erklären zu können, weshalb eine bestimmte Perspektive eingenommen wird, wie sich diese im Laufe des Lebens entwickelt hat oder auch wie diese im Interviewkontext erzeugt wird“" (Rosenthal 2005: 125-126). 
Diese auch „Prinzip der Offenheit“ (Rosenthal 2005: 126) genannte Technik bringt noch eine weitere Dynamik mit sich, die Jørgensen und Phillips treffend beschreiben:

„Advantages are that the researcher does not influence the material and the type of material collected opens up for an analysis of variation across social contexts. For instance, an individual can give one version of the world in an interview and another in a conversation with a friend or in something he or she writes." (Jørgensen und Phillips 2002: 120)

In diesem Zitat wird deutlich, wie groß die Bedeutung der Differenzierung für die Auswertung des Interviewmaterials ist. Die Interaktion zwischen Interviewtem und Interviewer ist Teil des Forschungsprozesses (Rosenthal 2005: 126) und erfordert deshalb eine hohe analytische Transparenz. So gehe ich beispielsweise davon aus, dass ich aufgrund meines Geschlechts spezifische Aussagen von weiblichen Künstlerinnen zu den Genderdebatten Japans erhalten habe, die ein männlicher Gesprächspartner mir gegenüber nicht geäußert hätte. Umgekehrt sind mir mit Sicherheit einige Aspekte verborgen geblieben, die für einen männlichen Gegenwartskünstler eine vergleichbare Rolle spielen.

Durch das narrative Interview eröffnet sich dem Forscher die Möglichkeit, sich für die hohe Komplexität der verschiedenen diskursiven Aushandlungen mit all ihren Überschneidungen und Wechselwirkungen zu sensibilisieren und noch im Feld entsprechende Rückfragen zu stellen. Durch diesen offenen Austausch im Feld ist es mir möglich, den individuellen Persönlichkeiten meiner Gesprächspartner in meiner Arbeit Ausdruck zu verleihen und ihre Erfahrungen nachvollziehbar zu machen. Dabei besteht die Wertigkeit der Erhebungen nicht in der Häufigkeit ihres Auftretens, sondern generiert sich aus der analytischen Tiefe und der Nachvollziehbarkeit der Genese der aufgedeckten Bedeutungssysteme (siehe Rosenthal 2005: 137).

\subsubsection{Biographieforschung}

„A single study analyses a limited number of discursive utterances, and in order to say something meaningful about them, for example, whether they contribute to reproduction or to change, it is necessary to set them against some kind of background. [...] that is, one needs to have an understanding of the kind of structure in relation to which it should be analyzed."

(Jørgensen and Phillips 2002: 140)

Um zu verstehen, wie der individuelle Gesprächspartner seine Lebenswirklichkeit herstellt, ist der von Jørgensen und Phillips angesprochene Hintergrund von entscheidender Bedeutung. Als Hintergrund begreife ich dabei diejenigen Bereiche des persönlichen Wissens, die einen Erfahrungsschatz für die befragte Persönlichkeit darstellen und der sowohl ihr Selbstbild als auch ihr Handeln maßgeblich mitbestimmt. Aus diesem Grund habe ich mich dafür entschieden, die Biographien 
meiner Gesprächspartner in der oben beschriebenen offenen Interviewführung zu erheben. Wie Spradley in seinem bereits zitierten Werk The Ethnographic Interview (1979) deutlich macht, bilden Lebensgeschichten einen möglichen Zugang zur ethnologisch zu erschließenden Lebenswirklichkeit der untersuchten Personengruppe. Nachfolgend möchte ich verdeutlichen, was die narrativ erhobene Biographie auszeichnet und wie sich das entsprechende Analyseverfahren darstellt.

Der Sozialwissenschaftler Rustin (2000: 49) formuliert die Überschneidungen zwischen Ethnographie und Biographie sehr treffend: „Ethnography and biography explore process, rather than merely structure." Es ist der prozesshafte Vorgang, der mich neben den Inhalten des Interviews besonders interessiert. Es geht mir darum, die „[...] Herstellung von Bedeutung und Wissen aufzuzeigen“ (Rosenthal 2005: 127).

Das auf diese Weise generierte biographische Datenmaterial stellt das Hintergrundwissen zur Verfügung, das in Bezug auf das Erfassen der Handlungsfähigkeit (agency), Identität, Selbst-Konstruktion und Reflexivität für den Forscher von entscheidender Bedeutung ist (siehe Chamberlayne et al. 2000: 5; Ortner 2006). Individuelle und strukturelle Ebene erfahren in der persönlichen Rahmung des Interviewten eine Überbrückung: Das persönliche Erleben sozialer Prozesse wird in einem kulturell-historisch einordbaren Rahmen verankert und stellt damit Hintergrundwissen dar.

Die biographische Arbeit bezieht dabei folgerichtig die zeitliche Dimension mit ein:

„Biographical work is a way to orient the individual and make him or her reliable for institutions in a historical social situation when static personal definitions (such as status) or quasi-natural phases of a life-cycle are not sufficient for this purpose." (Fischer-Rosenthal 2000: 115)

Das Narrativ der jeweiligen Lebensgeschichte enthält also zweierlei Charakteristika: Erstens ihre Bedeutung für die gesellschaftliche Integration und zweitens den Orientierungsrahmen für das Individuum im Verhältnis zu anderen Mitgliedern der Gesellschaft.

Ein biographisches Narrativ vereint darüber hinaus alle drei Zeitebenen in sich: Der Erzähler kann von der Gegenwart aus die Vergangenheit konstruieren, diese stringent in seine Lebensgeschichte einordnen und gleichzeitig eine Zukunftsperspektive entwerfen. Dem Forscher kann sich in der Analyse dieses Datenmaterials der Prozess des Werdens, der „process of becoming“, offenbaren (Fischer-Rosenthal 2000: 118).

Die geschilderte Verbindung des Zeitlichen mit dem gesellschaftlichen sowie dem individuellen Level schließt die Möglichkeit ein, dass eine persönliche Bedeutungszuschreibung von dominanten Diskursen abweicht. Basierend auf individuellen Erlebnissen und Wahrnehmungen erstellen Individuen eigenständige Orientierungsrahmen, die nach einem Ventil des Ausdrucks verlangen, um gesehen, gehört und 
umgesetzt werden zu können. Die Sozialwissenschaftler und Experten der biographischen Methode Chamberlayne et al. (2000: 7) drücken es wie folgt aus: „We make our own history but not under conditions of our own choosing, and we need to understand these conditions of action more if our future making of our own history is to produce outcomes closer to our intentions and projects."

Die von meinen Gesprächspartnern selbst gesteuerte Darstellung ihres „process of becoming" manifestiert sich in ihrem sprachlichem Ausdruck, ihrem äußerem Erscheinungsbild und ihren Haltungen der japanischen Gesellschaft gegenüber. Diese sichtbaren Elemente werden im Interview mit der subjektiven Bedeutung des Einzelnen aufgeladen und präsentiert, was es anderen Mitgliedern der Gesellschaft möglich macht, das Gegenüber einzuschätzen und Erwartungen zu formulieren. Die Möglichkeit, die latenten Bedeutungszuschreibungen herauszufiltern, stellt folglich einen besonderen Wert der biographischen Fallanalyse dar (Chamberlayne et al. 2000: 9). Der Vorgang der Selbstpräsentation kann auch als politische Handlung verstanden werden, zum Beispiel indem der Sprecher eine Identifizierung seiner Person mit Werten, Zielen und Maßnahmen anstrebt. Die Aushandlung des Handelns im Einzelfall zu beobachten, ist dabei für den Forscher sehr aufschlussreich.

Das Teilziel meiner Analyse in Bezug auf die Biographieforschung besteht also darin, herauszufinden, inwiefern die Katastrophe von 2011 das biographische Narrativ meiner Gesprächspartner bestätigt oder teilweise in Zweifel gezogen hat. Die Wechselseitigkeit von Diskurs und Individuum, Gesellschaft und Identität des Einzelnen wird damit zum theoretischen wie methodologischen Ausgangspunkt aller Zugänge zum Forschungsfeld und im Umgang mit den erhobenen Daten. Es geht mir um die zentralen Fragen, wie und in welchem Kontext sich sowohl die Realitäten als auch die Identitäten meiner Gesprächspartner generieren und welche Konsequenzen sie daraus für ihr Handeln ableiten.

Zusammenfassend verbinde ich also den phänomenologischen Ansatz der personalisierten, inneren Bedeutungswelten (Chamberlayne et al. 2000; Hollway und Jefferson 2000; Rustin 2000; Bernard 2011: 157; Rosenthal 2005;) mit einem diskursiven Zugang zu strukturgebenden Elementen (Foucault 1973/ 1974; Beck 1986; Habermas 1989).

\subsubsection{Biographieforschung in Japan}

Von diesen methodischen Ausführungen zur Biographieforschung ausgehend wird deutlich, dass das gesellschaftliche Umfeld ein wesentliches Element bei der Konstruktion und Artikulation des Selbstnarrativs eines Akteurs ist. Kulturspezifische Verhaltensskripte in Bezug auf Emotionen ${ }^{26}$ und Selbstkonzepte ${ }^{27}$ nehmen entsprechend großen Einfluss auf die Rahmung der erhobenen Aussagen.

\footnotetext{
26 Siehe Kapitel 4.2.1 „Die ,polarisierende Differenzgesellschaft - Eine Diskussion“.

27 Siehe Kapitel 4.1.1 „Der Kompass der Werte - Ethik und Selbst im japanischen Kontext“.
} 
In einem, wie die Japanologin Gebhardt (2012: 200) es nannte, „engen Diskursraum Japan" eine eigene Perspektive zu entwickeln und nach außen zu vertreten, stellt meine Gesprächspartner vor einige Herausforderungen. Diese werden kontextabhängig mal mehr mal weniger stark gesellschaftlich ,sanktioniert“. Das bedeutet im Einzelfall, dass die Aktivität als Künstler negative Auswirkungen für ihn oder sie haben kann. Das Spektrum reicht dabei von negativen Emotionen bis hin zu konkreten wirtschaftlichen und sozialen Konsequenzen, beispielsweise indem sich Kuratoren weigern, die Werke eines bestimmten Künstlers auszustellen. Der Rückbezug auf eine wichtige Aufgabe, wenn nicht sogar auf eine selbstdefinierte Schlüsselposition innerhalb eines solchen „Handlungskorsetts“, stellt einen umso wichtigeren Anker im Selbstnarrativ meiner Gesprächspartner dar. So beschrieben die Künstler ihre gesellschaftliche Position mir gegenüber zunächst nur als marginalisiert. Ihre Erzählweise macht angesichts des japanischen Skripts der Interdependenz von Individuen intrinsisch Sinn. ${ }^{28}$ Erst im Laufe der Zeit und mit einer wachsenden Vertrautheit beziehungsweise wachsendem Vertrauen mir gegenüber, wurde dieses Narrativ durch die Facetten der Handlungsfreiheit einer Künstlerrolle erweitert. Die Nutzung dieser Handlungsfreiheit stellt allerdings angesichts einer ,[...] - schon vor Fukushima offenbar festgefahrenen, konformistischen Gesellschaft" (Gebhardt 2012: 200) eine besondere Herausforderung dar, wie ich anhand der Einzelfallanalyse noch genauer erläutern werde.

Im Laufe der Gespräche und Befragungen wurde deutlich, wie sich die Darstellung der eigenen Person, Rolle und deren Verantwortung entwickelt, also einem Prozess unterliegt. Wichtige Einschnitte für die eigene Person wurden von meinen Gesprächspartnern in einem gesamtgesellschaftlichen Zusammenhang geschildert. Meist wurden diese diskursiven Thematiken so geschildert, dass sie als Anlass für eine vertiefte Selbstreflexion des jeweiligen Gesprächspartners dargestellt wurden. Mir fiel auf, dass es dabei sowohl um die Hinterfragung der persönlichen Rolle im Beziehungsgeflecht des unmittelbaren Umfeldes geht als auch um normative Vorstellungen bezüglich konkreter Aufgaben und Leistungen der Künstlerrolle im Allgemeinen.

Ich gehe davon aus, dass die Methode des biographischen Zugangs in Japan unter anderem deshalb von meinen Gesprächspartnern so gut angenommen wurde, weil es ihrer eigenen Haltung, die Geschichte einer Persönlichkeit wertzuschätzen und ihrer Weltanschauung entsprechenden Raum einzuräumen, stark entgegenkam. Mein Wunsch, ihre individuellen Lebensentscheidungen verstehen zu wollen, ermutigte sie dazu, mir auch sehr persönliche Facetten aus verschiedenen Lebensphasen anzuvertrauen. Diese Aspekte wären mir ohne diesen freien Zugang womöglich verschlossen geblieben und liefern mir nun „[...] fresh insights and creative approaches“ (Chamberlayne et al. 2000: 22).

Ich weise im Besonderen darauf hin, dass es mir weder um eine Homogenisierung im Sinne einer Typenbildung noch um einen allgemein gültigen Anspruch auf

\footnotetext{
${ }^{28}$ Siehe Kapitel 4.1 „Der kulturspezifische Ausdruck des Selbst im sozialen Gefüge“.
} 
Bedeutungszuschreibung geht. Wenn ich Verallgemeinerungen nutze, beziehen diese sich lediglich auf einige Gemeinsamkeiten, die die Vertreter dieser Kunstwelt aus meiner Sicht auszeichnen.

Objektivität, im wissenschaftlichen Arbeiten allzu häufig als Leistungsmaßstab angelegt, verstehe ich nicht als Anspruch der Neutralität gegenüber meinem Forschungsfeld und seinen Akteuren, sondern als Aufforderung, meine eigenen Werte, Normen und Meinungen transparent zu machen: „The goal is not for us, as humans, to become objective machines; it is for us to achieve objective - that is, accurate knowledge by transcending our biases“" (Bernard 2011: 278).

Die Erhebung der individuellen Kontexte meiner Gesprächspartner ergänze ich durch die Anwendung der teilnehmenden Beobachtung. Mein eigener unmittelbarer Eindruck von der Lebenswirklichkeit meiner Gesprächspartner gewährt mir eine weitere Perspektive auf ihren Alltag und ermöglicht mir eine Kontrastierung ihrer Aussagen mit meinen eigenen Beobachtungen. Häufig waren diese zum Teil unterschiedlichen Einschätzungen von Situationen eine weitere Basis des Austauschs zwischen mir und dem jeweiligen Künstler.

\subsubsection{Teilnehmende Beobachtung}

Die teilnehmende Beobachtung liefert zusätzliche, ergänzende Informationen zum erhobenen Textmaterial des Interviews. Es war mir sehr wichtig, einen direkten Eindruck vom Leben einzelner Gesprächspartner zu bekommen und zum Beispiel ihre Wohnverhältnisse oder ihren erweiterten Freundeskreis kennenzulernen. Rosenthal beschreibt die teilnehmende Beobachtung als eine Möglichkeit, dass die „[...] sozialen Lebenswelten oder -milieus, soziale Gruppen, Ortsgesellschaften, einzelne Personen oder auch Organisationen in ihrer ,natürlichen Umgebung', das heißt in ihren Alltagskontexten erforscht werden“" (Rosenthal 2005: 101).

Wie zu Beginn dieses Kapitels bereits erläutert, wird in der qualitativen Forschung zwischen explizitem und implizitem Wissen unterschieden. Die Narration der eigenen Lebensgeschichte bedarf einer Verbalisierung und stellt damit eine explizite Form des Wissens dar. Die teilnehmende Beobachtung hingegen ermöglichte mir einen Zugang zu den Bereichen des Alltagslebens, die für den Gesprächspartner selbstverständlich sind und daher in den Interviews größtenteils nicht artikuliert wurden. Aus Sicht des Forschers fungiert die teilnehmende Beobachtung also als ergänzende Methode zur Einschätzung sowohl des Milieus, in dem sich meine Gesprächspartner tagtäglich bewegen, als auch den dort vorherrschenden Annahmen, Einstellungen, Werten und so weiter. Ein Nebeneffekt dieser Form des Zugangs ist, dass der Forscher eine „tiefe Vertrautheit“ (Rosenthal 2005: 101) mit dem sozialen Umfeld der von ihm untersuchten Akteure gewinnt.

Die meisten meiner Beobachtungen fanden im öffentlichen Raum statt, beispielsweise wenn der Künstler eine Straße in der Nähe einer Bahnstation für die Ausstellung seiner Bilder ausgewählt hatte. So konnte ich viel über die Interaktion des Künstlers mit den Passanten lernen. Die mit dem interessierten Publikum geführten 
Gespräche vermittelten mir einen guten Eindruck von den Einschätzungen und Emotionen der jeweiligen Rezipienten und des Künstlers. Häufig wollten Besucher bei den öffentlichen Ausstellungen die jeweiligen Werke mit dem Künstler besprechen oder es kamen Themen wie „Fukushima“ und die damit verbundenen Ängste und Sorgen der Tokyoter Bevölkerung zum Ausdruck. Teilweise wurden aber auch allgemeinere Themen angeschnitten, die die normativ-moralischen Facetten der Katastrophendiskurse berührten. Beispielsweise tauchten in den Gesprächen häufiger Fragen danach auf, was es bedeutet, sich um andere zu sorgen und zu kümmern. Der sich an die Beobachtung anschließende Austausch mit dem Künstler über die gerade stattgefundene Situation verhalf mir zu tiefen Einblicken in dessen Wahrnehmung und Prioritätensetzung. Diese ergänzenden Eindrücke runden meinen Eindruck von der jeweiligen Person ab und verhelfen mir zu einer sicheren Basis für die Interpretation der im Interview gemachten Aussagen. Die Alltagsbegleitung meiner Gesprächspartner half mir darüber hinaus, implizites Wissen über das Alltagsleben meiner Gesprächspartner zu erhalten, „[...] das nur schwer in verbaler Form vermittelt werden kann" (Rosenthal 2005: 102).

Über diese inhaltlichen Aspekte hinaus war es für die Beziehung zwischen dem einzelnen Künstler und mir als Forschende von besonderer Bedeutung, ihn in seinem Alltag zu begleiten. So stellte sich im Nachhinein heraus, dass der hohe Zeitaufwand der Beobachtung für viele meiner Gesprächspartner als ein Ausdruck von authentischem Interesse meinerseits wahrgenommen wurde. Die Methode der teilnehmenden Beobachtung entwickelte sich auf diese Weise zu einer Möglichkeit für mich, meinerseits Wertschätzung für die teilweise sehr lange und intensive Mitarbeit zu vermitteln. Dies war umso wichtiger, als dass in den Interviews teilweise Thematiken besprochen wurden, die in der japanischen Öffentlichkeit sehr kontrovers diskutiert wurden. Ein starkes und stabiles Vertrauensverhältnis wurde folglich zu einer Grundbedingung für den Fortgang der Forschung. Die zunehmende Öffnung einzelner Gesprächspartner für mein Forschungsvorhaben führe ich deshalb unter anderem auf den Einsatz der teilnehmenden Beobachtung zurück.

\subsection{Meine Gesprächspartner}

Bei der Zusammenstellung meiner 20 Interviewpartner aus der japanischen Kunstszene und 3 Experten halfen mir einzelne Gesprächspartner, indem sie mich auf ihr Kontaktnetzwerk zurückgreifen ließen. Bei der Auswahl achtete ich auf die jeweiligen aktuellen Projekte, regionale Herkunft, Gender und Alter ebenso wie auf langjährige Verbindungen und Kooperationen untereinander. Aufgrund persönlicher Vorlieben herrschte beim einzelnen Gesprächspartner ein unterschiedlich großer Wunsch nach gemeinschaftlicher Arbeit und Gedankenaustausch mit Kollegen, so dass es mir mitunter möglich war, neue Verbindungen im etablierten Netzwerk anzustoßen - ein Umstand, der ebenfalls zur Vertrauensbildung beitrug. 


\subsubsection{Zugänge zur Kunstszene}

Obwohl sich die Analyse größtenteils auf meinen Standort Tokyo fokussiert, spielt auch die regionale Herkunft eine wichtige Rolle in der Untersuchung der biographischen Verarbeitung der Dreifachkatastrophe. Beispielsweise ist Tokyo im Unterschied zur Ostküstenregion nicht direkt von den Auswirkungen des Tsunamis betroffen. Die Gefahr, radioaktiv verstrahlte Lebensmittel zu konsumieren, ist hingegen sehr viel häufiger Gesprächsthema gewesen. Die Katastrophendiskurse sind also unter anderem aufgrund des Grades der gefühlten Betroffenheit der Menschen unterschiedlich gewichtet.

Deshalb wollte ich sowohl das Tsunamigebiet entlang der Ostküste besuchen als auch die Stimmen einiger Akteure der Präfektur Fukushima aufzeichnen. Entsprechende Zugänge eröffneten sich mir mithilfe der bereits interviewten Gesprächspartner, die ein persönliches Interesse am Gelingen meines Vorhabens zeigten. Jeweils lokal arbeitende Künstler stellten daher eine wichtige Ergänzung meiner Analyse in Bezug auf Alter, Gender und lokale Identität dar. ${ }^{29}$

Mit fortschreitender Zeit im Feld wurde mein Anliegen in der vergleichsweise kleinen Kunstwelt Japans weitergetragen und es entwickelte sich ein Netzwerk, in dem mir weitere Kontakte vermittelt wurden. Es folgten Einladungen zu Ausstellungen und Galerieeröffnungen, wodurch ich in entspannter Atmosphäre mit weiteren Künstlern ins Gespräch kommen und teilnehmende Beobachtungen durchführen konnte.

Bei der methodischen Vorbereitung hatte ich mich gegen eine Kategorisierung entlang von Kunstbereichen wie Malerei, Musik oder Installationskunst entschieden, um die biographische Bandbreite der Erhebung möglichst offen zu gestalten. Diese Vorgehensweise wurde vor Ort als die richtige bestätigt, als die Tendenz meiner Gesprächspartner deutlich wurde, die Definition als Künstler (z.B. „ätisto") lieber von Dritten vornehmen zu lassen, als sie sich selbst zuzuschreiben. Eine weitere Einteilung entlang der genannten Kunstkategorien spielte für sie selbst zunächst eine untergeordnete Rolle und fand erst in den biographischen Verortungen ihren Ausdruck. War ein Gesprächspartner von einem anderen Künstler als solcher identifiziert und mir vorgestellt worden, verkürzte sich der langwierige Prozess der Vertrauensgewinnung massiv. Mein Selbstverständnis als Lernende im Feld konnte ich sehr schnell transparent machen beziehungsweise war diese bereits durch die Kontakte vermittelt worden. In Verbindung mit meiner Bereitschaft, die verschiedenen Gesprächspartner miteinander bekannt zu machen, trug dies im Einzelfall zur Entstehung eines beinahe freundschaftlichen Verhältnisses bei.

Mit wachsendem Verständnis für die Situation meiner Gesprächspartner, meinem Eingehen auf japanische Gepflogenheiten wie dem Überreichen von Mitbringseln (jap.: omiyage) aus den bereisten Regionen, kleineren persönlichen Gefallen und meinem Beitrag zur Netzwerkbildung veränderte sich das Verhältnis zu denjenigen

\footnotetext{
29 Siehe Kapitel 8 „Ayako von Shiogama - Der Tsunami im lokalen Kontext der Ostküste“.
} 
Akteuren, mit denen ich über den gesamten Forschungszeitraum von 12 Monaten in Verbindung bleiben konnte. Dies machte sich entsprechend in zunehmend vertrauterer Ansprache mir gegenüber bemerkbar und zeigte meinen Gesprächspartnern, dass ich ihre Bewertung der Diskurse nachvollziehen konnte. Maynard, Professorin für die japanische Sprache, beschreibt die gemeinsame Behandlung eines Themas im Diskurs vom linguistischen Standpunkt aus als ein „target of emotion“: „Speaker and partner, by sharing the emotional target from the same perspective, co-experience the feelings" (Maynard 2005: 35).

Dieses Zitat beschreibt meine Erfahrungen im Feld sehr treffend. Das Annähern an eine gemeinsame Perspektive stabilisierte nicht nur das Vertrauensverhältnis zwischen mir und dem jeweiligen Künstler, sondern erweiterte auch mein persönliches Netzwerk an Interviewpartnern. Darüber hinaus ermöglichte mir die zunehmende Sozialisation im Feld durch die entsprechende Empathie auch einen Zugang zu weit zurückliegenden und schmerzlichen Abschnitten der jeweiligen Lebensgeschichte. Rückblickend konnte ich erleben, wie „[...] discourse itself aims to create empathy on the basis of speakers' and partners' shared experience" (Maynard 2005: 35). Umso höher gestaltete sich der analytische Aufwand im Feld, die eigene Perspektive von der meiner Gesprächspartner zu trennen und die Thesen entsprechend weiterzuentwickeln.

\subsubsection{Empathie als Leitlinie meiner Interaktion im Feld - Eine Heranführung}

In der Phase des Zugangs zum Feld stellt sich ein Ethnologe den Herausforderungen, die die Reflexion über eigene Realitätsvorstellungen und die des Gegenübers mit sich bringt. Dieser Adaptionsprozess vor Ort führte für mich zu einer zunehmenden Sensibilisierung für die vom kulturellen Kontext geprägten und individuell interpretierten Bewertungsprozesse. Meine Prozesse des Einfindens in Japans Kultur beziehen sich vor allem auf die Emotionalität und die darin implementierten Moralvorstellungen der jeweiligen Gesprächspartner. Mein Ziel in diesem Kapitel ist es, die subjektiv empfundenen biographischen Zäsuren der Interviewten ebenso zu erfassen wie die jeweiligen Bewertungen der desaströsen Ereignisse vom 11. März 2011. Dies erforderte, dass sich die jeweiligen Akteure öffneten und bereit waren, auch schmerzliche Phasen ihres Lebens anzusprechen.

Um den Rahmen für eine solche Tiefe der Erzählweise zu etablieren, war die Präsentation meiner Selbst als Lernende im konkreten Umfeld ebenso wichtig wie der sukzessive Aufbau eines tiefen Vertrauensverhältnisses zu den einzelnen Gesprächspartnern über längere Zeit. Die dafür erforderliche Empathie sensibilisierte mich schon früh für eine Facette des Forschungsprozesses, die sich sowohl in den einzelnen Interviews als auch im alltäglichen Miteinander stets aufs Neue zeigte: Im Laufe der Zeit löste sich die starre Rahmung gegenseitiger Zuschreibungen immer mehr auf. Je länger ich mich als verlässlich im Umgang mit emotionalen Verhaltenskodizes erwies, desto mehr eröffneten sich für beide Parteien neue Möglichkeiten, sich in verschiedenen Situationen und Rollen kennenzulernen. Erst auf der Basis 
dieses gegenseitigen Verständnisses war es allen Gesprächspartnern möglich, mir sehr persönliche Aspekte ihres Selbst zu zeigen. Das Eintreten in diese Phase markierte den Abschluss meiner Transformationsphase von einem fremden, höflich distanziert zu behandelnden, ausländischen Kontakt zu einer den kulturellen Regeln entsprechend handelnden und emotional mündigen Person. ${ }^{30}$ Mir wurde zugetraut, über ausreichend kulturelles Wissen zu verfügen, um keine eklatanten Normverletzungen zu begehen und somit keine negativen Gefühle wie Scham oder Schuld auszulösen. Damit zeigte sich in meinen konkreten Interaktionsphasen, was die japanische Kulturwissenschaftlerin Lebra (2010) als „exposure sensivity“ bezeichnet. Mit diesem Begriff beschreibt Lebra die besondere Rolle des öffentlichen Ausagierens von Scham und Schuld in der japanischen Gesellschaft unter psychokulturellen Gesichtspunkten und setzt sie in einen engen Bezug zu Moral- und Normvorstellungen. Die Sensibilität für die Offenbarung von Emotionen im japanischen Kontext und den daraus folgenden sozialen Konsequenzen sieht Lebra (2010: 102) als einen essentiellen Teil des japanischen Emotionskodex an, der sich aus der Interdependenz des Selbst ergibt: „Shame is a pervasive part because Japan [...] has its cultural norms well defined so that their violations are readily recognized, and partly because the Japanese individual is more surrounded by significant audiences to whom his action is exposed“ (Lebra 2010: 102). Als Forschende betrat ich also eine gesellschaftliche Bühne, in der negativ belegte Handlungsweisen durch das Gefühl der Scham sanktioniert werden. Diese Sanktionierung wird umso stärker vom Agierenden empfunden, da er sich der emotionalen Konsequenzen für sein Gegenüber sehr bewusst ist. „This tendency is derived from the allocentric empathy with which the Japanese actor is prone to take the role of audience and to stare at his own action as if he were an object of attention" (Lebra 2010: 103).

Die hier von Lebra so treffend beschriebene Gleichzeitigkeit der Perspektiven, sowohl Handelnder als auch Beobachter zu sein, ist in meinen Interviewdaten besonders evident und zeigt sich im Einzelfall in der Berücksichtigung der jeweilig involvierten Akteure sowohl innerhalb als auch außerhalb der Kunstszene. So nahm ich in den ersten Phasen des Aushandlungsprozesses zwischen mir als Forschender und den jeweiligen Gesprächspartnern rhetorisch-mimische Abwehr wahr, wenn wir uns in ambivalenten Themengebieten bewegten. Dies äußerte sich in betontem Lachen, Schweigen gefolgt von Themenwechsel oder starker Abschwächung des vorher Gesagten.

Abgesehen von diesen subtilen Hinweisen auf ihr Unbehagen bemühten sich die jeweiligen Akteure zu Beginn der Forschung sehr darum, mein möglicherweise nicht vorhandenes Wissen um die japanische Kunstszene und die entsprechenden sensiblen Bereiche auszugleichen, indem sie ihre Erzählungen mit zusätzlichen, sehr weit führenden Informationen ausschmückten. Ich interpretiere dies als einen Versuch meiner Gesprächspartner sicherzustellen, dass es zu keinen Missverständnissen

${ }^{30}$ Siehe Kapitel 4.1 „Der kulturspezifische Ausdruck des Selbst im sozialen Gefüge“. 
kommt. Nach Abschluss dieser Phase, ca. drei Monate nach meiner Ankunft im Feld, verwendeten meine Gesprächspartner keine solchen Ausführungen mehr.

Die Basis der sprachlichen Abwehr oder „defensive tactics“, wie Lebra (2010: 104) sie nennt, beruhte vor allem auf der Reflexionsfähigkeit meiner Gesprächspartner über die jeweiligen Standpunkte, Meinungen und Perspektiven sowie aus einer stark ausgeprägten Empathiefähigkeit. In der stufenweisen Abschwächung dieses Schutzes vor unangenehmen Situationen und des langsamen Übergangs von der äußeren zur inneren Selbstpräsentation des jeweiligen Akteurs zeigte sich die zunehmend positiv ausfallende Bewertung meines Enkulturationsprozesses durch mein japanisches Gegenüber.

Bei meiner anschließenden analytischen Betrachtung meines Enkulturationsprozesses zeigten sich subtil ablaufende Aushandlungsprozesse, die ich auch im japanischen Diskurs der Dreifachkatastrophe wahrnahm. So wie meine Gesprächspartner sichergehen wollten, dass ich ihre Haltung bezüglich kontroverser Themen einordnen konnte, so findet auch ein Abgleich unterschiedlicher Realitäten auf gesellschaftlicher Ebene statt.

\subsubsection{Die Auswahlkriterien meiner Gesprächspartner: Alter, Gender, Lokalität}

Ein wichtiges Steuerungselement bei der Auswahl meiner Gesprächspartner war die Spezifizierung meiner Auswahlkriterien, die ich auf der Basis meiner Vorkenntnisse der japanischen Gesellschaft traf. Einige dieser Kriterien bestätigten sich während der Forschung. So war "Gender" beispielsweise für viele meiner männlichen Gesprächspartner zunächst keine essentielle Thematik. Frauen hingegen sprachen die Geschlechterrollen ausnahmslos von sich aus an und machten deutlich, dass die Kunstszene diesbezüglich keine Ausnahme von der gesellschaftlichen Rollenverteilung darstellt.

Das Bewusstsein meiner Gesprächspartner in Bezug auf Gender oder andere gesellschaftliche Themen war zudem stark vom Alter des jeweiligen Gesprächspartners abhängig: Jüngere weibliche Künstler hatten sich aufgrund der an sie herangetragenen hohen Erwartungshaltung, beispielsweise die Rolle der Ehefrau und Mutter einzunehmen, intensiv mit diesen Fragen auseinandergesetzt. Meine männlichen Gesprächspartner, von denen alle ledig und zum Zeitpunkt der Forschung in keiner partnerschaftlichen Beziehung waren, reflektierten ebenfalls über diese Thematik, sprachen sie jedoch nicht von sich aus an. Dies könnte auf den in industriellen Gesellschaften gängigen Umstand zurückzuführen sein, dass die Mutterrolle mit einem beruflichen Wunsch nur unter erschwerten Bedingungen gelingen kann, wenn der männliche Partner als hauptverantwortlicher „breadwinner" definiert wird. Dieses Modell, welches in Japan nach wie vor gängig ist, erschwert es auch den weiblichen Künstlern, im Falle einer Heirat ihrem Beruf weiter nachzugehen. Umso wichtiger erschien es mir, diese und andere genderbedingten Aspekte in der Erhebung zu berücksichtigen. 
Um die Antworten meiner Gesprächspartner auf zeitgenössische Fragen im Kontext des 11. März 2011 einordnen zu können, gehe ich in den folgenden Kapiteln explizit auf einige Kulturspezifika Japans ein. Ich setze Normen und Wertvorstellungen mit den rekonstruierten Abläufen des 11. März 2011 in Beziehung, wie sie sich mir nach dem Studium von wissenschaftlicher Literatur, japanischer wie internationaler Medien und persönlichen Gesprächen darstellen. Diese Informationen setzen den Diskursrahmen, von dem aus ich mich dem Realitätsverständnis einzelner Individuen annähere und die Rückwirkungen auf das soziale Gefüge Japans untersuche. 



\section{Der sozio-kulturelle Kontext der Dreifachkatastrophe}

Aus meiner Sicht waren einige entscheidende Voraussetzungen für den Verlauf der Dreifachkatastrophe bereits lange vor dem 11. März 2011 gegeben. So erfuhr ich im Feld, dass einige Fragen für meine Gesprächspartner von großer Bedeutung waren: Inwiefern waren Verantwortliche über bauliche wie technische Missstände am AKW Fukushima informiert gewesen, lange bevor die Ereignisse von 2011 ihren Lauf nahmen? Wurden wichtige Informationen nicht weitergegeben? Wurde der Wirtschaftlichkeit mehr Priorität eingeräumt als der Sicherheit der Menschen? Diese Fragen beschäftigten meine Gesprächspartner sehr, betreffen sie doch unmittelbar die Aushandlung gesamtgesellschaftlicher Verantwortung für das Geschehene.

$\mathrm{Zu}$ den durch menschliches Versagen geschaffenen Voraussetzungen für die AKW-Katastrophe gehören neben einigen technischen Planungen des AKW Fukushima auch die gesetzlichen Sicherheitsbestimmungen, die die atomare Stromgewinnung betreffen, und das Machtverhältnis zwischen Wirtschaft und Politik. Diese auf allen gesellschaftlichen Ebenen diskutierten Aspekte der Katastrophe werfen ein Licht auf die Position des Individuums in einem nach wie vor hierarchisch geprägten Sozialgefüge und den damit verbundenen Wertvorstellungen.

Ich gehe davon aus, dass sich die kulturell geprägten Gestaltungen des Alltags in direkter Weise auch auf der gesellschaftspolitischen Ebene auswirken, beispielsweise in der Ausformung der politischen Parteienlandschaft, dem demokratischen Selbstverständnis und in Bezug auf alle Formen der freien Meinungsäußerung. Wichtige 
Aspekte in diesem Zusammenhang sind unter anderem der soziale Raum für Diskussionen und Auseinandersetzungen, soziale Bewegungen, Wissensgenerierung und zivilgesellschaftliche Strukturen. Die entsprechenden gesellschaftlichen Entwicklungen sind in Japan erst seit Ende der 1980er Jahre zu beobachten. Derzeit wird unter anderem durch verstärkte Straßenproteste und unabhängige Berichterstattung im Internet versucht, „[...] Widerstand denk- und lebbar zu machen sowie eine Verschiebung der Grenzen des Sagbaren in gesellschaftlichen Diskursen zu erwirken“ (Leser und Trunk 2013: 338).

Protestformen wie Demonstrationen existieren in Japan nicht erst seit den Ereignissen des 11. März 2011. Diese erfahren jedoch angesichts des katastrophalen Ausmaßes der Vorkommnisse und der enormen Beteiligung der Bevölkerung ${ }^{31}$ verstärkt mediale Aufmerksamkeit. Die Wurzeln dieser Hinwendung zu verstärktem zivilgesellschaftlichem Engagement verorten die Japanologen und Politikwissenschaftler Leser und Trunk (2013: 338) unter anderem in den sich verändernden Arbeits- und Lebensrealitäten der Menschen seit der wirtschaftlichen Krise der 1980er Jahre, das auch als „Platzen der Bubble“ bezeichnet wurde. Die wirtschaftliche Rezession der 1990er und 2000er Jahre macht es seitdem jungen Menschen schwer, dem nach wie vor herrschenden japanischen Ideal einer Festanstellung mit den entsprechenden finanziellen wie sozialen Sicherheiten gerecht zu werden. Gleichzeitig etabliert sich unter den jungen Arbeitnehmern zunehmend die Idee, sich verstärkt auf Selbstbestimmung und Selbstversorgung zu konzentrieren und sich nicht mehr von Großkonzernen und ihren Arbeitsmöglichkeiten abhängig zu machen. Diese so entstehende Unabhängigkeit manifestiert sich laut Leser und Trunk (2013: 342) unter anderem in der Produktion von unabhängigen Medien. Diese Entwicklungen in der Politik und Wirtschaft Japans bestärken meiner Meinung nach eine Diversifizierung der politisch-gesellschaftlichen Landschaft, was meine Gesprächspartner mir in ihren Aussagen spiegelten.

Aufgrund dieser Bedeutungszusammenhänge werde ich im folgenden Kapitel wichtige kulturelle Thematiken erläutern. Ich befasse mich darin ausführlich mit der gesellschaftlichen Ebene und den innerjapanischen Debatten um die Sozialstruktur. Auf diese Weise ordne ich die Dreifachkatastrophe in den bereits vor 2011 existierenden japanischen Diskurs ein und schaffe damit die Basis für eine Einordnung der Interviewaussagen meiner Gesprächspartner in eine emotional aufgeladene Diskussion, die sich insbesondere an „Fukushima“ entzündet.

\footnotetext{
${ }^{31}$ Die verfügbaren Zahlen der Demonstrationsteilnehmer in Tokyo im September 2011 beziffern sich beispielsweise auf 60.000 Beteiligte (Leser und Trunk 2013: 339). Im Juni 2012 sollen 150.000 Menschen vor die Residenz des damaligen Premierministers Noda Yoshihiko gezogen sein, um gegen die Wiederinbetriebnahme der Reaktoren in Oi zu protestieren (Leser und Trunk 2013: 346).
} 


\subsection{Der kulturspezifische Ausdruck des Selbst im sozialen Gefüge}

Wie ich bereits ausgeführt habe, verstehe ich Emotionen sowohl als Diskurs als auch als Kommunikationsmittel. Dies bedeutet, dass Emotionen ihrerseits kommuniziert werden müssen, um in Aushandlungsprozessen wirksam zu sein. In ihrer Kulturspezifik offenbart der Ausdruck von Emotionen wesentliche gesellschaftliche Umgangsregeln und -normen, die die persönliche Auseinandersetzung mit einem Diskurs entscheidend beeinflussen. Insbesondere die kontextgebundene Sprache kann viel darüber aussagen, in welchen Machtverhältnissen die jeweiligen Akteure zueinander stehen, wie sie die konkrete Situation einschätzen und auf welche Weise Spannungsverhältnisse gelöst werden können.

Aufgrund dieser Überlegungen ziehe ich erneut die Linguistin Maynard (2005) heran, die sich durch ihre Analyse des emotionalen sprachlichen Ausdrucks im Japanischen besonders hervorgetan hat. In ihren zahlreichen Publikationen zur japanischen Sprache untersucht sie den verbalen Ausdruck von Emotionen mit besonderem Augenmerk auf Kontext und aktuelle Diskursentwicklungen. „Language is not understood only for its informational content, but also felt for its emotion and empathy" (Maynard 2005: ix).

Der Einfluss der Sprache auf Emotionen spielt in meinem Forschungsansatz insofern eine sehr wichtige Rolle, als dass bei der Interpretation des von mir erhobenen Interviewmaterials zum einen das Verhältnis zwischen mir als Forscherin gegenüber meinen Gesprächspartnern zu berücksichtigen ist. Zum anderen ist auch der mit diesem Verhältnis zusammenhängende Einfluss von Vertrauen und Nähe auf die Länge und Tiefe der geschilderten Lebensgeschichten nicht zu unterschätzen. Während des gegenseitigen Kennenlernens haben meine Gesprächspartner und ich die gegenseitigen Gruppenzugehörigkeiten und Rollenverständnisse ausgehandelt, definiert und Handlungsoptionen ergründet. So konnten sich meine Gesprächspartner aufgrund von Gender, Nationenzugehörigkeit, Berufsgruppe, Klassenzugehörigkeit, Alter, Generation, regionalem Hintergrund (Dialekt) unter anderem definieren und mir gegenüber entsprechend auftreten.

Im Folgenden geht es mir im Speziellen um eine Kontextualisierung von Emotionen in Japan, wie sie sich sowohl in der gesellschaftlichen Diskursführung als auch in den geführten Interviews manifestieren. Die im vorangegangenen Abschnitt ausgeführten Aspekte von Kitayama und Markus (1994) sowie die von Hendry (1992) zum relationalen Selbst und der Rolle der Individualität darin werden dabei eine besondere Rolle spielen.

Die Ansichten, Perspektiven, Hintergründe und emotionalen Bewertungen von Erfahrungen des jeweiligen Individuums zu verstehen, ist für mich von besonderer Wichtigkeit. Die Bedeutungszuschreibung von Erfahrungen und der institutionell 
flankierten Lebensstationen von Kindheit, Jugend, Schule und Berufsausbildung ${ }^{32}$ erhalten im jeweiligen Narrativ meiner Gesprächspartner ihre individuelle Konnotation und Schwerpunktsetzung. Durch die biographische Erzählweise zu einem bestimmten Zeitpunkt verortet sich der jeweilige Akteur in seinem sozialen Umfeld, also im gesellschaftlichen Gefüge mit seinen Werten und Normen, und nimmt Bezüge zum aktuellen dominanten Diskurs.

Zum Verständnis dieser Vorgänge dienen die folgenden Kapitel, in denen ich auf den kulturspezifischen Kontext meiner biographischen Analyse eingehe. Die entsprechenden Zugänge erlauben tiefergehende Einblicke in die Form, Struktur und das Vorgehen der Verortung des jeweiligen Gesprächspartners im japanischen Kontext.

\subsubsection{Der Kompass der Werte - Ethik und Selbst im japanischen Kontext}

In meiner Auseinandersetzung mit Selbst und Person auf theoretischer Ebene ist mir eine bedeutende Schnittstelle mit der Biographieforschung bewusst geworden: Um verstehen zu können, wie und warum ein Individuum seine persönliche Sichtweise in einer spezifischen Weise darstellt, muss ich seine Sozialisation und die prägenden Ereignisse in seinem Leben erfassen. Eine retroperspektivisch hergestellte stringente Interpretation mit emotionalen Momenten und einem möglicherweise von gesellschaftlichen Konventionen abweichenden Umgang mit diesen Momenten kann ich auf diese Weise sichtbar machen und sowohl in ihrer Genese als auch in ihrer Prozesshaftigkeit darstellen.

Zum Zeitpunkt meiner Forschung waren seit der Dreifachkatastrophe bereits drei Jahre vergangen, was sich in entsprechenden Reflexionen meiner Gesprächspartner widerspiegelt. Ich mache in diesem Prozess zwei Bemessungsstränge von Erfahrungen aus, die eine wichtige Rolle spielen: Zum einen die kulturell geprägten Rollenvorstellungen von Mann und Frau im Allgemeinen und zum anderen die Erwartungen, Freiräume und Rahmungen, denen der jeweilige Akteur im Speziellen unterworfen ist. ${ }^{33}$

Darüber hinaus trat bei der Auswertung der Literatur und meines Datenmaterials ein Spannungsfeld zwischen einem inneren, privaten Selbst (honne) mit individuellen Fähigkeiten und Eigenschaften und einem äußeren, öffentlichen Selbst (tatemae) zutage. Dieses Konzept erachte ich als so wichtig für das analytische Verständnis meines Datenmaterials, dass ich an dieser Stelle ausführlicher darauf eingehen möchte. Lebra schrieb mit ihrem Werk Japanese Patterns of Behavior (1976) einen der ersten Klassiker über die Verbindung von Moral, Emotionen und Verhalten in der japanischen Gesellschaft. Wie auch Kitayama und Markus (1994) betont sie die Aushandlung emotionaler Äußerungen in der Beziehung (Lebra 1976: 16). Zusätzlich zeigt Lebra eine Trennung des japanischen Selbst in einen inneren (honne) und einen

\footnotetext{
32 Siehe dazu Okano und Tsuchiya (1999).

33 Siehe Kapitel 6 „Kunst und Künstler in Japan - Eine Milieuanalyse“.
} 
äußeren Teil (tatemae) auf, wobei letzterer für die Bewertung des Verhaltens durch Dritte herangezogen wird. Der innere Teil kann sich wenig oder gar nicht im Konsens mit dem gelebten Emotionskodex befinden:

„While conformity is thus maintained in socially expressed behavior, the individual's inner self is likely to lack such conformity. This kind of inner autonomy, as one might call it, is taken for granted in Japan. [...] The frustration of the unexpressed nonconfirming self can sometimes be released through social interaction without spoiling the united front. Sometimes, however, it builds up so much as to become irreversible. In that case the dissenting member may organize a splinter group or defect from the group entirely."

(Lebra 1976: 29-30)

Diese Diskrepanz zwischen Innen und Außen ist eine legitime Gestaltungsart zwischenmenschlicher Beziehungen in Japans Gesellschaft. Durch diese Aufteilung des Selbst kann eine soziale Sanktionierung, zum Beispiel durch Stigmatisierung oder Scham, vermieden werden.

Nichtsdestotrotz ist es von entscheidender Bedeutung für den Einzelnen, eine Dissonanz zwischen sich und dem Rest der Gruppe nicht zu groß werden zu lassen. Es entsteht eine internalisierte Form des Konformitätsdrucks, die von außen durch hierarchische Strukturen, wie zum Beispiel dem Schulsystem, verstärkt wird. Der eigene Wille zur Anpassung, so Lebra (1976: 29) weiter, sei an eine Intoleranz gegenüber dem Versagen oder der Verweigerung von Konformität gekoppelt. Der entstehende homogenisierende Effekt wird folglich gezielt gefördert, um sowohl die einzelnen sozialen Einheiten, wie beispielsweise Schulklassen, kontrollieren zu können als auch die gesamte Bevölkerung im Falle des Falles an einen gemeinsamen Moral- und Emotionskodex zu binden und sich nach außen abzugrenzen. „The pressure of conformity often results in a type of self-restraint called enryo, refraining from expressing disagreement with whatever appears to be the majority's opinion" (Lebra 1976: 29). Für meine Untersuchung ist es angesichts dieser Rahmung umso wichtiger, die Aussagen meiner Gesprächspartner im Hinblick auf ihren persönlichen Emotionsausdruck zu untersuchen - insbesondere, da im Zuge der Dreifachkatastrophe von 2011 die Konflikte im Inneren der japanischen Gesellschaft sichtbar wurden.

An dieser Stelle möchte ich zunächst das Individuum im japanischen Kontext betrachten. Dabei richte ich meinen Fokus zunächst auf die Verbindung des Individuums mit ethischen Dimensionen und Empathie, deren Verinnerlichung im Sozialisationsprozess wichtige Rahmungen des Selbst im Allgemeinen darstellen. ${ }^{34}$

Dazu ziehe ich im Folgenden Erläuterungen des Kulturwissenschaftlers Lambek heran, in denen er die Ethik nicht als metaanalytisches Theorem behandelt, sondern in der täglichen Praxis und als Teil des Daseins als Mensch verortet. Lambeks

\footnotetext{
${ }^{34}$ Diese Aspekte werde ich im Kapitel 4.1.2 „Das Verhältnis vom Individuum zur Gruppe“ wieder aufgreifen.
} 
Ansatz ist vorwiegend philosophisch geprägt und setzt sich mit den Wesenszügen ethischer Fragen und ihrer Behandlung in den Sozialwissenschaften im Allgemeinen auseinander. Seiner Definition zufolge ist Ethik „,...] not a matter of smoothly following the rules but of the exhilaration of self-transcendence, as well as the struggle with ambivalence and conflict" (Lambek 2010: 12). Lambek betont die Alltäglichkeit ethischer Fragen und Konflikte, die die Konfrontation mit ethisch-moralischen Soll-Zuständen und einem wahrgenommenen Ist-Zustand aufwerfen. Die Antwort auf die Frage, wie gelebt werden sollte, ist in seinem Verständnis ein tief in der sozialen Praxis verankertes Prinzip, das der täglichen Beurteilung durch die Handelnden unterworfen ist. Dabei spielt die strikte Einhaltung von ethischen Grundsätzen und Regeln, wie in der oben zitierten Aussage angedeutet, weniger eine Rolle als die individuelle Anwendung von Kriterien auf spezielle Handlungen und Umstände (Lambek 2010: 19).

Das „gute Leben“ zeichnet sich demzufolge durch Handlungen aus, die aufgrund dreier Kriterien als „tugendhaft“ eingestuft werden können: 1. Der Handelnde besitzt das praktische Wissen darum, was das Richtige ist. 2. Er entschließt sich, dementsprechend zu handeln. Passivität oder Inaktivität schließt dieses Verständnis von Ethik folglich aus. 3. Die Handlung unterliegt nicht dem Zufall, sondern hat ihren Ursprung in persönlicher Überzeugung (siehe Lambek 2010: 19).

Ethisches Verhalten setzt laut Lambek daher immer eine Auseinandersetzung mit den Thematiken des sozialen Alltags voraus. Das Verhalten eines Einzelnen wird danach beurteilt, was man über den Handelnden weiß und unter welchen Umständen er handelte. Dabei kommen sowohl explizite Regeln zum Tragen als auch implizit gelebte Kriterien, die die Wahrnehmung von ethischen Brüchen im sozialen Gefüge maßgeblich mit beeinflussen. Im Alltag ergibt sich daraus ein Spannungsfeld, das sich zwischen Kontinuität und Innovation, zwischen expliziten und impliziten Regeln bewegt (Lambek 2010: 28). Wie ein Individuum in diesem Spannungsfeld agiert, ist ein direkter Spiegel seines Aushandlungsprozesses.

In Bezug auf den japanischen Kontext möchte ich hervorheben, dass meiner Erfahrung nach die Wahrnehmung der Verletzung oder Einhaltung ethischer Grundsätze einen wesentlichen Bestandteil des japanischen Selbst-Konzeptes ausmacht und sich zum Teil als empathisches Verhalten im Alltag niederschlägt. Das Handeln nach impliziten wie expliziten Umgangsregeln positioniert den Empathie-Gebenden gegenüber dem Bedürftigen (siehe Hermann 2011:32). In meinem Forschungskontext sind es diese moralisch aufgeladenen Facetten des Verhaltenskodexes, die sich im Verhältnis des Gegenwartskünstlers zu den unmittelbar Betroffenen des 11. März 2011 niederschlagen.

Darüber hinaus zeigten sich im Laufe meiner Forschung Konflikte zwischen der Generation meiner Gesprächspartner und deren Eltern, die vor allem auf unterschiedlichen Werteausrichtungen basierten. Die Japanologin Lebra (1976) beschreibt die gültigen normativen Vorstellungen der sogenannten „Babyboomgeneration“. Deren Mitglieder wurden zwischen 1947 und 1949 geboren. Als Nachkriegsgeneration er- 
lebten diese Menschen ein starkes wirtschaftliches Wachstum und identifizieren sich besonders stark mit ihrer Generation.

Mit ihrem Fokus auf das Verhalten im kulturellen Kontext bezieht Lebra sich auf beobachtbare Handlungen, die von einem Wertesystem gespeist und gelenkt werden. Sie geht der Frage nach, was Glück und Unglück, Freude und Ärger auslöst: „In other words, I am interested in those aspects of cultural behavior that are ,valuecharged“" (Lebra 1976: xvi). Woran orientierte sich also das japanische Individuum der Elterngeneration meiner Gesprächspartner?

Lebra unterteilt ihre Forschungsergebnisse in drei Kategorien, die sich mit den sozialen, physischen und symbolischen Aspekten des Verhaltens auseinandersetzen. Sie beschreibt eine Ausrichtung der letzten beiden Kategorien am Sozialen. Das heißt, dass die sozialen Beziehungen diejenige Logik generieren, nach der konsumiert und interagiert wird. Lebra (1976: 5) bezeichnet dies als „social preoccupation“, die sich auch in der moralischen Ausrichtung der japanischen Gesellschaft zeige: „For the Japanese, goodness or badness is a relative matter, relative to social situation and impact, whose complexity may often be beyond any judge's comprehension" (Lebra 1976: 11). Damit sind alle sozialen Beziehungen dieser Generation auf eine möglichst harmonische, reibungsfreie Interaktion ausgelegt, die unter anderem auf einem starken Bewusstsein der Zugehörigkeit wurzelt.

Obwohl Lebras Ausführungen auf den ersten Blick sehr schematisch und stereotyp wirken, gelten Orientierungswerte wie die der „reference group“ (Lebra 1976: 22) auch heute noch als wichtige Stützpfeiler der japanischen Identität. Unter Referenzgruppe versteht Lebra (1976: 22) eine Zuschreibung von Zugehörigkeit, basierend auf Geburtsort, Arbeitsplatz, Generationszugehörigkeit, Alter etc., die das Individuum in den sozialen Rahmen einbettet. Ähnlich wie Kitayama und Markus (1994) beschreibt Lebra die Ausrichtung des japanischen Individuums an dieser sozialer Rahmung und Harmonie: „The sense of identity anchored in group belongingness is thus sustained by going along with peers. This goes with the desirability of being accepted by peers, anxiety about being left out, and a competitive urge for always being 'in'" (Lebra 1976: 29).

Im Handeln meiner Gesprächspartner spiegeln sich sowohl die persönliche Standpunktverortung des jeweiligen Künstlers in einem Diskussionsraum als auch latente Aspekte des gesellschaftlichen Selbstverständnisses. Brüche und Konfliktfelder treten in diesem Prozess deutlich zu Tage, ganz im Gegensatz zu ihrer Elterngeneration. Aus meiner Sicht stellt dies eine besondere Brisanz in der Situationsbeurteilung und der individuellen Aushandlung ethischer Regeln dar. Sollte ein Akteur wahrnehmen, dass seine Vorstellungen guten Handelns verletzt werden, wie kann er darauf öffentlich wirksam reagieren? Welche Verantwortung übernimmt der Einzelne damit für sich und andere?

Mit dem Wechselspiel von Handlungsvermögen, Ethik und der daraus erwachsenden Verantwortung befasst sich Laidlaw. In „Agency and Responsbility: Perhaps You Can Have Too Much of a Good Thing“ setzt sich Laidlaw kritisch mit der 
Praxistheorie von Sherry Ortner und der Akteur-Netzwerk-Theorie von Latour auseinander. Laidlaws Kritik entspringt seiner Auffassung, dass Handlungsvermögen (agency) sehr eng mit Eigenverantwortung verknüpft ist, auch im gesamtgesellschaftlichen Kontext: „My interpretation is not only why but actually what has happened is inseparable from, because partly constituted by, my judgements about responsibility for it" (Laidlaw 2010: 147). Kurz gesagt: Wenn ich die Verantwortung einer bestimmten Personengruppe zuschreibe, beeinflusst dies in eklatanter Weise meine Interpretation der Ereignisse und ihrer Ursachen.

Von dieser Prämisse ausgehend, übt Laidlaw Kritik an den theoretischen Überlegungen von Ortner und Latour. Er sieht deren Arbeit durch die ihnen zugrunde liegende Annahme kompromittiert, Handlungsvermögen würde sich vor allem im Widerstand gegen Strukturen zeigen. Laidlaws Ansicht zufolge verstecken sich in dieser Definition von agency bereits bestimmte Werte, die das Individuum entsprechend handeln ließen. Er jedoch ist der Auffassung, „[...] to think that there is a natural fact or quality of ,moral responsibility' against which our judgements and attributions might be measured, even in principle, is a category error" (Laidlaw 2010: 147).

Die enge Verbindung von Handlungsvermögen und Verantwortung in Laidlaws Konzept lässt ihn agency nicht als Qualität einer Person (Ortner 2006) verstehen, sondern er ordnet sie einem situativen Kontext zu. Die sich daraus ergebenden $\mathrm{Zu}$ ordnungen von Verantwortung sind für das Handeln des Einzelnen von entscheidender Bedeutung. Die Auseinandersetzung mit der strukturellen Rahmung durch eine Gesellschaft erfolgt Laidlaw zufolge nicht im aktiven Widerstand „[...] to change the structural systems in which they live by means of authentic self-expression" (Laidlaw 2010: 144), sondern in der alltäglichen Aushandlung des Einzelnen.

Ich kann mich Laidlaws Kritik nur teilweise anschließen, sehe ich doch keinen Widerspruch in der Kontextualität von Verantwortung und einem Widerstand gegen gesellschaftliche Strukturen. Auch wenn Laidlaw sowohl das alltägliche Element des Widerstands als auch die Reproduktion gesellschaftlicher Strukturen von Ortners agency-Definition (2006) vernachlässigt, ist seine Betonung des situativen Kontextes in der Betrachtung von Ethik und agency nichtsdestotrotz für meine Arbeit sehr gewinnbringend. Insbesondere vor dem Hintergrund von Katastrophen verschwimmen die Grenzen zwischen gutem, verantwortungsvollen Handeln und situativ notwendigen, teilweise schmerzhaften Entscheidungen. Dabei bleibt es nicht aus, dass gesellschaftliche Vorstellungen ethisch korrekten Verhaltens verletzt oder stark belastet werden.

Die Performanz der ethischen Überzeugungen verleiht den einzelnen Handlungen also erst ihre Bedeutung, zeigt darüber hinaus aber auch deren Grenzen auf: „Ethics, then, is not only about executing acts, establishing criteria, and practicing judgment, but also about confronting their limits, and ours" (Lambek 2010: 39). Die Erfahrung der Ausdehnung oder sogar Überschreitung ethischer Grenzen ist es letztendlich, die an den grundlegenden Selbstverständnissen einer Gesellschaft rührt und ein stark emotional aufgeladenes Moment in sich trägt. Deren Auswirkungen 
können wir derzeit unter anderem in der Diskussion um den Umgang mit den Betroffenen der Dreifachkatastrophe nachvollziehen. Zusammengefasst berühren die individuellen Bewertungen der ethischen Dimensionen einer Katastrophe sowohl das Selbstverständnis einer Person als auch deren gesellschaftliche Selbstpositionierung. Aus diesem Grund sind die im Post-Fukushima-Diskurs aufgeworfenen ethischen Fragen für meine Gesprächspartner von großer Bedeutung. Jedoch sind sie es nicht nur aufgrund einer gesellschaftlichen Rollenzuschreibung, in der Gegenwartskünstler als sozialkritische Akteure betrachtet werden. Welche anderen Aspekte eine Rolle spielen, zeige ich im folgenden Kapitel.

\subsubsection{Das Verhältnis vom Individuum zur Gruppe}

Die Autoren Kitayama und Markus (1994) beschäftigen sich als Vertreter der Kulturpsychologie mit Selbst, Kognition, Identitätskonstruktion und Emotionen. Bei der Analyse einer ganzen Bandbreite psychologischer Prozesse entlang einer geographisch gedachten kulturellen Ost-West-Achse legen sie in ihren Beiträgen eine besonders hohe Sensibilität für mögliche Missverständnisse und Gegensätzlichkeiten an den Tag.

In Kitayamas und Markus' Untersuchungen stehen jedoch nicht die Dichotomien zwischen „Ost“ und „West“ im Fokus. Stattdessen betonen sie Unterschiede und Gemeinsamkeiten gleichermaßen. Ein Ziel ihres Wirkens ist es, die sozio-kulturelle Basis von diversen psychologischen Prozessen nachvollziehbar zu machen, die sie im japanischen Kontext beobachten. So heben sie hervor, dass die jeweiligen Artikulationen einer Person von kulturellen Rahmenbedingungen geprägt werden, die ebenso wie andere Facetten einer Kultur starken Wandlungsprozessen unterliegen. Von dieser konstruktivistischen Prämisse ausgehend, beschreiben Markus und Kitayama die Einordnung des Selbst vor dem gesellschaftlichen Hintergrund eines Typus'. Sie unterscheiden zwischen einem unabhängig ausgerichteten Selbst (independent) und einem relationalen Selbst (interdependent). Im Falle des unabhängigen Selbst ist das individuelle Empfinden ausschlaggebend für das Verhalten im öffentlichen Raum (Markus und Kitayama 1991: 226). Diese Ausrichtung des Selbst verorten Markus und Kitayama in der westlichen Sphäre, wohingegen sie dem relationalen Selbst im japanischen Kontext eine entscheidende Rolle zuschreiben. In einem Selbst, das sich stark in Beziehung zu anderen setzt, spielen externe Funktionen und Eigenschaften eine wesentlich größere Rolle als im unabhängigen Selbst. Status, Rolle und Beziehungsgestaltung erhalten Priorität vor den individuellen Empfindungen und Einschätzungen:

„Key features of this intersubjectivity are a heightened sense of the other and of the nature of one's relation to the other and the expectation of some mutuality in this regard. The goal is not individual awareness, experience, and expression, but rather some attunement or alignment of one's reactions and actions with those of another, and intersubjective experience is a result of 
these efforts and, in turn, fosters these efforts. "(Markus und Kitayama 1994a: 101-102)

Was Kitayama und Markus hier als „Intersubjektivität“ bezeichnen, verstehe ich als das Resultat eines zwischenmenschlichen Verhaltens, dem die Fähigkeit vorausgeht, Gefühle, Erfahrungen und Erwartungen des Gegenübers zu erfassen und sich entsprechend darauf einzustellen. Ich interpretiere dies als Hinweis auf die besondere Rolle der Empathie für die Kommunikation und die Gestaltung guter Beziehungen im japanischen Kontext.

Das bedeutet, dass für eine gute Kommunikation neben dem erhöhten Bewusstsein für Status und Rolle des Gegenübers auch eine erhöhte Sensibilität für dessen emotionalen Zustand entscheidend ist. Aus diesem Grund gehe ich genauer auf die Empathie im japanischen Kontext ein. Zunächst jedoch möchte ich an dieser Stelle die Wirkungen des interdependenten Selbst als Verhaltensrahmung genauer darlegen und aufzeigen, wie dieses japanische Selbst-Verständnis in der Literatur mit einem „westlichen“ kontrastiert wird.

Kitayama und Markus beschreiben sehr treffend, wie das Bemühen um eine Einstimmung auf das Gegenüber dazu führt, dass sich Situationen stabilisieren. Den Ursprung für eine starke Kontrastierung dieser, in der Literatur als relationales SelbstVerständnis bezeichneten, japanischen Konzeptionalisierung mit dem westlichen Individualismus wird unter anderem in nationalstaatlichen Narrativen verortet. So stellen Autoren wie Ishikawa (2005: 20), ein Vertreter der Medienanthropologie, eine Verbindung zwischen einer zu starken Fokussierung auf das eigene Ich (jap.: kojinshugi) und entsprechenden negativen Konsequenzen im Staatskontext her: Es habe in der Vergangenheit eine Tendenz vorgeherrscht, Individualismus als eine Einladung zur Genusssucht zu betrachten, was die Einheit des Nationalstaates gefährdet habe. In diesem Verständnis von Individualismus läuft die Fokussierung auf die eigenen Wünsche den Interessen der Gruppe, in diesem Fall der nationalstaatlichen Gesellschaft, zuwider und ist deshalb negativ konnotiert:

„[...] the inner self was often conceptualized in opposition to the nation state, a collective entity to which one belonged and yielded. The anticipated tension between a pursuit of self-fulfillment and a commitment to the nation became a source for indigenous models of individualism during the interwar period."

(Ishikawa 2005: 21)

Die Ansicht, eine einzelne Person als einzigartiges Wesen mit essentiellem Selbst wahrzunehmen, intensivierte sich in Japan erst in den 1980er Jahren, was vor allem mit Japans wachsender wirtschaftlicher Macht zusammenhängt. Die Mehrheit der Bevölkerung, dereinst durch das staatliche Narrativ einer homogenen Gesellschaft vereint, diversifizierte sich zunehmend mit wachsendem Wohlstand und steigendem Selbstbewusstsein der Nachkriegszeit (Ishikawa 2005: 143). 
In der wissenschaftlichen Literatur gibt es jedoch auch Stimmen, die diese Betrachtungsweise als zu stark vereinfachend kritisieren. Die Sozialwissenschaftlerin Hendry (1992) sticht in diesem Zusammenhang mit ihrem Plädoyer für erhöhte Sensibilität gegenüber dem japanischen Selbst besonders hervor. Ihrer Analyse zufolge wird im Japanischen auch auf sprachlicher Ebene stark zwischen Individualismus (dem zuvor erwähnten Begriff kojinshugi für selbstsüchtig und kindlich) und der Individualität (jap.: kosei) unterschieden (Hendry 1992: 56). Kosei gilt Hendry zufolge als Ideal, bei dem man seine eigenen Talente und Fähigkeiten optimal entwickelt und der Gemeinschaft zur Verfügung stellt. Um die Umsetzbarkeit des sich bereits andeutenden Balanceaktes zwischen Individualität (gemeint sind individuelle Fähigkeiten, Vorlieben und Ressourcen), und der Notwendigkeit, sich der Gruppe unterzuordnen, zu erfassen, verwendet Hendry den Begriff der "Rolle“ als Ausdruck gesellschaftlicher Hierarchie: Man sei zwar als Person keiner anderen über- oder unterlegen, wohl aber in der zu erfüllenden Rolle (Hendry selbst empfindet den Begriff der „Rolle“ als missverständlich, kann aber keinen passenderen Terminus anbieten). Hendry argumentiert weiter, dass das entsprechende Verhalten in der öffentlichen Sphäre durch dieses Rollenverständnis klar definiert ist und daher nicht frei gewählt werden kann (Hendry 1992: 63).

In Hendrys Ausführungen wird deutlich, dass dem für das westliche Verständnis der Individualität so essentiellen Aspekt der „Freiheit“ in der Rollenausübung, bei dem das innere Selbst möglichst deckungsgleich mit der nach außen präsentierten Person auftritt, im japanischen Kontext einen anderen Stellenwert erhält. Sich im japanischen Sozialgefüge zu viele Freiheiten herauszunehmen würde implizit auf ein mangelndes Bewusstsein für die eigene Position innerhalb eines Beziehungsnetzwerks hindeuten. Dies ist jedoch nur für unmündige Mitglieder der Gesellschaft wie Kinder ein tolerierbares Verhalten. Hendry schreibt dazu: „[...] characteristics which make an American mature are precisely the same as those which are described as immature in Japan“ (Hendry 1992: 64).

Obgleich es wenige Einzelfälle gibt (wie zum Beispiel Künstler), werde die breite japanische Bevölkerung nach wie vor dazu ermutigt, sich den gesellschaftlichen Rahmenbedingungen anzupassen, diese auszuhalten und dadurch ihre Reife zu zeigen (Hendry 1992: 65). Jede Form von Gesellschaft, so Hendry weiter, bedeute schlussendlich eine Unterordnung des Individuums unter die Bedingungen sozialer Bindungen, und somit könne sich ein Individuum auch nur in der Gesellschaft als solches definieren.

Nichtsdestotrotz finden sich die Spuren einer stark dichotomen Betrachtungsweise bis heute in den japanischen Diskursen. Beispielsweise im Diskurs der "Japaneseness" (Nihonjinron), der mit japanischem Patriotismus gleichgesetzt wird. Im Nihonjinron-Diskurs werden die „Einzigartigkeit der japanischen Kultur“ eng mit einem relationalen Selbst verknüpft. Das heißt: In diesem Diskursstrang wird die große Bedeutung, die der Beziehung zu Anderen zugeschrieben wird, als japanspezifisch und einzigartig betrachtet. Diese strenge Überzeugung, Japans Kultur sei absolut einzigartig, mag nur von einer Minderheit vertreten werden. Nichtsdestotrotz 
hat die Identifikation mit einer relationalen Ausrichtung des Selbst nichts von seiner diskursiven Macht verloren.

Die hier von mir aufgezeigten wissenschaftlichen Stimmen diversifizieren das stark vereinfachte, polarisierende Konstrukt von einem westlichen, individualistisch geprägten Selbst auf der einen und einem relational ausgerichteten, japanischen Selbst auf der anderen Seite. Meine eigene Forschung und das Studium der einschlägigen Literatur zeigen, dass das Selbst-Verständnis des sich über Beziehungen definierenden Individuums von der Mehrheit der japanischen Bevölkerung nach wie vor geteilt und gelebt wird. Jedoch existieren viele Schattierungen der genannten Gegensatzpole. Die Diskussion um die individualistischen und relationalen Anteile der Interaktion erhöhte meine Sensibilität in Bezug auf die gesammelten Interviewdaten. Genauer gehe ich darauf in den entsprechenden Analysekapiteln 7, 8 und 9 ein. Jedoch greife ich nicht zu stark voraus, wenn ich sage, dass sich Gegenwartskünstler durch die öffentliche Ausstellung ihrer Werke und der damit verbundenen Gesellschaftskritik einer besonderen Spannung ausgesetzt sehen. Künstler bewegen sich zwischen dem Ausdruck einer individuellen Meinung und der gesellschaftlichen Einbettung mit Konformitätsdruck. Eine sich daran anschließende Frage stellte sich also in Bezug der Künstler: Wer genau ist ihre primäre Bezugsgruppe, wenn sie Individualität (die Hendry als kosei aufführt) als positiv beschreiben und auch so leben wollen? Wie sieht ihr praktisch gelebtes Konzept von Individualität im Dienste anderer aus? Und wird diese Lebensweise nicht vielleicht sogar im größeren Kontext der japanischen Gesellschaft in Anbetracht ihrer speziellen Rolle erwartet? Diese Fragen beantworte ich auf der Basis meiner Datenanalyse.

Die in der wissenschaftlichen Literatur stark betonte Wichtigkeit des kulturellen Kontextes bei der Analyse gibt Hinweise auf die Bedeutung der Bildungsprozesse des Selbst und insbesondere die der emotionalen Sozialisation. In dieser werden Bedeutungszuschreibungen von Erfahrungen einem spezifischen sozialen Umfeld gemäß erlernt. Hendry (1992: 59) hebt in ihrem Werk die Wichtigkeit von Sozialisationsinstitutionen wie Schule und Kindererziehung im Allgemeinen hervor, die wichtige Etappen des aktiven Aushandlungsprozesses zwischen einer Einzelperson und ihrem spezifischen Umfeld darstellen. Nachdem ich in diesem Kapitel den hohen Stellenwert der Empathie im japanischen Kontext hervorgehoben habe, stelle ich im Folgenden die Emotionen selbst in den Fokus.

\subsubsection{Konfliktlösungen in der sozialen Praxis}

In der Zuschreibung der Opferrolle stehen für die unmittelbar Betroffenen der radioaktiven Verstrahlung aufgrund der Neuartigkeit der Situation keine geeigneten Charakteristika zur Verfügung. ${ }^{35}$ Die Diskussion um den Umgang mit den sogenannten „Atomflüchtlingen“ wird zusätzlich dadurch belastet, dass sich im öffentlichen Diskurs die Attribute der Täter mit denen der Opfer vermischen: Die Anwohner be-

\footnotetext{
35 Siehe Kapitel 9.1 „Tsunami und Atomkraft: Eine Gegenüberstellung der emotionalen Diskurse“.
} 
troffener Gebiete hatten dem Bau von Atomkraftwerken in ihrer Region aufgrund wirtschaftlicher Profitmöglichkeiten in den 1960 und 1970er Jahren zugestimmt. In den japanischen Diskursen wird ihnen daher eine Teilschuld an den Ereignissen des 11. März 2011 angelastet. Daraus entstehen widersprüchliche Emotionen, nämlich sowohl Anteilnahme und Verständnis als auch Distanzierung und Stigmatisierung. Die individuelle Beantwortung der Frage nach Schuldzuschreibung und der darin enthaltenen Verletzung von ethischen Vorstellungen spielt also in diesem Zusammenhang eine besonders wichtige Rolle, wenn es darum geht, das Verhalten der jeweiligen Akteursgruppen zu kontextualisieren und zu verstehen.

Der von Lebra (2010: 107) beschriebene Akt der Reflexion als Voraussetzung für Schuldgefühle generiert sowohl ein Bewusstsein für das Selbst in der konkreten Situation als auch für Norm- und Regelverletzungen auf gesellschaftlicher Ebene. Diese Reflexion zieht meist einen Akt der Selbst-Beschuldigung und -Entschuldigung nach sich: „If an alter aggresses against an ego, it should be understood that the aggression originated from ego against the alter and now has returned to its origin" (Lebra 2010: 107).

Diese Zusammenhänge aus Schuldzuweisung und Selbst-Beschuldigung sind auch in einigen Äußerungen der Betroffenen der Strahlenkatastrophe zu finden, die eine Teilschuld einräumen, auch wenn die Entscheidung zur Nutzung der Atomenergie von vorangegangenen Generationen gefällt wurde (siehe Brandner 2014). Die teilweise vorgenommene Selbstidentifikation der Anwohner der Präfektur Fukushima als Profiteure der Atomenergie verlangt, im gesellschaftlichen Zusammenhang betrachtet, nach einer formal korrekten Wiederherstellung der harmonischen Verhältnisse. Sowohl einige der direkt Betroffenen als auch einige Amtsinhaber sahen sich in der Folgezeit des 11. März 2011 zu einem öffentlichen Schuldeingeständnis veranlasst. Die Formalität einer solchen öffentlichen Entschuldigung kann Lebra (2010: 109) zufolge, und hier stimme ich ihr aufgrund der Beurteilung insbesondere politischer Entschuldigungsrituale durch meine Gesprächspartner zu, als ein „perfunctory ritual lacking sincerity" angesehen werden, das sogar als strategische Vertuschung der Regelverletzung aufgefasst werden könne. ${ }^{36} \mathrm{In}$ dem öffentlich demonstrierten Bemühen, entstandene Normverletzungen auszugleichen und negative Gefühle zu befrieden, zeigen sich die zwei Seiten einer starken Betonung von Beziehungen eines sozialen Gefüges: Zum einen offenbaren sie die von Lebra (2010) aufgezeigte Kontrolle des eigenen Verhaltens durch den Akteur, um das Gegenüber nicht emotional zu verletzen und damit Disharmonie auszulösen. Zum Anderen werden die vergleichsweise starken Sanktionen im Falle eines Versagens, wie beispielsweise einer gesellschaftlichen Normverletzung, deutlich.

Die große Heftigkeit, mit der die Dreifachkatastrophe vom 11. März 2011 auf Japan einwirkt, löst nicht nur starke Emotionen, sondern, als Folge dessen, auch Impulse für gesellschaftliche Transformationsprozesse aus. Wie in Kapitel 2 bereits angedeutet, nimmt mit zunehmendem Informationsgehalt um die genauen Abläufe

\footnotetext{
36 Siehe Kapitel 4.1.3 „Konfliktlösungen in der sozialen Praxis“.
} 
der Katastrophe und den entsprechenden Reaktionen der Entscheidungsträger auch die Kritik an Kommunikations- und Interaktionsmustern im Allgemeinen zu.

Insbesondere in Bezug auf die Atomkatastrophe richten sich die Fragen meiner Gesprächspartner nach der eigenen Identität, dem Selbstverständnis als japanischer Bürger sowie der eigenen Verantwortung für das Geschehen im AKW „Fukushima“ an zwei Richtungen aus: Vergangenes wird aufgearbeitet und im Licht der neuen Erkenntnisse um Netzwerkbildung zwischen Wirtschaft und Politik, um persönliche Absprachen oder sogar bewusste Vertuschungsaktionen von kontroversem Datenmaterial neu bewertet. Gleichzeitig richtet sich der Gedankenfokus auf die Gegenwart und Zukunft.

Indem diese Fragen und erste Antworten in die Öffentlichkeit getragen werden, werden sie aus einem geschlossenen Personenkreis von Experten herausgelöst und auf breiter gesellschaftlicher Ebene diskutiert. Die allgemeine Betroffenheit der sozialen Folgen der Katastrophe wird also umso deutlicher in den Fokus gestellt desto aktiver die verschiedenen Akteure sich ihrer annehmen. Dieser Prozess führt zu tiefergehenden Fragen, wie sie auch die Protestforschung (siehe Stekelenburg und Klandermans 2010) zu beantworten versucht. Im japanischen Kontext kristallisiere ich folgende Fragen als die drängendsten im Diskurs um die Atomkatastrophe heraus:

- Zu welchem Anteil ist jeder Bürger persönlich für die Entscheidungen verantwortlich, die schlussendlich zur Katastrophe führten?

- Wie sind die Konsequenzen individuell und gesamtgesellschaftlich zu bewerten?

- Wie können verhängnisvolle strukturelle Entwicklungen in Zukunft verhindert werden und welchen Beitrag kann/sollte jeder Einzelne leisten?

Zunächst habe ich beobachtet, dass insbesondere von Seiten der Behörden versucht wurde, die negativen Emotionen im Zusammenhang mit der von der Katastrophe aufgedeckten Diskrepanzen zwischen einem moralischen Soll- und dem aktuellen Ist-Zustand zu befrieden. Dabei wurde häufig auf öffentliche Schuldbekenntnisse und Entschuldigungen zurückgegriffen. Lebra beschreibt die Strategien im Umgang mit Konflikten so:

„By conflict management I mean a reaction to a conflict situation without necessarily entailing a resolution. Management can involve procrastination, aggravation of conflict, or initiation of a new phase of conflict. [...] It is not that Japanese never risk confrontations but that, as long as harmony, or the appearance of harmony, is to be maintained, nonconfrontational modes must be exhausted first." (Lebra 1984: 41-42)

Im Falle eines bereits eingetretenen Konflikts, in dem offizielle Statements und die erlebte Situation der unmittelbar Betroffenen aufeinanderprallen können, steht Lebra zufolge nicht die unmittelbare Lösung im Mittelpunkt. Das Bemühen um 
die Aufrechterhaltung der Harmonie bezeugt bereits die Ausrichtung des Entscheidungsträgers an den Wünschen der Gruppe, in diesem Fall der Bevölkerung Japans und insbesondere der unmittelbar betroffenen Menschen an der Ostküste und der Präfektur Fukushima.

Als weitere Strategien des Umgangs mit Konflikten führt Lebra (1984: 41ff.) Schweigen, Vermeidung und Akzeptanz von Konfliktsituationen auf, sensibilisiert aber gleichzeitig für ungewollte Nebeneffekte der einzelnen Vorgehensweisen. Schweigen könnte beispielsweise nicht als Ablehnung oder Protest, sondern auch als stille Akzeptanz interpretiert werden, so Lebra (1984: 43-44). ${ }^{37}$ In meinen eigenen Forschungsunterlagen finden sich Hinweise auf und Reflexionen über beobachtetes Verhalten von Entscheidungsträgern, politischen wie wirtschaftlichen Akteuren und meinen Gesprächspartnern zu diesen Äußerungen. Die von Lebra (1984: 50 ff.) dargestellten Lösungsstrategien für Konflikte wie Selbstmord, Verinnerlichung des Konflikts (das heißt die schlussendliche Unterdrückung negativer Gefühle) oder Akzeptanz versagen angesichts der gewaltigen geographischen, sozialen und zeitlichen Dimension der Katastrophe von 2011. Dies ist ein wesentlicher Bestandteil der derzeitigen Diskurse um die Dreifachkatastrophe in Japan.

In Bezug auf die Dreifachkatastrophe interpretiere ich Lebras Ausführungen so, dass das Versagen der gängigen Konfliktlösungsstrategien im Fall des 11. März 2011 dazu beitragen könnte, die Bevölkerung für aufglimmende Konflikte stärker zu sensibilisieren, denn: „The norm of harmony may be precisely what makes people more aware of conflicts with others, conflicts between their self-interest and obligations, and so forth. [...] In other words, the cultural value of harmony may intensify, instead of mitigate, conflict" (Lebra 1984: 56).

Dafür spricht mein persönlicher Eindruck, dass die im japanischen Kontext gängige Strategie des Besänftigens durch Rituale, beispielsweise dem Rücktritt eines Entscheidungsträgers, angesichts des Ausmaßes und Andauerns der Katastrophe in der japanischen Öffentlichkeit als nicht mehr ausreichend empfunden wird. Unmittelbar Betroffene, Interessenvertreter unterschiedlicher Bürgerinitiativen, NGOs, Gegenwartskünstler und viele weitere Akteure liefern bis zum heutigen Tag stetig neue Informationen über interne Abläufe, zurückliegende Fehlentscheidungen und politische Fehltritte. Damit stellen diese Akteure sicher, dass die emotionale Verarbeitung stetig neue Nahrung erhält und sich die diversen Thematiken in den PostFukushima-Diskursen halten.

Ich beobachte, dass der von Lebra geschilderten Strategie des Schweigens von Seiten der genannten Akteure eine stetige Anfrage nach Informationen, Aufklärung und Erläuterung entgegengesetzt wird, so dass der Frage der Verantwortung schlussendlich bei vielen verschiedenen Vertretern aus Politik, Wirtschaft, Staat und Zivilbevölkerung nachgegangen wird. Diese vielen verschiedenen Akteure bringen ihre jeweilige Version der Abläufe und Geschehnisse in den Diskurs ein und diver-

37 Entsprechende Auswirkungen auf zivilgesellschaftliche Prozesse und die Demokratie Japans finden sich bei Patricia Steinhoff $(1984,2013)$ und MacLachlan (2008). 
sifizieren ihn somit. Der Übernahme von Verantwortung und Selbst-Beschuldigung, dem Prozess der Verinnerlichung wie Lebra (1984: 50) dies nennt, wird dadurch entgegengewirkt.

Für den japanischen Kontext ist ein Versuch des Austragens von Konflikten im öffentlichen Raum als zumindest ungewöhnlich zu beurteilen. Dieser Umstand gibt Hinweise auf die tiefgreifende und nachhaltige Wirkung von Katastrophen auf Gesellschaften im Allgemeinen. Für das japanische Emotionsskript im Speziellen manifestiert sich dies meiner Ansicht nach in einer Hinterfragung des Emotionskodex', in dessen Folge Emotionen nicht mehr umschrieben oder gar verschwiegen, sondern artikuliert und in ihren Konsequenzen sichtbar gemacht werden. Auch in meinen Interviews kam es zu Ausrufen, die in ihrer Direktheit nicht nur einen gewissen Vertrauensgrad mir gegenüber anzeigten, sondern auch auf eine beinahe kämpferische Stimmung des Betroffenen hindeuteten („Mitero!“- „Wait and see!“).

Im aktuellen Konfliktmanagement des 11. März 2011 beobachte ich, dass hier vor allem von ziviler Seite aus versucht wird, der politischen Legitimationsmacht eine moralisch-emotionale Diskursführung entgegenzuhalten. Wie meine Gesprächspartner erkennen lassen, kann dies die Krise des Systems nicht nur realitätsnah widerspiegeln, sondern die Notwendigkeit der Transformationsprozesse untermauern und damit das staatliche Diskursmonopol aufbrechen. Den Aussagen meiner Gesprächspartner zufolge vertreten gerade staatliche Behörden die geltenden Konventionen von augenscheinlicher Harmonie, an der meine Gesprächspartner ausdrückliche Kritik üben.

Es wird deutlich, dass mit diesem Umgang mit Emotionen, Konflikten und Lösungsangeboten ein moralisch aufgeladener Gegendruck zur politischen Gestaltungsmacht auf emotionaler Basis aufgebaut wird, der die Bürger Japans aktivieren und Verantwortliche in die Pflicht nehmen kann. Meiner Ansicht nach handelt es sich um einen in der japanischen Geschichte bisher seltenen Moment gesellschaftlicher Diskussionsführung, der durchaus als Legitimationskrise seiner staatlichen Vertreter und deren wirtschaftlicher Verbündeter interpretiert werden kann. In derart geführten Diskursen werden die aus den sozialen Folgen der Katastrophe entstandenen Forderungen nach finanzieller Entschädigung der unmittelbar Betroffenen, nach Offenlegung der wirtschaftspolitischen Netzwerke und nach Transformationen der Verwaltungsstruktur als unausweichlich darstellt. Gegenwartskünstler, als Vertreter federführender Akteure der "Grassroot-Ebene“, liefern in dieser gesellschaftlichen Debatte viel Material für die emotional geführten Katastrophendiskurse.

Die besondere Bedeutung ihres Handelns liegt in der Suche nach neuen Interaktionswegen in einer stark auf Hierarchien aufgebauten Gesellschaft, die alte Muster der "Differenzgesellschaft“ (kakusa shakai) ${ }^{38}$ aufbrechen und nachhaltige Strategien dauerhafter Kommunikation zwischen allen Ebenen der Gesellschaft ermöglichen sollen.

\footnotetext{
${ }^{38}$ Siehe Kapitel 4.2.1 „Die ,polarisierende Differenzgesellschaft - Eine Diskussion“.
} 


\subsection{Ein diskursiver Blickwinkel auf die japanische Gesellschaft}

Es ist bereits angeklungen, dass meine Gesprächspartner eine spezifische Auffassung von der japanischen Gesellschaft haben. In den folgenden Kapiteln ziele ich darauf $\mathrm{ab}$, die Einschätzungen, Meinungen und Emotionen meiner Gesprächspartner transparent zu machen, die diese Auffassung prägen. Dazu gehören neben der Berücksichtigung jener Rahmenbedingungen, die ihre Rolle als Gegenwartskünstler prägen, ${ }^{39}$ auch jene Diskurse, die die negativen Seiten einer Gesellschaft aus emischer Perspektive beleuchten. Die zutage tretenden Aushandlungsprozesse lassen nicht nur die Wirkmuster der japanischen Gesellschaftsstruktur sichtbarer werden, sondern setzen auch diskursive Rahmungen, die viel über die aktuelle Stimmung, die Paradigmen und das Selbstverständnis einer Gesellschaft aussagen.

Nachdem ich in den vorangegangenen Kapiteln vor allem auf die Konzepte eingegangen bin, die das japanische Selbst maßgeblich rahmen, geht es mir in den folgenden Abschnitten um die aktuellen diskursiven Repräsentationen Japans als Sozialgefüge. Die in öffentlichen Diskursen favorisierten Narrative weisen auf kontrovers diskutierte Themen wie Ethik, Verantwortung und interdependentes Miteinander auf gesellschaftlicher Ebene hin, die entscheidend für das Verständnis der vielfältigen Argumentationsstränge um das Thema „Fukushima“ und seiner sozialen Folgen sind. Diese Hintergründe reichen bis in die unmittelbare Nachkriegszeit mit ihren wirtschaftlichen Veränderungen, deren sozialen Konsequenzen und politischen Reaktionen zurück. Diese verschiedenen Bausteine des gesellschaftlichen Zusammenspiels bestimmen ein Lebensgefühl, das sich unmittelbar auf die individuelle Bewertung der persönlichen Situation innerhalb der Gesellschaft auswirkt. Vor dem Hintergrund meines biographischen Analyseansatzes erachte ich die Erläuterung dieser sozio-politischen Narrative als essentiell für die Kontextualisierung der zitierten Aussagen. Wichtige Fragen sind dabei: Durch welche Argumentationsstränge und -rahmungen werden die jeweiligen Aussagen meiner Gesprächspartner geprägt? Inwiefern ist das persönliche Selbstverständnis mit dem historischen Hintergrund einer Region oder einem lokalem Umfeld verbunden?

Im Folgenden beschränke ich mich in der Analyse auf diejenigen Diskursstränge Japans, die von meinen Gesprächspartnern als entscheidend erachtet werden. In der Diskussion um die verschiedenen Ausdifferenzierungen in der japanischen Gesellschaft mache ich zwei Argumentationslinien ausfindig.

Die eine zielt auf Differenzen der Werte und Normen zwischen den Generationen und den sich daraus ergebenen Konflikten ab. Die andere beschreibt Japan als „hope-divided society“ (Slater 2010: 8), also als eine von Ungleichheit in Bezug auf Bildung und den damit verbundenen sozialen Aufstiegschancen geprägten Gesellschaft. Dies ist in Japan deshalb von so herausragender Bedeutung, da Bildung nach

\footnotetext{
39 Siehe Kapitel 6 „Kunst und Künstler - Eine Milieuanalyse“.
} 
wie vor entscheidend für eine erfolgreiche Karriere und damit für die soziale Anerkennung ist. „Formal education in school and training within the company are the most obvious sites of socialization in Japan" (Slater 2010: 23).

Allen Diskursführungen gemeinsam ist jedoch die Betonung der sozialen Ungleichheit in der einen oder anderen Form, die sich im Begriff „polarisierende Differenzgesellschaft" (jap. kakusa shakai) zusammenfügen. Die Hervorhebung der Differenz gegenüber dem Ideal einer homogenen Gesellschaft verweist auf starke Risse und Brüche im Sozialgefüge, die nicht erst seit dem 11. März 2011 diskutiert werden.

\subsubsection{Die „polarisierende Differenzgesellschaft“ - Eine Diskussion}

Während der Auseinandersetzung mit den vorherrschenden Grundannahmen meiner Gesprächspartner über ihr alltägliches Leben, ihre Wahrnehmungen geltender sozio-ökonomischer Regeln und persönlicher Handlungsmöglichkeiten innerhalb dieses Gesellschaftssystems stieß ich auf den Begriff der „polarisierenden Differenzgesellschaft" (jap. kakusa shakai). In diesem Begriff verbindet sich die Beschreibung der aktuellen wirtschaftlichen Situation Japans mit der Kritik an den daraus resultierenden sozialen Umständen sowohl für den Einzelnen als auch für die Gesellschaft im Allgemeinen.

Diesen zwei Bedeutungsebenen des Begriffs entsprechend beschäftige ich mich im Folgenden zunächst mit den wirtschaftlichen Entwicklungen Japans und verorte diese in ihrem historischen Verlauf. Die insgesamt stark normativ geprägte Debatte um die strukturgebenden Kräfte der japanischen Gesellschaft und ihre historische Genese lässt sich um viele Aspekte erweitern, die hier jedoch nicht in aller Ausführlichkeit behandelt werden können. Ich beschränke mich auf die mir wesentlich erscheinenden Werke, die die Herausforderungen der japanischen Gesellschaft in diesem Zusammenhang veranschaulichen. Die individuelle wirtschaftliche Leistung ist insbesondere in der meritokratischen Leistungsgesellschaft Japans aufs engste mit der Verortung im sozialen Gefüge, dem Selbstverständnis als vollwertiges Mitglied der Gesellschaft und den damit verbundenen Rechten und Pflichten verbunden. Folgerichtig setze ich mich in einem zweiten Schritt mit den sozialen Folgen der ökonomischen Entwicklungen und deren Abbild im öffentlichen Diskurs auseinander. Dabei werde ich ein besonderes Augenmerk auf die im japanischen Diskurs deutlich werdende Problematisierung der Jugend legen, an deren konkreter sozio-ökonomischer Situation sich die ethische Diskussion um die Gegenwart und Zukunft Japans entzündet.

Spätestens seit den 1970er Jahren herrscht in Japan ein Disput über die Ursachen und Folgen der sogenannten ,atypischen Arbeitsverhältnisse“, die seit dieser Zeit zunehmen. Darunter werden in Japan, wie auch in anderen industriellen Ländern, jene Beschäftigungsverhältnisse verstanden, die keine längerfristige Garantie auf Erhalt des Arbeitsplatzes mit entsprechender Lohnanpassung geben. In Japan fallen darunter mittlerweile weit verbreitete Arbeitsplatzregelungen, die weder eine 40-Stun- 
den-Woche noch eine entsprechend den Qualifikationen angemessene Bezahlung beinhalten (Obinger 2009). Zu den gängigen Beispielen zählen Kurzzeitverträge, Leiharbeit, geringfügige Beschäftigung und andere vertraglich geregelte Arrangements, die sowohl die Sozialabgaben als auch das wirtschaftliche Risiko des Arbeitgebers minimieren. Die Folgen für die Arbeitnehmer hingegen manifestieren sich in einer als prekär wahrgenommenen wirtschaftlichen Situation, geprägt von einer geringeren Planungssicherheit für die Zukunft als es für die Elterngeneration der Fall war. Als Gründe für diese von Unsicherheit geprägten wirtschaftlichen Verhältnisse nennen Autoren wie der japanische Soziologe Kariya (2010) unter anderem die Veränderungen des Arbeitsmarktes aufgrund der anhaltenden Rezession der japanischen Wirtschaft und dem daraus resultierenden Druck, die Lohnkosten möglichst gering zu halten. Die bis in die 1970er Jahre als selbstverständlich erachteten Übereinkünfte zwischen Staat, Wirtschaft und dem einzelnen Arbeiter, namentlich die lebenslange Vollzeitbeschäftigung, gerieten unter starken wirtschaftlichen Druck. Seither hat sich die Vollbeschäftigung auf Lebenszeit von einer Norm zu einem Ideal gewandelt, das nur noch wenige durch kontinuierliche Anstrengung, hervorragende Leistungen und jede Menge Glück erreichen können.

Die Dramatik der im Begriff kakusa shakai zusammengefassten wirtschaftlichen wie sozialen Drift der japanischen Gesellschaft wird angesichts von Japans Aufstiegsgeschichte zur internationalen Wirtschaftsmacht deutlich. In den Jahren nach dem Ende des Zweiten Weltkriegs und dem Wirtschaftswunder der 1950er und 1960er Jahre galt in Japan das Kredo einer klassenlosen Gesellschaft. Im allgemeinen Aufschwung und Wiederaufbau nach dem Krieg verschwanden wirtschaftliche Differenzen fast völlig und die Mehrheit der Japaner zählte sich zur Mittelschicht. In den 1980er Jahren waren dies immerhin noch bis zu 80\% (Schad-Seifert 2007: 106). Die Ölkrise von 1973 offenbarte jedoch die sozialen wie wirtschaftlichen Schwachstellen des Systems, in dem die Familie die vorrangige Sicherungseinheit ist. Aus dieser Zeit, Ende der 1970er Jahre, stammt die Idee der japanischen Version der Wohlfahrtsgesellschaft (nihongata fukushi shakai). Die entsprechend der wohlfahrtsstaatlichen Ideen ergriffenen Maßnahmen konnten die Auswirkungen der systemischen Schwachstellen noch bis zur Asienkrise 1990 verschleiern, bis in der sich anschließenden Rezessionsphase die soziale Ungleichheit erstmalig offen thematisiert wurde (Schad-Seifert 2007: 112). Die in dem Begriff kakusa shakai zusammengefassten Thematiken von Heterogenisierung der Bevölkerung entlang von Einkommen, Ausbildung und Zielen beschreiben folglich ein erwachtes Bewusstsein für gesamtgesellschaftliche Zustände.

Dies ist insofern von zentraler Bedeutung, als dass an dieser Stelle deutlich wird, wie stark das dominante (meist staatliche) Narrativ von den Lebensrealitäten vieler abweichen kann und dennoch ein stark bindendes gesamtgesellschaftliches Element darstellt. Im dominanten Narrativ Japans hatte über vier Jahrzehnte lang ein Bild der Realität existiert, das sich in Zeiten der wirtschaftlichen Krise nicht halten konnte. Mit der Auflösung dieses Narrativs war die Schockwirkung auf die japanische Gesellschaft entsprechend groß. „Soziale Ungleichheit“, „soziale Polarisierung“ und „neue 
Klassengesellschaft" (shin-kaikyü shakai Nippon) wurden zu Schlagworten der neu einsetzenden Debatte (Schad-Seifert 2007: 112) und mündeten schlussendlich in dem seit 2004 etablierten Begriff der kakusa shakai. Die Soziologen Ishida und Slater definieren diesen Terminus wie folgt:

"The demise of the large-scale firm as the ideological structure of society and as the anchor of social identity has not particularly led the Japanese population into the floating signifiers of the consumer market (or at least, not any more than was the case pre-Bubble) or into an exclusionary identity politics. Rather, it has led them back to social class, as one way to understand the increasingly fractured social order and to situate social identity."

(Ishida und Slater 2010: 9)

In der wissenschaftlichen Ursachenforschung gibt es unterschiedliche Ansatzpunkte der Analyse. Obinger (2009: 172) vertritt als Japanologin in ihrem Beitrag „Working Poor in Japan: Atypische Arbeitsverhältnisse im aktuellen Diskurs" die Meinung, dass sich in Japan zunehmend das Phänomen der „working poor" ausbreitet. Als „working poor" gelten diejenigen Arbeitnehmer, die Vollzeit arbeiten, jedoch trotzdem unter dem Grundsicherungsniveau entlohnt werden. Hinzu kommen laut Obinger (2009: 164) auch Menschen mit mehreren Jobs gleichzeitig und jene, die gar keiner geregelten Arbeit nachgehen. Das Resultat ist, dass immer mehr Arbeitnehmer sich gezwungen sehen, mindestens zwei Jobs anzunehmen, um ihre Grundbedürfnisse wie Miete, Nebenkosten und Lebensmittel zu finanzieren.

In den Diskursen um prekäre Arbeitssituationen werden einzelne Phänomene mit Begriffen belegt, die neben einem geringen Lohn auch einen bestimmten Umgang der Betroffenen mit diesem Umstand beschreiben. Dazu gehört ein „Freeter“, ein Schulabgänger in prekären Angestelltenverhältnissen und sehr niedrigem Lohn, der sich stark um geregelte Verhältnisse bemüht. Als „Needs“ hingegen werden Individuen bezeichnet, die sich auf Kosten ihrer Eltern dem Arbeitsmarkt grundsätzlich verweigern. Beide Varianten werden als Beispiele für die unterschiedlichen Reaktionen der vor allem jungen Generation auf die wirtschaftlichen Rahmenbedingungen herangezogen. Die Auswirkungen der schwierigen Wirtschaftslage erhalten eine zusätzliche Brisanz durch die kulturspezifische Werteausrichtung der japanischen Gesellschaft, die sich als meritokratische Bildungsgesellschaft versteht. Ein „Versagen“ auf dem Arbeitsmarkt ist mit entsprechendem Schamgefühl und einem gewissen Maß an Ausgrenzung sanktioniert (siehe Matsumoto 1996). Es entsteht der Eindruck, dass die schwache Arbeitsmarktsituation dem Einzelnen von Seiten der Gesellschaft dennoch zur Last gelegt und ein Gefühl der Schuld hervorgerufen wird.

Die geringe Unterstützung sozial Schwacher durch den japanischen Staat ist dabei politisch gewollt und stützt sich auf die Annahme, jeder sei für sein eigenes Glück oder Unglück verantwortlich. Der Erfolg des Einzelnen beruht dieser Annahme zufolge zu einem entscheidenden Maße auf dessen Eigenverantwortlichkeit (jap.: jiko sekinin) und persönlicher Anstrengung, die im besten Falle noch von der Familie, 
Nachbarn und dem engeren sozialen Umfeld unterstützt wird (siehe Avenell 2009: 262). Dieses Modell der japanischen Wohlfahrtsgesellschaft (jap.: nihongata fukushi shakai) verknüpft so den wirtschaftlichen Erfolg mit einer moralisch-normativen Bewertung des Charakters eines Individuums und erhöht damit den adaptiven Druck. Entsprechend groß ist das Stigma, sollte die staatliche Hilfe zur finanziellen Grundsicherung dennoch benötigt werden (Obinger 2009: 173). Abhilfe leisten nichtstaatliche Netzwerke, die vor allem aus Familien bestehen. Laut Obinger (2009: 173) stellen sie in Japan noch stärker als in Europa die Basis der sozialen Sicherung dar. Die Familienmitglieder finanzieren, wie im Falle der "Needs“, ein arbeitsloses Individuum und tragen auf diese Weise dazu bei, dass der Schein eines geregelten Auskommens gewahrt werden kann. Die Bedeutung, die der Wahrung des öffentlichen Gesichts (jap.: tatemae) beigemessen wird, kann also nicht stark genug betont werden. Die Marktgesetze beeinflussen im Wesentlichen sowohl wie sich der Einzelne als Individuum und als Vertreter einer Generation in der Gesellschaft positioniert sieht als auch wie die soziale Wirklichkeit mit allen Chancen und Hindernissen vom Einzelnen wahrgenommen wird.

Ich konnte beobachten, dass der angesprochene prekäre Niedriglohnsektor auch in den Jahren 2014 und 2015 nach wie vor regen Zulauf hatte. Die Diskussion um dessen Auswirkungen stand unter anderem auch deshalb im Zentrum des öffentlichen Interesses, da sie nun auch Studierende betreffen, die ursprünglich als zukünftige Topverdiener, gesicherte Arbeitnehmer oder als die zukünftige Elite Japans gehandelt wurden. Auch aus Sicht der Japanologin Schad-Seifert (2007: 113-114) liegen die strukturellen Ursachen in der mangelnden Umverteilung der gesellschaftlichen Ressourcen und der Ausweitung des Niedriglohnsektors, die extreme Unterschiede in Lebensstandard und Zukunftssicherung hervorrufen. Aspekte wie die stark voranschreitende demographische Alterung der japanischen Gesellschaft, die genannte generationale Einkommensdifferenz und geringe soziale Mobilität bestimmen die graduelle Stärke dieser Auswirkungen. Die Folge ist: Die Mittel der Herkunftsfamilie haben wieder an Bedeutung gewonnen - ein Umstand, auf den sich vor allem Slater und Ishida (2010) beziehen, wenn sie von „Klasse“ sprechen. Die beiden Soziologen, die sich auch mit Technologie, Wirtschaft und mit der Dreifachkatastrophe Japans auseinandersetzen, nutzen den Begriff der Klasse, um die Rahmungen der Möglichkeiten zu beschreiben, die sich auf die Lebensgestaltung auswirken. Dabei lassen sie auch nicht außer Acht, wie sich dies auf die sozialen Beziehungen auswirkt. Slater und Ishida gehen davon aus, dass in Japan, mehr noch als in anderen Ländern, die Institutionen eine entscheidende Rolle bei der Wahrnehmung und Nutzung von Handlungsoptionen und damit bei der Gestaltung einer sozialen Identität spielen (Ishida und Slater 2010: 10). In einem vom Krieg zerstörten Japan wurde die Kategorie der „Mittelklasse“ zu einem Synonym für hart erarbeiteten Wohlstand und durch den damit verbundenen sozialen Status zu einem Maßstab für kommende Generationen.

Slater und Ishida hinterfragen in ihrer historischen Gesellschaftsbetrachtung allerdings nicht die Genese des Begriffs der „Mittelklasse“ im japanischen Kontext, die 
doch aus meiner Sicht vor allem auf ein Bewusstsein für die „Differenzgesellschaft“ hinweist: Im historischen Rückblick auf das gesellschaftliche Narrativ zeigen sich die generationellen Unterschiede im Verständnis von Wohlstand und Normalarbeitsverhältnissen, die nun den Kontrast zu gegenwärtigen Gesellschaftsverhältnissen drastisch hervortreten lassen. Dass es sich bei der Etablierung einer Mittelklasse tatsächlich mehr um eine Vorstellung als um ein Faktum handelte, zeigen die Auswertungen von Schad-Seifert (2007). Sie weist darauf hin, dass im Japan der 1950er Jahre keine soziologisch fundierten Kategorien erstellt wurden, sondern dass es sich vielmehr um einen ,[...] politisch induzierten Wunsch [...], den Lebens- und Konsumstil einer prosperierenden Industriegesellschaft führen zu wollen [...]" handelte (Schad-Seifert 2007: 108).

Schad-Seiferts Erörterungen verstehe ich dahingehend, dass das öffentliche Selbstbild des Japans der 1950er Jahre stark von der Sehnsucht nach einer homogenen, harmonischen Gesellschaftsstruktur geprägt war und dementsprechend proklamiert wurde - obwohl die gemessenen Daten zu ungenau waren, um einen Großteil der Bevölkerung der „Mittelklasse“ zuzuordnen. Wie auch Ishida und Slater (2010) schreibt Schad-Seifert den Begriff der „Mittelklassegesellschaft" demzufolge der sozialen statt der wirtschaftlichen Kategorie zu. Diese Zuordnung liegt ihrer Meinung nach vor allem an den nicht ausreichend kategorisierten Selbstwahrnehmungen der Befragten in den 1950er Jahren.

Letzten Endes wird das Bild einer Mittelklassegesellschaft von Schad-Seifert historisch widerlegt. Sie stellt indes fest, dass „[...] keineswegs erst in jüngerer Zeit eine feststellbare Differenzierung [...]" einsetzte (Schad-Seifert 2007: 110). Der richtungsweisende soziale Maßstab eines Normalarbeitsverhältnisses auf Lebenszeit in einer bestimmten Firma stammt folglich aus der japanischen Nachkriegszeit, ist jedoch, wie im vorangegangenen Abschnitt beschrieben, nichtsdestotrotz ein starkes Ideal bis zu heutigen Tag. Es ist dieses Ideal einer wirtschaftlich begründeten Sozialstruktur, das in seiner Antagonie die Rahmung für die „polarisierende Differenzgesellschaft" (kakusa shakai) legt und bis heute den innerjapanischen Identitätsdiskurs beeinflusst.

Es ist diese enge Verbindung von Identitätsverständnis und Wirtschaftlichkeit, die zu Spannungen zwischen den Generationen führt und deren Bedeutung für die Diskursrahmung sich auch auf das Narrativ meiner Gesprächspartner auswirkt. Im Vergleich zu ihren Eltern sieht sich die japanische Jugend (ab etwa 1980) ganz anderen Arbeitsbedingungen ausgesetzt: „Today, a much higher rate of fluidity and instability characterizes the youth labor market, with differing effects on the self-perception and subjective understanding of young people“ (Kariya 2010: 91). Diese starke „Verflüssigung" der Orientierungspunkte im Leben (siehe Bauman 2000; 2008) schlägt sich entsprechend auf die Selbstwahrnehmung junger Menschen nieder, die im Falle des Scheiterns unter dem Eindruck stehen, sich nicht ausreichend bemüht zu haben - obgleich sich die Rahmenbedingungen ihrer Handlungsmöglichkeiten gegenüber denen ihrer Eltern stark verändert haben. 
Die Soziologin Brinton (2010) ergänzt das Bild der polarisierten Verständnisse eines guten (Berufs-)Lebens mit dem Beitrag „Lost in Transition: Youth, Work, and Instability in Postindustrial Japan“. Bei der Analyse der Wirtschaftskrise der 1990er Jahre und von 2008 betont sie die besondere Betroffenheit der jungen japanischen Generationen, die sich zu diesen Zeitpunkten in der Übergangsphase von Schule und Beruf befanden:

"Those most affected are not the middle-aged and not the elite. Instead, the people who have been, lost in transition' as the Japanese economy tries to restabilize itself in the early twenty-first century are people in the younger generation, especially the non-elite among them." (Brinton 2010: xi)

Meiner Auffassung nach könnte man also sagen, dass eine Gesellschaft, die die Werte der Anstrengung für sozio-ökonomischen Erfolg vor allem beim Individuum verortet, Voraussetzungen für eine erhöhte Sensibilität bezüglich sozialer Differenzen schafft. Der inhärente Widerspruch von einem öffentlich weitestgehend als homogen präsentierten Gesellschaftsbild und der gelebten Realität ist im Laufe der Jahrzehnte auch für innergesellschaftliche Beobachter immer augenscheinlicher geworden. Die daraus resultierende Not der jungen Generationen führt zu einem Lebensstil, der „[...] a collective refusal or inability to follow an established path to adulthood" (Mathews and White 2004: 7) repräsentiert.

Um die emotionalen Effekte dieser Lage zu verstehen, ziehe ich die Ethnologen Mathews und White hinzu. Ihnen zufolge hängt eine Ablehnung des etablierten Weges zum Erwachsenwerden mit einer Legitimierungskrise des gesamten gesellschaftlichen Systems zusammen: Angesichts des stark nachlassenden Zusammenwirkens von Anstrengung und sozio-ökonomischem Gewinn verlieren die jeweiligen Attribute in der andauernden Wirtschaftskrise zunehmend an Bedeutung und führen zu einer "pluralization of values“ (Mathews und White 2004: 8). Gemeint sind damit alternative Wege der Lebensführung, die sich an der Verwirklichung eigener Ideen und Neigungen orientieren statt an bedingungslosem Fleiß und Hingabe. Diese Vorstellungen gewinnen im gleichen Maße an Anziehungskraft wie die Zuverlässigkeit gesellschaftlicher Zusagen abnimmt. Meine Analyse wird in den folgenden Kapiteln aufzeigen, wie sich dies für meine Gesprächspartner konkret darstellt.

Weitere Aspekte dieser Drift zwischen den Lebensvorstellungen der Generationen stellt der Juraprofessor und Philosoph Sakurai (2004: 15ff.) in den Mittelpunkt seiner Analyse. Er leitet die verschiedenen Phasen und Ansätze generationeller Widerstände gegen die Elterngeneration seit dem Zweiten Weltkrieg her und stellt damit den kontextuellen Rahmen für den derzeitigen japanischen Generationsdiskurs dar. Sakurai betrachtet die Diskursentwicklungen seit der Nachkriegszeit und benennt als Ursache für die „kakusa shakai“die Auswirkungen der demographischen Veränderungen in der japanischen Gesellschaft. Für ebenso bedeutsam hält er die teilweise gewalttätigen Studentenproteste der 1960er Jahre und die Entwicklung neuer Technologien wie die des Internets. Vor allem die steigende Nutzung der so- 
zialen Medien seit den 1980er Jahren schlug sich seiner Betrachtung zufolge in einer eigenen Bezeichnung für die neue Generation im japanischen Sprachgebrauch nieder. Sie verdeutlicht die wachsende Distanz zwischen den Realitäten innerhalb der Gesellschaft: Junge Menschen wurden fortan als shin-jinrui - „new species of human being“ oder „new breed“ (Sakurai 2004: 23) betitelt. All diese Entwicklungen lösten Sakurai zufolge bei den älteren Generationen großes Misstrauen und Kritik aus, änderten jedoch wenig an der gelebten Praxis der jungen Leute. Die in diese Zeit fallende zunehmend schwierigere wirtschaftliche Situation tat das Übrige, um die Lebenssituation der jungen Menschen mit der Elterngeneration zu kontrastieren.

Die jungen Generationen können nur in vermindertem Maße den Ansprüchen ihrer Eltern gerecht werden, die sich an den traditionell geprägten Markern eines japanischen Erwachsenen orientieren. Für Brinton sind es insbesondere die institutionellen Transformationen der letzten Jahrzehnte, die sowohl zu wirtschaftlichen wie auch psychologischen Problemen der jungen Generationen, wie dem hikkikomori ${ }^{40}$-Phänomen, geführt haben. Die kulturspezifische enge Verbindung zwischen den verschiedenen Lebensphasen und ihren sozialen Markern (Arbeit, Heirat und Kindern) bezeichnet sie als „, $[. .$.$] , social locations ' in which individuals find security$ and a sense of identity" (Brinton 2011: xvii). Folgt man Brintons Argumentation, hat die junge Generation ihre soziale Verortung verloren, ohne sie durch ein entsprechendes Äquivalent ersetzen zu können.

Die in diesen Diskursnarrativen deutlich werdende Problematisierung der Jugend wird auch bei Brinton auf sozio-ökonomische Ursachen zurückgeführt, die das Bild Japans als eine Kultur der Sicherheit statt eine Kultur des Risikos stützen (Brinton 2011: 18). Das bedeutet im Kontext der Jahre 2014 und 2015, dass das Vertrauen der Bürger in ihre Gesellschaftsform immer noch an deren Fähigkeit zur Bereitstellung von Langzeitarbeitsplätzen und den damit verbundenen "traditionell“ wichtigen Werten wie Sicherheit gemessen wurde - und damit heutzutage oft enttäuscht wurde. Die „Krise der Jugend“ (wakamono kiki) ist in diesem Narrativ im Wesentlichen von den Herausforderungen an die Jugend geprägt. Sie sollen sich den schnellen sozialen Transformationen anpassen und gleichzeitig unter schwierigen Rahmenbedingungen die volle Verantwortung für sich selbst tragen können.

\subsubsection{Die verstärkte Individualisierung und ihre Folgen für die soziale Mobilität}

Eine so starke Betonung der individuellen Verantwortung in einer auf Interdependenz ausgerichteten Gesellschaft wie Japan ${ }^{41}$ hat in ihrer Widersprüchlichkeit sowohl eine gewisse Verwirrung als auch psychische Erkrankungen wie das hikkikomori-Phänomen zur Folge. In meinem Bemühen, die sozialen Auswirkungen der sozio-öko-

\footnotetext{
${ }^{40}$ Als hikkikomori wird eine Person bezeichnet, die sich der Gesellschaft durch vollständigen Rückzug in seine Wohnung verweigert. Bekannt geworden ist das Phänomen durch eine steigende Anzahl an Schulverweigerern, die auch mit ihren Familienmitgliedern nur eingeschränkt Kontakt pflegen.

${ }^{41}$ Siehe 3.6.2 „Das Verhältnis vom Individuum zur Gruppe“.
} 
nomischen Entwicklungen auf das Individuum zu erfassen, ziehe ich erneut Slater und Ishida (2010: 7) hinzu. Zwar fehlt meiner Meinung nach in ihrer Analyse die historische Facette des gesamtgesellschaftlichen Zusammenhangs. Aber sie liefern durch ihre analytische Ausdifferenzierung wichtige diskursive Artikulationen der Bevölkerung im Zusammenhang mit den genannten gesellschaftlichen Thematiken.

„Class analysis allows us to see how individuals form coherent social identity and how those on different life courses develop distinctive goals and strategies for reaching those goals, and how these become disseminated through wide segments of the population." (Ishida und Slater 2010: 9)

Sie argumentieren, dass die individuelle Identität sich infolge der „Differenzgesellschaftsdiskurse“ nicht mehr an institutionellen Rahmungen, sondern an persönlichen Beziehungsnetzwerken orientiert, deren Gemeinsamkeiten in derselben Klassenzugehörigkeit zu finden sind: "These destinies are not shared among ,all Japanese“ or even those in the same company but among those in the same class position, a position that of course cuts across institutional contexts" (Ishida und Slater 2010: 12). Die Wechselwirkung zwischen der Position innerhalb des sozialen Gesellschaftsgefüges und der sozialen Mobilität spielt in dieser Argumentation eine entscheidende Rolle. Schad-Seifert (2007: 116) hingegen beschränkt sich in ihren Betrachtungen vor allem auf die generationalen Auswirkungen der individuellen psychologischen Effekte.

Beiden Ansätzen gemeinsam ist indes die Feststellung eines allgemeinen Trends zum wachsenden Gesellschaftsrisiko, dem sich nicht nur die Mitglieder der japanischen Gesellschaft ausgesetzt sehen. Dieses erhöhte Risiko sehe ich im Einklang mit Becks (1986) soziologischen Beobachtungen vor allem in Bezug auf die Individualisierung der Arbeit in vielen Gesellschaften weltweit und den damit zusammenhängenden irregulären und individuellen Leistungen. Dies schließt eine Ausbeutung des einzelnen Arbeitnehmers und eine Ausgrenzung von sozialen Positionen und Normen mit ein. (siehe auch Schad-Seifert 2007: 116-117). Wie auch Brintons Analyse (2011) aufgezeigt hat, wird die Begründung für diese Verhältnisse sowohl von Schad-Seifert (2007) als auch von Ishida und Slater (2010) im Wandel der Beschäftigungsstruktur gesehen, die „[...]den wachsenden Anstieg im Segment der nichtregulären Teilzeitarbeit verursacht hat" (Schad-Seifert 2007: 118).

Begriffe wie der des kakusa shakai erzeugen Gegenkräfte, sobald sie im Diskurs wirksam sind. Meine Gesprächspartner berichten von einer zunehmend starken Ausrichtung junger Menschen auf Selbstverwirklichung und individuellere Lebensgestaltung. Dies spielt auf die stets implizierten Relationen von starker Wirtschaftskraft, Konsumfähigkeit und Lebenszufriedenheit an, die im individuellen Fall nicht zwingend in diesem Wirkungszusammenhang stehen muss. Vielmehr entwickeln Akteure abweichende Einstellungen und Wertesysteme, die eher im Einklang mit den veränderten wirtschaftlichen Voraussetzungen stehen. So wird laut meinen Ge- 
sprächspartnern dem Sinn von Arbeit und persönlicher Erfüllung seitens der jungen Generation mehr Wert beigemessen als einem hohen Gehalt.

Von entscheidender Bedeutung für die Konstruktion der eigenen Identität in der lebensgeschichtlichen Erzählung ist dabei die Freiwilligkeit der sozio-ökonomischen Situation des jeweiligen Akteurs. Die gesellschaftliche Konnotation des Begriffs der kakusa shakai als Krise im japanischen Diskurs gründet sich auf einem fehlenden historischen Bewusstsein wie Schad-Seiferts Analyse hervorhebt. Hinzu kommen die als anormal bewerteten geringen finanziellen Möglichkeiten des Einzelnen, die zu weniger Haushalts- und Familiengründungen führen - mit entsprechenden Folgen für den Konsum und die Demographie Japans. In diesem Spannungsverhältnis zu traditionellen japanischen Werten wie Sicherheit, Stabilität und Harmonie liegt die Brisanz der entstandenen wirtschaftlichen Auswirkungen auf das soziale Gefüge.

Auf individueller Ebene jedoch kann dies zu Bewertungen der eigenen sozialen Position führen, die durchaus sinn- und identitätsstiftend sind. Als Chance begriffen eröffnen die prekären wirtschaftlichen Situationen viele Möglichkeiten der Entfaltung, was sich sowohl auf die Handlungsmöglichkeiten als auch auf das biographische Narrativ auswirkt. Auf diese, stark an die persönliche Wahrnehmung geknüpften, positiven Rahmungen der Lebensgestaltung gehe ich in Kapitel 6 im Speziellen für die Akteursgruppe der Gegenwartskünstler ein.

Ich halte fest, dass der Begriff der „Differenzgesellschaft“ weitaus differenzierter und kritischer hinterfragt werden muss als es auf den ersten Blick den Anschein hat. Die dominanten Diskurse verschleiern die historischen Zusammenhänge und missachten die seit langem existierenden Unterschiede in Einkommen, Ausbildung und Zielausrichtung zwischen den Bevölkerungsgruppen. Ich komme zu dem Schluss, dass eine Identitätskonstruktion des Individuums weder in den 1950er Jahren noch heute ausschließlich auf der Zuordnung zur Klasse der Mittelschicht basiert. Sie findet sich vielmehr in unterschiedlichen Artikulationsformen, Selbstzuschreibungen und der Bedeutungsaufladung von Erfahrungen. Auf diese Weise macht das 1980 geborene Individuum andere Ankerpunkte des Selbst ausfindig als es seine Eltern getan haben und konstruiert eine Identität entlang dieser verschobenen Fixpunkte.

\subsubsection{Eine Schwächung der sozialen Bindungen}

Abgesehen von dem augenscheinlichen Konfliktpotential, das sich aus den unterschiedlichen Lebensausrichtungen der Generationen ergibt, tritt noch eine weitere Folge in den Fokus der Aufmerksamkeit: Die sichtbar gewordenen sozialen Ungleichheiten und die prekären Arbeitsbedingungen werden in den Diskursen Japans für eine Schwächung der sozialen Bindungen verantwortlich gemacht. Die bereits aufgeführten Werte der Stabilität, Sicherheit und Harmonie werden unter den erschwerten sozio-ökonomischen Bedingungen nur noch selten in vollem Umfang in der Lebenspraxis umgesetzt. Damit werden nicht nur entsprechende Erwartungen an ein als "gut" definiertes Leben nicht erfüllt, sondern auch die ihnen zugrunde 
liegenden Werte und Normen verletzt. Inwiefern dies für meine Analyse relevant ist, erkläre ich im folgenden Abschnitt.

Die Japanologin Hommerich (2014) untersucht die Zusammenhänge der in Folge der kakusa shakai einsetzenden Schwächung der sozialen Bindungen für das soziale Kapital und das zivile Engagement in der japanischen Gesellschaft. Sie analysiert mit Bezug auf Böhnke, dass es bei einem zunehmenden Anteil der japanischen Bevölkerung zu einer Wahrnehmung der Marginalisierung kommt: „Growing shares of the population feel marginalized and disconnected from society“ (Böhnke 2004, zitiert in Hommerich 2014: 5). Diese Entwicklung wird unter dem Terminus der „society without bonds“ (jap.: muen shakai) zusammengefasst und bezieht sich vorrangig auf die zunehmenden Lockerungen von Bindungen in Familie, der lokalen Gemeinschaft und der Arbeitsplatzsituation.

Entsprechende Prognosen, die die Lebenssituation betreffen, sagen einen Anteil von 37 Prozent Alleinlebender für das Jahr 2030 voraus (Hommerich 2014: 6), was den starken Rückgang des Dreigenerationenhaushaltes unterstreicht. Spätere Heiraten und Familiengründungen werden im Bereich der lokalen Gemeinschaft von einer weniger starken Beziehung zu Nachbarn und Gemeindemitgliedern begleitet. Die Arbeitssituationen einzelner Angestellter einer Firma variieren so stark in Bezug auf Dauer der Anstellung, Entlohnung und sozialem Status, dass ein Beziehungsaufbau zusätzlich erschwert wird (Hommerich 2014: 7). Unter Zuhilfenahme offizieller Statistiken, die unter anderem die mentalen Erkrankungen der Arbeitnehmer abbilden, kommt Hommerich (2014: 7) zu dem Schluss, „[...] individuals have replaced communal activities with social isolation."

Das in diesem Zusammenhang verstärkte Auftreten von Unsicherheit, Isolation und Ängsten (Hommerich 2014) stützt die Ergebnisse der von mir bereits genannten wissenschaftlichen Studien und beschreibt ein von wachsender Angst geprägtes Lebensgefühl in der japanischen Gesellschaft. So sei der Prozentsatz derer, die im Alltag Angst und Besorgnis empfinden, von 47 Prozent im Jahr 1991 auf 66 Prozent im Jahr 2013 angestiegen (Hommerich 2014: 7). Die Ausführungen Hommerichs zu den Auswirkungen dieses Lebensgefühls auf das zivile Engagement darzulegen, würde an dieser Stelle zu weit führen. Hommerich liefert jedoch entscheidende Hinweise in Bezug auf die Kognition des Vertrauens und der Isolation auf sozialer Ebene. Dies sind essentielle Ergebnisse, die vor dem Hintergrund der Dreifachkatastrophe wichtige Rahmungen zur Analyse von Emotionen setzen.

Das Gefühl der sozialen Zugehörigkeit zu einem gesellschaftlichen Ganzen habe gelitten, so Hommerich (2014: 10) weiter: „The precarity of living circumstances enforces the negative impact of low family support and a weak social network on perceived social exclusion“ (Hommerich 2014: 10). Hommerichs Ausführungen stellen einen ergänzenden Blickwinkel auf die in Kapitel 4.1.2 „Das Verhältnis vom Individuum zur Gruppe“ erläuterten Untersuchungen Matsumotos (1996: 5) dar, in denen er gemeinsames Erleben von Emotionalität als „cultural glue“ bezeichnet. Ohne die Gemeinsamkeiten, die beispielsweise aus einem Arbeitsplatz, einer per- 
sönlichen Situation oder dem Tragen von Verantwortung bestehen, gehen wichtige soziale Bindungen verloren.

Auf die Ebene des Individuums übertragen bedeutet das fehlende Vertrauen in die Gesellschaft, ihre Institutionen und Würdenträger eine zusätzliche emotionale Belastung in einer ohnehin schon stark individualisierten Arbeitswelt, die bereits vor dem Eintreten der Dreifachkatastrophe von 2011 existent war. Die persönlichen Konsequenzen dieser gesellschaftlichen Entwicklungen, bestehend aus wirtschaftlichem Wandel, entsprechenden Folgen für die Lebenssituation und das gesellschaftliche Lebensgefühl, werde ich anhand meiner biographischen Erhebungen aufzeigen.

An dieser Stelle möchte ich betonen, dass die für die persönliche Bewertung der Lebenssituation zugrunde gelegten Werte und Normen sich aufgrund der aufgezeigten Transformationen der japanischen Gesellschaft seit den 1990er Jahren in einem verstärkten Hinterfragungs- und Aushandlungsprozess befinden. Es sind diese Diskussionen und generationalen Differenzen, die auch meine Interviewpartner in prägnanter Weise beeinflussen.

Zusammenfassend könnte man meiner Ansicht nach sagen, dass die Diskussionen um die Dreifachkatastrophe als Symptom dieses Gesellschaftsverständnisses bewertet werden können. Dieser Eindruck speist sich aus den Meinungen und Bewertungen des Status quo aller Interview- und Alltagssituationen während meines Forschungsaufenthaltes in Tokyo in den Jahren 2014 und 2015 und meiner Teilnahme an entsprechenden Diskussionen um Ideale, Normen und Moral. 


\section{Vertrauen und Risiko - Die vorläufige Bilanz einer gesellschaftlichen Katastrophe}

Meine Ausführungen zu den Konsequenzen der wirtschaftlichen Stagnation Japans für das Vertrauen in die Gesellschaft - und schlussendlich den Staat und seine Institutionen - haben verdeutlicht, wie eng wirtschaftliche Prozesse und soziale Transformationen miteinander verknüpft sind. Das Vertrauen in die Hierarchiestruktur ist insbesondere in einer vertikal organisierten Gesellschaft wie der japanischen ein markantes Charakteristikum des Sozialwesens und von entscheidender Bedeutung für das Funktionieren der einzelnen Institutionen. Wie in Kapitel 4.1 „Der kulturspezifische Ausdruck des Selbst im sozialen Gefüge" dargelegt, ist diese Gesellschaftsstruktur der Ausdruck der interdependenten Ausrichtung des Individuums und dessen Unterordnung unter die Ziele des Kollektivs. Wie sich der Verlust des Vertrauens auf ein derart strukturiertes Sozialgefüge auswirkt und wie es sich im Falle eines desaströsen Ereignisses darstellt, zeige ich in Kapitel 5.2 „Kulturelle Spezifika als Katastrophenrisiko?" auf.

In den folgenden Abschnitten geht es mir jedoch zunächst darum, die japanischen Post-Fukushima-Diskurse genauer zu betrachten. Damit nähere ich mich nach der allgemeinen Einführung in den japanischen Kontext den spezifischen $\mathrm{Zu}-$ sammenhängen der Dreifachkatastrophe an. Ich beziehe die internationale Analyseebene mit ein, da an ihr deutlich wird, dass es sich bei den Post-Fukushima-Diskursen nicht um reine Wissenschaftsdiskurse handelt. So steht in der Kontroverse um die Einschätzung der Strahlungswerte der Glaube an die Wissenschaft auf dem 
Spiel. Massive Unterschiede zwischen den lokalen Realitätswahrnehmungen und Prioritätensetzungen und der wissenschaftlichen Interpretation mancher Akteure und Institutionen (siehe UNSCEAR 2015) sind evident und verweisen auf Interessenverbände innerhalb des Geflechts von Wirtschaft, Politik und Öffentlichkeit.

\subsection{Der ,soziale Tsunami“ - Auswirkungen der Dreifachkatastrophe auf die japanische Bevölkerung}

In den vorangestellten Abschnitten habe ich einige wichtige Diskursstränge angerissen, wie sie in Japan in den Medien in Erscheinung traten. In diesem Kapitel möchte ich mich nun eingehender mit den Argumentationen beschäftigen, die sich mit dem Alltag nach dem 11. März 2011 befassen und die vor dem Hintergrund der in Kapitel 4.2 genannten Aspekte der Gesellschaftsdiskurse zu verstehen sind. Insbesondere die Auswirkungen der atomaren Strahlung in den direkt betroffenen Gebieten lösten eine Debatte um die schwer absehbaren Folgen für die ehemaligen Bewohner aus. Heimatlosigkeit, der Verlust von einem vertrauten Alltag, große Ungewissheit angesichts der gesundheitlichen Risiken und Trennung bestehender sozialer Kontakte durch die Verteilung der Gemeinden auf unterschiedliche Notunterkünfte führten zu starken psychischen Belastungen. Hinzu kommt eine finanziell ungesicherte $\mathrm{Zu}$ kunft: Wie viel, wann und für wie lange wird man die Entschädigungszahlungen erhalten, die die Regierung und TEPCO zugesagt haben?

All diese Faktoren summieren sich zu einer über den akuten Notstand hinausgehenden psychischen Dauerbelastung für die Geflüchteten. Die öffentliche Diskussion entzündet sich derweil auf der Suche nach der "Wahrheit" an den unterschiedlichen Versionen sowohl der institutionellen wie auch der zivilgesellschaftlichen Akteure, die sich jeweils teils sehr stark widersprechender Expertisen bedienen. Doch wie sieht die Lebenssituation vor Ort fünf Jahre nach den Ereignissen wirklich aus und welchen Schwierigkeiten begegnen die Menschen im Bestreben, einen neuen Alltag zu finden?

\subsubsection{Gesundheitliche Folgen der Verstrahlung und das Ringen um die Wahrheit}

Bis zum heutigen Tag ist es nicht einfach, an gesicherte Informationen zu gelangen, besonders, da das Thema „Fukushima“ gerne gemieden wird: „The issue has become a ,taboo" in the national discourse" (Bohn et al. 2015: 10). Die wissenschaftliche Aufarbeitung der Ereignisse wird noch Jahre dauern und wird mit Sicherheit auch dann keine hundertprozentige Gewissheit bringen können. Für meine Arbeit ist es jedoch entscheidender, die Genese der Diskurse in Japan mit den unterschiedlichen Stimmen, Interessen und den sich daraus ergebenden Einschätzungen der Lage aufzubereiten. In den Diskursen werden wissenschaftliche Einschätzungen häufig mit den subjektiven Erfahrungen der Opfer kontrastiert und erfahren so je nach Interessenlage eine unterschiedliche Bewertung. 
Eine Graphik des METI von 2015 veranschaulicht die verschiedenen Evakuierungszonen in ihren Dringlichkeitsstufen, ausgehend vom Kernkraftwerk Fukushima Daiichi. $^{42}$

Pinkfarbene Areale bezeichnen ,areas where it is expected that the residents have difficulties in returning for a long time" und erstrecken sich vom Kernkraftwerk in nordwestlicher Richtung über Okuma, Futaba bis einschließlich Namie. Die orangene Markierung zeigt Gebiete an, in denen es den Bewohnern derzeit nicht erlaubt ist zu leben. Dazu zählen Tomioka in südlicher Richtung sowie Iitate und Minamisoma im Nordwesten bzw. Norden. Diese Gebiete können jedoch stundenweise besucht werden, um zum Beispiel Familiengräber zu besuchen oder Erinnerungsstücke zu holen.

In den grünen, weiter umliegenden Bereichen hingegen sollen die Dekontaminationsmaßnahmen bald abgeschlossen und die Menschen in der Lage sein, in ihre alte Wohngegend zurückzukehren. Für das Jahr 2018 existierten Pläne, nach denen TEPCO den meisten der 2014 noch 160.000 Evakuierten (Asanuma-Brice 2014: 1) die Entschädigungszahlungen einstellt, insbesondere wenn es sich dabei um Menschen handelt, deren Gegend offiziell als dekontaminiert und damit als sicher gilt (die japanische Zeitung Asahi Shimbun berichtete). ${ }^{43}$ Im Jahr 2021 gibt es immer noch 36.000 Evakuierte, von denen 80 Prozent außerhalb ihrer Heimatpräfektur leben. ${ }^{44}$ Die Rechtsstreitigkeiten um Entschädigungszahlungen sind auch 10 Jahre nach dem Unglück noch nicht endgültig geklärt.

Dessen ungeachtet streiten sich nationale und internationale Experten noch heute öffentlich über Messmethoden, Datenauswertung und Interpretation der jeweiligen Strahlenpartikel, die am 11. März 2011 freigesetzt wurden. Dieser Schlagabtausch verunsichert die verängstigte Bevölkerung zusätzlich (Broinowski 2013). Die Ärzte Claußen und Rosen (2014), dem Verein „ippnw Internationale Ärzte für die Verhütung des Atomkriegs - Ärzte in sozialer Verantwortung e.V." angehörig, gaben am 28. Februar 2014 ein Informationsblatt heraus, in dem „Fukushima“ sowohl in ein Verhältnis zu Tschernobyl gesetzt als auch die Belastungen für die Bevölkerung erläutert werden.

Im Vergleich mit Tschernobyl sind in Fukushima zwischen dem 12. und 14. März 2011 20\% des Jod-131 bzw. 40-60 \% des Gesamtausstoßes von Cäsium-137 freigesetzt worden (siehe Rosen 2012: 4). Dabei sei zu beachten, dass es insbesondere die chronische Belastung großer Bevölkerungsteile durch niedrige Strahlendosen sei, die die größte Herausforderung der kommenden Jahrzehnte darstelle: „Ein Krebsfall trägt kein Herkunftssiegel, sodass man eine solche Erkrankung nie kausal auf ein be-

\footnotetext{
${ }^{42}$ Ministry of Economy, Trade and Industry (METI): „Areas to which evacuation orders have been issued“, 3.9.2015 (https://www.meti.go.jp/english/earthquake/nuclear/roadmap/pdf/150905MapOf Areas.pdf).

43 The Asahi Shimbun: „Ministry plans to end TEPCO compensation to 55,000 Fukushima evacuees in 2018“, 19.5.2015 (http://ajw.asahi.com/article/0311disaster/fukushima/AJ201505190055).

${ }^{44}$ Hiroshi Fukatsu: „Survey: Most evacuees still feel Fukushima plant poses a danger“, 18.3.2021 (http://www.asahi.com/ajw/articles/14258943).
} 
stimmtes Ereignis zurückführen kann" (Claußen und Rosen 2014: 64). Dies ist nicht nur für die wissenschaftliche Einschätzung der zukünftigen gesundheitlichen Folgen für die Bevölkerung äußerst relevant, sondern betrifft die Menschen bereits heute: Indem etwaig festgestellte Schilddrüsenkrebserkrankungen nicht eindeutig mit der entlassenen Strahlung aus Fukushima Daiichi in Verbindung gebracht werden können, schwinden die Chancen der Betroffenen, staatliche Entschädigungszahlungen zu erhalten. Dies ist einer der Gründe, warum sich die „ippnw“ dafür stark macht, dass eine Schwerpunktsetzung der öffentlichen japanischen Debatte auf das Schicksal und die Gesundheit der bereits heute betroffenen Bevölkerung gelegt wird.

Broinowski (2013: 3), Forscher der Australian National University im Bereich Kultur, Geschichte und Sprache, liefert Zahlen zu diesem Thema, die nicht nur die gemessenen Strahlenwerte in einen engen Zusammenhang mit der politischen Agenda Japans bringen, sondern auch erste gesundheitliche Erhebungen aus den Gebieten rund um das AKW Fukushima in ein Verhältnis zu Tschernobyl, Hiroshima und Nagasaki setzen. Seinen sehr kritischen Ausführungen zufolge sind die absehbaren gesundheitlichen Folgen der Langzeitwirkung geringer Strahlendosen verheerend: Forschungsergebnisse aus dem Jahr 2006 zeigten, dass chronische Müdigkeit, Herzerkrankungen, verschiedene Krebsarten, Hautveränderungen etc. bei den Überlebenden von Hiroshima und Nagasaki auf eine längere Aussetzung mit gering erhöhter Strahlung zurückzuführen seien. Von den im Jahr 2013 getesteten 210.000 Kindern in den Präfekturen Fukushima stünden bereits 25 unter Verdacht, Krebs zu entwickeln und weitere 43 hätten bereits Schilddrüsenkrebs, wobei die Dunkelziffer der Kinder mit bereits veränderten Zellen noch höher einzuschätzen sei (Broinowski 2013: 4). Angesichts einer bisher noch unbekannten Menge an freigesetztem radioaktiven Material und der geringen Diversifizierung der vorliegenden Forschungsergebnisse seit den 1940er Jahren anhand von Alter, Gender etc. hält Broinowski die für die Entscheidungen der japanischen Regierung herangezogenen Berichte von WHO und UNSCEAR für überaus optimistisch:

"Given that the different metabolism of pregnant women, children and adults have been exposed to radioactive contamination in excess of $250 \mathrm{mSv}$, whether accumulated or singular doses (Chernobyl mandatory evacuation level was $5 \mathrm{mSv}[\ldots])$ it seems clear that the risks from Fukushima are being downplayed by the Government and the IAEA-affiliated radiation protection institutions."

(Broinowski 2013: 4)

Die anhaltende Diskussion über die Auslegung der gemessenen Strahlenwerte führt folglich dazu, dass die Menschen nicht wissen, ob eine Rückkehr in ihre Häuser, sofern dies konkret debattiert wird, tatsächlich sicher ist. Stattdessen beobachten sie insbesondere an Kindern bereits auftretende Schwellungen an der Schilddrüse, bemerken Nasenbluten, Husten und Asthma.

Es sind diese konkreten Erfahrungen körperlicher Veränderungen, die die Distanz zwischen wissenschaftlicher Debatte und den Lebensrealitäten augenscheinlich 
werden lassen. Eltern sehen sich daher selbst dazu veranlasst, die Initiative zu ergreifen, sich zu informieren und politisch zu engagieren. ${ }^{45}$ In den mir zur Verfügung stehenden Artikeln unterschiedlichster Medienformate und in von mir persönlich vor Ort geführten Anwohnergesprächen werden in Fukushima ansässige Bürgerinitiativen erwähnt, die für selbst organisierte Messungen und Selbstinformation der Bevölkerung eintreten. Damit bringen die jeweiligen Akteure gleichzeitig ihr gewachsenes Misstrauen gegenüber den staatlichen Behörden zum Ausdruck (Broinowski 2013). Lokale Akteure erkennen die sich abzeichnende Tendenz in den offiziellen Diskursen, die entweder die unvorhersehbare Entfaltung der Naturgewalten für die Ereignisse verantwortlich machen oder die Zuständigkeit für die Bewältigung der sozialen Folgen an die Lokalregierungen abtreten. So argumentierte der Energiebetreiber TEPCO in einer Gerichtsverhandlung von 2012 bezüglich Kompensationszahlungen an den Betreiber einer zwangsgeschlossenen Golfanlage, dass „[...] as a private contractor its responsibilities to the public were limited and that it was no longer the owner of radioactive matter released from the Fukushima Daiichi NPP“ (Broinowski 2013: 1). Dieser Argumentation zufolge geht das radioaktive Material in den Besitz dessen über, auf dessen Land es sich absetzt. Nichtsdestotrotz werden TEPCO und der Zentralregierung Japans nach einem Urteil im Februar 2021 jeweils eine Teilschuld am Reaktorunglück zugesprochen. Die 43 Kläger aus der Präfektur Chiba erhalten 43 Millionen Yen (2,7 Millionen USD). ${ }^{46}$ Meiner Einschätzung nach wird dies jedoch nicht das letzte Urteil in der Frage der Schuld und der Haftung bleiben.

Beispiele wie dieses zeigen, wie sich seit der ersten offiziellen Freigabe einiger Wohngebiete Ende 2012 sowohl der finanzielle als auch psychische Druck auf die Geflüchteten erhöht. Gleichzeitig mit der Aufhebung des Gefahrenstatus wird die Bereitstellung mietfreier Wohnräume durch die Regierungsorgane stufenweise zurückgenommen (Asanuma-Brice 2014: 2). Diese Zwangslage wirkt sich verheerend auf die psychische Gesundheit der Bewohner der Notunterkünfte aus: „[...] their health has gradually deteriorated as time has passed. [...] no financial support is available for nuclear refugees seeking to relocate. Those who can, leave at their own expense“ (Asanuma-Brice 2014: 4). Besonders hart trifft es diejenigen, die keinen offiziellen Evakuierungsbefehl erhalten haben. „They are considered ,voluntary refugees" and thus the Government provides no financial assistance", was auch zur steigenden Anzahl von Selbstmorden beitrage (Asanuma-Brice 2014: 4). Auf die genaue Entscheidungslage der verantwortlich gemachten Akteure gehe ich im Kapitel 5.3 „Die Akteure der Diskurse um den 11. 03. 2011“ ein.

Zunächst halte ich fest, dass der japanischen Bevölkerung angesichts der Debatten nur wenige Informationen zu aktuellen gesundheitlichen Folgen zur Verfügung

\footnotetext{
45 Eine aktuelle Untersuchung dazu führt das Forschungsprojekt von Phoebe Holdgrün „Eltern gegen Radioaktivität - Eine Fallstudie“ vom Deutschen Institut für Japanstudien in Tokyo durch.

46 The Asahi Shimbun: „State again found liable for Fukushima nuclear accident“, 20.2.2021 (http:// www.asahi.com/ajw/articles/14206399).
} 
stehen. Der Umstand, dass die radioaktive Strahlung mit menschlichen Sinnen nicht wahrnehmbar ist, erhöht zusätzlich den psychischen Druck für die geflohenen wie verbliebenen Menschen in den betroffenen Zonen und in weiten Teilen des Landes. Das verbindende Element zwischen den verschiedenen Lebenssituationen bildet die Ungewissheit, die sich nicht nur auf eine ferne Zukunft bezieht, sondern auch auf die in der Gegenwart gelebte Praxis.

\subsubsection{Der mentale Stress: Die Lage der Betroffenen fünf Jahre danach}

Vor diesem Hintergrund der Unsicherheit und Verwirrung in den öffentlichen Streitgesprächen über die historische Einordnung von Fukushima bewegen sich die verschiedenen lokalen Akteure ebenso wie die Vertreter aus Politik und Wirtschaft. Trotz aller Bemühungen seitens der Behörden, die Stimmung in Japan positiv zu beeinflussen, weisen die Stimmen der Menschen vor Ort in eine andere Richtung.

Mein Eindruck der Situation vor Ort, der bis zum Antritt meiner Forschung im Jahr 2014 vor allem auf Medienberichten und ersten im Vorfeld geführten Gesprächen basierte, wurde schließlich von vielen meiner Gesprächspartnern vor Ort bestätigt: „People are stuck. They cannot understand what has happened and how they can go on with their lives. It's a crushing feeling" (Kogoro 02.05.2014: 13).

Der Musiker und Komponist Yoshihide Otomo hielt am 28. April 2011 eine Vorlesung in der Tokyo University of Arts, der sehr renommierten Tokyoter Kunstuniversität. Vor einer versammelten Menge junger Kunststudenten schilderte der 1959 in Yokohama geborene Otomo von seinen persönlichen Verbindungen zu Fukushima und beschrieb seinen persönlichen Eindruck von der lokalen Lage. „Right now, the direct damage caused by the radiation isn't so much the problem as the wound in people's hearts, the emotional disorders" (Otomo 2011: 7). Seine Erfahrungsberichte sind auf einen Zeitpunkt vier Wochen nach der Freisetzung des radioaktiven Materials in Fukushima datiert und decken sich weitestgehend mit den Erzählungen von Kogoro und anderen Akteuren meiner Forschung.

Otomo (2011: 8) berichtet von einer unterschwelligen Wut in der Bevölkerung, die jedoch von einem gleichzeitig vorhandenen Minderwertigkeitsgefühl gedämpft werde: ${ }^{47}$

"The current reality is that the main culture found throughout Japan is the one from Tokyo. [...] it's possible to go to Tokyo in a little under 2 hours, which places Fukushima almost completely within the sphere of Tokyo culture, but at its very edge. This is where Fukushima stood, so before the quake, information might have been sent out from Fukushima but nobody in Tokyo

\footnotetext{
47 Dies ist vor dem Hintergrund zu verstehen, dass sich die Bevölkerung der Präfektur Fukushima in den 1970er Jahren größtenteils für die Inbetriebnahme eines AKW aussprach. Anreize bildeten damals die sicheren Arbeitsplätze, ein gutes Gehalt und die Errichtung einer guten Infrastruktur in einem von Landwirtschaft geprägten Gebiet.
} 
thought to catch it. People in Tokyo didn't feel the need to do so, and the people of Fukushima had probably given up." (Otomo 2011: 12)

Diese emotionalen Diskrepanzen, die gleichzeitig das Machtverhältnis zwischen Tokyo und Fukushima widerspiegeln, finden jedoch in den Berichten der Energiebetreiber oder staatlichen Behörden keine Erwähnung. Offizielle METI-Berichte zur Lage Japans nach Fukushima fokussieren sich vor allem auf die wirtschaftlichen Kernbereiche ${ }^{48}$ und deren Stabilisierung. Die institutionellen Beiträge konzentrieren sich auf die behördlichen Anstrengungen bezüglich der Sicherheitslage in Fukushima Daiichi, der Energieversorgung Japans und der Dekontaminationsbemühungen. Dabei gestaltete sich die Lage der 136.000 Menschen, die 2014 nach wie vor in Notunterkünften untergebracht waren, aus psychologischer Sicht dramatisch, wie folgende Zahlen belegen: Der Japan Times vom 20. Februar 2014 zufolge starben aufgrund des von der Evakuierung und Ungewissheit ausgelösten Stresses und anderer Erkrankungen insgesamt 1.656 Menschen „[...] outnumbering the 1,607 whose deaths were directly tied to disaster-caused injuries [...] “. ${ }^{49}$ Der Artikel nennt die starken psychologischen Belastungen, denen sich insbesondere ältere Bevölkerungsanteile ausgesetzt sehen und die mit dem Verlust von Heimat, gewohntem Umfeld und dem sozialen Netzwerk einhergehen.

Auch mein Gesprächspartner Kogoro (siehe Kapitel 7) berichtete von vielen Selbstmorden in den von ihm besuchten Notunterkünften und der Verzweiflung der im AKW eingesetzten Arbeiter. Er weiß vor allem von der Angst derjenigen zu berichten, die höchstwahrscheinlich einer hohen Strahlendosis ausgesetzt waren. Laut Kogoro (02.05.2014: 17) legte ein Arzt einer besorgten Mutter, die ihr Kind auf Krebszellen untersuchen ließ, nahe, , ,[...] not to speak about this. When people know about this, your child will face problems finding a job or a marriage spouse." Eine Angst vor Stigmatisierung aufgrund der Herkunft verbreitete sich im nur knapp 280 Kilometer entfernten Tokyo auch unter Kindern spürbar und sorgte vier Jahre nach den Ereignissen auch in den japanischen Medien immer wieder für Schlagzeilen. Das ebenfalls aufgetretene Phänomen der sogenannten „Atom-Scheidungen“ (Genpatsu rikon) erreichte 2013 auch die deutschen Medien: „Oft trennen sich Familien, in denen die Frauen mit kleineren Kindern fortzogen, die Männer wegen ihrer Arbeit aber blieben ". ${ }^{50}$ Auch hier spielt die Einschätzung der Gefahrenlage eine entscheidende Rolle. Ist die Diskrepanz zu groß, führt sie zur Scheidung oder sogar dazu, dass bestehende Eheversprechen noch vor Vollzug aufgelöst werden: „Es sind Fälle

\footnotetext{
48 Siehe Government of Japan: „Economic Impact of the Great East Japan Earthquake and Current Status of Recovery“, August 2011 (http://japan.kantei.go.jp/incident/pdf/20110811_Economic_Im pact.pdf).

49 The Japan Times: „Fukishima stress deaths top 3/11 toll - Uncertainties amid nuclear crisis acutely felt by elderly.“, 20.2.2014 (https://www.japantimes.co.jp/news/2014/02/20/national/post-quake-ill nesses-kill-more-in-fukushima-than-2011-disaster).

${ }^{50}$ Carsten Germis: „Die Liebe im Schatten des Atoms“, Faz.net, 10.3.2013 (https://www.faz.net/ aktuell/gesellschaft/zwei-jahre-nach-fukushima-die-liebe-im-schatten-des-atoms-12108688.html).
} 
bekannt, in denen Eltern ihre Söhne dazu brachten, ihre Verlobung mit Frauen aus der Region zu lösen", so der Journalist Germis weiter. ${ }^{51}$

Dies sind nur wenige Beispiele dafür, wie sich die sehr starke Unsicherheit in Kombination mit dem Verlust der alltäglichen Ankerpunkte auf die Menschen auswirken. Die Suche nach Verantwortlichen, nach Anerkennung des eigenen Leids und der persönlichen Unschuld des Einzelnen bilden wichtige Eckpfeiler im Empfinden der unmittelbar Betroffenen, während die wirtschaftliche Perspektive für die Zukunft, vor allem im Hinblick auf die Olympischen Spiele, die 2020 in Tokyo stattfinden sollten, im nationalen Diskurs einen höheren Stellenwert einnimmt. Die ethischen Fragen des menschenwürdigen Lebens, des emotionalen Zustandes der ehemaligen Anwohner und die aus der Flucht entstandenen Leiden werden diskursiv von den nicht unmittelbar Betroffenen abgespalten.

Der Künstler Tadasu Takamine zeigt sich in seinem Beitrag zu der Ausstellung „Japan Syndrome - Kunst und Politik nach Fukushima" solidarisch mit Menschen, denen eine Opferrolle zugeschrieben wird, und sieht Kunst in der Verantwortung zu handeln: „Sie [die Kunst] beschäftigt sich mit der Frage, was es bedeutet zu leben und was das Leben lebenswert macht. Physische und psychische Entfernung sind zwei vollkommen unterschiedliche Dinge" (Takamine 2014: 23). Takamine ist damit ein Beispiel für den Versuch, die psychologischen Belastungen der Menschen vor Ort in den dominanten Diskurs zu integrieren. Indem er den Begriff der Betroffenheit selbst zur Diskussion stellt und eine Kategorisierung ablehnt, rückt er die lokalen Realitätswahrnehmungen wieder in den Mittelpunkt der Aufmerksamkeit.

„Mir erscheint schon die bloße Vorstellung widersinnig, dass sich irgendjemand von irgendeinem Aspekt der Situation persönlich ,nicht betroffen fühlen könnte. [...] Betroffenheit kann ein nützlicher Begriff sein, der einem den Rückzug in die Haltung ermöglicht, dass die anderen einen, einfach nicht verstehen' - als gäbe es eine emotionale Blackbox in unserem Inneren, die legitimiert, dass uns besondere Privilegien zustehen." (Takamine 2014: 23)

In seiner eingangs erwähnten Vorlesung erwähnt Otomo ebenfalls solidarische Züge der Betroffenheit über die Grenzen des Lokalen hinaus.

„People might say that it's the job of psychiatrists to heal wounded hearts and minds. But this isn't just about that kind of disorder. The wound isn't just afflicting each individual; everyone is collectively wounded. I think that the biggest reason for this is that everyone has lost confidence." (Otomo 2011: 9)

Schlussendlich ist nicht sicher, ob Folgeerkrankungen auch zu einer Anerkennung durch entsprechende Behörden und damit zu einer finanziellen Kompensation führen, da die Ursache für den Schilddrüsenkrebs einwandfrei auf die freigesetzte Radio-

${ }^{51}$ Germis wie in vorangegangener Fußnote, Seite 3. 
aktivität zurückzuführen sein müsste. Dies ist eine angesichts des internationalen Disputs über Grenzwerte unmöglich zu erbringende Beweispflicht. Internationalen Medien zufolge wurden seit 2011 um die 370.000 Kinder per Ultraschall auf Zellveränderungen untersucht. ${ }^{52}$ Die entsprechenden Ergebnisse bestätigen jedoch keineswegs den subjektiv wahrgenommenen Zusammenhang, den japanische Eltern zwischen erhöhter Strahlendosis und eventuell bereits jetzt sichtbaren zellulären Veränderungen sehen. Vom einfachen Beobachtungseffekt - noch nie zuvor wurden flächendeckend so viele Menschen auf Krebs untersucht - bis hin zur ungeklärten Strahlenhöchstdosis, die beim Menschen definitiv Krebs auslöst, sind viele Erklärungen für die Befunde möglich. Laut Stockrahm und Lüdemann verlieren sich

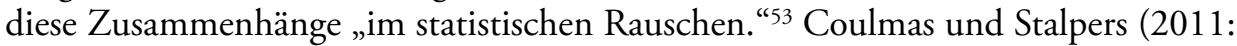
145) beschrieben die rechtliche Situation der Eltern in ähnlicher Weise: Die Grenze zwischen unbedenklichen und schädlichen Strahlenniveaus sei nicht trennscharf. Ebenso verhält es sich mit den nationalen Höchstbelastungsgrenzen für Zivilisten. In Deutschland beispielsweise liegt diese bei $1 \mathrm{mSv} / \mathrm{Jahr}$. Nur für ausgewiesene Risikogruppen wie medizinisches Personal gilt ein Höchstwert von $20 \mathrm{mSv} / \mathrm{Jahr}(\$ 56 \mathrm{der}$ Strahlenschutzverordnung Deutschland) ${ }^{54}$ - dies entspricht dem Wert, der nun in den kontaminierten Gebieten Japans von den Behörden als Belastungsgrenze für die Bevölkerung ausgewiesen ist.

Ohne einwandfreie Beweise für einen direkten Zusammenhang zwischen einer erhöhten Strahlenbelastung und den auftretenden Erkrankungen stehen die Chancen der Betroffenen auf Entschädigungen jedoch schlecht. Der zwischenmenschliche Umgang in Japan zeigt, dass dieses Thema zu einem Tabu im nationalen Diskurs geworden ist (siehe Bohn 2015: 10). Es ist jedoch nicht nur die mangelnde Information, die die Menschen dazu bringt, Dritte auszugrenzen. Die Ungewissheit über tatsächliches Auftreten von Krebserkrankungen etc. ist auch 30 Jahre nach Tschernobyl noch nicht ausreichend erforscht.

Hinzu kommt die psychische Belastung durch die fehlenden sozialen Netzwerke, da Familien, Freunde und Nachbarn zum Teil nur in sehr weit entfernten Unterkünften Zuflucht fanden:

„According to The Chernobyl Forum, held in 2006 (in Belarus and Ukraine), results of studies of adults from the areas contaminated with radioactivity showed that incidence of post-traumatic stress disorder (PTSD) and other mood and anxiety disorders doubled, and people had statistically significantly lower subjective ratings of health. [...] Likewise, a survey about mental health and lifestyle undertaken among residents of evacuation zones showed the sub-

\footnotetext{
52 Sven Stockrahm und Dagny Lüdemann: „Der Super-GAU in den Köpfen“, Zeit Online, 11.3.2016 (https://www.zeit.de/wissen/2016-02/fukushima-jahrestag-atomkraft-tsunami).

53 Stockrahm und Lüdemann wie vorangegangene Fußnote.

${ }^{4}$ Bundesamt für Strahlenschutz der Bundesrepublik Deutschland: „Grenzwerte im Strahlenschutz“, 21.6.2019 (https://www.bfs.de/DE/themen/ion/strahlenschutz/grenzwerte/grenzwerte.html, abgerufen 1.10.2020).
} 
stantial effect of the Fukushima Daiichi NPP accident on mental health. The survey identified the difficulties of evacuee families, who were separated from each other and moved to unfamiliar areas after the accident - similar to those reported by Chernobyl evacuees." (Hasegawa et al. 2015: 485)

Laut dieser Untersuchung leiden mit 14,6 Prozent fünf Mal mehr Menschen in den betroffenen Gebieten an Depressionen als im Rest der Bevölkerung. Der Professor der Universität Tokyo Yasuo Kobayashi (2015: 21-22) findet auf analytischer Ebene folgende Worte: „One lives in a temporality of exposure to risk, of uncertainties [...] existential construction of meaning is falling apart in a stochastic cloud. Our existential integrity is in crisis."

Im Abgleich des Kollektiven mit dem Individuellen wird deutlich, wie stark die Diskurse den Einzelnen beeinflussen und vice versa. Es ist dieser Aushandlungsprozess, der physischen wie psychischen Leiden der unmittelbar Betroffenen eine Bedeutung zuschreibt, sie im Diskurs berührt und den Aushandlungsprozess zwischen der gesellschaftlichen und persönlichen Ebene analytisch greifbar macht.

Ich habe betont, wie sehr der diskursive Umgang mit den gesellschaftlichen Folgen der Dreifachkatastrophe nicht nur von materiellem, sondern auch von emotionalem Verlust geprägt ist. Aus meiner Sicht zeigt sich in der Diskussion um wirtschaftliche Entschädigungen für ertragendes Leid ein Konkurrenzverhältnis zwischen materiellem und emotionalem Verlust, wobei letzteres bislang wenig Beachtung findet.

Gleichzeitig veranschaulicht es meiner Ansicht nach die bestehenden Machtverhältnisse zwischen Politik, Wirtschaft und lokaler Bevölkerung. Die aktuelle Aushandlung enthält nicht nur die implizite Frage, ob finanzielle Mittel zur Entschädigung für emotionale Verletzungen ausreichen und welches Maß dafür angebracht wäre, sondern auch die Frage, ob und wer die Verantwortung für den Gesamtverlauf der Ereignisse zu tragen hat. Die Befriedung der aufgewühlten Emotionen von Trauer, Wut und Verlust stellt die große Herausforderung im japanischen Post-Fukushima-Diskurs dar. Inwieweit dies im konkreten Fall als kulturspezifisches Phänomen zu betrachten ist, analysiere ich im folgenden Kapitel.

\subsection{Kulturelle Spezifika als Katastrophenrisiko?}

Insbesondere seit der einsetzenden Rekonstruktion der Zusammenhänge diverser menschlicher Fehler bei Konstruktion, Bau und Verwaltung der Atomkraftwerke seit den 1970er Jahren und der Verstrahlung ganzer Landstriche am 11. März 2011 werden die kulturspezifischen Prinzipien der japanischen Gesellschaftsorganisation im öffentlichen Diskurs in Frage gestellt. Die Definition der Atomkatastrophe als „man-made disaster“ (jap.: jinsai) hat dem Verlust des Vertrauens der japanischen Bevölkerung in ihre Regierungsgremien eine neue Dimension verliehen und führt zu einer gesellschaftsinternen Hinterfragung von Handlungsprinzipien, die zur aktuellen Situation beigetragen haben könnten. 


\subsubsection{Gestaltung der vertikalen Beziehungen}

Der 2014 verstorbene Professor Harutoshi Funabashi vom Institut für Sustainability Research an der Hosei Universität Tokyo und entschiedener Gegner der japanischen Atompolitik beschreibt einen klaren Zusammenhang zwischen dem Eintreten der Atomkatastrophe und einem bestimmten Beziehungstypus. Er bezeichnet diesen als in der japanischen Kultur vorherrschend: die „binary combination“. Eine solche Beziehung sei „[...] characterized by intimacy and a vertical relation. Binary combination tends to produce a group or organization that is closed to outsiders and often shows indifference as well as insensitivity to outside opinions" (Funabashi 2012: 65). Als Beispiel für derartige Beziehungen führt er vertikale zwischenmenschliche Beziehungen an wie sie typischerweise zwischen Eltern und Kind, Lehrer und Schüler sowie zwischen Chef und Mitarbeiter auftreten (Funabashi 2012: 71). Diese Hervorhebung der Vertikalität als charakteristisches Merkmal der japanischen Sozialordnung deckt sich mit den in Kapitel 4.1 „Der kulturspezifische Ausdruck des Selbst im sozialen Gefüge“ dargestellten Positionen von Kitayama und Markus (1994).

Im Zusammenhang mit der Atomkatastrophe hat, Funabashis Argumentation zufolge, diese Beziehungsstruktur eine verheerende Dynamik entwickelt, da sie die Architektur für ein Gruppen- und Organisationsverständnis der verantwortlichen Akteure (Hersteller, Energiekonzern TEPCO, Premierminister und der Nuklearkommission) erstellte, die schlussendlich zum Sicherheitsmythos der Atomenergie und $\mathrm{zu}$ schwerwiegenden Fehlentscheidungen führten. Beispielsweise seien zum einen in technischer Hinsicht seit den 1970er Jahren die auftretenden Sicherheitsschwierigkeiten in den Reaktoren nicht sachgemäß behoben worden. Die Anlage habe damit einem starken Erdbeben nicht standhalten können (Funabashi 2012: 66). Zum anderen bezeichnet Funabashi (2012: 67ff.) die aufgetretenen Sicherheitsmängel als einen Ausdruck des „nuclear complex“, einem Beziehungsnetzwerk zwischen den entscheidenden Institutionen (Ministry of Economy, Trade and Industry; dem Energiekonzern TEPCO und politischen Kräften), die zum gegenseitigen Vorteil agieren. Das genaue Zusammenwirken dieser drei Kräfte, auch als „Atomdorf“ (genpatsu mura) bezeichnet, führe ich in Kapitel 5.3.1 genauer aus.

Funabashis entscheidendes Argument bezieht sich in diesem Zusammenhang auf ein kulturelles Verständnis von der Position des Einzelnen im Sozialgefüge, das diesem wechselseitigen Beziehungsnetzwerk zugrunde liegt. Dem Individuum steht es Funabashi zufolge nicht zu, Skepsis oder gar Kritik an Entscheidungen zu äußern: „In organizations belonging to the nuclear complex, skepticism and criticism on nuclear development have become a kind of ,taboo"“ (Funabashi 2012: 70; siehe auch Matsumoto 1996: 9). Dies zeigt laut Funabashi, wie sich ein solcher etablierter Beziehungskodex auf das Verhalten des Individuums derart auswirkt, dass „[...] each individual tends to show assimilation with others, lacking independence and showing the immaturity of subjectivity. One's opinion in a binary combination tends to be dependent on another person" (Funabashi 2012: 71). 
Funabashis Bezeichnung dieses Verhaltens als Ausdruck von „Unreife“ und Mangel an Unabhängigkeit lässt auf den internationalen Kontext seiner Argumentation schließen. Wie in Kapitel 4.1 dargestellt, ist es im japanischen Verständnis ein Zeichen der Reife, sich entsprechend der von Funabashi aufgezeigten Norm zu verhalten und eigene Gefühle, Einschätzungen und Bewertungen zum Wohle des Kollektivs zurückzustellen. Diese im japanischen Diskurs positiv konnotierten Verhaltensweisen erhalten bei Funabashi eine negative Facette, indem ihre gegenteiligen Wirkungen aufgezeigt werden. So erwähnt er zum Beispiel den großen Zusammenhalt innerhalb der Gruppe, der laut Funabashi zu einer Gleichgültigkeit und Insensibilität gegenüber Außenstehenden führt. Der große Konformitätsdruck innerhalb dieses Personenkreises würde zudem eine Abneigung gegen abweichende Ideen und Meinungen generieren, was zu der „unverantwortlichen Haltung" führe, auch im Angesicht ungerechter und irrationaler Situationen zu schweigen (Funabashi 2012: 72). Diesem Fokus auf die inneren Interessen des Komplexes wird von Funabashi eine Teilschuld am Verlauf der Atomkatastrophe vom 11. März 2011 zugesprochen, da innere Interessen auch die öffentliche Diskussion und Meinung seit der Zeit vor der Grundsatzentscheidung für die Atomenergie in Japan entscheidend beeinflusst habe.

Für meine Analyse ist Funabashis Perspektive dahingehend wichtig, als dass er davon ausgeht, dass sozio-kulturelle Gründe entscheidend zur Atomkatastrophe beigetragen haben: , [...] generally speaking, a person in a binary combination dislikes a social relation with tension and prefers to avoid any tension“ (Funabashi 2012: 72).

Professor Funabashis Argumentation folgend könnte man davon ausgehen, dass zur Lösung der Probleme eine grundlegende Transformation kulturspezifischer Umgangsformen notwendig wären - und damit eine Veränderung des kulturellen Selbstverständnisses zu favorisieren wäre.

\subsubsection{Politik, Ökonomie und Verantwortung - eine Frage der Aushandlung}

Funabashis Ausführungen werden durch die Perspektive des Werks Bending Adversity: Japan and the Art of Survival (2014) des Journalisten Pilling ergänzt, der ebenfalls die politisch-ökonomischen Voraussetzungen der japanischen Gesellschaft für die Reaktorkatastrophe von 2011 untersucht. Er berücksichtigt die kulturellen Rahmungen Japans in ähnlicher Weise wie Funabashi und betont deren Wichtigkeit bei der Beurteilung der konkreten Verantwortungsfrage von 2011. Der Umgang der japanischen Behörden mit der Öffentlichkeit in Bezug auf die Ereignisse legen seiner Meinung nach das „highly flawed political and bureaucratic system“ (Pilling 2014: xxxv) Japans offen, das er in einen historisch gewachsenen Kontext einbindet.

$\mathrm{Zu}$ den von Pilling ausgemachten Störungen und Manipulationen des Informationsflusses zwischen Führungsriege und Gesellschaft zählen unter anderem die Beeinflussung der öffentlichen Meinung durch die Medien, die er als „elitist and secretive bureaucratic culture" (Pilling 2014: 260) bezeichnet. Nach dem Zweiten Weltkrieg habe dieses System Japan gut gedient als es darum ging, sich wirtschaftlich 
zu erholen. Dennoch sei es im höchsten Maße fehlerhaft: „According to the withering parliamentary inquiry [by Kiyoshi Kurokawa, born 1955, a medical doctor who had been president of the Science Council of Japan], Fukushima was not a natural disaster at all, but a ,profoundly manmade' catastrophe, the result of 'willful negligence'“ (Pilling 2014: 261).

Pilling ist damit eine weitere Stimme in den Post-Fukushima-Diskursen, die die Schuld und Verantwortung in einem Beziehungsgeflecht verortet sieht, das ohne Kontrolle durch Dritte aus den Fugen geraten zu sein scheint. Wie die meisten meiner Gesprächspartner ist auch Pilling der Vertreter eines Narrativs, das davon ausgeht, dass nicht das Versagen die Katastrophe bewirke, sondern die Systeme, die die Humanität des Irrtums in unbegreifliche Zerstörungskräfte verwandeln (Beck 1986: 8). Die Humangeographen Felgentreff und Glade sagen dazu:

„Ganz allgemein gelten Katastrophen (und dies gilt gleichermaßen für die sogenannten Natur- wie für man-made-Katastrophen) in unserer Gesellschaft dann als verhinderbar, wenn erstens die Bedingungen ihrers Eintritts bekannt sind sowie zweitens diese Bedingungen beeinflussbar sind."

(Felgentreff und Glade 2008: 4; Hervorhebung im Original)

Die Wandlung der Diskurse von der Naturkatastrophe zu einem „man-made disaster" setzte die Hinterfragung der Vernetzung der Entscheidungsträger im japanischen Diskurs in Gang. In einer der ersten Zusammenstellungen von kausalen Entscheidungen, die zum „Sicherheitsmythos der Atomenergie“ in der Bevölkerung beitrugen (zum Beispiel in Schulbüchern), geht Pilling (2014: 265) den genauen Hintergründen des Vertrauensverlustes der Bevölkerung in ihre politischen Institutionen und Repräsentanten nach 2011 auf den Grund. Er spielt auf die kulturspezifische Herangehensweise der Konfliktlösung beziehungsweise -vermeidung Japans an. Pilling beschreibt ein System, in dem sich der Einzelne durch das Berufen auf festgelegte hierarchische Entscheidungsstrukturen und den sich daraus ergebenen Gruppendynamiken aus der Verantwortung nimmt. Die Fokussierung auf die Wahrung eines augenscheinlichen harmonischen Miteinanders führt gemäß meiner eigenen Eindrücke, siehe Kapitel 4.1.3 „Konfliktlösung in der sozialen Praxis“, häufig zur Vermeidung unangenehmer Themen. Gestützt wird die diesbezügliche Meinung der genannten Autoren von Aussagen der Vertreter der japanischen NAIIC (Nuclear Accident Independent Investigation Commission), wie deren Vorsitzenden Kiyoshi Kurokawa, der den Vorfall als „made in Japan“ bezeichnet:

„What must be admitted - very painfully - is that this was a disaster 'Made in Japan'. Its fundamental causes are to be found in the ingrained conventions of Japanese culture: our reflexive obedience; our reluctance to question authority; our devotion to 'sticking with the programme'; our groupism; and our insularity." (Kurokawa 2012: 9) 
Seine Aussage deckt sich mit anderen wissenschaftlichen Beiträgen, die auch von einem hohen Risiko innerhalb der angelegten kulturellen Strukturen ausgehen. Die Soziologin Ute Volkmann spricht sich zum Beispiel für einen neuen Fokus in der Betrachtung der Katastrophe aus: „Um die organisierte Unverantwortlichkeit zu überwinden ist folglich ein Perspektivenwechsel erforderlich: von der System- zur Akteurperspektive" (Volkmann 2007: 35).

Dieser Forderung Volkmanns kommt die vorliegende Arbeit nach, indem ich hier die Bewertung der angesprochenen Konventionen der japanischen Kultur durch meine Gesprächspartner wiedergebe. Indem ich die bereits beschriebenen Narrative hinterfrage, werde ich das Gesellschaftsbild Japans diversifizieren und sowohl die Emotionalität der Diskurse aufzeigen, wie sie beispielsweise in Funabashis Analyse (2012) anklang, als auch die persönlichen gesellschaftlichen Folgen eines Lebensgefühls veranschaulichen, das unter anderem vom Vertrauensverlust in das Establishment geprägt ist.

\subsection{Die Akteure der Diskurse um den 11.03.2011}

„Despite Japan's long history with earthquakes and tsunamis, and it being regarded as a world leader in disaster preparation, the country was simply not ready for what happened on 11 March 2011.“ (Hobson 2014: 205)

Bei der Untersuchung der zahlreichen Medienberichte und öffentlichen Diskurse kann der Eindruck entstehen, Japans Experten seien bei den Risikoberechnungen für das Szenario eines gewaltigen Seebebens von wesentlich geringeren Krafteinwirkungen ausgegangen als es schlussendlich der Fall war. Diesem Eindruck zufolge wirkte sich diese Fehleinschätzung auch auf die Wahl des Standortes sowohl von Fukushima Daiichi als auch der anderen Kernreaktoren aus. Doch wer trägt tatsächlich die Verantwortung für die getroffenen Entscheidungen? Welche Interessen aus Politik und Wirtschaft haben die Atomenergiepolitik Japans geprägt?

Es lassen sich konkrete Akteursgruppen in den Machtzentren der japanischen Gesellschaft ausmachen, die sich bereits sehr früh für die Etablierung der Atomenergie in Japan einsetzten und dies bis heute tun. Dieses vor allem aus wirtschaftspolitischen Akteuren bestehende Beziehungsgeflecht wird in Japan "das Atomdorf" (genpatsu mura) genannt, das einen Verbund aus Politikern, METI-Bürokraten, Stromunternehmen, AKW-Herstellern, Baufirmen, Standortkommunen, Wissenschaftlern und Medienunternehmern umfasst (siehe Berndt 2012: 27).

Der Begriff des Atom-Dorfs ist gleichzeitig zur Bezeichnung eines Narrativs geworden, das die Voraussetzungen für den Verlauf der Dreifachkatastrophe beschreibt, zum Beispiel bei der Wahl des AKW-Standortes am östlichen Küstenstreifen. Damit verortet diese Argumentation die Verantwortung für die Vorgänge des 11. März 2011 in den elitären Beziehungsnetzwerken der japanischen Gesellschaft. Die Macht 
dieser Erzählweise verdeutlicht sich dadurch, dass sie in den Berichten meiner Gesprächspartner mehr als Realität denn als ein Narrativ präsentiert wurde.

Ich habe den Eindruck gewonnen, dass dieses Katastrophennarrativ eine gewisse Attraktivität bei meinen Gesprächspartnern genießt. Zum einen lässt sich auf diese Weise der menschliche Faktor der Dreifachkatastrophe bei einem konkreten Personenkreis verorten. Zum anderen wird veranschaulicht, wie aus den natürlichen und unbeeinflussbaren Zerstörungskräften des Erdbebens und des Tsunamis ein nie dagewesenes Ausmaß der Verseuchung durch nukleare Energie werden konnte: "Nuclear Village' is shorthand for the policy community in which energy policy has been made and which ,captured" the industry" (Samuels 2013: 118). So kann die Verseuchung der Umwelt mit einer moralischen Verdorbenheit einiger weniger mächtiger Personen in der Gesellschaft in Verbindung gebracht werden. Aus meiner Sicht birgt dies gleichzeitig die Hoffnung, mit der Veränderung des Entscheidungssystems auf hoher Ebene könne eine Wiederholung eines solchen Zwischenfalls in Zukunft verhindert werden.

Dieser konkret zu verortenden Verantwortung setzten die Befürworter der Atomenergie und "das Atomdorf" ihre eigenen Herleitungen der Geschehnisse vom 11. März 2011 entgegen, die vor allem auf die Unberechenbarkeit der Naturgewalten und die nun aus ihrer Sicht angebrachte Sachlichkeit im Umgang mit den Folgen verweisen (siehe Samuels 2013: 123). Da „das Atomdorf" eine so bedeutende Rolle im Realitätsverständnis sowohl meiner Gesprächspartner als auch im japanischen Diskurs im Allgemeinen innehat, will ich mich im Folgenden auf diese Narrative konzentrieren.

\subsection{1 „Das Atomdorf“ (genpatsu mura)}

Man mag sich die Frage stellen, wieso ausgerechnet ein Land, das zwei Atombombenabwürfe erlebte, sich der atomaren Energiegewinnung zuwandte. In den Argumentationssträngen sowohl der Gegner als auch der Befürworter der Atomenergie spielen vor allem die geringen Ressourcen Japans zur unabhängigen Energieversorgung eine entscheidende Rolle.

In Japan gibt es nur geringe Vorkommen fossiler Energielieferanten wie Erdöl und Kohle. Daher ließ sich die Atomenergie seit den 1950er Jahren als eine vielversprechende Alternative der Stromerzeugung propagieren. Schließlich wuchs mit der wachsenden Wirtschaft auch der Energiebedarf stetig an. Die "friedliche Nutzung“" der gewaltigen Energien der Atome sollte im kriegszerstörten Japan den entscheidenden wirtschaftlichen Anschub für einen Neubeginn schaffen. So waren es vor allem staatspolitische Interessen, die den Weg für die Verbreitung der Atomenergie in Japan bestimmten. Im Laufe der Zeit entwickelte sich daraus ein engmaschiges System, in dem staatliche Institutionen, genauer das METI (Ministry for Economy, Trade and Industry), eng mit Wirtschaftsvertretern zusammenarbeiten und unter anderem die Preise und Gewinnspannen genehmigen. 
In seinen Ausführungen zur Rolle des Staates beschreibt Berndt, Professor für internationales Management, Firmenkultur und Szenarienplanung an der Ritsumeikan Universität Kyoto, eine Verquickung mit Vertretern aus Stromunternehmen, Herstellern, Baufirmen, Wissenschaftlern etc.: Staatspolitik sei in Japan Wirtschafts- und Industriepolitik und umgekehrt (Berndt 2012: 27). Auch lokal auftretendem Widerstand gegen den Bau von neuen Atomkraftwerken wurde mit jahrzehntelangen Zahlungen hoher Subventionen, dem Bau von Infrastruktur und der Schaffung von Arbeitsplätzen von Seiten der Atomkraftbetreiber entgegen getreten. So wurden im Kontext der Erdölkrisen zwischen 1970 und 1994 „[...] pro Jahr durchschnittlich knapp zwei neue Atomreaktoren in Japan in Betrieb genommen" (Berndt 2012: 26). Insbesondere in landwirtschaftlich geprägten Gegenden, wie zum Beispiel der Präfektur Fukushima, boten die Atomkraftwerksbetreiber eine attraktive Arbeits- und Einkommensquelle, die zusätzlich die Infrastruktur ihrer Standorte deutlich verbesserte. ${ }^{55}$

Dies geschah ohne nennenswertes Risiko für die Betreiber, denn es kamen gesetzliche Absicherungen der Energiebetreiber durch den Staat hinzu: Spätestens seit der Verabschiedung des Gesetzes zur Entschädigung bei Atomschäden von 1961 sind AKW-Hersteller von jedweder Haftung ausgenommen. Dies bedeutet im Einzelnen, dass beim Auftreten von Schäden, die über das Höchstmaß der privaten wie staatlichen Versicherung hinaus gehen, die restliche Last vom Staat und damit vom Steuerzahler getragen wird. Das unkalkulierbare Risiko eines wie auch immer gearteten Zwischenfalles und den daraus resultierenden Zahlungsansprüchen wurde damit für die Unternehmen minimiert und steigerte ihr Interesse an der Einflussnahme auf Politik, Parlament und Bürokratie. Im Narrativ über „das Atomdorf“ wird ein roter Faden der „Subordination unter den Willen der Mächtigen“ (Berndt 2012: 74) konstruiert, der das schwer zu durchschauende Geflecht aus Beziehungen, Interessen, Motivationen und Zielen auf eine einfache Formel der Schuldzuweisung herunterbricht. Opfer- und Täterrollen sind dabei klar voneinander getrennt. Diese Klarheit übt meiner Meinung nach eine große Anziehungskraft angesichts einer nach wie vor verwirrenden Lage vor Ort aus.

Bei dieser Zuweisung von Verantwortung an eine bestimmte Personengruppe werden jedoch wichtige Faktoren für den Ausbau der Atomenergie in Japan vernachlässigt. So zum Beispiel die sozio-ökonomischen Entwicklungen seit der Nach-

\footnotetext{
55 Zwick und Renn (2008: 82) untersuchten die Frage, unter welchen Bedingungen Risiko für die Öffentlichkeit akzeptabel ist und nannten dabei folgende Faktoren: Das psychometrische Paradigma (die Bereitschaft, Risiken zu tolerieren), die Stigmatisierung von Risiken, Institutionsvertrauen bzw. die Beurteilung der institutionellen Performanz beim Risikomanagement, Wertorientierung und die Frage der Sensibilisierung gegenüber bestimmten Risiken sowie ausgewählte soziodemographische Charakteristika. Dem Risiko der Kernkraft liegt den Autoren zufolge ein „hohes Schadenspotenzial bei zufälliger oder sehr geringer Eintrittswahrscheinlichkeit" zugrunde, weshalb für das Laienurteil die Eintrittswahrscheinlichkeit weit weniger als das katastrophale Schadensausmaß wiege (Zwick und Renn 2008: 81). Wichtig für ein Risikomanagement sei in jedem Fall das umfassende Wissen über Risikowahrnehmung in der Öffentlichkeit (Zwick und Renn 2008: 94).
} 
kriegszeit, wie die wirtschaftlichen Krisen der 1970er, 1980er und 1990er Jahre, und die zunehmend wahrnehmbare Überalterung der japanischen Gesellschaft. Insbesondere innenpolitisch war in der Nachkriegszeit argumentiert worden, mithilfe der friedlichen Nutzung der nuklearen Energie die Abhängigkeit vom Ausland „zu akzeptablen Kosten zu reduzieren“ (Berndt 2012: 43). Folgerichtig wurden die Kosten der Wiederaufbereitung und die Gefahren sowohl des Betriebs als auch der Endlagerung nuklearer Brennstäbe in der japanischen Öffentlichkeit als alternativlos dargestellt. In der Retroperspektive scheint es gerade dieser Mangel an Informationen und Perspektiven zu sein, die die Menschen an die Realität und Macht des „Atomdorfs" glauben ließen.

Berndt (2012: 28) beobachtet, dass Vorfälle wie die AKW-Katastrophen von Three Mile Island von 1979 und Tschernobyl 1986 keinen großen Einschnitt in der Atompolitik Japans zur Folge hatten. Während nach 1986 in Europa und den USA die Nachfrage nach Kraftwerken deutlich zurückging, baute man in Japan noch bis Mitte der 1990er Jahre weitere Atommeiler. Eine genaue Erklärung kann Berndt dafür nicht anbieten und verweist stattdessen auf weitere politische Entwicklungen auf internationaler Ebene: Die Hinwendung der globalen Weltpolitik zu nachhaltiger Energiegewinnung und Senkung der CO2-Emissionen ab den 2000er Jahren ließ die Atomenergie als vermeintlich sauberer Lieferant erneut an Attraktivität gewinnen (siehe Berndt 2012: 28). Damit erhielten AKW-Befürworter neue Argumente. Aufgrund des vorangegangenen Rückbaus der Atommeiler in den USA und Europa wurde Japan damit zu einem der weltweit führenden Exporteure nuklearen Equipments und erlebte den prognostizierten großen wirtschaftlichen Aufschwung. Das Narrativ des „Atomdorf““ ist ein Ausdruck eines Erklärungsversuchs für die staatliche Unterstützung der Atompolitik, die sich in Form von gesetzlichen Anpassungen, Preisabsprachen und Kostenregelungen manifestiert. Hinzu kommt die nach dem 11. März 2011 verstärkt sichtbar werdende ungleiche Risikoverteilung zwischen Betreiber, Staat und Anwohnern zugunsten der Atomindustrie, die, wie oben erläutert, kein finanzielles Risiko im Falle eines Zwischenfalles trägt.

Berndt (2012: 58) stellt „das Atomdorf" in einen Argumentationskontext der allgemeinen politischen Regierungsstruktur Japans, die er "neoliberalen Dreiklang“ nennt. Gemeint ist damit die Deregulierung und Privatisierung der Wirtschaft bei gleichzeitigem Sozialabbau des japanischen Staates. Den Unternehmen wird es damit ermöglicht, Kosten zu senken und Verbindlichkeiten zu reduzieren. Der zeitgleich angekurbelte Wettbewerb wird von den Gegnern des „Atomdorfs“ als logische Konsequenz einer fehlgeleiteten Führung angesehen, die schlussendlich zur Katastrophe von 2011 beitrug: Anstatt auf die Sicherheit der Menschen und der Umwelt zu setzen und die entsprechenden Kontrollinstanzen unabhängig von wirtschaftlichen Interessen agieren zu lassen, gehen die Gegner des „Atomdorfs“ von engen Absprachen und sogar gegenseitigem Personalwechsel zwischen METI und entscheidenden Wirtschaftsgremien aus (siehe Samuels 2013: 121). Gerade weil die Grenzen zwischen Politik und Wirtschaft verschwimmen, stehen sowohl politische Akteure als auch Wirtschaftsvertreter aktuell im moralischen Kreuzfeuer der Gegendiskurse. 
Um es mit den Worten des Japanologen Samuels auszudrücken, wurden die Absprachen dieser konspirativen Gruppe dazu benutzt „,...] to take the teeth out of the regulation" (Samuels 2013: 119). Angesichts der angenommenen Auswirkungen dieser Vereinbarungen auf die Entwicklung des 11. März 2011 werden die Diskurse mit moralischen Untertönen geführt. Die fehlende Transparenz und der Zusammenschluss der Eliten bestärken nur noch den Eindruck einer innergesellschaftlichen Frontenbildung zwischen Machtinhabern und der Bevölkerung. Samuels (2013: 119) weiß von Vertuschungsaktionen und Datenfälschungen zu berichten, die die moralische Argumentationsposition der Gegner des „Atomdorfs“ unterstützen. Diese Aspekte verweisen auf die Emotionalität der geführten Diskurse, die durch die Schuldzuweisung an Einzelne von der Teilverantwortung aller, vor allem durch fehlende zivile Kontrolle, ablenken.

Ich möchte erneut darauf hinweisen, dass, ungeachtet der Wirkmächtigkeit des „Atomdorfes“, die genauen technischen und sozio-politischen Ursachen angesichts der Größenordnung der Vorkommnisse in mehr als nur einer Quelle zu suchen sind. Das vergleichsweise geringe öffentliche Interesse an der Hinterfragung der über Jahrzehnte dominanten Diskurse über die Atomenergie hat daran ebenso ihren Anteil, wie die geschilderten Absprachen zwischen den einzelnen Interessenvertretern auf höherer gesellschaftlicher Ebene. Berndt sieht in der Verfechtung der bürgerlichen Interessen einen Lösungsweg, der sich aus der Existenz des „Atomdorfs“ ergibt:

„Nur eine Vielfalt von basis-politischen, bürgerrechtlichen, gemeinnützigen, religiösen und beruflich-professionellen Bewegungen, die sich verbünden, kann staatliche und wirtschaftliche Macht sowie deren Verschränkung dort delegitimieren und einschränken, wo sie den Interessen der Gesellschaft und individuellen Grundrechten zuwider läuft.“ (Berndt 2012: 73)

Die eingangs erwähnten regelmäßigen Proteste seitens der lokalen Bevölkerung in manchen Orten oder einzelner NGOs gegen dominante Narrative fanden in der breiten japanischen Öffentlichkeit nur wenig Gehör. Ihre Rolle in den aktuellen Katastrophen-Diskursen war marginal. Auch die andauernden Bemühungen der Überlebenden des Atombombenangriffs auf Nagasaki und Hiroshima im Zweiten Weltkrieg, die hibakusha genannt werden, die japanische Bevölkerung für die Gefahren der Atomenergie zu sensibilisieren, konnten die japanische Öffentlichkeit bis zum März 2011 nicht in ausreichendem Maße erreichen. Hibakusha nehmen insofern eine besondere Position in den Post-Fukushima-Diskursen ein, als dass sie als Strahlenopfer bis zum heutigen Tag diskriminiert und stigmatisiert werden. Viele Menschen aus den verstrahlten Gebieten von 2011 müssen befürchten, dass sie selbst und ihre Nachkommen auf ähnliche Weise von der Gesellschaft benachteiligt und ausgegrenzt zu werden. Auch aus diesem Grund sind die Diskussionen über die Verantwortlichkeit für den atomaren GAU von 2011 stark emotional aufgeladen.

Ich möchte an dieser Stelle ergänzen, dass die alleinige Verantwortlichkeit für die Entwicklung des 11. März 2011 nicht beim „Atomdorf“ zu suchen ist. Dennoch 
weisen die kritischen Diskurse auf die gewaltige Macht hin, die diese wirtschaftspolitische Verbindung bis heute auf die meinungsbildenden Instanzen ausübt. Gemeint sind damit vor allem die japanischen Massenmedien bestehend aus Zeitung, Radio und Fernsehen. Deren Beeinflussung erläutere ich im folgenden Abschnitt und nehme Bezug auf die von Berndt aufgeworfene Frage nach der bürgerlichen Kontrollinstanz in einem Land, das sich offiziell als demokratisch versteht.

\subsubsection{Medien, die staatliche Öffentlichkeit und ihre Alternativen}

Wie die Debatten um den havarierten Atomreaktor Fukushima Daiichi zeigen, ist die Meinungsbildung der japanischen Bevölkerung wie auch im Rest der Welt im Wesentlichen von Informationsgehalt und -vielfalt abhängig, um die Lage in ihrer lokalen Darstellung beurteilen zu können. Da sich Radioaktivität nicht vorhersehbar ausbreitet und darüber hinaus mit den menschlichen Sinnen nicht wahrgenommen werden kann, bestimmen die zur Verfügung stehenden Informationen das Realitätsverständnis des Einzelnen (siehe Beck 1986). Dies führt dazu, dass es, wie in Japan geschehen, zu völlig unterschiedlichen Schlussfolgerungen bezüglich der gegenwärtigen und zukünftigen Lage kommen kann.

Meine Gesprächspartner betonten während meines 12-monatigen Aufenthalts in Japan die Abwesenheit einer nicht-staatlichen Öffentlichkeit und bemängelten ein Defizit an Informations- und Meinungsvielfalt. Meist äußerten sie dies in einem Atemzug mit Kritik an den staatlich dominierten Medien wie dem Fernsehsender NHK oder großen Tageszeitungen wie der japanischen Zeitung The Asabi Shimbun. So meint meine Gesprächspartnerin Kaeko, eine international weit gereiste Künstlerin:

„The newspapers don't support or report minority opinions. People are innocent about that which adds to the difficulty of expressing a different opinion. The government diverts peoples' attention and preoccupies them with other issues so they neither have the time nor the space in mind to think about the important issues that requires their attention." (Kaeko 25.07.2014: 2)

Die aus einer solchen Haltung resultierenden Schwierigkeiten der politischen Aktivierung ihrer Mitmenschen für die in ihren Augen notwendigen gesellschaftlichen Umwälzungen waren ein weiterer wichtiger Punkt in Kaekos Argumentation. Wie der Soziologe Calhoun (1992: 2) in seiner Diskussion von Habermas' Werk The Structural Transformation of the Public Sphere (1989) feststellt: „In a nutshell, a public sphere adequate to a democratic polity depends both on quality of discourse and quantity of participation." Habermas bezieht zwar sein soziologisches Werk auf den europäischen Raum und betrachtet die Entstehung der öffentlichen Sphäre und ihrer Bedeutung für die Entstehung einer Zivilgesellschaft vor diesem spezifischen Hintergrund. Dennoch lassen sich die Merkmale seiner Transformationsanalyse auch auf andere Gesellschaften übertragen. So ist Habermas' Betonung der Qualität 
eines Diskurses und die Quantität der Partizipation von besonderer Bedeutung (Calhoun 1992: 2-3). Die Sphäre des Öffentlichen bildet bei Calhoun eine Plattform der möglichen sozialen Integration, der Koordination und der Orientierung von privaten Individuen (Calhoun 1992: 5). Die Bedeutung des öffentlichen Raumes, die Habermas hervorhebt, soll an dieser Stelle ausreichen, um die folgenden Aspekte in ihrer Wichtigkeit einordnen zu können. ${ }^{56}$

Diese Vorüberlegungen sind wichtig, um die Rolle der (Massen-)Medien als erste Informationsquelle der breiten Bevölkerung in ihrer ganzen Bedeutsamkeit erfassen zu können. Medien sind im Idealfall die kritischen Informanten der lokalen wie globalen Bevölkerung, um Daten zu vermitteln, die Situation im Kontext zu verorten und Ermitteltes kritisch zu hinterfragen. Sie bilden damit eine spezifische Kommunikationsform zwischen den strukturellen Ebenen der Gesellschaft, die in ihrer Wichtigkeit unter anderem für zivilgesellschaftliche (Macht-)Prozesse nicht zu unterschätzen ist. Vor dem US-amerikanischen Hintergrund untersucht der Soziologe Castells (2009) die Verbindung zwischen Kommunikation und Macht und postuliert mit seiner Theorie der Netzwerk-Gesellschaft einen Ansatz, um Machtverhältnisse gegenwärtiger Gesellschaften zu verstehen. Macht wird dabei von Castells als Beziehung verstanden, die sich unter anderem, unter Rückbezug auf Foucaults Definition von Macht als Wissen und Sprache, in sozialer Praxis als Machtverhältnis manifestiert. Das in Kapitel 5.3.1 „Das Atomdorf“ geschilderte Beziehungsnetzwerk könnte hierfür als konkretes Beispiel dienen. Bei der genauen Betrachtung von politischer Macht und ihrer Entstehung definiert er Macht als „meaningful communication coded by culture" (Castells 2009: 24), zu deren wichtigsten Vertretern der Staat als einer der Hauptakteure der dominanten Diskursformung auftritt. Castells Darstellung des Kommunikationsmanagements in der Politik beschreibt vor allem die Massenmedien und ihr Verhältnis zu politischen Akteuren. Diese Personen formen mit dem Ausdruck ihrer Interessen nicht nur die Sprache und den diskursiven Rahmen, sondern gestalten auch den öffentlichen Raum als solchen. Besonders letzterem wird in Castells Ausführungen besondere Bedeutung verliehen. In der Kontrolle über den öffentlichen Raum sieht Castells (2009: 299) den Schlüssel zur sozialen Macht.

Mögliche Veränderungen auf allen gesellschaftlichen Ebenen sind den Zugangsbeschränkungen und Spielregeln der (Massen-)Medien unterworfen, denen Castells die Autonomie der „mass self-communication“, also der eigenverantwortlichen Informationsbeschaffung der Bürger, gegenüberstellt. Die damit unabhängigere Bedeutungskonstruktion gesellschaftlicher Prozesse ist ein wichtiger Bestandteil der von ihm geschilderten „counter-frames“ (Castells 2009: 164). Es handelt sich bei „counter-frames" also um eine alternative Perspektive auf Politik, Wirtschaft, Medien und deren Interaktion. Dieser Gegenentwurf zu staatlichen Diskursen ermöglicht es, die Bürger im zivilgesellschaftlichen Sinne zu mobilisieren. Alternative Rahmungen von Ereignissen bilden ein oppositionelles Gegengewicht zur dominanten Vorgehensund Sichtweise und ermutigen damit zu dessen Hinterfragung.

\footnotetext{
${ }^{56}$ Für weitere Ausführungen siehe Habermas (1989).
} 
Castells beschreibt das Kreieren von Gegendiskursen als eine Form der Herausforderung des etablierten Machtgefüges, für dessen Wirksamkeit die öffentliche Sphäre unabdingbar ist: „For the counter-frames to be powerful enough to challenge the elite-induced frames, they need to be culturally resonant with the public - or at least with the journalists' perception of public opinion" (Castells 2009: 164). Veränderungen im Allgemeinen benötigen demzufolge ein „reprogramming of communication networks" (Castells 2009: 302), was die Zusammenführung diverser Informationsquellen erfordert. „Thus, the rise of mass self-communication, as I call the new forms of networked communication, enhances the opportunities for social change, without however defining the content and purpose of such social change" (Castells 2009: 8). Den entsprechenden Mehrwert erhalten die Informationen aus Netzwerken gegenüber den üblicherweise generierten Daten durch ihre hohe Vielfalt an Emotionen, Interessen und Zielgruppenausrichtungen. Der diskursive Raum wird auf diese Weise angereichert mit einer Vielfalt an Meinungen und Informationsquellen, die der fundierten Meinungsbildung zuträglich sind.

Im japanischen Kontext der Post-Fukushima-Diskurse stellen „counter-frames“ meiner Ansicht nach eine Reaktion auf die in Folge des Disasters von 2011 offen zu Tage getretenen Entscheidungsstrukturen dar. Gegenwartskünstler tragen mit ihrem Bemühen um eine solch öffentliche Plattform alternativer Informationsbeschaffung, Datenauswertung und -diskussion entscheidend dazu bei. Gegenwartskünstler könnten aus meiner Sicht eine dieser neuen Formen der „mass self-communication" darstellen, da sie durch den Aufbau eines alternativen Bedeutungsrahmens einen „counter-frame“ in Castells Sinne mitgestalten. Meiner Erfahrung nach ist die Kommunikation ein entscheidendes Werkzeug der Kunst, das den Meinungsbildungsprozess all jener, die mit ihr in Berührung kommen, beeinflussen kann. Um Veränderungen im „System Japan“ (Gebhardt 2015) wirkungsvoll zu initiieren, benötigen „counter-frames“ laut Castells (2009: 164) eine kulturelle Resonanz mit der Öffentlichkeit beziehungsweise zumindest mit der journalistischen Wahrnehmung der öffentlichen Meinung.

Meine Analyse wird zeigen, dass die Ereignisse von 2011 eine Öffnung zu Diskursen über Japan als gesellschaftliches Gefüge bewirkt haben könnten, der die japanische Regierung trotz aller Bemühungen das Monopol der Bedeutungszuschreibung gekostet hat. Alternative Informationszugänge und Perspektiven auf politisches Leben hätten demzufolge einen Bedeutungszuwachs aufzuweisen.

Dies gewinnt umso mehr an Bedeutung, da es in der Medienlandschaft Japans, wie die Japanologin Liscutin bemerkt, um die Katastrophe vom 11.03.2011 sehr „still“ geworden ist:

„Die meisten der japanischen Massenmedien scheinen in ihren Inlandnachrichten nur dann von dem Kernkraftwerk zu berichten, wenn der Betreiber in seinen Pressekonferenzen auftretende Probleme benennt, wie zum Beispiel im Februar 2012 die plötzlich ansteigenden Temperaturen im Reaktor 2 [...].“ 
Hinzu kommt die Konstatierung einer gewissen „Atommüdigkeit“ der MainstreamMedien (Liscutin 2012: 136), die sich angesichts der schweren sozialen Krise und stark emotional aufgeladene Debatten anderen Themen zugewandt haben.

Die Medien stehen damit laut Liscutin in starkem Kontrast zu den sich nach 2011 organisierten Netzwerken und Informationsplattformen einzelner Akteure und internetbasierter Medien wie Twitter, Facebook und Blogs, die es sich zur Aufgabe gemacht haben, über die von den Massenmedien nicht abgedeckten Bereiche wie Schicksalsbewältigung und Protestaktionen zu informieren und die verschiedenen Sichtweisen zu diskutieren. Liscutin (2012: 137) beschreibt eine sich nach dem 11.03.2011 etablierende, in Castells Sinne stattfindende „Massen-Selbst-Kommunikation“, die einen „rapiden und entscheidenden Strukturwandel in der Massenkommunikation in Japan vollzogen [...]." Da die Regierung den Informationsfluss bewusst reglementiere, sei es zu einem Informationsvakuum gekommen, das diese neuen Medien zu füllen versuchten (Liscutin 2012: 144). Möglich wurde dies jedoch erst durch den scharfen Kontrast zwischen den Bildern der Katastrophe und den beschwichtigenden Meldungen der Regierung, die der Eigenwahrnehmung, insbesondere der Anwohner von Fukushima, Iwaki, Koriyama, Nihonmatsu etc. zuwider liefen.

Ich argumentiere, dass in der Zeit nach Fukushima eine von der Politik nicht zu beherrschende Dynamik in die Gesellschaftsdiskurse eintrat, die die Erfahrungen von Tschernobyl anstelle der verbreiteten verharmlosenden Meldungen der Regierung als Bewertungsmaßstab für die Vorgänge in Fukushima Daiichi anlegte. Unabhängige Informationen zu beschaffen und zu kontextualisieren wurde daher schnell zur Hauptaufgabe der genannten neuen Medienvertreter, die nicht nur ein wachsendes Bewusstsein für die notwendige Eigeninitiative schufen, sondern darüber hinaus den direkt Betroffenen in den entsprechenden Gebieten um Fukushima auch die Möglichkeit einer neuen Rolle gaben. Die Personengruppen, die sich in die Opferrolle gedrängt sahen, können sich von „passiven, aufnahmebereiten Empfängern" (Liscutin 2012: 149) zu Gehör einfordernden Akteuren wandeln. Damit handelt es sich meiner Auffassung nach um einen Akt des „Empowerment“, der die Hoheit über die Bedeutungszuschreibung einzelner Informationen von regierungsnahen Institutionen auf die Betroffenen selbst überträgt.

Bei der Analyse des von ihr konstatierten Strukturwandels der Medienlandschaft stellt Liscutin unter anderem fest, dass bei dieser neuen Form der Kommunikation mehrere Merkmale auftreten, die die Netzwerkbildung unter den Menschen stark begünstigen:

1. Es kommt zu einer horizontalen Kommunikation, die die Menschen durch das Teilen von Erfahrungen auf emotionaler Ebene anspricht und damit in besonders starkem Maße zu aktivieren vermag.

2. Die Vernetzung in „Echtzeit“ ermöglicht ein gemeinsames Erleben in gleichzeitigem Raum, was dazu beiträgt, eben jene kollektive Identität zu formen, 
die besonders wirksam ist, wenn man Druck auf die politische Ebene ausüben möchte (Liscutin 2012: 149).

Organisationen wie NGOs zielten bewusst darauf ab, eine „kommunikative Autonomie" der Bürger herzustellen, was wiederum ein entscheidender Faktor bei der aktiven Gestaltung der angestrebten Transformationsprozesse darstelle (Liscutin 2012: 157). Dieser Akt des „Empowerment“ bei der Auseinandersetzung mit den Folgen, aber auch mit den Prämissen der Katastrophe von 2011, zieht einen weiteren Prozess nach sich, den die Japanologin Gebhardt (2012) als „Politisierung“ beschreibt. In Bezug auf die sich im Licht von 2011 verändernde japanische Literaturszene thematisieren die Autoren und Denker der japanischen Gesellschaft die ungenügenden Maßnahmen der Regierung und von TEPCO, die ausbleibenden kritischen Medienreaktionen und die Folgen des sozialen Verfalls in lokalen Kontexten. Literarische Größen zählen in Japan zu den einflussreichen Akteuren des öffentlichen Lebens und haben sich im Kontext der Dreifachkatastrophe früh mit kritischen Äußerungen in Bezug auf das Vorgehen der Regierung und TEPCO zu Wort gemeldet.

Liscutins und Gebhardts Ausführungen legen nahe, dass die Vertreter der japanischen Literaturszene aufgrund ihrer zur Diskussion gestellten Beiträge und ihres zum Teil erheblichen Einflusses ebenfalls einen wichtigen Beitrag zur kritischen Meinungsbildung in der Öffentlichkeit leisten. Ihnen wird, wie in Kapitel 6 „Kunst und Künstler in Japan - Eine Milieuanalyse" dargestellt, eine streitbare Meinung zugestanden beziehungsweise wird ihnen aufgrund der Definition als „Denker“ kultureller Spielraum für kontroverse Themen eingeräumt. Gebhardts Beitrag bildet damit eine wichtige Kontextualisierung und Ergänzung zu meinen eigenen Ergebnissen in Bezug auf Gegenwartskünstler. 



\section{Kunst und Künstler in Japan - Eine Milieuanalyse}

People make themselves, but not in circumstances of their own choosing.

(Twine 1994: 7)

In diesem Kapitel geht es mir nach den theoretischen Überlegungen und den Darstellungen der Diskursrahmungen der japanischen Gesellschaft nach dem 11. März 2011 um die kulturspezifischen Kontexte meiner Gesprächspartner in der Kunstszene Japans. Wichtige Rahmungen der Selbstkonstruktion meiner Gesprächspartner zeige ich ebenso auf wie das Standing der Gegenwartskunst in Japan und den damit zusammenhängenden Herausforderungen in einer modernen Leistungsgesellschaft. Dabei ist mir besonders wichtig, die verschiedenen tiefgreifenden Verbindungen zu betonen, die jedes Individuum in diesem auf Interdependenz beruhendem Gesellschaftsverständnis verankert. Für die Analyse der Interviewdaten ist die Einbeziehung dieser Verbindungen essentiell, um ein Verständnis für die Darstellung vom Leben, Beruf und den Herausforderungen meiner Gesprächspartner zu entwickeln.

Die verschiedenen Facetten des Künstlerlebens zu erfassen und zu verstehen, stellte zu Beginn der Forschungsphase eine Herausforderung für mich dar und führte meinerseits zu einem tiefgehenden Reflexionsprozess. Meine Auseinandersetzung bezog sich nicht nur auf die gegenseitigen Identifizierungen im Feld als "westliche“ Forscherin beziehungsweise Künstler, sondern auch auf die entsprechenden Annahmen und Selbstpositionierungen. So stellte sich das Herausfiltern von Kriterien für die Bezeichnung „Künstler“ zunächst als schwierig heraus, da meine Gesprächspartner, insofern sie sich tatsächlich als Künstler bezeichnen, das Urteil über ihre Arbeit vorrangig ihrem Publikum überlassen: „Whether this is art or not, my audience 
decides" (Saburo 13.07.2014: 4). Nachdem ich den Zugang zum Milieu gefunden und das Vertrauen von einigen Gesprächspartnern erworben hatte, war es mir möglich, sowohl meine eigene Position im Feld zu klären als auch gegenseitige Vorurteile abzubauen. So hat es sich als sehr zielführend erwiesen, dass ich mich nicht als Wissenschaftlerin, sondern als Lernende präsentierte. Dies wiederum führte dazu, dass der Leistungsanspruch meiner Gesprächspartner, mir „gutes Interviewmaterial“ zu liefern, relativiert wurde. Die so aufgelockerte Atmosphäre erlaubte es mir schließlich, die entsprechenden Kategorisierungen und Selbstzuschreibungen der Künstler abzufragen, wenn sie nicht selbst darauf zu sprechen kamen.

\subsection{Was ist Kunst?}

Nach der Auswertung der entsprechenden Aussagen definiere ich eine Person als Künstler, wenn er oder sie der Kunst in ihren verschiedenen Formen (Malerei, Bildhauerei, Musik, Installation etc.) einen festen Platz in ihrem Leben einräumt und dies als ihr leitendes Lebensprinzip ansieht. Durch diese offene, personenbezogene Definition konnte ich dem jeweiligen Gesprächspartner größeren Raum zur Gestaltung seiner Selbstrepräsentation geben als wenn ich von vornherein von einer geschlossenen Kategorisierung ausgegangen wäre. Diese offene Gestaltung führte dazu, dass ich durch meine Erstkontakte an Künstler verwiesen wurde, die völlig unterschiedlichen Genres angehören, und meine Auswahl an Gesprächspartnern die von mir gewünschte Diversität von Kunstrichtungen aufweist. Nichtsdestotrotz sind es die Gemeinsamkeiten aller meiner Gesprächspartner, die mein Bild eines japanischen Gegenwartskünstlers prägen. Diese Gemeinsamkeiten beziehen sich auf Einstellung, Motivation, Zielausrichtung und Selbstdarstellung während der Interviews.

Spannenderweise äußerten sich die verschiedenen Künstler darüber hinaus in vielfältiger Weise zu den Fragen, was Kunst eigentlich ausmacht, worin sie ihre Aufgabe als Künstler sehen und wie sie in der Gesellschaft wirken wollen. Die Antworten meiner Gesprächspartner beschreiben ein Spannungsverhältnis entlang zweier Aspekte: Zum einen entlang ihrer persönlichen biographischen Zugänge zur Kunst und zum anderen entlang einer gesellschaftlichen Trennung zwischen Gegenwartskunst und „traditionellen“ Kunstformen. Die Herausforderung besteht also zunächst darin, die Kategorie „Gegenwartskunst“ möglichst genau zu beschreiben, bevor ich mich auf die Aushandlungspraxis zwischen gesellschaftlichem Kollektiv und dem jeweiligen Individuum eingehe.

Allen Gesprächspartnern war die Bezeichnung ihrer Kunst als Kommunikationsmittel besonders wichtig, was die geringe Trennschärfe von Kunst und ihrem täglichen (Er-)Leben noch unterstreicht. Der Ethnologe Geertz sagt in Bezug auf Kunst und Kommunikation: 
„[...] we characterize art as a language, a structure, a system, an act, a symbol, a pattern of feeling; we reach for scientific metaphors, spiritual ones, technological ones, political ones; and if all else fails we string dark sayings together and hope someone else will eludicate them for us." (Geertz 1983: 95)

Geertz' Zitat unterstreicht die Schwierigkeit, Kunst zu definieren, aber auch, wie viele Bereiche des menschlichen Daseins sie berührt. Ein Werk, egal welcher Gattung es angehört, ist immer ein organisch gewachsenes Produkt, das auf der Basis einer gesellschaftlichen Ordnung zu einem bestimmten Zeitpunkt steht. Dabei spielen die Erfahrungen des Alltags für den Künstler eine wesentliche Rolle: Er wird von seinen Erlebnissen angeregt, inspiriert oder durch Widrigkeiten motiviert. Meine Gesprächspartner vermittelten mir den Eindruck, dass das Werk dabei sowohl das emotionale Empfinden als auch den Intellekt anspricht und damit auf zwei Arten berührend und aktivierend wirkt. Laut den Aussagen meiner Gesprächspartner gelingt es dem Künstler dabei idealerweise, sich selbst als eine Art Instrument des Ausdruckes zu nutzen und verschiedene Stimmen und Ausprägungen einer Kultur, einer Gesellschaft oder einer Gruppe einzufangen. Diese Stimmen einer Kultur schlagen sich schlussendlich in einem Werk nieder:

„[...] the feeling a people has for life appears, of course, in a great many other places than in their art. It appears in their religion, their morality, their science, their commerce, their technology, their politics, their amusements, their law, even in the way they organize their everyday practical existence. The talk about art [...] is largely directed to placing it within the context of these other expressions of human purpose and the pattern of experience they collectively sustain." (Geertz 1983: 96)

Eben diese von Geertz beschriebene ständige Auseinandersetzung mit dem eigenen gesellschaftlichen Umfeld macht die Gegenwartskünstler Japans auch nach dem 11. März 2011 zu einer wertvollen Stimme. Die zum Teil für den Rest der Welt verdeckt ablaufenden sozial-politischen Prozesse werden dabei nicht nur sichtbar gemacht, sondern auch nachvollziehbar dargestellt. „It seems that art as art expresses a truth, an experience, a necessity which, although not in the domain of radical praxis, are nevertheless essential components of revolution" (Marcuse 1978: 1).

Die Ansatzpunkte zu meinem Verständnis von japanischer Gegenwartskunst lieferten die befragten Interviewpartner selbst, indem sie direkt oder indirekt auf wichtige Phasen ihres Lebens hinwiesen, die sie als maßgeblich für ihre persönliche wie berufliche Entwicklung erachteten. In ihren Äußerungen wird deutlich, dass Künstlern Fragen zu ihrer Kunst zu stellen immer auch bedeutet, sie indirekt an ihre Person zu richten und sie dazu aufzufordern, ihr persönliches Empfinden zu erläutern: „It [art] is not a new cryptography that we need, especially when it consists of replacing one cipher by another less intelligible, but a new diagnostics, a science 
that can determine the meaning of things for the life that surrounds them" (Geertz 1983: 120).

Da ich mich in dieser Arbeit ausschließlich mit Gegenwartskünstlern beschäftige, möchte ich im Folgenden auf die Begrifflichkeiten und ihre Unterscheidung zur sogenannten „traditionellen“ Kunst eingehen. Die damit verbundenen und im allgemeinen japanischen Sprachgebrauch verwendeten Verständniskonnotationen sind entscheidend für das soziale Setting und das Selbstverständnis der von mir untersuchten Künstler.

\subsection{Die Gegenwartskunst in Japan: Eine historische Einordnung}

Die Legitimität ihres Wirkens ist für zeitgenössische Künstler seit der beginnenden Einflussnahme ausländischer, sprich „westlicher“, Kunsttheorien in Japan eine Herausforderung gewesen. Von der Epoche der politischen und wirtschaftlichen Abschottung des Shogunats geprägt, waren andersartige Vorstellungen von Ästhetik und Auftrag der Kunst größtenteils ausgesperrt worden. Mit dem Fall des TokugawaShogunats und der damit einhergehenden Öffnung Japans im späten 19. Jahrhundert sickerten neben ideologischen und wirtschaftlichen Vorstellungen auch ausländische Auffassungen über Kunst und Künstler in die japanische Gesellschaft ein.

Die Japanologin Takeuchi (2004) beschreibt in „The Artist as Professional in Japan“ den epochalen Werdegang von Künstlern in ihren jeweiligen zeitgenössischen Definitionen und erläutert den Hintergrund für die bis heute andauernde Aushandlung des Wirkungsbereiches von Künstlern und Handwerkern. Dabei kommt sie zu dem Schluss, dass es vor der Meiji-Restauration von 1868 keinen Kunstbegriff, sehr wohl aber eine weit gefasste Bezeichnung für Handwerk gab (Takeuchi 2004: 3). Bijutsu beschrieb in diesem zeitlichen Kontext vor allem die visuelle Kunst, während geijutsu eine Kategorie kennzeichnet, zu der auch Musik und Performance-Künste zählten. Dies spiegelt sich unter anderem in den Berufsbezeichnungen jener Zeit wider, wie etwa in dem der Geisha, was so viel wie „Person der Künste“ bedeutet (siehe Grimmig 2012). Bis ins 19. Jahrhundert ist mit Kunst vor allem der Fokus auf die technologische Produktion gemeint, der sich auch in den Begrifflichkeiten niederschlägt. Die Meisterschaft (gei) und Herstellungsweise ( $k o$ ) werden bis heute von Generation zu Generation weitergegeben. Diskussion und Kritik, heute ein wesentlicher Teil der Definition von Gegenwartskunst, bezog sich vor allem auf die Literatur und war auch terminologisch vom Handwerk getrennt (Takeuchi 2004: 3-4).

Den Paradigmenwechsel von Kunst als ästhetisches und durch Herstellungstraditionen gerahmtes Ausdrucksmittel hin zu einer mit gesellschaftskritischen Elementen versehenen Anschauung vollzog sich Takeuchi zufolge erst im späten, von sozio-politischen Umwälzungen geprägten 19. Jahrhundert: „It is no coincidence that the earliest surviving discourse on artistic production in Japan appeared also 
at a time when issues of emerging statehood and national identity drove the official rhetoric" (Takeuchi 2004: 8).

In dem Bestreben Japans, sich zu modernisieren, wurde das über Generationen weitergetragene Handwerk in dieser Zeit bewusst von ausländischen Kunsttheorien abgespalten und in einen stark von nationalen Interessen geprägten Diskurs integriert. Diese „traditionellen Künste“ sollten „[...] as proofs of Japan’s past greatness“ (Takeuchi 2004: 7) fungieren und den japanischen Staat in einem neuen internationalen Kontext verwurzeln. Satō (2011), Autor über die moderne Kunst Japans, beschreibt diesen Werdegang detailliert und geht dabei auf die in den 1870er Jahren entstandene Verflechtung von Politik und Kunst sowie deren Auswirkungen auf das Verständnis von „moderner Kunst“ ein. Die Auslotung der Nutzbarmachung von Kunst für politische Zwecke und die „Wiedergeburt“ traditioneller Kunst angesichts eines zunehmend spürbaren ausländischen Einflusses auf Japan eröffneten die Diskussion um kulturelle Authentizität und nationale Identität (Satō 2011: 3ff.). Der Grundstein für das Verhältnis von traditioneller Kunst auf der einen zur modernen Kunst auf der anderen Seite wurde von wissenschaftlicher Seite in dieser Zeit verortet.

Die Wirkungen dieser Trennung der Kunstbegriffe sind bis zum heutigen Tage deutlich wahrzunehmen: Im nationalen Interesse sollten traditionelle Künste vor allem durch staatliches Protektorat unter Schutz gestellt werden, während die Vertreter einer Gegenbewegung diese Künste für Innovation und Interpretierung öffnen wollten (Satō 2011: 54ff.). Die in diesem Spannungsfeld stehende interkulturelle Diskussion ermöglichte erst die Experimentierfreudigkeit der Gegenwartskunst und führte zu einer zunehmenden Politisierung des Kunstdiskurses selbst. Die daraus entstehende Hierarchisierung der verschiedenen Kunstgenres führte laut Satō (2011: 76) dazu, dass zwar der aufblühende internationale Kunstmarkt mit Gegenwartskunst versorgt wurde, aber dem traditionellen Kunsthandwerk zugeordnete Objekte bis zum heutigen Tage eine besondere Wertschätzung im In- und Ausland erfahren. Diese politische Aufladung schlug sich auch in den Begrifflichkeiten nieder und orientierte sich an dem entsprechend etablierten Wertesystemen: Bijutsu als Ausdruck einer innovativen visuellen Kunst wird dem westlichen Ethos zugerechnet. Als „traditionell“ verstandenes Handwerk wird hingegen als gigei bezeichnet (Satō 2011: 193). Bei der Verortung des geijutsu stimmt Satō mit Takeuchi überein und beschreibt sie ebenfalls als breiter aufgestellte Kategorie, die über die visuelle Kunst hinaus weitere Genres umfasste.

Um die historisch bedingte Wertzuschreibung an „traditionelle“ Kunst verständlich zu machen, beziehe ich mich auf den Sozialwissenschaftler Havens (1982). Er widmet sich der nach dem Zweiten Weltkrieg entstandenen Mittelschicht und den Auswirkungen dieser neuen Gesellschaftsschicht auf die Kunst, die vor dem Hintergrund einer zunehmenden Kommerzialisierung der 1950er Jahre zu überleben versuchte. Havens' in den 1980er Jahren veröffentlichtes Werk beschreibt einen Kontext der erneuten Auseinandersetzung mit der japanischen Nationalidentität nach dem Zweiten Weltkrieg, die zu einem Aufblühen der Kunstszene führte. Gerade 
in dieser Zeit des erneuten gesellschaftlichen Umbruchs hätten Künstler mit ihrem Ausdruck einem sozialen System gedient, das auf der Suche nach Antworten war, so Havens (1982: 16). Die intensive Auseinandersetzung mit zeitgenössischen Fragen und einem sich wandelnden Wertesystem verortet Havens in einer moralischen Diskussion, in der es immer auch um die gesellschaftliche Rahmung von zukünftigen Entscheidungen geht. Laut Havens veränderte sich der Status der Gegenwartskünstler dahingehend, dass Kunst nach 1945 in Japan zunehmend als wertvoll für die Gesellschaft eingestuft wurde: „[...] after World War Two, did most Japanese stop thinking of art as singing, dancing, acting, or painting and start regarding the work as something to be esteemed in itself" (Havens 1982: 30). In Havens' Analyse der finanziellen Manifestierung dieser Anerkennung spielt vor allem der Staat als Hauptgeldgeber seit der Meiji-Restauration im Jahr 1868 eine Rolle. Aber auch Aristokraten werden als entscheidende Akteure genannt, die jene Kunsthandwerker förderten, die seit Generationen in den Diensten ihrer Familien standen.

\subsection{Gegenwartskunst heute}

Hier wird ebenfalls deutlich, dass Havens' Analyse nur eine geringe Trennschärfe zwischen gegenwärtiger und traditioneller Kunst aufweist. Die Bedeutung von Kunst in seiner weitgreifenden Definition spiegelt sich dementsprechend ausschließlich in der ihr von staatlicher Seite zugedachten Rolle als ,national treasure " und des damit zusammenhängenden Prestiges im In- und Ausland wider (Havens 1982: 62). In Havens' Ausführungen klingt die unterschiedliche Behandlung beider Kunstwelten seitens der staatlichen Förderer nur schwach an, die sich innerhalb des Kulturministeriums manifestierte: Die Unterstützung kreativer Aktivität wuchs aufgrund geringer finanzieller Mittel am langsamsten (Havens 1982: 63).

Trotz dieser Kritik von meiner Seite finden sich in Havens Recherchen Aussagen, die ich auch für das Jahr 2015 für hochaktuell halte und die auch für meine Gesprächspartner gelten: „Only a few hundred artists, at best, make a living exclusively from selling their works. Nearly all those who are considered professionals have side jobs in teaching, commercial design, advertising, or the other commercial mass media" (Havens 1982: 6).

Statements wie diese werden unter anderem durch Takeuchi (2004) und Satō (2011) gestützt. Kreativität, die Havens' Aussagen zufolge in der Gegenwartskunst einen höheren Stellenwert einnimmt als in den sogenannten „traditionellen“ Künsten, war in der Nachkriegszeit ein wesentlicher Bestandteil der Argumentation nationaler Kräfte, die die traditionelle Kunst vor Interpretationen zu schützen versuchten: „The traditional Japanese arts rarely prized innovation for its own sake, and even today they carefully regulate who may alter a time-honored script, technique, or piece of choreography" (Havens 1982: 70). Kreativität spielt aus dieser Perspektive nur dann eine Rolle, wenn es darum geht, verschiedene, als dichotom wahrgenommene Welten zu überbrücken (im damaligen historischen Kontext zwischen Japan und 
dem „Westen“) oder zwischen Vergangenheit und Gegenwart zu vermitteln. Dies ist ein Anspruch, den die als „traditionell“ bezeichnete Kunst nicht an sich stellt.

Der Aspekt des individuellen Ausdrucks stellt insofern eine Herausforderung für Gegenwartskünstler der Nachkriegszeit dar, als dass sie sich damit von gültigen sozialen Normen und Standards entfernen. Mein Eindruck des Kunstmilieus ist der eines von der Regierung und großen Firmen stark beeinflussten öffentlichen Raumes, der nach wie vor wenig Freiraum für eigenständige Ausdrücke zulässt. Havens (1982: 20) beschreibt diesen Zustand als „bedrohlich“ oder gar „einschüchternd“ (menacing), der dazu führe, dass Künstler ständig Gefahr liefen, nur als Unterhalter wahrgenommen zu werden. Finanzstarke Akteure der Kunstszene, wie beispielsweise $\mathrm{Mu}$ seen, bildeten die Hauptkonsumenten von Kunstobjekten im Allgemeinen, während die Avantgarde vornehmlich auf eine kleine Gruppe privater Sammler angewiesen sei (Havens 1982: 42). Havens führt diese Umstände auf die enge Identifizierung von Öffentlichkeit und Staat zurück, wie ich sie in Kapitel 5.3.2 „Medien, die staatliche Öffentlichkeit und ihre Alternativen" dargestellt habe, und wie sie von vielen Japanologen ebenfalls bewertet wird (siehe Gebhardt 2015; Richter 2012).

Zusammenfassend ergibt sich ein Bild der Gegenwartskunst, das von hoher sozialer wie ökonomischer Unsicherheit, Verwundbarkeit und von einem ständigen Kampf geprägt ist. Havens (1982: 252) ergänzt diese Darstellung der Gegenwartskunst durch weitere wichtige Aspekte: Hohe Solidarität untereinander, eine starke Bindung an die eigene Familie, ein hoher moralischer Anspruch, der Gesellschaft zu dienen, und eine narrative Abkopplung von einer Fixierung auf monetäre Mittel. Die persönliche innere Stärke gegen die normativen Kräfte des gesellschaftlichen Drucks aufrecht zu erhalten und den eigenen Rollenansprüchen zu genügen, muss also von jedem Gegenwartskünstler immer wieder ausgehandelt werden. Der jeweilige Akteur bewegt sich damit im Spannungsfeld von gesellschaftlichem Konformitätsideal und der Diversität seiner persönlichen Eindrücke. Havens trifft Aussagen, die meiner Ansicht nach auch heute noch gelten: „The habit of avoiding confrontation and criticism is deeply embedded in Japanese social structure and reinforces the group solidarity that undergirds all the arts" (Havens 1982: 252).

Die tief verankerten Regeln und Normen sowohl im künstlerischen Ausdruck als auch auf gesamtgesellschaftlicher Ebene zu beugen, auszudehnen und schließlich zu transformieren, ist eine der schwierigsten Aufgaben japanischer Gegenwartskünstler seit der Einführung eines Kunstbegriffes im späten 19. Jahrhundert. „Innovation“ als gesellschaftlich inhärentes Element wurde erst Ende der 1970er Jahre in Japan populär. Grundsätzlich gilt auch heute noch, was Havens (1982: 14) bereits in seiner historisch ausgerichteten Analyse betonte: „The arts have blossomed to this degree since the mid-1950s because they serve the social system, not just the artist's need for expression" (Havens 1982: 14). Dies gilt in besonderem Maße für eine Zeit, in der, wie im Nachkriegsjapan der 1940er Jahre, nach Antworten auf aktuelle Problemstellungen gesucht wird. 



\section{Kogoro aus Tokyo - „Fukushima ist ein Röntgenbild der japanischen Gesellschaft"}

Mit meinem Interviewpartner Kogoro ${ }^{57}$ erhielt ich einen ersten Zugang zu einem biographischen Narrativ, das stark von den sozialen Folgen der Atomkatastrophe geprägt ist. Kogoros Aussagen stellen eine persönliche Antwort auf die Frage dar, wie die Emotionalität und Wahrnehmung der „Fukushima-Katastrophe“ in die Formulierung der Selbstkonstruktion integriert wird. Die in diesem Kapitel aufgeführten Themenkomplexe orientieren sich an seinem Narrativ und an den darin sichtbar werdenden Schwerpunkten. Zum Beispiel konzentriert sich Kogoro auf jene Aspekte der Katastrophendiskurse, die die atomare Verstrahlung betreffen, und weniger mit den Auswirkungen des Erdbebens und des Tsunamis. Seine Darstellungen veranschaulichen sowohl sein Selbstbild als Künstler als auch die identifikatorischen Schnittstellen zwischen seiner persönlichen Lebensgeschichte, der Situation der sogenannten „Atomflüchtlinge“ und der japanischen Gesellschaft.

In der folgenden biographischen Rekonstruktion arbeite ich seine persönlichen emotionalen Bewertungsmuster heraus, die eine enge Verbindung zwischen seinem Selbst-Konzept, der kulturellen Emotionsspezifik und seinen Erfahrungen des 11. März 2011 herstellen. Erst seine persönlichen Bewertungsmuster geben Aufschlüsse

\footnotetext{
${ }^{57}$ Die Namen aller meiner Gesprächspartner wurden geändert, um ihre Anonymität sicherzustellen. Des Weiteren habe ich mir erlaubt, sämtliche Informationen, die zu einer Identifizierung ihrer Person führen könnten, abzuändern.
} 
über Kogoros Leitmotive für die Integration der Ereignisse vom 11. März 2011 in sein Bild der japanischen Gesellschaft.

Im Laufe unserer 12-monatigen Zusammenarbeit gewährte mir Kogoro tiefe Einblicke in die unterschiedlichen Aspekte seines Lebens, die weit über seine Rolle als Künstler hinausgehen. Mein Übersetzer Satoshi war bei unseren Interviews nicht anwesend, da Kogoro es vorzog, keine dritte Person dabei zu haben. So fanden unsere Gespräche auf Englisch und zum Teil an öffentlichen Orten wie in Cafés statt. Das während meiner Zeit in Tokyo entstandene enge Vertrauensverhältnis erleichterte es Kogoro und mir, nach Beendigung meines Forschungsaufenthalts in Kontakt zu bleiben, so dass die Daten durch einen umfassenden schriftlichen Gesprächsverlauf aus sozialen Netzwerken bis 2017 ergänzt wurden.

\subsection{Kogoro, der Künstler}

Ich hatte bereits zwei Wochen nach meiner Ankunft in Tokyo von Kogoro über meine im Vorfeld etablierten lokalen Kontakte gehört und konnte zeitnah erste Vorgespräche führen. Diese ersten Annäherungen waren wichtig, um unseren beiderseitigen Vorstellungen in Bezug auf das zu führende narrative Interview entsprechenden Raum zu geben. Bereits in dieser Phase erwies sich Kogoro als sehr engagiert. Schon auf dem Weg zum vereinbarten Gesprächsort in der Nähe seiner Privatwohnung berichtete er mir von seinen derzeitigen künstlerischen Aktivitäten und seiner Arbeitsweise auf offener Straße. Er bezeichnete dies als Arbeit in „open space“: „A rapper doesn't come to the Kantei [government building], but to Shibuya, Shinjuku, Harajuku" (Kogoro 02.05.2014: 2). Kogoro nimmt hier Bezug auf die Szeneviertel Tokyos, die mit ihren zahlreichen Geschäften und Einkaufsmöglichkeiten entsprechend regelmäßig und in großer Zahl von den Bewohnern frequentiert werden. Die Präsentation seiner Werke auf offener Straße steht seinen eigenen Aussagen zufolge im scharfen Gegensatz zu einer Ausstellung in einer Galerie. Ausstellungsräume beschreibt Kogoro als „enclosed spaces“. Diesen Ausdruck der „geschlossenen Räume" wird er noch bei anderen Gelegenheiten benutzen, wobei er jeweils eine etwas andere emotionale Konnotation zeigt. Bei der Beschreibung einer Örtlichkeit zum Beispiel steht für ihn die Möglichkeit im Vordergrund, die Menschen inmitten ihres Tokyoter Alltagslebens auf seine Werke und die darauf abgebildeten Realitäten von Fukushima aufmerksam zu machen. Auf die besondere Bedeutung, die Kogoro den Örtlichkeiten, dem Raum und den damit verbundenen Konzepten zuweist, werde ich an anderer Stelle genauer eingehen.

Für den an dieser Stelle wichtigen ersten Zugang zu Kogoros Realitätsbeschreibung ist es essentieller, sich seinem Selbstverständnis als Künstler anzunähern. Dieses ist zu großen Anteilen mit seinem Wohn- und Geburtsort Tokyo verbunden. Seine Beobachtungen in Tokyos Machtzentrum, in diesem Fall dem Regierungsgebäude, lassen ihn direkte Konsequenzen für sein eigenes Handeln und seine Tätigkeit als Künstler ziehen. 
„Tokyos people are losing interest for ,Fukushima' day by day. But the process is going on. For example, it has been difficult to find those who know anything about the nuke workers' payment. [...] Talking to them, many people are surprised to find out about these problems." (Kogoro 16.01.2015: 23)

In den Gesprächen mit Kogoro wurde mir bewusst, dass bei der Analyse der Katastrophendiskurse um den 11. März 2011 zwischen Argumentationssträngen unterschieden werden muss, die stark lokal geprägt sind. Da Tokyo 2,5 Zugstunden von Fukushima entfernt liegt, ist die Tokyoter Bevölkerung auf Informationen angewiesen, um die Lage vor Ort einschätzen zu können. ${ }^{58}$ Die Beurteilung der Lage durch einen Tokyoter Anwohner kann also massiv von der eines lokalen Anwohners abweichen. Dabei spielen die Machtverhältnisse zwischen Tokyo und Fukushima eine wichtige Rolle, wie ich im Folgenden ausführen werde.

\subsubsection{Die lokale Verortung der Katastrophendiskurse}

Die verschiedenen Realitätswahrnehmungen, die Kogoro im obrigen Zitat beschreibt, manifestieren sich zwischen Tokyo als sozio-ökonomischem Machtzentrum und der landwirtschaftlich geprägten Präfektur Fukushima, die mit ihren Kernkraftwerken seit der Etablierung der Atomenergie in Japan den Strom für die Hauptstadt mit ihren 30 Millionen Einwohnern produziert.

Dass sich insbesondere nach dem 11. März 2011 Spannungen zwischen den Gebieten Tokyo und Fukushima entfalten, sieht Kogoro in der Komplexität der Faktenlage rund um die Strahlenwerte begründet. „Maybe, too many people are affected by this problem by now and it is too complicated for them" (Kogoro 16.01.2015: 23). „People live in different realities. They [population of Tokyo] understand the structure problem now, but they cannot imagine the reality of the people of Fukushima“ (Kogoro 02.05.2014: 13). Wenn Kogoro hier von „Realitäten“ spricht, bezieht er sich auf seine Erfahrungen, die er als Reisender zwischen Tokyo und Fukushima macht. Er erhält in Tokyo Informationen, die er in seinen Gesprächen mit der lokalen Bevölkerung Fukushimas überprüft. „The Japanese want to believe that 3.11 is over. But in reality, there are still people living in temporary houses who are committing suicide. [...] Against all this hope [that 3.11 is over] there is this reality" (Kogoro 02.05.2014: 6).

Kogoro machte mir zu diesem Zeitpunkt der Forschung bewusst, wie stark eine Perspektive auf die Katastrophendiskurse durch Lokalität geprägt ist und wie sich dies auf den emotionalen Umgang mit den Ereignissen auswirkt. In den folgenden Kapiteln gehe ich genauer darauf ein.

Des Weiteren beschreibt Korogo die Anforderungen des Tokyoter Alltags und den damit einhergehenden Konsequenzen als wichtigen Faktor für das schwindende Interesse der Tokyoter am Fortlauf der Katastrophe: „For example, people [from

\footnotetext{
58 Siehe Kapitel 5.3.2 „Medien, die staatliche Öffentlichkeit und ihre Alternativen“.
} 
Tokyo] are too busy to go to Fukushima themselves [...] The long working hours are one reason for that" (Kogoro 16.01.2015: 23). Ein Tokyoter Angestellter hat laut Kogoro kaum eine Möglichkeit, die ihm zugetragenen Informationen zu überprüfen und sich ein persönliches Bild von der Lage zu machen. Dies sind wichtige Hinweise auf die Handlungsmöglichkeiten des Großteils der japanischen Bevölkerung. Kogoros Aussagen beziehen sich nicht nur auf Tokyos Einwohnerschaft allein, sondern spiegeln eine Alltagsrealität für Japans Vollzeitbeschäftigte wider. In Aussagen wie diesen zeigt Kogoro eine „Gleichzeitigkeit der Perspektiven“, die ich in Kapitel 4.1 „Der kulturspezifische Ausdruck des Selbst im sozialen Gefüge“ dargestellt habe. Anstatt die Menschen anzuklagen, die so mit ihrem Arbeitsalltag beschäftigt sind, erläutert Kogoro deren Situation. Indem er die langen Arbeitszeiten erwähnt, zeigt er Verständnis und vermeidet eine Verurteilung beziehungsweise starke emotionale Verletzung jener Personen. Er wahrt auf diese Weise ein harmonisches Gesamtbild. An anderer Stelle wiederum wird er trotzdem seinen eigenen Standpunkt deutlich machen.

\subsubsection{Die Aufgabe der Kunst in Krisenzeiten}

Kogoro beschäftigt sich mit der Gruppe der sogenannten „Atomflüchtlinge“, im Speziellen mit Müttern und Kindern, die entweder evakuiert wurden, auf eigene Kosten flohen oder nach wie vor in Gebieten mit umstrittenen Strahlenwerten leben. Es ist die Beschäftigung mit den sozialen Folgen der hohen Strahlenwerte, die ihn aufgrund der damit verbundenen hohen Emotionalität zu der Definition dessen führen, was Kunst für ihn ausmacht. Diese enge Verbindung zwischen beruflicher Beschäftigung mit einem Thema und dem persönlichen Bedürfnis Kogoros, sich auszutauschen, setzte starke Akzente in unserer gemeinsamen Arbeit. Er offenbarte in unseren Gesprächen seinen Willen, die Perspektive der „Atomflüchtlinge“ nachvollziehen und verstehen zu können. Er tat dies nicht nur, um Informationen zu überprüfen, die er aus den Medien erhielt, sondern auch, um die Gefühle der Mütter und Kinder der Region nachempfinden und in seine Kunst übersetzen zu können. Diese Gefühle sollten für andere Mitglieder der Gesellschaft transparent gemacht werden. Kogoro zeigt große Empathie mit seinen eigenen Gesprächspartnern vor Ort und vermittelt mir den Eindruck, dass deren Geschichten ihn sehr bewegen. Umso wichtiger ist ihm, diese Wahrnehmung auch vermitteln zu können.

Kogoro definiert seine Kunst wie folgt: „My paint is a communication tool. To help the communication among people, I need to go out of these closed spaces onto the street“ (02.05.2014: 11). Erneut nimmt er Bezug auf die „closed spaces“. In diesem Fall beschreibt er kulturelle Einrichtungen wie Galerien, die durch ihre zum Teil hohen Eintrittspreise in Kogoros Augen eine Hürde für das Publikum darstellen, sich die Werke anzusehen. Das ist für ihn der Grund, seine Werke auf der Straße auszustellen: „Young people seem to have little interest in anything. But they have an interest in art. So I show my paintings on the big streets where many people are passing by. So they come close and we can talk" (Kogoro 02.05.2014: 7). Noch ent- 
scheidender ist jedoch seine Aussage über die Kunst selbst. Dabei fächert sich seine Rolle vom Erschaffer des Werkes weiter auf und offenbart die verschiedenen Facetten seiner Tätigkeit.

Der erste, womöglich offensichtlichste Aspekt seiner Arbeit, ist das Sammeln, Hinterfragen und Verarbeiten von Informationen rund um den 11. März 2011. Indem er sich nicht auf die mediale Aufbereitung der Fakten verlässt, sondern seinen eigenen Gedanken nachgeht und vor Ort recherchiert, beginnt er die Abläufe Stück für Stück zu rekonstruieren und zu dokumentieren. „I live in the present but I create the past with my painting" (Kogoro 09.02.2015: 24). Kogoros intensive Auseinandersetzung mit den Themen der Katastrophe zeugt von seinem starken Bemühen um das Verstehen von Informationen in der Auseinandersetzung mit den Geschichten der Menschen vor Ort. Dabei werden seine Aussagen meist von starken Emotionen begleitet, auch wenn er sie nicht direkt anspricht. Besonders deutlich wurde dies, wenn Kogoro kurz vor einem erneuten Zusammentreffen mit mir von einer längeren Reise in die Präfektur Fukushima zurückgekehrt war und mir von seinen Eindrücken berichtete. So auch im Mai 2014, als er Menschen aus Koriyama in einer Notunterkunft besuchte. Koriyama ist ein kleiner Ort, der ca. $75 \mathrm{~km}$ vom AKW entfernt gelegen und unmittelbar von hohen Strahlenwerten betroffen ist. Bei dieser ersten Gelegenheit nach seiner Rückkehr berichtete er mir von dem sich etablierenden Alltag vor Ort:

„The people living there are asking the chairman 'Why did you allow these people to come here?' They [the people of Koriyama city] are isolated. They chose to stay among themselves. People have nothing to do. They had to give up their way of life, the place where they were born and grew up. The places they worked. So it is difficult for them to do something else."

(Kogoro 02.05.2014: 4)

In wenigen Sätzen zeichnet Kogoro hier ein intensives Stimmungsbild von der psychischen Lage der Menschen, die ihren Geburtsort aufgrund der hohen Strahlenwerte verlassen mussten. Wie ich mich im September 2014 persönlich überzeugen konnte, versammeln sich in den Notunterkünften Menschen verschiedenen Alters, die jede Orientierung im Leben verloren zu haben scheinen. Es sind wichtige persönliche Netzwerke weggebrochen, weil die entsprechenden Personen tot oder weggezogen sind oder sich in einer weit entfernten Unterkunft befinden. Jede Form von Sicherheit als Basis für ein geregeltes Leben scheint nicht mehr zu existieren.

Kogoro umreißt in unseren Gesprächen nicht nur die hinzukommende Stigmatisierung der Evakuierten, die sie auch dann betrifft, wenn sie in andere Teile des Landes gezogen sind. Er vermittelt mir zusätzlich einen intensiven Eindruck seiner persönlichen Emotionen beim Erzählen dieser Geschichten. So wird beispielsweise seine Stimme zunehmend leiser und er senkt den Blick als er von den Stigmatisierungen der Evakuierten berichtet. Diese Eindrücke scheinen ihn im wahrsten Sinne des Wortes zu bedrücken. 
Kogoro vermittelte mir im Laufe der Gespräche, dass sich seiner Meinung nach die Stigmatisierung der Betroffenen aus der Unsicherheit und Verwirrung der Bevölkerung angesichts widersprüchlicher Expertenaussagen zu Strahlengrenzwerten speist. Gestützt werden seine Aussagen durch meine Rechercheergebnisse. So berichtete die UNESCEAR, das unabhängige Untersuchungsgremium der Vereinten Nationen, im Jahr 2014 auf einer öffentlichen Veranstaltung in Tokyo von geringeren Strahlenwerten als beim Reaktorunfall in Tschernobyl 1986 (siehe auch UNSCEAR 2015: 4). Dieser Bericht ist unter anderem deshalb von Bedeutung, weil im Fall atomarer Strahlung die Informationsvermittlung zwischen Wissenschaft und Öffentlichkeit von entscheidender Bedeutung ist. Da die Eigenschaften der atomaren Strahlung nicht mit menschlichen Sinnen erfahrbar ist, sind diese Informationen umso wichtiger. Zudem müssen vorhandene Informationen für den Laien verständlich aufbereitet werden. Ohne dieses Verständnis bleibt den Menschen nur ihre eigene Einschätzung ihres persönlichen Risikos - und mündet im Zweifelsfall in eine beobachtbare Ablehnung betroffener Personengruppen.

Lokale Akteure wiesen bei der Veranstaltung auf die psychologischen Folgen hin, wie beispielsweise die entstandene Unsicherheit und die sich daraus generierende lähmende Wirkung auf ganze Bevölkerungsteile. Von Expertenseite werde diesen Aspekten der Gesundheit vergleichsweise wenig Bedeutung beigemessen, hieß es vor Ort. Damit wird deutlich, wie stark selbst auf einer internationalen Fachkonferenz die Realitätsvorstellungen der Lage vor Ort auseinanderdriften. Hier verorte ich die hohe soziale Sprengkraft der Dateninterpretation. ${ }^{59}$

Kogoro fokussiert sich in den ersten Schritten seines Zugangs zu den Menschen vor Ort nicht auf eine „allgemeingültige“ Aussagekraft der gemessenen Strahlendaten, sondern ausschließlich auf die lokalen Interpretationen dieser Werte in Fukushima. Anders als bei den Opfern des Tsunamis drehen sich Kogoros Berichte weniger um Verstorbene infolge einer Naturkatastrophe, sondern um die Opfer der Folgen der Evakuierung, des Verlustes der Heimat und der sozialen Netzwerke: „[...] almost all old people say they want to return to their homes. But when they did, they found their houses in the restricted areas are no more. These people in contemporary houses are heartbroken. [...] So the cases of suicide are increasing" (Kogoro 02.05.2014: 4-5). ${ }^{60}$ Kogoros Körperhaltung verändert sich in diesen Gesprächsabschnitten. Er richtet sich auf und sein Blick wird eindringlich. Im Gespräch deute ich dies so, dass er mir die verzweifelte Situation der Menschen nachdrücklich näherbringen möchte.

Es überrascht mich daher nicht, dass Berichte wie diese, die von einem starken Gefühl des Verlustes einer bisher gekannten Normalität und der eigenen Wurzeln zeugen, Kogoros derzeitige Tätigkeit als Künstler prägen. Während der ersten Arbeitsphase mit mir nahm er keinen Bezug auf Informationen, die von den Medien

\footnotetext{
59 Siehe Kapitel 5.1.1 „Gesundheitliche Folgen der Verstrahlung und das Ringen um die Wahrheit“.

${ }^{60}$ Aktueller Artikel zu dieser Thematik siehe Dahl (2020): „Governing through kodokushi. Japan’s lonely deaths and their impact on community self-government“"
} 
verbreitet wurden, sondern setzte den Fokus auf den unmittelbaren Kontakt mit den Betroffenen. „People in contemporary houses are so isolated. [...] I started to talk to them. [...] But I felt a need to know about this kind of stories and to tell them. So I want to go to Fukushima and I want to add their stories to my paintings" (Kogoro 02.05.2014: 6).

Die Emotionalität des intensiven Kontakts mit Betroffenen überträgt sich unmittelbar auf Kogoro. Zurück in seiner Wohnung in Tokyo sind es Kogoros eigener Aussage zufolge die lokal gesammelten emotionalen Eindrücke, die ihn motivieren weiter zu machen. „Just to use words is difficult. So I created my paintings as a way to communicate everything that people told me" (Kogoro 02.05.2014: 7). Dabei versteht er sein Tun unter anderem als Dienst an den Menschen, die ihm ihre Geschichte anvertrauten. Die Möglichkeiten des Ausdrucks von Unaussprechbarem prägt die zweite Facette seiner Rolle als Künstler.

„I give a voice to voiceless people. For example, if someone dies, let's say a worker suffers a heart attack. He can no longer speak for himself. That's what I do with my art. It is almost like religion. Like in Buddhism when a priest speaks with a spirit. Maybe it is a bit strange, but I am speaking for others. I show the grief of people and it makes me feel sad too."

(Kogoro 02.05.2014: 24)

In Kogoros Narrativ werden Verstummte oder aus anderen Gründen ungehörte Personen mithilfe seiner Vorstellungskraft wieder sichtbar. Sein Selbstverständnis in der Rolle des Künstlers sieht vor, diese Stimmen in andere Bereiche des Landes, beispielsweise nach Tokyo, zu transportieren und damit eine große Lücke („big gap“) in den Realitätsvorstellungen zu schließen. Die Unterschiede in den Realitätsvorstellungen basieren nicht nur auf unterschiedlicher Informationslage, sondern auch auf den damit zusammenhängenden emotionalen Bewertungen: Stetige Information, transportiert durch emotionale Ansprache, soll die Schicksale der Menschen vor Ort transparent machen und im Bewusstsein halten.

Vor dem Hintergrund der Katastrophe von 2011 ist es des Weiteren wichtig darauf hinzuweisen, dass im japanischen Verständnis die Grenze zwischen der Welt der Lebenden und der Toten durchlässig ist: Die Geister der Verstorbenen können Einfluss auf die Lebenden ausüben und Unterstützung sein oder Schaden anrichten: "There is not only separation (from the dead), but also integration, and the wellbeing of the living is intimately interwoven with that of the departed" (Formanek and LaFleur 2004: 35-36). Die Seelen (jap.: tamashii/ jinkon) treten bei rituellen Anlässen in Kontakt mit den Lebenden und müssen von ihnen besänftigt werden (vgl. Rotermund 2004: 369). Durch die Integration der Toten in den Alltag werden Leiden, gewaltsame Tode und negative Emotionen gereinigt und geheilt, so dass die Lebenden auf den Schutz und Fürsprache ihrer Verstorbenen bauen können statt ihre Wut zu fürchten. Dies ist ein wichtiger Aspekt der Emotionsarbeit von Künstlern wie Kogoro, der Empathie und Mitgefühl als Möglichkeit beschreibt, für andere 
zu sprechen. ${ }^{61}$ „My paint has an aspect which is aimed at giving response to these souls which exist outside of everyone's sight" (Kogoro 09.02.2015: 28). Das einzelne Werk fungiert damit sowohl als Transportmittel als auch als Auslöser von Emotionen im Rezipienten. Der Betrachter der Bilder wird dazu angehalten, sich mit der emotionalen Resonanz, die in ihm erzeugt wird, auseinanderzusetzen.

So konnte ich bei meiner teilnehmenden Beobachtung im Mai 2014 einige dieser Reaktionen der Passanten auf Kogoros Bilder miterleben. Kogoro hatte seine Bilder an einem Ausgang einer großen Bahnstation aufgestellt. Einige Passanten blieben stehen und fragten beispielsweise, warum dieses Bild dort stehe und was er [Kogoro] damit bezwecke. Auf seine Erklärung hin folgten Reaktionen des Unglaubens („Was soll das bringen?“) oder der Ermutigung („Mach weiter!“). Kogoro berichtete mir aber auch von Tagen, an denen er von der Polizei gebeten wurde, den Platz zu räumen.

Kogoro tritt bei seinen Ausstellungen auf offener Straße zum einen in der Rolle des Erschaffers der Werke besonders deutlich in den Vordergrund. Zum anderen verfolgt er seinen Aussagen zu Folge die Intention, mit seinen Bildern eine Antwort auf den Wunsch der Ungehörten zu geben, die ihren Gefühlen Ausdruck verleihen wollen:

„I wish to create something. I am giving birth to something. I believe people are driven by emotion. In Japan, it is normal to control emotion, to be rational. But human beings are animals too. [...] Of course, it is important to be rational but we still have emotions. So I believe social change is based on emotion. So that's what only art can do: To make people feel for an issue that is distanced from themselves." (Kogoro 02.05.2014: 25)

Die enge Verbindung zwischen Emotionen und Handeln entfaltet Kogoro hier auf zwei Ebenen. Zum einen sind es die Emotionen in ihm selbst, die ihn dazu befähigen, etwas Neues zu erschaffen. Er kontrastiert in seinen Aussagen auf subtile Weise seinen persönlichen emotionalen Umgang mit der kulturspezifschen Handhabung von Emotionen. Aus seiner Überzeugung heraus, dass Veränderungen auf Emotionen basieren, stellt er dem kulturspezifischen Verschweigen oder Vermeiden von emotionalen Auslösern das bewusste Wahrnehmen von Emotionen gegenüber. Kogoro räumt damit den Emotionen eine wichtige Rolle bei der Entstehung von Realitäten ein. Zum anderen setzt er auf diese Weise einen entscheidenden Fokus in seiner Arbeit, die eine Sensibilisierung für die sozialen Folgen der Verstrahlung auf gesamtgesellschaftlicher Ebene zum Ziel hat. Durch das Ansprechen der Emotionen

\footnotetext{
${ }^{61}$ An dieser Stelle möchte ich Frau Prof. Dr. Katja Triplett herzlich für ihre Unterstützung und die zahlreichen Literaturhinweise danken, die diese Arbeit bereichert haben. Sie hat unter anderem gemeinsam mit Michael Pye den Band Streben nach Glück: Schicksalsdeutung und Lebensgestaltung in japanischen Religionen herausgegeben (Pye und Triplett 2007).
} 
wird dem Rezipienten vor Augen geführt, dass er auf gesellschaftlicher Ebene ebenfalls von diesen Schicksalen betroffen ist.

Kogoros Perspektive auf Emotionen zieht sich durch sein gesamtes lebensgeschichtliches Narrativ. Aus analytischer Sicht untermauert dies die ethnopsychologische Perspektive, wonach Emotionen als starke Kraft für konkretes Handeln fungieren. ${ }^{62}$ Als Vermittler von Emotionen, welche in seinen Werken sowohl dargestellt als auch im Publikum erzeugt werden, kreiert Kogoro einen Raum für kontroverse gesellschaftliche Themen, die sonst nur wenig Raum im Diskurs finden. Dieser Raum ist geprägt von einer Transparenz, die sich sowohl auf konkrete Informationen als auch auf Emotionen bezieht.

Die Menschen „dazu zu bringen, etwas zu empfinden“ begreift Kogoro als eine einzig der Kunst vorbehaltene Möglichkeit der emotionalen Ansprache und „Aktivierung" der Menschen, sich mit Themen auseinanderzusetzen, die sie nur scheinbar nicht unmittelbar betreffen. Wenn er hier von „distanced issues“ spricht, so halte ich es für gesichert anzunehmen, dass es ihm nicht nur um die geographische Distanz, sondern auch um die angesprochene Distanz der verschiedenen lokalen Realitäten geht, die mithilfe seiner Werke überbrückt werden kann. Bevor ich darauf näher eingehe, möchte ich jedoch Kogoros enge Verbindung zwischen seiner Tätigkeit und seinem Selbst weiter ausführen.

\subsubsection{Authentizität als emotionaler Anker}

In seiner Aufgabenbeschreibung der Kunst artikuliert Kogoro die enge Verbindung zwischen seiner beruflichen Tätigkeit und ihm selbst. In folgenden Aussagen geht er jedoch noch einen Schritt weiter: „My painting is a part of myself that will exist even after I died. That is a huge motivation for me" (Kogoro 02.05.2014: 24). Die Basis seiner Identität als Person und Künstler wiederum stützt sich seinem Narrativ zufolge auf Empfindungen und Annahmen über seine gesellschaftliche Position, in der er sich - und Gegenwartskünstler im Allgemeinen - als „Außenseiter“ beschreibt: „As an artist, we don't have a place in society. We create the place through our existence and work" (Kogoro 09.02.2015: 26). Hinter der Form, wie er mit seinen eigenen Empfindungen und denen anderer Personen umgeht, sie in seinen Werken verarbeitet und sie in die Gesellschaft zurückspiegelt, verbirgt sich eine Funktion von Kunst, die das Fortbestehen persönlicher Botschaften auch über den Tod des Künstlers hinaus sicherstellt. Ein Teil von Kogoros Selbst ist in diesen Artikulationen enthalten und verleiht sowohl seiner eigenen Stimme als auch seinen Emotionen eine Form von Unsterblichkeit.

In seiner Rolle als Künstler kann Kogoro seine persönlichen Emotionen in den von ihm geschaffenen Diskursraum hineingeben. Wie ich während meiner teilnehmenden Beobachtungen an der Bahnstation Shinjuku im Zentrum Tokyos erleben konnte, nutzt Kogoro seine Bilder, um seine eigene Wahrnehmung der Situation in

${ }^{62}$ Siehe Kapitel 3.3 „Emotionsforschung“. 
Fukushima plakativ zu untermauern. Dabei geht er aktiv auf die Passanten zu, erklärt ihnen die Symbole der Bilder, beantwortet Fragen zur Situation der Menschen vor Ort und erfährt im Gegenzug auch einiges über die Zweifel und Beweggründe der Bewohner Tokyos.

Von der Wichtigkeit, die er dem Dialog mit den Vorübereilenden zuschreibt, zeugt seine Überlegung, Erklärungen zu seinen Bildern einfach auf die Ränder seiner Werke zu kleben. „I thought about using labels which could explain the symbolism, but I decided not to do it. The message would be poisoned. I am here to talk to people“ (Kogoro 02.05.2014: 3). Tatsächlich gelang es ihm bei der von mir beobachteten Gelegenheit jedoch nur im Falle eines kanadischen Pärchens, einen Meinungsund Informationsaustausch in Gang zu bringen. Die meisten japanischen Passanten liefen nach kurzer Zeit weiter ohne lange mit ihm im Gespräch gewesen zu sein. Dennoch bemühte er sich um einen lang anhaltenden Dialog, in dem Emotionen aller Beteiligten ihren Raum finden und ein Austausch der Perspektiven stattfinden soll - wichtige Aspekte der Definition von Empathie nach Hollan and Throop (2011). ${ }^{63}$

Sein empathisches Vorgehen könnte der Grund dafür sein, dass Kogoro Energie aus diesen Begegnungen zieht. Sie scheinen ihm das Gefühl zu geben, im Bewusstsein der Menschen wirksam zu sein. „That my paint is known by some people and that they start to feel something, that is my pleasure" (Kogoro 09.0.2015: 26). Als er dies sagt, lächelt er zum ersten Mal breit und wirkt zufrieden mit sich.

Dieser Teil seiner Arbeit, der sich nicht mehr auf das Erschaffen des Werkes selbst bezieht, wird zu einer sinn- und identitätsstiftenden Tätigkeit. Die Freude, die er dabei empfindet, wenn er in anderen Menschen Gefühle auslöst, motivieren ihn zusätzlich. Dadurch, dass Kogoro seiner Kunst eine Bedeutung zuschreibt, die auch seine Identität als Künstler einschließt, stellt daher eine Aufwertung seines Status als „Außenseiter" dar. Wie bedeutsam wiederum die Zuschreibung einer Außenseiterrolle für Kogoro und andere Künstler ist, zeigte sich erst im Laufe der biographischen Analyse. ${ }^{64}$

Ich möchte an dieser Stelle jedoch darauf hinweisen, dass seine teils leidvollen Erfahrungen, seine Emotionalität in den verschiedenen Lebensphasen und die darauf basierenden Entscheidungen durch die Außenseiterrolle eine positive Konnotation erhalten. Den verschiedenen Facetten seiner persönlichen Lebensgeschichte, die in der Auseinandersetzung mit seiner Rolle als Künstler bereits latent aufgetaucht sind, werde ich im Folgenden genauer nachspüren. Dabei werde ich verdeutlichen, wie stark sich seine lebensgeschichtlichen Erfahrungen in seiner Tätigkeit als Künstler niederschlagen.

\footnotetext{
${ }^{63}$ Siehe Kapitel 3.3.4 „Empathie - wissenschaftliche Perspektiven auf den ,social glue““.

${ }^{64}$ Siehe dazu Kapitel 9.2.3 „Widerstand gegen gesellschaftliche Konventionen“.
} 


\subsection{Eine Biographie der Emotionen}

Bevor ich mich den biographischen Themen zuwende, möchte ich hier einige Informationen zur Verfügung stellen, die Kogoros Aussagen nicht nur in kulturspezifischer Weise, sondern auch in historischer Hinsicht kontextualisieren.

Kogoro wurde 1976 in Tokyo geboren und war zum Zeitpunkt des Interviews im Jahr 2014 damit 39 Jahre alt. Er gehört einer Generation an, die die gesellschaftlichen Akzentuierungen der „Babyboomgeneration“ ihrer Eltern mitzutragen hatten. Der Japanologe Coulmas (2007: 36) beschreibt den prägenden Einfluss dieser Generation auf die japanische Gesellschaft. Aufgrund der starken Erhöhung der Geburtenrate um ca. 30 bis 50 Prozent in den Jahren zwischen 1947 und 1949 hat die damit einhergehende soziale Erfahrung zur Ausbildung einer gemeinsamen Identität geführt, „,...] deren vielfältige psychologische und soziale Folgen für ihre Sozialisation, ihre schulische Erziehung, ihren Einfluss auf Konjunkturzyklen und ihre Anforderungen an das Sozialsystem hat" (Coulmas 2007: 35). So ist die Elterngeneration von Kogoro die letzte, die sowohl das Ideal der Firmenloyalität als auch das der lebenslangen Beschäftigung ausleben konnte und bis Anfang der 2000er Jahre einen Großteil der erwerbstätigen Bevölkerung ausmachte (Coulmas 2007: 37). Diese Elterngeneration orientiert sich an der sogenannten "reference group“, basierend auf Geburtsort, Arbeitsplatz, Alter etc. (Lebra 1976: 22) und favorisiert Harmonie in Beziehungen sehr stark. ${ }^{65}$

Kogoro hat von seinen Eltern einen Wertekodex vorgelebt bekommen, der aufgrund gesetzlicher und kultureller Normen sowie der anders gelagerten wirtschaftlichen Rahmenbedingungen (siehe Coulmas 2007: 42) für seine Generation nicht mehr ohne Weiteres umzusetzen ist. Nach Kogoros eigener Aussage war es für ihn aufgrund dieses fest etablierten Wertesystems umso schwerer, mit den sozialen Folgen der Abweichung von diesen Vorstellungen umgehen zu lernen. Seine Artikulation des Gefühls „nicht wie alle anderen zu sein“ verweist direkt auf ein zentrales Thema seines lebensgeschichtlichen Narrativs.

\subsubsection{Isolation und Einsamkeit}

Dem fest verankerten hohen Stellenwert des Zusammenseins und -lebens in Japans Gesellschaft steht ein Gefühl gegenüber, das in den auf die „Babyboomgeneration“ folgenden Jahrgängen um sich gegriffen hat und das auch in Kogoros Artikulationen immer wieder Anklang findet. Einsamkeit (jap.: sabishii) und Isolation kristallisierten sich zu Leitthemen seiner Biographie heraus. „Many times in my life I felt isolated“ (Kogoro 02.05.2014: 27). Wenn wir bei diesen Phasen aus Kogoros Leben ankamen, wirkte er zunächst sehr zurückhaltend. Er schien meine Reaktionen aus dem Augenwinkel heraus zu beobachten und genau abzuwägen, was er mir erzählen wollte. Erst

${ }^{65}$ Siehe Kapitel 4.1 „Der kulturelle Ausdruck des Selbst im sozialen Gefüge“. 
im Laufe der Zeit baute er so viel Vertrauen zu mir auf, dass er ausführlicher auf diese für ihn schmerzhaften Abschnitte seines Lebens zu sprechen kam.

Kogoros Einsamkeitsgefühl beruht auf Erfahrungen in den unterschiedlichen Kontexten von Schule, dem Erleben des familiären Zusammenlebens und einigen Wohnortwechseln, die aufgrund der Wichtigkeit der „reference group“ in Japan von besonderer Bedeutung für das Verständnis seines prägenden Lebensgefühls sind. Für Kogoro gab es keine Zugehörigkeit aufgrund seines Geburtsortes wie für seine Klassenkameraden, da sein Vater noch vor Kogoros Eintritt in den Kindergarten von Tokyo in eine abgelegene Gegend der Präfektur Fukushima versetzt wurde. Damit ging der Bezug zu seinem Geburtsort zunächst verloren, was sich, wie ich später zeigen werde, stark auf Kogoros Erleben von Zugehörigkeit auswirkte.

Zunächst jedoch lebte die Familie in der Präfektur Fukushima. Die Lage der Region beschreibt Kogoro als isoliert und greift damit auf ein prägendes Stimmungsbild in dieser Zeit zurück. Die von Landwirtschaft abhängige Gegend hält für junge Menschen nicht viele Verdienstmöglichkeiten bereit und viele entschließen sich nach ihrer Ausbildung, nicht zurückzukehren.

Kogoro machte bereits im Kindergarten Bekanntschaft mit den abweichenden Konventionen zwischen der Region Fukushima und der Hauptstadt Tokyo. „I had long hair, so my hairstyle was different. I had a different kind of accent as well, so I experienced terrible abuse. I could do nothing. I can change my clothes but the cultural difference remains" (Kogoro 02.05.2014: 23). Kogoro beschreibt rückblickend ein erstes Gefühl des Andersseins in diesen jungen Jahren. Interessanterweise unterscheidet er zwischen seinem äußeren Erscheinungsbild, das der augenscheinlichste Marker für abweichende Konventionen war, und dem ihm zugrunde liegenden kulturellen Konzept, das sich nicht so schnell anpassen ließ. Der Wohnortswechsel hatte ihn zu einem Fremden in einer bestehenden „reference group“ von lokalen Schülern gemacht. Für diesen Konflikt gab es keine Lösung - eine Form von Harmonie ließ sich unter diesen Umständen nicht durch Schweigen und Vermeidung herstellen. „I had to defend myself, so sometimes I got violent. I had to hit them to put an end to that" (Kogoro 02.05.2014: 23). Bei dieser Aussage lacht er leise als wollte er das eben Gesagte abschwächen. Für mich unterstreicht er damit nur die Hilflosigkeit, die er in der damaligen Situation empfand - zumal er darüber hinaus eine gleichzeitig einsetzende Spaltung seines „Ichs“ beschreibt:

„I only got more balance when I made a difference between the behavior being at home and being outside. In Japanese, we have different terms for selfreference. In my home, I was using 'boku' which represents being very calm, being part of something. Outside I used 'ore' which symbolizes being more wild." (Kogoro 02.05.2014: 23)

Diese Aussage unterstreicht in besonders markanter Weise seine Anpassungsversuche an veränderte Bedingungen seines Umfeldes. Kogoro verinnerlichte gewissermaßen den Konflikt und versuchte, auf diese Weise eine Lösung zu finden: Da er aufgrund 
regionaler Unterschiede nicht von seinen Mitschülern akzeptiert wurde, bestand seine Lösung des Konflikts in einer Spaltung seines Selbst, versehen mit unterschiedlichen Verhaltenscharakteristika. Das innere Empfinden und sein Auftreten außerhalb seines Elternhauses beschreibt Kogoro als dementsprechend polarisiert. „My behavior at home and in school was very different. There was a huge gap between the two" (Kogoro 02.05.2014: 23) ${ }^{66}$ Kogoro spricht ohne lange Pausen und ohne lange nachzudenken von dieser Zeit. Er scheint sich oft Gedanken über diese Zeit gemacht zu haben. Seine Selbstbeschreibung ändert sich auch mit Blick auf seine Rückkehr in seine Geburtsstadt Tokyo in seiner Highschoolzeit nicht. Nun sei er so lange dem städtischen Kontext fern gewesen, dass man ihn erneut als „nicht von hier" wahrgenommen habe. „Tokyo is my birthplace but the teacher and my fellow students looked at me as one who comes from the countryside" (Kogoro 02.05.2014: 24). Seine Kindheitserzählung schließt mit der Bemerkung ab, dass die Entscheidungen der Erwachsenen immer unmittelbare Auswirkungen auf ihre Kinder hätten.

In Anbetracht dieser, von stetiger Entwurzelung geprägten Kindheitserinnerungen, wird aus meiner Sicht nachvollziehbar, warum sich Kogoro im Jahr 2014 so stark für Mütter und Kinder in den Evakuierungszonen Fukushimas einsetzt. Kogoros Einsamkeit hat ihren Ursprung sowohl in einer geographischen Entwurzelung, die ihn ohne Heimatgefühl aufwachsen ließ als auch in dem Umstand, dass die ihm zugeschriebenen Attribute ein Zugehörigkeitsgefühl zu Altersgenossen verhinderten. Seine damaligen Strategien, mit dieser Konfliktsituation umzugehen, entsprechen zwar den Möglichkeiten, die die kulturellen Emotionsspezifika zulassen. Meinem Eindruck zufolge haben sie Kogoro aber nicht geholfen, die Situationen nachhaltig zu klären.

Kogoros Bemühen, mir die Ausgangssituation seiner Familie detailliert zu erklären, wirft ein interessantes Licht auf die beschriebenen, von ihm als entscheidend empfundenen Dynamiken seiner persönlichen Prägung als jüngstes Mitglied der Familie. In einzelnen oben zitierten Passagen vermittelte er mir den Eindruck von großer Traurigkeit, was sich in Gesprächen außerhalb der Interviewsequenzen bestätigte. Im folgenden Kapitel werde ich darauf eingehen, welche Rolle Kogoros Erleben von Familie für seine Traurigkeit spielt.

\subsubsection{Die emotionalen Parallelen zwischen den Generationen}

Sein Vater ist Journalist, dessen beruflicher Werdegang laut Kogoro vor allem durch seine privaten Angelegenheiten beeinflusst wurde: Obgleich seit längerem verheiratet, ließ sich Kogoros Vater auf eine Affäre mit einer anderen Frau ein, was auch außerhalb der Privatsphäre für einige Aufregung sorgte. Die Firmenleitung sah sich angesichts der moralischen Verfehlungen ihres Mitarbeiters dazu gezwungen, diesen vor die Wahl zu stellen: Er solle entweder die Firma oder die Gegend verlassen (Kogoro 02.05.2014: 24). Kogoros Vater entschied sich dafür, weiterhin für die Firma

${ }^{66}$ Siehe Kapitel 4.1.3 „Konfliktstrategien in der sozialen Praxis“. 
zu arbeiten, musste dafür aber an abgelegenen Orten die Berichterstattung übernehmen. Diese erste Entscheidung, die bereits vor Kogoros Geburt gefällt wurde, führt Kogoro in seinen Erzählungen weiter zurück in die Vergangenheit. Um die Motive seines Vaters nachvollziehen zu können, hat sich Kogoro viele Gedanken über dessen Hintergrund gemacht. Wie weit seine Recherche der Familiengeschichte geht, ist in folgenden Aussagen belegt.

Sein Großvater sei in rechten Organisationen hervorragend vernetzt gewesen, die Japan als Nation gestärkt sehen wollten. Kogoros Vater habe mit 20 Jahren versucht, diesen Netzwerken zu entkommen und seinen eigenen Weg zu gehen: „My grandfather then introduced him to some companies and due to his connections my father could start working there straight away. It was a patriotic weekly magazine which also introduced the idea of nuclear power plants to Japan" (Kogoro 02.05.2014: 25).

Hier entsteht eine weitere wichtige Verbindung zwischen Kogoros Aufarbeitung seiner eigenen familiären Vergangenheit und aktuellen Ereignissen und Sinnzusammenhängen. Aufgrund der patriotischen Ausrichtung des Magazins lag es nur nahe, die Unabhängigkeit Japans von ausländischen Energieträgern zu fordern und die Atomenergie zu befürworten. Vor dem Hintergrund der Ereignisse vom 11. März 2011 gewinnen Kogoros eigene emotionale Themen an Bedeutung für seine biographische Erzählung. Er stellt Sinnzusammenhänge her, die seine Perspektive auf die Atomenergie und deren soziale Auswirkungen erhellen. Dass Kogoro den Anteil seiner eigenen Familie an der Propagandaarbeit der Atomindustrie aufzeigt, wäre vor dem Hintergrund einer anderen Forschungsfrage womöglich nicht zur Sprache gekommen. Seine ganze Familiengeschichte wird von ihm vor der emotionalen Schablone des 11. März 2011 bewertet und gespiegelt. Dies bezieht sich auch auf die Zeit der Kindheit seines Vaters. Vor dem Zweiten Weltkrieg sei sein Großvater zu großem Einfluss gelangt, weil er, ungewöhnlich für diese Zeit, nach New York gegangen sei und viel Geld verdient habe. Die günstigen Kurse zugunsten des Dollars hätten dazu geführt, dass seine Familie auch in den Notzeiten des Zweiten Weltkrieges sehr gut versorgt gewesen sei. Unter anderem dadurch habe eine Entfremdung seines Vaters von gleichaltrigen Kindern eingesetzt: „This kind of richness brought difficulty to my father. This gap made it difficult for him" (Kogoro 02.05.2014: 25). Während der gesamten Erzählung bezüglich seiner Familie sitzt Kogoro aufgerichtet da und bewegt sich kaum. Auf mich macht dies einen strengen Eindruck. Gleichzeitig scheint es mir, als wolle er sich aufrecht halten und keinen Zweifel an seiner Erzählung aufkommen lassen. Schließlich wird seine Stimme sanfter als er biographische Parallelen zwischen den Erfahrungen seines Vaters und sich selbst zieht: „My father must have felt isolated, too" (Kogoro 02.05.2014: 25).

Aus Kogoros Perspektive haben er und sein Vater gemeinsam, dass sie sich auf eine Art und Weise von ihrem sozialen Umfeld unterscheiden, die es ihnen stark erschwerte, sich in gesellschaftlich favorisierter Weise in die sozialen Netzwerke einzubinden. Im Falle seines Vaters war es der vergleichsweise große Wohlstand der Familie. Für Kogoro war es sein fremdartiges Aussehen für seine Schulkameraden. Diese, ich nenne es ,soziale Heimatlosigkeit', wird von Kogoro als eine Art Familien- 
tradition dargestellt, die eine Stringenz mit vorangegangenen Generationen aufbaut und wichtige Aspekte seines Selbstverständnisses und damit auch seiner Handlungsweisen in den verschiedenen Rollen erklärt.

Die in Kogoros Erzählung als unverschuldet präsentierte Sonderrolle der männlichen Mitglieder aus seiner Familie findet sich auch in den Geschichten der Menschen von Fukushima, zu denen Kogoro direkt im Anschluss an seine Familiengeschichte überleitet. Dieses enge Verweben seiner eigenen persönlichen Erfahrungen von Einsamkeit, Wurzellosigkeit und Ausgeschlossenheit mit seinen Eindrücken aus den Katastrophengebieten Fukushimas geschieht nicht zufällig. Es bezeugt Kogoros stark persönlich motivierte Herangehensweise an die Auseinandersetzung mit „Fukushima“ als Ereignis.

\subsection{Traurigkeit und Wut: Die Verknüpfung biographischer Emotionen mit aktuellen Ereignissen}

Kogoro setzt sich insbesondere mit den psychischen Folgen auseinander, die die Flucht aus betroffenen Gebieten bei vielen ehemaligen Anwohnern nach sich zieht. Durch intensive Gespräche mit Menschen in den verschiedenen Unterkünften diversifiziert er das Bild, das die breite japanische Öffentlichkeit von der Situation dieser Menschen hat und bringt sie ihnen emotional näher. Wie alle meine Gesprächspartner arbeitet auch Kogoro nicht zufällig in einem gesellschaftlich so kontrovers diskutierten Bereich. Im Laufe der Gespräche mit mir wurde deutlich, dass einige Thematiken wie die Stigmatisierung, die die Diskurse um den Umgang mit Anwohnern aus den verstrahlten Gebieten beleuchten, auch in Kogoros Biographie zu finden sind.

\subsubsection{Empathische Identifikation: Kogoros Sicht auf Atomkraft und ihre Folgen}

„As I said to you, these nuclear power plants are very close to war business. It's a heavy industry, which involves companies like Mitsubishi, Chitoshi, Toshiba and so on. [...] The construction of plants is complicated and Japan experiences too many earthquakes. It is difficult to have a functioning nuclear power plant." (Kogoro 02.05.2014: 26)

Mit diesen Ausführungen leitet Kogoro seine Ansichten über Atomenergie im Allgemeinen und im japanischen Kontext im Besonderen ein. Er nimmt sowohl Bezug auf die Expertenkultur, die die Atomenergie umgibt, als auch auf deren offensichtliches Versagen angesichts der spezifischen geographischen Lage Japans auf dem Pazifischen Feuerring. Die damit einhergehenden regelmäßig auftretenden Erdbeben seien nicht ausreichend berücksichtigt worden. „The professional people who are exporting this technology out of Japan never think regionally. They write an report 
saying there is no risk. But there is too much risk for Japan, ne? It's too much of a problem for a society" (Kogoro 02.05.2014: 26-27).

Die Fehleinschätzungen des Risikofaktors im Falle eines Zwischenfalls schlagen sich auch in den Zahlen zu den Kosten des Unglücks nieder. Laut eines Artikels der Japan Times vom 28.11.2016 werden die Kosten von ursprünglich 11 Billionen Yen auf mindestens 20 Billionen Yen (201 Milliarden USD) steigen und damit doppelt so hoch sein wie veranschlagt. ${ }^{67}$ Autoren wie der Japanologe Steineck (2015: 39) stellten ebenfalls vorsichtige Berechnungen an, wonach sich allein die Lagerungsund Entsorgungskosten auf vier bis sieben Billionen Yen (ca. 60 bis 86 Milliarden USD) belaufen.

Wieder sind es die Autoritäten wie Experten, Professoren, Mediziner und Techniker in Kogoros Narrativ, die die Lage falsch einschätzten und die für das Leid von Dritten verantwortlich sind. Die unter anderem in Artikeln von 2015 angesprochene ungeklärte finanzielle Absicherung der Evakuierten auch fünf Jahre nach dem Reaktorzwischenfall macht die Vertriebenen zu abhängigen Akteuren, die scheinbar nur wenige Entscheidungsmöglichkeiten haben. Die sozialen Auswirkungen dieses Zustandes erlebt Kogoro bei jedem seiner Besuche in den Notunterkünften. „People get addicted to alcohol and gambling because they lost their home, the place they belonged“ (Kogoro 02.05.2014: 6). Ebenso berichtet er wiederholt von Selbstmorden, von denen ihm vor Ort erzählt wurde.

„They have to go back because the support from the government is going to subside. But there are only old people left. So they commit suicide. One man I heard of was 50 years old. He was forced to come back but there is nothing."

(Kogoro 02.05.2014: 6)

Mit den hier beschriebenen Selbstmorden nimmt Kogoro auf eine Möglichkeit des Umgangs mit Konflikten Bezug, die in Japan durchaus akzeptiert wird, um Situationen zu lösen. Der Selbstmord einer Person kann dabei für die große emotionale Last, die derjenige gefühlt haben muss, stehen. Im Falle einer Schuldzuschreibung ist der Selbstmord aber auch eine legitime Art, um Verantwortung für sich und die verletzten Gefühle anderer zu übernehmen.

In Kogoros Aussagen ist auffällig, dass er im Kontext seiner biographischen Erzählung immer wieder enge Bezüge zu aktuellen Ereignissen einwebt. Seine eigene soziale Heimatlosigkeit, wie ich sie im vorangegangenen Kapitel umschrieben habe, findet Ausdruck in seiner Empörung über die Behandlung der „Atomflüchtlinge“ von Fukushima. Seine Körperhaltung verstärkt meinen Eindruck von Empörung auf Kogoros Seite noch zusätzlich: Er sitzt aufrecht vor mir und gestikuliert, für ihn untypisch, mit den Händen.

\footnotetext{
${ }^{67}$ The Japan Times: „Cost of Fukushima disaster expected to soar to ¥20 trillion“, 28.11.2016 (https://www.japantimes.co.jp/news/2016/11/28/national/cost-fukushima-disaster-expected-soar- $¥ 20$ -trillion).
} 
Bei folgenden Passagen wird seine Empörung besonders deutlich: Wollen die „Atomflüchtlinge“ nicht in die verstrahlten Gebiete zurückkehren, stehen sie vor dem finanziellen Ruin. Nicht zuletzt an der Frage der Entschädigung entzünden sich die Debatten um die unterschiedliche Auslegung der Strahlendaten und deren Bedeutung für die gesundheitliche Beeinträchtigung der Menschen. Kogoro verfolgt seit 2011 aktiv die laufenden Prozesse von Highschoolschülern und deren Familien gegen den Staat. Diese Familien hatten nicht auf einen offiziellen Aufruf gewartet, sondern waren „freiwillig“ evakuiert. ${ }^{68}$ Seit 2014 läuft eine zweite Prozesswelle, die noch weitere Gebiete der Präfektur Fukushima mit einschließt. Als treibende Kraft nennt Kogoro (02.05.2014: 13) die große Wut der Eltern, die, entgegen den allgemeinen Aussagen der Ärzteschaft, auftretendes Nasenbluten ihrer Kinder mit den erhöhten Strahlenwerten in Verbindung bringen. ${ }^{69}$

In Kogoros Erzählung verbinden sich seine persönlichen Themen der Isolation mit den Geschichten der Betroffenen, die sich mit ihren Ängsten vor gesundheitlichen Schäden vom japanischen Staat allein gelassen fühlen. Diese empathische Identifikation Kogoros mit den betroffenen Familien bildet den Hintergrund für die folgenden Aussagen.

Kogoro hatte sich zu diesem Zeitpunkt meiner Forschung sehr für die Eltern engagiert und im Vorfeld der Prozesse Informationsflyer für alle Interessierten gedruckt, die er vor dem Gerichtsgebäude verteilte.

„People [of Fukushima] are feeling a great disconnectedness. They are quite isolated. No one is taking responsibility. At that time, I thought maybe we are not minority. So many people came. But in Tokyo I found we are minority still.“ (Kogoro 02.05.2014: 15)

Wie stark Kogoros empathische Bezüge zum politischen Umgang mit den Betroffenen sind, zeigt sich daran, wie er sich in dieser Aussage mit dem Wechsel der Personalpronomina von einem Beobachter und aktiven Bürger zu einem persönlich Betroffenen wandelt. Seine Realität nähert sich den Ansichten der Menschen vor Ort immer mehr an und generiert einen Grad an Zugehörigkeit, der es ihm in seiner biographischen Erzählung schwer macht, sich artikulativ von ihnen zu lösen. Er ist in dieser Aussage zu einem Mitglied der Gruppe der Betroffenen geworden, indem er sagt: "Wir sind immer noch eine Minderheit."

Die Eltern der von der Strahlung belasteten Kinder, vor allem aber die Mütter, beschreibt er als Kämpfer für die Wertigkeit dieser anderen Realität. „By trial, they

\footnotetext{
${ }^{68}$ Als „freiwillig Evakuierte“ werden diejenigen Personen bezeichnet, die nicht explizit von der Regierung Japans dazu aufgefordert wurden ihre Häuser und Wohngebiete zu verlassen. Dieser Auslegung zufolge bestand zu keinem Zeitpunkt akute Gefahr durch Verstrahlung, so dass diesen Personengruppen Entschädigungszahlungen verweigert werden (siehe u.a. Justin McCurry: „Fukushima evacuees face ,forced" return as subsidies withdrawn “, The Guardian, 11.3.2017, https://www.theguardian.com/ world/2017/mar/10/japan-fukushima-nuclear-disaster-evacuees-forced-return-home-radiation).

69 Siehe Kapitel 5 „Vertrauen und Risiko - Die vorläufige Bilanz einer gesellschaftlichen Katastrophe“.
} 
are fighting to get enough compensation from TEPCO. [...] I feel the number of people who underwent a mind change is not major. [...] So I try to create a place for those who try to speak out" (Kogoro 16.01.2015: 23).

In diesen Aussagen umreißt Kogoro seine Rolle in diesen gesellschaftlichen Vorgängen bereits genauer. „I feel that because 3.11 occured, it is time to take responsibility. [...] We need to speak out and to express ourselves. Silence only tends to stress isolation" (Kogoro 02.05.2014: 31). Er wird zum Gestalter eines Raumes für den Austausch, indem er neben der Bereitstellung von wichtigen Informationen auch die Emotionen der Menschen anspricht. Gleichzeitig arbeitet er wieder einer Strategie des Verschweigens entgegen, die, wie bereits erwähnt, in der Kulturspezifik Japans durchaus ein Lösungsweg ist. ${ }^{70}$ "Through the emotions I try to paint, people can relate. A highschool-student in Fukushima and one in Tokyo, for example" (Kogoro 02.05.2014: 8). Ich sehe in der von Kogoro beschriebenen Beziehung eine empathische Verbindung, die er durch seine Werke herzustellen versucht. In Kogoros Bildern wird die Mutter, die für die Gesundheit ihrer Kinder kämpft, gleichzeitig zu einem moralischen Appell, der in anderen Teilen der Gesellschaft eine Resonanz erzeugt. Indem er in dieser Weise tätig ist, kann auch Kogoro gehört werden und sich gegen die Dynamiken, wie die der Stigmatisierung, aussprechen.

\subsubsection{Identifikationspunkte von Diskriminierung und Stigmatisierung}

Zu den Themen des öffentlichen Diskurses zählt die aktive Ausgrenzung der Evakuierten durch ihr neues Umfeld auch Jahre nach dem Reaktorvorfall. Ausgrenzungen haben laut Kogoro ihre Wurzeln in dem Unwissen der Menschen über die Auslegung der Strahlenwerte und über die Charakteristika der Verbreitung von atomarer Strahlung. Kogoro beschäftigt sich unter anderem mit der gesundheitlichen Überwachung der Kinder aus den entsprechenden Gebieten, die mit den Strahlungsdaten aus Aomori, Yamanashi und Nagasaki verglichen werden. Die Ergebnisse betrachtet er sehr skeptisch. „One thyroid cancer was detected among the tested children between 2012 and 2013. One in 4.365 children. [...] But the doctors say that it is difficult to recognize a distinct radiation effect" (Kogoro 02.05.2014: 16). Nicht nur sind die Ergebnisse der Untersuchungen nicht eindeutig, sie führen bereits zur Beeinträchtigung der Lebensführung. „One doctor said to a mother of a child who underwent thyroid tests: ,I recommend that you do not speak about this. As people know this, your child may get problems finding a job or a partner for marriage "“ (Kogoro 02.05.2014: 17). Sich auch nur dem Test zu unterziehen birgt bereits die Gefahr einer Stigmatisierung in sich, so dass viele Familien diese Untersuchungen nur heimlich vornehmen lassen. Coulmas und Stalpers (2011: 149) berichteten bereits 2011 von der Abweisung von sogenannten Reaktorflüchtlingen an Notunterkünften und vom Ausschluss von Kindern aus Klassengemeinschaften. ${ }^{71}$

\footnotetext{
70 Siehe Kapitel 4.1.3 „Konfliktlösungen in der sozialen Praxis“.

${ }^{71}$ Siehe Kapitel 5.1.2 „Der mentale Stress: Die Lage der Betroffenen fünf Jahre danach“.
} 
Kogoros Gewichtung dieses Themas in seiner Erzählung ist auffällig und betont seine persönlichen Erfahrungen von Isolation und Einsamkeit. Dabei zeigen sich die emotionalen Parallelen zwischen ihm und den unmittelbar Betroffenen: Wie so viele Menschen in den Notunterkünften versuchte auch Kogoro, einen endgültigen Ausweg aus seiner zunehmend als untragbar empfundenen Situation zu finden. Ich verstehe Kogoros Aussagen dahingehend, dass er eine emotionale Nähe zu den unmittelbar Betroffenen aufbaut, in dem er ihre Emotionen nachempfindet. Die Bedeutung dieser Empathie Kogoros wird dadurch umso deutlicher, dass sein eigenes Empfinden von Isolation und Einsamkeit ihn zu einem drastischen Schritt bewogen haben.

\subsection{3 „Es gibt keinen Platz für mich“: Der Selbstmordversuch}

Durch die Auseinandersetzung mit der Lage der Mütter und Kinder in Fukushima erinnerte sich Kogoro in unserem Gespräch an seine Jugend und die damit zusammenhängenden Herausforderungen. Hier verlassen wir im Gespräch den aktuellen Kontext wieder und er setzt seine biographische Erzählung fort. Obwohl Kogoro zum Zeitpunkt seines Studiums wieder in seiner Geburtsstadt Tokyo lebte, erwarteten ihn auch hier soziale Hürden, die er glaubte nicht bewältigen zu können:

„In school, I was loosing motivation and I didn't have a real friend. When I went to university, I couldn't understand the other students around me who just wanted to get a good job and lots of money. I couldn't find the interest for work and making money. I felt like there is no place for me. [...] Then my father had a car accident and suffered a severe head injury. At that time, I became too isolated and took many pills." (Kogoro 02.05.2014: 29)

Die hier deutlich werdende Distanz, die Kogoro zu seinem Umfeld aus Mitstudenten und gesellschaftlicher Ausrichtung auf eine gute Stellung und entsprechendes Gehalt empfand, markiert den Beginn eines dunklen Kapitels in seiner biographischen Erzählung. Der Selbstmordversuch markiert so etwas wie einen Wendepunkt und bildet den Auftakt zu weitläufigen Ausführungen zu sich als Künstler, seiner gesellschaftlichen Rolle und seinen Anliegen. In diesen Passagen spricht er leise und wird nicht sehr ausführlich in seinen Erzählungen. So bleibt unklar, wie er diesen Selbstmordversuch überlebte. Mit der knappen Bemerkung „I woke up“ (Kogoro 02.05.2014: 29) beendet er die Erzählung dieses Abschnitts abrupt. Dass er diese Zeit als eine Phase der nichtexistenten sozialen Verortung umschreibt, lässt erneut die Parallelen zur Situation der Atomflüchtlinge erkennen. Gleichzeitig zeigt er hier, dass er die Möglichkeit des Selbstmords zur Herstellung von Harmonie selbst versucht hat anzuwenden. ${ }^{72}$

72 Siehe Kapitel 4.1.3 „Konfliktlösungen in der sozialen Praxis“. 
Es ist auffällig, dass Kogoro seine eigenen Erfahrungen in einen größeren Zusammenhang setzt und meine Aufmerksamkeit auf die gesamtgesellschaftliche Verantwortung für den Einzelnen lenkt. So kommt er auf die Tendenz, sich aus der Gesellschaft zurückzuziehen und sich nicht um eine weitere Eingliederung zu bemühen, in einem Kontext zu sprechen, der nichts mit der Katastrophe zu tun hat.

„Do you know the term hikkikomori? It describes people of the Japanese society who lock themselves in their rooms and don't speak to nobody. It's a feeling like: 'There is no place for me in this society.' But in reality there is. [...] It's what the world is like to some people after 3.11. So through my paintings I want to communicate." (Kogoro 02.05.2014: 31)

Mir fällt auf, dass Kogoros Stimme kräftiger wird je mehr wir uns thematisch von Kogoros persönlicher Erfahrung des Selbstmordversuchs entfernen. Indem Kogoro die gesellschaftliche Thematik des Rückzugs aus der Gesellschaft anspricht, stellt er einen emotionalen Zusammenhang zwischen einem Phänomen der japanischen Gesellschaft und der Situation der Atomflüchtlinge her. Bei mir entsteht der Eindruck, dass er mir auf diese Weise eine für ihn wichtige Botschaft vermitteln will, ohne sie direkt anzusprechen: Was den Einzelnen betrifft, betrifft schlussendlich alle.

Diese Grenze zwischen dem Einzelnen und dem Kollektiv wird auf diese Weise transparent. Das wachsende Verständnis für einander kann in weiterer Folge dazu beitragen, die Kluft zwischen den von ihm beschriebenen Realitäten zu überbrücken. Die Situation der Menschen in den Notunterkünften mag speziell sein, ihre Emotionen und sozialen Konsequenzen hingegen sind sehr wohl bekannt und bedürfen laut Kogoro lediglich einer tiefergehenden Betrachtung.

In diesem Zusammenhang kommt Kogoro immer wieder auf die junge Generation zu sprechen, deren Interesse er mithilfe seiner Kunst wecken möchte. Das Charakteristikum seiner Werke, nicht nur faktische, sondern auch emotionale Botschaften zu vermitteln, entfaltet sich erst in der Auseinandersetzung seiner Rezipienten mit seiner Person. Die Sensibilität für die Verantwortung des Einzelnen, sich Kontroversen zu stellen und die entsprechenden Konsequenzen für das gemeinschaftliche Handeln zu ziehen, entspringt seinen eigenen Erfahrungen der Hilfslosigkeit, der Ohnmacht und der tiefen Trauer angesichts seiner Einsamkeit in Kindheit und Jugend. Die Verantwortung des Einzelnen ist ein Thema, dem er in Form von Kunst gerecht werden möchte. „,...] to overcome this kind of problem is very important. Looking at the art scene in Japan, there is not much of such an art that is treating this kind of isolation, so I don't care about doing it without the proper education" (Kogoro 02.05.2014: 29).

In diesem Abschnitt seines Narrativs wandelt sich Kogoro vom Opfer seiner Empfindungen zu einem aktiven Mitglied der Gesellschaft. Damit wird ein ihm persönlich sehr wichtiges emotionales Thema zur Initialzündung für sein weiteres Engagement. „I woke up [after trying to commit suicide] and then I felt that I want to do my art. To make music, to start painting again. [...] The problem I encountered 
might not be my problem alone. Maybe many of the young Japanese generation are feeling this" (Kogoro 02.05.2014: 29). Kogoros Selbstbeschreibung als Außenseiter erhält eine positive Konnotation, indem er den besonderen Wert seiner Perspektive in den Mittelpunkt rückt:

„So, as an outsider, there is so much to do. We can speak about and express the silence that tends to increase the stress of people. [...] All the experts, scholars and professors do not realize what is happening in Fukushima. All the anxiety. So this is a fight against mainstream." (Kogoro 02.05.2014: 31)

Kogoros hohe Sensibilität für die psychologischen Auswirkungen von Entwurzelung, für die er aufgrund seiner eignen Biographie sensibilisiert wurde, erlauben es ihm im Umkehrschluss die Vorzüge seiner eigenen Rolle und damit die Wichtigkeit seiner Tätigkeit für die Gesellschaft hervorzuheben. Indem er seine eigene Emotionalität beschreibt und dabei Raum für Parallelen zu Dritten zulässt - in diesem Fall den hikkikomori - ermöglicht er dem Zuhörer einen Perspektivwechsel auf die Gefühlswelt anderer. Mit anderen Worten: Der Zuhörer wird in die Lage versetzt, Empathie für Kogoro und diejenigen zu empfinden, auf die er sich bezieht. Meiner Meinung nach bewegt sich Kogoro hier in der kulturellen Emotionsspezifik Japans, die auf Harmonie durch Empathie abzielt. Er ermöglicht die dafür notwendige Empathie für Dritte jedoch erst durch die Überbrückung räumlicher Distanz sowie durch die Bereitstellung von Informationen. Darüber hinaus kann Kogoro seine Zugehörigkeit zu einer Szene von Außenseitern formulieren und übt Kritik am Status quo der Gesellschaft. Damit liefert er eine Umschreibung für sein Dasein als Künstler, die auf diesen Grundannahmen fußt: Obwohl er sich selbst am Rande der Gesellschaft positioniert, ist er doch tief verbunden mit den sie prägenden Themen und versucht, entsprechende Antworten auf die Bedürfnisse der Menschen zu finden.

\subsection{Die persönliche Bedeutung des 11. März 2011 - „I am one of the seeds of change“"}

Kogoros Kunst besteht seiner Auffassung nach darin, persönliche Geschichten der Atomflüchtlinge und der sozialen Folgen dieser Entwicklung in bildhafte Symbole zu übersetzen. Dies spricht viele Aspekte seiner persönlichen Lebensgeschichte an. Der 11. März 2011 hat nicht nur auf technischer und historisierender Seite viele Fragen aufgeworfen, sondern auch in der Folgezeit viele Bruchstellen der japanischen Gesellschaft sichtbar werden lassen. Diese wurden vorher vor allem von der betroffenen Minderheit betrachtet, ohne dass dies entscheidende Impulse im öffentlichen Diskurs bewirkt hat. Da durch die Katastrophe das Leben so vieler Menschen zerstört oder massiv beeinträchtigt wurde, sind dringende Fragestellungen nach Verantwortung und Umgang mit bereits vor dem Desaster existierenden $\mathrm{Zu}$ ständen sichtbarer geworden. Kogoro drückt es folgendermaßen aus: „There are so 
many problems in the Japanese society now. Fukushima was like an $\mathrm{x}$-ray for society and now we can see a picture of our society's bones. The problems are visible now" (Kogoro 02.05.2014: 19). Kogoro spricht damit einen Eindruck an, den er bereits in seinen bisherigen Ausführungen in unterschiedlichen Kontextualisierungen anklingen ließ: Die Dreifachkatastrophe, und insbesondere der atomare GAU, ist zu einem Aufdeckungsereignis geworden, in dessen Symptomatik sich bereits zuvor existierende Problematiken konzentrieren. Kogoro kann in seiner Rolle als Künstler diese unterschiedlichen Zeit- und Sinnzusammenhänge herstellen, miteinander in Beziehung setzen und in den öffentlichen Raum zurückspiegeln.

Damit wird der Einzelne, und das schließt Kogoro als Erschaffer der Werke mit ein, wieder Teil eines gesellschaftlichen Gesamtgefüges, dessen Wirkungsdynamiken sich auch in ihren individuellen Spannungsverhältnissen zeigen. Auf diese Weise kann Kogoro am Ende unseres letzten Gesprächs auch positive Impulse des 11. März 2011 erkennen: „I feel something positive looking at the children who were evacuated by their mothers. I feel that the experience of 3.11 caused a mind change. They are looking outside [of their own reality] and are expressing their own thoughts" (Kogoro 16.01.2015: 22). Die Wirkung von Kogoros Kunst richtet sich auf Gegenwart und Zukunft, aber es ist die Vergangenheit, für die er eine neue Perspektive zu erschaffen versucht. „I live in the present but I create the past with my paintings. [...] Every painting is the mother of the past" (Kogoro 09.02.2015: 24).

Die eingangs aufgezeigte enge Verbindung seiner Bilder mit seinem Selbst und deren Wirkung über seinen physischen Tod hinaus fügt diesen Überlegungen die Zeitdimension der Unendlichkeit hinzu. Wie auch die atomare Strahlung auf unbestimmte Zeit ihre Wirkung entfalten wird, so hofft Kogoro, dass dies seine Bilder für die Gesellschaft tun werden. Die Bedeutung seiner Rolle als Künstler, Dokumentar der Vergangenheit, Vermittler zwischen den emotionalen Realitäten und Initiator von Gedanken und Handlungen offenbart sich für ihn deshalb nicht im finanziellen Erfolg, sondern in der Zuschreibung von Sinnhaftigkeit. „I want to do something meaningful. [...] And art has a value on its own. Art exists because of the artist. And it will still exist when I died“" (Kogoro 09.02.2015: 25-26). Er wirkt zu diesem Zeitpunkt des Interviews sehr nachdenklich und nimmt sich Zeit, die passenden Worte zu finden. Häufig schaut er in die Ferne und sucht nach einem Ausdruck, der seine Emotionen am treffendsten beschreibt.

Seiner Existenz als Außenseiter spricht Kogoro positive Eigenschaften zu, die der Gesellschaft nicht nur derzeit nützlich sind, sondern die auch in ferner Zukunft von Bedeutung sein könnten. Als einer derjenigen Akteure, die sich aktiv und kritisch mit sozialen Themen beschäftigen, festigt er sowohl seine Zugehörigkeit zur japanischen Gesellschaft als auch seine Selbstzuschreibungen als Künstler und Persönlichkeit. In dieser Dynamik wirkt sein Narrativ für seine Persönlichkeit wie auch für sein Handeln stabilisierend und sinnstiftend. „I am like a tree so I have to grow. If some fruits will proceed from this tree, it's good. I am one of the seeds of change" (Kogoro 06.03.2015: 27). 
An Kogoros Metaphorik eines „Samenkorns der Veränderung“ wird deutlich, dass er sich der zukunftsgerichteten Wirkung seiner Tätigkeit durchaus bewusst ist. Indem er seinen eigenen Emotionen in Bezug auf die Ereignisse vom 11. März 2011 Ausdruck verleiht, hofft Kogoro, den kulturspezifischen Umgang mit Konflikten zu verändern. „I made a book which contents collected information and my paintings. [...] This book is working against the mainstream. [...] The Japanese government is throwing people away, but I want to confirm what is really happening so younger generations will know what has happened. This is what it means to take responsibility" (Kogoro 02.05.2014: 31).

Die gegenwärtigen Stimmungen, Emotionen und Erfahrungen zeitnah aufzunehmen und in dieser Weise zu verarbeiten, ermöglicht es ihm auch, als Erschaffer in sehr wirksamer Weise sichtbar zu werden. Dafür nimmt er zum Teil negative Auswirkungen in Kauf, wie er mir nach meiner Rückkehr nach Deutschland mitteilt:

„My exhibitions have caused either attacks or affirmative actions from various sides. [...] I know that the general interest in Fukushima has decreased only five years after the disaster. But because people are willing to forget about it, I have to talk about this incident and its problems even louder."

(Kogoro 03.11.2016: 31)

Vor dem Hintergrund der zu diesem Zeitpunkt für 2020 geplanten Olympischen Spiele werden die Maßnahmen der japanischen Regierung immer massiver, der internationalen Gemeinschaft ein sicheres und genesendes Japan zu präsentieren. ${ }^{73} \mathrm{Da}$ her sollten nach offiziellen Plänen der Regierung im Jahr 2018 die Notunterkünfte geräumt und abgerissen, die Ausgleichszahlungen eingestellt und die Menschen nach Möglichkeit in ihre Wohnhäuser zurückgekehrt sein. ${ }^{74}$ Bereits am 07. September 2013 hatte Premierminister Shinzo Abe mit dem Satz „The situation is under control“ Werbung für Japan als Austräger der Olympischen Spiele gemacht. ${ }^{75}$ Im März 2020 gab es jedoch immer noch Gebiete, für die der Evakuierungsbefehl nur teilweise aufgehoben wurde: In Okuma, südlich vom Kernkraftwerk Fukushima Daiichi sind es knapp 40\% des Distrikts. ${ }^{76}$ Die weltweite Corona-Pandemie seit 2020 hat zudem dazu geführt, dass die Olympischen Spiele unter dem offiziellen Namen „Games of Recovery“ um ein Jahr verschoben wurden.

\footnotetext{
${ }^{73}$ Zur anti-olympischen Bewegung in Japan siehe Sonja Ganseforth (2020).

${ }^{74}$ Kanna Mitsuta: „Fukushima Evacuees abandoned by the government“, Citizens’ Nuclear Information Center, 31.3.2017 (http://www.cnic.jp/english/?p=3486).

${ }^{75}$ Felix Jawinski: „Erläuterung und Kommentar zum Werk 'Gastfreundschaft hat auch ihre Kehrseite von Yosomia Takashi“", Textinitiative Fukushima, 3.3.2014 (http://www.textinitiative-fukushima.de/ pages/posts/erlaeuterung-und-kommentar-zum-werk--gastfreundschaft-hat-auch-ihre-kehrseite--von -yosomiya-takashi-343.php).

${ }^{76}$ Caitlin Stronell: „Fukushima and the Recovery Olympics“, Citizens' Nuclear Information Center, 24. März 2020 (https://cnic.jp/english/?p=4828).
} 
Wie Kogoros Perspektive und meine eigenen Beobachtungen zeigen, handelt es sich dabei aus Sicht der Betroffenen um eine Politisierung der Strahlungsdaten aufgrund von machtpolitischen Interessen. Als Vertreter der nicht-staatlichen Öffentlichkeit gestaltet Kogoro gemeinsam mit anderen Akteuren aus Literatur, Kunst und unabhängigen Medien (siehe Gebhardt 2012) einen diskursiven Raum zur differenzierteren Auseinandersetzung mit den durch den 11. März 2011 aufgeworfenen gesellschaftlichen Themen. 


\section{Ayako von Shiogama - Der Tsunami im lokalen Kontext der Ostküste}

Ayako ${ }^{77}$ stellt eine wichtige perspektivische Ergänzung zu Kogoros Erfahrungshintergrund und Arbeitskontext dar. Sie hat mit ihren 32 Jahren zum Zeitpunkt des Interviews einen Bezug zu ihrer Tätigkeit als Gegenwartskünstlerin entwickelt, der sich stark von Kogoros Narrativ unterscheidet. Ihre im Jahr 2014 und 2015 durchgeführten Kunstprojekte richten sich, im Unterschied zu Kogoros Arbeit, an die lokale Bevölkerung ihrer unmittelbaren Nachbarschaft und sollen in erster Linie die Verarbeitung der Ereignisse befördern und das Bewusstsein für die Geschichte des Landstrichs und die Bindung innerhalb der kleinen Gemeinschaft verstärken.

Ayako hat im Laufe ihres Lebens einige Zeit im englischsprachigen Ausland verbracht und wollte unsere Gesprächssituation nutzen, sich in englischer Sprache auszudrücken, so dass wir auf die Unterstützung meines Übersetzers Satoshi verzichten konnten.

Während der Zusammenarbeit tauchten weitere Facetten von Ayakos Narrativ auf, die ihr Selbstverständnis als betroffene Anwohnerin ihrer Rolle als lokale Künstlerin gegenüberstellen. Im Gegensatz zu Kogoro, der sich zum Zeitpunkt des Bebens 2011 in Tokyo aufhielt, war Ayako am Ort des Geschehens und musste vor dem Tsunami fliehen. Das Erleben der teilweisen Zerstörung ihres Heimatortes innerhalb weniger Minuten und des Todes einiger Bekannter und Freunde ließ Ayako

77 Es handelt sich um ein Pseudonym, um die Identität meiner Gesprächspartnerin zu schützen. 
einen anderen emotionalen Rahmen für ihre Arbeit setzen als Kogoro. Ayakos Arbeit löst weit weniger Kontroversen aus als Kogoros. Dies liegt aus meiner Sicht auch daran, dass ein Tsunami ein in Japan bekanntes Phänomen darstellt - im Gegensatz zu den Folgen atomarer Verstrahlung. In den Gesprächen mit Ayako spiegelt sich dies beispielsweise in ihren ungezwungenen Schilderungen ihrer eigenen Reaktionen während und nach den Ereignissen des 11. März 2011 wider. Im immer größer werdenden zeitlichen Abstand findet sie zudem unterschiedliche Erzähl- und Ausdrucksweisen. Dieser Reflexionsprozess tritt deutlich zu Tage und bettet Ayakos psychischen Genesungsprozess in den Kontext ihrer lokalen Schicksalsgemeinschaft aus Familie, Nachbarn, Freunden und Kunden ein.

Dass die Erdbeben- und Tsunamikatastrophe in den japanischen Diskursen anders bewertet und emotional behandelt wird, wirkt sich auch stark auf Ayakos Arbeit aus. $^{78}$ Sie stellt in gewisser Weise auch auf biographischer Ebene einen Gegensatz zu Kogoro dar: Ayako ist eine junge Frau von 32 Jahren, die sich, im Gegensatz zu Kogoro, tief in ihrer lokalen Familiengeschichte verwurzelt sieht und seit ihrer Kindheit fortwährende Unterstützung in ihren Zukunftsplänen erfährt. Dies wirkt sich sowohl stark auf ihre Darstellung einer Gegenwartskünstlerin in Japan aus als auch auf ihr Empfinden der gesellschaftlichen Resonanz auf das Leiden der lokalen Bevölkerung Shiogamas.

\subsection{Ayako, die Künstlerin}

Ayako ist eine junge Frau, die in einem kleinen Ort in der Präfektur Miyagi/Tohoku geboren wurde und aufwuchs. Diese knapp 55.000 Einwohner zählende Stadt liegt an der Ostküste Japans und wurde 2011 direkt vom Tsunami getroffen. Die zerstörerische Kraft der Welle ließ sich auch bei meinem Besuch der Region im Jahr 2014 noch deutlich an zerstörten Häusern und Straßenzügen ablesen.

Ich wurde auf Ayako durch ein in Tokyo veröffentlichtes Projekt aufmerksam, das künstlerisches Engagement in den unmittelbar betroffenen Küstenregionen behandelte. Als Mitwirkende war Ayako mit einer Kurzbiographie aufgeführt, die sowohl ihre Auslandserfahrung als auch eine Schwerpunktsetzung auf lokale Netzwerke und Anliegen verdeutlichte.

Die von mir bis Juni 2014 gesammelten Erzählungen enthielten noch keine Stimmen von Personen, die im Katastrophengebiet der Ostküste lebten und den Tsunami miterlebt hatten. Ayako erklärte sich bereit, mir ihr Erleben zu schildern und meine Forschung um eine wertvolle Facette zu erweitern.

Mich interessierte vor allem die Frage, ob Künstler, sobald sie sich selbst eine Opferrolle zuschreiben, im lokalen Kontext wesentlich andere Themenschwerpunkte und -ansätze verfolgen als diejenigen, die sich mit der atomaren Katastrophe beschäftigen. Inwiefern wirken sich die dabei empfundenen Emotionen auf ihr Selbstbild

78 Über die Geschichte Japans als „Earthquake Nation“ siehe Clancey (2006). 
und ihr Kunstverständnis aus? Darüber hinaus beschäftigte mich der möglicherweise vom Tokyoter Setting abweichende Konformitätsdruck in ländlicheren Gebieten während den entscheidenden ersten 10-15 Jahren der Sozialisation. Vorangegangene Interviews hatten diesen Lebensabschnitt bereits als entscheidende Phase im Leben eines späteren Gegenwartskünstlers aufgedeckt. Viele meiner Gesprächspartner haben sich spätestens im Teenageralter für ein Leben als Künstler entschieden beziehungsweise darin ihre Rolle gefunden.

Nach einer kurzen Terminabsprache per E-Mail begegnete Ayako mir das erste Mal im Juni 2014 an der Bahnstation in Shiogama. Gleich beim ersten Gespräch nahm sie sich mehrere Stunden Zeit für mich und blieb auch über ein erstes Treffen hinaus sehr aufgeschlossen und kooperativ. Es folgte ein reger E-Mail-Austausch und weitere Gespräche über soziale Netzwerke. Dieser lange Austausch ermöglichte es uns, eine stabile Bindung aufzubauen, die sich überaus positiv auf den Prozess der Datengenerierung auswirkte. Ayako erzählte offen über ihre Erlebnisse, Perspektiven, Probleme und Herausforderungen bei ihrer Arbeit in vergleichsweise kleinen, ortsgebundenen Projekten. Sowohl ihr junges Alter als auch ihr Geschlecht in Verbindung mit ihrer Tätigkeit als Künstlerin sichern Ayako einen besonderen Status innerhalb dieser lokalen Gemeinschaft, den sie, ganz anders als die Mehrzahl meiner Tokyoter Gesprächspartner, überaus positiv darstellt.

Ihr unmittelbares Erleben des 11.03.2011 in Kombination mit ihrem biographischen Narrativ bildet einen teilweise starken Kontrast zu Kogoros Erzählweise. Worauf diese Unterschiede im Erleben beruhen, werde ich in den folgenden Kapiteln aufzeigen.

\subsection{Eine lokal geprägte Lebensgeschichte}

Das Hauptinterview fand am 25.06.2014 von 10 bis $13 \mathrm{Uhr}$ in ihrer Galerie in ihrem Heimatort Shiogama statt. Drei Jahre nach dem Tsunami sind ihre Räumlichkeiten zu einer Art inoffiziellem Gemeindezentrum geworden, in dem sich viele Angehörige von Verstorbenen und Überlebende einfinden. Die ruhige Atmosphäre lädt dazu ein, über das Erlebte zu sprechen, und das trifft auch auf Ayako selbst zu. Ihr Narrativ ist gekennzeichnet durch eine enge Verbundenheit mit ihrem Geburtsort und den dort lebenden Menschen. Wie sehr sie sich insbesondere nach der Katastrophe mit den Schicksalen der Menschen identifiziert, wurde immer wieder deutlich. Während des Interviews bat sie mich beispielsweise um eine kurze Unterbrechung unseres Gesprächs, als eine junge Frau in ihre Galerie trat. Ayako behandelte diese Frau sehr zuvorkommend, sprach mit leiser Stimme und bot ihr an, später erneut bei ihr vorbeizukommen, sollte sie Gesellschaft brauchen. Wie sich später herausstellte, handelte es sich bei dieser Frau um die einzige Überlebende einer vierköpfigen Familie, der Ayako ihre Hilfe in alltäglichen Belangen angeboten hatte. Diese enge Beziehung zwischen ihr und den Menschen vor Ort spiegelt sich auch zu Beginn 
ihrer biographischen Erzählung wider, in der sie über die Beschreibung von Orten zu ihrem eigenen Erleben und dem Genesungsprozess überleitet.

In den folgenden Abschnitten beschäftige ich mich in größtmöglicher Dichte mit den von Ayako aufgezeigten Themen ihres biographischen Narrativs. Dabei berücksichtige ich die von Ayako gewichteten Akzente ihrer Erzählung, wie sie sie drei Jahre nach der Katastrophe in den Kontext ihrer persönlichen Erfahrungen integriert hat.

\subsubsection{Erinnerungen und lokale Verbundenheit}

Den Einstieg in Ayakos Biographie bildet eine Erinnerung an ein Kunstprojekt in einem Café, dessen Gebäude vom Tsunami 2011 vollkommen zerstört beziehungsweise fortgespült wurde. Offenbar handelte es sich um einen wichtigen Treffpunkt für die Gemeinde, so dass die lokale Bevölkerung weiterhin hofft, dieses Gebäude als "Community space“ wieder aufzubauen. Ayako verbindet mit diesem Wiederaufbau alte Erinnerungen mit neuen Möglichkeiten und Perspektiven: Der Treffpunkt wird am selben Ort entstehen, der der lokalen Gemeinschaft bereits in der Vergangenheit einen Raum zum Austausch geboten hat. Neue Erinnerungen können an einem vom Tsunami sehr in Mitleidenschaft gezogenen Ort entstehen und den Menschen eine Möglichkeit geben, zusammenzukommen.

Ayako berichtet mir zunächst sehr frei und ohne zu zögern. Sie lächelt viel und fühlt sich meinem ersten Eindruck nach wohl. In ihrer Galerie, umgeben von kleinen Gemälden und Bildern, ist sie in ihrem Element. Es ist ein bunter Raum, der nicht viele Möbel oder Sitzgelegenheiten enthält, nichtsdestotrotz auf mich aber sehr einladend wirkt. Die Stimmung ist gleich zu Beginn des Interviews sehr hoffnungsvoll und spiegelt Ayakos Tatkraft und Energie wider.

Als Ayako mit der Thematik des neuen „Community space“ das Gespräch eröffnete, hatte sie bereits sehr wichtige Themen ihres Lebens und Arbeitens genannt: Alte Erinnerungen, die sich mit Orten auch dann noch verbinden lassen, wenn einige Mitglieder der Gemeinschaft nicht mehr am Leben sind, werden zur Basis für neue Erinnerungen. Das neu entstandene Café ist ein Ort der Begegnung, der auch als Schauraum für Kunstprojekte genutzt werden soll. So soll das Geschehene dokumentiert werden und gleichzeitig zur Verarbeitung beitragen. Ayakos Bestreben, die Erinnerungen an die Katastrophe in einen positiven Narrationsrahmen zu setzen, weitet sich auch auf ihre Auslandserfahrungen aus: Ganz bewusst besuchte sie das im Jahre 2013 von einem Erdbeben geschüttelte Christchurch in Neuseeland, um eine Vergleichsperspektive für die Situation in ihrem Heimatort aufzubauen. Ayako beschreibt ihren Versuch, die Umstände der Katastrophe in Christchurch mit denen Shiogamas und anderer Regionen Sendais zu vergleichen, um Möglichkeiten der Zusammenführung der jeweiligen lokalen Bevölkerungen zu entdecken. So geplant Ayako ihre Vorgehensweise auch darstellt, betont sie doch die Facette der Entdeckung auf ihrer Reise nach Neuseeland. Die Aktivitäten vor Ort hätten sie „die Energie der Künstler" sehen lassen, die den zerstörten Raum der Stadt neu gestalte (Ayako 25.06.2014: 3). Im Unterschied dazu sei in Japans Region Sendai die Stadt 
Shiogama selbst recht schnell nach der Katastrophe weitestgehend intakt gewesen, so dass viele Menschen mit ihrem alltäglichen Leben hätten fortfahren können. Vor diesem Hintergrund bringt Ayako ihre Missbilligung gegenüber der Haltung japanischer Künstler in der unmittelbaren Zeit nach dem Desaster zum Ausdruck: „The artists said, they lost their energy, they could not make any paintings, bla bla bla. They actually can do it" (Ayako 25.06.2014: 3).

Dieses Thema liegt ihr offensichtlich am Herzen, denn sie spricht diese Sätze lauter und sehr viel energischer. Ihr Eindruck von den „kreativen Energien“ von Neuseelands Künstlern haben sie angesichts der enormen Schäden durch das Erdbeben nachhaltig beeindruckt. So beschreibt Ayako eine Stadt im Wiederaufbau, die vor allem von diesen positiven Energien der Menschen getragen wird und sich in Gemeinschaftsprojekten, Ausstellungen etc. manifestiert: „I could see what would happen. I could see the energy of the artists. [...] I don't know the previous scenery. But, it was really a ghost town" (Ayako 25.06.2014: 3). Der Eindruck des Zusammenkommens der Menschen in Christchurch und der kreativen Nutzung der noch zur Verfügung stehenden Ressourcen wirkt für Ayako bis heute nach. Die Schilderung dieser Erlebnisse bildet den direkten Übergang zu ihrem eigenen, von der Tokyoter Regierung gesponserten Projekt an Japans Ostküste. Sie will das umsetzen, was sie in Neuseeland erfahren und gelernt hat. Ihr nächstes Projekt soll die Menschen zusammenbringen und ihnen etwas über die Geschichte ihres Heimatortes beibringen. „This project provides the opportunity to learn more about the place we live in. The cultural history, the geographical situation and so on" (Ayako 25.06.2014: 4).

In Ayakos Satz, den sie mehrmals während unserer Gespräche nannte, „so there is life in there" (25.06.2014: 4), schwingt ihre Hoffnung für den Wiederaufbau ihrer eigenen Region mit. Bereits in diesem ersten Interview zeigt sich Ayakos enge Verwurzelung in ihrem lokalen Kontext. „The audience consists of people from this area. Not of people who love art, not the typical art audience" (Ayako 25.06.2014: 4).

Das erste Mal kommt Ayako auf die Geschichte der Region und seiner Menschen zu sprechen und offenbart einen historisierten Aspekt ihrer Erzählung. Ihre Aussagen zeugen von einem Bewusstsein für die tiefen kulturellen Wurzeln der lokalen Bevölkerung in der Landschaft einer Region, die sich angesichts eines weiteren Tsunamis in seiner Geschichte auf diese Wurzeln besinnt. „This coast line has been the same since the Shogun period. [...] We see the same scenery like our ancestors, it's beautiful" (Ayako 25.06.2014: 26). Gestützt wird diese erste Annahme von Ayakos folgenden Aussagen, die sich mit der näheren Beschreibung der öffentlichen Wirkung des von ihr geleiteten Projekts befassen: „It's not a typical big art project. It's more like a quiet process for local people“ (Ayako 25.06.2014: 4).

In Ayakos Darstellungen ihres Engagements und ihrer Rolle vor Ort verbinden sich zwei Dinge, die Einblicke in ihr Selbstbild erlauben: Zum einen besteht der bereits erwähnte starke Fokus auf ihre Mitmenschen vor Ort, die nicht nur ihre Rezipienten, sondern auch Freunde, Nachbarn und Bekannte sind. Dieser enge Kontakt definiert in maßgebender Weise die Zielgruppe ihres Wirkens und wird mit dem Zusatz „not the people who love art“ (Ayako 25.06.2014: 4) versehen. Kunst, 
wie Ayako sie praktiziert und anwendet, verlangt nach bestimmten Ressourcen, um Verständnis für diese Ausdrucksform bei Menschen zu erzeugen, die nicht mit Kunst im Allgemeinen vertraut sind. Besonders deutlich wird Ayakos Standpunkt anhand der wiederholten Betonung, sie würde aus diesem Grund auch keine „Kunstbegriffe“ oder auch nur den Terminus „Kunst“ für ihre Projekte verwenden: „The conversation, the dialogue, is very important. I cannot really use art and art terms" (Ayako 25.06.2014: 4). Ayako bezeichnet diesen Aspekt ihrer Arbeit als „sehr herausfordernd" („very challenging") und unterstreicht damit gewissermaßen den intellektuellen Anspruch ihrer Arbeit, deren Herausforderungen in der Übersetzungsleistung zwischen den Erzählungen der Betroffenen und dem kreativen Ausdruck liegen. Sie beschreibt sich als eine Art Dolmetscherin, die den verbalen Ausdruck von Wut, Trauer und Verzweiflung in ein Projekt umwandelt, um der Gemeinde das unterschiedliche Erleben der Katastrophe, aber auch Möglichkeiten der Heilung näherzubringen. Mit diesem Verständnis ihrer Rolle zeigt Ayako, wie so viele meiner Gesprächspartner, den Wunsch, sich mit den Emotionen auseinanderzusetzen, statt sie zu verschweigen oder vollständig zu vermeiden.

Ihr Ziel hat Ayako zum anderen mit der Schwerpunktsetzung auf den Dialog und die Konversation definiert und damit gleichzeitig ihre eigene Rolle in der Gemeinschaft umrissen. Die Rezipienten ihrer Arbeit stammen vorwiegend aus ihrer unmittelbaren Umgebung und gestalten gemeinsam mit Ayako den neuen Alltag nach dem 11. März 2011. Das wird umso bedeutungsvoller, als dass Ayakos Kunst wichtige Impulse für die Wegbereitung aus akuten psychischen Notsituationen setzen kann.

Ayakos persönliche Betroffenheit und Mitgliedschaft in dieser Gemeinschaft tritt immer wieder deutlich im Gespräch hervor. So tritt sie neben ihrer Rolle als Künstlerin auch als eine Privatperson auf, die wie ihre Rezipienten den Tsunami unmittelbar erlebt hat und emotional verarbeiten muss. Deutlich wird dies besonders bei ihrer Schilderung eines neuen Kunstprojektes, das von Seiten eines berühmten Künstlers an sie herangetragen wurde: „I refused. [...] He [the artist] wanted to do it in Shiogama. I am living here and I was like ,Eh?! An art project in an affected area? No way!'“ (Ayako 25.06.2014: 6).

Da sich die Rollen als Künstlerin, Privatperson, Anwohnerin, Nachbarin und Opfer in keiner Form trennen lassen, wird Ayako auch in diesen unterschiedlichen Facetten ihres Selbst von ihrem Umfeld wahrgenommen und angesprochen. Gleichzeitig sind es ihre Fähigkeiten der Kommunikation und ihre überregionale Vernetzung (und nicht zuletzt der ihr damit gewährte Zugang zu finanziellen Ressourcen zur Verwirklichung ihrer Projekte), die es ihr ermöglichen, ihrer Gemeinschaft auf besondere Weise dienlich zu sein. Bei unserem ersten Treffen bietet Ayako mir an, mit ihr gemeinsam einen Spaziergang durch die teilweise verwüstete Stadt zu machen. Dabei wird mir klar, wie viele Menschen Ayako persönlich kennt. Ständig wird sie angesprochen oder begrüßt jemanden, erkundigt sich nach Befinden, Gesundheit oder wie die Geschäfte laufen. Als ich Ayako im Gespräch mit anderen Betroffenen beobachte, fällt mir auf, dass die Wahrnehmung Ayakos durch Dritte einerseits 
durch ihre Rolle als Künstlerin mit Auslandserfahrung geprägt ist. Sie wird gezielt auf ihre nächsten Projekte angesprochen oder es wird sich erkundigt, was gerade in ihrer Galerie zu sehen sei.

Andererseits ist sie darüber hinaus ebenfalls ein Opfer der Naturgewalten und damit ein Teil der Schicksalsgemeinschaft: „,After 3.11] I think, I had a shock. [...] I didn't really think about art. I know creative people, I have a crew, so I had a use us' kind of feeling" (Ayako 25.06.2014: 20). ${ }^{79}$ Diese unmittelbare Betroffenheit bildet ein erst vor kurzem geschmiedetes, aber nichtsdestotrotz sehr starkes emotionales Band, das sie mit ihren Mitmenschen verbindet. Für die anderen Bürger Shiogamas scheint sie eine legitime Mitgestalterin des lokalen emotionalen Diskurses rund um den 11. März 2011 zu sein.

Aus meiner Sicht ist dieses emotionale Band einer Schicksalsgemeinschaft eine Facette von Ayakos Biographie und Arbeit, die sie aus dem Kreis meiner Gesprächspartner hervorhebt. Ayako erhält aufgrund ihrer persönlichen Verankerung in der lokalen Gemeinschaft einen anderen Zugang zur Emotionalität und deren Verarbeitung als Künstler von außerhalb es könnten. Dies bringt jedoch auch einige Schwierigkeiten für Ayako mit sich.

\subsubsection{Rollenkonflikte}

Ayakos Empfindungen als Opfer scheinen in der Zeit kurz nach dem Tsunami hin und wieder mit ihrer Arbeit als professionelle Künstlerin kollidiert zu sein. Ein ganzes Kaleidoskop an Gefühlen trat bei der Diskussion eines Projektvorschlags eines sehr bekannten japanischen Künstlers sehr deutlich zu Tage. Ayako sträubte sich zunächst vehement gegen die Idee, ein Künstler von außerhalb könne einen Ausdruck für das lokale Leid finden. In dem Moment, in dem ein anderer Interpret „,on außen“ an die Gemeinschaft herantrat, offenbarte sich ihre Identifikation als Teil der lokalen Schicksalsgemeinschaft:

„I'm living here [...] I know, maybe they [die anderen Betroffenen] don't want that, right? [...] Actually, we [der Künstler und Ayako] really fought, we kind of, well we didn't fight. But we had so many arguments at the beginning of this project. But the other artist really wanted to do this project and then it was fixed, so I had to accept." (Ayako 25.06.2014: 6)

Sie beschreibt eine Situation, in der sie von einer örtlichen Autoritätsperson vor vollendete Tatsachen gestellt und damit gezwungen wurde, dieses Projekt zuzulassen. Dennoch verstand sie zunächst nicht, worum es ging und warum sie sich auf die Idee eines Nicht-Betroffenen einlassen sollte. Ayakos Ausdruck „I'm living here“ verdeutlicht, dass sich Ayako im Moment des Antrags vorrangig nicht als Künstlerin angesprochen fühlte, sondern als Anwohnerin und Opfer in einer zerstörten Region.

79 Siehe Kapitel 8.4 „Das Erleben des 11. März 2011 - Eine andere Realität“. 
Erst im Laufe der Zeit wandelte sich ihre Sichtweise durch das Heraustreten aus ihrer Rolle als Betroffene in die einer kunstverständigen Kollegin, die zunehmend die Möglichkeiten des Projektes wahrnahm: ${ }^{80}$ „I was like, I really want to quit this project. [...] But as time went by we tried to learn more about the town itself. [...] So, we tried to learn more about ourselves. [...] Anyway, I really appreciate this project" (Ayako 25.06.2014: 6-7).

Schlussendlich war es diese künstlerische Initiative von außen, die ihr den Anreiz gab, mehr über die Geschichte der Region zu lernen, mit den Menschen in der unmittelbaren Nachbarschaft ihres Wohnortes in Kontakt zu treten und sich mit ihren eigenen familiären Wurzeln auseinanderzusetzen. Diesen persönlichen Lernprozess, der in die Akzeptanz des Projekts und in die Auseinandersetzung mit der regionalen Geschichte mündete, wird sie auch zu einem späteren Zeitpunkt des Interviews wieder erwähnen. Letztendlich habe sie sich aufgrund der vielen Lernoptionen für eine Zusammenarbeit entschieden und es auch nicht bereut: „We will continue this project for this year, too" (Ayako 25.06.2014: 7).

Dieses Projekt scheint eine der wichtigsten Möglichkeiten für Ayako zu sein, ihre „Mission“ nach der Katastrophe zu beschreiben: „,...] this year's mission is to train people“ (Ayako 25.06.2014: 7). In Anbetracht der Umstände, dass es vor Ort keine Kunst-Nonprofitorganisation gibt, will Ayako andere Optionen des Austausches erarbeiten, damit die Menschen in einen Dialog miteinander treten. Ayako betont mehrmals, dass es ihr dabei nicht um oberflächliche, sinnentleerte Vergnügungen geht, die den Anstrich eines Kunstprojekts bekommen, sondern um einen Begegnungsraum lokaler Menschen aus allen Bereichen des Lebens. „We should take advantage of those opportunities more and it has to relate more to the local context" (Ayako 25.06.2014: 8), ist einer ihrer zentralen Punkte beim Thema Lokalität. Die Bedeutungs- und Sinnzuschreibung an Festivals und an sonstigen Veranstaltungen mit Beteiligten des gesamten Küstenstreifens könnten ganze Landstriche miteinander verbinden, so ihre Hoffnung. So werden auch Inseln in die Projektplanung einbezogen, die der Küste vorgelagert sind und nur von wenigen Menschen bewohnt werden: „We try to see, we see these islands as a basecamp [...] We are learning so much" (Ayako 25.06.2014: 8).

Als eine der Künstlerinnen und Künstler der Region greift Ayako mit ihrer Suche nach Verbindungen, Fertigkeiten und zwischenmenschlichen Anknüpfungspunkten verschiedene lokale Diskurse auf und verbindet sie in einem Diskursraum, in dem die Gemeinsamkeiten der Küstenbewohner im Vordergrund stehen. Dass sie selbst während dieser Tätigkeit viel über die Region, die Vergangenheit und die Lebensweise vorangegangener Generationen gelernt hat, hat sie im Laufe des Gesprächs immer wieder angeführt. Ayako präsentiert sich damit nicht in einer Expertenrolle, sondern als Lernende in einem Diskursrahmen, der ihr durch ihre verschiedenen Zugangs-

\footnotetext{
${ }^{80}$ Um die Anonymität meiner Gesprächspartner zu wahren, behalte ich mir vor, nicht genauer auf das Projekt eingehen. Andernfalls könnten möglicherweise Rückschlüsse auf die involvierten Personen geschlossen werden.
} 
möglichkeiten, und nicht zuletzt aufgrund ihrer unmittelbaren Betroffenheit und Emotionalität nach 2011, verschiedene Türen zu anderen lokalen Netzwerken öffnet. Bereits in dieser frühen Phase des Interviews wird deutlich, dass sich Ayako an einer Schnittstelle zwischen den verschiedenen lokalen Netzwerken und ihren Diskursräumen bewegt, die ihre eigene Lernmotivation nähren. Wie tief Ayakos regionale Wurzeln greifen, wird jedoch erst im folgenden Abschnitt des Interviews deutlich, in dem wir uns verstärkt mit ihrer Herkunft und Lebensgeschichte befassen.

\subsection{Biographische Hintergründe}

Ayako erzählt flüssig, als sie beginnt, von ihrer Geburt in dem kleinen Ort an Japans Ostküste und ihren, bereits in jungen Jahren aufkeimenden, Interessen an unterschiedlichen Lebensperspektiven zu berichten. Damit setzt sie ihren lokalen Kontext bereits sehr früh in einen größeren Bezugsrahmen und ergründet retroperspektiv ihre Beweggründe für ihre Begeisterung für das Bereisen anderer Länder:

„I have been interested in various ways of thinking, especially since I am living in Japan, it's like one way and, you have to follow the rule, so, it was uncomfortable for me. When I was in elementary school, this just wasn't right, you know? [...] The world must be bigger." (Ayako 25.06.2014: 9)

Aus diesem Zitat spricht ein früh einsetzender Wunsch Ayakos, die Welt zu entdecken und die an sie herangetragenen Regeln der japanischen Gesellschaft zu hinterfragen. Rückblickend verortet sie diesen Wunsch bereits in ihrer Grundschulzeit. So beschreibt Ayako es als „lucky“, dass ihre Mutter ihr bereits in diesen jungen Jahren die Möglichkeit bieten konnte, sich mit diversen Perspektiven auf die Welt auseinanderzusetzen: „[...] my mom is interested in overseas exchange students and they stayed over night at my place, so I could see that [Japan] is not the only world" (Ayako 25.06.2014: 9). Dieser frühe Kontakt mit ausländischen Perspektiven scheinen ihre Neugier auf eine Welt außerhalb Japans geweckt zu haben, denn sie fährt fort: „And I wanted to get out of this country as soon as possible, so I studied English" (Ayako 25.06.2014: 9).

Ayako stellt hier einen Sinnzusammenhang her zwischen der Aufgeschlossenheit ihrer Mutter gegenüber ausländischen Studenten und ihrem persönlichen Lebensweg. Dieser Zusammenhang zieht sich durch ihre gesamte biographische Darstellung und stellt Ayako in einen unmittelbaren narrativen Bezug zu ihrer Familie. Damit schloss sie den Einfluss anderer Beteiligter auf ihre folgenden Entscheidungen ein und nahm sich selbst aus dem unmittelbaren Fokus ihrer Erzählung. Zunächst entschloss sie sich im Alter von 15 oder 16 Jahren, an einem Austauschprogramm teilzunehmen. „And my parents said, they let me go, they paid“ (Ayako 25.06.2014: 9).

Mit der Unterstützung ihrer Familie konnte Ayako ihren Wunsch, die Welt zu entdecken, bereits früh erfüllen, was erklären könnte, warum sie, im Gegensatz zu 
Kogoro, ihren durchaus unkonventionellen Weg in ein positives Licht rückt. Sie zeichnet das Bild einer Familie, die Ayakos Wünsche nicht nur wahrnimmt und respektiert, sondern sie sogar aktiv bei der Umsetzung ihrer Pläne unterstützt. Ayakos Schilderungen zufolge war es daher nur ein logischer Schritt von einem ersten Austauschprogramm zu einem Studienplatz an einer Kunstuniversität in Kanada. „That university is an art university and teaches young culture like contemporary art. So it was right for me" (Ayako 25.06.2014: 9). Die Unterstützung ihrer Familie für ihren eingeschlagenen Weg basiert unter anderem darauf, dass in ihrer Familie ein Verständnis für Kunst existiert, auf das sie später zu sprechen kommt.

Ihre Erzählung folgt zunächst einem chronologischen Strang, der ihre Rückkehr nach Japan und den Abschluss der High-School beschreibt, bevor sie sich dem Kunststudium in Vancouver widmete. Diesen Abschnitt ihres Lebens und ihre Gefühlslage zu jener Zeit hält sie sehr knapp. Ihrem schnellen Fortschreiten in diesem Teil des Narrativs nach zu urteilen könnte es gut sein, dass sie zu diesem Zeitpunkt in Japan nicht wieder richtig Fuß gefasst hat: „And right after graduation, I moved to Canada" (Ayako 25.06.2014: 9). Der von ihr gewählte Kunststudiengang der "youth culture" und "contemporary art" gab ihr die Möglichkeit, ihren Fokus auf „art around culture“ zu legen.

„I was working at the gallery, like a non-profit artist at the center for two years. So I learned about the gallery system and I met a lot of art people. At the time, I was a reporter for a Japanese online magazine to inform about what was going on in Canada." (Ayako 25.06.2014: 10)

Ihre verschiedenen Tätigkeiten in der kanadischen Kunstszene mit den diversen Verbindungen zu Künstlern lieferten ihrer Erzählung zufolge nicht nur wichtige Impulse für ihren weiteren Werdegang, sondern hielten gleichzeitig den Bezug zu Japan aufrecht. „Working for this Japanese online magazine, I could meet great artists right from the start" (Ayako 2014: 10). Die Tätigkeiten für das Online-Magazin legen nahe, dass es ihr nicht um einen vollständigen Bruch mit ihrem Herkunftsland geht, sondern um eine Erweiterung ihres Horizonts. Aus dieser Zeit stammt auch ihr Plan, eine eigene Galerie zu eröffnen und mit Kunst zu arbeiten:

„I wanted to have my own gallery. I wanted to work with art. So I decided to go back to Japan. Vancouver is beautiful but nothing is going on. I was still young, so I wanted to try something. So I was coming back to Japan and was working at a publishing company in Tokyo." (Ayako 25.06.2014: 10)

Vor dem Hintergrund ihrer bisherigen Schilderungen wird deutlich, dass Ayako zum damaligen Zeitpunkt etwas bewegen und ihre eigenen Fähigkeiten im Kunstkontext testen wollte. In dieser ersten Phase der Erprobung ihrer Möglichkeiten im heimatlichen Kontext führte es sie in einen Job, der sie zwar finanzierte, ihr jedoch 
keinerlei kreativen Freiraum ließ. Entsprechend fällt ihr Urteil aus: „I was making books, but I was needed to do editing [...] it wasn't right for me" (Ayako 25.06.2014: 10). In diese Zeit fällt ein Angebot ihrer Künstlerfreunde aus Kanada, die Ayako die Möglichkeit einer eigenen Ausstellung boten, die sie jedoch aufgrund ihrer beruflichen Verpflichtungen ausschlagen musste.

„My friends in Canada gave me the opportunity to create a show in Canada. The asked me two times and they said 'We have the money, we pay for everything', but I was working. I started thinking about my life. What's important? There are real opportunities but I am stuck in this office."

(Ayako 25.06.2014: 10)

Die Verhinderung dieses Projektes aufgrund ihrer eng gesteckten Rolle als Angestellte spielt meines Erachtens eine wesentliche Rolle bei allen folgenden Entscheidungen ihres weiteren Lebens. Diese Möglichkeit verstreichen lassen zu müssen, setzte einen tiefgreifenden Reflexionsprozess in Gang, der Ayako über die Ziele ihres Leben nachdenken und Entscheidungen treffen ließ: „And really, this is not right. So I decided to quit." (Ayako 25.06.2014: 10). Mit der Kündigung ihres Verlagsjobs nimmt sie wieder engeren Kontakt zu Tokyos Kunstszene auf. Ihr Job als Editor bei einem Verlagshaus hat ihr vor Augen geführt, dass sie mehr Freiraum zum Agieren braucht. Doch nicht nur deshalb verleiht sie diesem Schritt ihres Lebens entsprechende Bedeutung:

„During my stay in Tokyo, I met a lof of art people [who were] working in museums, art directors and now I know art people can go anywhere. So I didn't need to be in Tokyo just to make money to pay the rent."

(Ayako 25.06.2014: 11)

Diese Phase von Ayakos Leben ist geprägt von ihrem zunehmenden Verständnis der Möglichkeiten und Vorteile einer ortsunabhängigen Künstlertätigkeit, wie ihre Kollegen es ihr vorleben. „At that time I thought, that location is not important for me" (Ayako 25.06.2014: 11). In der folgenden Erzählung ihrer beruflichen wie privaten Orientierungsphase betont sie erneut die Unterstützung durch ihre Familie, die es Ayako ermöglichte, ihren ursprünglichen Plan aus der Zeit in Kanada zu verfolgen und umzusetzen. Sie hebt mit einem Augenzwinkern hervor, sie sei wahrlich verwöhnt (,spoiled“), da sie auf einen Raum im Haus ihres Vaters für eine eigene Galerie zurückgreifen konnte:

„He [ihr Vater] wanted to have that art space, he wanted to do something with art, so I was like Hey, I could stay in my hometown. [...] I didn't care about the location for my career. I just need to try myself. [...] so at the beginning, I didn't care about anything." (Ayako 25.06.2014: 11) 
In diesem Abschnitt verdeutlicht Ayako eine Konstellation von Entscheidungen, mit denen sie einen entscheidenden Sinnzusammenhang konstruiert. Das eine ist die Haltung ihres Vaters gegenüber der Kunst und des damit verbundenen Verständnisses für Ayakos Beweggründe und Wünsche. Das andere ist Ayakos betonte Gleichgültigkeit gegenüber der Lokalität ihres Wirkens.

Ihrem Vater fiel es nicht schwer, ihr den Raum zur Verwirklichung ihres GalerieTraumes zur Verfügung zu stellen, da er selbst schon über solch eine Option nachgedacht hatte. „He [ihr Vater] made the space, but before I came back here“ (Ayako 25.06.2014: 11). Die Hilfestellung seitens ihrer Familie hielt also weit über die Zeit ihres Studiums hinaus an und setzte einen entscheidenden Impuls für Ayako, nach jahrelanger Abwesenheit in ihre Heimatstadt zurückzukehren. Grundsätzlich ist ihre Familie sowohl als Einheit als auch in Form verschiedener Einzelpersonen in ihrer Biographie sehr präsent. Das unterscheidet Ayakos biographische Erzählung von den meisten anderen meiner Interviews mit Künstlern.

Die 2014 so überaus wichtige Rolle der lokalen Verwurzelung hingegen scheint zu diesem Zeitpunkt noch keine große Bedeutung gehabt zu haben. Die Aussage „I didn't care about the location of my career" (Ayako 25.06.2014: 11) betont in aller Deutlichkeit die gerade erst gelernte Lektion eines ortsunabhängigen Künstlers, auf dessen Wohn- und Galeriestandort es nicht ankommt. Der Ort des Wirkens orientiert sich zu diesem Zeitpunkt noch nicht an geographischen Merkmalen, sondern ist noch ganz auf Ideengenerierung und Projektkonzeptionen fixiert. Ayakos Verankerung in einem überregionalen Diskursraum, der sich bis auf die internationale Ebene erstreckt, hat den lokalen Bezug ihres Handelns weit in den Hintergrund rücken lassen. „I just wanted to hide and train myself" (Ayako 25.06.2014: 11).

$\mathrm{Zu}$ diesem Zeitpunkt ist sie eine 25-jährige, weit gereiste Künstlerin, die gerade erst dabei ist, sich und ihre Wirkmöglichkeiten zu entdecken. Auch möchte Ayako zu diesem Zeitpunkt zunächst gar nicht weiter in Erscheinung treten, geschweige denn im Mittelpunkt einer Gemeinschaft stehen: „I didn't care about the location or my career. I just needed to try what I could do. [...] I just wanted to practice [my art]" (Ayako 25.06.2014: 11). Ayako zeichnet das Bild einer jungen Frau, die sich zunächst ausprobieren und keinesfalls eine professionalisierte Rolle einnehmen möchte. Ayakos Perspektive auf die sich bietende Möglichkeit der Rückkehr in ihren Heimatort erfährt in ihrer Reflexion jedoch langsam einen Wandel, als ihr die Reaktion ihres direkten Umfeldes vor Augen geführt wird: Die Tatsache, dass eine junge Frau in ihren Heimatort zurückkommt und einen „kulturellen Ort“ erschafft, führte zu entsprechendem Engagement auf Seiten der Stadt, die Ayako bis heute mit finanziellen Mitteln unterstützt. Ayako jedoch bleibt bei ihrer Version, die Belange der Stadt bei ihrer Entscheidung nicht berücksichtigt und dies den entsprechenden Stellen auch mitgeteilt zu haben: „I am just trying to do what I am feeling [...] the location is not important to me, [...] but really, they don't really care, ne" (Ayako 25.06.2014: 11). Umso mehr überrascht sie die Reaktion der entsprechenden Akteure, sie unterstützen zu wollen: „,What?' [...] At the beginning I was saying, I don't 
care about payment. I'm not trying to make Shiogama city better with this art" (Ayako 25.06.2014: 11).

Interessanterweise spricht Ayako bei der Beschreibung ihres Arbeitsansatzes im Präsens und nutzt erst dann wieder die Vergangenheitsform. Mit dem Satz „nur tun zu wollen, was sie empfindet" drückt sie aus, was viele Gegenwartskünstler von sich sagen: Privates Empfinden und berufliche Ausrichtung verschmelzen zu einer Lebensweise, deren jeweilige Bestandteile den Sphären aus Privatheit und Profession im Rückblick nicht mehr trennscharf zuzuordnen sind. Mit der Akzeptanz dieser unerwarteten Förderung seitens der Stadt erfährt auch Ayakos Erinnerung eine neue Form der Betrachtung: Erst zu diesem Zeitpunkt habe sie entdeckt, dass das Arbeiten in einer Gemeinschaft wirklich schön sei und dass sie genau dies in Kanada gelernt habe: „In Canada, no big things were happening, but there are so many unique art communities and I could see the energy of people" (Ayako 25.0.6.2014: 11-12).

Hier zeigt sich Ayakos besonders ausgeprägte Fähigkeit zur Reflexion. Sie vermischt die Zeitebenen nur geringfügig und kann sich in ihre jeweiligen Gefühlslagen zurückversetzen, die ausschlaggebend für ihre Entscheidungen waren. Der erneuerte Kontakt zu den Menschen ihrer Heimatstadt stellt den Beginn einer engen Beziehung dar und schließt den Kreis mit ihrer Vergangenheit. Dieser Bezug zu ihrer eigenen Geschichte spiegelt sich auch in Ayakos Sensibilität für Historizität im Allgemeinen wider: Sie setzt die Aktivitäten der Künstler in Kanada mit deren Intentionen und Empfinden in Verbindung und zieht neue Lehren aus bereits gemachten Erfahrungen: „[...] in art, young people think they are making culture and history. [...] They are believing in it" (Ayako 25.06.2014: 12). Kanadas Künstler werden in ihrer Narrative zu Produzenten von Kultur und Geschichte, während sie sie gleichzeitig leben.

In ihrer eigenen Erzählung setzt Ayako diesen neuen Denkansatz in einen engen Zusammenhang mit ihrem eigenen Engagement, das bereits vor 2011 stark im Wachsen begriffen war: Mit der Rückkehr in ihren Heimatort legte sie besonderen Wert auf die Etablierung einer vertrauensvollen Beziehung zu ihren Mitmenschen und den offiziellen Vertretern der Präfektur Miyagi. Dies wiederum eröffnete ihr neue Möglichkeiten, diesen lokalen Kontext auch nach Tokyo zu tragen und über ihre Arbeit zu sprechen: „I had the opportunity to talk about my art, my town, and my activities. So art people are paying attention to my activity, even so I am here in this small little town" (Ayako 25.06.2014: 12).

An dieser Stelle zeigt sich eine Besonderheit in der Art und Weise, wie Ayako die Begrifflichkeiten „lokal“ („local“) und „Ort“ („location“) verwendet.

„I still think that location is not really important, but people's mind is really important. Wherever you are, if you have guts and mind and spirit and purpose, you can do whatever you want [...] art people can go anywhere. So, I don't need to be in Tokyo, just to make money." (Ayako 25.06.2014: 12) 
Dem Ort ihrer Tätigkeit räumt sie einen geringeren Stellenwert sein, da er sich auf ihre eigene Anwesenheit vor Ort in Shiogama bezieht. Gleichzeitig lässt sie durchklingen, dass sie mit ihrer Arbeit eine weitere Facette von Freiheit in ihrer künstlerischen Arbeit entdeckt hat.

In greifbaren Initiativen durch die „lokale“ Bevölkerung kommt dieser „spirit“ zum Tragen, in denen die enge wechselseitige Beziehung mit Menschen in Ayakos unmittelbarer Umgebung im Mittelpunkt steht. Im Anstreben dieser engen Beziehung und dem Erreichen der Menschen durch ihre Arbeit erfüllt sich ihre selbstdefinierte Aufgabe: Mit Nicht-Materiellem etwas Essentielles im Leben der Menschen anzustoßen. Auf diesen überaus wichtigen Aspekt wird sie zu einem späteren Zeitpunkt erneut zu sprechen kommen, wenn es um ihre Erfahrungen vom 11. März 2011 geht.

Ayakos Offenheit im direkten Umgang mit den Herausforderungen des Lebens prägt ihre lokalen Initiativen. Um die internationale Bindung dennoch nicht zu verlieren, arbeitet sie nach wie vor mit Künstlern aus Kanada zusammen. Ich habe den Eindruck gewonnen, dass Ayako damit einen für sie stimmigen Kompromiss zwischen ihrem Wunsch der Beziehungspflege vor Ort und der gelegentlichen Nutzung von Bühnen außerhalb ihres Heimatortes schaffen will, um in der überregionalen Kunstszene Japans präsent zu bleiben.

Diese, in meinen Augen überaus wichtige Interviewpassage zur Lokalität, führt entscheidende Facetten von Ayakos Selbstbild und ihres Narrativs zusammen, die vor dem Hintergrund der nun folgenden Beschreibung ihres Erlebens des 11. März 2011 entscheidend sind. Mit ihrer Selbstdarstellung als moderne, international gereiste und weit vernetzte junge Frau verankert Ayako sich selbst in einem größeren Rahmen als ihn ihre Heimatstadt bieten kann. Gleichzeitig schreibt sie sich auf diese Weise positive Eigenschaften ihrer Generation wie Flexibilität zu und verdeutlicht, wie wichtig ihr der enge Bezug zu ihrer Familie und den Menschen in ihren Umfeld ist. Auch wenn Ayako die Rückkehr an ihren Heimatort der besonderen Unterstützung ihrer Familie zuschreibt, gibt sie mir durch die Erwähnung ihrer Künstlerkollegen und überregionalen Aktivitäten dennoch das Gefühl, dass sie weiterhin selbstbestimmt arbeiten will, um ihre Individualität als Künstlerin auszuleben.

Ayakos Verbundenheit mit dem Ort ihrer Herkunft wird in den folgenden Passagen ihrer biographischen Darstellung noch an Bedeutung gewinnen. Das Zusammenführen der lokalen Ebene mit der internationalen Sphäre verleiht Ayako einen hervorgehobenen Status innerhalb ihrer Gemeinschaft, der ihrer Identität als Künstlerin eine besondere Note gibt. Gleichzeitig setzt sie sich selbst in ein enges Wechselwirkungsverhältnis mit ihrer Umwelt, indem sie ihre Entwicklung als maßgeblich durch ihr Elternhaus beeinflusst beschreibt. Daher legt sie den Fokus ihres Narrativs verstärkt auf die interdependenten Beziehungen, die ihr Leben in dieser Form begünstigen.

Diese enge Verbindung zwischen ihrer Familie und ihrer Kunst legt Ayako auch in Ausführungen dar, die eine künstlerische Familientradition erkennen lassen sollen: Ayako beschreibt ihre Familie als „kulturell“ (Ayako 25.06.2014: 13), da auch 
ihr Großvater und ihr Onkel kreative Interessen hatten. Ihre Situation innerhalb ihrer Geschwisterkonstellation als mittleres von drei Kindern verleiht ihr nach eigener Aussage eine Sonderrolle, gekennzeichnet durch geringe Verantwortung gegenüber ihren Eltern. So konnte sie früh ihrer Neigung nachgeben, ihren eigenen Weg zu gehen, ihre Wünsche umzusetzen und Chancen für Auslandsaufenthalte nutzen. Interessanterweise schreibt Ayako sich selbst eine beinahe passive Rolle in der frühen Gestaltungsphase ihres Lebens zu, indem sie die Initiative, fast schon entschuldigend, in ihre Familiengeschichte verlagert: „My grandfather is interested in [...] foreign culture, that's why he was showing foreign films. [...] I'm not the person who started this thing. More like my family, probably. I have like a gene" (Ayako 25.06.2014: 13). Sie fährt weiter fort: „[...] there are so many creative people in my relatives, so probably my family understands art. [...] I have a kind of cultural family" (Ayako 25.06.2014: 13). In ihrer biographischen Darstellung wirkt dies wie eine Grundvoraussetzung für ihre persönliche Entwicklung und zugleich wie eine Rechtfertigung für ihre Aktivitäten außerhalb Japans. Besonders der Satz „I am not the person who started this thing " wirkt, als sei ihr beruflicher Werdegang eine logische Konsequenz aus dem familiären Umfeld und ihren Angeboten und habe sich damit ihrer Kontrolle entzogen. Besonders im Vergleich zur Situation ihrer Geschwister tritt der Aspekt der geringen Verantwortung gegenüber ihrer Familie (beispielsweise deren Erwartungshaltung an sie) in ein besonderes Licht: „My brother probably has to take over my dad's business. My sister can, I don't know... and I thought, I want to see the world" (Ayako 25.06.2014: 13).

Ayakos beiden Geschwistern hingegen attestiert sie lachend ein geringes Interesse an Kunst: „My brother and sister usually don't get what I am doing" (Ayako 25.06.2014: 13). Die treibenden Faktoren der Familie scheinen vor allem die Offenheit ihrer Mutter für ausländische Perspektiven und einige männliche Mitglieder ihrer Familie mit besonders großem kulturellem Interesse zu sein (Vater, Onkel und Großvater). Sie alle beeinflussten Ayako, da sie ihr nicht nur theoretische Möglichkeiten boten, sondern sie ermutigten, die Welt für sich zu entdecken. „My dad, my grandfather, they tell me culture is very important. [...] And my grandfather said: 'You have to go out [of Japan] and see the world'" (Ayako 25.06.2014: 13).

Mit ihrer Erzählung bringt Ayako gleichzeitig ihre große Dankbarkeit für die Freiheiten zum Ausdruck, die sie sowohl ihrer Familie, ihrer eigenen Position in der Geschwisterabfolge als auch dem breiteren gesellschaftlichen Umfeld zuschreibt. In diesem familiären Klima fühlte sich Ayako bereits in sehr jungen Jahren in ihren Wünschen ernst genommen. Man kann also argumentieren, dass es ihr gelang, sich in der Kunst eine entsprechende Nische zu suchen, in der sie ihre Perspektiven gesehen und geachtet fühlte.

Unabhängigkeit und Freiheit kommen neben ihrer familiären Ungebundenheit allerdings auch in anderer Hinsicht zum Ausdruck: wenn Ayako über ihre sonstige, von ihren Eltern abweichende Lebensführung spricht, die auch im Alter von 32 Jahren noch keinen Lebenspartner oder gar Heiratspartner vorsieht. Auch im modernen Japan ist dies ein eher ungewöhnlicher Umstand. 1970 betrug das durchschnittliche 
Heiratsalter von Frauen 24 Jahre, das von Männern 26,9 (Salamon 1986: 131). Bis heute hält sich das Ideal einer Heirat vor dem 24. Lebensjahr für Frauen. Es scheint jedoch trotz diesbezüglicher Gedanken ihrer Eltern keine großen Einmischungen in Ayakos Leben zu geben, die ihr Verhältnis zu ihnen trüben könnte. Ayako zeichnet von sich das Bild einer jungen Frau, die sich weitestgehend als modern und aufgeschlossen versteht und dabei ihre Bande an Heimat, Familie und gemeinschaftliches Umfeld nicht als einengend betrachtet. Dieses Narrativ setzt eine entscheidende Rahmung bei der Betrachtung ihrer Erzählung über den März 2011, der sowohl Ayako selbst als auch das gesamte Gefüge ihrer Lebenswirklichkeit bedrohte.

\subsection{Das Erleben des 11. März 2011 - Eine andere Realität}

Ayakos vergleichsweise kurzer Überblick über ihre näheren Familienverhältnisse wird begleitet von einer detailreichen Erzählung über den 11. März 2011, den sie vor Ort erlebte. Sie ist deshalb nicht nur eine Gesprächspartnerin, die mir wertvolle Hinweise auf das Wirken einer Künstlerin in einer lokalen Gemeinde an der Ostküste Japans gab, sondern kann gleichzeitig aus Sicht einer unmittelbar Betroffenen sprechen. In ihrem Narrativ zeigt sie, wie sie den Schock ihrer Erfahrungen mit Hilfe ihrer Aktivitäten als Künstlerin Stück für Stück zu einer neuen Realität formte und auch anderen Gemeindemitgliedern half, mit Verlust, Schmerz und Traurigkeit umzugehen.

\subsubsection{Direkt nach dem Tsunami - Ein neuer Alltag}

Eine Tsunamiwarnung seitens der örtlichen Behörde hatte Ayako nicht ernst nehmen wollen, als sie das Beben spürte. Erst auf Drängen ihres Bruders war sie mit in dessen hoch gelegenes Apartment gekommen. Kaum dort eingetroffen, konnte sie von dort aus die Welle und die sich entfaltende Zerstörung beobachten. Der Schreck über ihr sehr knappes Entkommen findet noch heute ihren Ausdruck: „If we were five minutes late, we would have been in the wave. Scary, ne? It was actually dangerous" (Ayako 25.06.2014: 15). Für fünf auf die Katastrophe folgende Tage blieb Ayako im Apartment ihres Bruders und beobachtete, wie vor allem alte Häuser weder der Gewalt der Welle, noch den daraus entstandenen Schäden hatten standhalten können. Ayakos Galerie war von Schlamm und Unrat zerstört worden und erst eine Woche später stand ihnen wieder Strom zur Verfügung.

Für die Gefühle von Fassungslosigkeit und einer gewissen Realitätsferne angesichts dieser plötzlich über sie hereinbrechenden Katastrophe findet sie folgende Worte: „I thought, I couldn't wear a skirt. My mind was in a real survival mind set. [...] Wherever I go, I need everything" (Ayako 25.06.2014: 15).

Für besonders interessant halte ich die enge Verbindung zwischen Alltagskleidung und ihrem psychischen Schockzustand, die Ayako hier aufzeigt. Das Tragen eines Rockes, in Japan als weibliches Attribut und als angemessene Alltagskleidung 
betrachtet, setzt Ayako mit einer Normalität gleich, die sie zum damaligen Zeitpunkt nicht mehr empfindet. Ihr neues Lebensgefühl, ausgelöst durch die völlige Hilflosigkeit und Ohnmacht angesichts der entfesselten Naturgewalten, lässt ihr ein solches Attribut des normalen, unbeeinträchtigten Alltags als nicht angebracht erscheinen. Wie eine zeitliche Zensur hat sich der Tsunami in ihre Realität gedrängt und hat etwas Neues erschaffen, das nach einem anderen Ausdruck verlangt: „A real backpack. With jeans, and you know?" (Ayako 25.06.2014: 15). Die Jeanshose, zusammen mit allen weiteren nötigen Gegenständen für einen weiteren Notfall, trug sie noch wochenlang mit sich herum, um vorbereitet zu sein. Diese Hose zu tragen ist damit Ausdruck ihres „survival mind set“, wie sie es selbst nennt (Ayako 25.06.2014: 15). Der von ihr gewählte Kleidungsstil veranschaulicht Ayakos innere Gefühlswelt außerhalb jeder ihr bekannten Realität. Ihre neue Wahrnehmung ist geprägt von den Gefühlen des Schocks, der Angst und der Hilflosigkeit, zu denen sich nach einigen Wochen noch Ungläubigkeit gesellt: Auch am Ende des Monats fuhren weder Züge noch gab es Gas oder Benzin. Diese eingeschränkten Bewegungsmöglichkeiten verstärkten ihr Gefühl der Ohnmacht: „We were kind of stuck in this town [...]“ (Ayako 25.06.2014: 16).

Ayako erschafft an dieser Stelle ihres Narrativs mit wenigen Worten eine Atmosphäre, in der ihre Versuche deutlich werden, sich in einer Welt zurechtzufinden, die sich schlagartig um sie herum verändert hat: Ganze Straßenzüge existieren nicht mehr, Häuserreihen sind mitsamt ihren Bewohnern verschwunden und die stehengebliebenen Wohnungen sind zum Großteil unbewohnbar. Unter der Annahme, dass sich jeder entlang der Ostküste in einer ähnlichen (Gefühls-)Lage befände, ließ Ayako sich Ende März 2011, drei Wochen nach dem Tsunami, in die nahegelegene Stadt Sendai mitnehmen. „I was wearing, you know, everything. But people [there] were wearing skirt and [...] I was like: ,What?' [...] The train wasn't running and we didn't have gas, no gasoline. I thought everyone was having this kind of life, but actually that was not the case" (Ayako 25.06.2014: 16).

Die Konfrontation mit einer Realität außerhalb ihrer eigenen, in der die Normalität noch weitestgehend existierte, scheint ihr geholfen zu haben. Der Rucksack mit den von ihr ausgesuchten Materialien zum Überleben wurde in der Folgezeit schrittweise reduziert: „[...] I don't know whether it was good or not, but, I was kind of reducing the survival kit" (Ayako 25.06.2014: 16). Die langsame Rückeroberung eines Alltags und einer neuen Normalität wird von Ayako als eine echte Anstrengung beschrieben, für die sie sich eine Partnerin suchte:

„I tried to wear a skirt. It was more like a mental training. [...] But I couldn't really recover. [...] That lady [sie nimmt Bezug auf eine Besucherin während des Interviews], she is one of my team. We were in the same kind of situation. [...] So we tried, whenever we see each other, ok, let's wear a T-Shirt [...] We try for our mentality. Like: 'Let's wear a skirt! Maybe we will be ok', that kind of thing." (Ayako 25.06.2014: 16) 
Die gegenseitige Unterstützung ermöglichte es Ayako, ihre Emotionen zu verarbeiten und langsam eine neue Routine zu entwickeln. Die Betonung des „mental training“ und der schrittweisen Rückeroberung ihrer weiblichen Kleidung gleicht einer Suchbewegung nach einem neuen Selbst. Dennoch sollte es noch weitere drei Monate bis zum Juli 2011 dauern, bis sich beide Frauen in ihren Röcken wieder wohl zu fühlen begannen. Der Schnelligkeit, mit der der Tsunami ihr bisheriges Leben zerstörte, steht der Langsamkeit der emotionalen Verarbeitung gegenüber.

\subsubsection{Die Verarbeitung von Ohnmacht, Verlust und Trauer}

In Ayakos Fall fällt das Auftauchen aus dem emotionalen Schockzustand zeitlich mit der Reinigung und Inbetriebnahme ihrer Galerie zusammen, was ihr eine tägliche Routine während dieser Zeit des Wiederaufbaus und der seelischen Verarbeitung der Geschehnisse verschaffte: „In July, I could feel comfortable again. I feel like I'm back" (Ayako 25.06.2014: 17). Eindrücklich beschreibt sie in diesen Passagen die enge Verbindung, die die empfundenen Emotionen zwischen ihr und ihrem Umfeld erschaffen haben, aber auch die zu bewältigende Heftigkeit dieser Gefühle, die ihre Zeit brauchen, um verarbeitet werden zu können: „I took one step at a time [...] So I tried to run the gallery again, I made flyers and clothes, that kind of thing" (Ayako 25.06.2014: 16). Erst im Juli 2011 erlebte sie sich zunehmend wieder als aktiv gestaltende Persönlichkeit, die Einfluss auf ihr Leben nimmt. Auch wenn die von ihr eröffnete Ausstellung in ihrer Galerie nur wenige Menschen anlockte, erfüllte die Arbeit ihren Zweck: „Not so many people came to this [first] exhibition. But, it was important for me. To have my life back. [...] I was happy and the people [...] were happy to see art pieces, so they were ok. Because they think it's getting better" (Ayako 25.06.2014: 17). Ayako lächelt viel und lacht auch hin und wieder, während sie mir von dieser ersten Erholungsphase berichtet. Dabei wirkt sie auf mich wie jemand, der noch nicht ganz mit den Ereignissen abgeschlossen hat. Der Rock als gegenständlicher Ausdruck ihres Findungsprozesses in eine Rolle als Frau, Galeristin, Künstlerin und Gemeinschaftsmitglied markiert einen entscheidenden Wendepunkt in ihrem Leben, der ihr nicht zuletzt auch diejenigen Rollen vor Augen führt, die sie nicht ausfüllt: „I don't have family [...] I don't know where to go to if something happens, I don't know where to go to. Probably, Sendai people have a similar mind“ (Ayako 25.06.2014: 17).

In dieser Aussage spiegelt sich eine Kehrseite der ihr eingeräumten Freiheit und Lebensweise und die Bedenken ihres Umfeldes, dass sie mit ihren 32 Jahren noch keine eigene Familie hat und die Rollen der Ehefrau und Mutter noch nicht erfüllt. Ayako verwendet diese Bedenken jedoch innerhalb ihres Narrativs geschickt als Anknüpfungspunkt zu der sich möglicherweise auch in anderen Regionen abzeichnenden Hilflosigkeit angesichts eines möglicherweise nach wie vor drohenden Verlustes der Heimat: Auch im Juni 2014 ist ihr diese Möglichkeit einer erneuten zukünftigen Katastrophe nach wie vor präsent und wirkt sich auf ihre Lebenseinstellung und die von ihr initiierten Projekte aus. 
Die Verbundenheit mit ihrem Heimatort ist durch die Geschehnisse im März 2011 gewachsen und verstärkt Ayakos Drang, positive wie negative Auswirkungen der Katastrophe auf ihre Gemeinschaft zu dokumentieren. Ihren Status als ledige junge Frau, die im Unterschied zu anderen niemanden aus ihrer Familie verloren hat, führt sie dabei als vorteilhaft für ihre Projektarbeit an: „I didn't lose anyone, so that's why I can work on those projects" (Ayako 25.06.2014 19). Gleichzeitig ermöglichen ihr ihre eigenen Erfahrungen mit dem Tsunami ein empathisches Empfinden für jene Personen in ihrem Umfeld, die weniger Glück hatten und zum Teil zahlreiche Angehörige verloren haben. „I don't know whether I'm in a regular mindset or not. I still don't know, maybe. But that's why I can do this [art projects]. Because I experienced this, [...] that's why I am part of this affected area" (Ayako 25.06.2014: 20). Besonders der Vergleich zu nahegelegenen Regionen, die es ebenfalls schwer getroffen hat, lässt sie ihr eigenes Schicksal in einem entsprechenden Verhältnis betrachten: „,...] whenever I go [there] I feel like: ,Ok, I have everything. I have my blanket, my order, just in case." I still have that mind" (Ayako 25.06.2014: 17). Damit wird deutlich, dass Ayakos Verarbeitungsprozess der Ohnmacht und des Verlustes zum Zeitpunkt des Interviews noch nicht abgeschlossen ist. „So I still feel negative effects. I still feel like an affected person" (Ayako 25.06.2014: 20).

Dennoch wandelt sich ihr anfänglicher Schock zunehmend zu der Erkenntnis, dass diese Erfahrungen für sie persönlich auch sehr wichtig sind und Auswirkungen auf ihre Arbeit haben. Im Erzählstrang ist Ayakos eigene Emotionalität eng mit dem Ort der Geschehnisse verknüpft, und der Aufgabe, die der 11. März 2011 hinterlassen hat: „Rebuilding the place is important and also documenting" (Ayako 25.06.2014: 19). Der Verlust der Menschen, die diesen Ort ausmachen, steht dabei im Vordergrund ihrer Überlegungen. „I can imagine that for these people [who lost someone] time may have stopped. I can think about the disaster based on my own experience. But in case [of family loss] my imagination is still limited" (Ayako 25.06.2014: 19). Ich gewinne in unserem Gespräch den Eindruck, dass Ayako stark zwischen dem Ausmaß ihres eigenen Leids und dem von Menschen unterscheidet, die Familienmitglieder verloren haben. Ihr eigenes Gefühl des Verlusts entsteht unter anderem aus einem starken Mitgefühl für diese Personen, die mehr als nur ihr Hab und Gut verloren haben. Dies führt sie in ihren Erinnerungen erneut zum Ausgangspunkt ihrer eigenen Emotionen zurück: „It was shocking, looking at the city. [...] I think I had a shock, I couldn't think of anything" (Ayako 25.06.2014: 19). Erst rückblickend erhalten diese blockierenden Empfindungen ihre Wichtigkeit. „Those little experiences were really important to me" (Ayako 25.06.2014: 20). Worin genau diese Bedeutung liegt, erschließt sich erst aus den folgenden Passagen, in denen Ayako sich wieder in der Orientierungsphase unmittelbar nach dem Tsunami befindet. Das Bedürfnis, mit den eigenen Ressourcen etwas zum Wiederaufbau und zur Betreuung der traumatisierten Menschen beitragen zu können, stammt aus dieser Zeit. Die entsprechende Passage halte ich für so wichtig, dass ich sie in voller Länge zitiere: 
„So at the beginning, there were a lot of art projects in the affected areas. So if I can do something, then I wanted to be part of it. So, I didn't really think about the art itself or of the art gallery itself. It was more like: I know creative people. I have a friend who can build a house or [...] And supporting or helping affected people is not a special thing for us. It's more like a daily thing now. Just like rising up, all together, including us, including me." (Ayako 25.06.2014: 20)

Im Hinblick auf Ayakos Emotionalität geschehen hier gleich mehrere Dinge. Das Augenscheinlichste ist ihr bereits erwähntes Bedürfnis, eine Hilfe für die Betroffenen zu sein. Interessanterweise nimmt sie sich selbst im Fortlauf ihrer Erzählung zunächst aus dieser Gruppe der Betroffenen heraus, indem ihre Verortung eher in der Crew der Menschen liegt, die ihre Fähigkeiten zur Verfügung stellen. Die Kunst als Ressource spielt laut ihrer Aussage noch keine große Rolle. Es geht primär um handwerkliche Fähigkeiten derjenigen Künstler, die beispielsweise ein Haus wiederaufbauen können. Sie sind damit in der Lage, dringend benötigte Fertigkeiten anzubieten, bei denen es noch nicht um die emotionale Verarbeitung geht, sondern um praktisches Anpacken vor Ort. Dass die tägliche Arbeit mit den Traumatisierten auch ihr selbst hilft und sie damit wieder in den Kreis der Betroffenen rückt, wird von ihr erst im Nachsatz erwähnt. „So, for me, working on these projects, it's like rehabilitation" (Ayako 25.06.2014: 20).

Diesen Abschnitt erachte ich deshalb als besonders aussagekräftig, weil er Ayakos Zugehörigkeiten zu zwei verschiedenen Personengruppen, Opfer und Künstler, verdeutlicht, die sie sowohl gegenüberstellt als auch miteinander verbindet. Als Künstlerin hat sie Zugang zu einem Netzwerk von Personen, als dessen Teil sie sich fühlen und durch das sie Unterstützung für lokale Projekte erfahren konnte. Die Tätigkeit als aktive Helferin geht über ihre persönlichen Belange hinaus und erlaubte Ayako, sich selbst als Werkzeug für die seelische Wiederaufrichtung ihrer Mitmenschen zu begreifen. Gleichzeitig offenbart sie ihre Ansicht, sich mit Emotionen auseinandersetzen und diese bewältigen zu wollen. Die Projekte helfen dabei sowohl ihr als auch anderen bei der Verarbeitung.

\subsubsection{Die Zeit danach: Gestärktes Zugehörigkeitsgefühl und Reflexion}

Die hier deutlich werdende Verzahnung von der Person Ayakos mit den verschiedenen Gesellschaftsebenen bildet meiner Meinung nach eines der anschaulichsten Merkmale für die Werte von Gegenwartskünstlern. Als kritische Akteure stehen sie angesichts großer gesellschaftlicher Unruhe und Unordnung stärker im Vordergrund und zeigen die Stärke von Kreativität inmitten widriger Umständen auf. Ayako empfindet sich im Juni 2014 als Teil der Betroffenen, hebt sich in ihrem Narrativ jedoch zumindest in Teilbereichen deutlich von ihnen ab. Indem sie diese Rolle aktiv gestaltet und so abwandelt, dass sie sie als Teil ihres Selbst integrieren und stringent in ihre Vergangenheit inkorporieren kann, prägt sie ihre Identität nach dem 11. März 
2011. Die für Ayako so wichtige Selbstverwirklichung findet durch ihre Arbeit ebenso einen Platz wie das Bedürfnis, ihren Mitmenschen zu helfen. Dass ihre Betroffenheit für Außenstehende deutlich wahrnehmbar ist, wird in einer Randbemerkung deutlich, als es um die Hilfe geht, die sie persönlich von anderen Künstlern erfahren hat. Da sie die Gewalten der Natur erlebt habe, hätten ihre Freunde ihr Verhalten als „komisch“ (,weird“) empfunden. „They are still supporting me and I feel I am supporting something. These art projects are helping. [...] I still feel affected. That's why I am part of this affected area" (Ayako 25.06.2014: 20).

Ayako beschreibt hier etwas sehr wichtiges: Sowohl die Gegend als auch die mit ihr verbundenen Menschen sind „betroffen“ („affected“) und werden von ihr mit demselben Adjektiv umschrieben. Es findet eine sprachliche Verknüpfung zwischen der Landschaft und ihren Bewohnern statt, die Ayako selbst mit einschließt. „I am part of the affected area" bedeutet für Ayako aber auch, sich stark mit den Traumatisierten zu identifizieren und auch diesen Teil ihres Lebens als Teil ihrer Biographie anzuerkennen. „This is why I wanted to keep on working on those projects. They are like a part of my life. Not like: I'm doing volunteer work to support, not like that. More like, this is my life, this is part of my daily life" (Ayako 25.06.2014: 20).

Dadurch, dass Ayako ihre Fähigkeiten und Netzwerke als Künstlerin der Gemeinschaft zur Verfügung stellt, scheint sich eine tiefere Verbindung zu ihrem Umfeld entwickelt zu haben. Bereits vor der Katastrophe war sie ein Teil der Gemeinde, verankert durch ihre familiären Bindungen und Erinnerungen an die Region. Diese Verbundenheit wurde auch in der Zeit ihrer internationalen Reisen aufrechterhalten bis sich ihr die Gelegenheit bot, zurückzukehren. Durch den 11. März 2011 werden nun rückblickend diese Facetten ihrer Identität besonders hervorgehoben und verdichten sich in dem starken Gefühl, Teil einer Schicksalsgemeinschaft zu sein. Vor diesem Hintergrund ist Ayakos Aussage zu verstehen, freiwillige Arbeit zu leisten gehöre zu ihrem täglichen Leben. Ihre künstlerische Tätigkeit ist ebenso Teil ihres Lebens wie die Gestaltung eines neuen Alltags mit all diesen Erfahrungen und Erinnerungen, für die erst die passenden Worte gefunden werden müssen. Eine Trennung von beidem macht aus ihrer Sicht keinen Sinn und verdichtet sich zu einer neuen Pointierung ihrer Rollenbeschreibung, in der sie nicht nur wie zuvor Künstlerin, Angehörige und Nachbarin ist, sondern auch Opfer, Freiwillige und Schicksalsgefährtin. Dieses gestärkte Gefühl der Zugehörigkeit lässt sie nicht nur die Region und ihre Bewohner mit anderen Augen sehen, sondern erlaubt ihr, im Laufe des Gesprächs auf eine Neubewertung der katastrophalen Ereignisse zu sprechen zu kommen:

„I shouldn't say that but [...] I needed the experience. 3.11 [1 $11^{\text {th }}$ of March 2011] was really needed. I shouldn't say that but [...] some of them lost their family and these are not good things but [...] it caused me to think about art, about society, about my life. I think if you live in Japan you are very happy. I didn't know, maybe I didn't know what sadness is. But now I know." 
Erst jetzt, als das Gespräch schon weit fortgeschritten ist, kommt Ayako auf ihr Gefühl der Trauer zu sprechen, die sie angesichts der Katastrophe empfindet. Dabei vollbringt Ayako eine bedeutende Reflexionsleistung, indem sie es schafft, über ihre eigenen traumatischen Erfahrungen hinaus den Einfluss dieser Ereignisse für die Zukunft Japans zu formulieren und mit ihrer persönlichen Vergangenheit in einen Kontext zu setzen. In der Zuschreibung von Sinn an ihre traumatischen Erlebnisse verbleibt sie nicht in der eng gesteckten Rolle des passiven Opfers, sondern versucht hilfreiche Lektionen für sich und ihr unmittelbares Umfeld abzuleiten. Diese beschreibt sie vor allem als einen Gewinn auf emotionaler Ebene. Deshalb entsteht bei mir der Eindruck, diese Erlebnisse hätten sie auf emotionaler Ebene reifen lassen. Gefühle wie Traurigkeit kann Ayako nicht nur formulieren und ihnen damit Ausdruck verleihen. Sie kann der Existenz dieser Emotionen auch eine positive Facette abgewinnen.

Gleichzeitig ist Ayako sich der Tatsache bewusst, dass eine solche Formulierung derzeit die Gefühle vieler Menschen verletzen würde. Gekennzeichnet wird dies durch ihre wiederholte Aussage „I shouldn't say that", die auf der einen Seite die Hemmung ausdrückt, sich in dieser Weise zu äußern. Ayako zeigt hier, dass sie sich des normativen Umgangs mit ihren Emotionen bewusst ist, der einen positiven Ausdruck angesichts von Trauer nicht gut heißt. Dennoch macht sie mir gegenüber deutlich, dass sie ihre persönlichen Lehren aus negativen Gefühlen wie Wut und Trauer gezogen hat und dies in ihrer Kunst auch umsetzt.

Ihre Sensibilität für die Belange ihres direkten Wirkungskreises kann sie sowohl aus ihrer eigenen Emotionalität als auch aus der engen Kommunikation mit ihrem Umfeld schöpfen. Die Verortung ihrer Trauer und Hilflosigkeit in einem zeitlichen Rahmen führt Ayako in ihrem Narrativ zu einer weiteren zukunftsgerichteten Argumentationslinie, bei der es vor allem um die Vergänglichkeit materieller Dinge im Gegensatz zu menschlichen Bindungen geht: „I really think that people are non-material. If we have creativity and guts, even without money, we can make something" (Ayako 25.06.2014: 21).

\subsubsection{Ayakos persönliche Lektionen des 11. März 2011}

Die inneren Ressourcen, Fähigkeiten und persönlichen Beziehungen in einen Gegensatz zu monetären Mitteln zu stellen, bildet eine entscheidende Argumentation, die es Gegenwartskünstlern im Allgemeinen und nicht nur in dieser aktuellen Situation ermöglicht, das eigene Selbstbild positiv zu artikulieren. In Ayakos Fall der unmittelbaren Betroffenheit scheint diese Einstellung durch die Erfahrungen des 11. März 2011 nur noch unterstützt worden zu sein.

Auf meine Nachfrage, was die Menschen vor Ort nun brauchen, antwortet Ayako sowohl mit einer gegenwartsbezogenen Aussage als auch mit einer zukunftsgerichteten Perspektive: „They need motivation. The convenient life comes back and people are easy to go back to the way of thinking before 3.11. But some people try to 
keep what we realized. The new value. [...] We don't know how to keep the new way of thinking" (Ayako 25.06.2014: 22).

Der „neue Wert“, wie Ayako es nennt, beschreibt eine anders gewichtete Fokussierung auf Emotionen und Beziehungen als noch vor dem Tsunami. Das NichtMaterielle, also dasjenige, was nicht mit Geld zu kaufen oder zu ersetzen ist, steht im Mittelpunkt der emotionalen Konnotation ihrer Erfahrungen. Durch die Herstellung einer Sinnhaftigkeit im Leid und der Umwandlung in „Lektionen“ für alle werden diese Erfahrungen erträglicher. Sie erhalten den Charakter einer Weichenstellung für die Zukunft, in der es nicht mehr nur allein um ein angenehmes Leben geht. Wie auch immer die Zukunft der Gemeinschaft aussieht, Ayako enthüllt in diesem Abschnitt des Interviews ihre Hoffnung, dass die Zukunft von den erfahrenen Emotionen vom 11. März 2011 bestimmt ist.

Ihre Beobachtung, dass diese neue Art zu denken von einem „convenient life“ erneut überdeckt werden könnte und die Menschen nur allzu gern vergessen wollen, stellt gleichzeitig eine Aufforderung an die Gesellschaft dar, genau dies nicht zuzulassen. Erneut artikuliert Ayako in ihrem Narrativ zwei Rollen, die sich in ihrer Person vereinen. In der Aussage „We don't know how to keep the new way of thinking “ lässt sich der Ausdruck der Zugehörigkeit in Form von „Wir“ („We“) sowohl den Künstlern als auch den Betroffenen im Allgemeinen zuschreiben. Eine Unterscheidung nimmt Ayako selbst nicht vor. Ich betone dies deshalb, weil Ayako an dieser Stelle sehr deutlich aufzeigt, wie sie artikulativ zwischen der Perspektive des Opfers und einer distanzierteren Außenperspektive hin- und herwechselt.

Mit anderen Worten hinterfragt und analysiert sie nicht nur ihre Emotionalität, sondern versucht, ihre Emotionen gleichzeitig als Orientierungspunkt für ihr Handeln in Gegenwart und Zukunft zu nutzen. Die Gespräche, die sie mit anderen Künstlern und ebenfalls Betroffenen führte, hatten ihr geholfen, sich zu stabilisieren. Diese Erfahrung im Umgang mit starken Emotionen setzt sie nun in einem größer angelegten Projekt um, das nicht nur ihre Gemeinde, sondern den gesamten Küstenabschnitt einbezieht. Sie ist Organisatorin eines Festivals, bei dem die „neuen Werte“ des Zusammenhaltens mit gemeinsamen Mahlzeiten, Musik und Kunst zelebriert und im Gedächtnis gehalten werden sollen:

„It's challenging, but that's what's real. That's why we are living in this area. Having a dialogue is important. A dialogue and an open place to talk. To share ideas. Of course we need money. The financial support is always needed. But besides that people are getting really engaged in promoting these new values. [...]" (Ayako 25.06.2014: 23)

In Ayakos Aktivitäten kommen folglich verschiedene Ansätze zusammen, die den Menschen vor Ort ihren nach wie vor existierenden Verbund vor Augen halten sollen. Kurzfristig betrachtet geht es um die Aufrichtung der Menschen, die alles verloren haben. Längerfristig zielen Ayakos Bemühungen auf eine Zukunft ab, in der diesen vertieften Beziehungen ein erhöhter Stellenwert gegenüber Geld und 
materiellen Dingen eingeräumt wird. „First, only people who share the values will gather. And then [we] will try to make it bigger" (Ayako 25.06.2014: 23). Sie beschreibt damit einen hoffnungsvollen Entwurf eines gesellschaftlichen Gefüges, das sich mehr denn je um das Individuum im Kontext kümmert und damit maßgeblich dazu beiträgt, die Ressourcen eines jeden unabhängig von seiner wirtschaftlichen Kraft zu würdigen.

Im Zusammenhang mit ihrer bisherigen biographischen Darstellung veranschaulicht Ayako die Deutung ihres eigenen Lebens als einen solchen Glücksfall. Ihre eigene Familie und die besondere Konstellation in ihrem Heimatort ließen ihr genug Raum zur Selbstentfaltung und zur Entwicklung von Fähigkeiten, die sie nun wiederum in diesen, von der Krise geschüttelten Raum zurückgeben kann. Diese Darstellung eines Gebens und Nehmens in einem Beziehungsgeflecht weist markante Merkmale ihres kulturspezifischen Skriptes auf, das sie dazu anhält, zum Wohle der Gemeinschaft beizutragen. Im gleichen Augenblick bringt sie damit ihre von internationalen Erfahrungen geprägte Ansicht zum Ausdruck, dass diese Ziele auch anders als auf den stark von neoliberalen Ideen geprägten Wegen der japanischen Gesellschaft erreicht werden können. Die Motivation, Veränderungen anzustoßen und die Denkweise der Menschen zu transformieren, findet sich meinen Erkenntnissen zufolge auch bei anderen Gegenwartskünstlern und scheint mir daher ein grundlegender Aspekt ihrer Identitätskonstruktion zu sein. In Ayakos Fall basiert sie auf ihrer tiefen Verbindung mit lokalen Themen und Herausforderungen. Ihre Wurzeln und die Auseinandersetzung mit den daraus resultierenden spezifischen Aufgaben und Fragestellungen bilden die Basis sowohl für ihre Arbeit und als auch für ihre Identitätskonstruktion als Künstlerin.

In der Passage, die den Abschluss des Interviews am 25.06.2014 einläutet, nimmt Ayako verstärkt Bezug auf ihre Rollen als Künstlerin, Initiatorin und Organisatorin: Das geplante Event in der Region, das zur Auseinandersetzung mit lokalen Traditionen, Fertigkeiten und Persönlichkeiten einlädt, soll nur eine von vielen Optionen darstellen, diesen bewohnten Landstrich neu zu entdecken. „We are not trying to make one answer, or, one idea; more like, first making the communities [itself]" (Ayako 25.06.2014: 23).

Diesen Veranstaltungen liegt also laut Ayako ein betont kommunikativer und partizipatorischer Leitfaden zugrunde, der alle Teilnehmer zum Austausch einladen soll. Es geht dabei um die Erschaffung eines Raumes und einer Gelegenheit, die individuellen Gefühle auszudrücken. Die emotionale Bindung an den Ort Shiogama und seine Umgebung basiert schlussendlich auf gemeinsamen Erinnerungen seiner Bewohner und langsam gewachsenen Beziehungen. Diese Bindungen weiter zu erhalten, indem man den Emotionen der Menschen einen Platz im Leben einräumt, kann gegen das Muster des Vergessens wirken, das Ayako in dieser Situation fürchtet. Die Diversität und verschiedenen Schattierungen der Erfahrungen und der dadurch entstehende Reichtum an individuellen Konsequenzen für das zukünftige Leben kann die Welt nach dem 11. März 2011 für alle unmittelbar Betroffenen erträglicher 
machen, so Ayakos Gedankengang. Ein Gemeinschaftsgefühl, so Ayakos Hoffnung, kann die Gemeinschaft auch überregional stärken:
"[...] trying to create opportunities, creating a dialogue, and then these strong communities might work with other communities to understand each other. So we can make the situation better. [...] Oh, I really feel this is important. If people feel this maybe [they think] 'if I stick to this idea I will be back and live here'." (Ayako 25.06.2014: 23)

Hier spielt Ayako auf die Kraftreserven im zwischenmenschlichen Miteinander an. Sobald Emotionen sichtbar gemacht und einen legitimen Artikulationsraum erhalten haben, können sie ihr Potential als Motivator und Antrieb entfalten. Für diesen Vorgang beobachtet Ayako bereits erste Regungen in ihrem Umfeld: „So, people are getting active now" (Ayako 25.06.2014: 24).

Nicht nur sich selbst kann Ayako damit ihre Handlungsfähigkeit und Gestaltungsmöglichkeiten vor Augen führen. Auch die Gemeinschaft um sie herum kann durch diese gemeinsame Emotionsarbeit wachsen und auch diejenigen Probleme in Angriff nehmen, die nicht nur sie selbst betreffen. Ayako meint dazu: „We don't share that problem, not really, but if we learn about the history of the area" (Ayako 25.06.2014: 24). Erneut kommt Ayako auf die Suche nach Gemeinsamkeiten zu sprechen, die über die Erfahrungen von 2011 hinausgehen. Dazu wird in der geschichtlichen Vergangenheit der Orte gesucht, um zum Beispiel die tiefe Verbundenheit der Anwohner, die auf den vorgelagerten Inseln leben, mit dem Meer zu verstehen.

\subsubsection{Die Historisierung des Erlebten: Aus Mitleid wird Stolz}

Der beschriebene Prozess der Historisierung der Katastrophe ermöglicht es Ayako, ein tieferes Verständnis für den Umgang zu gewinnen, den vorangegangene Generationen mit solchen Ereignissen pflegten. Die Verknüpfung von Gegenwart und Vergangenheit erweitert nicht nur die Bindung an die Lokalität an sich, sondern vermittelt auch eine Verbundenheit durch die Zeit hinweg. Ayako fand dafür diese Worte: „So, if you understand that history or the problem or the memory, you understand the characteristics of the people there. I don't feel sorry for them. Now, I feel pride“ (Ayako 25.06.2014: 25).

Hier beschreibt Ayako einen interessanten Umwandlungsprozess ihrer emotionalen Bewertung aufgrund der Auseinandersetzung mit der Geschichte der Region. Mit wachsendem Verständnis für die historisch gewachsene Existenz an Japans Ostküste wachsen auch Ayakos Gefühle des Stolzes. Auch vorangegangene Generationen haben Tsunamis erlebt, überstanden und den Ort wieder aufgebaut. Diese historische Stringenz erzeugt ein tief empfundenes Gefühl der Gemeinsamkeit über die Zeit hinweg und stellt die aktuelle Situation in den Kontext einer langen historischen Kette von ähnlichen Erfahrungen. 
Das Kunstprojekt, das im Wesentlichen für die Entdeckung dieser Historizität verantwortlich war, läuft auch im Jahr 2014 noch. Menschen aus allen Küstenabschnitten der Region können sich an diesem Projekt beteiligen und erhalten damit die Gelegenheit, sich untereinander zu vernetzen. Auch Ayako sieht für sich als Mitwirkende einige Vorteile: „[...] I got to know because of this project. [...] I went to the islands every day because of the project. [...] So it's like kinship or neighborhood to me now" (Ayako 25.06.2014: 25). Die besondere Härte der aktuellen Situation wird in ein historisches Verhältnis gesetzt und erfährt so eine stückweise Relativierung: „So once every 20 years, the island is affected. But people are still living there. Oh, then I undestand: Japan is like that. [...] We are not special, you know? We should just understand where we are living" (Ayako 25.06.2014: 25).

Hier scheint die Grundlage für das von Ayako artikulierte Gefühl des Stolzes zu liegen: Seit Jahrhunderten trotzen die Menschen dem Meer nicht nur ihre Existenz $\mathrm{ab}$, sondern widerstehen immer wieder aufs Neue seinen Gewalten: „I mean, a tsunami is a big deal, but we live with the tsunami from back then, for thousands of years. [...] We understand how to live with nature [...]" (Ayako 25.06.2014: 26). Die Vergangenheit wird in Ayakos Narrativ nicht nur wiederentdeckt, sondern vor dem Hintergrund aktueller Ereignisse rekonstruiert. Dieser Wandel der emotionalen Akzentuierung speist ein neues Identitätsbewusstsein, das die Folgen der Katastrophe als Auslöser für einen positiven Wandel zu akzeptieren vermag. Durch dieses Narrativ verliert das Desaster während des Verarbeitungsprozesses zunehmend an Sinnlosigkeit und hilft Ayako, den entstandenen gesellschaftlichen Raum für bis dato nur schwer denkbare Veränderungsmöglichkeiten wahrzunehmen. In diesem Prozess kann Ayako sogar Schönheit erkennen: „And [...] we see the same scenery like our ancient people. It's beautiful“ (Ayako 25.06.2014: 26).

Das Mitleid mit den Menschen, die über das Materielle hinaus auch Angehörige, Freunde und Nachbarn verloren haben, wird dabei nicht in den Hintergrund gedrängt. In der aktuellen Situation ist es sogar essentiell. Indem Ayako ihr Mitgefühl mithilfe ihrer Aktivitäten, Kunstinitiativen und im direkten Dialog transparent macht und die Ereignisse darüber hinaus in einen historischen Kontext eingebettet werden, unterstützt sie den Prozess, Mitleid in ein Gefühl des Stolzes auf die eigene Identität umzuwandeln. Auf diese Weise entstehen aus einer katastrophalen Erfahrung positive Energien. Das Erleben von Katastrophen über Generationen hinweg begründet gewissermaßen eine soziale Gemeinschaft mit den verstorbenen Vorfahren, die ihre Widerstandskraft bereits unter Beweis gestellt haben. Die Geschichte, Landschaft, Tradition, Vorfahren und die aktuellen Entwicklungen verbinden sich in Ayakos Erzählung zu einer ununterbrochenen Geschichte aus Leid und Wiederaufbau, die die aktuelle Katastrophe in einen neuen Kontext setzt und für die Betroffenen erträglicher macht. 


\subsection{Die Kunst als Form persönlichen Handlungsvermögens}

Wie ich in der Einleitung zu Kapitel 8.4 „Das Erleben des 11. März 2011 - Eine andere Realität" bereits angesprochen habe, nutzt Ayako ihre Kreativität in der Kunst, um sich und andere vom Schock des Erlebten zu erholen und sich ein neues Leben aufzubauen.

Die direkte landschaftliche Verbindung und damit auch den Link zu den Vorfahren dauerhaft im Bewusstsein der Menschen halten zu können, stellt eine Seite von Ayakos Bestreben dar, die negativen Gefühle von Trauer und Verlust in lokalen Stolz zu verwandeln. So verstünde die Gemeinschaft vielleicht die einzelnen Kunstbegriffe nicht, jedoch in jedem Fall die Stoßrichtung, die die einzelnen Projekte zum Ziel hätten, meint Ayako: „I don't know whether the community really understands art. I am not using the word, art'" (Ayako 25.06.2014: 26). Immer wieder kommt sie in unserem Gespräch auf die Menschen zurück, für die sie diese Projekte initiiert. Sie vermittelt mir damit den Eindruck, dass richtungsweisende Möglichkeiten der einzelnen Vorhaben und Projekte für sie im Mittelpunkt stehen und nicht die Durchsetzung eines bestimmten Kunstbegriffs. Seit 2012, so Ayako weiter, wollten die Menschen neue Visionen ihrer Region und ihres Lebens entwickeln - ein Wunsch, der aus Ayakos Sicht sehr gut durch die Kunst beantwortet werden kann. "They don't know how to plan the new future. Those art projects actually give hints" (Ayako 25.06.2014: 27). Dieser von ihr beschriebene Orientierungsprozess wird viel Zeit in Anspruch nehmen, dessen ist sich Ayako bewusst. Sie glaubt jedoch fest an die Wirkungsmöglichkeit der Kunst und die Wandlungsfähigkeit der Menschen: „I believe in art. And I believe that art brings individual's skills, and possibilities, for themselves. I believe that's why I use art. This kind of comments or beliefs is really abstract, in a way [...] So I am still struggling with the way of showing the power of art" (Ayako 25.06.2014: 27).

Diese Aussage veranschaulicht, auf welche Weise Ayako mit sich ringt, um die entsprechende, Übersetzung' ihrer Ideen in einer Sprache zu bündeln, die die Menschen verstehen und annehmen können. Sie verletzt den kulturspezifischen Umgang mit Emotionen nicht, weil sie die Emotionen in einer Art und Weise sichtbar macht, die die Menschen nicht abstößt. Dennoch spricht sie Emotionen in ihrer Kunst direkt an. So entsteht eine Balance, die Ayako halten muss, um im direkten Austausch mit ihren Rezipienten bleiben zu können. Es geht ihr um die Herstellung von Bedeutung, die die Menschen während dem Zusammenfinden bei diesen Projektarbeiten vornehmen. „The activity among young people kind of shows or proves that those art cultural activities are movements and that they are meaningful" (Ayako 25.06.2014: 27). Um die Zielrichtung ihrer Kunst transparent zu machen, nutzt sie auch andere Medien:

„Yesterday, we had an article in the local newspaper featuring my activities. So I hope community people read this and they say 'Oh, I see! I understand.' [...] 
The moment we are working on it, little is seen, but later on the meaning and the purpose will be more meaningful. It takes time."

(Ayako 25.06.2014: 27)

Ayakos Ansicht, dass dies ein langsamer Prozess ist, wird an dieser Stelle besonders deutlich. Damit sich die „Macht der Kunst“, wie Ayako sie hier bezeichnet, entfalten kann, reichen starke, kurzfristige Impulse nicht aus. Vielmehr betont sie den nachhaltigen Effekt ihrer Arbeit. Wie ein Instrumentarium setzt sie die Kunst ein, um die Beziehungen der Menschen in der Gemeinschaft zu stärken. Ayako bringt das in folgender Aussage gezielt zur Sprache: „It's like raising people. [...] Really. You know? How to make a good community. How to make strong, creative people" (Ayako 25.06.2014: 28). Die Artikulationen, die Ayako hier zur Beschreibung ihres Alltagskontextes wählt, geben Aufschluss über ihr emotionales Standing ihren Mitmenschen gegenüber. Ihre Aussagen spiegeln Ayakos Hoffnung wider, dass sich alle Küstenbewohner, sie selbst eingeschlossen, in der Auseinandersetzung mit dem Erlebten zur einer neuen Gemeinschaft finden können. Die Erfahrungen vom März 2011 werden dabei von ihr mit einem besonderen Fokus auf die Emotionen betrachtet, die den entsprechenden Raum erhalten, um Außenstehenden gegenüber repräsentiert werden zu können: „You felt the end of life, right? You feel something, right? It's right so see the result, really. It's just like that" (Ayako 25.06.2014: 28). Den Menschen zunächst eine Möglichkeit zu geben, sich zu offenbaren und ihren diversen Gefühlen im Zusammenhang mit nahen Todeserfahrungen und Verlust Ausdruck zu verleihen, ist in Ayakos Augen der entscheidende Schritt, um die Gemeinschaftsmitglieder zusammenzuhalten. „These are slow movements, slow activities. I still believe in that" (Ayako 25.06.2014: 28). Diese beständige Betonung des Langsamen, des ruhigen Arbeitens hat einen beinahe therapeutischen Unterton, bei dem Ayako sich in ihrer Rolle als Initiatorin dieses Prozesses aus der Rolle der Betroffenen herausnimmt. So betont sie explizit, dass es nicht um sie selbst oder um die Möglichkeit geht, viel Geld zu verdienen:

"It's not like selfing or to make that big money, you know? It's not like that. I'm not dealing with high art here or anything like that. [...] I believe that the community here really understands what I'm doing and what we are trying to do. So that's why I have jobs, and I have stuff to do cause they need our help to make the community better or the city." (Ayako 25.06.2014: 28)

Das Personalpronomen "wir“ bezieht sich an dieser Stelle erneut ausschließlich auf ihre Professionsrolle als Künstlerin, nicht auf sie als Betroffene. Ihre Katastrophenerfahrungen nutzt Ayako, um ihre Mitmenschen auf eine bestimmte Art und Weise anzusprechen und um sich selbst immer wieder zu reflektieren. Das ist ihr wichtig, um die Reaktionen anderer Betroffener angesichts bestimmter Projektideen einschätzen zu können. „Now I believe that the community here really understands what I'm 
doing and what we are trying to do. [...] I have stuff to do, I have a job because they need our help to create a better community" (Ayako 25.06.2014: 28).

Ayako ist damit Ausführende, Medium und Rezipientin zugleich. In dieser Rollendeckung mit ihren unterschiedlichen emotionalen Voraussetzungen schafft sie in ihrer Galerie eine Atmosphäre der Ruhe, die sehr beeindruckend auf den Eintretenden wirkt. Ayako zeigt keine Scham beim Austausch über ihre Erfahrungen und Gefühle vor, während und nach der Katastrophe, wie es andere Anwohner der Region zeigten. Meine weiteren Gesprächspartner bestätigten meinen Eindruck, dass die meisten Betroffenen mit nur sehr wenigen Menschen über das Erlebte sprechen möchten. Meine Gesprächspartner sehen darin eine Möglichkeit, die Erfahrungen nicht erneut durchleben und darüber hinaus ihre Mitmenschen nicht belasten zu müssen.

Das Gespräch mit Ayako zeichnet sich hingegen durch eine große Offenheit und Akzeptanz aus. Wie ich in Kapitel 8.4.3 beschrieben habe, zieht sie sogar Lehren und Kraft aus der Katastrophe.

Meine eigene Reaktion auf das Ambiente ihrer Galerie inmitten all der zerstörten Straßen und Häuser bestand in einer gewissen Erleichterung. In Ayakos Galerie kann man die Szenen der Katastrophe teilweise hinter sich lassen und sich auf sich selbst konzentrieren. Diese Möglichkeit des kurzen Rückzugs aus dem anstrengenden Alltag wurde während unseres Interviews auch von einigen Passanten genutzt, die sich nach Ayako erkundigten. Ayakos stets offene Eingangstür ist eine beständige stumme Einladung einzutreten, sich umzusehen und sich bei einer Tasse Tee auszutauschen. So entstanden auch neue Ideen für gemeinsame Aktivitäten während eines Festivals, bei dem Interessierte die alte Kunst des Fischernetz-Knüpfens erlernen konnten. „Learning how to knit nets is a great feeling. 'Cause I live here, you know, it's like a local skill. [...] And it's universal" (Ayako 25.06.2014: 29). Auf diese Weise fanden sich im wahrsten Sinne des Wortes neue Anknüpfungspunkte mit lokalen Traditionen, denen erst die Katastrophe einen Impuls der Revitalisierung gab. Ayakos Kunst vereint meiner Ansicht nach viele Facetten in sich, die diverse Ansätze für Handlungsoptionen liefern und die darin therapeutischen Ansätzen gleichen: Kommunikationsform, Übersetzungsmöglichkeit (z. B. von Emotionen in Bilder) und Raum für Emotionen als identifikationsstiftendes Element. Indem sie ihre Kunst einsetzt, um transparent zu machen, dass Emotionen wie Trauer und Wut, aber auch Mitleid und Stolz, von den allen Mitgliedern der Gemeinschaft geteilt werden, unterstützt sie den Prozess der aktiven Neuformung der Gemeinschaft und das gemeinsame Entdecken von Vergangenheit, Gegenwart und Zukunft. 



\title{
9 Die biographischen Antworten der Gegenwartskünstler im Kontext des nationalen Post-Fukushima-Diskurses
}

\author{
Maybe you need to find your own strength, your own value, \\ what you are good at and the place where you are good at. \\ (Kogoro 02.05.2014: 21)
}

Viele der Aspekte, die in meiner Tiefenanalyse von Ayakos und Kogoros lebensgeschichtlichen Einschnitten deutlich geworden sind, treffen grundsätzlich auf die Mehrheit meiner Gesprächspartner zu. In diesem Kapitel lasse ich einige Gesprächspartner aus meinem Datenmaterial zu Wort kommen, die in meinen Augen ein gutes Beispiel für die Darstellungsweise essentieller biographischer Aspekte darstellen. ${ }^{81}$ Insbesondere die Aushandlung des Ausdrucks individueller Beobachtungen und Gefühle zählt zu den Gemeinsamkeiten. Darüber hinaus ist allen Beteiligten meiner Forschungsarbeit die Eigenschaft gemein, bereits früh ein Gefühl für die eigene „Andersartigkeit“ im Vergleich zu ihrem unmittelbaren und gesamtgesellschaftlichen Umfeld entwickelt und ihr Leben entsprechend gestaltet zu haben. Dies bezieht sich vor allem auf einen biographischen Entwurf, der von der dominanten japanischen Vorstellung eines „guten Lebens" abweicht. Dabei handelt es sich meiner Meinung nach im Fall meiner Gesprächspartner neben spürbaren Unterschieden zwischen Generationen um eine Besonderheit der sozialen Rolle des Künstlers. Es ist meiner Meinung nach essentiell zu verstehen, wie Empathie und Handlungsvermögen in

\footnotetext{
${ }^{81}$ Ich widme nicht jedem meiner Gesprächspartner ein eigenes Kapitel, um den Rahmen dieser
} Arbeit nicht zu sprengen. 
der Rolle des Künstlers ineinandergreifen. Innerhalb der mit den Vorstellungen von einem „guten Leben“" verbundenen, kulturell konstituierten Rahmenbedingungen und Konventionen entwickeln Künstler ihre persönliche Anschauung eines erfüllenden Daseins im Dienste der Gesellschaft. Diese ermöglicht es ihnen, mehr Individualität auszuleben als es ihnen außerhalb ihres Kunstkontextes möglich erscheint. Aus meinen Interviews geht beispielsweise hervor, dass viele Künstler wie Ayako durch ihre Erfahrungen mit einem Normalarbeitsverhältnis auf dem Arbeitsmarkt desillusioniert sind. Sie erschaffen sich eine eigene Form des finanziellen Auskommens, indem sie Kunst zu ihrem Broterwerb machen. Der entsprechende Lebensstil bietet ihnen zwar keinen ökonomischen Überfluss, verschafft ihnen aber dafür Freiräume, die alle meine Gesprächspartner als essentiell für ihr Wohlbefinden erachten. Die Definition eines Gegenwartskünstlers beinhaltet laut ihren Aussagen daher immer eine Verweigerungshaltung in Bezug auf Regeln oder Normen, die ihnen diese Freiräume des Denkens und Handelns verweigern oder zumindest erschweren.

Im Narrativ des Einzelnen ist eine Selbstdarstellung als Mitglied einer gesellschaftlich marginalisierten Personengruppe daher sehr auffällig. Dieses Selbstbild, sowohl des Individuums als auch der Gemeinschaft der Gegenwartskünstler, ist im Kontext der meritokratisch und neoliberal ausgerichteten Gesellschaft Japans zu verstehen. Leistung als Teil des ökonomischen Erfolges hat in der marktabhängigen Gesellschaftsausrichtung Japans einen besonders hohen Stellenwert. Künstler erbringen ihn nur in einem schwer zu erfassenden Maße. Dementsprechend gering fällt zunächst ihre gesellschaftliche Anerkennung aus.

Die Erfolgsdefinitionen meiner Gesprächspartner orientieren sich vielmehr an selbstgesetzten und schwer mit Zahlen zu stützenden Indikatoren wie Verständniserzeugung, Informationsbereitstellung und gesellschaftlicher Aktivität. Es ist daher nicht verwunderlich, dass sich das vom einzelnen Künstler geleistete Engagement in der Wahrnehmung der japanischen Öffentlichkeit oftmals in einem Graubereich zwischen Berufung und Hobby bewegt. Nach einer Gegenüberstellung von Kogoro und Ayako in Kapitel 9.1 befasse ich mich in 9.2 mit weiteren biographischen Herleitungen der Lebensweise von Künstlern in Japan und werde mich dazu an meinem weiteren Interviewmaterial orientieren. Bei der Gesprächsführung wurde ich teilweise von meinem Übersetzer Satoshi unterstützt, was ich an den jeweiligen Interviews kenntlich mache.

Ich mache nachfolgend deutlich, dass die lebensprägende Entscheidung, ein Künstler zu werden, in höchstem Maße mit den jeweiligen biographischen Selbstkonstruktionen und Erfahrungen zusammenhängt. Diese Konstruktionen der jeweiligen Identität geben Hinweise auf jeweilige emotionale Verbindungen zu bestimmten Teilaspekten der Dreifachkatastrophe und den Motivationen des einzelnen Künstlers, Emotionen sichtbar zu machen. Meine Gesprächspartner sind damit aus meiner Sicht aktiv Teilnehmende an den geführten Katastrophendiskursen, in denen die verschiedenen Akteure aus Politik, Wirtschaft, Kultur und Gesellschaft die abzuleitenden Folgen für Japans Zukunft verhandeln. 


\subsection{Tsunami und Atomkraft: Eine Gegenüberstellung der emotionalen Diskurse}

Kogoro und Ayako veranschaulichen in ihren biographischen Erzählungen die drei Facetten der Katastrophe vom 11. März 2011 und deren Auswirkungen auf ihr berufliches wie privates Leben. In diesem Kapitel möchte ich auf die Abweichungen in den Emotionsdiskursen bezüglich der Naturkatastrophe (Erdbeben und Tsunami) und der menschengemachten Katastrophe (atomarer GAU) zu sprechen kommen. Im direkten Vergleich wird deutlich, wie diese Diskurse zu wichtigen Rahmungen sowohl für die Emotionen meiner Gesprächspartner als auch für ihren künstlerischen Ausdruck von Emotionen werden.

Ayako übt ihre Tätigkeit als Künstlerin in einem lokalen Kontext aus, zu dem sie sehr enge persönliche Beziehungen pflegt. Ihr Wirken in ihrem direkten Umfeld stellt die Möglichkeiten einer lokal wirkenden Künstlerin in den Mittelpunkt. Im direkten Vergleich mit der Arbeit von Kogoro fällt auf, dass Ayakos Wirkungsrahmen sehr viel enger gesteckt ist. Dieser Wirkungsrahmen bezieht sich vor allem auf den lokalen und ihr größtenteils bekannten Personenkreis, mit dem Ayako täglich in Interaktion tritt, während Kogoro mit einer weitestgehend gesichtslosen Masse an Rezipienten in der Breite der Gesellschaft arbeitet. Ayakos eigenes unmittelbares Erleben des 11. März 2011 spielt darüber hinaus nicht nur in der Verarbeitung ihrer eigenen Emotionalität und mit der ihr als „passend“ erscheinenden Projektauswahl eine große Rolle, sondern zeigt sich auch in der sorgfältigen Wahl der Zugänge zu ihren Rezipienten. Sie zeichnet das Bild eines engen wechselseitigen Austauschs zwischen ihr als Initiatorin von Projekten und ihren Mitmenschen. Ayakos eigene Betroffenheit verleiht ihr in den Augen ihrer Rezipienten die für diese Rolle notwendige Legitimität. Dieser direkte Zugang lässt Ayako nicht nur alle aktuellen Entwicklungen vor Ort hautnah miterleben, sondern schließt sie als Akteurin in diesen Prozess mit ein. Sie ist als Betroffene direkt emotional involviert und überblickt gleichzeitig als Ansprechpartnerin vor Ort die Gesamtentwicklung der Gemeinschaft.

Kogoros Engagement hingegen basiert zwar auf biographischen Identifikationspunkten mit den Menschen in den verstrahlten Gebieten, doch weist er keine für die direkt betroffenen Menschen erkennbaren Verbindungen zu ihnen auf. Man könnte argumentieren, dass er auf diese Weise von der lokalen Gruppe der Opfergemeinschaft ausgeschlossen bleibt. Ayakos Anspruch, Veränderungen zu initiieren, ist damit, im Unterschied zu Kogoros Ansatz, stark auf ihren lokalen Kontext fokussiert. Selbst wenn Ayako überregional denkt, artikuliert sie, nie den Anspruch gesamtgesellschaftliche Veränderungen im Blick zu haben. Sie beschränkt sich auf den lokalen Kontext Shiogamas. Es fällt ihr deshalb leichter, konkrete Resultate als Ergebnis ihrer empathisch motivierten Bemühungen auszumachen, sobald sich diese in ihrem Umfeld zu manifestieren beginnen. Kogoro hingegen muss sich, unter anderem aus finanziellen Gründen, immer wieder aus der Region Fukushima zurückziehen. 
Ayakos unmittelbare Einbindung in das alltägliche Leben der Menschen bringt sie mit zukünftigen positiven Veränderungen direkt in Verbindung. Bereits zum Zeitpunkt des Interviews, knapp drei Jahre nach dem Desaster, ist Ayakos Galerie zu einer Art Gemeinschaftszentrum geworden, in dem sich die Menschen treffen, diskutieren, Projekte erörtern und sich austauschen. Ich konnte vor Ort beobachten, dass der von Ayako in Gang gesetzte Austauschprozess bereits angenommen und von den Anwohnern eigenständig weitergeführt wird, was sich in enger werdenden Netzwerken manifestiert. Ayako berichtet bei weiteren Treffen von neuen Kooperationen sowohl innerhalb als auch außerhalb Shiogamas, die zum Teil nur aufgrund des Engagements der Gemeindemitglieder zustande kommen. Ayako selbst gestaltet und flankiert die bereits existierenden Projekte mit eigenen weiterführenden Ansätzen und Ideen, um die Menschen auf aktuelle wie zukünftige Aspekte aufmerksam zu machen, die sie für die gemeinsame Gestaltung ihrer Gemeinschaft als notwendig erachtet. Dabei präsentiert sie sich selbst als offen für neue Impulse aus der Gemeinschaft. Sie grenzt sich dadurch maßgeblich von jenen Bauunternehmern ab, die in ihre Region kommen und ohne Vorkenntnisse der lokalen Bewältigungsstrategien ihre Vorhaben umsetzen wollen. „For the government, it's like make a construction, so they can make money. Concrete, you know?" (Ayako 25.06.2014: 26).

Ayako präsentiert damit das Selbstbild einer regional verwurzelten Künstlerin mit unterschiedlichen Rollenfunktionen in der Gemeinschaft, deren Anforderungen sie durch die Kunst als Form ihres persönlichen Ausdrucks gerecht wird. Die Kunst wird damit für sie selbst wie auch für ihren Wirkungsbereich zu einem nützlichen Werkzeug - sowohl was den konkret zu leistenden Wiederaufbau als auch die seelische Genesung angeht.

Bei der Betrachtung der unterschiedlichen Rollen von Ayako und Kogoro fiel mir auf, welche Formen der Artikulation Ayako insbesondere in Bezug auf ihre Mitmenschen und Rezipienten ihrer Kunstprojekte verwendet. In ihren Rollen als Anwohnerin, Betroffene, Opfer und Geschädigte vermag Ayako in Bezug auf Kunst einen anderen Part für sich zu entdecken und auszufüllen. Sobald sie diese Aufgabe in den Mittelpunkt ihrer Selbstdarstellung rückt, verändert sich auch ihre Artikulation dahingehend, dass sie fast mütterliche Emotionen zum Ausdruck bringt. So spricht sie beispielsweise von „raising strong, creative people“ (Ayako 25.06.2014: 28). Ungeachtet der Volljährigkeit der meisten ihrer Nachbarn ist ihr ihre selbstgewählte Aufgabe als Künstlerin in diesem Kontext sehr präsent, die sie vor allem in der Vermittlung neuer Gedanken und konstruktiver Ideen sieht. In der Auseinandersetzung, Dokumentation und Reflexion über das Geschehen jener Tage und Wochen im März 2011 ist sie ein ganz wesentlicher Akteur, wenn es darum geht, die lokale Gemeinschaft neu zusammenzuführen und Verluste zu verarbeiten.

In ihren Ausführungen zu ihrem Erleben des 11. März berichtet Ayako, wie sie selbst mithilfe der Kunst aus ihrer Opferrolle herausfand und sich selbst wieder als aktives, produktives Mitglied der Gemeinschaft erleben konnte. Durch die starke lokale Anbindung ist es ihr möglich, die von ihr getragenen Initiativen diesbezüglich unmittelbar zu verfolgen und sich persönlich mit ihren Rezipienten zu verknüpfen. 
Das kommt für Kogoro durch seinen anders gelagerten Anspruch, die Gesellschaft auf höherer Ebene zu aktivieren, nicht in Betracht.

Ayako hat sich mit einer Form des Verlustes auseinandergesetzt, die, wie sie selbst sagt, Japan bereits seit Jahrtausenden heimsucht und dessen Folgen dementsprechend im Allgemeinen abzuschätzen sind. Dieser Rückgriff auf bereits existierende Erfahrungen erleichtert nicht nur den unmittelbar Betroffenen das Zurückfinden in einen Alltag. Es sichert auch, wie eingangs erwähnt, die Anerkennung der Betroffenen auf gesamtgesellschaftlicher Ebene. Die durch den Tsunami verursachten Schäden an Gebäuden und Straßen sind nach wie vor deutlich sichtbar und die emotionalen Traumata sind angesichts des gewaltigen Verlustes an Menschenleben und Erinnerungen leicht nachvollziehbar.

Kogoros Aufgabe indessen, den Menschen in Tokyo die körperlichen wie psychischen Folgen der atomaren Verstrahlung zu vermitteln, gestaltet sich in dieser Hinsicht völlig anders. Er muss erst dazu beitragen, ein gesellschaftliches Verständnis und Bewusstsein für nicht unmittelbar wahrnehmbare Verletzungen des Körpers und der Psyche als Folge von Flucht, Unsicherheit und Entwurzelung zu schaffen. Das weitgehende Ausbleiben einer gesellschaftlichen Anerkennung des Leids und des damit zusammenhängenden sozialen Rückhalts spüren Kogoros Gesprächspartner somit zu einem ganz anderen Grad als Ayakos Rezipienten - ein aus meiner Sicht ganz entscheidender Punkt in der emotionalen Bewältigungsarbeit, der sich direkt auf die Genesung der Menschen und auf die Arbeitsansätze der Künstler auswirkt.

In der Auseinandersetzung mit den Folgen der Katastrophe kann Ayako sowohl auf persönlicher wie auch beruflicher Ebene auf eine weitestgehend intakte soziale Infrastruktur zählen, während unter Kogoros Gesprächspartnern viele Mitglieder von Familien- und/oder Dorfgemeinschaften waren, die sich auf der Flucht vor atomarer Kontaminierung über das ganze Land verteilt hatten.

Kogoro versucht in seinen Werken, ein anhaltendes Bewusstsein der Bevölkerung Tokyos für die Entwicklungen in der Region Fukushima zu etablieren. Die gesundheitlichen, wirtschaftlichen und sozialen Folgen der Atomkatastrophe werden erst im Laufe einer langen Zeit sichtbar werden. Kogoro sieht sich folglich mit einer Situation konfrontiert, in der er sein Publikum zunächst für die komplexen Problematiken der verstrahlten Gebiete sensibilisieren und interessieren muss - eine Voraussetzung, die in Ayakos lokaler Rahmung von vornherein gegeben ist.

Zum Komplex der Lokalität gehört ebenfalls der Umstand, dass Kogoros Arbeitsansatz auf Interviews mit Menschen basiert, die aus den verstrahlten Gebieten geflohen sind. Er kann sich, unter anderem aufgrund beschränkter finanzieller Mittel, nur für kurze Zeit dort aufhalten und erlebt sich so nicht als Teil der lokalen Gemeinde. Sein weit verzweigtes Beziehungsnetzwerk aus Betroffenen stellt für ihn die Hauptinformationsquelle dar, wenn es darum geht, persönliche Eindrücke, Erfahrungen und Emotionen zu sammeln. Die kurzen Aufenthalte finden zwar regelmäßig statt, können jedoch die Intensität des alltäglichen Erlebens wie in Ayakos Fall nicht ersetzen. 
Die unterschiedlichen Ebenen persönlicher Erfahrungen aufgrund der an Orte gebundenen Ereignisse werden durch eine weitere wichtige Facette ergänzt. Ayakos Erleben des Tsunamis ist nicht nur durch die historisierbare Erfahrung vorangegangener Generationen geprägt, sondern auch von dem Wissen um die Endlichkeit des akuten Katastrophenzustandes. In einem absehbaren Zeitraum wird ihr Heimatort wieder aufgebaut und bewohnbar sein. Eine bereits bekannte Form der Normalität kann sich einstellen. Dies ist eine Aussicht, die in der gegenwärtigen chaotischen Situation Trost spendet.

Kogoro hingegen, der sich mit den Konsequenzen der Atomkatastrophe auseinandersetzt, kann sich nur in einem stark von moralischen Aushandlungsprozessen flankierten Diskursrahmen bewegen. Die Neuartigkeit des atomaren Desasters mit unbekannten Folgen der inneren wie äußeren Verstrahlung erschweren sein Bemühen um Empathie, Solidarität und Handlungsbereitschaft seitens der Gesellschaft. Kogoro betätigt sich in Diskursfeldern, auf denen die verschiedenen gesellschaftlichen Machtinstanzen der japanischen Gesellschaft um die Dominanz im Diskursnarrativ ringen, was die Brisanz seines Wirkens nur noch erhöht. Es mehren sich die Stimmen, die von mehreren hundert Jahren sprechen, in denen das Land nur zeitweise betreten, jedoch keinesfalls gefahrlos bewohnt werden könne. ${ }^{82}$ Diese potentielle „Unendlichkeit der Katastrophe“ (siehe Beck 1986, 2015) belastet die japanischen Diskurse um die Atomkatastrophe insofern, als dass sie die Frage der Verantwortung für das Geschehene nicht nur in die Vergangenheit, sondern in die ferne Zukunft projiziert. Es ist noch nicht klar, ob die Langzeitfolgen für die körperliche und psychische Gesundheit jemals vollständig erfasst und entschädigt werden können.

Aufgrund dieses Umstandes rückt Kogoros Fokussierung auf die Atomkatastrophe die Auswirkungen auf das moralisch-ethische Selbstverständnis Japans viel mehr in den Mittelpunkt als Ayakos Arbeit. In diesem thematischen Kontext ist Kogoros Rolle von vornherein nicht von einer kritischen Auseinandersetzung mit gesellschaftspolitischen Strukturen der japanischen Gesellschaft zu trennen. Die Spiegelung der von ihm erfahrenen Situation und Herausforderungen der Betroffenen vor Ort wirken in einer Metropole wie Tokyo schnell wie eine Provokation und erfahren an diesem geographisch entfernten Ort die Konnotation der Subversivität. Damit läuft Kogoro sehr viel stärker als Ayako Gefahr, das kulturspezifische Emotionskonzept zu verletzen und mit Ausgrenzung und Scham sanktioniert zu werden. Kritik an den gesellschaftlichen Strukturen zu üben lässt Kogoros Arbeit zu einer großen persönlichen Herausforderung werden, die sich stark von Ayakos Arbeitskontext unterscheidet.

Die Voraussetzungen, unter denen die beiden Künstler arbeiten, sind also nicht nur aufgrund ihrer regionalen Schwerpunktsetzung und biographischen Erlebnisse völlig unterschiedlich gelagert, sondern werden auch durch die unterschiedlichen emotionalen Bewertungen und Konnotationen seitens der japanischen Gesellschaft

\footnotetext{
82 Siehe Kapitel 5.1 „Der ,soziale Tsunami` - Auswirkungen der Dreifachkatastrophe auf die japanische Bevölkerung“.
} 
beeinflusst. Die Konstellation aus persönlichem Erleben, der Wandlung der eigenen Betroffenheit in lokalen Stolz und die Entdeckung neuer Rollen scheint sich in Ayakos Fall entscheidend auf ihr Selbstnarrativ auszuwirken. Bereits bei unserem zweiten längeren Treffen im November 2014, drei Monate nach unserem ersten Interview, beschreibt sie sich nicht länger als Opfer. Sie sei darüber hinausgewachsen und plane nun weitere Auslandsaufenthalte in Europa, um sich noch weitere Perspektiven aneignen zu können, die nicht zwingend etwas mit der Katastrophe zu tun haben. „I am still affected but I gained something as well. It's hard to put into words. I learned to let go. I take things as they are now" (Ayako 20.11.2014: 1).

Ayako und Kogoro sind Beispiele für eine Gruppe von Akteuren, die sich dem Kredo des Verschweigens nicht beugen, sondern ihre selbstempfundene Andersartigkeit und Herangehensweise an Herausforderungen als Stärke betrachten. In der Kunst haben sie eine Sprache gefunden, die sie ihre eigene Identität im nicht zu starken Widerspruch zu geltenden Emotionskonzepten erscheinen lässt. Ihre jeweiligen Rezipienten erhalten so die Möglichkeit, sich mit neuen Perspektiven auseinanderzusetzen. Die Dinge verändern zu wollen, stellt besonders in einem Land wie Japan mit seinen vergleichsweise engen gesellschaftlichen Rahmungen und Rollenvorstellungen eine Herausforderung dar. Dennoch gewann ich den Eindruck, dass Gegenwartskünstler gerade vor dem Hintergrund der tragischen Ereignisse von 2011 die entstandene gesellschaftliche Unruhe aufzugreifen wissen, um mit Kreativität neue Wege aufzuzeigen und so wichtige Impulse für nachhaltige Transformationen in Japans gesellschaftlichen Diskursen zu geben. Diese Impulse entstehen aus ihrem persönlichen Erleben der Gesellschaft Japans heraus. Indem sie ihren persönlichen Umgang mit diesen Erfahrungen finden, können sie andere dazu ermutigen, sich ebenfalls stärker mit ihren eigenen Gestaltungsmöglichkeiten auseinanderzusetzen. Wie dieser Umgang aussieht, zeigen die folgenden Beispiele.

\subsection{Vor und nach 2011 - Biographische Antworten auf gesellschaftliche Fragen}

In der Analyse weiterer Interviews mit Künstlern und deren biographischer Facetten befasse ich mich sowohl mit der wirtschaftlichen und gesellschaftlichen Position der Künstler als auch mit der Bewertung ihrer Kunst vor und nach 2011, sofern die Dreifachkatastrophe in die biographische Erzählung einfließt.

Nur eine einzige Gesprächspartnerin gab an, sie wolle sich aufgrund der gesellschaftlichen Brisanz nicht mit dieser Thematik auseinandersetzen. Begründet wurde dies mit der Angst, man könne in Zukunft noch seltener Engagements finden. Eine persönliche Betroffenheit hatte demzufolge keinerlei Einfluss auf ihr professionelles Handeln - die einzige Ausnahme in meinem 20 Fälle umfassenden Datenmaterial. ${ }^{83}$

${ }^{83}$ Siehe Kapitel 9.3 „Die Ausnahme der Regel - Tasha aus Tokyo“. 
Die von mir zusätzlich interviewten Experten wie Kuratoren, Galeristen und Manager bestätigen meinen Eindruck: Die große Mehrheit der Gegenwartskünstler Japans beschäftigten sich bereits vor dem 11. März 2011 mit sozialkritischen Themen und demzufolge auch mit Aspekten im Spektrum der durch die Dreifachkatastrophe deutlich zu Tage getretenen Problemstellungen der japanischen Gesellschaft.

Im Kontext der Katastrophe von 2011 verwenden Künstler die Sprache der Kunst, um eben jene Aspekte in den dominanten Diskurs einfließen zu lassen, die sie thematisch in den meisten Fällen bereits zu früheren Zeitpunkten beschäftigt haben und nun den Einzelnen dazu inspirierten, sich genauer mit gesamtgesellschaftlichen Belangen zu beschäftigen. Kimiko, eine Animationskünstlerin aus Tokyo, stellt beispielsweise einen Wandel in ihrem persönlichen kreativen Fokus fest: „Before 2011 I was quite self-centered. Now I want to find expression for other people" (Kimiko 07.10.2014: 2). Die Diversität biographischer Lösungsansätze meiner Gesprächspartner hat ihren Ursprung in einer lebensgeschichtlichen Suche nach einer geeigneten Rolle innerhalb der japanischen Gesellschaft.

Besonders aufschlussreich in diesem Zusammenhang war folgende Aussage eines weiteren männlichen Gesprächspartners, Jahrgang 1979: „Art can make society change. The artist is the proposer to society" (Saburo 13.09.2014: 3). Es geht folglich weniger darum, einen alternativen Weg in unverrückbare Bahnen zu lenken, sondern darum, Vorschläge zur weiteren Gestaltung des Zusammenlebens zu machen, die nebeneinander existieren können ohne zwingenderweise in Konkurrenz zueinander zu stehen.

Im Folgenden zeige ich, wie die von meinen Gesprächspartnern behandelten gesellschaftskritischen Thematiken mit ihren jeweiligen biographischen Meilensteinen verwoben sind und dabei in Wechselwirkung zueinander stehen. Die Mitgestaltung der normativ aufgeladenen Debatte um die gesellschaftliche Entwicklung Japans nach der Dreifachkatastrophe, die in wissenschaftlichen Kreisen unter dem Begriff „Post-Fukushima-Diskurs“ zusammengefasst wird, ist dabei ein wesentliches Aktivitätsfeld meiner Gesprächspartner. Die Diskurse um die Dreifachkatastrophe und ihre Folgen bilden dabei meiner Meinung nach ein weiteres Moment im persönlichen Hinterfragungsprozess gesellschaftlicher Rahmenbedingungen meiner Gesprächspartner.

\subsubsection{Identität und Zugehörigkeit}

Gegenwartskünstler zu sein wird von meinen Gesprächspartnern als ein Zustand beschrieben, der ihr Sein ausdrückt. Ein solcher Zustand ist gekennzeichnet durch entsprechende Charakteristika, die die Individuen im Spannungsfeld mit japanischen Idealvorstellungen einer weitestgehend an die Normen der Gesellschaft angepassten Person entwickeln. Die Abweichung des jeweiligen Verhaltens wird in meinen Interviews aber in eine Quelle der Inspiration und des Ressourcenreichtums umgedeutet, die sowohl für den Künstler selbst als auch für die Rezipienten genutzt werden kann. Es handelt sich also um eine Betrachtungsweise, die es meinen Gesprächspartnern 
ermöglicht, das Gefühl, aus dem Schema zu fallen, positiv auszulegen. Im jeweiligen Narrativ passen meine Gesprächspartner das, was sie im Innersten ausmacht, bewusst nicht dem äußeren Rahmen an. Stattdessen bemühen sie sich, ein Arbeitsumfeld zu schaffen, in dem sie ihre persönlichen Kompetenzen zum Wohle aller ausleben können.

\subsubsection{Yasuko}

Dazu zählt auch der Durst des einzelnen Künstlers nach Wissen und nach der Hinterfragung bestehender, als selbstverständlich verstandener gesellschaftlicher Muster. Yasuko ist ein Beispiel für die Fortführung ihres künstlerischen Themas nach „Fukushima": Nach der Teilnahme an einer freiwilligen Hilfsaktion im vom Tsunami zerstörten Gebiet Ishinomaki und der Konfrontation mit Trauer, Wut und Verwirrung der Anwohner vertiefte sich ihr Wunsch, sich in ihrer Kunst weiter mit Identität, Erinnerung und Lokalität auseinanderzusetzen und die Menschen für diese Themen zu sensibilisieren. Sie zog es vor, unsere Gespräche ohne meinen Übersetzer Satoshi auf Englisch zu führen. Ich gewann den Eindruck, dass es ihr besonders wichtig war, eine emotionale Nähe zu mir herzustellen, bei der sie eine dritte Person als störend empfand.

„I use the coordinates from google earth to find and document new places in art. I want to give a little presentation about Fukushima with art and thinking. It might be a very little project, but I want to keep doing something."

(Yasuko 12.02.2015: 2)

Yasuko nutzt in ihrer Kunst die geographischen Koordinaten, um ihre Rezipienten auf verschiedene Orte aufmerksam zu machen. Beispielsweise bedruckte sie 2014 Kekse eines kleines Cafés mit den geographischen Daten des Standorts dieses Cafés und verband auf diese Weise ihre Kunst mit einem lokalen Kontext.

Selbst in Borneo, Malaysia, geboren, ergab sich Yasukos Faszination für verschiedene Orte und ihre eigene Selbstfindung vor dem Hintergrund ihrer Familiengeschichte: Ihre Eltern zogen nach Japan zurück, doch die Arbeit ihres Vaters als international tätiger Architekt ließ sie in ihrer Jugend oft umziehen. „When I was young, I didn't have many places to turn to. In this respect, artists are convenient people. I can make communication with many people, it's success" (Yasuko 12.02.2015: 6). In dieser Aussage verbinden sich mehrere Aspekte von Yasukos Selbstverständnis zu einem Ganzen. Das Leben als Künstlerin wird von ihr vor dem Hintergrund ihrer viel gereisten Familie auch unter dem Aspekt der örtlichen Flexibilität bewertet und liefert ihr damit ein Thema zur kreativen Auseinandersetzung mit ihrer eigenen Person. Wohin Yasuko gehört, welche Orte in ihrer Erinnerung eine wichtige Rolle spielen und wie sie dies kommunizieren kann, sind Fragen, die sie mit dem alltäglichen Leben der Menschen in ihrem Umfeld verbindet: „We have to listen to people's stories“ (Yasuko 12.02.2015: 3). Im Hinblick auf die Ereignisse vom 11. März 2011 
und ihren eigenen Erfahrungen vor Ort während ihrer freiwilligen Arbeit gewann ihre künstlerische Tätigkeit eine neue Dimension. Die Vergänglichkeit von Orten und den an sie geknüpften Erinnerungen wird Yasuko nach eigener Aussage auch in ihrem Wohnort Tokyo immer wieder vor Augen geführt: „In Tokyo, when you look at the architecture, everything is new and shiny. This might be a problem for people, because we lose track of time and history very quickly. Then we also lose memories connected to these places" (Yasuko 12.02.2015: 2).

\subsubsection{Saburo}

Ähnlich äußerte sich ein anderer in Tokyo lebender Künstler, der Freiluftmonumente aus verschiedenen Erdschichten erstellt, um den Bewohnern kleinerer Ortschaften ihre lokale Geschichte und deren historische Bedeutung plastisch vor Augen zu führen. Als ich Saburo auf einer Ausstellung in einer kleinen Galerie kennenlernte, war er schnell für ein Gespräch zu gewinnen. Sie fanden vorwiegend in seiner Galerie auf Englisch statt.

Saburo begann seine Karriere aus dem Wunsch heraus, eine tiefere Bindung der Menschen zu öffentlichen Orten wie Parks herzustellen: „I was wondering: How can I help the community? I don't know why I make sculptures. It just appeals to me" (Saburo 2014: 11). Der 35-jährige stammt aus einer Familie, in der ein großes Interesse für Kunst herrschte. Seine Mutter hatte Design studiert und hätte laut Saburos Aussage selbst gerne in dieser Branche gearbeitet. „My great-grandfather and grandfather were interested in art as well. When I went to art university I didn't know about all this, but maybe my mother and I inherited this" (Saburo 13.07.2014: 11). Saburo führte seine ersten Projekte als Künstler, in denen er sich bereits mit Lokalität und Identität und den mit ihr verbundenen Personen auseinandersetzte, nach dem 11. März 2011 weiter und offenbart dabei seine starke intrinsische Motivation: „When I went to Tohoku to see for myself I was in shock. In Tokyo nobody was dead but in the area it was quite different. People lost so many things and relatives, friends and neighbors. I was wondering ,Is this still Japan?" The realities of Tokyo and Tohoku are totally different. It's unnatural“" (Saburo 13.07.2014: 10). Diese Eindrücke, Erfahrungen und Gefühle des Schocks und der Verwunderung waren der Anlass für Saburo, die Rolle der Kunst in Zeiten der Katastrophe zu hinterfragen. Besonders der atmosphärische Druck in Fukushima-Stadt, die Kunst ausschließlich zur Aufheiterung zu nutzen, beschäftigte ihn lange: „Fukushima people want to think about the future. People from Tokyo are so careful, but Fukushima-people also want fun. They asked the artists to make a festival. I decided that I can participate in this kind of art as a human being. I had no experience with that until then" (Saburo 13.07.2014: 10).

Interessanterweise ist dies das einzige Mal während unserer Zusammenarbeit, dass Saburo eine Differenzierung zwischen seiner Rolle als Künstler und Privatperson vornimmt. Bis zu diesem Zeitpunkt hatte er die Deckungsgleichheit von persönlichem und professionellem Leben betont und verdeutlicht, dass sich diese beiden 
Sphären gegenseitig in seiner kreativen Tätigkeit befruchten. In der Konfrontation mit großer Zerstörung und menschlichem Leid musste Saburo sich jedoch, wie viele meiner Gesprächspartner, einer neuen Realität und einer Hinterfragung seiner Tätigkeit in diesem Kontext stellen. Die daraufhin eintretenden Veränderungen in seinem Denken und Handeln beschreibt Saburo so:

"The real situation in Tohoku made my mind [kokoro] for artwork change. I don't want to make people happy with my art. I want to do something more serious. I want to give something to people, to make them think or to give them an emotional effect." (Saburo 13.07.2014: 13)

Auf meine Nachfrage, was genau er mit kokoro in diesem Kontext meint, betont Saburo die Tiefe dieses Begriffes im Gegensatz zu einem oberflächlicheren „Gefühl“, das sich auch schnell verflüchtigen könne: „I made a decision about what kind of artist I want to be. I want to make serious art, nothing for fun. My experiences in Tohoku had that lasting effect on me" (Saburo 13.07.2014: 13).

Als ich das Interview mit meinem Übersetzer Satoshi durchsprach, wählte er „mind“ als Übersetzung für den von Saburo im Japanischen gebrauchten Begriff kokoro, was auch in der Literatur so üblich ist. Genau genommen umfasst der japanische Begriff jedoch nicht nur „Geist“ im Sinne von Denken, sondern beschreibt auch eine Ebene des Gefühls und der Emotion (vgl. Moeran 1986: 72; Maynard 2005: 56).

Diese neue Klarheit über seine gesellschaftliche Rolle manifestiert sich auch in Aussagen, die sich mit der Aufgabe von Kunst im Allgemeinen beschäftigen. Sie verdeutlichen Saburos Reflexion über die Ansprüche und emotionalen Facetten, die die Kunst seiner Meinung nach veranschaulichen kann: „We already knew that emotion of art can change something, not just people. [...] Art can make society change. Maybe the artist is also a part of society and proposes things to society. Pointing things out, making them visible" (Saburo 13.07.2014: 14).

Diese Aussage deckt sich weitestgehend mit der von Kogoro (02.05.2014: 26) und anderer von mir interviewten Künstler, die ihren Platz in der Gesellschaft vor allem über ihre Tätigkeit, das Ungesehene zu reflektieren, definieren. Saburo geht noch einen Schritt weiter, indem er der Kunst in ihren emotionalen Transformationseffekten eine Exklusivität einräumt: „To influence people in this way is something only art can do" (Saburo 13.07.2014: 14). Diese große gesellschaftliche Macht wird für den einzelnen Künstler nur erfahrbar, indem er sich direkt mit seinen Rezipienten auseinandersetzt. Erst in der Verbindung mit der emotionalen Reaktion des Rezipienten wird die Kraft der erschaffenen Kunst sichtbar.

Die hohe Bedeutung des „Sichtbarwerdens“ von starken Emotionen und Erfahrungen zeige ich auch im nächsten Abschnitt, in dem durch die Künstler Erinnerungen auf konzeptionelle Weise angesprochen werden, die sich ins kollektive Bewusstsein eingeschrieben haben. In den zum Teil sehr gesellschaftskritischen Werken der jeweiligen Künstler werden nicht nur Perspektiven aufgrund persönlicher 
biographischer Sensibilität erschlossen. Auch zeigt sich, wie die eigene Persönlichkeit im sozialen Gefüge verortet und in der Erzählsituation mit mir hergestellt wird. Die besondere Macht dieser Verknüpfung aus gesellschaftlicher Wirksamkeit und individueller Existenzbegründung beruht damit auf der, in den Biographien rekonstruierten Notwendigkeit, das eigene Selbst in den größeren gesellschaftlichen $\mathrm{Zu}$ sammenhang zu setzen und dort wirksam zu sein.

\subsubsection{Sichtbarkeit von Emotionen}

Die folgenden Aussagen der Künstler beschreiben Aspekte der biographisch umgesetzten Aushandlung ihrer individuellen Wirksamkeit im gesellschaftlichen Rahmen von Japans Konzept vom Umgang mit Emotionen. In diesem Prozess der individuellen Verortung kommt das Konzept meiner Gesprächspartner zum Ausdruck, sowohl ihre persönlichen Emotionen als auch die ihrer Rezipienten zu verarbeiten und transparent zu machen. Dabei nutzen sie unterschiedlichste, ihnen zur Verfügung stehende Materialien und Räume, um dem Unsichtbaren eine Gestalt zu geben.

\subsubsection{Mariko}

Die 50-jährige Mariko arbeitet seit zwei Jahrzehnten mit Photographien und Büchern, um ihr Konzept von Erinnerung sichtbar zu machen. Mariko gehört zu denjenigen Künstlern, die sich auf internationaler Bühne bewegen und die ihre Korrespondenz vorwiegend auf Englisch führt. Über Saburo war der Kontakt mit ihr zustande gekommen, so dass sie keinerlei Hemmungen hatte, gleich bei unserem ersten Kontakt tiefer in die Thematik einzusteigen.

Ihre Installationen strukturieren den Raum, in dem sie sich befinden, und sollen eine Komposition ihrer Gedanken darstellen (Mariko 23.03.2015: 1). Wie bei Saburo, Kogoro und Ayako wird die enge Verknüpfung zwischen Privatperson und professioneller Rolle deutlich: „In order to realize that theme I encounter myself, my family and also people who are strangers to me" (Mariko 23.03.2015: 1). Anders als die oben genannten Künstler geht Mariko vergleichsweise wenig auf die Dreifachkatastrophe ein, sondern konzentriert sich in ihren Aussagen vorwiegend auf ihr persönliches Verhältnis zur japanischen Gesellschaft. Ihre Erfahrungen im wirtschaftlichen Überlebenskampf als Künstlerin sind der Ausgangspunkt ihrer Thematik der Erinnerung und wie sie Einfluss auf die Charakteristika einer Person oder einer Gesellschaft nehmen. „I built this theme up gradually. I want to share some basic emotions from our daily life with people. What kind of common memories do people share from other areas, countries etc.? I want to focus on that" (Mariko 23.03.2015: 5). Diese Passage verdeutlicht die von Mariko gezogene Verbindung zwischen persönlichen Emotionen und Erinnerungen, die durch das Teilen mit Anderen ein verbindendes, zwischenmenschliches Element erschaffen. Ein Verbindungselement, das angesichts der Dreifachkatastrophe an besonderer Bedeutung gewinnt. 
Der Umgang mit Büchern und Photographien, die sie vorwiegend als Arbeitsmaterialien für ihre Installationsarbeit benutzt, hat sie auf ähnliche Weise wie ihre Thematik gefunden. Ihre Faszination für Bücher ließ sie darüber nachdenken, was genau das Erinnern ausmacht und wie sie die damit verbundenen Gefühle in ihrer Kunst umsetzen könnte: „Social issues, history, it is important to get this knowledge from books, but it is difficult to share the feelings. Such things I want to realize with my art: A dialogue with anyone living anywhere" (Mariko 23.03.2015: 5). Die Diskrepanz zwischen der äußeren Erscheinung von Büchern, die sie nur als „a bunch of paper and writing“ (Mariko 23.03.2015: 5) bezeichnet, und deren innerem Potential, das Bewusstsein der Menschen zu beeinflussen, interessiert sie unter anderem auch wegen ihrer Aussagekraft über die Persönlichkeit eines Menschen: „When we view someone's bookshelf it lets us imagine the kind of life they live. Collections of books are an invisible portrait of us" (Mariko 23.03.2015: 2). Bücher spiegeln aus ihrer Sicht also ein inneres Abbild ihres Besitzers wider.

Das Abbild menschlichen Lebens in seinen Facetten und Formen beschäftigt Mariko auch auf persönlicher Ebene. Sich in seiner Einzigartigkeit zeigen und dabei von anderen gesehen zu werden, ist ein starkes Thema ihrer Biographie. In einem sehr konservativen Haushalt aufgewachsen, kämpft Mariko seit ihrer Kindheit darum, sich individuell entfalten zu können. Ihr erster Kontakt mit Gegenwartskünstlern fand erst in einer persönlich turbulenten Zeit statt, als sie sich mit 27 Jahren von ihrem Ehemann scheiden ließ: „I met an independent artist at that time. He was smoking and drinking all the time and I really wanted to do art. During the following years art became really important to me" (Mariko 23.03.2015: 7). Ihre künstlerische Tätigkeit scheint Mariko in dieser Zeit stabilisiert zu haben und gab ihr darüber hinaus die Chance, ihr gewohntes Umfeld Japan zu verlassen, um sich international zu erproben. Verschiedene Ausstellungen in der Schweiz oder Australien ermöglichten ihr Einblicke in die dortige lokale Kultur und gaben ihr neue Erinnerungen, von denen sie bis heute emotional zehrt: „I change my memory by myself. I think memories are my life, the making of life" (Mariko 23.03.2015: 5). Mariko ist ein gutes Beispiel für eine Person, die Ideen und Inspirationen aus der Reflexion über die eigene Emotionalität bezieht. Sie vermeidet ihre eigenen Emotionen nicht, sondern verwendet sie gezielt für ihre Kunst. Darüber hinaus ist es vor allem der Austausch mit anderen, der sie auch nach der Dreifachkatastrophe dazu anhielt, sich weiter mit dem Teilen von Erinnerungen zu beschäftigen. „Right now Japan is at war with technology. To make artworks I need my own feeling. I have to update my feelings in new artworks because the feelings are also changing with age. I am more relaxed now than when I was 20 years younger. I only do what I want to do" (Mariko 23.03.2015: 7).

In dieser Aussage spiegelt sich meines Erachtens Marikos Distanzempfinden zur Technologie wider, die das zwischenmenschliche Zusammenleben rund um den Globus beeinflusst. Marikos Betonung der Wichtigkeit eines persönlichen Gefühls für die Ereignisse, um dieses Gefühl in Kunstwerke zu übersetzen, zieht sich durch das gesamte Interviewmaterial. Ihre persönlichen Eindrücke werden nicht mit der 
Agenda der Künstlerrolle gesammelt, sondern sie stellen vielmehr ein Ventil des Ausdrucks für diese private Emotionalität dar.

So nutzt Mariko mit der Photographie einen weiteren Ausdruck ihrer Emotionalität. Photographie als Medium fasziniert sie dabei vor allem wegen ihrer bildlichen Präsenz, die die Vorstellungskraft des Publikums beflügelt: „[...] because the photo has lost its specific information and identity, we can only imagine the surrounding story of the instant in time which otherwise ought to be certain" (Mariko 23.03.2015: 2). Die Zeit- und Kontextlosigkeit ihrer Bilder verdeutlichen Marikos Ansichten über die Flüchtigkeit von Erinnerungen. Da es jedoch gleichzeitig Erinnerungen sind, die ihrer Meinung nach das Selbst bestimmen, erhält ihre Arbeit vor dem Hintergrund des 11. März 2011 eine zusätzliche Dimension. Die Art und Weise, wie sich in Zukunft an die Katastrophe erinnert werden wird, entscheidet sich laut Marikos Narrativ auf der persönlichen Ebene eines jeden Einzelnen: „This means that the image of human life is constructed merely from personal memories" (Mariko 23.03.2015: 3).

Auf diese Weise nimmt Mariko in ihrer Rolle als Künstlerin gleichzeitig die Position der Dokumentarin und Archivarin von Ereignissen ein. Die durch das Objektiv der Kamera widergespiegelten Subjekte sollen das Publikum dazu veranlassen, sich neuen Emotionen und Wirkungen zu öffnen. Der Zeitfaktor hat in Marikos Arbeit eine weitere Komponente, die auch Saburo ähnlich artikulierte: „Because looking into the past is facing the present, not looking back at our life" (Mariko 23.03.2015: 3).

Dies umschreibt meiner Ansicht nach in vortrefflicher Weise einen in Kapitel 5 aufgeführten wesentlichen Aspekt, der die Gegenwartskunst in ihrer Gesellschaftskritik eng an den Diskurs um die Dreifachkatastrophe bindet: Die Rekonstruktion der Vergangenheit ermöglicht es, die Gegenwart zu bewerten, was wiederum zu unterschiedlichen Ansichten über die zu ziehenden Konsequenzen für die Zukunft führt. Im Falle der Künstler geschieht dies als gezielte Handlung, die sich aus ihren eigenen Emotionen und Empathie speist. Es entstehen viele verschiedene Perspektiven und emotionale Bewertungen derselben Situation. Diese nur schwer von institutioneller Seite zu kontrollierende Perspektivenvielfalt eröffnet erst den Raum für gesellschaftskritische Ansätze, wie sie auch die Künstler in kreativer Sprache in den öffentlichen Raum zurückspiegeln.

Hinzu kommt die in Marikos Aussagen deutlich werdende Ansicht, dass Vergangenheit und Gegenwart ständig neu konstruiert werden. Die von Mariko erwähnten persönlichen Erinnerungen, die das menschliche Erleben prägen, werden in ihrem Narrativ in Fotos festgehalten:

„Taking pictures is definitely the most efficient action in order to log those memories we don't want to lose. We know potentially that 'permanence' doesn't exist anywhere on earth. [...] Instead, when we re-encounter a photo after a long absence we often find something new or different from before in that image although nothing changed in the recorded fact." (Mariko 23.03.2015: 3) 
Der hier von Mariko angesprochene Perspektivenwechsel macht deutlich, dass es ihr, wie vielen anderen meiner Gesprächspartner, nicht um eine Wiedergabe von Fakten geht, sondern um die Aufarbeitung persönlicher Eindrücke. Folglich steht für Mariko nicht ein situativ favorisiertes Bild des öffentlichen Diskurses im Fokus, sondern die Realität wie sie der jeweilige Rezipient wahrnimmt.

Zusätzlich werden alltägliche Eindrücke in Marikos Arbeit durch den zeitlichen Abstand zu etwas Besonderem erhoben, obwohl sich an den Tatsachen nichts verändert hat. Vergangenes kann dadurch in der Gegenwart einen neuen Stellenwert bekommen, oft allein aufgrund der Tatsache, dass sich die Perspektive des Betrachters verändert. Marikos Narrativ betont die gewaltige Kraft der individuell erfahrenen Emotionen aufgrund eines einzigen Eindrucks. Die persönliche Vorstellungskraft des jeweiligen Rezipienten gestaltet die Wirkungsweise ihrer Kunst und damit auch dessen Erinnerung an vergangene Ereignisse mit. Die alltägliche Emotionalität kann auf diese Weise in den Mittelpunkt gerückt und geteilt werden, so dass vor den Augen des Rezipienten eine andere Lebenswirklichkeit ausgebreitet wird.

Dieser ständig ablaufende Prozess der Neuerschaffung von Erfahrungen, Erinnerungskonstruktionen und Entdeckung von Gemeinsamkeiten bildet das Kernstück von Marikos Arbeit. Sie verschafft dem scheinbar Trivialen einen besonderen Platz in ihren Installationen aus Fotos und Büchern, um es dem Publikum zu ermöglichen, in die Tiefe zu gehen und Erinnerungen neu zu erleben. In der graphischen Darstellung erschafft sie einen Raum, in dem sie auch ihr eigenes Leben immer wieder neu betrachten und sich mit den Eindrücken konfrontieren kann: „We face our misshapen lives and I think we [as human beings] are adorable for that very reason. It is that element of life filled with both heartache and warmth that I wish to convey with my artwork" (Mariko 23.03.2015: 1). Marikos Perspektive auf das Leben, bestehend aus koexistierender Wärme und Schmerz, ermöglicht gewissermaßen eine friedliche, hochgradig empathische Auseinandersetzung sowohl mit biographisch-persönlichen wie auch kollektiven Themen, die unter anderem durch zeitlichen Abstand in ihrer Existenz als Erinnerung verändert werden können. Marikos Leistung liegt ihrer eigenen Aussage zufolge in der Sichtbarmachung dieses Prozesses und im Teilen von Erfahrungen und täglichen Emotionen, die ihre eigene Aussagekraft außerhalb der Zeit besitzen (Mariko 23.03.2015: 1).

Emotionen einzufangen und visuell darzustellen ist eine Thematik, mit der sich eine weitere Künstlerin in Form von Animationsfilmen, basierend auf Handzeichnungen, auseinandersetzt.

\subsubsection{Kimiko}

Kimiko, geboren 1985 in Tokyo, genoss eine Ausbildung an der renommierten Geidai-Universität in Tokyo in Film und Animation. Bei dem Besuch einer Veranstaltung an der Geidai war ich von einer anderen Teilnehmerin auf Kimikos Kunst aufmerksam gemacht worden und der Kontakt gestaltete sich als sehr unkompliziert. Vermutlich durch den geringen Altersunterschied gelang es uns schnell, eine ver- 
trauensvolle Ebene aufzubauen, und sie stellte im Gegenzug viele Fragen zu meinen bisherigen Ergebnissen. Ich hatte den Eindruck, dass sie unter anderem aufgrund ihres Interesses für meine Arbeit sehr stark an einem Austausch interessiert war und auch deshalb meinen Übersetzer Satoshi, der ebenfalls Künstler ist, sehr gerne dabeihaben wollte. Satoshi war seinerseits von ihrer Form der animierten Kunst und ihren Möglichkeiten fasziniert. Unsere Gespräche im Anschluss an die Interviews waren entsprechend angeregt und führten zu einem andauernden Kontakt zwischen Satoshi und Kimiko.

Durch ihre konservative Familie erfuhr Kimiko „[...] not much encouragement for art" (Kimiko 07.10.2014: 2). Die anfängliche Unsicherheit über ihre eigenen Fähigkeiten legte sie mit 19 Jahren ab, als sie die Aufgabe bekam, ein „Life-Story-Book“ anzulegen, in dem sie ihre eigene Lebensgeschichte darstellen sollte. „It was a time of self-expression. I came to the realization that there is no way to measure talent. So I said: 'Just do it!'“ (Kimiko 07.10.2014: 2). In dieser Zeit verortet Kimiko im Rückblick massive charakterliche Veränderungen ihrer Person: Sie sei vom introvertierten, sehr stillen Mädchen zu einer extrovertierten Frau geworden (Kimiko 07.10.2014: 2). Ihre ersten Schritte als Künstlerin bestanden in der Arbeit für eine NGO, die sie beauftragte, Workshops für Kinder mit Asperger-Syndrom zu entwerfen und durchzuführen. Die Konfrontation mit den Lebensrealitäten anderer Menschen haben sie nach eigener Aussage stark beeinflusst und dazu gebracht, sich den Situationen des Lebens entsprechend anzupassen. „I feel like I was reborn at 22 years old“ (Kimiko 07.10.2014: 2).

Für ihre Abschlusspräsentation an der Universität Tokyo war eine Auseinandersetzung mit Kimikos eigener Familiengeschichte für den 01.04.2011 angesetzt worden. Aufgrund der Dreifachkatastrophe, die nur wenige Wochen zuvor stattfand, sah sich Kimiko jedoch veranlasst, ihr ursprüngliches Projekt zugunsten einer Animation über „Fukushima“ fallen zu lassen. Sie fuhr an die vom Tsunami betroffenen Gebiete und besuchte die Menschen in ihren Notunterkünften, die vor der atomaren Verstrahlungsgefahr geflohen waren. Mit unzähligen Zeichnungen und Erzählungen kam sie zurück nach Tokyo und startete ihr Projekt. „Nuclear power is invisible. Unlike in a movie, it can be expressed in animation. I want to create a memory of what happened“ (Kimiko 07.10.2014: 1).

Kimiko sieht einen entscheidenden Darstellungsvorteil in der Animationstechnik gegenüber anderen Medien wie beispielsweise der Photographie. „Animation anonymizes people. They are not shown in a recognizable manner so they can still tell their story even if they are afraid" (Kimiko 07.10.2014: 3). Kimiko beschreibt hier eine Art der Sichtbarmachung, die die Menschen zwar in Erscheinung treten lässt, sie jedoch nicht identifizierbar macht. Es ist eine Erzählweise von Erinnerungen, die die Geschichte und Emotionen der Betroffenen selbst in den Fokus stellt. Angesichts der mit der Verstrahlung einhergehenden Stigmatisierung von Betroffenen stellt Kimikos kreativer Zugang eine besonders sensible Form der Verarbeitung dieses brisanten sozialen Themas dar. Kimiko vermeidet mit der Animation, dass ihre Gesprächspartner aus Scham über das Erlebte oder aus Angst vor Identifizierung schweigen. 
Die Emotionen können also abgebildet werden, ohne dass der Einzelne negative Emotionen befürchten muss. Die Gefühle können trotzdem einen sehr kraftvollen Ausdruck in animierten Gesichtern, Landschaften und Behausungen finden. Wie in Marikos und Saburos Arbeit hat auch Kimikos Ausdruck ihrer Erfahrungen mehrere zeitliche Dimensionen. Zum einen besteht die Möglichkeit, mit Hilfe von Animationen vergangene Zustände, Landschaften und andere emotionale Bezugspunkte wieder auferstehen zu lassen. Wie bei einer Reise in die Vergangenheit können Erzählungen von Betroffenen visualisiert und anderen nähergebracht werden.

Zum anderen braucht die Erstellung eines Animationsfilms sehr viel Zeit. Normalerweise benötigt der Prozess von der Idee bis zum fertigen Projekt zwei bis drei Monate. Im Fall des „Fukushima“-Themas benötigte Kimiko sogar ein ganzes Jahr. Es ist kein Material, das sich so schnell wie ein Photo generieren und verarbeiten lässt, doch Kimiko sieht darin auch Potential: „It takes time but this way I can bring up issues that might have been forgotten otherwise. Issues hidden from society come into light and people can make up their mind" (Kimiko 07.10.2014: 3).

In Kimikos Aussage werden ihre Ansprüche an sich als Künstlerin deutlich. Die Sichtbarmachung verdeckter gesellschaftlicher Themen und bisher verschwiegener Emotionen ist nur einer davon. Der 11.03.2011 wird von ihr als eine Zäsur in ihrem eigenen Leben beschrieben, die den Wendepunkt in ihrer Verwendung von Kunst beschreibt. Hatte Kimiko die Kunst vor 2011 noch ausschließlich dazu genutzt, herauszufinden, wer sie ist und sich mit ihrem Leben auseinandergesetzt, will sie seitdem für die Gesellschaft arbeiten (Kimiko 07.10.2014: 1). Die Animationstechnik eröffnet ihr Möglichkeiten, auch die negativ konnotierten Aspekte des Lebens wie Tod, Zerstörung und Verlust in eine bildhafte Sprache zu übersetzen, die die Menschen nicht abschreckt, sondern zum Nachdenken anregt: „I want to initiate actions through raising questions. And in some cases it works. People who are watching my work want to visit the place for themselves. That is success" (Kimiko 7.10.2014: 4).

Kimikos Aussage ist ein gutes Beispiel dafür, wie aus ihrer persönlichen Perspektive die Handlungsfähigkeit für sich selbst und andere generiert wird. Sie berichtet von insgesamt sehr positivem Feedback für ihre Arbeit, wenn sie auch manchmal Kritik erhält. In ihrem Narrativ fällt diese Kritik jedoch nicht weiter ins Gewicht. Im Gegensatz zu anderen Künstlern weiß sie nichts von Repressalien in Form von Ausschlüssen von Galerien und Ausstellungsprojekten zu berichten. Ihre Arbeit scheint ihre Popularität sogar noch erhöht zu haben: Seitdem hat sie einige kritische TVSpots designen dürfen, die im nationalen Fernsehen ausgestrahlt wurden.

Anders als Mariko, die ebenfalls freiberuflich arbeitet, hat Kimiko weniger Schwierigkeiten, sich zu finanzieren. Werbeaufträge großer Firmen und gelegentliche Stipendien des Kulturministeriums ermöglichen es ihr sogar, längere Auslandsreisen zu finanzieren: „Once a year I want to get out of Japan. I need to meet new people and get new impressions. This way I can get my imagination started“ (Kimiko 07.10.2014: 1). Der finanzielle Aspekt ihres Daseins als Künstlerin nimmt bei Kimiko einen verschwindend geringen Anteil in ihrer biographischen Erzählung ein. Ihr Narrativ ist vielmehr von dem Durchsetzungskampf gegenüber ihrem konservativen 
Elternhaus auf dem Weg zu ihrer Berufung als Künstlerin geprägt. Finanzielle Sorgen und Nöte stehen hinter dem Gefühl zurück, entgegen aller negativer Stimmen aus ihrem Umfeld ihren Weg gegangen zu sein. Kimiko bringt ihre Haltung, insbesondere gegenüber ihrem als sehr korrekt beschriebenen Vater, folgendermaßen zum Ausdruck: „In art there is no correct answer, only my own“ (Kimiko 07.10.2014: 4).

Die öffentliche Verarbeitung ihrer Emotionen steht teilweise im Gegensatz zum japanischen Konzept des Umgangs mit Emotionen, aber Kimiko hat in ihrer Kunst einen Weg gefunden, der Individualität des Ausdrucks eine positive Note zu geben. Die überwiegend positiven Rückmeldungen zu ihrer Kunst legen nahe, dass Kimikos Arbeit weniger als Verletzung der Norm, sondern als Diversifizierung der Stimmen aufgefasst wird.

\subsubsection{Widerstand gegen gesellschaftliche Konventionen}

Wie bei Kimiko besonders deutlich geworden ist, ist Auflehnung gegen bestehende Regeln, Normen und Lebensentwürfe einer der grundlegenden biographischen Themen meiner Gesprächspartner und taucht in ihren Erzählsträngen immer wieder auf. Häufig wird von Widerstand im Zusammenhang mit ihrer künstlerischen Tätigkeit für die japanische Gesellschaft gesprochen, doch in der Rekonstruktion ihrer Biographien weisen die meisten bereits vor ihrer beruflichen Rollenfindung einen Hang zur Auflehnung auf. Dieses Aufbegehren richtet sich vor allem gegen den normativ vorgeschriebenen Umgang mit ihren Emotionen.

\subsubsection{Takeshi}

So berichtet es auch Takeshi, Jahrgang 1970, der versucht, seine Skulpturen und Gebilde in verschiedenen Galerien auszustellen. Ein erstes Vorgespräch hatte ich auf Englisch mit ihm geführt. Als er sich bereit erklärte, mich in meiner Forschung zu unterstützen, war ihm jedoch sehr wichtig, einen Übersetzer dabei zu haben. Meinem Eindruck zufolge ist dies darauf zurückzuführen, dass er sich im Englischen nicht sicher genug fühlte, um sich differenziert und präzise ausdrücken zu können. Satoshis Übersetzung aus dem Japanischen ins Englische verfolgte er sehr aufmerksam und bestätigte jede Passage ausdrücklich als stimmige Wiedergabe seiner Worte.

Takeshis Leidenschaft für das Kreative führt er auf den Beruf seines Vaters als Drucker zurück: „I watched my fathers' work as a printer, but it didn't touch me. I wanted to do something different. Nevertheless, I was surrounded by artwork since I was a baby because my father collected art from around the world as well" (Takeshi 20.12.2014: 2). Im Unterschied zu seinem älteren Bruder erlebte Takeshi den Vater als Hauptverdiener der Familie durch Kunst, was ihn zu der Überzeugung brachte, er selbst könnte von Kunst leben. Zuvor hatte die Mutter mit dem Gehalt einer Grundschullehrerin die Familie finanzieren müssen. Laut Takeshi führte diese Erfahrung zu unterschiedlichen Eindrücken der beiden Brüder in Bezug auf Kunst: „When I grew up my father had established his business as a printer and could make money. So the 
impressions were different" (Takeshi 20.12.2014: 5). Takeshi berichtet von einem Bruch in der Geschwisterbeziehung seit ihren gemeinsamen Kindertagen. Sein sechs Jahre älterer Bruder wohnt zwar am anderen Ende Tokyos, sie sehen sich jedoch nie. Es ist vor allem dieser Beziehungsbruch, der Takeshi in seiner künstlerischen Tätigkeit antreibt und ihn zu Themen wie Kommunikation und Beziehung, Grundlagen der Identität und unbewusstes Gruppenverhalten veranlasst. „He [his brother] is very strange. It makes me sad. We have no relationship at all. He is working for a pharmaceutical company which is testing new medicine" (Takeshi 20.12.2014: 4). In einer seiner Schriften beschreibt Takeshi seinen Anspruch an sich als Künstler und die grundlegende Frage, die sich durch all seine Arbeiten zieht: Welchen Grund könnte es haben, dass wir so leben, wie wir es tun?

Seinen Wunsch, das Leben in seinen Merkwürdigkeiten und Zufällen ebenso zu reflektieren wie die darin enthaltene Gleichzeitigkeit von Schmerz und Freude, entspringt unter anderem Erfahrungen wie denjenigen mit seinem Bruder: Aufgrund seiner späteren Geburt konnte Takeshi den Beruf seines Vaters als erfüllend erleben und sich demzufolge für die Kunst begeistern, während sein Bruder der Kunstszene nur Missachtung aufgrund der finanziell prekären Situation seiner Familie zur Zeit seiner eigenen Kindheit entgegenbringt.

Takeshi meint, er habe nie einen anderen Lebenstraum entwickelt als den, ein Künstler zu sein. „From early childhood on I drew. So I had no choice [laughs]. But at the end of artschool [for final exhibition], I thought I had to decide which kind of art I should do. Then I realized I didn't have to choose" (Takeshi 20.12.2014: 5). Die Möglichkeit, die die vierjährige Ausbildung an der Art School bot, ins Ausland zu gehen und zum Beispiel US-amerikanische Kunst der 1960er Jahre zu betrachten, habe ihn nach eigner Aussage sehr berührt. Besonders die Zeit in einem der „West Base"-Apartments in New York im Jahr 2013, das während des Zweiten Weltkrieges für den Bau der ersten Atombombe genutzt wurde, haben einen starken Eindruck bei ihm hinterlassen. „I took a video there, but connecting artwork with physics is difficult. I don't have enough knowledge" (Takeshi 20.12.2014: 2). Auf meine Frage, wie er die Kunst zum Thema Atomenergie einschätzt, antwortet er schlicht: „Art itself is polluted by radiation. I want to describe the effects of atomic bombs without using stereotypes" (Takeshi 20.12.2014: 2). Während dieser Zeit in New York mischte sich der Tod auf sehr persönliche Weise in Form einer schweren Krebserkrankung des Vaters in Takeshis Leben und künstlerisches Wirken. Im Versuch, die Gefühle von Verlustangst, Tod und atomarer Strahlung in einem Werk zu vereinen, fand er sich in seinen Bemühungen um die Erschaffung einer tiefen Bedeutung bestätigt: „I want to do something against the mainstream, against the most obvious opinion. Outside influences are interpreted by individuals who create stereotypes and group behavior. So anxiety is nutrition for artwork" (Takeshi 20.12.2014: 2).

Diese Passage halte ich für essentiell bei der Analyse seiner biographischen Aussagen. Sie beschreibt die Vermischung von Takeshis privaten und beruflichen Ambitionen und zeigt, auf welche Weise er sich seine Umwelt erschließt. Die Angst vor dem Verlust seines Vaters und die angestrebte Projektarbeit über Atombomben 
verbindet sein inneres Erleben mit der äußeren Welt. Diese Kombination konnte in Form eines Kunstwerks sichtbar gemacht werden und andere Menschen stimulieren. Damit greift Takeshi jene negativen Emotionen wie starke Sorgen auf, die zum Teil durch den kulturell favorisierten Umgang mit Emotionen entstehen. Für ihn sind diese Emotionen der Anlass, um die Auslöser genauer zu betrachten anstatt sie zu missachten: „An artist is like a shaman. Human beings live in an internal world. The artist connects this inner world with the outside world which can be brutal. Through this new perspective on the outside stereotypes are destroyed" (Takeshi 20.12.2014: 3). Diese Beschreibung seiner Arbeit als Künstler mit einem Begriff wie „shaman“ erlaubt sowohl Einblicke in Takeshis Selbstpositionierung innerhalb Japans Sozialgefüge als auch in den spirituellen Aspekt seiner Arbeit. Der ständigen Kontrolle der Bevölkerung durch Technologien wie Smartphones, deren exzessive Nutzung er täglich in den öffentlichen Verkehrsmitteln beobachtet, setzt Takeshi den Versuch entgegen, die Menschen auf eine andere Weise emotional anzusprechen: „I want to create some tingling feeling down the spine" (Takeshi 20.12.2014: 3). Indem er sich vor allem mit Selbstverständlichkeiten des Gruppenverhaltens, wie er es nennt, befasst, nimmt er die Rolle des Individuums im gesellschaftlichen Zusammenspiel genau unter die Lupe: „In Japan people try to behave normal, like stereotype. Everyone else is an outsider" (Takeshi 20.12.2014: 3). Des Weiteren nimmt er, wie auch Kogoro, aus meiner Sicht einige Elemente der Befriedung auf, die ich bereits bei Kogoro in Kapitel 7.1.2 „Die Aufgabe der Kunst in Krisenzeiten“ am Beispiel der spirituellen Befriedung von Seelen beschrieben habe. Die Sichtbarmachung und Verarbeitung von Emotionen, und damit ermöglichte Befriedung, weist insofern Parallelen im Umgang mit Emotionen von Verstorbenen auf (vgl. Rotermund 2004).

Die Außenseiter, wie sich Takeshi auch selbst bezeichnet, die einen eigenen Weg entwickelt haben die Ereignisse des Lebens anzugehen, seien in Japans Gesellschaft immer weniger akzeptiert je konservativer die Gesellschaft werde. Die etablierten Wege würden so nicht in Frage gestellt oder gar obsolet (Takeshi 20.12.2014: 4). Die derzeitige Lebenssituation in Japan empfindet er daher für sich als jemanden mit selbst zugeschriebenen Außenseiter-Attributen als sehr schwierig: „There is no place for a shaman and there is no tolerance anymore. There is no place for an outsider" (Takeshi 20.12.2014: 3). Ein Wohlbefinden kann sich solchen Aussagen zufolge für ihn nicht einstellen. Nichtsdestotrotz kann er nicht aufhören mit seiner kritischen Kunst, die genau diese ausgetretenen gesellschaftlichen Wege des Zusammenlebens hinterfragt und herausfordert.

Meine Gesprächspartner nutzen Kunst als eine Möglichkeit der Abgrenzung vom gängigen japanischen Umgang mit Emotionen und dessen gesellschaftlichen Auswirkungen, ohne sich völlig aus ihnen zu lösen. Die Thematiken, die sich aus meiner Sicht diesem Widerstandsnarrativ unterordnen lassen, sind dabei vielfältig. Das schnelle Tempo einer Konsumgesellschaft wird von Takeshi ebenso thematisiert wie die in seinem Empfinden schnell um sich greifende Taubheit in der Emotionalität: „I want people to feel. Stimulation is concentrated on the brain in this internet-age. People are numb. They are always checking their smartphones, being there and at 
the same time not being there" (Takeshi 20.12.2014: 4). Wie kein anderer meiner Gesprächspartner spricht Takeshi hier den feinen aber entscheidenden Unterschied bei der Rezeption von Kunst im Gegensatz zu anderen Stimulationsquellen an. Die Langsamkeit des Herstellungsprozesses, die die tiefe persönliche Reflexion des Künstlers und "the artist's conversation with his art“ (Takeshi 2014: 6) beinhaltet, entfaltet im Idealfall auf direkte Weise seine Wirkung in der Emotionalität des Publikums. Um diese Wirkung zu erzielen, nimmt Takeshi einige persönliche Opfer auf sich, die sein Ansehen in der Gesellschaft beeinflussen. Dazu gehören unter anderem negative Reaktionen auf Kunstwerke, die er selbst als „disgusting“ bezeichnet, die aber einen bestimmten Zustand in der Gesellschaft aus seiner Sicht widerspiegeln. Er verweigert sich dem dominanten Konzept, über diese Missstände hinwegzusehen oder sie verschönert darzustellen: „I used to see people who are upset about my work and who look down on me. Japanese people prefer more clean and moderate art" (Takeshi 20.12.2015: 6). Hier kommt der Teil des Emotionskonzeptes Japans zu Anwendung, das ich in Kapitel 4.1 beschrieben habe: Durch negative Reaktionen soll der Auslöser von Irritationen sanktioniert werden, zum Beispiel durch ein öffentliches Beschämen. Doch es gibt auch Stimmen, vor allem aus der Kunstszene selbst, die ihn in seinem Handeln bestätigen: „On the other hand, art people often get in contact with me for their show several years after they saw my work. So I can survive in the society narrowly so far" (Takeshi 20.12.2015: 6).

In diesem Erzählstrang erwähnt Takeshi die oben erwähnte Gemeinschaft aus Rezipienten und anderen Künstlern, die er aufgrund seiner Arbeiten findet. In seinem gesamten Narrativ scheint immer wieder Takeshis Annahme durch, dass er aufgrund seiner öffentlich in den Werken zur Schau gestellten Sichtweisen auf die Welt und die Emotionalität von Menschen gefunden wird, die ihn verstehen und akzeptieren. Dieses Element der Authentizität erreicht die Rezipienten in besonderem Maße, gerade, weil die Ausstellung von Takeshis Werken mit dem hohen Risiko der Ablehnung einhergeht. Diese Ablehnung resultiert unter anderem aus Abscheu und Ekel, die er beim Publikum hervorrufen möchte: „I would say when I make my work which touches the viewer deeply the work looks disgusting, but it remains in viewers mind and memory a long time" (Takeshi 20.12.2015: 6).

Interessanterweise spricht Takeshi im Gegensatz zu den meisten anderen meiner Gesprächspartner nicht vom finanziellen Auskommen, wenn er von überleben spricht. Vielmehr geht es ihm um das Berühren seines Publikums. Ein starkes Gefühl, und sei es auch Ekel angesichts seiner graphischen Umsetzung, reicht in Takeshis Augen aus, um das Publikum in seinem Sinne emotional zu stimulieren und einen längerfristigen Effekt in Gang zu setzen. Es ist der Versuch, den Menschen ihre eigene Emotionalität wieder näherzubringen, diese eingehender wahrzunehmen und mit gesellschaftlichen Rahmungen in Verbindung zu bringen. Die zu Beginn zitierte Aussage, Angst oder Beunruhigung seien Nahrung für die Kunst (Takeshi 20.12.2014: 3) zielt genau auf diesen wesentlichen Faktor von Takeshis Arbeit ab. Gefühle wie diese sind starke Triebfedern, die Takeshi zufolge das Potential haben, die Menschen dazu zu bringen, ihren stark von Normen flankierten Weg zu über- 
denken und neue Möglichkeiten des Denkens, Fühlens und Handelns in Erwägung zu ziehen. Eine Perspektiveneröffnung, die in Takeshis Augen nur durch den Widerstand gegen Althergebrachtes und Selbstverständliches („stereotypes“) erreicht werden kann. Ich denke, es ist an dieser Stelle besonders deutlich geworden, dass damit auch der Umgang mit Emotionen gemeint ist, der sich auf das gesellschaftliche Zusammenleben auswirkt.

Nach der Dreifachkatastrophe hat sich für Takeshi nichts an dieser Haltung geändert. Vielmehr ist er umso stärker von der Notwendigkeit und der Aufgabe seiner selbst definierten Außenseiterrolle als Künstler überzeugt, um die Passivität und „numbness“ insbesondere junger Menschen zu überwinden: „They [young people] tell me Japan is safe'. I work with young people from time to time during the preparation of exhibitions where they serve as assistants. They are so very passive [...], very quiet and calm" (Takeshi 20.12.2014: 5).

\subsubsection{Nanako}

Nanakos Geschichte hingegen enthält weniger Aspekte des Aufbegehrens gegen den Mainstream als vielmehr Hinweise auf innerfamiliäre Widerstände, die von Gegenwartskünstlern gerade zu Beginn ihrer Karriere überwunden werden müssen. Nanako ist eine 30-jährige Straßenkünstlerin, die sich mit Gelegenheitsjobs und den geringen Einkünften ihrer Performances finanziert. Ihr war wichtig, sich unter vier Augen mit mir zu unterhalten und sich dabei weiter im Englischen zu üben.

Nanako hat einen fünf Jahre jüngeren Bruder, der nach ihrer Aussage mit seiner abgeschlossenen Familiengründung die konservativen Eltern zufriedenstellt: „He is driving huge trucks up and down the coast. It's quite a demanding job" (Nanako 02.12.2014: 1). Die Eltern waren angesichts Nanakos Wunsch, von Kunst zu leben, entsetzt: „They kept telling me ,Be considered about the future. Art won't give you money'. But I am not like that. I always spoke up as a child. I was always like that. My brother is more like the standard" and he could not understand my lifestyle" (Nanako 02.10.2014: 2). Hier zeigt sich Nanakos Narrativ einer Außenseiterin sehr deutlich, welches aber auch die Konnotation einer Rebellion gegen einen Druck von außen enthält.

Nanako verknüpft ihr Selbst untrennbar mit ihrer Lebensweise als Künstlerin, indem sie sich eine Neigung zuschreibt, sich gegen ,Standards' aufzulehnen. Da sie in der Kunst eine Möglichkeit gefunden hat, sich auf gesellschaftlich legitimierte Weise gegen Rahmenbedingungen zu wehren beziehungsweise diese zu beugen, hebt sie den Widerwillen zur Anpassung als ausschlaggebendes Charakteristikum ihrer Identität hervor. Diese Form von Rebellion habe sie während der Schulzeit vor ihren Eltern und dem Rest ihres Umfelds größtenteils verbergen müssen. Erst nach ihrem Abschluss habe sie andere Menschen gefunden, die ihre Wesenszüge zu teilen scheinen. „After graduation I joined the design school and met more people like me. I didn't have to wear two masks anymore. It [creativity and self-expression] just burst out" (Nanako 02.10.2014: 3). 
In dieser Aussage wird deutlich, dass sich Nanakos biographisches Verständnis ihrer Selbst an der Annahme orientiert, sie hätte gar nicht anders handeln können, als sich der Kunst zuzuwenden, um sich selbst in all ihren Charakteristika und Emotionen authentisch zeigen zu können und zu dürfen. In ihrer Erzählung ist sie keine Person, die sich gesellschaftlichen (Emotions-)Normen anpasst, sondern eine, die sich eine passende Nische für eine authentische Existenz sucht oder erschafft. Nun gestaltet Nanako ihr Leben, indem sie für drei Monate hart arbeitet, um anschließend für drei Monate die Welt zu bereisen, andere Menschen zu treffen und sich mit ihnen auszutauschen - sehr zum Unverständnis ihrer Familie: „Now my family doesn't really understand my lifestyle. Work is supposed to be a constant thing. But they accept it. We don't talk about it anymore" (Nanako 02.10.2014: 3). Nanako berichtet, dass ihr dieser Zustand sehr viel lieber ist als die emotionale Kälte, die ihr insbesondere von ihrer Mutter entgegen gebracht worden sei. Sie habe Nanako ständig für ihren Lebensstil verspottet und sie als „Hippie“ bezeichnet: „She said I am not part of this family. With all my travelling and street activities, she could not understand what I am doing" (Nanako 02.10.2014: 2). Hier zeigt sich meiner Ansicht nach, wie stark von Seiten der Familie versucht wurde, Nanako in eine angepasstere Lebensführung zu drücken. Das schwierige Verhältnis zur Mutter wurde weiter verkompliziert, als diese während meiner Arbeit mit Nanako eine schwere Krebserkrankung entwickelte und stationär im Krankenhaus behandelt werden musste. Für Nanako traten damit die familiären Belange wieder verstärkt in den Vordergrund und beeinflussten unsere Gespräche sehr stark. „The family was so worried. We all love her very much. She is holding everything together. She is doing everything" (Nanako 02.10.2014: 3).

Interessanterweise erwähnte Nanako ihren Vater und ihren Bruder nur äußerst selten. Dies erweckt bei mir den Eindruck, die Frauen der Familie, neben der Mutter vor allem die Großmutter, hätten einen sehr viel stärkeren Einfluss auf ihr Leben ausgeübt als die männlichen Mitglieder ihrer Familie. 2014 kämpft Nanako nach eigener Aussage zudem mit Anpassungsschwierigkeiten an die impliziten Verhaltensregeln für japanische Frauen, nachdem sie sich längere Zeit im Ausland aufhielt: „Speaking English I am much louder and don't have such a delicate body language. When I come back from abroad, I speak Japanese again but altogether I am bolder with my body language. I get the feeling that people are irritated: 'You are Japanese but speak like that'" (Nanako 02.10.2014: 1-2). Nanako kommt hier auf jenen Anteil des japanischen Umgangs mit Emotionen zu sprechen, der Frauen eine vor allem zurückhaltende Körpersprache zuspricht. Offenbar steht dies im Gegensatz zu Nanakos Verhalten nach ihren Auslandsaufenthalten.

Das Ausleben eines spezifischen Verständnisses von Weiblichkeit wird auch in Bezug auf ihre Kunst nicht nur während der Performance zum Thema. Zu viel Engagement für gesellschaftliche Belange, so Nanako, sei unweiblich. Einen Raum für den persönlichen Ausdruck zu haben, ist einer der Gründe, weswegen sie nach eigener Aussage die Kunst sehr anziehend findet. Diese trägt für sie noch eine weitere Komponente in sich: 
„I need more space. Tokyo is so small - not enough space. There are so many people here, but whenever I leave Tokyo to go to the countryside, people are taking care of each other. It is a very warm and friendly atmosphere. I can communicate with people very easily. Here are so many people but it is cold. When I moved to Tokyo 14 years ago, I had this mask of not caring too much. Then I fit in, but now it changed [...]." (Nanako 02.10.2014: 4)

Diese warme Atmosphäre, wie Nanako sie umschreibt, ist die Artikulation eines persönlichen Wunsches nach Gemeinschaft, den sie durch den Kontakt zu gleichgesinnten Menschen zu erreichen versucht. Auch wenn Nanako, wie die meisten meiner Interviewpartner, vorwiegend allein arbeitet, findet sie in der Künstlerszene doch eine Form der Community und des Netzwerks, das ihr vermittelt, mit ihrer Lebenseinstellung nicht allein zu sein. Im Kontakt mit anderen Künstlern entwickelte auch Nanako ihre Lebensphilosophie und rekonstruierte ihre Biographie entlang dieses Rahmens, den sie vor der Schablone geltender Normen weitestgehend in Form von Widerstand entwirft. Damit erhält nicht nur das biographische Narrativ eine entscheidende Facette, die erklären kann, warum sich Individuen trotz deutlicher Entmutigungen durch ihr soziales Umfeld für dieses Leben entscheiden.

Die Bezüge von individueller Biographie und Gesellschaft im Spannungsfeld von Widerstand gegen Konventionen sind zum Teil sehr bewusste Lebenskonstruktionen einzelner Gesprächspartner. In der Verweigerung, bestimmten gesellschaftlichen Verhaltensnormen zu entsprechen, verorten meine Gesprächspartner sogar ihre besondere Hingabe an ihr soziales Umfeld. Diese Facette der Kunst tritt in Beispielen von Nanakos und Takeshis Narrativen besonders deutlich zu Tage, da sie sich nicht nur gesellschaftlichen Konventionen entgegenstellen, sondern auch den Erwartungen einiger Mitglieder ihrer Familien trotzen. Ihre Individualität findet in der Gegenwartskunst eine Nische, in der sie auf legitime Weise mit dem eigenen emotionalen Ausdruck experimentieren können und der sie dennoch fest in den sozialen Kontext einbindet. Diese biographisch prägende und für das Selbstverständnis als Künstler deshalb so essentielle Facette hat im Kontext der Dreifachkatastrophe laut meinen Gesprächspartnern noch an Bedeutung gewonnen. Insbesondere die Aufdeckungen der letzten Jahre, die sich vor allem an den internen Absprachen des „Atomdorfes" entzünden, erhöhen im Empfinden der Gegenwartskünstler die Bereitschaft der Bevölkerung, mehrere Stimmen zu diesen Themen nicht nur zu zulassen, sondern sogar aktiv einzufordern.

Zusammenfassend kann ich feststellen, dass im überwiegenden Teil der Biographien die Dreifachkatastrophe als Bestätigung dafür betrachtet wird, dass wirtschaftspolitische Absprachen und gesellschaftliche Strukturen hinterfragt werden müssen.

\footnotetext{
${ }^{84}$ Siehe Kapitel 5.3.1 „Das Atomdorf (genpatsu mura)“
} 


\subsection{Die Ausnahme der Regel - Tasha aus Tokyo}

Die meisten meiner Gesprächspartner setzen den Fokus nach eigener Aussage also sowohl vor als auch nach den Ereignissen von 2011 auf gesellschaftskritische Kunst. Sie charakterisieren ihre Aufgabe als Künstler größtenteils als eine Hinterfragung dominanter Diskurse. Den Ursprung ihrer gesellschaftlichen Aufgabe verorten sie in ihren Interessen, persönlichen Erfahrungen und Emotionen. Unter den 20 von mir geführten Interviews mit Künstlern gibt es nur eine Person, die diese Hinterfragung ablehnte.

Tasha, zum Zeitpunkt des Interviews 38 Jahre alt, beschäftigt sich mit dem Medium Film, entwirft Konzepte für Ausstellungen und Installationen und zählt auch Photographie zu ihrem Repertoire. In Osaka geboren, zog sie mit ihrer Familie zunächst nach Kyoto, wo sie die Highschool abschloss. Nach vielen weiteren Stationen ihres beruflichen Lebens kehrte sie 2013 nach Tokyo zurück. „It's exciting to live here. I enjoy my life in Tokyo" (Tasha 29.07.2014: 2), ließ sie gleich zu Beginn des Gesprächs verlauten. Ihre von ihr als traditionell beschriebenen Eltern arbeiten im familieneigenen Betrieb in Osaka und auch Tashas wesentlich jüngeren Geschwister - ihr Bruder ist sieben, ihre Schwester zehn Jahre jünger - streben danach, in den Familienbetrieb einzutreten. Obwohl Tasha diese Familienkonstellation erwähnt, nimmt sie während des Gesprächs nur wenig Bezug zu ihrem familiären Hintergrund. Viel ausführlicher spricht sie über ihre Klienten, die sie als freiberufliche Künstlerin anwerben konnte: „Some of my clients are big companies and their products get awards. They are very famous, in Japan and around the world. I am proud of my clients" (Tasha 29.07.2014: 4). Bereits in diesen ersten Aussagen treten Unterschiede zu bisherigen Gesprächen deutlich zu Tage. Bis zum Zeitpunkt des Treffens mit Tasha hatte ich schon mit einigen Künstlern gesprochen, die ihre weiterführende Familiengeschichte ebenfalls nur am Rande betrachten wollten, um sich verstärkt ihrer eigenen Perspektive zu widmen. Dadurch fiel mir gleich zu Beginn unserer Zusammenarbeit auf, dass Tasha weniger ihre eigene Perspektive direkt erläuterte, sondern vielmehr andere Personen zitierte, die sie als Persönlichkeit beschreiben. Dies ließ bereits zu einem frühen Zeitpunkt des Gesprächs erahnen, dass Tasha einen anderen Zugang zu ihrer biographischen Erzählung wählen würde als der Rest meiner Gesprächspartner. Wenig später stellte sich heraus, dass Kunst zu studieren nicht ihr eigener Wunsch gewesen war:

„My mother recommended I should go to an art university, even though I wanted to go to a pharmaceutical or dance university. Even when I was a kid I could make clothes or draw manga. I got angry about my mothers' plan, but she liked my talent for the arts so she wanted me to become an artist, maybe even a famous one. And I didn't like to study hard, so I agreed."

(Tasha 29.07.2014: 3) 
In dieser Passage wird ganz deutlich, dass Tasha keine persönliche Motivation oder Notwendigkeit gesehen hatte, ihre Emotionen und Erfahrungen über die Kunst auszudrücken. Im Unterschied zu meinen anderen Gesprächspartnern scheint sie geringen emotionalen Gewinn aus dieser Arbeit zu ziehen. Nichtsdestotrotz zeigt Tasha Ambivalenzen bezüglich ihrer Entscheidung für die Kunst und der Verwicklung der Wünsche ihrer Mutter auf:

„It's a difficult question for me because good and bad things happened to me. I think, I want to have more money, so I regret choosing the art university. On the other hand, my life is very exciting with my artist friends. I get to know western art, history and also the feeling of living in different countries. [...] Usually, people tend to care only about getting married and having children, but to have an interest in every culture is quite rare." (Tasha 29.07.2014:3)

Die Kombination aus Reisen und den Möglichkeiten, viele verschiedene Menschen und Kulturen kennenzulernen, übt also durchaus ihren Reiz auf Tasha aus. Sie kann die Vorzüge ihres Lebensstils gegenüber Biographien, die sie hier als „usual“ bezeichnet (gemeint sind Normalarbeitsverhältnisse), wertschätzen und entsprechend positiv bewerten. Dabei ist zu beachten, dass Tasha als Frau von 38 Jahren unverheiratet und kinderlos ist. Ich habe im Verlauf unseres Gesprächs den Eindruck gewonnen, dass Tasha sich unter anderem vom Leben ihrer 10 Jahre jüngeren Schwester abgrenzen möchte. Diese hat einen gänzlich anderen Lebensweg eingeschlagen und ist inzwischen eine verheiratete Mutter und Hausfrau. Dabei erweckt Tasha bei mir den Eindruck, dass sie sich ein Leben wünscht, das auch den geltenden Konventionen entsprechend von Außenstehenden als erfolgreich bewertet werden kann. In der zitierten Passage wird deutlich, dass sie Erfolg an einem entsprechenden Gehalt misst. Meine Vermutung, ihr Verhältnis zu Kunst könnte durch eine Prioritätensetzung zugunsten von Geld geprägt sein, bestätigt sich. Auf die Frage, wie sie ihre derzeitige Situation einschätzt, antwortete sie:

„Now I have a job and audience, so I think I don't want to loose money. Money first, then artwork. So I would like to have more money [...] When my friends started to plan a new art project or exhibition I was willing to create a new concept. But now I stop. I don't like thinking about the earthquake."

(Tasha 29.07.2014: 3)

Gleich mehrere Facetten von Tashas Selbstbeschreibung klingen hier an, die eng an Erwartungen an sich selbst gebunden sind. Zum einen würde sie gern wesentlich mehr Geld verdienen als es für einen freiberuflichen Künstler in Japan wahrscheinlich ist. Zum anderen tritt bei Tasha, im Vergleich zu meinen anderen Gesprächspartnern, der gesellschaftskritische Aspekt der künstlerischen Tätigkeit stärker in den Hintergrund. Konkrete Gehaltsvorstellungen nennt sie nicht, aber es wird deutlich, dass sie sich mit berühmten Künstlern vergleicht, die nicht nur Geld, sondern auch 
Anerkennung und internationales Prestige bekommen. In diesen Vergleichen sieht sie sich in der schwächeren Position. In Kapitel 9.4.2 „Prekäre Lebensumstände“ werde ich noch genauer auf den ökonomischen Aspekt der Gegenwartskunst eingehen. An dieser Stelle sei nur erwähnt, dass Geld, wie in anderen kapitalistischen Gesellschaften, auch in Japan mit Macht und Einfluss verbunden wird. Dies sind Aspekte, die Tasha offenbar sehr wichtig sind.

Die Gesellschaftskritik ist Tasha hingegen fremd. Einige ihrer Freunde planten ein Projekt anlässlich der Dreifachkatastrophe, aus dem sich Tasha mit der Begründung zurückzog, sie wollte nicht darüber nachdenken. Auf diese Ablehnung von kontroversen Themen wie der Dreifachkatastrophe komme ich später erneut zu sprechen.

Zunächst halte ich es für zielführend, Tashas Einschätzung der japanischen Kunstszene genauer zu betrachten. Aus eigenen Erfahrungen auf ausländischen Kunstmärkten zieht Tasha folgende Schlüsse: „We have a lot of complains about the Japanese art industry. It's very difficult. It's a problem of Japanese society and culture. [...] But we have to make our living. It's a great difficulty in Japan" (Tasha 29.07.2014: 4). Die wiederholte Betonung der Schwierigkeit, als Künstler in Japan seinen Lebensunterhalt zu verdienen, zeigt nicht nur Tashas Bewusstsein für ihre finanziellen Möglichkeiten. Vielmehr sind ihre Aussagen auch vor dem Hintergrund ihrer internationalen Reisen, beruflichen Stationen und Netzwerke zu sehen. Sie vergleicht ihre beruflichen Optionen mit denen ihrer Kollegen in New York, Berlin und Kanada: „In fact, I leave my artwork because Japan is in a recession. Especially after this earthquake and after, Fukushima'. It has become much harder [to get by on art alone]. Now even housing is becoming much more expensive in Japan" (Tasha 29.07.2014: 5).

Dies ist die einzige Artikulation, in der Tasha die Dreifachkatastrophe in einen Bezug zu ihrer Person setzt. In darauf folgenden Gesprächen wird der 11. März 2011 nur noch als Kontrastierungspunkt herangezogen, um sich von den Arbeiten ihr bekannter Künstler oder von Aussagen von Freunden zu distanzieren. Als Beispiel nennt sie das Engagement eines Künstlerfreundes, der längere Zeit in Malaysia lebte und seit 2011 in Tokyo aktiv ist: „He is very nice and friendly, but political. I could not understand his attitude. He went to demonstrations and no Japanese friend could understand it. He put pictures on Facebook. I was amazed" (Tasha 29.07.2014: 6).

Diese Aussage ist in vielerlei Hinsicht interessant. Zum einen veranschaulicht sie Tashas Gegenüberstellung positiver Eigenschaften wie Freundlichkeit mit politischem Interesse, das damit eine negative Konnotation erhält. Im Zusammenhang mit Tashas Unverständnis für die Meinungsäußerung und Teilnahme ihres Freundes an Demonstrationen scheint sie des Weiteren zu erstaunen, dass er dies über das soziale Medium „Facebook“ auch öffentlich bekannte. Mit der Einstellung seiner Bilder im Internet zeigt er sehr direkt seine persönliche Haltung. Angesichts der in Kapitel 4.2.1 aufgeführten Konfliktlösungsstrategien im öffentlichen Raum ist es nicht sehr verwunderlich, dass Demonstrationen in Japan nach wie vor kein sehr beliebtes Mittel der Meinungsäußerung sind. Dennoch überraschte mich die Heftig- 
keit, mit der Tasha das Verhalten ihres Freundes ablehnte. Es bedurfte einiger weiterer Nachfragen und mehrerer Treffen, um Aussagen von ihr zur Thematik der Dreifachkatastrophe zu erhalten. Ich gewann den Eindruck, dass sie nur ungern sowohl über die Katastrophe als auch über die gesellschaftlichen Folgen sprechen wollte. Als ob sie das Thema möglichst schnell beenden wollte, sagte sie: „I don't know ,Fukushima' and Tohoku after 2011 because I live in Kansai area" (Tasha 29.07.2014: 2). Tasha beschreibt ihren Schock, als sie die Auswirkungen des Bebens und des Tsunamis im März 2011 im Fernsehen verfolgte: „I watched TV and realized ,It's a very big earthquake'. I was shocked to see Kesennuma [area on the eastcoast of Japan that was hit hard by the tsunami] after the earthquake" (Tasha 29.07.2014: 2). In den Momenten, in denen es um die Dreifachkatastrophe geht, lacht sie auffällig viel, wirft mir nur flüchtige Blicke zu und versucht, die meiste Zeit den Blickkontakt zu vermeiden. Sie scheint die gesamte Thematik lieber vermeiden zu wollen.

Dies ändert sich erst, als sie von Künstler-Kollegen berichtet, die sich zu Aufräumarbeiten in die Gegenden von Tohoku als Freiwillige gemeldet haben: „They went to Tohoku for two or three months, took pictures and videos to create some artwork. But I don't know why they went to a dangerous area" (Tasha 29.07.2014: 2). Tasha selbst zog es vor, die Distanz zu wahren und einen gewissen Betrag an eine wohltätige Organisation zu spenden. Sie vermeidet das Thema nach Möglichkeit und berichtet lieber von ihren beruflichen Träumen: „[...] I would like to make use of my artist skill and my English skills. So I want to study web-design or media planning" (Tasha 29.07.2014: 2). Zu diesem Zeitpunkt weiß ich bereits von Tasha selbst, dass sie gerade erst dabei ist, die oben genannten Fähigkeiten zu erwerben, die sie für essentiell für ihren beruflichen Erfolg hält. Im Anschluss an unsere Gespräche bittet sie mich, ihr beim Verständnis eines englischen Textes zu helfen oder ihr Vokabeln zu erklären.

Mir fällt auf, dass Tashas berufliche Zukunftswünsche sie sowohl räumlich als auch finanziell unabhängiger machen könnten, da sie ihre internationalen Kontakte und bisherigen Werke für sich nutzen kann und nicht auf den japanischen Markt beschränkt ist. Das Desinteresse ihrer Familie an ihrer Kunsttätigkeit könnte zu Tashas Entscheidung beigetragen haben, eine internationale Karriere mit entsprechendem Gehalt der gesellschaftskritischen Kunst meiner anderen Gesprächspartner vorzuziehen. Dafür spricht die Heftigkeit, mit der sie auf meine Frage nach dem Interesse ihrer Familie an ihrer Kunst reagiert: „They are totally not interested. They don't talk about my artwork since I am doing it on a freelance basis" (Tasha 29.07.2014: 2). Ich halte es für aufschlussreich, dass Tasha diesen Zusammenhang zwischen FreelanceTätigkeit und Desinteresse seitens ihrer Familie herstellt. Meinem Eindruck zufolge glaubt sie, das Desinteresse ihrer Familie sei an ihre Freelance-Tätigkeit geknüpft und nicht so sehr an ihre Tätigkeit als Künstlerin selbst. Hier scheint mir Tashas Annahme durchzuscheinen, ihre Familie würde sich mehr für sie interessieren, wenn sie eine dauerhafte, rentable Anstellung hätte. Diese Annahme wird insofern durch Tashas Aussage gestützt, als dass sie ihre Biographie weniger als Lebensgeschichte 
denn als beruflichen Werdegang begreift: „My biography is stopping now because I stopped making artworks, because I lost money“ (Tasha 29.07.2014: 2).

Es dürfte deutlich geworden sein, wie sehr sich Tashas Aussagen von den Narrativen meiner anderen Gesprächspartner unterscheiden. Anders als diese nimmt Tasha den 11. März 2011 nicht nur nicht als Einschnitt in ihrem persönlichen Leben wahr, sondern wendet sich bewusster denn je von den verstärkt auftretenden gesellschaftskritischen Tätigkeiten ihrer Kollegen ab. Sie möchte mir gegenüber nicht näher auf ihre eigenen Emotionen eingehen, geschweige denn diese öffentlich in Form von Kunst zeigen. Stattdessen bleibt sie fest im kulturspezifischen Umgang mit Emotionen verhaftet, indem sie die Strategien des Vermeidens und Verschweigens anwendet.

\subsection{Weitere essentielle Facetten der gesellschaftlichen Verortung}

In den folgenden Abschnitten beschäftige ich mich mit Facetten des Künstlerlebens in Japan, die von meinen Gesprächspartnern unterschiedlich stark hervorgehoben wurden, jedoch bei allen Beteiligten eine Rolle spielen. Es handelt sich dabei in erster Linie um zusätzliche Aspekte zu den bereits genannten, die das Bild eines Künstlers in Japan prägen und für die emotionale Verortung des jeweiligen Künstlers im japanischen Sozialgefüge wichtig sind. Ich stelle fest, dass es nicht zuletzt das kollektive Bild eines Gegenwartskünstlers ist, das für meine Gesprächspartner Anreize, aber auch Herausforderungen bereithält. Dabei kristallisiert sich eine weitestgehend von allen Akteuren geteilte Wahrnehmung und Beurteilung des Lebensstils von Gegenwartskünstlern im japanischen Kontext heraus.

\subsubsection{Gender}

Insbesondere die Frauen unter meinen Gesprächspartnern kamen während der Interviews auf Geschlechterrollen zu sprechen. Bei allen Interviewten, die diese Thematik ansprachen, handelte es sich um Jahrgänge aus den 1970er Jahren. Jüngere Gegenwartskünstler hatten sich zwar auch eine Meinung dazu gebildet, äußerten sie jedoch erst auf meine ausdrückliche Nachfrage. Denjenigen Interviewpartnern, die sich von sich aus zum Gender-Aspekt äußerten, schien es hingegen ein Bedürfnis zu sein, sich darüber auszutauschen. In den Gesprächssituationen empfand ich es als Vorteil, dass ich als international reisende Frau in Erscheinung trat, die sich explizit für die Wahrnehmungen meines Gegenübers interessiert.

In den Geschlechterrollen spiegelt sich ein Spannungsverhältnis zwischen individuellen Wahrnehmungen und Zielen auf der einen sowie sozialen Rahmungen auf der anderen Seite wider. In den einzelnen Aussagen meiner Gesprächspartner zu Gender zeigt sich gleichzeitig eine klare Trennung zwischen Privatperson und pro- 
fessioneller Rolle als Künstler, die in anderen Passagen des biographischen Narrativs häufig vollkommen verwischt wird.

Aus allen gesammelten Aussagen möchte ich Yasukos Darstellung (siehe Kapitel 9.2.1.1), geboren 1972, besonders hervorheben. Sie beschäftigt sich mit Projektarbeiten, in denen sie Erinnerung, Lokalität und Identität zusammenführt. Aufgrund ihrer Kinderlosigkeit sah sich Yasuko veranlasst, das Thema Gender anzusprechen. Sie teilt sich ihren Arbeitsraum mit mehreren Künstlerinnen und bespricht die Auswirkungen von Gender auf ihren Arbeitsalltag regelmäßig mit ihnen. „Female artists have to do things at their own pace. We [women] are having children etc. so we have to slow down unlike men. We [husband and herself] don't have children so it is a bit easier but my friend in this gallery has got children and they are still small so it's complicated“" (Yasuko 12.02.2015: 2).

Yasuko spricht hier ein wesentliches Element des japanischen Rollenverständnisses an, zu dem Salamon bereits 1974 in ihrer Untersuchung von Ehen für die Jahre 1965-66 feststellte:

„In the culturally dictated life course in Japan, when one marries it follows that one has children, and the sooner the better. The husband continues at his job; so does the wife unless marriage involved a geographic move for her. [...] and who is therefore isolated until she has a baby, and thus gains entrance into the sorority of neighborhood mothers." (Salamon 1986: 133)

Obwohl einige Jahrzehnte zwischen Salamons Ergebnissen und meiner Forschung liegen, legen Yasukos Aussagen eine gewisse Konstante in der geschlechtlichen Aufgabenteilung nahe. So scheint es nicht in Frage zu kommen, das Kind durch Dritte betreuen zu lassen, damit auch die Mutter in gewohnter Weise ihrem Beruf nachgehen kann. Dies kann an den finanziellen Einschränkungen liegen ${ }^{85}$ hängt aber auch im Wesentlichen mit dem moralischen Druck zusammen, der auf die japanische Mutter in ihrer Funktion als Betreuerin der Kinder ausgeübt wird. Caudill und Weinstein sprechen von einer „kulturelle Dimension“ des Verhaltens und meinen damit „,...] historically derived patterns of thinking, feeling, and behaving that are shared in large part by all members of society [...]" (Caudill und Weinstein 1986: 203). Die Rolle der Versorgung des Kindes wird im kulturellen Konzept Japans überwiegend der Mutter zugesprochen. Die Väter der Kinder hingegen sind gesellschaftlich betrachtet vorwiegend für die finanzielle Absicherung der Familie zuständig und entsprechen damit aus heutiger Sicht einem konventionellen Bild von familiärer Aufgabenteilung.

Yasukos Aussagen zufolge haben sich die Genderrollen bis heute im Wesentlichen nicht verändert. Yasuko nimmt starke Auswirkungen von Gendervorstellungen auf die Berufstätigkeit als Künstlerin wahr. Auch die Kunstszene sei stark von Männern dominiert und bilde keineswegs eine Ausnahme im Vergleich zu anderen Berufs-

\footnotetext{
${ }^{85}$ Siehe Kapitel 9.4.2 „Prekäre Lebensumstände“.
} 
feldern (Yasuko 12.02.2015: 2). Es sei für Künstlerinnen schwieriger, sich auf dem stark umkämpften Markt zu behaupten, als für Künstler. Dennoch glaubt Yasuko erste Veränderungen zu erkennen:

„Females have a lot of ideas but they have a lot of roles so they are busy. Recently female supporters are increasing. Most of the volunteers and members of organizations are female. It's like a female circle around the male artists. [...] Also, the number of female project directors and curators are increasing. But the male artists still outnumber the female." (Yasuko 12.02.2015: 2)

Auf meine Nachfrage, welche Gründe sie für diese Entwicklung ausmacht, meint Yasuko: „I don't know - I think female artists want to say something to society using the power of art" (Yasuko 12.02.2015: 2).

Die oben bereits erwähnte 50-jährige Mariko (siehe Kapitel 9.2.2.1) hingegen würde sich vielleicht zu einer Generation von Frauen zählen, die sich lieber über ihre Tätigkeit ausdrücken wollen, als den Konventionen Japans zu entsprechen. Sie kontrastiert ihr eigenes Leben mit dem ihrer acht Jahre älteren Schwester, die sie als „more conservative than me" beschreibt. In Nagoya geboren und aufgewachsen erlebt sie selbst dieses Umfeld als sehr konservativ und traditionell. „Marriage is the most important thing in Nagoya and very expensive. You have to show off with titles and the luxury you can afford. Also, my brother who is 12 years older than me has to take care of my fathers' business. I always wanted to be released from that concept " (Mariko 23.03.2015: 7). In Bezug auf die Mutterrolle sagt sie: „My sister has two children and is often jealous of me because of my freedom. I don't have kids or other obligations. I can travel and do my artwork" (Mariko 23.03.2015: 7). Mariko zeigt hier, dass es ihrem Empfinden zufolge eine Entscheidung zu treffen gibt zwischen der Erfüllung einer Mutterrolle und der Verfolgung beruflicher Ziele. Yasukos und Marikos Äußerungen zur Ausgestaltung von Familienplanung und Karriere machen deutlich, dass sie sich diese Fragen auch in ihrem Leben haben stellen müssen und dass sie sich gegenüber ihren Kollegen aufgrund ihrer Kinderlosigkeit freier fühlen. Der einzige männliche Künstler, der sich diesbezüglich äußerte, stellte ebenfalls gesamtgesellschaftliche Veränderungen fest:

„Before we had a man-dominated society. 'Meat-eaters' as we called the men: aggressive, full of energy. Nowadays, it's changing; turning upside down. Now women are climbing mountains or are fond of creating train-models which was a male domain before, whereas the men are becoming the calm and passive 'vegetable-eaters' [laughs]. The boundaries between male and female spheres are melting; they are getting blurry." (Takeshi 20.12.2014: 5)

Dieses Aufbrechen der von meinen Gesprächspartnern als konservativ bezeichneten Genderrollen in Japan wird nur von wenigen wahrgenommen. Das „Verschwimmen der Grenze zwischen männlicher und weiblicher Sphäre“, wie Takeshi (20.12.2014:5) 
es hier nennt, wird von einem weiteren Interviewpartner hingegen klar abgestritten: „Curators are only inviting females if they want their ,kawaii' [süß, niedlich] status for advertisement. If they invite two or more female artists it has to be a feminist show, it's creepy. The status of women is lower than men's" (Kaeko 25.07.2014: 5).

Kaeko, eine Installationskünstlerin, wurde 1976 in Tokyo geboren und hat seitdem viele Länder bereist, darunter auch Deutschland. Sie äußerte sich wiederholt sehr verärgert angesichts der Situation ihres Geschlechts im Allgemeinen, nicht nur in der Kunstszene. Für sie ist Japans ,kulturelle Dimension', wie Lebra es nannte, geprägt von einem einzigen Weg des Umgangs: „Believe it, then it will be.“ This is so conservative even though it is even worse in rural areas. This way of dealing with a challenge is not for me" (Kaeko 25.07.2014: 3).

Es sind Frauen wie Kaeko, Mariko und Yasuko, die durch einen Perspektivenwechsel mit Herausforderungen und Zugehörigkeiten spielen. Angesichts der aufgezeigten Schwierigkeiten für Frauen und Männer, alternative Lebenswege einzuschlagen und in einem von ihnen als konservativ beziehungsweise traditionell bezeichneten Umfeld zu leben, fanden andere Genderkonzepte wie Transsexualität etc. in den Interviews erst Recht keine Erwähnung. Die Rolle der Frau in Abgrenzung zum Mann prägte unseren Austausch über die Verstandortung von Künstlerinnen in Japans Sozialgefüge. Zum Zeitpunkt meiner Forschung gab es Statements von Premierminister Abe, laut denen mit Hilfe des verstärkten Einsatzes von Frauen der japanische Arbeitsmarkt zu mobilisieren und zu revitalisieren ${ }^{86}$ sei. Diese Fragen wurden angesichts der Dreifachkatastrophe in unseren Gesprächen zurückgestellt.

\subsubsection{Prekäre Lebensumstände}

Wie ich im Kapitel 4.2.1 „Polarisierende Differenzgesellschaft - Eine Diskussion“ analysiert habe, wird die wirtschaftliche Situation Japans und ihre sozialen Auswirkungen in verschiedenen Diskurssträngen behandelt. In ihnen allen wird die zunehmende Diversifizierung der Gesellschaft als Ausgangspunkt für die aktuelle soziale Situation der Menschen betrachtet.

Die persönlich empfunden Realitäten der in der Wissenschaft als prekär definierten atypischen Arbeitsverhältnisse, die in Japan seit den 1970er Jahren bestehen, bilden einen wesentlichen Aspekt der Selbstkonstruktion meiner Gesprächspartner. An dieser Stelle möchte ich darauf hinweisen, dass eine prekäre Situation ${ }^{87}$ nicht nur auf der Beurteilung des Grundeinkommens beruht, sondern sich auch am subjektiven Empfinden von Sinnverlust, Anerkennungsdefiziten und Planungsunsicherheiten orientiert (siehe Seifert 2009: 38). Wirtschaftlich prekär sind dieser Definition zufolge Arbeitsverhältnisse, „wenn die Beschäftigten aufgrund dieser Tätigkeit deutlich unter das Einkommens-, Schutz- und soziale Integrationsniveau sinken, das in der

\footnotetext{
86 The Japan Times: „Still a struggle for working women“, 8.4.2016 (https://www.japantimes.co.jp/ opinion/2016/04/08/editorials/still-a-struggle-for-working-women/).

${ }^{87}$ Siehe auch Götz und Lemberger (2009).
} 
Gegenwartsgesellschaft als Standard definiert wird“ (Seifert 2009: 38). Eine Armutsgrenze gibt es für Japan offiziell nicht. Laut OECD-Definition liegt die Armutsgrenze bei weniger als 50\% des Durchschnittseinkommens. In Japan belief sich dieser Einkommenssatz 2008 auf 15.000 Euro Jahreseinkommen pro Jahr (Obinger 2009: 164).

In diesem wirtschaftlichen Klima verdingen sich meine Gesprächspartner nebenberuflich als Lehrer, Angestellte in einem Restaurant oder anderen Bereichen des Dienstleistungssektors. Viele geben aufgrund der finanziellen und zeitlichen Belastungen ihre künstlerische Tätigkeit auf, wie Kaeko ebenfalls betont: „Some artists give up being an artist because of the job situation. To have a good standard of living in Tokyo, we would have to sell artwork which is worth 10.000 Euro every month. We also have to pay for galleries, materials etc. That's hard to accomplish" (Kaeko 25.07.2014: 1). Das vorwiegend in der japanischen Gesellschaft negativ diskutierte Bild eines von mehreren Jobs finanzierten Lebens einer schrumpfenden Mittelklasse (siehe Slater 2010) brachte viel Frustration und Ängste mit sich. Die diskursiv tief verwurzelte Verknüpfung von sicherer Vollzeitstelle und Heirat (Brinton 2011: xv; siehe auch Deutschmann 1991) als Ausdruck des Erwachsenwerdens kollidiert zunehmend mit der wirtschaftlichen Realität des Landes. Umso auffallender waren Aussagen meiner Gesprächspartner, die diesen gesellschaftlichen Druck zwar widerspiegelten, sich jedoch in ihren Artikulationen davon frei zu machen versuchten.

Saburo ist ein gutes Beispiel für diesen Balanceakt, den er zwischen gesellschaftlicher Norm und subjektiver Wertschätzung vollführt: „I am working as a teacher of sculpture for young kids. I like kids but I don't like my students [...] Maybe I like teaching but I don't like to explain how to do this and that. It is difficult to find the right balance between what I want and the need to make money" (Saburo 13.07.2014: 7).

Für Saburo scheint dieser Job als Lehrer vor allem die Funktion der finanziellen Absicherung zu erfüllen. Gleichzeitig bringt er einige für ihn unbequeme Aspekte wie das Erklären der einzelnen Gestaltungsprozesse mit sich. Bei einem unserer letzten Treffen äußerte Saburo den konkreten Plan, das Lehren deshalb zugunsten einer langen Reise nach China und Russland vorerst aufzugeben. „This might cause some confusion for my students [...] but I'd like to think less about what other people think about my artwork and my lifestyle" (Saburo 13.07.2014: 4).

Saburos weitere Aussage unterstreicht seine Auffassung von den Herausforderungen eines Lebens als Künstler: „As an artist, you need to have inner strength, a strong heart, inner resilience. Maybe that's why we can express things" (Saburo 13.07.2014: 5). Saburos Meinung bezieht sich aus meiner Sicht nicht nur auf die Arbeit als Künstler, die meist von starken Emotionen und langen Reflexionsprozessen begleitet wird, sondern ist auch vor dem Hintergrund ihrer finanziellen Situation zu verstehen.

Diese Situation bildet eine weitere Facette ihrer Existenz als Künstler, die laut meinen Gesprächspartnern ,ausgehalten ' werden muss. Wie in Saburos Aussage deutlich wird, ist es gerade diese Fähigkeit des Aushaltens, die ihn dazu befähigt, sich künst- 
lerisch auszudrücken (Saburo 13.07.2014: 5). Sich selbst als Künstler definieren und ausdrücken zu können scheint ihm zwar weniger finanzielle, aber dafür emotionale Vorzüge zu verschaffen. In seinem subjektiven Empfinden ist Saburo gesellschaftlich wirksam, indem er eine wichtige Emotionsarbeit für sein Publikum leistet. Deshalb möchte er seine Tätigkeit auch nicht als Beruf verstanden wissen: „Being an artist is no occupation like a designer. It is a lifestyle" (Saburo 13.07.2014: 4).

Die eingangs zitierte Komponente der finanziellen Planungsunsicherheit als Teil des Prekariats (Seifert 2009: 38) wird in Saburos Narrativ wie auch in denen meiner anderen Gesprächspartner durch das sinnstiftende Element ihrer künstlerischen Tätigkeit und der Anerkennung ihres Selbst durch Dritte aufgewogen.

Man kann also durchaus argumentieren, dass sich die von mir interviewten Gegenwartskünstler objektiv betrachtet zwar durchaus in die Kategorie des Prekariats einordnen ließen, sie diese Zuschreibung subjektiv gesehen aber nur beschränkt gelten lassen. Indem sie die finanziellen Defizite nicht nur als Teil ihres Künstlerdaseins akzeptieren, sondern sogar in ihre Selbstdarstellung integrieren, wird ein gesellschaftlich definierter Makel in eine Facette ihres Lebensstils umgewandelt und erfährt dadurch eine Aufwertung. Beim Prekariat handelt es sich im Verständnis meiner Gesprächspartner um eine Konsequenz, die unter anderem aus ihrem Widerstand gegen gesellschaftliche Normen resultiert. Die subjektiv erfolgreiche Suche nach alternativen Lebenskonzepten macht den Erfolg ihrer Lebensweise aus. Ich hatte während der Gespräche den Eindruck, dass in den jeweiligen Aussagen auch ein gewisses Maß an Stolz angesichts ihres Umgangs mit der Situation mitschwingt.

Hinzu kommt eine narrative Finesse, durch die einige Gesprächspartner ihre Entscheidung für ein Leben als Künstler als ,Schicksal' präsentieren. Auf meine Frage, warum sie sich für dieses Dasein entschieden haben, häuften sich Aussagen wie die von Kogoro, der meint, dass er trotz des teilweise großen Erschöpfungsgefühls einfach nicht aufhören könne: „I have no choice“ (Kogoro 02.05.2014: 14). Andere wie Saburo antworteten in ähnlicher Weise: „Maybe I didn't chose. I had to [become an artist]. If I had actually chosen artwork I would have worked with photography or something like that" (Saburo 13.07.2014: 4).

Mit Aussagen wie diesen stellen meine Gesprächspartner eine Schicksalsergebenheit in den Vordergrund. Sie nehmen dabei vor allem Bezug auf ihren angeborenen Charakter. „I suffered from bullying in school. I didn't provoke anyone. Nevertheless, I always wanted to voice my opinion" (Saburo 13.07.2014: 5). Auf diese Weise werden auch negative Emotionen und Erfahrungen zum Teil ihres Selbstbildes, die sie weiter in ihrer Identitätskonstruktion bestärken.

Es sind Passagen wie diese, die bei mir den Eindruck entstehen lassen, dass meine Gesprächspartner die Radikalität ihrer Entscheidung für ein Leben als Künstler bewusst relativieren wollen. Meine Gesprächspartner tun dies, indem sie ihre Entscheidung vor dem Hintergrund gesellschaftlicher Normen - und nicht zuletzt vor dem Hintergrund des kulturspezifischen Umgangs mit Emotionen - kontextualisieren. Sie stellen ihre Tätigkeit damit als eine angeborene, natürliche Fähigkeit dar, die zwar nicht immer dem kulturell favorisierten Umgang mit Emotionen entspricht, diesen 
Umgang aber auch nicht gänzlich ablehnt. Der jeweilige Künstler kann sich auf diese Weise in der Mitte der Gesellschaft verorten, ohne zum Beispiel sein Empfinden der Situation verleugnen zu müssen. Für viele bringt der Verzicht auf wirtschaftliche Absicherung auch Vorteile. Diese bestehen aus normativen wie auch geographischen Bewegungsfreiräumen, wie für Saburo, der für einige Monate nach China und Russland gehen möchte (Saburo 13.07.2014: 6). Hinzu kommen erweiterte Möglichkeiten der Netzwerkbildung, der intensiven Beschäftigung mit persönlich relevanten Themen und der sinnstiftenden Arbeit im gesellschaftlichen Kontext.

Im Zusammenhang mit der Betrachtung der Dreifachkatastrophe von 2011 und den sozialen Folgen habe ich deutlich gemacht, dass sich Gegenwartskünstler nicht zuletzt aufgrund der genannten Aspekte dafür prädestiniert sehen, sich mit gesellschaftskritischen Themen auseinanderzusetzen. Meine Gesprächspartner haben sich in vollem Bewusstsein der Schwierigkeiten und Herausforderungen für den Beruf des Künstlers entschieden. Sie sehen sich aber in einer Position, die die persönlichen Vorteile überwiegen lassen. 



\section{Gegenwartskünstler als Experten der Bedeutungsherstellung}

In den vorangegangenen Ausführungen habe ich die gesellschaftlichen Rahmenbedingungen Japans beschrieben, wie sie mir meine Gesprächspartner vermittelt haben. Das persönliche Narrativ des jeweiligen Künstlers veranschaulicht sowohl die Schnittstellen und Spannungsfelder zwischen Individuum und Gesellschaft als auch die jeweiligen Handlungspotentiale. In einer Zeit der emotionalen Aufarbeitung der Dreifachkatastrophe liefern die Perspektiven und emotionalen Bewertungen meiner Gesprächspartner wichtige Hinweise sowohl auf dominante als auch auf subversive Diskurse. Ich habe aufgezeigt, wie die jeweiligen Akteure diese Diskurse in ihre persönliche Lebensgeschichte integrieren und in ihrer Kunst einsetzen, um ihre Erfahrungen und Emotionen der Öffentlichkeit zur Verfügung zu stellen. Jetzt bleiben noch einige letzte Fragen zu beantworten, die die gesellschaftliche Ebene betreffen. Nachhaltige gesellschaftliche Veränderungen nach einer schweren Katastrophe entstehen nur langsam. Sie manifestieren sich im Spannungsfeld der Meinung vieler Bürger auf der einen sowie bereits etablierten Machtinstanzen der Gesellschaft auf der anderen Seite. Wie deutlich geworden ist, verbinden sich in der gesellschaftskritischen Tätigkeit meiner Gesprächspartner ihre individuellen Bedürfnisse nach emotionalem Ausdruck, Teilung von Erfahrungen und dem Wunsch nach gesellschaftlicher Wirksamkeit mit den Erwartungen und Ansprüchen der japanischen Gesellschaft. 
Doch wie lassen sich diese Schnittstellen zu einer Erkenntnis über die Arbeits- und Lebensweise der Gegenwartskünstler zusammenfassen? Um diese Frage zu beantworten, werde ich im Folgenden die biographischen Aspekte und gesamtgesellschaftlichen Rahmungen in ihrer Wechselwirkung genauer betrachten. Damit möchte ich der Komplexität der verschiedenen Wechselwirkungen zwischen den Ebenen Rechnung tragen und verdeutlichen, wie Künstler ihre biographischen Lösungswege zur Verfügung stellen.

\subsection{Die Rolle des Gegenwartskünstlers in der gesellschaftlichen Aushandlung von Emotionen}

Aus meiner Sicht spielt die individuelle Perspektive in einer kollektiv-normativ ausgerichteten Gesellschaft eine entscheidende Rolle in der gesellschaftskritischen Arbeit von Gegenwartskünstlern. Der normativ festgelegte Ausdruck lässt noch Raum für einen alternativen Umgang mit Emotionen und Erfahrungen. Zusammenfassend ausgedrückt: Die mit den Aussagen der Werke verbundene Sprengkraft kann sich entfalten, weil die Wahrheit des Einzelnen das Potential hat, zu einer Wahrheit der Gesellschaft zu werden. Es braucht jedoch ein Individuum, das nicht nur fähig ist, diesen Emotionen durch handwerkliches Geschick ihren Ausdruck zu verleihen, sondern das auch Eindrücke aus den verschiedenen Bereichen der Gesellschaft verarbeiten kann. Mit den verschiedenen Zitaten meiner Gesprächspartner habe ich herausgearbeitet, dass meine Gesprächspartner sich dazu veranlasst sehen, sich den Raum selbst zu erschaffen, in dem ihre Werke wirken können. Dies nimmt Einfluss auf die Perspektive des einzelnen Betrachters bezüglich der dominanten Diskurse und trägt dazu bei, diese zu diversifizieren. Diese Vielfalt der Perspektiven aufzuzeigen, stellt in meinen Augen eine entscheidende Facette im Selbstnarrativ eines Künstlers dar, wobei er unterschiedliche Rollen für die Gesellschaft einnehmen kann. Die wesentlichen Aspekte dieser Rollen, die dafür eingesetzten Ressourcen und Lösungswege führe ich in den folgenden Kapiteln mit verschiedenen theoretischen Aspekten zusammen. Auf diese Weise führe ich auf das Narrativprinzip meiner Gesprächspartner hin, in dem sich ihre Arbeitsweisen mit ihrer persönlichen Lebensgestaltung verbinden.

\subsubsection{Künstler als Erschaffer eines sozialen Aushandlungsraumes}

In diesem Abschnitt betrachte ich das Zusammenspiel von subjekter Wahrnehmung und kulturellen Emotionskonzepten im Hinblick auf die Dreifachkatastrophe von 2011 genauer. Bisher habe ich erläutert, wie meine Gesprächspartner den kulturellen Umgang mit Emotionen in ihrem Arbeitsalltag auslegen beziehungsweise teilweise beugen, um ihren Emotionen Ausdruck zu verleihen.

Unter Berücksichtigung der von Röttger-Rössler (2004: 99) aufgezeigten Wirkungsebenen von Emotionen stelle ich fest: Zwischen kulturellen Konzepten, sozia- 
ler Interaktion und individuellem Empfinden muss im gesellschaftlichen Kontext eine möglichst harmonische Wechselwirkung bestehen, um Konflikte zu vermeiden. Dies gilt meiner Auffassung nach in besonderem Maße für den japanischen Kontext, wie meine Ausführungen zur interdependenten Ausrichtung verdeutlicht haben. ${ }^{88}$ Die sozialkritische Kunst, wie sie vom überwiegenden Teil meiner Gesprächspartner praktiziert wird, stellt jedoch eine direkte Herausforderung für die auf der Basis des japanischen Umgangs mit Emotionen geführten Diskurse dar, indem sie verschiedene Ansichten und Perspektiven bereitstellt.

In der japanischen Gesellschaft wird, wie in anderen Gesellschaften auch, mit Konformitätsdruck reagiert, sobald Widersprüche, Widerstände oder gar Konflikte auftreten. In vorangegangenen Kapiteln habe ich dargelegt, wie zum Beispiel Scham eingesetzt wird, um Künstler an einen als angemessen betrachteten Emotionsausdruck zu binden. Für meine Gesprächspartner geht es jedoch nicht darum, sich anzupassen, sondern stattdessen eine Antwort darauf zu finden, wie persönliche Einzigartigkeit und gesellschaftlich geteilte Werte in eine möglichst konkurrenzlose Beziehung zueinander treten können. Meine Gesprächspartner finden diese Antwort, indem sie mit ihrer Kunst Räume für Diskurse schaffen. Dafür stellen sie ihre eigenen biographischen Lösungsstrategien bereit. Ihre Werke sind dabei der Anlass für einen Aushandlungsprozess, der verdeutlicht, dass jedes Individuum in der japanischen Gesellschaft, trotz der vorhandenen Emotionskonzepte und den damit verbundenen Normen, individuelle Handlungsoptionen hat. Meine Gesprächspartner sprechen einen wichtigen Punkt für die Wirksamkeit von Kunst als Ausdruck von individueller Kreativität an, wie ihn Liep (2001) betont: Wenn Kunst ihre sozialkritische Wirkung entfalten soll, muss sie in die breite Öffentlichkeit getragen und dort diskutiert werden. Privates, individuelles Denken muss in das Licht der Öffentlichkeit treten und trotz seiner Einzigartigkeit Verbindungen zu anderen Ansichten und Perspektiven herstellen. Nur so ist sichergestellt, dass Identifikationen mit dem Neuen ermöglicht werden, um alte Wege des Denkens zu verlassen und eine nachhaltige Transformation anzustoßen. ${ }^{89}$

Ein gutes Beispiel für eine Aushandlung von Handlungsoptionen liegt in der Aufspaltung des Selbst in einen privaten und einen öffentlichen Teil. Nanako, deren Erzählung ich bereits in Kapitel 9.2.3 „Widerstand gegen gesellschaftliche Konventionen“ ausführlich beschrieben habe, ist ein Beispiel dafür. „From senior school to the end of Highschool, at the age from 13 to 17, I listened to Indie-Rock music and after 5 pm I met friends who I didn't go to school with. [...] I had a second life. My parents knew about this but they didn't mind as long as I didn't quit school“ (Nanako 02.10.2014: 3). Nanako hat in ihren Teenagerjahren ein Ventil für ihre Persönlichkeit gefunden, indem sie sich ein zweites Leben erschuf. Ihre Eltern haben dies stillschweigend toleriert, wollten aber von Nanakos Aktivitäten möglichst wenig mitbekommen. „Before coming home, I switched back into my schooluniform“ (Nanako

\footnotetext{
${ }^{88}$ Siehe Kapitel 4.1.2 „Das Verhältnis vom Individuum zur Gruppe“.

${ }^{89}$ Siehe Kapitel 10.1.2 „Kreativität als eine Ressource für Empathie und Handlungsvermögen“.
} 
02.10.2014: 3). Sie sagt, sie habe dies auf sich genommen, um einem Bedürfnis nachzugehen, das sie bis heute hat: „I need my life to be colorful.“ Auf meine Nachfrage nach den Gründen fährt sie fort: „[...] The government wants people to be the same, on the same level. You are not supposed to be below or above the standard. Everyone should be on the same [level]" (Nanako 02.10.2014: 4).

Nanako wendet bis heute mit ihrer Kunst diese Praxis eines zweiten Lebens an. Einige meiner Gesprächspartner berichten allerdings von sehr starker Ausgrenzung. Sie gehen mit dieser Situation um, indem sie ihrer Arbeit ausschließlich außerhalb Japans nachgehen. Yoshinori ist so ein Fall. Er ist ein Anfang 30-jähriger Künstler, der sich aufgrund seiner kritischen Arbeit innerhalb der japanischen Kunstszene nicht mehr willkommen fühlt: „Curators hate me because people look at my work and get angry. So I have to go out [of Japan] and back, out and back" (Yoshinori 05.08.2014: 3). Yoshinori empfindet den gesellschaftlichen Druck als zu stark, um sich in Japan weiterhin wohlzufühlen. Die meisten meiner Gesprächspartner jedoch vollführen den Balanceakt zwischen dem Ausdruck innerer Autonomie und Konformitätsdruck.

Psychologische Untersuchungen wie die von Swann und Hill (1982) legen nahe, dass ein Selbst sich auch an Kritik und Widerstand stabilisiert, insbesondere wenn es die Möglichkeit hat, die Kritik zu entschärfen (z. B. durch Versuche, das Gegenüber umzustimmen). Diese Möglichkeit wird auch im japanischen Kontext genutzt, wie Künstler mir von Ausstellungsbesuchen und angeregten Unterhaltungen mit dem Publikum berichten. Der Austausch zwischen Künstler und Rezipienten betont das Anliegen des Künstlers, in den Dialog treten zu wollen statt emotionalen Widerstand zu erregen. In diesen Fällen werden die Werke vom Publikum als Teil der sozialkritischen Rolle des Künstlers wahrgenommen. Der Künstler selbst erhält durch diese Zuschreibung ein gewisses Maß an Legitimität für das Hinterfragen des Status quo. Damit wird dem jeweiligen Künstler aus meiner Sicht eine entsprechende Auslegungsmöglichkeit des gültigen Umgangs mit Emotionen zugesprochen. Künstler werden also weniger im negativen Sinne als individualistisch wahrgenommen, sondern als Personen, die auf diese Weise der Gesellschaft dienen wollen.

So kann ein neuer Aushandlungsraum entstehen, der Veränderungspotentiale in der Gesellschaft sichtbar werden lässt. Dies betrifft in direktem Maße sowohl Emotionen als auch den Umgang mit ihnen.

Die Verbindung aus gesellschaftlicher und privater Sphäre in einer Person angesichts der sonst geltenden Trennung der öffentlichen (tatemae) und privaten (honne) Präsentationsebene wirkt daher wie eine starke Triebfeder für den einzelnen Künstler. ${ }^{90}$ In seiner Individualität, sichtbar gelebter Herausforderung des bestehenden Umgangs mit Emotionen und der Hinterfragung des gesellschaftlichen Status quo wird ihm ein hohes Maß an Authentizität zugesprochen, das sich je nach Artikulation positiv auf seine gesamtgesellschaftliche Wirkung und Rolle auswirkt.

\footnotetext{
${ }^{90}$ Siehe Kapitel 4.1.1 „Der Kompass der Werte - Ethik und Selbst im japanischen Kontext“.
} 


\subsubsection{Kreativität als eine Ressource für Empathie und Handlungsvermögen}

Bei der Analyse des Interviewmaterials tauchten in Bezug auf die Situation, die Rolle und das Selbstverständnis der Gegenwartskünstler Japans wiederholt Begrifflichkeiten wie ,Neuerschaffung', die Wichtigkeit von „Bedeutung' und ,die Entwicklung eines Gefühls' auf. Diese Termini beziehen sich auf eine Situation und/oder die Gestaltung von gesellschaftlichen Beziehungen. Ein häufiges Beispiel meiner Gesprächspartner dafür war ihr Bedürfnis, den engeren Kontakt mit evakuierten Bewohnern von Fukushima zu suchen und deren neuen Alltag verstehen zu wollen.

Kreativität tritt in den verschiedenen Interviews als wichtige Ressource sowohl für die emotional geprägte Arbeit des jeweiligen Künstlers in Erscheinung als auch für dessen Realitätsverständnis. Aus diesem Grund möchte ich Kreativität in ihren Wirkungsweisen und Anwendungen genauer betrachten.

Wie bereits Mageo und Knauft ausführen, illustriert die Fähigkeit, eine eigene Realität zu erschaffen, die Kapazität „,[...] to innovate upon if not to reimagine existing schemata; these innovations and reimaginings are integral to the activity of selfmaking" (Mageo und Knauft 2002: 8).

Die Kreierung neuer Perspektiven setzt voraus, dass Künstler sich in die Lage versetzt sehen, ihrer Neugier auf „das Andere" nachzugehen. Wie in den vorangegangenen Kapiteln beschrieben, ist die Schaffung der dafür notwendigen Freiräume mit einigen Hindernissen und Herausforderungen verbunden. Doch die von meinen Gesprächspartnern oftmals als persönliche Opfer beschriebenen Kompromisse der Lebensführung, beispielsweise in der als schwierig empfundenen finanziellen Situation, werden in der Präsentation der jeweiligen Biographie durch einen emotionalen Gewinn und tief empfundene Beziehungen innerhalb ihres Netzwerks aufgewogen. In ihren Werken schlägt sich ihre Fähigkeit der Empathie als kreativer Ausdruck nieder und erscheint in vielen Formen. Dies hängt meiner Ansicht nach mit dem in Kapitel 4.1 „Der kulturspezifische Ausdruck des Selbst im sozialen Gefüge“ beschriebenen japanischen Umgangs mit Emotionen zusammen, in dem negative Gefühle nicht direkt an den entsprechenden Adressaten gerichtet werden.

Meine Gesprächspartnerin Yoko macht deutlich, dass an ein erwachsenes Individuum gewisse Erwartungen im Umgang mit seiner Emotionalität gerichtet werden, die darauf abzielen, andere nicht mit den eigenen Gefühlen zu belasten:

„In Japan, even when people are furious, you can't really tell unless they are super-angry. But they don't express their anger that much. To the Japanese people, this is not being untruthful but it's more like being respectful. It's rude to be direct. In Britain for example, it's the other way around as they understand directness as a way of being honest." (Yoko 11.02.2015: 9)

Für Yoko, die lange Jahre ihrer Jugend in den USA verlebte und daher ein besonderes Verhältnis zur japanischen Sprache hat, spiegelt sich dies in der Sprache selbst wider: „The language has a lot of rules. You are to be very polite, cover your main idea rather 
than throwing it into peoples' face - it's a lot of beating around the bush" (Yoko 11.02.2015: 6).

Man könnte also sagen, dass das Erspüren des emotionalen Zustandes des Anderen die emotionale Reife der involvierten Gesprächspartner verdeutlicht. Gegenwartskünstler übertragen die für sie entscheidenden emotionalen Themen in ein für andere sichtbares Format, das es ihnen erlaubt, direkter mit ihren Gefühlen umzugehen. Das persönliche Erleben des Künstlers wird durch seine Kreativität für Dritte erlebbar beziehungsweise transparent. Empathie und Kreativität vereinen sich so in der Lebens- und Arbeitsweise meiner Gesprächspartner zu einer fruchtbaren Kombination. Dabei schwingen Emotionen auf eine Art mit, die dem Rezipienten einen unmittelbaren Eindruck vermitteln. Dennoch entscheidet der Rezipient selbst, wie er mit seinen Emotionen bezüglich der Werke umgeht. Kunst ist eine Form der Kommunikation, die Emotionen vermittelt, jedoch nicht auf eine unmittelbare Antwort angewiesen ist wie beispielsweise in einem Gespräch.

Konzeptionell betrachtet weisen Empathie und Kreativität viele Schnittpunkte auf. Künstler sehen sich mithilfe ihrer Kreativität und Empathie in der Lage, ihren Rezipienten die gesellschaftliche Notwendigkeit einer Beschäftigung mit dem „Anderen" bewusst zu machen. Dies ist ein Aspekt ihrer Arbeit, der vor dem Hintergrund der Dreifachkatastrophe an Bedeutung gewonnen hat. Die kulturspezifischen Rahmenbedingungen, unter denen Veränderungen wiederum stattfinden können, werden unter anderem in der Kreativitätsforschung diskutiert. Kreativität wird dabei als jenes Element menschlichen Handelns verstanden, das durch das innovative Herstellen von Zusammenhängen auch gesellschaftliche Transformationen initiiert. Der Kulturwissenschaftler Liep (2001: 2) definiert Kreativität „[...] as activity that produces something new through the recombination and transformation of existing cultural practices or forms."

Ich argumentiere, dass die Anstöße zur Neukombination und Transformation von kulturellen Praktiken und den damit verbundenen emotionalen Ausdrucksmöglichkeiten im öffentlichen Raum im Allgemeinen von Empathie getragen werden. Insbesondere im Katastrophenkontext ist es die Kombination aus Information und intuitivem Mitfühlen, die es Dritten ermöglicht, sich in die Situation der unmittelbar Betroffenen zu versetzen und einen persönlichen Handlungsbedarf abzuleiten. In meinen Interviews finden sich wiederholt Formulierungen, die auf eben diese Verknüpfung von Kommunikation beziehungsweise emotionaler Wissensaneignung mit der Beziehungen zwischen Menschen und der dafür nötigen inneren Haltung abzielen: „Normally artists focus on things that non-artist people don't care about, but artists focus on that kind of aspect. So artists' task is to make normal people to focus on that kind of things" (Kaeko 25.07.2014: 1). Dies deckt sich mit Hastrups ethnologischen Überlegungen zum Thema Kreativität im gesellschaftlichen Kontext: „To be creative is not merely to invent or to innovate but to make a new kind of understanding possible by revealing what is already partly sensed“ (Hastrup 2001: 40).

Wie ich in Kapitel 9 bereits beschrieben habe, umfasst das Engagement meiner Gesprächspartner insbesondere jene gesamtgesellschaftlichen Themen, die sich mit 
dem politischen System Japans und dem gesellschaftlichen Zusammenleben befassen. Um Veränderungen zu gestalten, braucht es eine Vorstellungskraft, die die Notwendigkeit und Art der Handlungen verdeutlicht.

Der allgemeine Nutzen von Kreativität für Gesellschaften liegt Vertretern der Kreativitätsforschung wie Hastrup (2001) und Liep (2001) zufolge in der empathischen und imaginativen Bereitstellung der individuellen Perspektive für das Verständnis der Andersartigkeit eines Gegenübers. Die damit verbundene Einsicht der Notwendigkeit eines Handlungsbedarfs erschafft schließlich das Transformationspotential einer Gesellschaft: „Imagination is both constitutive and creative. It is a process, central to any event of understanding" (Hastrup 2001: 41). Hastrup setzt Kunst als Synonym für Kreativität im Besonderen ein und erläutert: „The poetic power of language, and of art, marks difference while also overcoming it" (Hastrup 2001: 42).

Die Herangehensweise meiner Gesprächspartner, implizite und unausgesprochene Emotionen in eine andere Kommunikationsform zu übertragen, betrachte ich als eine Möglichkeit, Differenzen im hastrupschen Sinne zu überwinden. Einer meiner Gesprächspartner sagt dazu: „In Japan, we don't discuss. It's artificial harmony. The way I can talk with you, I usually don't have much opportunity to do so with Japanese friends" (Kaeko 28.02.2014: 6).

Demzufolge könnte man also argumentieren, dass Kreativität, Empathie und Handlungsvermögen in einer engen Wechselbeziehung zueinander stehen und die weitere Genese des Emotionsausdrucks in Zeiten ermöglichen, in denen die verbale Sprache versagt. Darin liegt meiner Meinung nach das große gesellschaftliche Transformationspotential der Arbeit meiner Gesprächspartner, aber auch ihr persönliches Risiko: „Generally you cannot expect to be supported as an artist and by choosing this occupation you take the risk of being alone" (Kaeko 25.07.2014: 3).

Kaeko arbeitete zum Zeitpunkt unseres Kennenlernens im Jahr 2014 zu dem weltweiten Bestreben wirtschaftlichen Wachstums. Sie nutzt dafür vor allem Photographien, die sie in Galerien in den USA und Japan ausstellt. In den Gesprächen mit ihr ist auffällig, wie klar sie ihre Meinung mitteilt und sich teilweise sehr leidenschaftlich darum bemüht, weitere Treffen mit mir zu arrangieren und die Situation der Künstler darzustellen. So betont sie mir gegenüber:

„Artists have a hunger for sharing. Politeness and caring is on the surface but the loneliness is quite common in Japan, even though it's not exclusive to us [artists]. It's a modern problem that might have its roots in the family constellation." (Kaeko 25.07.2014: 4)

In den Kapiteln 7 und 8 haben die Analysen der einzelnen Aussagen und meine teilnehmenden Beobachtungen vor Ort gezeigt, dass die Kreativität als Ausdruck der in den Diskursen mitschwingenden Emotionen gefragt war, um mit den Ereignissen umgehen zu können. Aussagen wie die von Kaeko machen deutlich, dass aus ihrer Sicht die Wurzeln für viele persönliche Themen wie Einsamkeit auf gesellschaftlicher 
Ebene zu suchen sind. Dies gilt in besonderem Maße für die Auseinandersetzung mit der Dreifachkatastrophe: Durch die Vermittlung von Informationen und Emotionen haben meine Gesprächspartner dazu beigetragen, den dringenden Handlungsbedarf in den verschiedenen Regionen offen zu legen: „The Japanese way of dealing with a challenge is this: ,It's not for me' which means, ,I cannot argue so well since I don't know enough about it. And my expressions might not be so perfect so I stick to the majority.' [...] Art can be a way around that" (Kaeko 25.07.2014:3).

Die langfristige Wirkung kultureller Kreativität und die transparente Verarbeitung von Emotionen obliegt Akteuren wie Künstlern, die das dominante Verständnis von Gesellschaft zugunsten neuer Entwürfe hinterfragen (siehe Liep 2001: 6): „Creative action is promoted or hindered by institutions in society and to ,move the world' it must resonate with deep emotions of people“ (Liep 2001: 1).

Diese Facette der ,Resonanz mit tiefen Emotionen' zeigte sich nach der Dreifachkatastrophe in einer Welle der Solidarität und der Anteilnahme in Japan und über den japanischen Inselstaat hinaus. Durch die Verarbeitung der Geschehnisse in der Gegenwartskunst wurden aber auch Aspekte der japanischen Gesellschaft transparent, die deren kulturelle Diversität und unterschiedliche Diskurse aufzeigten. Der Widerstand der staatlichen Institutionen gegen subversive Diskurse zeugt von einem Verständnis von Differenz, die eine Gefahr für die gesellschaftliche Stabilität bedeutet. Meine Analyseergebnisse zeigen jedoch auch, dass die Agenda der kreativen Akteure nicht nur darin besteht, die bereits vorhandene Diversität offenzulegen, sondern gleichzeitig allzu große gesellschaftliche Differenzen zu überbrücken. Dies geschieht beispielsweise, indem meine Gesprächspartner alternative Eindrücke zu den aktuellen Vorgängen und deren sozialen Folgen aus den unmittelbaren Katastrophengebieten anbieten. Damit laufen sie Gefahr, in ein Spannungsverhältnis mit staatlichen und wirtschaftlichen Akteuren zu treten, die sich in den Post-FukushimaDiskursen als Gegenspieler dieser kreativen Agenda zeigen.

In meinen Interviews präsentieren sich Künstler als Akteure, die einer politischökonomischen Macht ihre Authentizität entgegensetzen, indem sie kollektive Erfahrungen in ihrem eigenen Empfinden widerspiegeln und diese Verbindung in einem öffentlichen Raum ausdrücken. Kogoro aus Kapitel 7 ist dafür ein gutes Beispiel: Seine eigenen Erfahrungen der Einsamkeit und Traurigkeit sowie seiner Außenseiterrolle befähigen ihn dazu, die emotionale Verbindung zwischen den unmittelbar Betroffenen der Atomkatastrophe und den Bewohnern Tokyos aufzubauen. Auch Ayako und viele andere meine Gesprächspartner nutzen ihre eigene Emotionalität, um sich selbst und die Erfahrungen ihrer Mitmenschen authentisch in eine kreative Form zu bringen und den Bezug zu einem gemeinsamen Emotionskonzept herzustellen. Ich schließe mich Ortner (2006: 46) an, die den Begriff der Authentizität für die Beschreibung einer in sich logisch konstruierten historischen Lebenswirklichkeit heranzieht. Auf mein Forschungsfeld übertragen ist Authentizität an ein bestimmtes Rollenverständnis als Künstler geknüpft und bietet eine Leinwand für gesellschaftliche Erwartungshaltungen. 
Hier sehe ich die Schnittstelle zwischen individuellem Handeln und gesellschaftlichem Wirken. Diese Kombination aus Ausdruck, emotionaler Ansprache und dem Rückbezug auf Gemeinsames macht die Kraft des kreativen Ausdrucks in der Bedeutungsherstellung aus (siehe Friedman 2001: 60). Die im Wirken der Künstler deutlich werdende Verbindung zwischen Empathie und kreativem Handlungsvermögen entfaltet ihre Kraft auf der gesellschaftlichen Bühne und erschafft Bindungen über die persönliche Realität hinaus. Diese biographische Wahrheit sieht der einzelne Künstler im direkten Kontakt mit Rezipienten bestätigt. Die Rückwirkung von starken emotionalen Erfahrungen in das gesellschaftliche Regelwerk Japans drückte sich unter anderem in den Folgemonaten der Katastrophe aus, als sowohl in den nationalen als auch in den internationalen Medien die Sicherheit der Atomkraft als „Mythos" dargestellt wurde. Die Menschen fanden unter anderem in der Kunst den Zugang zu einem öffentlichen Raum für ihre sonst im Privaten verbleibenden Emotionen wie Wut, Ärger, Enttäuschung, Angst und Trauer. Auf diese Weise können sie sich als Gemeinschaft erfahren, so wie es auch Ayako aus Shiogama berichtete. Dies zeigt die große Sprengkraft der Verbindung von Empathie, Kreativität und Handlungsvermögen, aber auch ihr Potential, tiefe Verbindungen zu erschaffen. Mithilfe der Empathie verliert die Unterschiedlichkeit der Realitäten ihren Schrecken und wird zu einem Ankerpunkt für Gemeinsamkeiten.

Als Vertreter einer Personengruppe, die die Kreativität in den Mittelpunkt ihres Lebens gestellt haben, haftet meinen Gesprächspartnern eine gewisse Ambivalenz an, die sich aus der polarisierenden Wirkung der Kreativität selbst ergibt: Gesellschaftskritische Werke können sowohl als Bereicherung als auch als Bedrohung empfunden und ihre Erschaffer entsprechend behandelt werden. Deshalb sehen meine Gesprächspartner sich dazu veranlasst, provozierende Botschaften in der Öffentlichkeit weniger scharf zu formulieren. Viele betonen mir gegenüber ihre Absicht, zwar ihre Meinung zum Ausdruck bringen zu wollen, dies jedoch auf eine Art und Weise zu tun, die es ihren Rezipienten erlaubt, sich in der Auseinandersetzung mit den aufkommenden Gedanken und Gefühlen ein persönliches Bild von der Situation zu machen: „It's all about kickstarting the thinking process“ (Saburo 13.09.2014: 3). Auf diese Weise zeigen sie Handlungs- und Gestaltungspotentiale auf und erschaffen die Voraussetzungen für eine Neuorientierung (siehe Liep 2001: 12). 


\subsection{Biographische Lösungswege für die Gesellschaft?}

Wie ich in den vorangegangenen Kapiteln ausgeführt habe, gilt es im Leben eines Künstlers, verschiedene Herausforderungen zu bewältigen. Eine der größten besteht im Ausbalancieren der persönlichen Ressourcen und Wünsche auf der einen sowie den Beschränkungen des individuellen Handlungsvermögens in ihrer Arbeit und in ihrem Leben in der Rolle des Künstlers auf der anderen Seite. Die Unabsehbarkeit der Konsequenzen einer solchen Entscheidung bildet ein großes Risiko für den einzelnen Künstler.

Aufgrund meines Interviewmaterials komme ich zu dem Schluss, dass dieses Risiko nur dann eingegangen wird, wenn es durch die Bewertungsgewichtung zugunsten anderer Werte, wie zum Beispiel größerer individueller Freiräume und der Bereitstellung biographischer Lösungswege für die Gesellschaft, aufgewogen wird. Doch worin besteht der Preis der Auflehnung gegen gesellschaftliche Konventionen und inwiefern dienen die persönlichen Antworten auf gesellschaftliche Fragen als Handlungsschablone auf gesellschaftlicher Ebene?

Einen wesentlichen Faktor bei der Arbeit als japanischer Gegenwartskünstler bilden die zur Verfügung stehenden, meist überwiegend immateriellen Ressourcen und Fähigkeiten, die sie selbst innehaben. Ich konnte sowohl in Tokyo als auch in Shiogama Netzwerke aus sozialem Kapital ausmachen, die von entscheidender Bedeutung für den Verlauf des persönlichen wie beruflichen Lebens eines Künstlers sind. Diese Netzwerke zur gegenseitigen Unterstützung erleichtern es dem Einzelnen, einen individuellen Umgang mit Herausforderungen zu finden. Gleichzeitig bildet die Gemeinschaft dieses Netzwerkes die Basis für die Rollen, die Künstler auch als gesellschaftliche Akteure einnehmen.

\subsubsection{Japans Künstler als ethisches Korrektiv der Gesellschaft?}

Da Empathie in der Gestaltung sozialer Beziehungen in Japan einen besonders hohen Stellenwert einnimmt, stellen sich noch einige theoretisch-analytische Fragen bezüglich der ethischen Dimension des alltäglichen Handelns. Wie positionieren sich die Gegenwartskünstler in ihrem alltäglichen Erleben zu ihren eigenen ethischen Überzeugungen? Wenn meine Gesprächspartner in ihrem jeweiligen Narrativ manche Kontrastierung mit ihrem unmittelbaren oder mittelbaren gesellschaftlichen Umfeld aufzeigen, welche Konsequenzen ziehen sie daraus bezüglich der sozialen Verantwortung anderen gegenüber?

Wie ich in den vorausgegangenen Kapiteln erläutert habe, kann der Künstler mit der Bereitstellung von Emotionen ein großes emotionales Potential in einer breiten Öffentlichkeit wachrufen. Dieses Potential berührt auch ethische Überzeugungen. ${ }^{91}$ Kulturelle Rahmenbedingungen wie die der Ethik und individuelles Selbstverständ-

\footnotetext{
${ }^{91}$ Siehe Kapitel 4.1.1. „Der Kompass der Werte - Ethik und Selbst im japanischen Kontext“.
} 
nis finden im Moment der Aushandlung von Handlungsvermögen starke Berührungspunkte.

Ich habe bereits dargelegt, wie der Zugang der Gegenwartskunst zu gesellschaftlichen Themen auf den kulturspezifischen Umgang mit Emotionen Bezug nimmt und so betrachtet im Einklang mit der kulturellen Rahmung Japans steht (vgl. Kitayama und Markus 1994: 8). Jedoch überschreiten die Künstler einige gesellschaftlich gezogene Grenzen, indem sie jedem Bürger Japans (und in einigen Fällen der ganzen Welt) ihre Betroffenheit vor Augen führen und nicht unmittelbar Betroffene durch die Einbindung von Emotionen wie Traurigkeit, Wut und Scham in die ethische Verantwortung für die Folgen der Dreifachkatastrophe nehmen. Damit wird die Verbindung von Handlungsvermögen und Verantwortung auf ethischer Ebene wirksam. Gleichzeitig erweitert sich der Aushandlungsraum für die von Rosaldo (1984: 147ff.) betonte gegenseitige Einflussnahme von Individuum und kulturell-gesellschaftlichem Kontext.

Die übergeordnete gesellschaftliche Struktur betrachte ich nicht ausschließlich als Einschränkung für das Handeln des Einzelnen, sondern auch als Möglichkeit zur Erschließung von Handlungsmöglichkeiten. Strukturelle Rahmungen eröffnen die Bühne für die Betrachtung von eben jenen Aushandlungsdiskursen, die diese Strukturen erst erschaffen beziehungsweise in ihrem Fortbestand legitimieren. Damit muss auch die Handlungsfähigkeit des Einzelnen berücksichtigt werden.

Insbesondere im japanischen Kontext ist es die Gestaltung der Aushandlungsprozesse von Transformationen im Ortnerschen Sinne (2006), die die Artikulationen zwischen den Praktiken einzelner sozialer Akteure und der übergeordneten Struktur so aufschlussreich gestaltet: „It restored the actor to the social process without losing sight of the larger structures that constrain (but also enable) social action" (Ortner 2006: 3).

In den Ausführungen der Japanologin Lisette Gebhardt zum Beitrag der japanischen Literaturszene zu den Post-Fukushima-Diskursen wird ebenfalls diese Verbindung zwischen Individuum und Struktur hergestellt. Dabei verbindet Gebhardt Handlungsvermögen wie beispielsweise Laidlaw (2010) mit Überlegungen zur ethischen Dimension und zieht in Betracht, dass Schriftsteller „[...] perhaps fulfill the antiquated role of an ethical corrective. They act thus as intellectual celebrities' who are able to stimulate the audience to think things over" (Gebhardt 2015: 250).

Die Funktion des, ethischen Korrektivs' trifft laut Gebhardt auch auf andere kulturelle Akteure wie die Gegenwartskünstler zu, die sich in gesellschaftskritischer Weise mit sozialen Thematiken auseinandersetzen. Ebenso wie Schriftsteller beschäftigen sie sich mit dem gesamten emotionalen Spektrum der Post-Fukushima-Diskurse, die Themen beinhalten wie

"[...] confrontation with a waste land, the lingering feeling of threat, life in the emergency accommodation, the preservation of the memory of the dead, the fear of radiation, damage done by radiation, discrimination against Fuku- 
shima victims, flight from Japan, the hope of recovery and the strength of a community." (Gebhardt 2015: 251)

Die besondere Leistung aller kulturellen Akteure liegt in der Sichtbarmachung von Verletzungen expliziter wie impliziter ethischer Vereinbarungen einer Gesellschaft und den sich daraus ergebenen Konsequenzen für das Handeln des Einzelnen. Diese Herstellung von Transparenz erfordert, wie auch Gebhardt (2015: 250) feststellte, besonderen Mut und, so möchte ich hinzufügen, eine feste Überzeugung, das ethisch Richtige zu tun. Die Besonderheit in der Rolle des Künstlers liegt in der bewussten Strapazierung des Bandes zwischen Individuum und Kollektiv in Bezug auf den kulturspezifischen Umgang mit Emotionen. Indem Künstler Selbstverständlichkeiten, Wertvorstellungen und Realitäten kontrastieren und hinterfragen, wird das harmonische Bild des Miteinanders in Zweifel gezogen: „[...] art makes sense, if at all, only when it uses personal perception to counter the power of some collective belief" (Kuryluk 1994: 13). Ein gewisses Maß an Subversivität, also die bestehenden sozialen Ordnungen zu hinterfragen und mit einem Gegenmodell zu konfrontieren, ist demzufolge einer Künstlerrolle inhärent wie sie meine Gesprächspartner verstehen. In der Lebensweise und im Ausagieren ihres Handlungsvermögens verdeutlichen Gegenwartskünstler sowohl in ihrem biographischen Narrativ als auch in ihrer Arbeit im Katastrophenkontext, dass Ethik „,...] is vulnerable to - but also achieved in the face of - rupture, erosion, and skepticism" (Lambek 2010: 63). Meine Gesprächspartner machen sehr deutlich, dass sie den Hinterfragungsprozess nicht als konfliktstiftend verstanden sehen wollen. Vielmehr vermittelten sie mir ein Selbstbild, in dem sie auf eine nachhaltige, tiefgreifende Harmonie in Beziehungen hinarbeiten: „It's about bringing people together, sharing feelings and perspectives“ (Ayako 20.11.2014: 2).

Der Ethnologe Stafford (2010: 188) bezeichnet Handlungen, wie sie meine Gesprächspartner zeigen, als ein ethisches Verhalten, das gleichzeitig unmoralisch ist. Moral bildet bei Stafford den strukturellen Überbau, während Ethik die gelebte Praxis repräsentiert. Daher sei es möglich „[... [o do what we think is right, even if the rules say otherwise" (Stafford 2010: 188). Gesprächspartner wie Kaeko machen ihre Perspektive in Bezug auf die Chancen zur Veränderung besonders deutlich, die Japan nach der Katastrophe ihrer Ansicht nach hatte: „It's just been four years since ,Fukushima' but we forget very quickly. After 3.11 we recognized we could live using less electricity but as soon as we could we got back to the standard we had before. Japan missed a chance" (Kaeko 28.2.2015: 5).

Die analytische Sicht auf Handlungsvermögen im Zusammenhang mit Ethik eröffnet den Blick auf eine Facette der Gegenwartskünstler in ihrer Rolle als gesellschaftlicher Spiegel: Die Präsentation eines Ist-Zustandes, der in starkem Kontrast zum ethisch-moralischen Soll-Zustand der Gesellschaft stehen kann. Ich bin der Auffassung, dass dieses Spannungsverhältnis von Ist- und Sollzustand dem gesellschaftskritischen Aspekt der Arbeit meiner Gesprächspartner ein besonderes emotionales Gewicht verleiht. Das Streben nach einer Form der Transparenz zwischen 
persönlicher Erfahrung und gesellschaftlicher Realität bildet die Basis für ihr ethisch motiviertes Handlungsvermögen im gesamtgesellschaftlichen Kontext.

Nichtsdestotrotz erachte ich die Aussage Gebhardts, dass Künstler als „ethisches Korrektiv" fungieren, als zu stark. Aufgrund meiner Analyse und der darin deutlich gewordenen Kulturspezifik Japans komme ich stattdessen zu dem Schluss, dass sich meine Gesprächspartner vielmehr als Kompass gesellschaftlicher Ausrichtungen betrachten würden. Ein ethisches Korrektiv zu sein, würde eine klare ethisch-politische Agenda nahe legen, die als Schablone für aktuelle Vorgänge herhalten würde. Diesen Aspekt behandle ich im nächsten Kapitel.

\subsubsection{Ist ein Künstler ein politischer Aktivist?}

Bei der Verbindung von Politik, Gesellschaft und Kunst und ihren fortlaufenden Transformationen ist es Künstlern mit gesellschaftskritischer Agenda nahezu unmöglich, nicht in irgendeiner Form politisch Position zu beziehen. Zwar betonen meine Gesprächspartner wiederholt, sie seien unpolitisch, jedoch spezifizieren sie auf meine Nachfrage hin, dass sie sich mit dieser Aussage einer Zuordnung zu einer bestimmten Partei oder politischen Ausrichtung verweigern wollen. Die meisten sind sich jedoch unsicher, was einen politischen Aktivisten ausmacht: „Sometimes it's hard to tell if someone is an activist. Maybe not an activist, but they are certainly doing a lot" (Kogoro 09.02.2015: 25).

Mir wurde in den verschiedenen Gesprächen ein Bild eines japanischen Politikverständnisses vermittelt, in dem Politik als eine Art übergeordneter Steuerungsmechanismus gesehen wird, auf den der Bürger in seinem alltäglichen Leben wenig Einfluss ausübt. Bei mir entstand der Eindruck, dass politisches Handeln in Japan mit der Verantwortung und den Pflichten des Regierens und damit mit der Personengruppe der Mächtigen und (wirtschaftlich) Großen synonym behandelt wird. Wie ich im Kapitel 2.2 „Arbeiten über die Narrative zu ,Fukushima““ bereits dargestellt habe, haben die Ereignisse von 2011 an diesen Vorstellungen der Machtverhältnisse gerüttelt: die Erwartungen der Menschen an den Staat, zum Beispiel in Bezug auf Fürsorgepflicht und Verantwortung, wurden nicht erfüllt. Die Vorstellungen der Bevölkerung von ethisch richtigem Handeln wurden dementsprechend stark verletzt und stehen im Fokus der aktuellen Diskurse.

Vor dem Hintergrund des angenommenen Versagens institutioneller Akteure am 11. März 2011 weitet sich der Fokus der Aushandlung von persönlicher Schuld und Verantwortung auf die gesellschaftliche Ebene aus. Die Kulturwissenschaftler Collier und Lakoff bezeichnen eine solche Ebene als Zwischenstufe zwischen Staat und Individuum, auf dem die Gesellschaft selbst als zentrales Element der ethischen Verortung wieder auftaucht: „At the same time, ,politics" is reconfigured in more partial and provisional forms around problems of collective existence related to life itself" (Collier und Lakoff 2005: 28-29).

Collier und Lakoffs theoretischen Zugang halte ich deshalb für erkenntnisreich, da ich in ihren Ausführungen viele Aspekte der sich derzeit entfaltenden Diskurse in 
Japan wiedererkenne. Die Diskursräume, die sich nun verstärkt durch die verschiedenen Akteure der japanischen Gesellschaft generieren, bauen sich entsprechend Collier und Lakoffs Verständnis antagonistisch zu den von politischen und wirtschaftlichen Kräften bevorzugten Narrativen auf. Meine Erhebungen verdeutlichen, dass dabei auf der von Collier und Lakoff beschriebenen gesellschaftlichen Ebene angesetzt und agiert wird: Angesichts der eingetretenen Desillusionierung bezüglich des staatlichen Orientierungsrahmens sucht die Bevölkerung nach einem zuverlässigen Kompass für ihr weiteres Leben - und greift dabei unter anderem auf ein ethisches Verständnis als Maßstab für zukünftige politische Entscheidungen zurück. Es sind folglich unter anderem Emotionen wie Enttäuschung, Wut und Trauer im Katastrophenkontext der aktuellen Diskurse, die eine Politisierung im weitesten Sinne vorantreiben. Kogoro fasst seine Meinung dazu in folgende Worte: „It's like I am part of a tree so I have to grow. If some fruits will come in the future, it's good. [...] I have the feeling the next generation will have to express their opinion about social issues more and more" (Kogoro 06.03.2015: 26-27).

Trotz aller begrifflichen Unschärfen in der Kategorisierung ihrer Werke vermitteln Künstler über die von ihnen geleistete Emotionsarbeit in diesem Kontext auch entsprechende politische Ansichten, die sich an einem individuellen Verständnis von ethisch richtigem Handeln orientieren. Emotionen wie Enttäuschung und Wut werden von ihnen zum Anlass genommen, um über entsprechende Verletzungen des Emotionskonzepts zu reflektieren und allgemeine sozio-politische Forderungen zu formulieren. In der Kunst verbinden sich meiner Auffassung nach also Emotion und Politik zu einer kraftvollen persönlichen Aussage des jeweiligen Künstlers.

Auf theoretischer Ebene verbindet der Soziologe Marcuse das oppositionelle Potential mit den subversiven, revolutionären Anlagen dieser Haltung: ,[...] the political and aesthetics, the revolutionary content and the artistic quality tend to coincide" (Marcuse 1978: 2). Das Bemühen, die sozialen Diskurse zu verarbeiten und in einer symbolischen Sprache in den öffentlichen Raum zurückzuspiegeln, lässt Künstler in gewisser Weise in die Oppositionsrolle gegenüber der homogenisierenden Macht des Staates rücken. Der „Diskurs des Anderen“, in dem sie sich selbst mehr oder weniger stark verorten, spielt in ihrer Lesart die ethischen Fragen des Zusammenlebens in den Vordergrund (siehe Kapitel 7 und 8). Dabei bilden Künstler jene Aspekte ab, die im dominanten und, wie im japanischen Fall, stark staatlich geprägten Gesellschaftsbild nicht oder nur marginal auftauchen. Marcuse beschreibt die ästhetische Form folgendermaßen: „Aesthetic form, autonomy, and truth are interrelated. [...] The truth of art lies in its power to break the monopoly of established reality" (Marcuse 1978: 9). Die solchermaßen universell verstandene Sprache der Kunst neigt also in Japan wie auch in anderen Gesellschaften dazu, die sozialen und politischen Gräben aufzuzeigen, diskutierbar zu machen und ihre Überwindung anzuregen. Subjektiv empfundenes Recht und Unrecht wird dabei den gesellschaftlich gültigen ethischen Kategorien von „richtig“ und „falsch“ gegenüber gestellt (siehe Marcuse 1978: 28) und kann in ihrer Folge oppositioneller, zivilgesellschaftlicher Arbeit wie der von NGOs zuträglich sein. In der Spiegelung der praktisch gelebten Umsetzungen von 
moralisch-ethischen Grundsätzen treten Widersprüche zutage, die im besten Falle ein geschärftes Bewusstsein für die diversen Lebenswirklichkeiten von verschiedenen Gesellschaftsteilen nach sich ziehen. Wird dieser Impuls weiter getragen, können die aufgedeckten Transformationspotentiale auf allen gesellschaftlichen Ebenen umgesetzt werden.

Meiner Meinung nach greift es nicht zu weit, Gegenwartskünstler als Akteure zu bezeichnen, die politische Basisarbeit in dem Sinne betreiben, als dass sie die Menschen zur Diskussion über die Umsetzung ethisch-moralischer Wertvorstellungen auf gesellschaftlicher Ebene anregen. Das als abstrakt wahrgenommene Politikgeschehen wieder in den gelebten Alltag zu integrieren und in einer bestimmten gesellschaftlichen Situation zu konkretisieren, ist die selbstverstandene Aufgabe von Gegenwartskünstlern auf einer politisch-gesellschaftlichen Ebene.

Der von Collier und Lakoff (2005) geschilderte Fokus auf eine bestimmte Lebenssituation und einen speziellen Kontext ist die Voraussetzung für die wirksame und flexible Orientierung in einem unsicheren, bisher nie dagewesenem gesellschaftlichen Zustand. Durch die Erweiterung der Diskurse mithilfe diverser Akteure werden Werte, Moralvorstellungen und als gegeben betrachtete Normen in den Mittelpunkt der gesellschaftlichen Diskurse gerückt. Individuelle, also auch innerstaatliche, Positionierungen werden dabei in Zweifel gezogen (vgl. Ong und Collier 2005). Dieser Aushandlungsprozess weckt durch die Berührung des fundamentalen Selbstverständnisses einer Gesellschaft Emotionen.

Mir scheint, dass es das geschilderte Zusammenwirken aus der Hinterfragung bestehender Machtverhältnisse und subjektiver Rückwirkung auf gesellschaftlicher Ebene ist, das die zugeschriebenen subversiven und politisierten Charakteristika der Gegenwartskünstler Japans ausmacht.

\subsection{Emotionen als moralischer Kompass für eine Gesellschaft}

Bei der Betrachtung des Arbeitsumfeldes der Gegenwartskünstler kristallisieren sich ihre sozio-ökonomischen Rahmenbedingungen und Möglichkeiten zur öffentlichen Diskussion kontroverser Themen als entscheidend für ihr biographisches Erleben und ihre Selbstpositionierung im sozialen Gefüge heraus. Die damit einhergehende Rollenzuschreibung eines Gegenwartskünstlers in Japan spiegelt sich in den Aussagen meiner Gesprächspartner wider, die davon zeugen, unangenehme und damit diskussionswürdige Aspekte der Gesellschaft in die entsprechenden Diskurse einleiten und dort halten zu wollen. Die enge Verbindung zwischen ihrer beruflichen Tätigkeit, ihrem Selbstverständnis und der ihnen zugeschriebenen Rolle wurde dabei deutlich und weist eine gemeinsame Quelle der Motivation und des Antriebs auf, auf die ich hier zurückkommen möchte: Emotionen, durch die, wie Kitayama und Markus es formulieren „[...] individuals try to accomplish, collectively and person- 
ally, a form of adaption and adjustment to their own immediate sociocultural, semiotic environment" (Kitayama und Markus 1994: 1).

In den Aussagen meiner Gesprächspartner treten die von Kitayama und Markus erwähnten Anpassungs- und Gestaltungsprozesse im Kontext der Dreifachkatastrophe besonders deutlich zutage und veranschaulichen eine Möglichkeit des emotionalen Umgangs mit deren sozialen Folgen. Das Aufgreifen angrenzender Themen wie Verantwortungsbewusstsein, die Suche nach angemessenen Konsequenzen, Ethik, Empathie und die sich daraus ergebenen Forderungen nach gesellschaftlichen Veränderungen machen Gegenwartskünstler zu einem wichtigen Sprachrohr für eine ansonsten weitestgehend stumme Akteursgruppe der unmittelbar Betroffenen: „My paintings have aspects which aim at giving response to these souls which exist out of everyone's sight" (Kogoro 11.03.2015: 28).

Der kreative Ausdruck von empathisch nachempfundenen Emotionen findet auf zwei Ebenen statt, der privaten (honne) und der öffentlichen (tatemae), so dass die Hinterfragung der behandelten Gefühle immer auch entsprechende Fragen an die Gesellschaft beinhaltet.

Mit der Artikulation von Emotionen werden Reibungspunkte transparent, die das Potential zur Auseinandersetzung mit kontroversen und bereits seit geraumer Zeit vorhandenen Widersprüchlichkeiten einer Gesellschaft haben. Dabei handelt es sich meist um Widersprüche, für die im gesellschaftlichen Zusammenleben Japans bisher wenig Platz eingeräumt wurde (z.B. dem Umgang mit Personen, die „anders“ sind). Das Bild einer oberflächlich harmonisch erscheinenden Gesellschaft hat durch die Erschütterungen des Erdbebens vom 11. März 2011 Risse bekommen. Die darunter liegenden Konflikte werden durch kulturelle Akteure wie meine Gesprächspartner freigelegt und zur Diskussion gestellt. Dadurch erhalten negativ konnotierte Emotionen wie Wut oder Trauer einen legitimen Raum für verschiedene Interpretationen und Bedeutungszuschreibungen. Gleichzeitig werden diese Emotionen von meinen Gesprächspartnern als Hinweise für die Gestaltung des weiteren Weges der japanischen Gesellschaft verstanden.

In einer Zeit großer Verwirrung, Unsicherheit und einer unzureichenden Informationslage sind es vor allem Emotionen, die eine erste Orientierung bieten. Sie fungieren als Antrieb und moralischer Kompass und bilden gleichzeitig ein verbindendes Element zwischen einzelnen Individuen und dem Kollektiv, das ein gemeinsames Realitätsverständnis zu schaffen vermag. Das Mobilisationspotential von Emotionen für Veränderungen ist damit massiv, besonders wenn man die kulturspezifischen Bedeutungsaufladungen von Emotionen in Verbindung mit gesellschaftlichen Werten und Moralvorstellungen in Betracht zieht. Kitayama und Markus (1994) und insbesondere Taylor (1989) betonen diese Aspekte der Emotionalität und damit die wahrgenommene Einhaltung oder Verletzung gesellschaftlicher Regeln und ethischer Vorstellungen. In Bezug auf „Fukushima“ wurde dies besonders deutlich, da sich entsprechend moralisch aufgeladene Gesellschaftsgruppen mit Protestlegitimation ausgestattet sahen, wie zum Beispiel Mütter, die für die Sicherstellung der Gesundheit ihrer Kinder demonstrierten. Die den Müttern zugeschriebene 
Rolle als Verteidigerinnen der nächsten Generation verleiht ihnen die Legitimität, ihre Emotionen von Wut, Verunsicherung und Empörung zu äußern.

Eine vergleichbare, wenn auch gänzlich anders gelagerte Erwartungshaltung wird den Gegenwartskünstlern entgegengebracht. Aufgrund der Subversivität ihrer Tätigkeiten wird in der breiten Öffentlichkeit erwartet, dass sie sich eine eigene Meinung bilden und diese auch darstellen. Die Fokussierung der Gegenwartskünstler auf einen Ausdruck von Emotionen verleiht dem Künstlermilieu eine subversive oder sogar eine rebellische Konnotation, wie ich es in Kapitel 6 dargestellt habe. Nach Heine et al. (1999: 774) treten die eigenen Emotionen im japanischen Kontext stets hinter die Wünsche und Bedürfnisse der Gruppe zurück und erlangen keine eigene Autonomie. Im Wirken der Künstler geschieht meiner Meinung nach aber genau das: Ihre transparent gemachten persönlichen Emotionen stellen eine ausgestreckte Hand für all diejenigen dar, die hinsehen wollen, und beherbergen damit ein Potential sowohl für empathisch empfundene Solidarität als auch für zivilgesellschaftliches Engagement.

Wie bereits beschrieben, können Emotionen ihr Potential jedoch nur dann wirkungsvoll entfalten und zu gewünschten Ergebnissen führen, wenn sie auch gesehen, wahrgenommen und ernst genommen werden. Im Falle marginalisierter Gruppen, wie den unmittelbar Betroffenen der atomaren Katastrophe, verschwindet diese öffentliche Bühne im Laufe der Zeit und ihre Wünsche, Bedürfnisse und Herausforderungen drohen im neu entstehenden Alltag unterzugehen. Für nachhaltige Veränderungen auf breiter gesellschaftlicher Ebene müssen die ausgemachten Fehler weiterhin im Fokus der öffentlichen Aufmerksamkeit stehen und es muss dadurch Druck auf die machtvollen Akteure einer Gesellschaft ausgeübt werden.

Meiner Ansicht nach sind Gegenwartskünstler Experten für das Bereitstellen von Reibungsflächen, die ein Anstoß für Diskussionen sein können, jedoch nicht für ideale Lösungsvorschläge. Ihr Beitrag besteht darin, die essentiellen Fragen zu stellen, andere dazu zu bringen, über sie nachzudenken und in ihrer individuellen emotionalen Wirkung zu erforschen. Was es für jeden einzelnen Rezipienten bedeutet, diese Fragen gestellt zu bekommen, liegt außerhalb der Kontrolle des Künstlers. Ihr Anspruch an ihr Wirken ist viel bescheidener. Es geht nicht um einen bestimmten, wohl kalkulierten Effekt ihrer Arbeit, sondern um das Vertrauen in deren Wirkung überhaupt. In ihren biographischen Narrativen wird ihre Position deutlich, ihre Gedanken, Empfindungen und Ansichten auf legitime Weise einer breiten Öffentlichkeit zur Verfügung stellen zu wollen.

Ich gelange zu dem Schluss, dass es Künstlern in der Veranschaulichung von Emotionen um die Einsicht geht, dass jeder die Zukunft aktiv mitgestalten kann und soll. Durch diese Grundhaltung gelangt der jeweilige Gesprächspartner zu der Ansicht, dass jeder, unabhängig von Klassenzugehörigkeit und finanziellem Auskommen, jene Ressourcen und Fähigkeiten in sich trägt, die, unsichtbar für ökonomische Statistiken, ihren eigenen Wert besitzen. Meine Gesprächspartner leben dieses Konzept, indem sie die Verwundbarkeit ihrer Person durch den Ausdruck ih- 
rer Emotionalität transparent machen und versuchen, ihr Leben mit größtmöglicher Authentizität zu gestalten. Es ist ein Leben in Herausforderung des Status quo.

Das Leben des einzelnen Künstlers steht im Kontext eines historischen Zeitpunktes wie dem 11. März 2011, in dem bestimmte Handlungsoptionen und Hoffnungen wahrscheinlicher erscheinen als andere. Die Fähigkeit der Gegenwartskünstler, diese Optionen auszuloten und dabei verschiedene Perspektiven einzunehmen, wird vom Soziologen Mills als „sociological imagination“ beschrieben: „It is the capacity to range from the most impersonal and remote transformations to the most intimate features of the human self - and to see the relations between the two. [...] that men now hope to grasp what is going on in the world, and to understand what is happening in themselves as minute points of the intersections of biography and history within society" (Mills 1959: 7).

Diese Verschränkung von Biographie mit der Geschichte einer Gesellschaft wird meiner Ansicht nach am Beispiel der Gegenwartskünstler besonders deutlich. Transformationspotentiale werden von ihnen vor dem Hintergrund einer kulturspezifischen emotionalen Bedeutungsaufladung von Moral- und Wertvorstellungen betrachtet und an ihr Publikum zurückgespiegelt.

Die privaten Problematiken des Einzelnen verschwimmen durch die Bedrohung gesellschaftlicher Werte, wie beispielsweise Gesundheit, mit den Problemstellungen des Kollektivs. Diese spezifischen Verknüpfungen wahrzunehmen und in ihrer Wechselwirkung zu erkennen, ist laut Mills (1959) ein Zeichen für die soziologische Vorstellungskraft, die es braucht, um zunächst die Problematiken genauer zu formulieren. Die eigenen biographischen Themen der Gegenwartskünstler und aktuelle Diskussionsthemen im japanischen Diskurs ergänzen und decken sich und können mithilfe des kreativen Ausdrucks dieser Verbindung einen Legimitationsanspruch im öffentlichen Raum geltend machen. Private Herausforderungen finden so ihren Platz in öffentlichen Diskussionen, da sie den moralischen Wertekompass der Gesellschaft im Allgemeinen betreffen.

\subsection{Das Prinzip der narrativen Transparenz}

Künstler nehmen in ihren Bemühungen um die gesellschaftliche Verarbeitung der Katastrophe von 2011 Rollen ein, die die Bereitstellung von Informationen, des entsprechenden emotionalen Interpretationsrahmens und die Dokumentation der Ereignisse beinhalten. In der Kombination mit der Kreativität als Ressource für Empathie ${ }^{92}$ und der Sichtbarmachung ethischer Fragestellungen erschafft der Künstler mit jedem Werk auch ein Abbild seiner Individualität im gesamtgesellschaftlichen Gefüge.

Gleichzeitig wird in den jeweiligen biographischen Erzählungen meiner Gesprächspartner ein spezifisches Narrativ deutlich, das im Vergleich zu gleichaltrigen

\footnotetext{
${ }^{92}$ Siehe Kapitel 10.1.2 „Kreativität als Ressource für Empathie und Handlungsvermögen“.
} 
Mitmenschen einige Besonderheiten aufweist. In meinem Interviewmaterial tauchen immer wieder Passagen auf, in denen die jeweiligen Akteure ihre Eigeninitiative im biographischen Prozess deutlich machen. Jeder Einzelne meiner Gesprächspartner, bis auf die in Kapitel 9.3 porträtierte Ausnahme Tasha, suchte während seiner Jugend diejenigen Arbeitsbedingungen, die es ihm ermöglichten, seine Neugier, Kreativität und Interessen auszuleben. Der unter einem starken Konformitätsdruck negativ bewertete Aspekt der Individualisierung wird von den Künstlern zu etwas Positivem umgedeutet, der sich in ihrer kreativen Arbeit ausdrückt. Dies erklärt, warum sie Beruf und Privatleben in ihrem Narrativ demonstrativ untrennbar miteinander verbinden.

Die einzelnen Analyseabschnitte verdeutlichen die Tendenz meiner Gesprächspartner zu betonen, Künstler zu sein sei ein Lebensstil oder Schicksal und damit viel mehr als ein Job. Ferner weißt dies darauf hin, dass sie sich in einem Wertekodex verorten, in dem nicht eine bezifferbare Leistung (Geld, Produktion von Werken, Besucherzahlen) im Mittelpunkt steht, sondern eine Verifizierung ihres Selbst, die sich im Fall der Ausstellung, der Betrachtung durch Dritte und emotionalem Feedback einstellt.

Die Artikulationen meiner Gesprächspartner zielen dabei in zwei Richtungen. Zum einen richten sie sich an die Zielgruppe ihrer Rezipienten. Zum Anderen bestätigen sie sich selbst in ihrer Rolle als Künstler, indem sie sich entsprechend präsentieren. Das Image des interessanten, da unangepassten Künstlers wird auch von meinen Gesprächspartnern in unterschiedlich stark ausgeprägter Weise gepflegt, indem sie zum Beispiel Marker wie einen auffallenden Kleidungsstil bevorzugen. Auch mir als Forschende gegenüber trat der Einzelne vor allem in der Öffentlichkeit in dieser betonten Art und Weise auf, bis das Vertrauensverhältnis groß genug war, um sich auch in privaten Räumlichkeiten zu treffen. In der öffentlichen Präsentation des privaten Selbst (honne) zeigen sich die Künstler als authentische Personen und werden von den Rezipienten vorwiegend auch als solche wahrgenommen. Die zusätzlich an die Rolle des Gegenwartskünstlers geknüpften Erwartungen der Gesellschaftskritik und Meinungsäußerung eröffnen ihnen diskursive Nischen, in denen sie ihre persönliche Vorstellungskraft im Dienste gesellschaftlicher Thematiken verwenden können.

Bei der Absicherung ihres Selbstbildes handelt es sich um eine zwischenmenschliche Dynamik. Sie ist Teil des biographischen Narrativs, auf das ich in der Analyse eingegangen bin. Vor dem kulturspezifischen Hintergrund Japans halte ich dies für besonders erwähnenswert, da meine Gesprächspartner durch den von mir gewählten narrativen Forschungsansatz die maximale Freiheit erhielten, ihre Biographie zu präsentieren. In den einzelnen biographischen Darstellungen finden sich daher auch Anzeichen dafür, dass sich meine Gesprächspartner mir gegenüber ihres Selbstbildes zu versichern versuchten (vgl. Swann und Hill 1982). So tauchen im Japanischen wie auch im Englischen immer wieder linguistisch signifikante Satzpassagen auf, in denen meine Gleichgesinnung und Zustimmung angenommen beziehungsweise eingeholt wurde. Am auffälligsten war dies bei Kogoro (2014), der seine Sätze häufig 
mit einem japanischen „ne?" als Mischung aus Betonung und Rückversicherung beendete.

Ich habe die Erkenntnis gewonnen, dass sich in der Rolle des Künstlers Authentizität, Empathie und subversives Handeln vereinen. Meine Gesprächspartner verbinden ihre eigenen biographischen Erfahrungen auf empathische Weise mit den Erlebnissen der unmittelbar Betroffenen der Katastrophe und zeigen dabei gleichzeitig die große Vielfalt und Komplexität der Narrative zum 11.03.2011 auf. Dieses Vorgehen basiert meiner Ansicht nach auf tiefen Überzeugungen bezüglich ihrer Rolle als Künstler, Wertvorstellungen und einer Reflexion über eigene Erlebnisse, weswegen ich es als „Prinzip der narrativen Transparenz“ bezeichne.

Bei der Suche nach einem etablierten Platz in der japanischen Gesellschaft scheint es eher zuträglich zu wirken, dass eine Facette des künstlerischen Selbstverständnisses auf dem Aushalten eines persönlich als wirtschaftlich prekär empfundenen Zustandes beruht. Bei der Analyse der verschiedenen Narrative eröffneten sich mir im Einzelfall auch starke persönliche Abgründe und biographische Brüche, die meine Gesprächspartner schlussendlich jedoch nur in der Verfolgung ihres Weges bestätigten. ${ }^{93}$ Ihre Lebensgeschichten verstehen Künstler teilweise als die praktisch erprobte Alternative zu bisherigen Denkansätzen, zum Beispiel in Bezug auf zivilgesellschaftliches Engagement. In den Interviews wird das unter anderem dadurch deutlich, dass der jeweilige Künstler einen Handlungsbedarf in den Katastrophengebieten wahrnimmt und entsprechend im Rahmen seiner Möglichkeiten handelt.

Auch die Rollen als Bereitsteller von Vorschlägen, Informant und Dokumentar inkorporieren sie in ihre Identität als Künstler: „The artist is also a part of society so he can only propose to society. Art is just a drop of ,now' of society, but also the base for the future" (Saburo 13.07.2014: 3).

Indem sie sich diesen Platz in der Gesellschaft erschaffen, gestalten Künstler auch ihr eigenes Selbst in Beziehung zu Menschen ihres direkten Umfeldes. Gleichzeitig umreißen sie ihre Position innerhalb der japanischen Gesellschaft. Das Bild von Japans Gesellschaft, das meine Gesprächspartner in ihren Erzählungen mir gegenüber zeichnen, ähnelt mehr der Idealvorstellung einer Gemeinschaft von Individuen als einer meritokratischen Gesellschaft. In diesen Vorstellungen sind vor allem die inneren Qualitäten, Fähigkeiten und Charakteristika von entscheidender Bedeutung, was insbesondere im Angesicht der aktuellen katastrophalen Verluste durch den 11. März 2011 an Attraktivität gewinnt: Das soziale Kapital, bestehend aus persönlichen Beziehungen und Netzwerken, hat in der Lebensanschauung meiner Gesprächspartner weitaus mehr Wert als zum Beispiel monetäres Kapital oder ein hoher Posten in einer renommierten Firma.

Hier wird die Bedeutung des „Prinzips der narrativen Transparenz“ deutlich: Es sollen möglichst viele verschiedene Akteure die kollektive Erinnerung an die Dreifachkatastrophe und insbesondere an „Fukushima“ mitgestalten und es soll keine

\footnotetext{
93 Siehe Kapitel 7 „Kogoro aus Tokyo -,Fukushima ist ein Röntgenbild der japanischen Gesellschaft".
} 
dominante Erzählweise unreflektiert übernommen werden. Um die Erinnerung zu gestalten, halten es meine Gesprächspartner für essentiell, ihrer Stimme in Werken und anderen Diskursbeiträgen Ausdruck zu verleihen und damit andere zu ermutigen, es ihnen gleichzutun: „It's the Japanese way of dealing with things: 'Believe it, then it will be" (Kaeko 25.07.2014: 1). Um der Gefahr einer Verkümmerung des „Ereignisses Fukushima“ zu einem einzigen Narrativ entgegenzuwirken, seien die Grenzen zwischen Performance-Künstler, politischem Aktivist und anderen Künstlergenres verwischt (Kaeko 25.07.2014: 3). Einfacher Glaube schaffe jedoch keine Fakten, so Kaeko weiter, und so liege die Funktion der Kunst in der Darstellung der sozialen Komplexität (Kaeko 25.07.2014: 3).

Die Betonung der Wichtigkeit eines persönlichen Erlebens von Freiheit, das heißt weitestgehende Freiheit von eng gesteckten Rollenzuschreibungen, hebt einen Strang des Narrativs hervor, in dem das eigene Selbst des Künstlers zum Kunstwerk wird. Durch seine Existenz angesichts der als widrig dargestellten gesellschaftlichen Bedingungen stellt das Selbst des einzelnen Künstlers ein hohes Maß an Widerstandsfähigkeit unter Beweis. Saburo drückte es so aus: „As an artist, you need to have inner strength, a strong heart, inner resilience. Maybe that's why we can express these things" (Saburo 13.09.2014: 5).

Die Übergänge der privaten wie der beruflich-öffentlichen Ebene verlaufen hier fließend. Die „innere Kraft“, wie Saburo es ausdrückt, speist sich meiner Ansicht nach unter anderem aus der Sinnhaftigkeit, die meine Gesprächspartner in der Auflösung der kulturspezifischen Trennung von innerem (honne) und äußerem Selbst (tatemae) sehen. Ihre Arbeit löst emotionale Resonanzen aus, die die vorhandenen Beziehungen zwischen den genannten Akteursgruppen sichtbar und erfahrbar machen und damit auch sie selbst als gesellschaftspolitische Akteure positioniert. Die Herausforderungen des Alltags fungieren dabei unter anderem als Gradmesser ihrer Leidenschaft für den kreativen Ausdruck ihrer Erfahrungen und Emotionen.

Aufgrund dieser Ausführungen sehe ich in der Arbeit meiner Gesprächspartner nicht zuletzt auch eine strukturell-kreative Funktion, die unter anderem in ihrem subversiven Vorgehen begründet ist. Indem sie die große Vielfalt der Katastrophennarrative zum 11.03.2011 sichtbar machen, wirken ihre Werke sinnstiftend für ihre Rolle als Akteure, die gesellschaftliche Beziehungen, Bindungen und Verantwortlichkeiten transparent machen.

Auf theoretisch-analytischer Ebene rücke ich die Arbeit meiner Gesprächspartner in die Nähe der „Narratologie der Krise“, die der Literatur- und Kulturwissenschaftler Ansgar Nünning (2013) beschrieben hat. ${ }^{94}$ In seinen Ausführungen zur Metaphorik der Krisen und Katastrophen bezieht er sich zwar auf eine literarischsprachwissenschaftliche Ebene, jedoch erkenne ich viele Übereinstimmungen mit meinen eigenen Ergebnissen. So betont Nünnung unter anderem die kreative und schöpferische Funktion von Sprachbildern, wie sie in der Rhetorik rund um desa-

\footnotetext{
${ }^{44}$ Ich danke an dieser Stelle Herrn Prof. Dr. Gerrit Jasper Schenk für seine fachliche Unterstützung und seine Verweise auf entsprechende Literatur zum Thema Krise und Katastrophe.
} 
ströse Ereignisse von den wirtschaftlichen, politischen und medialen Akteuren eingesetzt werden (Nünning 2013: 137). Meine Gesprächspartner nutzen Metaphorik sowohl im Austausch mit ihren Rezipienten als auch in der vergegenständlichten Form ihrer Werke.

Auch im Hinblick auf historisch-politische Deutungen, der Erzeugung von Sinnhaftigkeit und der Verdeutlichung der normativen Verbindung zwischen allen Beteiligten einer Katastrophen- und Krisengeschichte erkenne ich Parallelen zu der Arbeit meiner Gesprächspartner: „Indem Krisenmetaphern das niemals beobachtbare oder übersehbare Geschehen, auf das sie sich beziehen, bildlich veranschaulichen, machen sie einen komplexen historischen und politischen Zusammenhang überhaupt erst vorstellbar" (Nünning 2013: 137). ${ }^{95}$

Im Unterschied zur Katastrophenmetaphorik der Akteure aus Japans Wirtschaft und Politik geht es meinen Gesprächspartnern nicht um eine vereinfachte Darstellung eines einzelnen Narrativs, sondern, im Gegenteil, um das Aufzeigen von Komplexität, Vielfalt und Pluralität verschiedener Erzählweisen, die nicht zuletzt in den diversen emotionalen Bewertungen der Situationen begründet liegen. In den verschiedenen Narrativen sehen sie kein trennendes Element, sondern eine Betonung gesellschaftlicher Beziehungen, für die Empathie essentiell ist, um die Heilung der leidvollen Erfahrungen des 11.03.2011 zu bewirken.

\footnotetext{
95 In diesem Zusammenhang möchte ich auch auf den Beitrag „Historical Disaster Experiences. First Steps toward a Comparative and Transcultural History of Disasters across Asia and Europe in the Preindustrial Era“von Prof. Dr. Gerrit Schenk verweisen (Schenk 2017).
} 


\section{Schlussfolgerungen - Die Kunst zu leben}

Each person is the best judge of his own happiness.

(Taylor 1989: 82)

In dieser Arbeit habe ich diverse Aspekte der Künstlerbiographien vor dem Hintergrund der Post-Fukushima-Diskurse in Japan beleuchtet und in einer qualitativen Analyse zugänglich gemacht. Ich ging der Frage nach, wie sich die Enthüllungen von Mängeln und Fehleinschätzungen in der Folgezeit der Dreifachkatastrophe vom 11. März 2011 emotional in innerjapanischen Diskursen niederschlagen und welche persönlichen Konsequenzen sich für meine Gesprächspartner daraus ergeben. Dabei habe ich den kulturspezifischen Umgang mit Emotionen in Japan und die entsprechenden Auswirkungen auf die jeweilige Betrachtungsweise meiner Gesprächspartner untersucht. Des Weiteren habe ich die persönlichen Rollenzuschreibungen und Interpretationen der gesellschaftlichen Situation meiner Gesprächspartner mit den gesellschaftlichen Strukturen in einen engen Zusammenhang gesetzt. Ich habe auf diese Weise nicht nur kulturspezifische Kontexte, sondern auch den jeweiligen persönlichen Bezugsrahmen meiner Gesprächspartner für die emotionale Bewertung der aktuellen Situation nach dem 11. März 2011 in der Analyse berücksichtigt. Dabei habe ich herausgearbeitet, auf welche Weise Gegenwartskünstler ihre Handlungsmöglichkeiten in ihre biographische Darstellung einflechten.

Vor dem Hintergrund der Ereignisse von 2011 tritt der jeweilige Gegenwartskünstler in seiner biographischen Selbstdarstellung vor allem in der Rolle eines Gegenspielers zu dominanten, vor allem staatlich geprägten Diskursen auf. Diese Facette der Subversivität übt gerade in den Jahren nach einer Katastrophe einen be- 
sonderen Einfluss auf die Bedeutungszuschreibung an Erfahrungen aus und bildet ein wesentliches Element des „Prinzips der narrativen Transparenz“. Die Herstellung eines emotionalen Bezugs zum 11.03.2011, begleitet durch das Aufzeigen von Vielfalt, Komplexität und Pluralität der Katastrophennarrative, bildet das Kernelement der selbst zugeschriebenen gesellschaftlichen Rolle meiner Gesprächspartner.

Für das analytische Schlaglicht auf die Jahre 2014 und 2015, das diese Arbeit auf Japan geworfen hat, habe ich zunächst die relevanten gesellschaftsspezifischen Strukturbedingungen beschrieben, die unter anderem das politische System, wichtige Träger der öffentlichen Meinungsbildung und das Verständnis des Individuums im Verhältnis zum Kollektiv beinhalten. Das komplexe Geflecht aus institutionellen Rahmungen, dem gesellschaftlichen Umgang mit Individualität und den sich daraus ergebenden moralisch-ethischen Widersprüchen ist ebenso Bestandteil der vorliegenden Betrachtungen wie die Rolle der Öffentlichkeit in der Meinungsbildung. In der Auseinandersetzung mit den durch die Dreifachkatastrophe aufgeworfenen Themenkomplexen habe ich einen biographischen Ansatz gewählt, um die Beweggründe, Motivationen und Motive von Gegenwartskünstlern zu ergründen und vor ihrem spezifischen gesellschaftlichen Hintergrund zu beleuchten. Die analytische Verknüpfung der Betrachtung von gesellschaftlicher Struktur und einer qualitativen Untersuchung persönlicher Realitäten und Emotionen hatte zum Ziel, die Komplexität der Post-Fukushima-Diskurse zu veranschaulichen und die diversen Spannungsfelder aus Sicht meiner Gesprächspartner zu beleuchten.

Die Stimmen internationaler Sozialwissenschaftler, unter anderem aus der Ethnologie, Japanologie und Soziologie, bilden das theoretische Fundament dieser Arbeit, während die von mir in der Forschungsphase der Jahre 2014 und 2015 erhobenen biographischen Daten den erweiterten empirischen Kern bilden. Die Ansichten meiner Gesprächspartner beschreiben eine individuelle Rückkopplung mit den strukturellen Rahmenbedingungen und beleuchten sowohl ihr persönliches Erleben als auch ihre gesellschaftliche Verortung.

Aus diesem Grund habe ich die theoretische Perspektive auf die kulturellen Strukturen durch zwei intensiv analysierte Biographien um die Akteursebene ergänzt. Dies dient dazu, den persönlichen Umgang der Individuen mit dem „System Japan“" (Gebhardt 2015) im aktuellen Spannungsfeld von Zugehörigkeit und Unabhängigkeit darzustellen. Die Dreifachkatastrophe stellt einen wesentlichen Einschnitt in den meisten Lebenserzählungen meiner Gesprächspartner dar. Je nach individueller Rekapitulation der Erfahrungen findet die Dreifachkatastrophe in unterschiedlich starker Ausprägung einen Niederschlag im jeweiligen lebensgeschichtlichen Narrativ. Darüber hinaus verbinden sich die Erfahrungen im Katastrophenkontext mit bereits im Vorfeld wahrgenommenen Widersprüchlichkeiten, Themen und Herausforderungen des persönlichen Lebens. Das von meinen Gesprächspartnern geschilderte Bild der japanischen Gesellschaft findet dabei in den Interviews ebenso seinen Widerhall wie die persönlichen Ausgangspositionen für die Bewertung der Dreifachkatastrophe. Durch diese Verbindung der Analyse von Struktur- und Akteursebene konnte ich der Forschungsfrage nachgehen, wie meine Gesprächspart- 
ner ihre Emotionalität und die Wahrnehmung der Post-Fukushima-Diskurse in ihre Lebensgeschichte integrieren. Auffallend war, dass meine Gesprächspartner sehr viel über ihr Leben nachdachten. Die Darstellung ihrer Erfahrungen und Emotionen vor und nach dem 11. März 2011 verbinden sich mit dem Erleben des Umgangs mit aktuellen umweltpolitischen, sozialen und politischen Themen und bilden entscheidende Zugänge zur persönlichen Position meiner Gesprächspartner im sozialen Gefüge Japans.

Bei der Betrachtung der Rolle der Gegenwartskünstler in der japanischen Gesellschaft fallen einige weitere Besonderheiten ins Auge, die in der Verbindung mit der Dreifachkatastrophe die spezifische Sicht der Künstler auf das japanische Sozialgefüge nahelegen. So persönlich die Schwerpunktsetzung der kreativen Arbeit des einzelnen Künstlers auch ist, weisen sie als Akteursgruppe doch einige Gemeinsamkeiten in der Konstruktion ihres Selbst auf, die für das Verständnis ihrer Post-Fukushima-Narrative essentiell sind. Dazu gehören explizite wie implizite Abgrenzungen gegenüber normativen gesellschaftlichen Handlungsrahmungen und dem Umgang mit Emotionen ebenso wie ihre Selbstverortung in der Mitte der Gesellschaft, ungeachtet so mancher gegenteiliger Fremdzuschreibung.

Die Spannungsverhältnisse zwischen persönlichem Empfinden und gesellschaftlichen Erwartungen werden in der jeweiligen persönlichen Lebensgeschichte sowohl mit negativen als auch mit positiven Attributen versehen und spiegeln die ambivalente Haltung des einzelnen Gesprächspartners gegenüber seiner persönlichen Wirkungs- und Handlungsweise wider. Die Auswirkungen dieser Ambivalenzen zeigen sich wiederum in den einzelnen emotionalen Artikulationen, wenn sich meine Gesprächspartner im größeren Gesellschaftskontext verorten: Unsicherheiten, Ängste, geringes Zugehörigkeitsgefühl und wiederkehrende Identitätsfragen prägen ihre Biographien ebenso wie ein hohes Maß an Freiheitsdenken, großer Empathie, Reflexionsfähigkeit und ihr Wille, mit den eigenen Fähigkeiten einen Beitrag zur Transformation der Gesellschaft zu leisten. Diese Facetten ihrer Existenz werden zudem mit Hoffnungen auf einen Wandel der japanischen Grundhaltung verbunden, die vor dem Hintergrund der Diskurse um die Dreifachkatastrophe in der japanischen Öffentlichkeit verstärkt Gehör finden. Die Gegenwartskünstler nehmen also im Sinne von Abu-Lughod und Lutz (1990: 15) Einfluss auf die „politics of emotion discourse“, der Verbindung und gegenseitigen Beeinflussung von Emotionsdiskursen, Politik und der sozialen Hierarchie.

Die qualitative Auswertung der narrativen Interviews bestätigt meine These, dass Gegenwartskünstler ihre biographischen Lösungswege der japanischen Gesellschaft in Form ihrer Werke zur Verfügung stellen. Trotz aller lebensgeschichtlicher Unterschiede verbindet meine Gesprächspartner die Annahme, dass aufgrund der Geschehnisse und den sozialen Folgen des 11. März 2011 ihre biographischen Lösungsansätze auf gesellschaftlicher Ebene an Relevanz gewonnen haben. Die von meinen Gesprächspartnern behandelten Thematiken der Stigmatisierung, der Rolle und des Werts von Außenseitern, des Umgangs mit Andersartigkeit und des Verantwortungsbewusstseins des Einzelnen wurden mit dem Tsunami und dem atomaren 
Super-GAU erneut zu wichtigen moralisch-ethischen Orientierungsfragen in den japanischen Diskursen. Gegenwartskünstler bieten nicht nur diverse Informationen über Vorgänge der Vergangenheit an, sondern liefern auch emotional aufgeladenes Material, das unterschiedliche Realitäten abbildet. Aus meiner Sicht ist es dieses verbindend wirkende Element ihrer Arbeit, das für meine Gesprächspartner den Wert ihrer Arbeit für die japanische Gesellschaft darstellt. Ihre Lebenskunst kann für das interessierte Publikum eine Form des „Selbst-Bewusstseins“ reflektieren, das durch genaues Beobachten, Nutzen von Empathie und konsequentem Denken zum Ablehnen des unhinterfragten Konsums von Informationen und Meinungen anleitet.

Die Frage, warum gerade Gegenwartskünstler in Japan eine so entscheidende Rolle bei der Beeinflussung der öffentlichen Meinung und damit der Machtbasis einer demokratischen Gesellschaft spielen können, orientiert sich an den Schwerpunktsetzungen der dargelegten biographischen Narrative: Die individuellen Wertvorstellungen der Gegenwartskünstler stehen teilweise im Gegensatz zur gelebten Wirklichkeit der gesellschaftlichen Mehrheit. Die entstehende Reibungsfläche wird von ihnen als Quelle ihres kreativen Wirkens und als emotionale Motivation beschrieben. Indem Künstler versuchen, alternative Möglichkeiten des gesellschaftlichen Lebens gleichzeitig zu entwerfen und zu verwirklichen, bietet ihre eigene Lebenspraxis eine beispielhafte Projektionsfläche, die ihr Handeln trotz aller Ambivalenzen wahrhaftig und damit attraktiv wirken lässt. Der Umgang meiner Gesprächspartner mit Widersprüchen, Herausforderungen und den entsprechenden Emotionen spiegelt sich auch in ihren Werken wider und bildet einen Kontrast zu der oftmals als Strategie der Verschleierung wahrgenommenen Vorgehensweise der machthabenden Akteure Japans: Die Künstler machen ihre Emotionen sichtbar und stellen ihr Wissen und ihre Erfahrungen zur Verfügung. All dies bildet die Basis sowohl für ihre Lebenseinstellung als auch für ihre berufliche Ausrichtung. Diese enge Verbindung zwischen innen und außen, privatem Leben und Öffentlichkeit sowie Person und Umfeld, stellt einen im japanischen Kontext selten auftretenden, sehr engen Bezug zwischen Selbst und Öffentlichkeit her.

Dabei geht es meinen Gesprächspartnern nicht um das Erreichen eines bestimmten politischen Ziels oder um die Mobilisierung möglichst vieler Menschen für eine festgelegte Agenda, sondern um die Erschaffung eines Bewusstseins, das über die persönliche Lebenswirklichkeit hinausreicht und ethisch-moralische Facetten aufweist. Durch die Verbindung von Empathie, Kreativität und Handlungsvermögen lassen sich durchaus Parallelen zu zivilgesellschaftlichem Engagement mit demokratischer Ausrichtung in ihren Aussagen feststellen. Meine Gesprächspartner scheuen jedoch jede Kategorisierung ihres Handelns, auch wenn sie aus meiner Sicht im weitesten Sinne politisch aktiv sind. Sie schätzen ihre subjektiv empfundene Unabhängigkeit im Denken, Fühlen und Handeln und verzichten bewusst auf eine Anbindung an Parteien, Gruppen oder Firmen. Wie aus meinem Interviewmaterial hervorgeht, macht dies für sie den Reiz ihres Lebensstils aus, der gleichzeitig die Qualität ihrer Arbeit sicherstellen soll. Es ist eine Arbeit, die sich um das Erweitern 
der Vorstellungskraft und der Handlungsoptionen der Mitglieder der japanischen Gesellschaft dreht.

Auch wenn Autoren wie die Japanologen Foljanty-Jost und Haufe (2006: 20) angesichts eines „historischen Demokratiedefizits“ Japans davon ausgehen, dass man nicht von einer „Rekonstruktion des Phänomens der japanischen Zivilgesellschaft in seiner ganzen Komplexität" sprechen kann, so hat die Dreifachkatastrophe der Notwendigkeit von Veränderungen in der Denk- und Herangehensweise an gesellschaftliche Herausforderungen eine erhöhte Dringlichkeit verliehen. Eine Dringlichkeit, auf die die Gegenwartskünstler Japans mit ihrer eigenen Lebenskunst antworten. Das Bemühen meiner Gesprächspartner um einen Raum für Emotionsdiskurse nach 2011 kann dazu beitragen, den einzelnen Rezipienten in die Lage zu versetzen, sich auf die Herausforderungen der Zeit nach der Dreifachkatastrophe einzustellen und sich der eigenen Handlungsfähigkeit bewusst zu werden. Im Sinne Hardcastles (2008: 158) verbinden sich in den Werken meiner Gesprächspartner Emotionen, Wahrnehmungen, Erfahrungen und Erinnerungen zu einem Selbst, das sich seiner eigenen Handlungsfähigkeit bewusst ist und entsprechend agiert.

Folglich wird auf gesellschaftlicher Ebene insbesondere die Atomkatastrophe „Fukushima“, um es mit den Worten meines Interviewpartners Kogoro (02.05.2014: 19) auszudrücken, zu einem „Röntgenbild der japanischen Gesellschaft“, durch das die bereits im Vorfeld vorhandenen strukturellen Probleme sichtbar und damit veränderbar geworden sind. Im Jahr 2020, fünf Jahre nachdem ich Japan verlassen hatte, erreichte mich folgende Nachricht von Kogoro auf meine Anfrage nach seiner derzeitigen Einschätzung der Lage:

„I get information about the situation in Fukushima because my mother, who is still living there, tells me. I think the government is making very hasty and dangerous decisions. I feel this way because we are dumping highly contaminated water into the sea and residents have to return to their homes without decontamination [...] Many people who told me their stories have developed cancer. It is still hard for me to see this, but I will continue to do everything I can." (Kogoro 10.05.2020: 1) 



\section{Literaturverzeichnis}

Abu-Lughod, Lila und Catherine Lutz (1990): Introduction: Emotion, Discourse, and the Politics of Everyday Life. In: Catherine Lutz und Lila Abu-Lughod (Hg.): Language and the Politics of Emotion, 1-23. Cambridge: Cambridge University Press.

Asanuma-Brice, Céline (2014): Beyond Reality - or - an Illusory Ideal:

Pro-Nuclear Japan's Management of Migratory Flows in a Nuclear Catastrophe. The Asia-Pacific Journal 12 (47,1): Article ID 4221.

Avenell, Simon A. (2009): Civil Society and the New Civic Movement in Contemporary Japan: Convergence, Collaboration, and Transformation. The Journal of Japanese Studies 35 (2): 247-85.

Bauer, Lars (2013): Hibakusha, Strahlungsopfer - Ein globales Phänomen: Kamanaka Hitomi im Gespräch. In: Lisette Gebhardt und Steffi Richter (Hg.): Lesebuch „Fukushima“: Übersetzungen, Kommentare, Essays, 235-52. Berlin: EBVerlag.

Bauman, Zygmunt (2000): Liquid Modernity. Cambridge: Polity Press.

-. (2008): The Art of Life. Malden: Polity Press.

Beck, Ulrich (1986): Risikogesellschaft: Auf dem Weg in eine andere Moderne. Frankfurt am Main: Suhrkamp.

-. (2015): Weltrisikogesellschaft: Auf der Suche nach der verlorenen Sicherheit.

Frankfurt am Main: Suhrkamp. 
Bernard, H. Russell (2011): Research Methods in Anthropology: Qualitative and Quantitative Approaches. Lanham: AltaMiraPress.

Berndt, Enno (2012): Im strahlenden Schatten der Macht - Zur Politischen Ökonomie der nuklearen Katastrophe von Fukushima. In: Steffi Richter und Lisette Gebhardt (Hg.): Japan nach „Fukushima“: Ein System in der Krise, 9-90. Leipzig: Leipziger Universitätsverlag.

Birkhan, Barbara (2012): Foucaults ethnologischer Blick: Kulturwissenschaft als Kritik der Moderne. Bielefeld: Transcript Verlag.

Bohn, Thomas M., Thomas Feldhoff, Lisette Gebhardt et al. (2015): Preface: Introducing „Fukushima and Chernobyl“. In: Thomas M. Bohn et al. (Hg.): The Impact of Disaster: Social and Cultural Approaches to Fukushima and Chernobyl, 9-16. Berlin: EB-Verlag.

Bourdieu, Pierre (1987): Pierre Bourdieu Die feinen Unterschiede: Kritik der gesellschaftlichen Urteilskraft. Frankfurt am Main: Suhrkamp.

Brandner, Judith (2014): Zuhause in Fukushima - Das Leben danach: Porträts. Wien: K\&S.

Brinton, Mary C. (2010): Social Class and Economic Life Chances in Post-Industrial Japan: The „Lost Generation“. In: Hiroshi Ishida und David H. Slater (Hg.): Social Class in Contemporary Japan: Structures, Sorting and Strategies, 114-33. Milton Park: Routledge.

-. (2011): Lost in Transition: Youth, Work, and Instability in Postindustrial Japan. New York: Cambridge University Press.

Broinowski, Adam (2013): Fukushima: Life and the Transnationality of Radioactive Contamination. Asia-Pacific Journal 11 (41,3): Article ID 4009.

Calhoun, Craig (1992): Introduction: Habermas and the Public Sphere. In: Craig Calhoun (Hg.): Habermas and the Public Sphere, 1-48. Cambridge: MIT Press.

Castells, Manuel (2009): Communication Power. Oxford: Oxford University Press. Caudill, William und Helen Weinstein (1986): Maternal Care and Infant Behavior in Japan and America. In: Takie S. Lebra und William P. Lebra (Hg.): Japanese Culture and Behavior: Selected Readings, 201-46. Honolulu: University of Hawai'i Press.

Chamberlayne, Prue und Joanna Bornat and Tom Wengraf (2000): Introduction: The Biographical Turn. In: Prue Chamberlayne, Joanna Bornat und Tom Wengraf (Hg.): The Turn of Biographical Methods in Social Science: Comparative Issues and Examples, 1-30. Andover: Routledge.

Chong, Dennis und James N. Druckman (2007): Framing Theory. Annual Reviews of Political Science (10): 103-26.

Clancey, Gregory K. (2006): Earthquake Nation: The Cultural Politics of Japanese Seismicity, 1868-1930. Berkeley: University of California Press.

Claußen, Angelika und Alex Rosen (2014): IPPNW-Report: 30 Jahre leben mit Tschernobyl, 5 Jahre mit Fukushima. Gesundheitliche Folgen der Atom- 
katastrophen von Tschernobyl and Fukushima. https://www.ippnw.de/com monFiles/pdfs/Atomenergie/IPPNW_Report_T30_F5_Folgen_web.pdf.

Collier, Stephen J. und Andrew Lakoff (2005): On Regimes of Living. In: Aihwa

Ong und Stephen J. Collier (Hg.): Global Assemblages: Technology, Politics, and Ethics as Anthropological Problems, 22-39. Malden: Blackwell.

Coulmas, Florian (2007): Die Gesellschaft Japans: Arbeit, Familie und demographische Krise. München: Beck.

Coulmas, Florian und Judith Stalpers (2011): Fukushima: Vom Erdbeben zum Super-GAU. München: Beck.

Dahl, Nils (2020): Governing through kodokushi. Japan's Lonely Deaths and their Impact on Community Self-Government. Contemporary Japan 32 (1): 83-102. doi:10.1080/18692729.2019.1680512.

Deutschmann, Christoph (1991): Die japanischen Arbeitszeiten in sozio-kultureller Sicht. In: Deutsches Institut für Japanstudien (Hg.): Japanstudien. Jahrbuch des Deutschen Instituts für Japanstudien: Beiträge zum Thema Zeit, 89-101. München: Iudicium.

Felgentreff, Carsten und Thomas Glade (2008): Naturrisiken - Sozialkatastrophen: zum Geleit. In: Carsten Felgentreff und Thomas Glade (Hg.): Naturrisiken und Sozialkatastrophen, 1-10. Berlin: Spektrum Akademischer Verlag.

Fischer-Rosenthal, Wolfram (2000): Biographical Work and Biographical Structuring in Present-Day Society. In: Prue Chamberlayne, Joanna Bornat und Tom Wengraf (Hg.): The Turn of Biographical Methods in Social Science: Comparative Issues and Examples, 109-25. Andover: Routledge.

Foljanty-Jost, Gesinde und Karoline Haufe (2006): Bürgerliche Gesellschaft versus Zivilgesellschaft - die neuere Debatte in Japan. Arbeitspapiere des Institutes für Politikwissenschaft und Japanologie 1 (1). http://nbn-resolving.de/urn: nbn:de:gbv:3:2-2844.

Formanek, Susanne und William R. LaFleur (2004): Introduction. In: Susanne Formanek und William R. LaFleur (Hg.): Practicing the Afterlife: Perspectives from Japan, 7-48. Wien: Verlag der Österreichischen Akademie der Wissenschaften.

Foucault, Michel (1973): Archäologie des Wissens. Frankfurt am Main: Suhrkamp. - . (1974): Die Ordnung des Diskurses. Frankfurt am Main: Fischer.

Friedman, Jonathan (2001): The Iron Cage of Creativity: An Exploration. In: John Liep (Hg.): Locating Cultural Creativity, 46-61. London: Pluto Press.

Frijda, Nico H. und Batja Mesquita (1994): The Social Roles and Functions of Emotions. In: Shinobu Kitayama und Hazel R. Markus (Hg.): Emotion and Culture: Empirical Studies of Mutual Influence, 51-87. Washington, DC: American Psychology Association.

Funabashi, Harutoshi (2012): Why the Fukushima Nuclear Disaster Is a ManMade Calamity. International Journal of Japanese Sociology 21 (1): 65-75. doi:10.1111/j.1475-6781.2012.01161.x. 
Furukawa, Hiroko and Ranya Denison (2015): Disaster and Relief: The 3.11 Toho$\mathrm{ku}$ and Fukushima Disasters and Japan's Media Industries. International Journal of Cultural Studies 18 (2): 225-41. doi:10.1177/1367877914559301.

Ganseforth, Sonja (2020): The Difference between Zero and One: Voices from the

Tokyo Anti-Olympic Movements. In: Barbara G. Holthus et al. (Hg.): Japan through the Lens of the Tokyo Olympics, 110-15. New York: Routledge.

Gebhardt, Lisette (2012): „Ich habe die ganze Zeit darauf gewartet, dass dieser

Tag kommen möge“: Positionen japanischer Autoren nach „Fukushima“.

In: Steffi Richter und Lisette Gebhardt (Hg.): Japan nach „Fukushima“" Ein

System in der Krise, 171-206. Leipzig: Leipziger Universitätsverlag.

-_. (2013): Der Mut zur Meinung - Eine Miniaturskizze der kritischen Öffent-

lichkeit. In: Lisette Gebhardt und Steffi Richter (Hg.): Lesebuch „Fukushima": Übersetzungen, Kommentare, Essays, 313-25. Berlin: EBVerlag.

- - (2015): Japanese Literature After Fukushima: Between Protest and 'Healing'.

In: Thomas M. Bohn et al. (Hg.): The Impact of Disaster: Social and Cultural Approaches to Fukushima and Chernobyl, 227-60. Berlin: EB-Verlag.

Gebhardt, Lisette und Steffi Richter (2013): Vorwort. In: Lisette Gebhardt und Steffi Richter (Hg.): Lesebuch „Fukushima“: Übersetzungen, Kommentare, Essays, 9-11. Berlin: EBVerlag.

Geertz, Clifford (1968): Thinking as a Moral Act: Ethical Dimensions of Anthropological Fieldwork in the New States. The Antioch Review 28 (2): 34-59. doi: $10.2307 / 4610913$.

-.. (1983): Local Knowledge: Further Essays in Interpretative Anthropology. New York: Basic Books.

Glaser, Barney G., Anselm L. Strauss und Axel T. Paul (1972): Grounded Theory: Strategien qualitativer Forschung. Bern: Huber.

Götz, Irene und Barbara Lemberger (Hg.) (2009): Prekär arbeiten, prekär leben: Kulturwissenschaftliche Perspektiven auf ein gesellschaftliches Phänomen. Frankfurt am Main: Campus.

Grimmig, Wiebke (2012): Die Identität der Geisha: Transformationsprozesse bis ins 21. Jahrhundert. Magisterarbeit. Georg-August-Universität Göttingen.

Habermas, Jürgen (1989): The Structural Transformation of the Public Sphere: An Inquiry into a Category of Bourgeois Society. Cambridge: MIT Press.

Halpern, Jodi (2001): From Detached Concern to Empathy: Humanizing Medical Practice. New York: Oxford University Press.

Hardcastle, Valerie G. (2008): Constructing the Self. Amsterdam: John Benjamins Publishing Company.

Hasegawa, Arifumi, Koichi Tanigawa, Akira Ohtsuru et al. (2015): Health Effects of Radiation and Other Health Problems in the Aftermath of Nuclear Accidents, with an Emphasis on Fukushima. The Lancet 386 (9992): 479-88. doi:10.1016/S0140-6736(15)61106-0. 
Hastrup, Kristen (2001): Othello's Dance: Cultural Creativity and Human Agency. In: John Liep (Hg.): Locating Cultural Creativity, 31-45. London: Pluto Press.

Havens, Thomas R. H. (1982): Artist and Patron in Postwar Japan: Dance, Music, Theater, and the Visual Arts, 1955-1980. New Jersey: Princeton University Press.

Heine, Steven J., Darrin R. Lehman, Hazel R. Markus und Shinobu Kitayama (1999): Is There a Universal Need for Positive Self-Regard?. Psychological Review 106 (4): 766-94. doi:10.1037/0033-295X.106.4.766.

Hendry, Joy (1992): Individualism and Individuality: Entry into a Social World. In: Roger Goodman und Kirsten Refsing (Hg.): Ideology and Practice in Modern Japan, 55-71. London: Routledge.

Hermann, Elfriede (1995): Emotionen und Historizität: Der emotionale Diskurs über die Yali-Bewegung in einer Dorfgemeinschaft der Ngaing, Papua New Guinea. Berlin: Reimer.

-. (2011): Empathy, Ethnicity, and the Self Among the Banabans in Fiji. In: Douglas W. Hollan und C. J. Throop (Hg.): The Anthropology of Empathy: Experiencing the Lives of Others in Pacific Societies, 25-41. New York: Berghahn Books.

Hermann, Elfriede und Birgitt Rötter-Rössler (2003): Einleitung: Persönliche Handlungsmöglichkeiten im lokal-globalen Kontext. In: Elfriede Hermann und Birgitt Röttger-Rössler (Hg.): Lebenswege im Spannungsfeld lokaler und globaler Prozesse: Person, Selbst und Emotion in der ethnologischen Biografieforschung, 1-24. Munster: Lit Verlag.

Hobson, Christopher (2014): Life after the Triple Disaster: Human Security and the Future. In: Paul Bacon und Christopher Hobson (Hg.): Human Security and Japan's Triple Disaster: Responding to the 2011 Earthquake, Tsunami and Fukushima Nuclear Crisis, 198-207. London: Routledge.

Hollan, Douglas W. and C. Jason Throop (2011): The Anthropology of Empathy: Introduction. In: Douglas W. Hollan und C. J. Throop (Hg.): The Anthropology of Empathy: Experiencing the Lives of Others in Pacific Societies, 1-24. New York: Berghahn Books.

Hollway, Wendy und Tony Jefferson (2000): Biography, Anxiety and the Experience of Locality. In: Prue Chamberlayne, Joanna Bornat und Tom Wengraf (Hg.): The Turn of Biographical Methods in Social Science: Comparative Issues and Examples, 167-80. Andover: Routledge.

Hommerich, Carola (2014): Feeling Disconnected: Exploring the Relationship Between Different Forms of Social Capital and Civic Engagement in Japan. Working Paper: Deutsches Institut für Japanstudien 14 (4): 1-29. https:// www.dijtokyo.org/publications/WP1404_Hommerich.pdf.

Hornbacher, Annette (2013): Von der Naturkatastrophe zur Modernisierungskrise? Ein ethnologischer Blick auf kulturspezifische Varianten im Umgang mit Erdbeben und Tsunamis. In: Carla Meyer, Gerrit J. Schenk und Katja Patzel- 
Mattern (Hg.): Krisengeschichte(n): „Krise“ als Leitbegriff und Erzählmuster in kulturwissenschaftlicher Perspektive, 97-116. Stuttgart: Franz Steiner Verlag. Ishida, Hiroshi (2010): Does Class matter? Demographies of Class Structure and Class Mobility from a Comparative Perspective. In: Hiroshi Ishida und David H. Slater (Hg.): Social Class in Contemporary Japan: Structures, Sorting and Strategies, 33-56. Milton Park: Routledge.

Ishida, Hiroshi und David H. Slater (2010): Social Class in Japan. In: Hiroshi Ishida und David H. Slater (Hg.): Social Class in Contemporary Japan: Structures, Sorting and Strategies, 1-30. Milton Park: Routledge.

Ishikawa, Satomi (2005): Seeking the self: Individualism and Popular Culture in Japan. Bern: European Academic Publishers.

John, Franziska (2013): Tsuchida Takashi und die Problematik der Gefälligkeitsgelehrten. In: Lisette Gebhardt und Steffi Richter (Hg.): Lesebuch „Fukushima": Übersetzungen, Kommentare, Essays, 70-77. Berlin: EBVerlag.

Jørgensen, Marianne und Louise Phillips (2002): Discourse Analysis as Theory and Method. London: Sage.

Kariya, Takehiko (2010): From Credential Society to „Learning Capital“ Society - A Rearticulation of Class Formation in Japanse Education and Society. In: Hiroshi Ishida und David H. Slater (Hg.): Social Class in Contemporary Japan: Structures, Sorting and Strategies, 87-113. Milton Park: Routledge.

Kimura, Shuhei (2012): Lessons from the Great East Japan Earthquake: the Public Use of Anthropological Knowledge. Asian Anthropology 11 (1): 65-74. doi:10.1080/1683478X.2012.10600856.

Kitayama, Shinobu und Hazel R. Markus (1994): Introduction to Cultural Psychology and Emotion Research. In: Shinobu Kitayama und Hazel R. Markus (Hg.): Emotion and Culture: Empirical Studies of Mutual Influence, 1-22. Washington, DC: American Psychology Association.

Kobayashi, Yasuo (2015): Disaster and Philosophy. In: Thomas M. Bohn et al. (Hg.): The Impact of Disaster: Social and Cultural Approaches to Fukushima and Chernobyl, 19-24. Berlin: EB-Verlag.

Kurokawa, Kiyoshi (2012): Message from the Chairman. The Official Report of the Fukushima Nuclear Accident Independent Investigation Commission - Executive Summary, 9. https://cjwalsh.ie/wp-content/uploads/2012/07/Fukushima -NAIIC-Executive-SummaryEnglish_2012.pdf.

Kuryluk, Ewa (1994): A Plea for Irresponsibility. In: Carol Becker (Hg.): The Subversive Imagination: Artists, society, and Social Responsibility, 13-19. Oxfordshire: Routledge.

Laidlaw, James (2010): Agency and Resposibility: Perhaps You Can Have Too Much of a Good Thing. In: Michael J. Lambek (Hg.): Ordinary Ethics: Anthropology, Language, and Action, 143-64. New York: Fordham University Press. 
Lambek, Michael (2010): Introduction. In: Michael J. Lambek (Hg.): Ordinary Ethics: Anthropology, Language, and Action, 1-38. New York: Fordham University Press.

Lebra, Takie S. (1976): Japanese Patterns of Behavior. Honolulu: University of Hawaii Press.

-. (1984): Nonconfrontational Strategies for Management of Interpersonal Conflicts. In: Ellis S. Krauss, Thomas P. Rohlen und Patricia Steinhoff (Hg.): Conflict in Japan, 41-60. Honolulu: University of Hawai i Press.

—. (2010): Shame and Guilt in Japan. In: Robert A. LeVine (Hg.): Psychological Anthropology: A Reader on Self in Culture, 102-11. Chichester: Wiley-Blackwell.

Lebra, Takie S. und William P. Lebra (Hg.) (1974): Japanese Culture and Behavior: Selected readings. Honolulu: University of Hawai'i Press.

Lepowsky, Maria (2011): The Boundaries of Personhood, the Problem of Empathy, and the „Natives Point of View“ in the Outer Islands. In: Douglas W. Hollan und C. J. Throop (Hg.): The Anthropology of Empathy: Experiencing the Lives of Others in Pacific Societies, 43-68. New York: Berghahn Books.

Leser, Julia und Maria Trunk (2013): Radioactivists - Wurzeln und Dynamiken von Protest und Dissidenz in Japan seit Fukushima. In: Lisette Gebhardt und Steffi Richter (Hg.): Lesebuch „Fukushima“: Übersetzungen, Kommentare, Essays, 337-48. Berlin: EBVerlag.

Liep, John (2001): Introduction. In: John Liep (Hg.): Locating Cultural Creativity, 1-14. London: Pluto Press.

Liscutin, Nicola (2012): Vernetzt Euch! Fukushima, Neue Medien und die AntiAKW-Bewegung. In: Steffi Richter und Lisette Gebhardt (Hg.): Japan nach „Fukushima“: Ein System in der Krise, 135-70. Leipzig: Leipziger Universitätsverlag.

Lohmann, Roger I. (2011): Empathetic Perception and Imagination among the Asabano: Lessons for Anthropology. In: Douglas W. Hollan und C. J. Throop (Hg.): The Anthropology of Empathy: Experiencing the Lives of Others in Pacific Societies, 95-118. New York: Berghahn Books.

Lutz, Catherine (1988): Unnatural Emotions: Everyday Sentiments on a Micronesian Atoll and Their Challenge to Western Theory. Chicago: University of Chicago Press.

Lutz, Catherine und Lila Abu-Lughod (Hg.) (1990): Language and the Politics of Emotion. Cambridge: Cambridge University Press.

MacLachlan, Patricia L. (2008): Review of „Site Fights: Divisive Facilities and Civil Society and Japan and the West". The Journal of Japanese Studies 35 (2): 479-83. doi:10.1353/jjs.0.0098.

Mageo, Jeannette M. und Bruce M. Knauft (2002): Introduction: Theorizing Power and the Self. In: Jeannette M. Mageo (Hg.): Power and the Self, 1-28. Cambridge: Cambridge University Press. 
Marcuse, Herbert (1978): The Aesthetic Dimension: Towards a Critique of Marxist Aesthetics. Boston: Beacon Press.

Markus, Hazel R. und Shinobu Kitayama (1994a): The Cultural Contruction of Self and Emotion: Implications for Social Behavior. In: Shinobu Kitayama und Hazel R. Markus (Hg.): Emotion and Culture: Empirical Studies of Mutual Influence, 89-132. Washington, DC: American Psychology Association.

- - (1994b): The Cultural Shaping of Emotion: A Conceptual Framework. In: Shinobu Kitayama und Hazel R. Markus (Hg.): Emotion and Culture: Empirical Studies of Mutual Influence, 339-51. Washington, DC: American Psychology Association.

--. (1991): Culture and the Self: Implications for Cognition, Emotion, and Motivation. Psychological Review 98 (2): 224-53. doi:10.1037/0033-295X.9 8.2.224.

Mathews, Gordon (2000): What in the World is Japanese? On the Cultural Identities of Kotoists, Calligraphers, Bebop Pianists, and Punk Rockers. In: Gordon Mathews (Hg.): Global Culture - Individual Identity: Searching for Home in the Cultural Supermarket, 30-75. London: Routledge.

Mathews, Gordon und Bruce White (2004): Introduction: Changing Generations in Japan Today. In: Gordon Mathews und Bruce White (Hg.): Japan's Changing Generations: Are Young People Creating a New Society? 1-12. London: Routledge.

Matsumoto, David R. (1996): Unmasking Japan: Myths and Realities About the Emotions of the Japanese. Stanford: Stanford University Press.

Maynard, Senko K. (2005): Expressive Japanese: A Reference Guide to Sharing Emotion and Empathy. Honolulu: University of Hawai'i Press.

Mills, Charles W. (1959): The Sociological Imagination. Oxford: Oxford University Press.

Moeran, Brian (1986): Individual, Group and Seishin: Japan's Internal Cultural Debate. In: Takie S. Lebra und William P. Lebra (Hg.): Japanese Culture and Behavior: Selected readings, 62-79. Honolulu: University of Hawai'i Press.

Mundt, Lisa (2013): Ungehorsam ist die erste Künstlerpflicht - „Fukushima“ als Zäsur in der zeitgenössischen japanischen Theater- und Performanceszene. In: Lisette Gebhardt und Steffi Richter (Hg.): Lesebuch „Fukushima“: Übersetzungen, Kommentare, Essays, 101-25. Berlin: EBVerlag.

Nünning, Ansgar (2013): Krise als Erzählung und Metapher: Literaturwissenschaftliche Bausteine für eine Metaphorologie und Narratologie von Krisen. In: Carla Meyer, Gerrit J. Schenk und Katja Patzel-Mattern (Hg.): Krisengeschichte(n): "Krise" als Leitbegriff und Erzählmuster in kulturwissenschaftlicher Perspektive, 117-44. Stuttgart: Franz Steiner Verlag.

Obinger, Julia (2009): Working Poor in Japan: „Atypische“ Beschäftigungsformen im aktuellen Diskurs. In: Irene Götz und Barbara Lemberger (Hg.): Prekär arbeiten, prekär leben: Kulturwissenschaftliche Perspektiven auf ein gesellschaftliches Phänomen, 163-83. Frankfurt am Main: Campus. 
Okano, Kaori und Motonori Tsuchiya (1999): Education in Contemporary Japan: Inequality and Diversity. Cambridge: Cambridge University Press.

Oliver-Smith, Anthony (1996): Anthropological Research on Hazards and Disasters. Annual Review of Anthropology 25: 303-28. doi:10.1146/annurev. anthro.25.1.303.

Oliver-Smith, Anthony (2002): Theorizing Disasters. Nature, Power, and Culture. In: Susanna M. Hoffman und Anthony Oliver-Smith (Hg.): Catastrophe and Culture: The Anthropology of Disaster, 23-48. Santa Fe: School of American research press.

Oliver-Smith, Anthony und Susanna M. Hoffman (2002): Introduction: Why Anthropologists Should Study Disaster. In: Susanna M. Hoffman und Anthony Oliver-Smith (Hg.): Catastrophe and Culture: The Anthropology of Disaster, 3-22. Santa Fe: School of American research press.

Ong, Aihwa und Stephen J. Collier (Hg.) (2005): Global Assemblages: Technology, Politics, and Ethics as Anthropological Problems. Malden: Blackwell.

Ortner, Sherry B. (2006): Anthropology and Social Theory: Culture, Power, and the Acting Subject. Durham: Duke University Press.

Otomo, Yoshihide (2011): The Role of Culture: After the Earthquake and ManMade Disasters in Fukushima. Lecture at the Tokyo University of Arts on April 28, 2011. http://www.japanimprov.com/yotomo/fukushima/lecture. html.

Pharr, Susan J. (1984): Status Conflict: The Rebellion of the Tea Pourers. In: Ellis S. Krauss, Thomas P. Rohlen und Patricia Steinhoff (Hg.): Conflict in Japan, 214-40. Honolulu: University of Hawai'i Press.

Pilling, David (2014): Bending Adversity: Japan and the Art of Survival. London: Penguin Books.

Pye, Michael und Katja Triplett (Hg.) (2007): Streben nach Glück: Schicksalsdeutung und Lebensgestaltung in japanischen Religionen. Münster: Lit.

Repp, Martin (2015): Einführung zur Konferenz in Arnoldshain. In: Dokumentation der Konferenz „Die ,Energiewende“ vom Atomausstieg zu erneuerbarer Energie - Was können religiöse Gruppen zum Klimaschutz beitragen?", veranstaltet vom Zentrum für Ökumene. Frankfurt. https://doam.org/projekte/ veranstaltungen/konferenzen/2015/2015-atomausstieg/2973-einfuehrung-in -die-konferenmz.

Richter, Steffi (2012): Das Ende des „endlosen Alltags“? Post-Fukushima als JapanDiskurs. In: Steffi Richter und Lisette Gebhardt (Hg.): Japan nach „Fukushima": Ein System in der Krise, 91-134. Leipzig: Leipziger Universitätsverlag.

Richter, Steffi (2015): Comparing Fukushima and Chernobyl: Social and Cultural Dimensions of the Two Nuclear Catastrophes. In: Thomas M. Bohn et al. (Hg.): The Impact of Disaster: Social and Cultural Approaches to Fukushima and Chernobyl, 51-66. Berlin: EB-Verlag. 
Rosaldo, Michelle (1984): Toward an Anthropology of Self and Feeling. In: Richard A. Shweder und Robert A. LeVine (Hg.): Culture Theory: Essays on Mind, Self, and Emotion, 137-58. New York: Cambridge University Press.

Rosen, Alex (2012): Gesundheitliche Auswirkungen der Atomkatastrophe von Fukushima. http://www.ippnw.de/commonFiles/pdfs/Atomenergie/Gesund heitliche_Auswirkungen_der_Atomkatastrophe_von_Fukushima_final.pdf. Rosenthal, Gabriele (2005): Interpretative Sozialforschung: Eine Einführung. Weinheim: Beltz Juventa.

Rosler, Martha (1994): Place, Position, Power, Politics. In: Carol Becker (Hg.): The Subversive Imagination: Artists, society, and Social Responsibility, 55-76. Oxfordshire: Routledge.

Rotermund, Hartmut O. (2004): The Afterlife in Meiji-Period Sermons. In: Susanne Formanek und William R. LaFleur (Hg.): Practicing the Afterlife: Perspectives from Japan, 365-82. Wien: Verlag der Österreichischen Akademie der Wissenschaften.

Röttger-Rössler, Birgitt (2004): Die kulturelle Modellierung des Gefühls: Ein Beitrag zur Theorie und Methodik ethnologischer Emotionsforschung anhand indonesischer Fallstudien. Hamburg: Lit.

Rustin, Michael (2000): Reflections on the Biographical Turn in Social Science. In: Prue Chamberlayne, Joanna Bornat und Tom Wengraf (Hg.): The Turn of Biographical Methods in Social Science: Comparative Issues and Examples, 33-52. Andover: Routledge.

Sakurai, Tetsuo (2004): The Generation Gap in Japanese Society Since the 1960s. In: Gordon Mathews und Bruce White (Hg.): Japan's Changing Generations: Are Young People Creating a New Society? 15-30. London: Routledge.

Salamon, Sonya (1986): „Male Chauvinism“ as a Manifestation of Love in Marriage. In: Takie S. Lebra und William P. Lebra (Hg.): Japanese Culture and Behavior: Selected readings, 130-41. Honolulu: University of Hawai'i Press.

Samuels, Richard J. (2013): 3.11: Disaster Response and Political Change in Japan. Ithaca: Cornell University Press.

Sarasin, Philipp (2006): Michel Foucault zur Einführung. Hamburg: Junius.

Satō, Dōshin (2011): Modern Japanese Art and Meiji State: The Politics of Beauty. Los Angeles: Getty Research Institute.

Schad-Seifert, Annette (2007): Japans Abschied von der Mittelschichtsgesellschaft: Auflösung des Familienhaushalts oder Pluralisierung der Lebensformen?. In: Peter Backhaus (Hg.): Familienangelegenheiten, 105-28. München: Iudicium.

Schenk, Gerrit J. (2017): Historical Disaster Experiences: First Steps Toward a Comparative and Transcultural History of Disasters Across Asia and Europe in the Preindustrial Era. In: Gerrit J. Schenk (Hg.): Historical Disaster Experiences: Towards a Comparative and Transcultural History of Disasters Across Asia and Europe, 3-44. Cham: Springer. 
Seifert, Manfred (2009): Prekarisierung der Arbeits- und Lebenswelt: Kulturwissenschaftliche Reflexionen zu Karriere und Potenzial eines Interpretationsansatzes. In: Irene Götz und Barbara Lemberger (Hg.): Prekär arbeiten, prekär leben: Kulturwissenschaftliche Perspektiven auf ein gesellschaftliches Phänomen, 31-55. Frankfurt am Main: Campus.

Slater, David H. (2010): The „New Working Class“ of Urban Japan: Socialisation and Contradiction from Middle School to the Labor Market. In: Hiroshi Ishida und David H. Slater (Hg.): Social Class in Contemporary Japan: Structures, Sorting and Strategies, 137-69. Milton Park: Routledge.

Spradley, James P. (1979): The Ethnographic Interview. Belmont: Wadsworth Group, Thomson Learning.

Stafford, Charles (2010): The Punishment of Ethnical Behaviour. In: Michael J. Lambek (Hg.): Ordinary Ethics: Anthropology, Language, and Action, 187206. New York: Fordham University Press.

Steineck, Raji C. (2015): Nuclear Power: A Techno-Ethical Perspective. In: Thomas M. Bohn et al. (Hg.): The Impact of Disaster: Social and Cultural Approaches to Fukushima and Chernobyl, 25-50. Berlin: EB-Verlag.

Steinhoff, Patricia (1984): Student Conflict. In: Ellis S. Krauss, Thomas P. Rohlen und Patricia Steinhoff (Hg.): Conflict in Japan, 174-213. Honolulu: University of Hawai'i Press.

—. (2013): Memories of New Left Protest. Contemporary Japan 25 (2): 127-65. doi:10.1515/cj-2013-0007.

Stekelenburg, Jacquelien van und Bert Klandermans (2010): The Social Psychology of Protest. Current Sociology 61 (5-6): 886-905. doi:10.1177/0011392113479314.

Stephens, Sharon (2002): Bounding Uncertainty: The Post-Chernobyl Culture of Radiation Protection Experts. In: Susanna M. Hoffman und Anthony Oliver-Smith (Hg.): Catastrophe and Culture: The Anthropology of Disaster, 91-111. Santa Fe: School of American research press.

Swann, William B. (1983): Self-Verification: Bringing Social Reality into Harmony with the Self. Psychological Perspectives on the Self 2: 33-66.

Swann, William B. und Craig A. Hill (1982): When Our Identities Are Mistaken: Reaffirming Self-Conceptions Through Social Interaction. Journal of Personality and Social Psychology 43 (1): 59-66. doi:10.1037/0022-3514.43.1.59.

Takamine, Tadasu (2014): Was ist ein Betroffener?. In: HAU Hebbel am Ufer (Hg.): Japan-Syndrome - Kunst und Politik nach Fukushima, 22-23. Berlin. https://www.hebbel-am-ufer.de/fileadmin/Hau/HAU3000/Publikationen/ JAPAN_ISSUE.pdf.

Takeuchi, Melinda (2004): Introduction. In: Melinda Takeuchi (Hg.): The Artist as Professional in Japan, 1-16. Stanford: Stanford University Press.

Taylor, Charles (1989): Sources of the Self: The Making of the Modern Identity. Cambridge: Harvard University Press. 
Twine, Fred (1994): Citizenship and Social Rights: The Interdependence of Self and Society. London: Sage.

UNSCEAR (United Nations Scientific Committee on the Effects of Atomic Radiation) (2015): Developments since the 2013 UNSCEAR Report on the Levels and Effects of Radiation Exposure due to the Nuclear Accident following the Great-East Japan Earthquake and Tsunami. https://www.uns cear.org/docs/publications/2015/UNSCEAR_WP_2015.pdf.

Volkmann, Ute (2007): Das schwierige Leben in der „Zweiten Moderne“ - Ulrich Becks „Risikogesellschaft“. In: Uwe Schimank und Ute Volkmann (Hg.): Soziologische Gegenwartsdiagnosen, 23-40. Wiesbaden: VS Verlag für Sozialwissenschaften.

Vosse, Wilhelm (1999): The Emergence of Civil Society in Japan. In: Ines Günther, Susanne Kreitz-Sandberg und Johann Nawrocki (Hg.): Japan im 21. Jahrhundert: Zivilgesellschaft und Staat in der postindustriellen Moderne, 31-53. München: Iudicium.

White, Geoffrey M. (1990): Moral Discourse and the Rhetoric of Emotions. In: Catherine Lutz und Lila Abu-Lughod (Hg.): Language and the Politics of Emotion, 46-68. Cambridge: Cambridge University Press.

- . (1994): Affecting Culture: Emotion and Morality in Everyday Life. In: Shinobu Kitayama und Hazel R. Markus (Hg.): Emotion and Culture: Empirical Studies of Mutual Influence, 219-40. Washington, DC: American Psychology Association.

Wiemann, Anna (2017): Networks and mobilization processes: The Case of the Japanese Anti-Nuclear Movement after Fukushima. München: Iudicium.

Zwick, Michael M. und Ortwin Renn (2008): Risikokonzepte jenseits von Eintrittswahrscheinlichkeit und Schadenserwartung. In: Carsten Felgentreff und Thomas Glade (Hg.): Naturrisiken und Sozialkatastrophen, 77-98. Berlin: Spektrum Akademischer Verlag. 
De er „11. März 2011“ oder „Fukushima“ wurde zu einem Synonym für eine Katastrophe und Unsicherheit über mögliche Folgen in der ganzen Welt. Ausgehend von der Frage nach der persönlichen und gesellschaftlichen Bedeutung der Ereignisse untersucht Wiebke Grimmig die Rolle der Gegenwartskünstler bei der Verarbeitung der Dreifachkatastrophe (Erdbeben, Tsunami und atomaren GAU) in Japan. Sie setzt ihren Fokus auf die emotionale Verarbeitung der Katastrophe durch ihre Gesprächspartner und bezieht deren biographischen Hintergrund, die Rolle der Gegenwartskunst in Japan und wirtschaftspolitische Interessen mit in ihre Betrachtung ein. Durch den ethnologischen Zugang einer 12-monatigen Feldforschung in Tokyo sowie in den unmittelbar betroffenen Gebieten der japanischen Ostküste und unter Einbezug der Tiefenanalyse narrativer Interviews gewinnt sie ein vielschichtiges Verständnis der Ereignisse und ihrer Folgen. Die Arbeit liefert mit Beschreibungen und Erläuterungen zu gesellschaftlichen Themen wie Emotion, Individualität, Macht, Resilienz, Politik, Wirtschaft und Kommunikation einen wichtigen Beitrag zur sozialwissenschaftlichen Katastrophenforschung.

Wiebke Grimmig ist Ethnologin mit einem regionalen Schwerpunkt auf Japan und einem besonderen Interesse an Emotionsund Biographieforschung im Kontext von Katastrophen. Sie studierte Ethnologie, Geschlechterforschung und Sozialpolitik an der Georg- August-Universität Göttingen, wo sie mit der vorliegenden Arbeit ihre Promotion abschloss. Während ihrer einjährigen Forschungsphase in Tokio wurde sie vom Deutschen Institut für Japanstudien (DIJ) gefördert.

ISBN: 978-3-86395-518-2

ISSN: $2199-5346$

eISSN: 2512-6881

\section{Universitätsverlag Göttingen}

